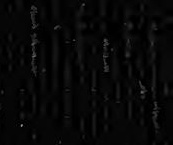







BS Bible, N.T. creek. 1887

\section{0.}

\section{PU PUCASE MOMET}

PU

\section{HESAYECV CESVS KLIST}

EN TESTEMENT MUCVSAT.

KLEK VLKE EM PUWVKV

MV OFV ENHVTECESKV COHOYVTE AOSSEN TOHTVLECICVHOTET OS.

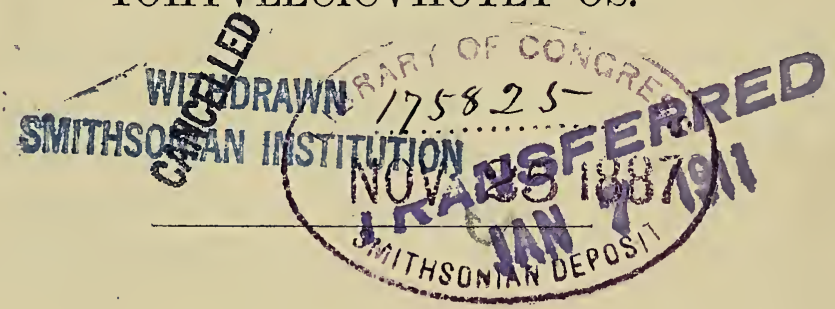

NEW YORK :

AMERICAN BIBLE SOCIETY, INSTITUTED IN THE YEAR MDCCCXVI.

$188 \%$

[. Thesskokee, New Testament.] 


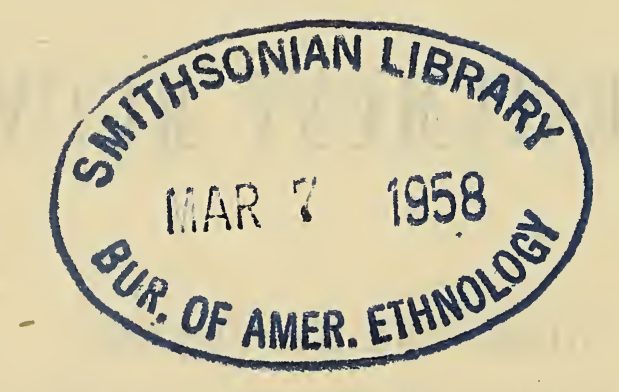

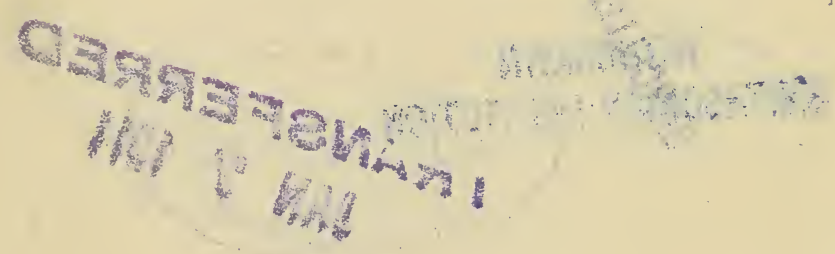


Setemwarke.

Setemwarke.

MARO • . . . . . $28 \mid$ Temore I. . . . . 6

Mak . . . . . . 16 Temore II. . . . . 4

Luk . . . . . . 24 Filemvn . . . . . 1

Cane . . . . . . 21 Titvs . . . . . . 3

Vpastelvlke em fulletv 28 Hepluvlke . . . . . 13

Lomvlke . . . . 16 Cems . . . . . . 5

Kvlenrvlke I. . . . 16 Cane I. . . . . . . 5

Kvlenrvlke II. . . . 13 Cane II. . . . . . 1

Kelesvlke • . . . 16 Cane III. . . . . . 1

Efesvnvlke . . . . 6 Petv I. . . . . . 5

Felepvlke . . . . . 4 Petv II. . . . . . 3

Kolasvlke . . . . . 4 Cutvse . . . . . . 1

Resvlonikvlke I. • . 5 Lefelesvn . . • . . 22

Resvlonikvlke II. . . 3 



\section{O P U N V K V - HER V \\ MARO COYVTE.}

\section{SETENTVCKE I.}

Heyv nakcokv Tewe e'ppuce, Eplehame e'ppuce, Cesvs Klist heckvrane vtecicvtet os.

2 Eplehame Iseken en heckuehocrtes; momen Iseke Cekvpen en heckuehocvtes; momen Cekvpe Cutvn en heckuehocvtes, momen etecakkakateu ;

' 3 Momen Cutv Felesen hvtvm Selvn Temvt en heckuecrtes; momen Felese Eslvmen en heckuehocrtes; momen Eslvme Elvmen en heckuehocvtes;

4 Momen Elvme Vmenetapen en heckuehocvtes; momen Vmenetape Neasvn en heckuehocrtes; momen Neasv Salmvn en heckuehocvtes;

5 Momen Salmv Poosen Lekapet en heckuecrtes; momen Poose Open Luret en heckuecrtes; momen Ope Crsen en heckuehocrtes;

6 Momen Cvse Tewe mekkon en heckuehocrtes; momen Tewe mekko Salomvn Yuliv e'hiwv tatet en heckuecrtes;

7 Momen Salomv Lopoen en heckuehocrtes; momen Lopoe Epivn en heckuehocrtes; momen Epiv Esen en heckuehocvtes ;

8 Momen Ese Cosefaten en heckuehocvtes; momen Cosefate Colvmen en heckuehocvtes; momen Colvme Osivn en heckuehocvtes;

9 Momen Osiv Corvmen en heckuehocrtes; momen 
Corvme . .asen en heckuehocvtes; momen Ekase Esekivn en heckuehocvtes;

10 Momen Esekiv Menasen en heckuehocvtes; momen Menase Emvnen en heckuehocvtes; momen Emvne Cosivn en heckuehocvtes;

11 Momen Papelvn svpehoyofv mahen Cosiv Cekonivn en heckuehocvtes, momet etecakkakateu :

12 Momen Papelvn resorihohcofvn, Cekoniv Svlatevn en heckuehocvtes; momen Svlatev Solopepen en heckuehocvtes :

13 Momen Solopepe Epivten en heckuehocvtes; momen Epivte Elieken en heckuehocvtes; momen Elieke Esvn en heckuehocvtes;

14 Momen Esv Setvken en heckuehocrtes ; momen Setvke Ekemen en heckuehocvtes; momen Ekeme Elivten en heckuehocvtes;

15 Momen Elivte Eleasvn en heckuehocrtes; momen Eleasv Marrvn en heckuehocvtes ; momen Marrv Cekvpen en heckuehocvtes;

16 Momen Cekvpe Cose, Mele e'hen, en heckuehocvtes ; momen Mele aossen Cesvs, mv Klist kihocat, heckvtes.

17 Monkv Eplehame vteke Tewe erorat honvpse omvlkat palen-ostohkakes; momen Tewe vteke Papelvn svpehoyvte erorat honvpse palen-ostohkakes; momen Papelvn svpehoyvte vteke Klist erorat honvpse palenostohkakes.

18 Momen Cesvs Klist heckvte hiyomēt omvtes : E'cke Mele Cose temfvecet ehiwvranet omof, tohvhoyeko munken, Puyvfekev Vcakat enaken es nvrkese tates.

19 Mohmen Cose, hokte e'he este fvcevt omet, Vlesketv en hayvkot, ehkusen. wikares, komvtes.

20 Momis heyv nanvken vkerricof, hecrs, Pucase em encelvt vpueckv ofvn em e heckuecet, Cose, Tewe e'p- 
puce toyetskat, ce 'hiwv Mele evpryetv ce penkahlats, mv ofv hocackat Puyvfekcv Vcakat enaket okv.

21 Momen cepvnusen heckuecvres, momen CESVN kicet hocefetskvies; em estvlken en holwryeckv enkvpvken hesahuecvret okv, kicvtes.

22 Momen heyv omvlkv hiyomeçat Pucase owalv en kerkuecen nake onayvtet esfvckvranaten omvtes, hvmmaket,

23 Hecvs, fvcenvt nvrkehset, cepvnusen heckuecvres, momen e hocefkv EMANYUEL kicvkvres, mv ohyvtekhoyat, Hesaketvmese epu'pakat, maketvt omes.

24 Momof Cose nocvten ahuyiret, Pucase em encelvt em punayvten momvtes, momet e 'hiwrn evpayvtes:

25 Momen, kerreko emunken, cepvnuse hvteceskvn heckuecvtes; momen e hocefkv CESVN kicvtes.

\section{SETENTVCKE II.}

Momen Helot mekko like nettv take ofvn, Cesvs Perehem Cutev ofv heckof, hecvs, este hoporrenvlke hvsossvfvecv awet Celuslvmen yicvtes,

2 Estvn Cusvlke em mekko heckat like haks? en kococumpv hvsossv-fvecrn heceyvtet, vrakkuecvranet yicēkv, makaket.

3 Momen, Helot mekko pohat, Celuslvmvlke omvlkv svprket fekhervkekates.

4 Momet este em plest enhomvhhotat omvlkvn, en nakcokv-hayvlkeu vteloyof, estvmvin Klist heckvranvte em 'pohvtes.

5 Momen em onvyakat, Perehem tvlofv Cutev ekvnv; hiyomēn owalv coyvtet omekv,

6 Momen Perehem, Cutv em ekvnv ofv liketskat, Cutv em mekkvlke vpaket cotkus mahat toyetskekos; mekko vm este Eslevl vfvstvranat a ec ossvret ok, kicakvtes. 
7 Momof Helot, este hoporrenvlke ehkusen enhohiket, estofvn kococumpv hecket omvte hericet em 'pohvtes.

8 Momet Perehem ohtotvtes, Vpeyet, hericet estuce mvnettusan hopoyvkes: momet eshecatskof, punvkv hvtvm 'svn yicatskvres, vneu ahyit vrakkuecaren, kicet.

9 Momen mekko em apohihcofvt, enkvpvkakvtes; momen, hecvs, mv kococumpv hvsossv-fvecv hecakvte enhomahtet aye emunket, estuce mvnettuse wakkat onvprn er oh fekhonnvtes.

10 Momen kococumpv hecakat afvcketv rakken tayen es afvckakvtes.

11 Momen cuko seceyof, hopuewuce e'cke Melen vpaken hecakvtes, momet tvkpvlatket vrakkuecakvtes: momet nakvtehkv en hauhicof; nanvke e'makvtes, cvtokunaplane, lipenv helukfvmecvn, smvnv esyomen.

12 Momen vpueckv ofvn Helot oh fulecvranekon Hesaketvmeset en kerkuehcen, vyetv etvmin em ekvnv yoh fulecepvtes.

13. Momen vpeyehpof, hecrs, Pucase em encelvt vpueckv ofvn Cosen em e heckuecvtes, Ahuyiret hopuewuce e'cke tepakvn evpayet, Ecepsen 'soh letkvs, momet mvn liketske munken cem onvyares: Helot hopuewucen elecetvn komet hopoyvranekv, kicrtes.

14 Momen ahuyiret, hopuewuce e'cke tepakvn evpayet, aeren es ayvtes, Ecepsen.

15 Momen, Ecepse osse cv'ppuce enhuehkvyvtes, make Pucase em punvkv owalv kerkuecvte esohfvckvren, mvn like munken Helot elvtes.

16 Momof Helot, este hoporrenvlket nakhayekot fullvten hecof, tetayen cvpakkvtes, momet vtotet, cepvavkuce ohrolope en hokkolan vteken 'ra en kvncapat Perehem; mornet em vtekakat vtehkat omvlkvn. 
pvsatvtes, este hoporrenvlke hericet em 'pohvte vcrkvyen.

17 Momof Cvlemiv owalv opunayvte esfackvtes, hvmmake,

18 Lemv ofv en haket pohkvtes, waketvt, momen hvkihketvt, momen hvkihketv rakket; Lecvlet en hopuetakucen es hvkihket, emahlvpvtkvre tayekates, svkepekokv.

19 Momis Helot ehlof, hecrs, Pucase em encelvt vpueckv ofvn Cose em e heckuecrtes, Ecepse ofvn,

20 Ahuyiret, hopuewucen, e'cke tepakvn evpayet, Eslevl ekvnvn es vyvs: estuce en hesaketv em vrahkvte pvsvtkepekv; kicet.

21 Momen ahueret, hopuewuce e'cke tepakvn evpayvtes, momet Eslevl ekvnvn 'res orvtes.

22 Momis Akelaet, e'rke Helot vyoposket, Cutev ofvn mekkvcuken pohat, vyetv penkalvtes: momis Hesaketvmese vpueckv ofvn en kerkuecekv, fiyiket Kalvlen ayvtes.

23 Momen tvlofv Nasvle hocefken ayet er likvtes: Nasvlvlken kihocvres, owalvlke opunahoyvte sohfvckvren.

\section{SETENTVCKE III.}

Mv nettr take omof Cane paptisvt, Cutev vnrvwv ofvn erkenaket,

2 Momet, Cem vkerrickvn eticvks, hvlwe em ohmekketvt vwolicusekv, maket;

3 Momet, Opunvkv en haket vnrvwvn huehket, Hesaketvmese em vyetvn etetakuecrks, en nenen lvpotlvken hahicvks, maket, owalv Hesiv opunvyece okvte heyv estet mvt omekv, maket vlakvtes.

4 Momen mv Cane rvcce-kuphe essen accet, cusse sewvnaketvn etetackv sewvnvket omvtes : momen tvffonoksv momen fo-semvnole en crmpe en hompetv tates. 
5 Momof Celuslvme, Cutev, Cotvne hvcce afopke este omvlkvt esosset oh vpeyvtes,

6 Momet, en holwryeckvn e ohonvyaken, Canet Cotvnen vk paptisvtes.

7 Momis Falvsevlke, Satusevlke esyomat sulket paptisan en yicen hecof, Cetto honvpse toyatskat, Cvpvkketv vlvkvranan em pefatkvks, estimvt ce kicvke haks?

8 Monkv vkerrickv etickv oricen em etten hayvkes;

9 Momen, Eplehame pu'rken ocēs, maketv vkerrihcatskvs; Hesaketvmese heyv cvto asosse hopuetake Eplehame em mahmice tayet omen, ce kicrkit okikv.

10 Momen hiyomis pucuswv eto eceskv take vwakkēs: monkv eto em ette heren hayekat vtekat tacket, totkvn tvk wihoket os.

11 Vne mehenwrn uewrn es ce paptisvkit omis, vkerrickv etickvn: momis ev yupv vlakat vne 'svn yekces, em estelepikv en crwvye taye heretv orvks: mvt Puyvfekev Veakan, momet totkrn es ce paptisvkvres.

$12 \mathrm{Mr}$ este es em weskv e'nke ofvt's, momen naken en nerkv en cesketv heren hvsvtecvres; momet en teleko vtelohyet, tohton vtehvres: momis en tvleesse totkv vsleko tayan es nekricvres, kicakvtes.

13 Momof Cesvs Kalvlen atet Cotvnen Canen em vlakvtes, paptisvren.

14 Momis Cane em vsehvtes, Vne min ov paptisetske tayes, momat vnen vm vlaketske haks? kicet.

15 Momen Cesvs em vyoposket, Hiyomat $v m$ vkvsvmvs; hiyomēn fvccetv omvl sohfveeceyvre momvre tayet omekv, kicvtes. Momof em vkvsamvtes.

16 Momen Cesvs paptiskan vpakusen newvn enkvpaket vcemkvtes; momen hecrs, sutvt en hauhakvtes; momen Hesaketvmese em Puyvfekevt prcehowe omēt hrtapket, oh liken hecvtes. 
17 Momen hecvs, opunvkv en hake hvlwe atet, Heyvt Cv'ppuce vnokecke, mv ofv vfackusvyat tes, maket pohkvtes.

\section{SETENTVCKE IV.}

Mомоғ Cesvs, Puyvfekcvt evprhyet, vnrvwrn es ossvtes, puyvfekev holwakat eskerrvren.

2 Momen nettr pale-osten nere pale-osten eelvwehcof, renyupvn elawvtes.

3 Momen eskerrvt em vlakof, Hesaketvmese E'ppuce toyetsken omat, maketsken, heyv crtot tvklike hakvkekvs, kicvtes.

4 Momis em vyoposket, Este tvklike tvlken vhesahkekos, momis opunvkv Hesaketvmese e cukwv aossat vtekan vhesakvres, maket coket os, kicvtes.

5 Momof puyvfekcv holwakat evprhyet, tvlofv vcakan 'res ceyvtes; momet cuko-vcakat sohvpettv oh licvtes.

6 Momet, Hesaketvmese E'ppuce toyetsken omat, a e tvk wikvs; Ec ohfvecvn em encelvlken vhakvn emvres; momen e'nke taken es ce kawvpvkvres, ce'le crton es vnaf ketskekaren, maket coket omekv, kicrtes.

7 Hvtvm, Pucase cen Hesaketvmese eskeriyetskvs, maket coket os, Cesvs kicvtes.

8 Hvtrm puyvfekev holwakat ekvnv hvlwe mahen es veemkvtes, momet yvmv ekvnv tvlofv ocakat omvlkvn, en rakketvu hecicvtes;

9 Momet, Heyv nanvke omvlkvn ce'mares, tvk latket ve vrakkuecetsken omat, kicvtes.

10 Momof Cesvt okat, Setvne toyetskat, etvn vyvs; Pucase cen Hesaketvmesen vrakkuecet, mv tvlken em vtotketskvres, maket coket omekv, kicvtes.

11 Momof puyvfekev holwakat enkvpakvtes: momen hecrs, encelvlket yicet, vfvstakvtes. 
12 Momen Canen cuko-yekcvn pihokvcuken Cesvs pohhet, Kalvlen ayvtes ;

13 Momet Nasvlen enkvpaket ayet, Kvpvnevn, vkhvse vfopke Sepulvn Naftelem em vtekakat ton, erlikvtes ;

14 Sepulvn ekvnv, momen Naftelem ekvnv, vkhvse en nene, Cotvne tvpalv, Kalvle Centilvlke em ekvnv;

15 Este yomuckofv vpokvte hvyayvke rakken hecen; elkv em ekvnv momet em vpette oce vpokvte hryayvket em osses,

16 Hesiv owalv opunayvte sohfvckvren.

17 Mvn vteken Cesvs erkenvketvn, momet, Vkerrickv eticvks; hvlwe em ohmekketv vwolicusekv; maket, vlicecrtes.

18 Momen Cesvs, Kalvle em vkhvse vfopken yvkapet aret, tecakkvte hokkolet, Simv Petv kihocat, etecakkat Vntolun tepaket hoyvn vkhvse vk wikaken hecvtes; rvro-poyvlket omekv.

19 Momen, Vc vevkvhoyvks; momen este em vkwiyvlken ce hayvkares, kicvtes.

20 Momen moman vpaken hoyv wihket, vcrkvhoyvtes.

21 Momen mv ayat, tecakkvte eti hokkolen, Cems, Sepete e'ppuce etecakkat Canen tepaket e'rke Sepeten vpaket perro-cuko ofvn en hoyv taken mvhericaken hecrtes; momet enhuehkvtes.

22 Momen moman vpakan perro-cuko momet erkeu wihket, vcrkvhoyvtes.

23 Momen Cesvs Kalvlen hvmecicet arvtes, en senekake take ofvn emvhayet, momet hvlwe em ohmekketv em opunvkv-hervn es erkenaket, momet enokketv vtekat, momet este em enanokketv vtekat omvlkvn wicecet.

24 Momen ohonvkv Selev omvl etehoyanvtes, momen este enokhokat enokketv momet estemerketv cahmelike 
en heckakat, momen puyvfekcv-holwakat vtehkakateu, momen hacohakakateu, momen palse es enokhokat, omvlkvn 'sen yicen, em wicecrtes.

25 Momen este sulsuke mahet Kalvle, Tekapole, Celuslvme, Cutev, momet Cotvne trpalv vwe tis rcvkvpeyrtes.

\section{SETENTVCKE V.}

Momen este sulsuke hecat, ekvnhvlwen ohcemkvtes: momet likof, en kerrepvlket en yicvtes.

2 Momen e cukwv hrwvklehcet, emvhayvtes, hvmmaket,

3 Puyvfekev ofv kvnevpakusat en herakes; hvlwe em ohmekketvt enaket okv.

4 Hvkihhokate en herakes; emahlvpvtkvkvret okv.

5 Eyaskakusate en herakes; yvmv ekvnvt enake taret okv.

6 Fvccetv elvwe ewvnhkakat en herakes; fvcecvkvret okv.

7 Merrakate en herakes; merretvn en heckvkvret okv.

8 Efeke ofv hvsvthakusat en herakes; Hesaketvmesen hecvkvret okv.

9 Herkv hayvlke en herakes; Hesaketvmese en hopuetaken kihocvret okv.

10 Fvecetv vrahkv assehocat en herakes; hvlwe em ohmekketvt enaket okv.

11 Cen hervkes, holwvken ec opunvyecrket, ece'ssecvket, nak holwvke vtekat omvlkvn 'sec oh laksvket ve vrahkvn ce kihocrke estomis:

12 Afvckvkes, momen ece 'fackusvkekvs; feketv hrlwe-tvlofv cem ocat rakket omekv; matvpomen owalvlke ce homv take fullvten assecakvtet omekv.

13 Ekvnv em okcvnwv toyatskes; momis okcvnwvt en yekcet en sumken omat, naken svprtecvr haks? ohvtv- 
lakat estomeckv toks, vpvlvthohyen, estet oh svpvklvranat tokon omat.

14 Ekvnv en hvyayvke toyatskes. Tvlofv ekvnhvlwen oh likat vrvnahkekos.

15 Momen estet kulke kulehcet, svkerkv elecvn eslicvkekot os, momis kulke sohhuerickvn sohhuericaken, cuko vtehkat omvlkvn en hvyayicet omes.

16 Mv omēt cen hvyayvke take este ehomvn hvyayvkekvs, cem vtotketv take herakan hecet, Ce'rke take hvlwe likan vrakkuecvkvren.

17 Vhakv, monkat owalvlke em punvkv sumecicvranet vlaket os, cv kohmatskvs : sumecicvranvyat tokot, momis sohfvceevranit vlvkvyet os.

18 Mehenwvn ce kicvkikv, Sutv ekvnv esyomat sumkeko vtekat letv cotkus mahat, monkat letv enake cotkus mahat estomaten vhakv en sumkeko emunken, omvlkvt sohfvckvres.

19 Monkv estimvt o estomet heyv vhakv lopockus mahat hvmkvteken kacet, momēn este mvhayat, hvlwe em ohmekketv ofv cotkus mahan kihocvres; momis estimvto estomet vfastet, momēn este 'semvhayat hvlwe em ohmekketv ofv rakken kihocvres.

20 Ce kicvkikv, Cem fvccetv taket nakcokv-hayvlke, momet Falvsevlke em fvccetvn emuntvlekon omat, estomēt hvlwe em ohmekketv escehyatskekos.

21 Elehcetskvs; momen estimvto estomet elecat ohfvece tetaye em penkvlke tares, hofunvlke makvtet omen, pohatskvtes :

22 Momis vne ce kicvkis, Estimvt o estomet etecakkvte nake omecice sekon en homecat, ohfvece tetaye em penkvlke tares: momen estimvto estomet etecakkvte, Ehvperkv toyetsks, kicat, en nvkvfhote tayen em penkvlke tares:- momis estimvt o estomet, Ce hopor- 
renekat, makat, totkv-rakko eceye taye em penkvlke tares.

23 Monkv nake Hesaketvmese emvranetskat altvn svlaketskis, mvn, etecakketskvte nake ce'nrvpuecet on vkerricetsken omat,

24 Nake emetv altv ehomv wihket, ahyet, etecakketskvte taten tenheriket, rvlahket, nake emetv emvs.

25. Ce'nrapv lvpkusan etenherkvs, mometske tetayet etepvket vhoyatskof, monkon omat, estofvtis ce'nrapv ohfvececv ehomvn ce huericen, ohfvececvt este-wrnayv e'nke ofvn ce wihken, cuko yekcvn ce 'pike wites.

26 Mehenwvn ce kicis, Estofvn aosseko emunket, kotenv cotkus mahat espoke mahen fehketske tvlkusvres.

27 Hoktahretskvs, hofunvlke makvtet omen pohatskvtet os.

28 Momis vne ce kicvkis, Estimvt o estomet hokten hecet, eyacen omat, e feke ofvn es hoktare hoyvneps.

29 Momen ce turwv vkvpervt ce 'lentappuecen omat, ossicet, ce'nkvpvken vwikvs: ce'na omvlkv estemerkv hute tvk wihokekon, ce 'nalvcce hvmkvtekis sumkat min cen heret okv.

30 Momen ce'nkvpervt ce 'lentappuecen omat, tacet, ce'nkvpvken vwikvs; ce'na omvlkv estemerkv hute tvk wihokekon, ce 'nalvece hvmkvtekis sumkat min cen heret okv.

31 Estimvt o estomet e 'hiwv wiken omat, nakcokv'setenkvpvketvn e'mekvs, mahokvtet os:

32 Momis vne ce kicvkis, Estimvt o estomet e 'hiwv hoktarekofvn wiken omat, hoktarepuecet omes; momen estimvt o estomet wihokat epayen omat, hoktaret omes.

33 Hvtvm, Hesaketvmese es hocefe frcceko opunahyetskvs; momis Hesaketvmese etemfaccetskat momecrranetskēs, hofunvlke makvtet omen pohatskvtet os. 
34 Momis vne ce kicvkis, Estofvn nake es hocefet opunahyetskvs; hvlwe tateu, Hesaketvmese em ohliketv veaket omekv :

35 Momet ekvnv tateu, ele em ohsehoyickvt omekv; Celuslvme tateu, Mekko rakkat eme tvlofvt omekv.

36 Ce'kv tateu es vhocefet opunahyetskvs, ce'kvesse hvmkis hvtke, lvste tateu hayetskeko tayet omekv.

37 Momis cem opunvkv take, Henka, henka; Hekus, hekus; maket omekvs: naket o estomet heyv'sen hoyanat holwvke vtet omekv.

38 Eturwvn eturwvt vyoposken, enuten enutet vyoposken, mahokvtet omen pohatskvtet os.

39 Momis vne ce kicvkis, Holwvyecv vnrahpatskvs; momis estimvt o estomet ce yvnrwv 'kvpervn tepken omat, pvlhvmkateu em fulotkvs.

40 Momen estimvt, kapv cen crwetvn komet, vhakv 'sec oh ayen omat, kapv-rakkou crwekvs.

41 Momen estimvt o estomet vkerkv hvmken ayetskvren ec ohyekcicen omat, hokkolen vevkvyvs.

42 Cem 'pohat emvs, momen cem pvlepetv komat em e fuliketskvs.

$43 \mathrm{Ce}$ 'wolicv vnokecet, cen horren en homecetskvres, mahokvtet on pohatskvtet os.

44 Momis vne ce kicvkis, Cen horrvlken vnokecvks, ce hanvkat heren opunvyecvks, cen homecvkat en lopicvks, momet ece 'holwryecrkat, momet ece'ssecrkat em emekusvpvks :

45 Ce'rke take, hvlwe likat, en hopuetake toyatskvret; en hvse holwvhokat momet herakateu a em ossicet, momet fvecakat, momet fvecvkekateu em oskicet omekv.

46 Ece 'nokecvkat vnokecatsken omat, feketv estomen ocatske haks? Tokunawv-vteloyvlke tis matan momecet omeko haks? 
47 Momen tecakkatskat tvlken vsekatsken omat, naken vpvlwv 'semuntalatske haks? Tokunawv-vteloyvlke tis momēn momaket omeko haks?

48 Momekv Ce'rke take hvlwe likat temvpokat omēt temvpokvket omvkes.

\section{SETENTVCKE VI.}

Heromatsinat, este ehomvn, estet ce hecvkvren omatskekaret vketecvkes; monkon omat, feketv Ce'rke take hvlwe likat vte ocatskekos.

2 Monkv herometskat mekusapv-vhayvlke senekake take momet tehoyvnkv take ofv, estet vkvsvmvkvren momecakat omēt pofketv ce homv hayehcetskvs. Mehenwvn ce kicvkis, Em feketv ocakes.

3 Momis herometskat, ce'nkvskvnv naken c'enkvperv celayan keriyates.

4 Cen heromkv ehke taren; momen Ce'rke, ehke tis hecat, eme mahusat hecken cem fekvres.

5 Momen mekusapetskiof, mekusapv-vhayvlke ohmetskvs; senekake take momet tehoyvnkv take kvnowv ofv svpakle mekusape, estet hecvre 'mvlostvket omekv. Mehenwrn ce kicvkis, Em feketv ocakes.

6 Momis ceme mekusapetskof, nvthofuce eceyvs, momet rhauke vkhottetskof, Ce'rke ehke likan mekusvprs; momen Ce'rke ehke tis hecat hecken cem fekvres.

7 Momis mekusapatskof, Centilvlke momakat omēt, opunvkv ehvperkvke oh apvlehcatskvs, Pum opunvkv sulkan pum apohihocvres, komvket omekv.

8 Monkv mv ohmatskvs; Ce'rke take nanvken ce vacvke tat em 'pohatskeko munken kerret omekv.

9 Monkv, hiyomen mekusvpvks: Pu'rke hvlwe liketskat, ce hocefkvt vcakekvs. 
10 Cem ohmekketvt vlvkekvs. Mimv hvlwe nake kometske momat etvpomet yvmv ekvnvn oh momekvs.

11 Nettv vrahkv tvkliken mucv nettrn pu'mvs.

12 Momet pum ahuervn es pum wikvs, vhuericeyat es em wikakeyat, etvpomen.

13 Nake pu naorkepuece taye eskerretv 'sep oh ahyetskvs; momis holwakat a sepu'ssicvs. Ohmekketvt, yekcetvt, momen rakketvt cenake emunkvt omekv. Emén.

14 Este nake cem mvttecicakat es em wikatsken omat, Ce'rke take hvlwe likateu es cem wikvkvret okv.

15 Momis este cem mvttecicakat es em wikatskekon omat, Ce'rke takeu em mvttecicatskat es cern wihkvkekos.

16 Momen eelvwecatskof, mekusapv-vhayvlke en heckv efeknokhoke omakat ohmatskvs; e turofheckv taken mvrahkuecet, esten eelvwece omēt em e heckuecvret omakekv. Mehenwvn ce kicvkis, Em feketv ocvket os.

17 Momis ceme eelvwecetskof, ce'kv vfeyet, ce turofvn okkosvs.

18 Esten eelwwece omēt em e heckuecekot, momis Ce'rke ehke likan em e heckuecetskvret: momen Ce'rke, ehke tis hecat, hecken cem fekvres.

19 Yvmv ekvnv sukehtv momet nake vhopanat yvmahken, horkopvlke vhopanet ropottet esohceyet horkopakan nakeockv e en herihcatskvs.

20 Momis cen nakockv hvlwe min, sukehtv momet nake vhopanat yvmahkekon, momen horkopvlke vhopanet ropottet esohceyet horkopvkekan, e en hericet omvkes.

21 Estrmvn cen nakockv ocen omat, ce feke takeu mvn ocvret okv.

22 Eturwvt ena en kulket os: monkv ce turwvt heren omat, ce'na omvlkvn hvyayvket fvcke tares. 
23 Momis ce turwvt holwaken omat, ce'na omvlkvn yomucket fvcke tares. Monkv hryayvke ece 'pikat yomucket on omat, mv yomucke en rakke estomvteke haks!

24 Este estomet pucase hokkolen em vtotkeko tayes: hvmken en homecet, momet hvmkan vnokecvret okv; monkat hvmken hvlatet, momet hvmkan vhepakvret okv. Hesaketvmese nakockv eshokkolen em vtotketv umatskeko tayes.

25 Monkv ce kicvkis, Cen hesaketv ohfvecv, nake hompvranatskat, monkat nake eskvranatskat, hvtvm ce'na ohfvecv, nake vecvranatskat senaoricet vkerrihcatskvs. Hesaketvt hompetvn muntvleko haks? momen ena acevken muntvleko haks?

26 Hecvkes fuswv hvlwe fullan, fokayet, monkat tacet, monkat vteloyet, tohto vtehvkekot omekv : momis Ce'rke take hvlwe likat hompvkuecēt os. Centake mv 'sem vcacvke mahet omatskeko haks?

27 Estomatskat vkerricatskatet cem mahe kupetv hvm-* ke ohvtvlvyatske taye haks?

28 Momen estomēn accvken senaoricet vkerricatske haks? Pvkpvke lele kihoce vnrvwv ocakat, estomēt mahan, vkerricvkes; vtotkvkekot os, momet fvllecvkekot os.

29 Momis ce kicvkis, Salomv tisem en rakketv omvlkv ofv $\mathrm{yv}$ hvmkvtekat omēt vccekates.

30 Momekv Hesaketvmese prhe vnrvwv ocat, mv mucv-nettv ocet, pvkse tvklike-esnorickv ofv vpvlvthoyat tis momēn accecen omat, ceme semuntvle mahen ce'ccecvkekare te, vkvsvmkv estomuse ocatskat?

31 Monkv, Naken hompeyvr haks? monkat, Naken eskeyvr haks? monkat, Naken vceeyvr haks? maket senaoricet vkerrihcatskvs. 
32 (Heyv nanvke omvlkvn Centilvlke hopoyaket omekv:) Ce'rke take hvlwe likat, heyv nanvke omvlkvn ce yacvkat kerret omekv.

33 Momis Hesaketvmese em ohmekketv, momet em fvecetvn tāten hopoyvks, momen heyv nanvke omvlkvt cem ohvpvkvkvres.

34 Monkv pvkse vkerrice senaorihcatskvs: pvkset nanvken ēmē tat e em vkerrice senaoricvret okv. Nettr en holwvketv ocat 'sem etetayes.

\section{SETENTVCKE VII.}

Ohfvecencatskvs, ec ohfvecvkekaren.

2 Ohfvececkv estomēn 'sohfvececatskat 'sec ohfvcevken, momen vhopakv estomēn 'svhopayatskat, hvtvm ece 'hopakvkvret ok.

3 Momen estomēn tecakketskat e turwv eto-kvckuce vk ocan hecetskat, momis tohkakv ce turwv vk ocan vkerricetskeko haks?

4 Monkat estomēt tecakketskat, Ce turwv eto-kvckucen cem vk ossihcvrs, kicetskvr haks? momat hecvs, tohkakvt ce turwvn vk oces.

5 Mekusapv-vhayv toyetskat, ce turwv tāten tohkakvn vk ossicvs; momofvt heren hecetskvres, etecakketskat e turwv eto-kvckuce em vk esetv.

6 Nake hvsvtkat efv ehmatskvs; momet cem makelitv taken sukhv em vpvlahtatskvs, oh svpaklet ele elecvn hahyet, afulehcet, ce selahlecvke, witet ok.

7 Vpohvks, momen ce'mhoyvkvres: hopoyvks, momen eshecatskvres; vnvfkvks, momen cen haukvkvres.

8 Vpohat vtekat en hecket omekv; momen hopoyat esheces; momen vnaf kat en haukvres.

9 Monkat este estomatskatet, e'ppucet tvkliken em 'pohen omat, cvton eh met o haks?. 
10 Momen rvron vpohen omat, cetton ehmet o haks?

11 Monkv centake, holwvhokatskat, emetv herakat cen hopuetake emetv kerratsken omat, Ce'rke take hvlwe likat māhvkvu em 'pohakat nake herakat emvkekar haks?

12 Monkv nanvke omvlkv este cem momecvkvre komatskat, cemeu matvpomusen em momecvks: heyvt vhakv momen owalvlket omekv.

13 Vhauke cotkan esceyvks : sumketv erorat vhauke taphet on, momen neneu taphet omen, momen mv esceyat sulket omekv.

14 Hesaketv erorat vhauke cotket on, neneu cotket omen, nvcomet eshecet omekv.

15 Owalv laksvlke, yvpefikv em accvke vchoye, momis ofv yvhv noksvlke cen yicakat vketecvks.

16 Em ette taken es kerratskvres. Etofvsken pvrko em mvmhoyet omen, vkacon ke-rakko em mvmhoyet ome haks?

17 Matvpomusen eto herat vtekat em ette heren hayet omes: momis eto holwakat em ette holwvken hayet omes.

18 Eto herat em ette holwvke hayeko tayes, momet eto holwakat em ette heren hayeko tayes.

19 Eto em ette here hayekat vtekat tacket, totkvn tvk wihoket os.

20 Momekv em ette taken es kerratskvres.

21 Pucasē, Pucasē, cv kicakat vtekat hvlwe em ohmekketv ceyvranet omekos; momis Cv'rke hvlwe likat komate momecat tvlket.

22 Sulket mv nettv omof, Pucasē, Pucasē, ce hocefkv ofv erkenvkēkat haks? momet ce hocef kv ofv puyvfekcv holwvhokat sossicēkat haks? momet ce hocefkv ofv vtotketv yekcrke sulke momecēkat haks? cv kicvkvres. 
23 Momen momof, Ce kerrvkvyvte sekos; holwvyeckv rtotkvlke toyatskat, v'nkvpvkvks, kicvkares.

24 Monkv estimvt o estomet heyv vm punvkv pohet, vfastat, este hoporrēne mv en cuko cvto oh hayvte svhopayares ;

25 Momen oske hvtapken, uelauke vlaken, momen hotvle hotalet, mv cuko oh latkvtes; momen lvtkekates; cvton oh liket omekv.

26 Momen heyv vm punvkv pohet vfvstekat vtekat, este hoporreneko, mv en cuko oktahv oh hayvte svhopayares:

27 Momen oske hvtapken, uelauke vlaken, hotvle hotalet, mv cuko oh latkvtes; momen latkvtes, momen en lvtketv rakke tates.

28 Momen momēt ocvtes, Cesvs heyv opunvkv es yukpoyof, em emvhakvt estomahes este komakvtes.

29 Nakcokv-hayvlke omēkot, yekcetv oce omēt emvhayakekv.

\section{SETENTVCKE VIII.}

EnvNhvlwe a hvtvpikof, este sulsuke mahet vcrkvpeyvtes.

2 Momen hecvs, lepvt vlaket, vrakkuecvtes, Pucasē, kometsken omat, hvsvtken $\mathrm{cv}$ hayetske tayes, maket.

3 Momen Cesvs e'nke awiyet, celayvtes, Komis ; hvsvtkvs, kicet. Momen moman vpaken lepvse en hvsvtkvtes.

4 Momen, Vketecet, estimvn 'monahyetskvs : momis ahyet, plest em e heckuecrs, momet nake emetv Mose makvte es en kerkueckvn sryvs, Cesvs kicvtes.

5 Momen Cesvs Kvprnevn cehyof, sentulevt en kusvpepet em vlakvtes:

6 Momet Pucasē, vm vtotkv palse es enokket, tayen estemerket, cukon wakkes, maket. 
7 Momen rorit em wicecares, Cesvs kicvtes.

8 Momen sentulev em vyoposket makvtes; Pucasē, vn cuko ceyetske taye heretv orvkot os: momis opunvkv tvlkusen opunvyvs, momen vm vtotkv em wikvres.

9 Vneu hunvnwr yekcetv elecr huervyet omit, suletawvlke cv'lecv ocikv; momet heyvn, Vyvs, kicin, ayet os; momet ètvn, Vtes, kicin, atet os; momet vm vtotkvn, Heyvn momecvs, kicin, momecet os.

10 Cesvs pohof, em estomahvtes, momen vcrkfullaten, Mehenwvn ce kicvkis, Vkvsvmkv rakke momat eshecvkates, momes, Eslevl ofv tat eshecvkos, kicvtes.

11 Momen ce kicvkis, Sulket hvsossv-fvecv momen hvsvklatkv-fvccvu awvres, momet Eplehame, Iseke, Cekvpe esyoman vpaket hvlwe em ohmekketv ofvn vpokvres.

12 Momis ohmekketv en hopnetake fettv yomuckofvn vpvlvthoyvres; mvn hvkihketv, momen nute setetēkketvu ocvres:

13 Momen Cesvt sentulevn, Vyepvs; momen vkvsametskat etvpomen cem momekvs, kicvtes. Momen em vtotkv mv hvse-vkerkv etvwvn em wikvtes.

14 Momen Cesvs, Petv en cukon er ceyof, e 'hiwv e'cket hepetkēt wakken hecvtes :

15 Momen enken en celayvtes; momen hepetketv em wihken, aliket vfvstakvtes.

16 Yafkofv, puyvfekev holwvhoke vtehkakat sulken 'sen yicakvtes; momen em punvkvn puyvfekcvlke esossicvtes; momet enokhokat omvlkvn em wicecakvtes :

17 Emet pum ehvperketv cawvtes; momen pum enokketv e oh wikvtes, owalv Hesiv opunayet makvte sohfvckvren.

18 Momen Cesvs este sulsuke mahet em afoloten hecof, tvpalv vyetvn makvtes. 
19 Momen nakcokv-hayv hvmket vlaket, Mvhaya, estvmv estomen ayetskis, ece 'crkvyares, kicvtes.

20 Momen, Culv ekvnhauke ocvkēpēs, momen hvlwe fuswv puknvkv ocvkēpès; momis este E'chuswv estvmvn e'kv licvranat tis ocekos, Cesvs kicvtes.

21 Momen en kerrepvlke ētvt, Pucasē, ahyit, cv'rke taten hericaren vm etektvnecvs, kicvtes.

22 Momis, Vc vcrkvtes; momen prsvtkat em prsvtkan hericvkekvs, Cesvs kicvtes.

23 Momen perro-cuko ecehyof, en kerrepvlke vcvkvpeyvtes.

24 Momen hecvs, vkhvse hotvle-rakkot kvwapkvtes, svheremahekv perro-cuko uemelohlvt ohranvtes: momis nocepe tates.

25 Momen en kerrepvlke en yicet, ahonecicaket, Pucasē, pu hesahuecvs; pu sumecvranes, kicakvtes.

26 Momen, Vkvsvmkv estomusen ocatskat, estomēn ce penkvlakvket o haks? 'kicvites. Mohmen ahuyiret, hotvle vkhvse tepaken em vsehtites; momen lekuthe rakket ocvtes.

27 Momis hunvntake em estomahakvtes, Heyv hunvnwv estomēt ot omen, hotvle tisem momen uehvtkv tisem. em apohicakes! makaket.

28 Momen tvpalv Kvkesenvlke ekvnvn rorof, este hokkolet, puyvfekcv holwvboke vtehket, cvpvkhoke mahet, este estomet $\mathrm{mv}$ hoyvneko tayaten, este herickv vlkan awolket, vnrvpakvtes.

29 Momen hecvs, huehkakvtes, Cesvs Hesaketvmese E'ppuce toyetskat, naken pu tenrvwv ocēya? oketv oreko munken pu 'stemerricvranet yvmv vlaket ometska? kicaket.

30 Momen mv hopvyen sukhv sulke fulle hvmket nanopet fullvtes. 
31 Momen puyvfekev holwvhokat, Sepu'ssicetsken omat, sukhv-fullan 'sen ceyeyvren pum etektvnecvs, kicaket, en kusvpakvtes.

32 Momen, Vpeyvks, kicvtes. Momen esossof, sukhvfullan es en ceyvtes; momen hecrs, sukhv-fulle hvmkat omvlkvt pvfpvnet pefatket, vknvthen vkhvsen vk tasecet, uewvn vk prsatkvtes.

33 Momen vhecicakvte pefatket, tvlofvn vpeyepvtes, momet nanvke omvlkvn onayakvtes, momen puyvfekev holwvhoke vtehkakvte nake em estomakateu.

34 Momen hecvs, tvlofv este omvlkvt Cesvs er vnrvpvranet a sossvtes; momen hecakof, em ekvnv em vtekakat enkvpvkvren en kusvpakvtes.

\section{SETENTVCKE IX.}

Momen perro-cuko ecehyet, tikvtes, momet eme tvlofvn ceyvtes.

2 Momen hecvs, hunvnwv palse es enokket pvtakv oh wakken sen yicvtes: momen Cesvs em vkvsvmkv taken hecof, palse es enokkat, $C v$ 'ppucē, ce mvhlvpvtkekvs; cen holwvyeckv ce'nkvpvkakes, kicvtes.

3 Momen hecvs, nakcokv-hayvlket, Heyv este Hesaketvmesen vtvkleces, eme ofvn makakat sasvtes.

4 Momen Cesvs em vkerrickv taken kerret, Estomēn ce feke ofvn holwvken vkerricatske haks? kicvtes.

5 Cen holwryeckv ce'nkvpvkakes, maketv, monkat Ahuyiret yvkvpvs, maketv tepakat estomat 'senyekcet ome haks?

6 Momis este E'chuswv yvmv ekvnv yekcetv ocet holwvyeckv kvpakice tayat kerratskvren; (momof palse es enokkat,) Ahuyiret, cem pvtakv cahwet, cen cukon vyvs, kicvtes.

7 Momen ahuyiret, en cukon ayvtes. 
8 Momis este sulsukat hecof, em estomahet, Hesaketvmese vkvssumakvtes, yekcetv momē este emvte.

9 Momen Cesvs mvn a ossof, hunvnwv, Maro hocefket, crtokunawv vtelokv cukon liken hecvtes; momet Vc vcrkvyvs, kicvtes. Momen ahueret, vcrkayvtes.

10 Momēn momē ocvtes, Cesvs hompet nuthofvn likof, hecvs, cvtokunawv-vteloyvlke holwvyecvlken esyomet sulket yicet, Cesvs en kerrepvlken esyomen vpaket vpokvtes.

11 Momen Falvsevlke hecof, Estomēn ce Pucase take cvtokunawv-vteloyvlke holwvyecvlke esyoman vpaket hompe haks? en kerrepvlken kicakvtes.

12 Momis Cesvs pohof, Cvfencakat vlekev eyacrkekos, momis enokhokat eyacakes, kicvtes.

13 Momis vpeyet, heyvt naken okat kerrvkes, Nake ohnekrickv tokon, merretv mimvn cvm vlostes: este fvecakan enhuehkvranit vlakit omvkokv; momis holwvyecvlke em vkerrickv eticvren.

14 Momof Cane en kerrepvlket en yicvtes, Pume Falvsevlke esyomeyat tewolen eelvweceyan, estoman cen kerrepvlke eelvwecvkekot ome haks? kicaket.

15 Momen Cesvs kicvtes, Hokte-epayvranat vpake vtekat hopuetake hokte-epayvranat en cuko vtehkvlke hvkihhohke taye haks? momis hokte-epayvranat em eshoyvre nettv crkkakvres, momen momoftan eelvwecvkvres.

16 Estimvt vccrke leske nake mucvse em vlicekot os; $\mathrm{mv}$ emvhericvrane em vlihocat vecvke en cawet on, cetakkat svheremahe hakekv.

17 Monkat este sukcv lesken prrko-opuswv mucrsen vcanvkekot os; momēn omat, sukcv setken, pvrkoopuswr fihnes, momen sukev hopvnkes: momis prrkoopuswr mucrsat suker mucrsen vevnhoyes, momen estrmitis yvmahikekos. 
18 Heyv nanvke kicakof, hecvs, este evpayvt vlaket en tokkekvtes, Cv'chuste entis elēps; momis atet ce'nken oh wvkecvs, momen vhesakvres, maket.

19 Momen Cesvs ahueret, vcrkayvtes, momen en kerrepvlkeu.

20 Momen hecrs, hokte, cate-fihne ohrolope pale-hokkolohkake es enokket, topvrvn vlahket, en kapv em vteken celayvtes :

21 En kapv celayin omat tvlkusis, ev cvfeknvres, eme ofv makvtet okv.

22 Momis Cesvs afulotket hecvtes, momet hecof, Cv'chustē, heren ce mahlvpvtkekvs; cem vkvsvmkvt cvfeknen ce hayes, kicvtes. Momen mv hvse-vkerkvn vteken hokte cvfekne hakvtes.

23 Momen Cesvs evpayv en cukon ecehyet, wahokvlke momen este sulke estokemahen hecof,

24 Tektvnecvks; hoktuce ēlekot, momis nocekv, kicvtes. Momen vpelicakvtes.

25 Momis este sossihocof, eceyet, enken en hvlatutes, momen hoktuce ahuervtes.

26 Momen mv opunvyèckv mv ekvnv omvlkvn hvmecicvtes.

27 Momen Cesvs mvn enkvpakof, hunvntake eturwvhecvkeko hokkolet vcrkvhoyet, huehkakvtes, Tewe e'ppucē, pum merrvs, makaket.

28 Momen cuko ecehyof, hunvntake eturwv-hecvkekat em vlahokvtes; momen, Heyv momece tayes, cv komet vkvsvmatsket o haks? Cesvs kicvtes. Henka, Pucasē, kicakvtes.

29 Mohmen e turwv en celayvtes, Cem vkvsvmkv take vevkvyen cem momvkekvs, kicet.

30 Momen e turwv en hauhakvtes ; momen Vketēcrks, estimvt keriyates, maket, Cesvs yekcen kicvtes. 
31 Momis vhoyof, mv ekvnv omvlkvn ohonvkv hvmecicakvtes.

32 Esossof hecrs, este opunayeko, puyvfekev holwvke vpiken, es en yicakvtes.

33 Momen puyvfekcv holwakan ossihcof, opunvyekate opunayvtes; momen este sulsukat em estomahakvtes, Eslevl ofv momē hechoyvte seks, makaket.

34 Momis Falvsevlket, Puyvfekev holwvhokat 'sem evpayv eteropotten puyvfeker holwvhokan sossicet os, makakvtes.

35 Momen Cesvs tvlofv tvlofucvlkeu omvlkvn arvtes, en senekake take ofvn emvhayet, ohmekketvt -em opunvkv-hervn es erkenaket, momet este em enokketv vtekat, momen evkvlkv vtekat em wicecakvtes.

36 Momis este sulsokan hecof, em merrusvtes, hotosakuset, momet vwahet yvpefikv vhecicv ocvkeko omakekv.

37 Momof, Mehenwusan aklokce sulket os, momis vtotkvlke nvcomuis:

38 Monkv naklokcat e pucasen ernekusvpvks, vtotkvlke en naklokce ohtotvren, en kerrepvlken kicvtes.

\section{SETENTVCKE X.}

Momen en kerrepvlke pale-hokkolohkakat enhuehkof, puyvfekcv hvsvthvkekat ohfvnke, sossice tayen, momet enokketv mvrahrvkv omvlkvn, momet evkvlkv mvrahrvkv omvlkv wicece tayen yekcetvn emvtes.

2 Tokvs, vpastelvlke pale hokkolohkakat hocefhokv heyv tes; Enhvteceskv Simv, Petv kihocat, momen etecakkat Vntolu; Cems Sepete e'ppuce, momen etecakkat Cane ;

3 Felvpe, momen Paralome; Tamvse, momen Maro 
crtokunawv-vteloyv; Cems, Alfer e'ppuce, momen Lepev, Ratev en hocef kv ;

4 Simv Kenvnvlke, momen Cutvse Eskalev, mv Cesvs erwiyvte.

5 Heyv pale-hokkolohkakan Cesvs vpeyicet, yekcen kicakvtes, hvmmaket, Centilvlke em ekvnv ofv vpehyatskvs; momèt Semēlevlke eme tvlofv hvmkis escehyatskvs :

6 Momis Eslevl en cuko hvmecvte yvpefikv sumecat min em vpeyvks.

7 Momet vpeyatskat, erkenvkvks, Hvlwe em ohmekketv vwolices, makaket.

8 Enokhokat em wicecet, lepvvlke en hvsvtecet, pvsvtkat asvpvklecet, puyvfekev holwvhokan sossicvks; enrahkv sekon cen heckvtes, enrahkv sekon wiyvks.

9 Cvtokunaplanet ot, cvtokunaphvtke, cvtolane tateu cen crtokunawr en sukev vtehken ehopakekot,

10 Monkat ehvlewv en sukcvt ot, kapv tateu hokkole, estelepikv tateu, setekkekv tateu hokkole ehopahkatskvs; vtotkv en hompetv en hecke taye heretv oret omekv.

11 Momet tvlofv, tvlofuce tateu estomis esceyatskat, estimvt mv ofv hērat, vpohvks ; momet mvn fekhonnvtet vpeyatskvres.

12 Momet cuko esceyatskof, herkvn oh opunvyvks.

13 Momen cuko heretv oren omat, cen herkv take oh vlvkekvs; momis heretv orekon omat, cen herkv take ec oh fulkvkekvs.

14 Momen estimvt o estomet ce yvpayekot, hvtvm cem opunvkv pohekon omat, mv cúko, tvlofv tateu enkvpakatskof, ce'le essu vlikan fekēyvks.

15 Mehenwvn ce kicrkis, Ohfvececkv nettv omof Satvme Komalv tepakatet mv tvlofvn 'sen here tares. 
16 Hecvkes, yvpefikv yvhv sulke em etenrvwv omatsken ece 'totvkis; monkv hoporrenvke cetto omēt, momet nake vhopvneko pvcehowe omvks.

17 Momis este vketecrks; tekueketvn 'sece'peyet, momet en senekake take ofvn ce tepkvkrret ok.

18 Momen vne $v c$ vrahkvn este em evpayvlke mornen mekkvlke ehomvn es ce vihocvkvres, Centilvlke, momen emetake vnrvpe esohkerkueckvn.

19 Momis mv enke ofv ce wikvkof, Estomēn, hvtvm naken opunahoyeyvr haks? vkerricet senaorihcatskvs; $\mathrm{mv}$ hvse-vkerkv ofv mahusan naken opunayvranatskat ce'mhoyvkvret okv.

20 Cemet opunayet omatskekon, momis Ce'rke take em Puyvfekcvt ec ofv take opunayet omekv.

21 Momen etecakkatet etecakkaten elkvn em wikvres, momen erket hopuewrn: momen hopuetaket erkvlken vnrvpaket prsvtepuecvkvres.

22 Momen cv hocefkv vrahkvn este omvl em en homeckatskvres: momis mv momis kome emeyuksicat hesakvres.

23 Momis heyv tvlofvn ece'ssehocvken omat, tvlofv etvn vpefatkvkes: mehenwvn ce kicvkikv, Eslevl eme tvlofv vlkan fulle poyatskekon, este E'chuswvt vlvkvres.

24 En kerrepv mvhayv es en hvlwekon, vtotkvu e pucase es en hvlweks.

25 En kerrepv e'mvhayv etvpomat em etetayen; momen vtotkv e pucase etrpomat em etetayet os; cuko e pucasen Pelsepvp kicakvten omat, en cuko vtehkvlke mahvkvu kicvke tayet omeko haks!

26 Monkv em penkvlahkatskvs, nake ohrvnke enrvmkvranekat sekon, ehke kerkvranekat sekot omekv.

27 Nake yomuckofv ce kicvkvyat hryayvke ofvn 
onvyvks; momen nake ce hvcko ofvn pohatskat, mvn cuko onvpvn erkenvkvks.

28 Momen ena elecaket, momis puyvfekev elecvkeko tayat em penkvlahkatskvs; momis puyvfeker ena eshokkolv estemerkv hute ofv sumecice tayat min em penkvlvks.

29 Cutkucvlke hokkolat kotenv hvmken wihoyet omeko haks? momat mv hvmkusis C'erke take kerret omekon ekvnv oh Ivtikekos.

30 Momis ce'kv take esse tis omvl vhonkvtkes.

31 Monkv ce penkahlvkates, cutkucvlke sulke es em veakatsket os.

32 Monkv estimvt o estomet este ehomvn cv kerkuecen omat, mvn vneu Cv'rke hvlwe likat ehomvn kerkuecares.

33 Momis estimvt o estomet este ehomvn ev hepakat, mvn vnen Cr'rke hvlwe likat ehomvn vhepvkares.

34 Herkvn ekvnvn ohtotvranet vlakes, ev kohmatskvs; herkvn vtotvranit vlakit omvks, momis eslafkv-cvpkon.

35. Este-hunvnwrn e'rken vnrvpēn etekvpicvranit, momet echuswrn e'cken vnrvpēn, momet em ehiwrn e'hee'cken vnrvpēn huericuranit vlakit omikv.

36 Momen este en horre e hutvlke mahusat omvres.

37 Estimvt erket ot, ecke tateu vne semuntvlen vnokecat crnake orekot os; momen estimvt eppucet ot, echuste tateu vne semuntvlen vnokecat, cvnake orekot os.

38 Momen estimvt tohweklepkv ëset, vc vcvkvtekon omat, evnake orekot os.

39 Estimvt en hesaketv eshecat sumecicvres; momen estimvt vne ve vrahkvn en hesaketv sumecicat eshecvres. 
40 Estimvt ce yvpayvkat, vnen cv yvpayet omes; momen $\mathrm{cv}$ yvpayat $\mathrm{vc}$ vtotvte evpayet ornes.

41 Estimvt owalvn owalv en hocefkv ofvn evpayat owalv es em feketvn en heckvres; momen estimvt este fvecvn este fvece en hocefkv ofvn evpayat este fvecv es em feketvn en heckvres.

42 Momen estimvt o estomet heyv lopockusat hvmken, en kerrepv en hocefkv ofvn, uekvsvppe es esketv hvmkusen es eme estomis, mehenwrn ce kicvkis, Em feketv estomaten en sumikekos.

\section{SETENTVCKE XI.}

Momen momē ocvtes, Cesvs en kerrepvlke pale-hokkolohkakat heyv vhakucvlke emat poyof, eme tvlofv vilkaten emvhayet, momet erkenvkvranet mvn enkvpakvtes.

2 Momen Cane cuko-yekcr ofvn Klist em vtotketvn pohof, en kerrepvlke hokkolen atotvtes,

3 Momet, Vlvkvranvte toyetska, monkat ètvn en nene hecē haks? kicvtes.

4 Momen Cesvs em vyoposkat, Vhoyet nake pohe momet hecatskat Canen en kerkuecvks :

5 Eturwv-hecvkekat hecakes, momen yvkvpvkekat yvkvpakes, lepvvlke en hvsvthakes, momen pohvkekat pohakes, pvsvtkat akvwrphoyet os, momen opunvkvhervn estemerrakat em erkenvkhoyes.

6 Momen estimvt o estomet ve vrahkvn elentappekat en heret omes, kicvtes.

7 Momen vhoyehpof, Cesvs Cane ohfvecvn este sulsokan 'sem punvyvketvn vlicecet, Naken hecvranet vnrvwvn esossatskvt haks? Kohv hotvle fvmoyicēn?

8 Momis naken hecvranet esossatskvt haks? Hunvnwv 
vccvke lowaloke vecen? Hecvs, vccvke lowaloke es fullat, mekkvlke en cuko taken vpokes.

9 Momis naken hecvranet esossatskvt haks? Owalvn? Momes, ce kicvkis, momen owalv semuntvlet omes.

10 Hecvs, vm punvkv-sarv, ce turofv ehomv cen nene etetakuecvranat, ce homvn vtotis, opunvyece cohoyvte, heyvt mvt omekv.

11 Mehenwrn ce kicrkis, Hoktvke heckuecrte vpvke Cane paptisv' 'sen rakke kvwrpkvte sekos; momis hvlwe em ohmekketv ofv cotkus mahatet es en rakket os.

12 Momen Cane paptisv en nettv vtekat vte hiyome orat hvlwe em ohmekketv ohyekhoces, momen ohyekcakat vyekcet eenakuecakes.

13 Owalvlke vhakv esyomat omvlkvt Cane vteken owalvkvtet okv.

14 Momen eenakuecetv komatsken omat, heyvt Elivs mv vlvkvranvte tes.

15 Estimvt e hvcko es pohetv ocat, pohekvs.

16 Momis naken heyv honvpse es vhopayar haks? Hopuetake nakwikv vlkan vpoke, momet vpvltake oh huehket,

17 Momet, Cem pofkvkēyis, opvnatskeks; cen hvkihhokēyis, hvkihhokatskeks, makake omēs.

18 Cane hompekot, eskekot vlakvtet omen, Puyvfekcvholwvken oces, makakekv.

19 Este E'chuswvi hompet momet ēsket vlakvtes, momen, Hecvs, hunvnwv hompetv vnoksat, momet pvrko opuswv ëskvt, cvtokunawv-vteloyvlke momen holwvyecvlke en hesset ares! makaks. Momis hoporrenkv e'chustaket fvececakes.

20 Momof mv tvlofv take, em vtotketv yekcakat sulke mahat momecvtet, vkerrickvn eticvkekat ohfvecvn vnra. pet em punvyetvn vlicēcrtes: 
21 Cen holwakes, Kolesa! cen holwakes, Persita! vtotketv yekcrke ec ofv take momakvte Tilv Sitvne tepakat ofvn momakvten omat, hofunen sukcv-hakvn vchoyet, momet essun trkvpoket em vkerrickvn eticakvnt omekv.

22 Momis ce kicvkis, Ohfvececkv nettv omof Tilv Sitvne tepakat es cen hervke tares.

23 Momen Kvprnev toyetskat, sutv oren kvwapketskat, estemerkv huten vkhvtapketskvres; vtotketv yekcvke ec ofv momakvte Satvme ofvn momakvten omat, mucv nettv oren likvnt omekv.

24 Momis ce kicvkis, Ohfvcceckv nettv omof Satvme ekvnvt es cen here tares.

$25 \mathrm{Mv}$ omof Cesvs opunayet, makvtes, $C v^{\prime}$ rke hvlwe ekvnvu e Pucase toyetskat, ece 'kvsamis, heyv nanvke kerrat momet hoporrenakat em ēhet, hopuetakucen em enrametskekv.

26 Momes, $C v^{\prime}$ rke, momēt cen renakv ehomv here es cem momvtet okv.

27 Nanvke omvlkvn Cv'rket cv'nke ofvn hayet os : momen hunvnwv estomēt Eppuce kerrekon, Erke tvlket kerres ; Erkeu hunvnwv estomet kerrekon, Eppuce, momen estimvt o estomēn Eppucet komet hecicat tvlket kerrakes.

28 Vtotket momet cen honhoyvkat omvlkvt ve oh vwvkes, momen fekvpkvn ce'mvkares.

29 Nokwvohwakkv crnaken e oh wvkecvks; momet 'svn kerrvks; eyaskvyet omit, cv feke ofvu kvncvpvyet omikv; momen cem puyvfekev fekvpkvn 'sen hecatskvres.

30 Nokwvohwakkv crnake yulohet on, vn sapokvu tahoknet omekv. 


\section{SETENTVCKE XII.}

Mv omof Cesvs nettvcakon ayet telekon etehoyanvtes; momen en kerrepvlket elawvket ot, en lokcen es mamet, prpetv vlicecakvtes.

2 Momis Falvsevlke hecof, Hecvs, cen kerrepvlke nettvcako mometv tokon momakes, kicakvtes.

3 Momis, Tewe momen vpvkakat elawakof estomvte,

4 Estomēt Hesaketvmese en cuko ecehyet, hecetv tvklike, mv hompeko tetayvte tat, momen vpvkakateu, momis plestvlke tvlke hompvkvranat hompvte ohonvkv ohonayatskekat haks? kicvtes.

5 Monkat estomēt plestrlke cuko vcakat ofvn em mvttekate nettvcako vhopanat, vhakv ofv ohonvkv ohonayatskekat haks?

6 Momis ce kicvkis, Heyvmvn cuko vcakat 'sen rakket likes.

7 Momis, Nake-ohnekrickv tokon, merretvn cvm vlostes, make naken okat kerratskvten omat, em mvttvkekate ohfvececatskekvnt os.

8 Este E'chuswr nettrcako tis e Pucaset omekv.

9 Momen mvn enkvpakof, en senekake taken eceyvtes:

10 Momen hecvs, hunvnwr e'nke yuknet likvtes: momen, Nettvcako nake wicecetv fvccvt o haks? kicaket, em 'pohakvtes, vnrvpvkvret.

11 Momen, Ece 'pakvkat hnnvnwv estomet yvpefikv hvmken ocen, nettrcakon ekvnhauken vk latken omat, hvlatet ossicekare te? kicakvtes.

12 Monkv estomomusen hunvnwvt yvpefikvn 'sem vcaket o haks? Monkv nettrcako here momeckv vfvstetv fvecvt omes.

13 Momof hunvnwvn, C'enken rvtosecvs, kicvtes. Momen rvtosecrtes: momen temvpoke hvmkate ome hakvtes. 
14 Momof Falvsevlke sosiyet, vnrvpēt estomecet sumecice tetayat ete'm opunvyecakvtes.

15 Momis Cesvs kerrof, mvn enkvpakvtes; momen este sulsuke mahet vcrkvpēyen, omvlkvn em wicecakvtes;

16 Momet ohkerkuecvkekaren yekcen kicvtes ;

17 Hesiv owalv hvmmaket opunvyvte 'sohfvckvren,

18 Hecvs, vm vtotkv, mv ensatkvyvte; vm vnokeckv, mv ofv vm puyvfekev heren afvekat: vm Puyvfekcvn oh ocares, momen fvcceckvn Centilvlken kerkuecvres.

19 Cevllekot, huehkekares; momen estimvt em opunvkv en hake tehoyvnkv vlkate pohekares.

$20 \mathrm{Kohv}$ tonufke kvcekot, afusyekcv ekkucat vsleceko munket, fvccetvt ohfvnketvn vtohte tvlkvres.

21 Momen en hocefkv ofvn Centilvlke vfekrakkvkvres.

22 Momof puyvfekcv-holwvke oce, heceko, momet opunayekon 'sen yihocen, em wicecvtes; monkv heceko, opunayekate opunayet, hecrtes.

23 Momen este omvlkvt em estomahaket, Heyv Tewe e'ppuce toko haks? makakvtes.

24 Momis Falvsevlke pohof, Heyv hunvnwvt puyvfekcv holwvhoke emet sossicet omekon, momis Pelsepvp, puyvfekcv holwvhokat em mekko, eteropotten sossicet os, makakvtes.

25 Momen Cesvs em vkerrickv taken kerret, hvmmakvtes; Tvlofv em etvwv vnrvpe tekvpakat vtekat hopvnketvn eroret os; momen tvlwvt ot, cuko tateu em etvwr vnrvpe tekvpakat vtekat huyirekos.

26 Momen Setvnet Setvnen ossicen omat, em etvwv e vnrvpēt tekvpakēt os; monkv estomēt em ohmekketv huervr haks?

27 Momen Pelsepvp eteropotten puyvfekev holwvhoke sossicin omat, estimv eteropotten cen hopuetake sossice haks? monkv mvt cem fvececvlke tares. 
28 Momis Hesaketvmese em Puyvfekcv eteropotten puyvfekev holwrhoke sossicin omat, Hesaketrmese em ohmekketvt ec oh vlakvket os.

29 Monkat estimvt, este yekce cuko en ceyet, nakoce em vhopvne tetaye haks? este yekcat taten wrnayet omekon omat, momen omofvt cuko em vhopanvres.

30 Estimvt vc vpvkekat, vc vnrvpes; momen estimvt ve vpaket vteloyekat, vwahecet omes.

31 Monkv ce kicrkis, Holwryeckv vtekat, momen Hesaketvmese tvkleckv omvlkvn este enkvpvyecihocvres; momis Puyvfekev Vcakat tvkleckv este opunayat enkvpvyecihocekares.

32 Momen estimvt o estomet opunvkv este E'chuswv vnrvpen opunayat, enkvpvvyecihocvres; momis estimvt ot Puyvfekev Vcakat vnrvpet opunayat, yvmv ekvnvt ot, ekvnv vlvkvranat to estomis, en kvpryecihocekares.

33 Eto tateu heren hayet, momet em etteu heren hayvks; monkat eto tateu holwvken hayet, em etteu holwvken hayvks; eto em etten es kerhoyet omekv.

34 Eketnv cetio honvpse toyatskat, estomēt ceme holwahokatske tat nake herake opunahoyatske taye haks? efeke sulke nake vtehkat aosse ecukwv opunayet omekv.

35 Este herat e feke ofv em enakockv herat aossen nake herakan 'sossicet omes; momen este holwakat e feke em enakockv holwakat aossen nake holwahokan 'sossicet omes.

36 Momis ce kicvkis, Opunvkv ehvperke vtekat este opunahoyat ohfvececkv nettv omof ohkerkuecvres.

37 Cem opunvkv verkvyen ce fvccrket, cem opunvkv vevkvyen ec ohfvecrkvret okv.

38 Momof nakcokv-hayvlke, momen Falvsevlket em vyoposket, Mvhaya, nake eskerretvn cen hecetv pu yaces, makakat sasvtes.

Muskokee, Matthew, 
39 Momis em vyoposket, Este honvpse holwvhoke momet hoktarvke nake eskerretvn hopoyaket os; momen nake eskerretv e'mhoyekarēs, momis nake eskerretv owalv Convse enake tvlken.

40 Convse nettv tutcenen nereu tutcenen rvro rakke e nvrke ofvn vpikvte etvpomen, este E'chuswv ekvnv e feke ofvn nettv tutcenen nereu tutcenen vpikvret ok.

41 Nenefv hunvntake ohfvcceckv omof akvwvpket, heyv este honvpse ohfvccecvkvres: Convse em erkenvketvn em vkerrickv eticakvtet okv; momen hecvkes, Convse es en rakket heyvmvn likes.

42 Wahvlv mekko-hokte ohfvcceckv omof akvwvpket, heyv este honvpse ohfvccecrres: ekvnv vtekakat vtet Salomv en hoporrenkv pohvranet vlakvtet okv; momen hecvkes, Salomv es en rakket heyvmvn likes.

43 Puyvfekcv hvsvtkekat este osiyof, fekvpkvn hopoyet, momet eshecekot, ekvnv kvrkapan ares.

44 Momof, $\mathrm{Cr}$ hute aossvyvtet oh fulkares, makes; momet vlakof, tvnket, pasket momet mvhericken esheces;

45 Momof ayet, puyvfekcr holwvhoke eme senholwvhoke kolvpaken evpayes, momen esceyet mvn vpokes; momen mv este en hueretv espoket hvteceskvn 'sen holwakes. Heyv este honvpse holwvyecakat em matvpomvres.

46 Este em punaye munkof, hecrs, e'cke momen etecakkakat fettvn svpaklvtes, em punvyetvn eyacaket.

47 Momof, Hecvs, ce'cke, momen ce tecakkakat fettvn svpakles, cem punvyetvn eyacaket, hvmket kicvtes.

48 Momis em vyoposket, Estimvt cv'cket o haks? momen estimvt tecakkakvyat to haks? em onayan kicvtes.

49 Momen e'nke en kerrepvlke oh rvtosehcet, Hecvkes cv'cke, momen tecakkvyat! makvtes. 
50 Estimvt o estomet Cv'rke Hvlwe likat komaten momecat, matvwvt tecakkvyat, momen cv wvnwvt, momen cv'cket omekv, makvtes:

\section{SETENTVCKE XIII.}

Mv Netrv etvwrn Cesvs cuko osset, vkhvse vfopken likvtes.

2 Momen este sulsuke mahet em etohvtelokvtes; monkv perro-cukon eceyet, likvtes; momen este sulsokat omvlkvt lvpvtken svpaklvtes.

3 Momen punvkv svhopakvn nanvke sulken 'sem punayvtes, kicet, Hecvkes, fokayvt ayvtes, fokayvranet;

4 Momen fokayof, nerk $v$ nene vfopken pvlatke sasen, fuswrt yicet, lokakvtes.

5 Cvtovlke, fakke oce mahekan, oh pvlatke sasat lvpkusan hontvtes, fakke en sufken ocvkekokv:

6 Momen hrse kvwapkof, ettopkvtes; momet yuknvtes, yvlonkvn ocvkekokv.

7 Momen etofvske vlkan pvlatke sasen, etofvske hontet, nokcepelakvtes.

8 Momis ekvnv heren oh pvlatke sasvtes; momen em ette, vprlwvt cukpe-hvmken, vpvlwvt pale-epaken, vpvlwvt pale-tutcenen hayakvtes.

9 Estimvt hvcko es pohetv ocat pohekvs.

10 Momen en kerrepvlke yicet, Estomēn opunvkv svhopakvn 'sem punayetske haks? kicakvtes.

11 Em vyoposket, Centake hvlwe em ohmekketv enake ehkakat kerrvranatsken ce'mhoyvket omekv, momis heyv e'mhoyekot omakes, kicvtes.

12 Estimvt o estomet ocat, mvn e'mhoyvret okv, momen sulke mahen ocvres ; momis estimvt o estomet ocekat, mv ocat tis en crwhoyvres.

13 Monkv opunvkv svhopakvn 'sem punayvkit okis; 
hecakat hecvkekot, momet pohakat pohekot, momet kerrvkekokv.

14 Momen mv ofvn Hesiv em owaletv esfuckes, Pohatskat pohatskvres, momet kerratskekares; momen hecatskat hecatskvres, momet kerratskekares :

15 Heyv este e feke take wrnwvhen, momen e hvcko take pohetv tefnet on, e turwv akhottakekv; monkon omat, estofis e turwv es hecet, e hvcko es pohet, e fekeu es kerret, fulotecen em wicecrkvye tayen, makat.

16 Momis ce turwv take en herakes, hecakekv; momen ce hvcko takeu, pohakekv.

17 Mehenwvn ce kicvkikv, Owalvlke sulket, momen este faccvlke, hecatskate- hecetv eyacakis, hecvkekates; momet pohatskat pohetv eyacakis, pohvkekates.

18 Monkv fokayv esvhopakvn pohvkes:

19 Estimvt hvlwe em ohmekketv em punvkv pohis, kerrekat, holwvyecat vlaket, $\mathrm{mv}$ e feke ofv fokahoyvte vkonahes. Heyv tes nene vfopke nerkv cawvte.

20 Momis crto vlkat nerkv cawvte opunvkv pohet, momet lvpece es afvcke cawat tes:

21 Momis eme ofv yvlonkv ocekot, estomusen hofunes : estemerkv. hvtvm asseckv tateu opunvkv omecicen kvwapkof, lvpkusen elentappet omekv.

$22 \mathrm{Mv}$ etofvske vlkat nerkv cawvteu, mv opunvkv pohat tes; momen yvmv ekvnv senaorickvt, momen nakocetv em mvkerretvt opunvkv nokcepeles, momen etteko taye hakes.

23 Momis mv ekvnv here nerkv cawvte, mv opunvkv pohat tes; momet kerret, em etteu ocet, vpvlwvt cukpehvmken, vpvlwvt pale-epaken vpvlwvt pale-tutcenen hayes. 24 Opunvkv-svhopakv etvn e'makvtes, kicet, Hvlwe em ohmekketv este-hunvnwv eme crpofv nerkv heren fokayvte omēt os ; 
25 Momis este nocicof, en horret vlaket, teleko vpvken teleko-vhaken fokahyet, ayepvtes.

26 Momis esse hontet, em ette hayof, teleko-vhakeu heckvtes.

27 Monkv cuko-pucase em vtotkvlke yicet, Pucasē, ceme cvpofv nerkv heren fokayet ometskekat haks? momat estvmv vten teleko-vhake ocet ome haks? kicakvtes. $28 V_{n}$ horret heyv momēcet os, kicvtes. Monkv rpehyet, vteloyeyvre ce yacet o haks? vtotkvlket kicakvtes.

29 Momis, Hekos, kicvtes: teleko-vhake vteloyatskof, teleko svprken lematske witet ok.

30 Hokkolvt etohkvlket mahen, vtelokv orekvs: momen vtelokv oketv orof, vteloyvlke kicares, Telekovhake taten vteloyvks, momet nekrvkueckvn wrnawicvks: momis teleko vn tohto ofvn vtehvks.

31 Ópunvkv-svhopakv ètvn e'makvtes; Hvlwe em ohmekketv este, mvstv enerkv ehset, eme crpofvn ahocvte omēt os:

32 Nerkv omvlkv'sen cotket omat mehenwvt's : momis mahof, vhockuce omvlkvn 'sen rakket os, momet vpe eto ome haken, hvlwe fuswv yicet elvccen oh vpokes, kicvtes.

33 Opunvkv-svhopakv ètvn es em punayvtes: Hvlwe em ohmekketv espakkueckv hoktet cawet, hockvte vkerkv tutcene ofvn ehen, oce munken omvlkvt pakkēs; mv omēt os.

34 Heyv nanvke omvlkv opunvkv-svhopakv taken Cesvs este sulkat 'set'em punayvtes; momet opunvkvsvhopakv tokat te'm punayekates:

35 Opunvkv-svhopakv take ofvn cv cukwv hvwvklecares; ekvnv hocackvte vtekat nake ehkvke ocvte sossicares, maket, owalv opunayvte sohfvckvren. 
36 Momof Cesvs este sulkat vpeyecihcet, cukon eceyvtes; momen en kerrepvlket en yicet, Opunvkv telekovhake crpofv ocat svhopakvn pum oh kerkuecrs, kicakvtes. $37 \mathrm{Em}$ vyoposket, kicvtes; Nerkv here fokayat este E'chuswr tes;

38 Crpofvt yvmv ekvnv tes; nerkv herat hvlwe em ohmekketv en hopuetake tes; momis telekohake holwvyecat en hopuetake tes ;

39 En horre nerkv fokayvte puyvfekev holwakat tes; nakevtelokv yvmv ekvnv em epuksv tes; momen vteloyvlket, encelvlke tes ;

$40 \mathrm{Monkv}$ telekohake vteloyet, totkvn tvk nekrihocet omekv; yvmv ekvnv em eyuksv etvpomvres.

41 Este E'chuswv em encelvlken vtotvres; momen nake elentappuecakat omvlkvn, momet holwvyecakat em ohmekketv asossen vteloyvkvres.

42 Mornet totkv fenken tvk pvlvtvkvres; mvn hvkihketvt enute setetekketvu ocvres.

43 Momof este faccrlke E'rke take em ohmekketvn hvse omēt hvyayvhokvres. Estimvt hvcko es pohetv ocat, pohekvs.

44 Hvtvm, hvlwe em ohmekketv nake vcake crpof ehke omēs; mvn este eshecet, ehēs; momet es afvckat ahyet, nake ocat omvlkvn wiyet, $\mathrm{mv}$ cvpofvn nesēs.

45 Hvtvm, hvlwe em ohmekketv esnesv-hunvnwv, makelitv herake hopoyat, mv omēt omes.

$46 \mathrm{Mv}$ este, makelitv hvmke enrahkv rakken eshecof, ahyet, nake ocat omvlkvn wiyet, nesvtes.

47 Hvtvm, hvlwe em ohmekketv hoyv uehvtkv vk wihoken, rvro mvrahrvkv omvlkv vteloyvte omēs.

48 Momen fackof lvpvtken esacayakvtes ; momet vpoket, herakan ensatket, sak.kvn vtehakvtes; momis holwvhokat vpvlvtakvtes. 
49 Ekvnv em eyuksv momē tares: encelvlket yicet, holwvyecvlken faccvlken etekvpicvkvres,

50 Momet holwvyecvlken totkv fenken tvk pvlvtvkvres; mvn hvkihketvt, enute setetekketvu ocvres.

51 Heyv nanvke omvlkvn kerratskvt haks? Cesvs kicvtes. Henka, Pucasē, kicakvtes.

52 Momof, Monkv nakcokv-hayv, hvlwe em ohmekketv ohfvecv emvhahoyat vtekat, hunvnwv cuko-pucase, nake mucvsvke tis, nake vculvke tis a sossicēs; mv omēt os, kicvtes.

53 Momen mome ocrtes, Cesvs, heyv opunvkvsvhopakv take poyof, mvn enkvpakvtes.

54 Momet em ekvnvn er orof, en senekake take ofvn mvhayakvtes, momen momē mvhayakat estomahes komakvtes; momet, Estvmv vten heyv hunvnwv heyv hoporrenkv, vtotketkv yekcakateu en hecke haks?

55 Heyvt cuko-hayv e'ppuce toko? e'cke Melet hocefkv toko? momen etecakkakat, Cems, Cose, Simv, momen Cutvse esyomakat toko?

56 Momen e wvntakeu omvlkvt epu 'pvkeko? Momat estrmv vten heyv hunvnwv heyv nanvke omvlkvt en hecke haks? makakvtes.

57 Momet eme ofv elentappakvtes. Momis, Owalv em ekvnv momet en cuko ofv tvlken vrakkuehocekot omes, Cesvs kicakvtes.

58 Momen mv vkvsvmvkekat svrahkvn vtotketv yekcvke sulke mvn vtotkekates.

\section{SETENTVCKE XIV.}

$\mathrm{Mv}$ омоғ Helot tetlak Cesvs ohonvkvn pohvtes.

2 Momet, Heyv Cane paptisv tes; pvsatkan a kvwapket omes; monkv vtoketv yekcrket $\mathrm{mv}$ ofvn e heckuecaket omes, em vtotkvlken kicvtes. 
3 Helot, etecakkat Felvpe e'hiwv, Helotev vrahkvn Canen hvlahtet, wrnahyet, cuko-yekcrn vpikvtet okv. 4 Canet, Epayetske tayat cem etektvnkekot omes, kicvtet omekv.

5 Momen elecetv eyacof, este sulkan em penkalvtes, Owalvt's, komvket omekv.

6 Momis Helot heckvte-nettvn vrakkuehocof, Helotev e'chuswv hoktet ehomv taken opanvtes, momet Helot afveecicvtes.

7 Momaten naket o estomen em 'pohis e'mvranet etemfvccetv vcakan 'setemfaccrtes.

8 Momen, enhomvn e'cket emvhayen, Heyvmvn Cane Paptisv e'kvn pvlvknv vpiken v'mes, makvtes.

9 Momen mekko e feke en nokke tates: mome estomis etemfvccetv vcakat 'sopunvyat, vpake hompe vpokate hake vrahkvn, E'mvks, makvtes.

10 Momen vtotet; Cane cuko-yekcv ofvn ekv en tacvtes.

11 Momen e'kv pvlvknv vpiken hokte mvnette 'sen yicet e'mhoyvtes; momen e'cken 'sem vlakvtes.

12 Momen en kerrepvlke yicet, ena ehset, hericakvtes, momet vpehyet, Cesvs em onvyakvtes.

13 Momen Cesvs pohet, mvn enkvpaket perro.cukon 'piket, vnrvwrn entvlket ayvtes: momen este pohakof, yvkvpaket tvlofv esosset vcrkvpeyvtes.

14 Momen Cesvs ayet, este sulke mahen hecet, em merrakusvtes, momet em enokhokat em wicecvtes.

15 Momen yafkof, en kerrepvlket en yicvtes, Heyv vnrvwvt omes, momen nettr hoyvnepet os; este sulkat vpeyecicvs, tvlofuce vlkan vpeyet, hompetvn e en nesvkepvren, makaket.

16 Momis Cesvs, Vpeyekis estōhkos; hompetv e'mvks, kicakvtes. 
17 Momen, Tvklike vnore cahkepat, momet rvro hokkolusat tvlkusen yvmv ocēs, kicakvtes.

18 Yvmvn 'svm vwvks, kicvtes.

19 Momen este sulkat, Pvhen oh vpokvkes, kicvtes, momet tvklike cahkepat rvro hokkolateu cawet, hvlwen es vhecet, ohvkvsamet kvlkayvtes, momet tvklike en kerrepvlken emvtes, momen en kerrepvlket este sulkan emakvtes.

20 Momen omvlkvt hompaket fvcecakvtes; momen wokocke vhoskat svmpv fvcke palen-hokkolohkaken cvwakvtes.

21 Momen $\mathrm{mv}$ hompakat, hunvntake cukpe-rakko cahkepat mahe tates, hoktvke, hopuetakeu svpvkekat.

22 Momen moman vpaken Cesvs en kerrepvlke perrocukon vtehkepuecet, ehomvn tvpalvn vpeyecicvtes, este sulsokat vpeyecicof.

23 Momen este sulsokat vpeyecihcof, ekvnhvlwen entvlkuset ohcemkvtes, mekusvpvranet: momen yafke orof, mvn entvlkusvtes.

24 Momis hiyomat perro-cuko vkhvse vknvrkvpvn, uemelohlvt fekhericekon, vklikvtes, hotvlet vnrvpet omekv.

25 Momen nere vketecetv esostof, Cesvs, vkhvsen oh yvkapet, er em vyakvtes.

26 Momen vkhvsen oh yvkapen en kerrepvlket hecakof, fekhervkekates, Puyvfekcvt's, makaket; momen penkvlaket huehkakvtes.

27 Momis moman vpaken Cesvs em punayet, Ce mahlvpvtkvkekvs; vne tes ; ce penkahlvkates ; kicvtes. 28 Momen Petv em vyoposket, Pucasē, cent on omat, Uewvn oh yvkapet, vc oh vtes, ev kicvs, makvtes.

29 Momen, Vtes, kicvtes: momen Petv perro-cuko osset a vk hvtapkof, uewv oh yvkapvtes, Cesvs oh ayvranet. 
30 Momis yekcetvt hotalen hecof, penkalvtes; momet vksumketvn vlicecof, huehkvtes, Pucasē, cv hesayecrs, maket.

31 Momen moman vpaken Cesvs enke awiyet, hvlatvtes ; momet, Vkvsvmkv estomuse ocetskat, estomēn cen kerkekisa? kicvtes.

32 Momen perro-cuko cukolahkof, hotvle wikvtes. 33 Momof perro-cuko vtehkat yicet, vrakkuecakvtes, Mehenwvn Hesaketvmese E'ppuce toyetskes, kicaket. 34 Momen tiyehcof, Cenesvle ekvnvn yicvtes. 35 Momen, mv tvlofv hunvntake ohkerrakof, mv twlofv afulotat omvlkvn vtotet, enokhokat omvlkvn 'sen yicvtes; 36 Momet en kapv em vtekat tvlkusis celayvkvren em 'pohakvtes; momen celayakat en sulke estomomusat cvfencvke hakakvtes.

\section{SETENTVCKE XV.}

Момоғ nakcokv-hayvlke Falvsevlke esyomat, Celuslvmvlket ot, Cesvs en yicvtes,

2 Estomēn cen kerrepvlke vculvke em punvkv kvcake haks? enke okkosvkekot tvklike hompakekv, kicaket.

3 Momis em vyoposket, Estomēn centakeu, vculvke em punvkv eteropotten, Hesaketvmese em vhakvn kacatske haks? kicvtes.

4 Hesaketvmeset, Ce'rken, ce'ckeu vrakkuecvs; momet, Estimvt e'rke, e'cke tateu ehanen omat, elkvn elekvs, maket opunvyvtet omekv.

5 Momis, Estimvt o estomet erket ot, ecke tateu, Nake cen lopicvye tayat Hesaketvmese emetv tes, kicet;

6 Erket ot, ecke tateu vrakkuecekis, en hervres, centaket makatskes: hiyomēn vculvke em punvkv eteropotten Hesaketvmese em vhakv ehvperken hayatskvtet os. 
7 Mekusapv-vhayvlke toyatskat, heren Hesiv ec oh owalvket okvtes,

8 Heyv este e cukwrn 'svc vwolicaket, e cukhvrpen 'sve vrakkuecakes; momis e feke vn hopvyeces;

9 Momis ehvperkvn vc vrakkuecakes, este em vhakvn 'seste mvhayet, maket.

10 Momen este sulkan enhuehkvtes, momet, Pohet, kerrvkes ;

$11 \mathrm{Mv}$ ecukwv eceyat este vhopanat tokos; momis ecukwv ossat, heyvt vhopanes; kicakvtes.

12 Momof en kerrepvlke en yicet, Falvsevlke heyv maketv pohakof eyacvkekis, kerretska? kicakvtes.

13 Momis em vyoposket, Vhockv Cv'rke Hvlwe likat vhocekate vtekat lemkvres;

14 Fullekvs; turhecvkeko turhecvkekvlke evpayvlket's. Momen turhecekat turhecekan evpayen omat, hokkolvt sufkan vk yorkvres, kicvtes.

15 Momof Petv em vyoposket, Heyv opunvkv svhopakvn pum oh hryvyicrs, kicvtes.

16 Momen, Centakeu kerretv ocatskeko munke haks?

17 Naket o estomet ecukwv eceyat, envrken eceyen, vpvlvthoyet omat kerratskeko munke haks?

18 Momis mv nanvke cukwv asossat efeken a vwet omes; momen mvt este vhopvnaket os.

19 Vkerrickv holwvhokat, eleckv, empvlse oce hoktarkv, empvlse seko hoktarkv, horkopkv, lakse ohkerkueckv, holwvke opunvyetv, efeke asosset omekv.

20 Nanvke este vhopanat heyvt omakes; momis enke okkoseko hompetv este vhopanekos, Cesvs kicvtes.

21 Momof, Cesvs mvn enkvpaket, Tilv, Sitvne tepakat em vtekakan rorvtes.

22 Momen hecvs, hokte Kenvnvlket matvwv em vtekakan aossvtes; momet oh huebket, Vm merrvs, Pucasē, 
Tewe E'ppuce toyetskat; cv'chuswv-hokte tayen puyvfekev holwvken es estemerkes, kicvtes.

23 Momis opunvkv hrmkis em ayoposkekates. Momen en kerrepvlket en yicet, en kusvpakvtes, Etvn vyecicvs, epu 'cvk huehkekv, kicet.

24 Momis em vyoposket, Eslevlke en cuko hvmecate yvpefikv sumecat tvlken ve ohtothoyet os, kicvtes.

25 Momof vlaket, vlakkuecvtes, Pucasē, vm vnicvs, kicet. 26 Momis em vyoposket, Hopuetake en tvklike eset, efv em awiketv fvecekos, kicvtes.

27 Momen, Mehenwrt's Pucasē; momis efv e pucase em ohhompetv oh wokocke a pvlatkat hompaket os; makvtes.

28 Momof Cesvs em vyoposket, Hokte toyetskat, cem vkvsvmkv rakkes; kometskat mahusat cem momekvs, kicvtes. Momen e'chuswv-hokte mv hvse-vkerkv mahusat vteken cvfekne hakvtes.

29 Momen Cesvs mvn enkvpaket, Kalvle em vkhvse vnakvn erorvtes: momet ekvnhvlwen ohçemket, mvn likvtes.

30 Momen este sulsuke mahet en yicvtes, elenokhokat, turhecvkekat, opunahoyekat, vnvttakat, momen etv sulken evpayaket, momet Cesvs e'le tempen tvk pvlvtakvtes; momen em wikvkuecvtes.

31 Momekv opunvyekate opunayen, vnvttakate cvfencvke haken, elenokhokat yvkvpaken, turhecvkekate hecvke haken hecakof, este sulkat em estomahakvtes; momet Eslevl en Hesaketvmese vkvsvmakvtes.

32 Momof Cesvs en kerrepvlke enhohiket, momet, Este sulkan em merrusis, vc vpake munken, momet hompetv ocvkekon, hiyomat nettv tutcenat orekv; elawvke vpeyecihcrkos, vpeyof hotosake witet okv, kicvtes. 
33 Momen, Estvmv vten tvklike sulke momvtekat vnrvwr ofv ocet, este sulke momvtekat fvcecvkueceye taye haks? en kerrepvlket kicakvtes.

34 Momen Cesvs, Trklike vnore nvcomen ocatskc haks? kicvtes. Momen Kolvpaken, rvrocvlkeu nivcomusen, kicakvtes.

35 Momen este sulkan, Ekvnvn tvk vpokvkes, kicvtes.

36 Momet tvklike vnore kolvpakat, rvrou cawet, Hesaketvmesen vkvsamvtes, momet-kvlkayet en kerrepvlken emvtes; momen en kerreprlket este sulkan emakvtes.

37 Momen omvlkvt hompaket, fvcecakvtes; momen hompetv wokocke vhoskat svmpv fvcke kolvpaken crwakvtes.

38 Momen inv hompakat hunvntake cukpe-rakko ostvtes, hoktvke hopuetakeu srpvkekat.

39 Momen este sulkat vpeyecicvtes, momet perrocukon eceyet, Maktelv em vtekakan rorvtes.

\section{SETENTVCKE XVI.}

Momen Falvsevlke, Satusevlken esyomet en yicet, eskerret, hvlwe vte nake-eskerretvn hecicvren komakvtes.

2 Em vyoposket, Yafkofv, Nettv here tares; sutv catet omekv.

3 Momen huthvyvtke, Nettv holwvke tares, mucvnettv; sutv catet ot yomucket okv, makatskes. Mekusapv-vhayvlke toyatskat, sutv en heckv kerratskes; momis oketv eskerretv kerratskeko taye haks?

4 Este honvpse holwvyecvke hoktarakat, nake-eskerretvn hopoyes; momen nake-eskerretv e'mhoyekares; momis nake-eskerretv owalv Convse enake tvlken e'mhoyvres, kicrtes. Momet wikaket, enkvpakvtes.

5 Momen en kerrepvlke tvpalv roricof, tvklike svpeyetv ehosvke tates. 
6 Momof Cesvs, Vketecet, Falvsevlke, Satusevlke esyomat enake espakkueckv em e vfekcakhvkes, en kerreprlken kicvtes.

7 Momen, emetvwvt te'm opunayet, Tvkliken cawēkisekv omes, makakvtes.

8 Momis Cesvs kerret, Vkvsvmkv estomusen ocatskat, estomēn tvkliken es awatskekat omecicen te'm opunahoyatske haks?

9 Kerratskeko munke haks? momet tvklike cahkepe cukpe-rakko cahkepvte, momen svmpv nvcomen cawatskvte,

10 Monkat, tvklike kolvpake cukpe-rakko ostvte, momen svmpv nvcomen cawatskvte vkerricatskeko haks?

11 Estomēt on, Falvsevlke, Satusevlke esyomat enake espakkueckv em e vketecvks, makvyat, tvkliken cem opunv-yecvkit okvkat kerratskeko haks? kicvtes.

12 Momof, tvklike espakkueckvn okekot, momis Falvsevlke, Satusevlke esyomat em emvhakvn oken kerrakvtes.

13 Cesvs, Seselev Felepi em vtekakan rorof, Este E'chuswv toyvyat, estimvt's, este makake haks? kicet en kerrepvlken em 'pohvtes.

14 Momen, Cane paptisvt makakat sasen; vpvlwvt Elivt; momen etvt Cvlemiv, monkat owalvlke hvmkat omes, makakes ; kicakvtes.

15 Momis ceme, estimvt's, cv kicatske haks? kicvtes.

16 Momen Simv Petvt em vyoposket, Mv Klist, Hesaketvmese wenakat E'ppuce toyetskes, kicvtes.

17 Momen Cesvs em vyoposket, Cen heres, Simv Paconv toyetskat: enavpeswv, catv esyomat cem enramet omekokv, momis Cv'rke hvlwe likat.

18 Momen ohvtvlakat ce kicis, Petv toyetskes, momen heyv cvton vm mekusapvlke oh hayares; momen estemerkv-hute em vhauke take emuntvlvkekares. 
19 Momen, hvlwe em ohmekketv es en hauweckvn ce'mares : momen naket o estomen ekvnv oh wrnayetskat hvlwe mimv wrnvke tares; momen naket o estomen ekvnv oh enrecopetskat, hvlwe miruv enrecopke tares.

20 Momof Cesvs, mv Klist tat estimvn em onvyvkvranekon en kerrepvlken yekcen kicvtes.

21. Mvn vteken, Cesvs vlicecet, estomet Celuslvme oh ayvranat; momen nanvke sulken vculvke, momen plestvlke enhomvhhotat, momen nakcokv-hayvlket 'sestemerricvkvranat elecen, momen nettv tutcen of akvwrpkvranat, en kerrepvlke en kerkuecvtes.

22 Momof Petvt hvlahtet em vsehetvn vlicecvtes, Cen hopvyecet omekvs, Pucasē; heyv cem momekares, kicet.

23 Momis afulotket, Petvn, $\mathrm{Cr}$ topvrvn vyvs, Setvnē, vm vnvttecv toyetskes: nanvke Hesaketvmese ohfvecv ocakat cem vlostekot, momis este ohfvecv nanvke ocan, cem vlostet omekv, kicvtes.

24 Momof Cesvs, Estimvt vc vev.kvyetvn komen omat, e em ehvperkuecet, em etohweklepkvn kvwapet, vc vcrkvtekvs, en kerrepvlken kicvtes.

25 Estimvt o estomet en hesaketv vcryecetv komen omat, sumecicen, estimvt o estomet vc vrahkvn en hesaketv sumecicen omat, vcryecrranet omekv.

26 Ekvnv omvlkvn este heckuecen omis, em puyvfekcvn sumecicen omat, naken en lopice haks? monkat naken este em puyvfekcr es vyoposkvr haks?

27 Este E'chuswv em encelvlken evpayet, E'rke en rakketv ofvn vlvkvranet omekv: momen omof em vtotketv vcrkvyen este vtekat em fekvres.

28 Mehenwvn ce kicrkis, Yvmv svpakle sases, elkv lasvkeko munket, este E'chuswv em ohmekketv ofv vlaken thecahke tvlkvranat. 


\section{SETENTVCKE XVII.}

Momen nettv epakat hoyanof, Cesvs, Petv, Cems, momen Cems etecakkat Canen esyomen evpahyet, kvpvken ekvnhvlwe mahen es vcemket,

2 Ehomvn emvrahkvtes, momen e. turofv hvse omèt hryayakvtes; momen em accvke en hvtke hvyayvke omēt omvtes.

3 Momen, hecvs, Moses, Elivs tepaket em e heckuecakvtes, ete'm punvyaket.

4 Momof Petvt em vyoposket, Cesvn kicvtes, Pucasē, yvmv fulleyat heres; kometsken omat, yvmvn cukovhake tutcenen hayvkeres; hvmket cenaket on, hvmket Moses enaket on, momen hvmket Elias enaket on.

5 Punaye munkof, hecrs, vholoce hvyayvket ohranvtes; momen hecvs, opunvkv en hake vholoce aosset, Heyvt Cv'ppuce vm vnokecket, mv ofv vfackusvyat tes; mvn em apohicvks, makvtes.

6 Momen en kerrepvlke pohakof, e turofvn oh pvlatkvtes; momet tayen penkvlvke tates.

7 Momen Cesvs vlaket, celayakvtes, Asvpvklvks, momet ce penkahlvkates, kicet.

8 Momen ahecakof, estimvn hecvkekot, Cesvs tvlkusen hecakvtes.

9 Momen ekvnhvlwe ahvtrpecof, Cesvs, Este E'chuswvt pvsatkan a en kvwvpkeko vtekat heyv hecatskise estimvn em onahyatskvs, yekcen kicakvtes.

10 Momen en kerrepvlket em 'pohaket, Moman estomēn nakcokv-hayvlke Elivs tātet vlvkvranes, makaket oke haks? kicakvtes.

11 Momen Cesvs em vyoposket, Mehenwusen Elivs țātet vlvkvres ; momet nanvke omvlkvn ohcvfeknicvres.

12 Momis ce kicvkis, Elivs vlake hoyvneps, momen Elivs kerrvkekates, momis naket o estomen komakat em 
momecakvtes. Matvpomen este E'chuswvn estemerricvkvres.

13 Momof en kerrepvlket Cane paptisvn em opunvyecet oken kerrakvtes.

14 Momèn este sulkat en yicof, hunvnwvt em vlakvtes, momet en tokkeket,

15 Pucasē, cv'ppucen em merrvs; sokhetvt enokkicet on, tayen estemerkekv : totkv tis tvk latke mahet, uewv tis vk latke mahet omekv, kicvtes.

16 Momen cen kerrepvlken es em vlahkis, moman em wicecvkeko tayis.

17 Momof Cesvs vyoposket, Vkvsvmkv oceko, honvpse yenahatskat, en hofune estomusen ece 'pakvranvki haks? en hofune estomusen vm muntvlkekot 'sece 'yvranvki haks? yvmvn 'svm vwvkes? makvtes.

18 Momen Cesvs puyvfekcr holwakat em vsehvtes; momen aosset enkvpakvtes; momen cepanat mv hvsevkerkv mahusan vteken em wikvtes.

19 Momof Cesvs en kerrepvlke kvpvket en yicvtes; momet, Estomēn pumet ossicēko tayvt haks? kicakvtes.

20 Momen vkvsvmatskekat omecices; mehenwvn ce kicvkikv, Vkvsvmkv mvstv nerkv omusis ocatsken omat, heyv ekvnhvlwe, Yvmvn vkueket, vsvn rorvs, kicatsken omat, vkuekvres; momen nake momecatskeko taye cen svkekares; Cesvs kicvtes.

21 Momis heyv omat eelvwece mekusvpetv ofv tvlken osset omes.

22 Momen Kalvlen vpokof, Este E'chuswv este enke ofvn wihoyvres ;

23 Momen elecvkvres, momen nettv svtutcenof hvtrm akvwrphoyvres, Cesvs kicvtes. Momen e feke tayen en nokhokvtes. 
24 Momen Kvpvnevn roricof, ekv feketv cvtokunawv vteloyvlket Petvn en yicvtes, momet, Ce pucase ekv feketv feket omeko? kicakvtes.

25 Henka; Petvt makvtes. Momen cuko ceyof, Cesvt 'sen homv hakvtes, Simā, naken vkerricetske haks? estimvn ekvnv em mekkvlke vtelokv feketv, monkat ekv feketv en cawe haks? en hopuetaken, monkat tvleporvlken? maket.

26 Tvleporvlke, Petvt kicvtes. Monkv hopuetaken enrecopkes, Cesvs kicvtes.

27 Mome estomis, elentappuecëkaren, vkhvsen ahyet, cufokunhen vkwikvs, momet rvro enhvteceskv akvwapkan esvs, momet e cukwvn en hvwecetskof, cvtokunawvn eshecetskvres; mvn ehset, vc vrahkv ece 'rahkvu emvkvs.

\section{SETENTVCKE XVIII.}

Mv HVSE-vKerkv omof en kerrepvlket Cesvs en yicvtes, Estimvt hvlwe em ohmekketv ofv rakke mahat to haks? kicaket.

2 Momen Cesvs hopuewucen enhohiket, en nvrkvpvn licvtes,

3 Momet, Mehenwvn ce kicvkis, Fulotiket, hopuetakuce omẽ hakatskekon omat, hvlwe em ohmekketv escehyatskekos, kicvtes.

4 Monkv, estimvt o estomis heyv hopuewuce omēt e kvncvpuecen omat, matvwrt hvlwe em ohmekketv ofv rakke mahat tes.

5 Momen estimvt o estomet hopuewuce heyv omẽ hvmken $\mathrm{cv}$ hocefkv ofv evpayen omat, vnen cv yvpayet os.

6 Momis, estimvt o estomet heyv vc oh vkvsvmvke lopockusat hvmken elentappuecen omat, emfulotkv en crtot e nokwvn vtarken, uehvtkv en laukaten vk êlat min en hervnt omes. 
7 Ekvnv en holwakes, elentappueckv take omecicen! elentappueckvt ocvkvranat tvlket omekv: momis en holwakes, mv hunvnwv omecice elentappueckv vlakat! .

8 Monkv ce'nket ot, ce'le tateu, ce 'lentappuecen omat, waret, vpvlvtrs; cekunnet, monkat vnvttetske estomis hesaketv. eceyetskat min heres, ce'nke hokkolen ocet, $c e$ 'leu hokkolen ocet, totkv eme-yuksv-seko ce tvk wihokeko munket.

9 Momen ce turwvt ce 'lentappuecen omat, ossicet vwikvs: ce turwv hvmkusen ocet, hesaketv eceyetskat min heres, ce turwv hokkolen ocet, estemerkv hute ce tvk wihokeko munket.

10 Vketecet, heyv lopockusat hvmkis en homehcatskvs; ce kicvkikv, Hvlwe tvlofv em encelvlket, Cv'rke hvlwe likat e turofv hecake munkvt os.

11 Sumkvten hesayecrranet este E'chuswr vlvket omekv.

12 Estomēn vkerricatske haks? este, yvpefikv cukpehvmken ocen, hvmket em ehosen omat, pale-ostvpakenostvpohkakat wihket, cefahlofvn ayet, mv em ehosan hopoyekot o haks?

13 Momen eshehcvt haken omat, mehenwvn ce kicvkis, $\mathrm{mv}$ yvpefikvn pale-ostvpake-ostvpohkake ehosvkekate semuntvlen oh afvckes.

14 Matvpomen, heyv lopockusat hvmkis sumkvre Ce'rke take hvlwe likat komat tokot omes.

15 Momen etecakketskat cem mvttecicen omat, ahyet em mattan emen tepakusatsken em onvyvs; cem apohicen omat, etecakketskat epoyetskes.

16 Momis cem apohicekon omat, hvtvm hvmiken, monkat hokkolen evpryvs, momat opunvkv vtekat akerrvlke hokkole monkat tutcene e cukwv ofvn yekce taren.

17 Momer em apohicetv komekon omat, mekusvpkv- 
cukon em onvyvs : momis mekusvpkv-cukon em apohicetv komekon omat, Centilvlke momet cvtokunaẉv vteloyv omët ce homvn huerekvs.

18 Mehenwvn ce kicvkis, Naket o estomen ekvnv oh wrnayatsken omat, hvlwe mimv wvnvke tares; momen naket o estomen ekvnv oh enrecopatsken omat, hvlwe mimv enrecopke tares.

19 Hvtvm ce kicvkis, Yvmv ekvnun hokkolet nake vpohvranatskat ohfvecvn etem vkvsvmatsken omat, Cv'rke Hvlwe likatet em momecvkvres.

20 Estvmvn hokkolet monkat tutcenet ev hocef $k v$ ofvn etohnvkaften omat, mvn vnet en nvrkvpvn huerikv.

21 Momof Petvt em vlakvtes, momet, Pucasē, em etewole estomusen etecakkvyat vc vnaorkat, em merrar haks? Kolvpake oren? kicvtes.

22 Cesvs kicvtes, Kolvpake oren ec kicvkos; momis, Pale-kolvpakvke kolvpakat oren.

23 Monkv hvlwe em ohmekketv mekko hvmke, mv em vtotkvlke em vhuere 'sen kerkuecvranate omēs:

24 Momen es kerkuecetv vlicecof, hvmken talentv cukpe-rakko palen em e vhuericen 'sen yihocvtes.

25. Momis ocet fekeko tayckv, e pucaset, E hiwv, en hopuetakeu, nake ocat omvlkv svpvken wihoyen, feketvt hakekvs, makvtes.

26 Monkv em vtotkv tvk latket vrakkuecakvtes; $\mathrm{Pu}$ casē, v'mehakvs, momen omvlkvn cem fekares, kicet.

27 Momof, mv vtotkv e pucase, merkvt nekeyicen, enrecopet em vhuere 'sem wikvtes.

28 Momis $\mathrm{mv}$ vtotkv etvwvt osset, vtotkvlke em etohkvlketv hrmken, tenelyv cukpe hvmke em e vhuericen eshecrtes.: momen hvlahtet, e nokwvn em eset, Ece 'huerēn vm fekvs, kicvtes.

29: Momen vtotky em etohkvlkety e'le tempen tvk lat- 
ket, en kusapvtes, V'mehakvs, momen omvlkvn cem fekares, kicet.

30 Momen komekates; momis ayet, enko-yekevn 'rvpikvtes, em vhuere em fehke tvlkvren.

31 Monkv vtotkvlke em etohkvlketv, nake estomat hecakof, e feke nokhokusvtes, momet yicet, nake estomat omvlkvn e pucasen em onvyakvtes.

32 Monkv e pucase enhohikof, Vtotkv holwryece toyetskate, $m v$ vhuere omvlkvn es cem wikryvnks, rm 'pohetskekv:

33 Vne cem merrvyvnke omēt, cemeu vtotkr cem etohkvlketv em merretskeko tayvt haks?

34- Momen e pucase crpakkvtes; momet, em e vhuericat omvlkvn em fehke twlkvren, estemerriculken em wikvtes.

35 Momen etecakkat naorkat ce feke take vten 'sem wikatske vlke tokon omat, Cr'rke hvlwe likat matvpoinen cem momecrkvres.

\section{SETENTVCKE XIX.}

Momen mome ocvtes, Cesvs heyv opunvkv espoyof, Kalvlen enkvpakvtes, momel Cotvne tvpalv Cutev em vtekakan rorvtes;

2 Momen, este sulsuke mahet vovkvpeyvtes; momen mvn em wicecakvtes.

3 Falvsevlkeu en yicet, Hunvnwv nake omecicat vtekat e hiwv wiketv em etektvnke te? eskerraket kicakvtes.

4 Momen em vyoposket, Mr enhyteceskv hayat, hunvnwv, hokteu hayvte,

5 Momet, Heyv omecicen hunvnwv erke, eckeu wihket, e hiwvn hvlvtvres; momen mv hokkolat vpeswv hvmkusvres, kicvte, ohonayatskvte seko haks? kicvtes.

6 Monkv $\mathrm{mv}$ svnvcomvn hokkolet omeks, momis 
enavpeswv hvmkus; monkv mv Hesaketvmese tehvlvtepuecvte estet etekvpihcates; kicvtes.

7 Moman, Estomēn Moses, Nakhocce esetewiketvn ehmet, wikekvs, makvt haks? kicakvtes.

8 Ce feke take em wvnhe omecicen Moses ce hitake wiketv cem etektvnecvtes; momis enhvteceskv atat momekot omvtes, kicvtes.

9 Momen ce kicvkis, Estimvt, e hiwv hoktarekofvn wiket, etvn epayen omat, hoktaret omes; momen mv wihokat estimrt epayen omat, hoktaret omes.

10 Hunvnwv e hiwv ohfvecv em momẽt on omat, etepvyetv hereks, en kerrepvlket kicakvtes.

11 Momis, Este omvlkvt heyv maketv esvkeko tayes; momis e'mhoyvte tvlket, kicvtes.

12 Yunvkvlket sasekv, e'cke e nvrke ofv mome hocvckvtet; momen yunvkvlket este hahicvtet sases; momen hvlwe em ohmekketv vrahkv emetvwv e hayvtet sases. Estimvt ese tayen omat, esekvs.

13 Mómof hopuetakucen 'sen yihocvtes, enken oh wvkhokicet, mekusvpvren ; momen en kerrepvlket em vsehakvtes.

14 Momis, Hopuetakuce vc oh vwepvre em etektvnecet, em vsehhatskvs; mv omakat hvlwe em ohmekketvt omekv, Cesvs makvtes.

15 Momet enke oh wvkhokicahket, mvn enkvpakvtes.

16 Momen hecvs, hvmket em vlaket, Mvhayv heretskate, hesaketv emunkv ocaret, nake here estoman momecare te? kicvtes.

17 Momen, Estoman here cv kicetslie haks? este here sekos, momis hvmkuset, Hesaketvmese tes : momis hesaketv ceyetvn kometsket on omat, vhakv taken vfvstvs, Cesvs kicvtes. 
18 Estomaten? kicvtes. Elehcetskvs, Hoktahretskvs, Horkohpetskvs, Fvccekon akeriyetskvs,

19 Ce'rke, ce'ckeu vrakkuecvs : momet, $\mathrm{E}$ vnokecetskat etvpomen cem vwolicvn vnokecrs, Cesvs kicvtes.

20 Hunvnwv mvnettat, Heyv nanvke omvlkvn cv mvnette vteke vfaste vtecicvyvtes: naket vm vhoske munke haks? makvtes.

21 Temvpoke haketvn kometsken omat, ahyet, nake ocetskat wiyet, estemerrakaten emvs; momen hvlwetvlofvn nake ockv ocetskvres: momet atet, vc vcrkvyvs, Cesvs kicvtes.

22 Momis hunvnwv mvnettat mv maketvn pohof, efeke nokket ayvtes; enake sulece mahet omekv.

23 Momof en kerrepvlken, Mehenwvn ce kicvkis, Este enakocr hvlwe em ohmekketv ceyetv en yekce tares.

24 Momen hvtvm ce kicvkis, Rvcce-kuphe esropottv e turwv ropotte tayat en yekce mahekon, este enakocr mimvt Hesaketvmese em ohmekketv eceyetvt en yekces, Cesvs kicvtes.

25 En kerrepvlke pohakof, tayen em estomahakvtes, Monkv estimvt hesake taye haks? makaket.

26 Momis Cesvs oh hecaket, Estet on omat heyv em momvkeko tayuses: momis Hesaketvmese nanvke omvlkvt em momvke tayes, kicvtes.

27 Momof Petvt em vyoposket, Hecvs, pume omvlkvn wihket, ece 'crkvpeyemvts; monkv naket pun heckvr haks? kicvtes.

28 Momen, Mehenwrn ce kicvkis, ceme svnvcomv hecketv omof, vc vcrkvpeyatskvte este E'chuswv em ohliketv-vcakan oh likof, centakeu, Eslevl em vliketv pale hokkolohkakan ohfvececet, ohliketv vcacvke palenhokkolohkakan oh vpokatskvres.

29 Momen cv hocefkv vrahkv, cuko tis, etecakkvte tis 
ewvntake tis, erke tis, ecke tis, ehiwv tis, hopuetakuce tis, ekvnv tis, wikakvte vtekat, cukpe-hvmke erohvtvlvket en heckvkvres, momen hesaketv yuksv-sekon epucasvkvres.

30 Momis enhvteceskvt sulket espoke tares; momen espoket enhrteceskv tares.

\section{SETENTVCKE XX.}

Hvwwe em oHмerketv hunvnwv cuko epucase omët omes, vtotkvlke em pvrko-cvpofv sohceyvre pvlvrane hvthvyvtkuse ossvte omẽt omekv.

2 Momen vtotkvlke tenelyv hvmke nettv vrahkv etemvkvsahmof, em pvrko-cvpofvn ohtotvtes.

3 Momet hvse-vkerkv svtutcenat mahen osset, etvt vtotkekot nakwikv ocan svpaklen hecvtes,

4 Momet, Cemeu prrko-crpofv seceyvks, momen estomuset fvecvt on omat ce'mvkares, kicvtes. Momen vpeyvtes.

5 Hvtvm hvse-vkerkv sepakat momet ostvpakat mahen osset, matvpomecrtes.

6 Momet hvse-vkerkv palen-hvmkontvlakat mahen ossvtes, momet etvt vtotkekot svpaklen hecvtes, momet, Estomēn vtotkekot svpaklatsken yafke haks? kicvtes.

7 Estimvt pu pvlekokv omes, kicakvtes. Cemeu pvrko-cvpofv seceyvks; momen estomuset fvecvt on omat, cen heckvkvres, kicvtes.

8 Monkv yafke orof, pvrko-crpofv e pucase, Vtotkvlken enhohiket, em fekvkvs, espoken vlicecet hvteceskvn vteken, en nakvfastvn kicvtes.

9 Momen hvse-vkerkv pale-eshvmkontvlake mahe pvlhoyat yihcof, este vrahkv tenelyv hvmke vlket en heckakvtes.

10 Momis enhvteceskv pvlhoyat yicof, 'senhoyvnet pun 
heckvranēs, komakvtes: momis mvu, este vrahkv tenelyv hvmke vlket en heckakvtes.

11 Momen en heckahkof, cuko epucase em ecayakvtes,

12 Heyv espoke hvse-vkerkv hvmkuse vtotkakat, pume, hotoskv, hiyye entekkekeyat etvpomen hayetskes, kicaket.

13 Momis hvmken em vyoposket, Vn hesse, naken cem vhopanvks; tenelyv hvmken vm vkvsvmetskekat haks?

14 Cenaken esehpet, vyepvs; ce'mvyat etvpomen heyv espoke emares.

15 Crnake naken komvyat momecryis fvccut omeko haks? $\mathrm{Cv}$ herekv, ce turwvt holwake haks?

16 Monkv espoket hvteceskvt omen, hvteceskvt espoke tares: sulken enhuehokvte tisem, nvcomet enhopoket omekv, kicvtes.

17 Momen Cesvs, Celuslvmen ayof, en kerrepvlke pale-hokkolohkakat nenen vpeyof, kvpvken evpayvtes:

18 Momet, Hecvks, Celuslvmen vpeyēs; momen este E'chuswv plestvlken nakcokv-hayvlken esyomen em wihokvres, momen ele tayen ohfvececrkvres,

19 Momet Centilvlken em wikahken, nakhayekot, tepket momet tohweklepkvn vtvrvkvres; momen nettr svtutcenof hvtvm akvwvpkvres, kicvtes.

20 Momof, Sepete e'ppuce take e'cket e'chushunvntaken evpayet, es em vlakvtes, vrakkuecet, momet nake hvmken em eyacet.

21 Momen, Naken kometske haks? kicvtes. Heyv cv'chushunvntake hokkolat, cem ohmekketv ofv, hvmket cem vkvpervn liken, hvmkatet cem vkvsnvnvn likvren emvs, kicvtes.

22 Momen Cesvs vyoposket, Naken vpohatskat kerrats- 
keks. Sesketv 'seskvranvyat 'seskatske tayet, momet paptiskvn es paptiskvyat, es paptiskatske taye haks? kicvtes. Momeye tajes, kicakvtes.

23 Momen, Mehenwusen sesketv 'svm eskatskvres, momet paptiskvn es paptiskvyaten es paptiskatskvres; momis vm vkvperv mornet vm vkvskvnv likvre emetv crnake tokos; momis estimvn Cv'rket em etetakuecvten e'mvkhoyvres, kicvtes.

24 Momen $\mathrm{mv}$ palat pohakof, tecakkvte hokkolaten vnrvpet crpvkhokvtes.

25 Momis Cesvs enhohiket, Centilvlke em mekkvlke ohfvnketvn, momen mv rvkrakat yekcetvn 'sohfvnken, kerratskes, kicvtes.

26 Momis cem etenrvwr take momvkekares; momis ece 'pakvkat estimvt rakke haketvn komen omat, cem vtotkv taket omekvs :

27 Momen estimvt ce'nhomvhtetv taken komen omat, cem vtotkv taket omekvs :

28 Este Ec'huswv tisem, em vtothokvranekon, momis vtotkvranet, momet en hesaketv sulke es nesetvn est' emvrane vlakvte etvpomen.

29 Momen Cvleko enkvpvkakof, este sulke mahet vevkvpeyvtes.

30 Momen hecvs, este turhecrkeko hokkolet nene tempen kaket, Cesvs hoyanen pohakof, Pucase, Tewe E'ppucē, pum merrvs, makaket huehkakvtes.

31 Momen este sulkat em vsehakvtes, cryayvhokvren : momis svheremahen huehkaket, Pucase, Tewe E'ppucē, pum merrvs, makakvtes.

32 Momen Cesvs huyiret, enhuehket, Naken cem momecvkaren komatske haks? kicvtes.

33 Pucasē, pu turwvt en hauhvkvren, kicakvtes.

34 Monkv, Cesvs, em meriyet, e turwv en celayakvtes; 
momen moman vpaken e turwv renakv en heckahken, vcrkvhoyvtes.

\section{SETENTVCKE XXI.}

Momen Celuslvmen vwolicaket, Perfec, ekvnhvlwe Olefvn yicof, Cesvs en kerrepvlke hokkolen vtotet,

2 Tvlofuce ce homuse ocan cukolvkvks, momen moman vpaken corakko-wakv wvnvken eshecatskvres, momen echuswvt vpvken; enrecohpet, 'svm vthoyvks.

3 Momon estimvt naken ce kicvken omat, Pucaset eyaces, kicatskvres; momen moman vpaken atotvres, kicvtes.

4 Momen heyv omvlkvt momvtes, owalv hvmmaket, opunayvte esfvckvren,

5 Sivn e'chushokten 'monvyvkes, Hecvs, cem Meklko cem vlaket os, eyaskuset, corakko-wakv, corakko-wakv e'chuswr tisem oh liket.

6 Momen mv en kerrepvlke vhoyet, Cesvs kican momecakvtes,

7 Momet corakko-wakv, echuswv tepakvn svlahokvtes, momet em vchoyetvn oh ocaket, mvn oh licakvtes.

8 Momen este sulke mahet em vchoyetvn nenen oh pvtapicen, vpvlwvt eto lvecen a waret, nenen oh pvtapicakvtes.

9 Momen este sulke homv vpeyen, momen yupv vpeyateu huehkaket, Tewe e'ppuce Hosanv em ocekvs: Hesaketvmese hocefkv ofv vlakat vkvsvmket omes: Hosanv hvlhvwe mahat ofvn.

10 Momen Celuslvme er oh ceyof, Tvlofv omvlkvt nekeyvtes, Heyv estit o haks? makaket.

11 Momen este sulkat, Heyvt Cesvs, Nasvle owalv Kalvle ekvnv aossat tes, makakvtes.

12 Momen Cesvs Hesaketvmese en cuko vcakan ceyv- 
tes; momet $\mathrm{mv}$ ofv vpoke, nake wiye nesakaten sossicet, cvtokunawv esyoposkvlke em ohhompetv, prce-howe wiyakat em ohvpoketvu en cektulcecrtes.

13 Momen, Vn cuko mekusvpetr cukon kihocvres, maket coket os, momis centake horkopvlke em vpoketvn hayatskes, kicvtes.

14 Momen turhecvkekat, yvkvpvkekateu cuko rcakat ofvn en yicen, em wicecakvtes.

15 Momen nake estomahvke momècen, momen cuko. vcakat ofvn hopuetakeu, Tewe e'ppuce Hosanv em ocekvs, makaket huehkaken, plest enhomvhhotat, nakcokrhayvlkeu hecakof, eyacake sekates,

16 Momet, Heyv nak makakat pohetske haks? kicakvtes. Momen Cesvs, Henka; Hopuetakuce, momen cokakusat e cukwv ossen vkvsvmkv etetakuecetskes, makvte ohonayatskvte seko haks? kicvtes.

17 Momet enkvpaket, tvlofv ossvtes, momet Pervnen ayet, mvn fekhonnvtes.

18 Momet hvthvyvtken, tvlofv ohfulket, elawvtes.

19 Momet ke-rakko em vpe nene tempen hecof, oh ayvtes, momis naket oh sekon, esse tvlket oh ocen eshecvtes, momet, Mucv ayat ette ec oh ocvte sekot es rnunkekvs, kicvtes. Momen moman vpaken ke-rakko em vpe yuknepvtes.

20 En kerrepvlke hecakof, em estomahakvtes, Vyvkhvmkusen ke-rakko em vpe yuknepa! makaket.

21 Momen Cesvs em vyoposket, Mehenwrn ce kicvkis, vkvsvmkvn ocet, cen kerkvket on omat, heyv ke-rakko em vpe em momat tvlken momecet omatskekares; momis heyv ekvnhvlwe, Vkueket, uehvtkvn ce'k wihokekvs, kicatsken omat, momvres.

22 Momen nanvke vtekat omvlkv mekusvpkv ofv vkvsamet vpohatskat cen heckvkvres. 
23 Momen cuko-vcakan cehyet, invhayet likof, plest enhomvhhotat, este em vculvke esyomat en yicvtes, momet, Yekcetv estomen heyv nanvke es momecet ometske haks? momen estimvt heyv yekcetv ce'me haks? kicakvtes.

24 Momen Cesvs em vyoposket, Vneu nake hvmken ce 'mpohvranvkis, momen mvn vm onayatsken omat, vneu matrpoinen yekcetv estomen heyv nanvke es momecit omvyat cem onvyvkares, kicakvtes.

25 Cane em paptiskv, estrmvn vte tat haks?- hvlwen vte tat haks? monkat este enake tāt haks? kicvtes. Momen e etem punvyaket, Hvlwe vten; makēn omat; Monkv, estoman vkvsvmatskekat haks? pu kicvres.

26 Momis, Este enaket's; makēn omat, esten em pu penkvlvkës; omvlkvt Canen owalv omecvket omekv.

27 Momen Cesvs em vyoposkaket, Kerrēkos, makakvtes. Momen, Vneu, yekcetv estomen heyv nanvke es momecit omvyat, cem onvyvkvkos, kicvtes.

28 Momis estomen vkerricatske haks? Este hvmket eppucetake hokkolicet ot, enhvteceskvn em vlahket, $C v$ 'ppucē, vm pvrko evpofvn ayet, mucv-nettvn vtotkvs, kicvtes.

29 Em vyoposket, Momvranvkos, kicvtes. Momis renyupvn, em vkerrickvn etihcet, ayvtes.

30 Momen 'svhokkolan em vlahket, matvpomen kicvtes. Momen em ryoposket, Vyares, Pucasē; kicet, ayekates.

$31 \mathrm{Mv}$ hokkolat estomatet erke komat momecvt haks ? Enhvteceskv, kicakvtes. Mehenwrn ce kicvkis, Crtokunawv-vteloyvlke hoktarvke esyomat ce homv taken Hesaketvmese em ohmekketv esceyet os; Cesvs kicvtes.

32 Cane frccetv vyetv ofvn cem vlakvkvtet omekv; 
momen vkvsvmatskekvnkes; momis cvtokunawv-vteloyvlke hoktarvke esyomat vkvsvmakvnkes; momen ceme hecatskof, rohyupv cem vkerrickv eticatskekvnkes, oh vkvsvmatskvret.

33 Opunvkv-svhopakv etvn pohvkes; Cuko-epucase hvmket pvrko cvpofvn vhocvtes; momet em vtohopkihcet, ofvn pvrko esopecetvn korvtes; momet cuko hvlwen hahyet, vfastvlken em wikvtes; momet ekvnv hopvyen ayvtes.

- 34 Momis, ette em oketv vwolicof, em vtotkvlken vfastvlken ohtotvtes, em ette en heckvkvren.

35 Momen vfastvlke em rtotkvlken cahwet, hvmken tepiket, hvmken elehcet, momet hvmken cvton es nvfkakvtes.

36 Hvtvm vtotkvlke etv enhvteceskv 'sen sulken atotvtes; momen matvpomecakvtes.

37 Momis espoke e'ppucen ohtotvtes, Cv'ppuce rrakkuecvke tares, maket.

38 Momis eppucen vfastvlke hecakof, e etem punahoyet, Heyvt epucase taranat tes, vwvkes, elecvkeres; momet en heckvranvten cvwepvkeres; makakvtes.

39 Momet ehset, pvrko cvpofv ossihcet, elecakvtes.

40 Momekv pvrko crpofv e pucase vlakof, heyv vfastvlke estomecvr haks?

$41 \mathrm{Mv}$ holwvyecvlke tayen estemerrvkuecet, pvsatet, pvrko cvpofv vfastvlke etv em ette em oketv orof emvkvren em wikvres; kicakvtes.

42 Tohtarvlke cvto wikvte, matvwvt kvnowv em ekv hakes: heyvt Pucase nake momecat tes: momet pun renakv ehomv estomahet os, makat, Nakcokv-rakko ofv ohonayatskvte seko haks?

43 Monkv ce kicvkis, Hesaketvmese em ohmekketv cem ehset, tvlwr etv em ette es yican emhoyvres. 
44 Momen estimvt heyv cvton oh latkat kvckvres; momis estimvn oh latkat lopotvres, Cesvs kicvtes.

45 Momen plest enhomvhhotat momen Falvsevlke em opunvkv-svhopakrn pohakof, emen opunvyecet oken kerrakvtes.

46 Momis esetvn komakof, este sulsokan em penkvlakvtes; Owalv tes, komvket omakekv.

\section{SETENTVCKE XXII.}

Momen Cesvs em vyoposket, opunvkv-svhopakv taken hvtvm 'sem punayvtes, hvmmaket,

2 Hvlwe em ohmekketv mekko etepvyetv e'ppuce en hayvte omēs.

3 Momet em vtotkvlke etepvyetv oh vwrrane kihocvte enhuehkvren vtotvtes: momen vwetv eyacrkekates.

4 Hvtvm vtotkvlke etvn vtotvtes, Enhuehhokvte em onvyvks; Hecvkes, vn hompetv-rakko etetakuecis; vm wakvlke, momen nake nehakat prsvthoyes: momen nanvke omvlkvt etetakes; etepvyetv oh vwvkes, kicvtes.

5 Momis vrakkuecvkekates; momet vpeyepvtes, hvmket eme crpofvn ayen, hvmket en nakneskvn ayen;

6 Momen vhoskat em vtotkvlken cahwet, vlesketr en hayet pvsvtakvtes.

7 Momis mekko pohof, cvpakkvtes; momet en suletawvlke vtohtet, mv este prsatvlke prsatvtes, momet eme tvlofv nekricvtes.

8 Mohmet em vtotkvlken, Etepvyetv etetakes, momis $\mathrm{mv}$ enhuehhokvte heretv oricvkekvnks;

9 Momekv nene tvleme vlkan vpeyvks; momet en sulke estomēn eshecatskat etepryetv enhuehkvks, kicvtes.

10 Momen mv vtotkvlke nene tvleme vlkan sossvtes, 
momet hecat vtekat omvlkvn, holwvhokat herakat esyomen vteloyakvtes: momen etepryetv hompvlket tetayet heckvtes.

11 Momen mekko hompvlken hecvranet eceyof, hunvnwv hvmket etepvyetv accrke vccekon hecvtes:

12 Momet, Vn hessē, estomaten etepryetv accvke vccekot ye cehyetske haks? kicvtes. Momen opunvyeko tayvtes.

13 Momof, Elen enken esyomen wrnayvks; momet etvn svpeyet, fettv yomuckofvn er vwikvks; mvn hvkihketvt, momen enute setetekketvt ocvres, mekkot vtotkvlken kicvtes.

14 Sulken en huehhokvtet omis nvcomen en svthoket omekv, Cesvs kicvtes.

15 Momof Falvserlke vpeyvtes, momet estomecet em opunvkvn es ese tayat etem punahoyvtes.

16 Momet en kerrepvlken, Helotvlken esyomen ohtotakvtes, hvmmaket, Mvhayā, mehenwetsket ot, Hesaketvmese em vyetv mehenwvn este mvhayet, hunvnwv estoskometskekat kerrēs; este en heckv moskometskekot omekv.

17 Momekv pum onvyvs, Estomen vkerricetske haks? Sesv ekv feketv emetv fvccut o haks? monko haks?

18 Momis, Cesvs en holwvyeckv taken kerrof, Mekusapv-vhayvlke toyatskat, estomēn es cv kerratske haks?

19 Ekv feketv cvtokunawrn cv hecicvkes, kicvtes. Momen tenelyvn 'sen yicrtes.

20 Momen, Heyv estimv vhakēt on, momen en hoccvt o haks? kicvtes.

21 Sesv enakē, kicakvtes. Momof, Monkv Sesv enanvken Sesvn emvks; momet Hesaketvmese enanvken Hesaketvmesen emvks, kicvtes. 
22 Momen pohahket, em estomahakvtes; momet enkvpaket, vpeyepvtes.

$23 \mathrm{Mv}$ nettv Satusevlket en yicvtes, mv, Elkv akvwvpketv sekos, makakat, momet em 'pohaket,

24 Mvhayā, Hunvnwv hopuetake ocekot elen omat, etecakkatet ehiwv epayet, etecakkan honvpse em mahecvres, Moses makvtes.

25 Momen etecakkvte kolvpaket epu 'paket fullvtes; momen hvteceskvt hokte epahyof, elvtes, momet, honvpse ocekot, e hiwv etecakkan em wikvtes :

26 Esvhokkolateu, momen esvtutcenateu, eskolvpakan vteket etvpomusvtes.

27 Momen omvlkv 'sem poken hokteu elvtes.

28 Monkv akvwrpketv omof, mv kolvpakat estoman e hiwv tar haks? omvlkvt epayvkvtet omekv.

29 Momen em vyoposket, Nakcokv-rakkon, momet Hesaketvmese en yekcetvu kerrekot, cem etehoskvket os.

30 Akvwvpketv omof, etepayvkekot, etepayepuehohcekos, momis hvlwe tvlofv Hesaketvmese em encelvlke omvket omakes.

31 Momis este prsatkat a en kvwrpketv ohfvecrt on omat, Hesaketvmese nake cem punayat,

32 Vne Eplehame en Hesaketvmese, momet Iseke en Hesaketvmese, momet Cekvpe en Hesaketvmese toyis, makvte, ohonayatskvte seko haks? Hesaketvmese pvsatkat en Hesaketvmese tokot os, momis hesahokat en Hesaketvmese tes, Cesvs kicvtes.

33 Momen este sulkat pohakof, Cesvs nake emvhayat em estomahakvtes.

34 Momis Satusevlken cryayicen Falvsevlket pohahket, etohvtelokvtes.

35. Momof mv hvmket, vhakv mvhayvt ot, eskerret em 'pohvtes, hvmmaket, 
36 Mvhayā, vhakv ofv estrt vhakv rakkat to haks?

37 Pucase cen Hesaketvmesen ce feke omvlkvn, cem puyvfekev omvlkvn, momet cem vkerrickv omvlkvn es vnokecrs.

38 Heyvt vhakv enhvteceskv, momet rakkat tes.

39 Momen esvhokkolat mv omēt omes, E vnokecetskat etvpomen cem vwolicvn vnokecvs.

40 Heyv vhakv hokkolan vhakv owalvlke esyomat omvlkvt vtvrtvkes, Cesvs kicvtes.

41 Falvsevlke etohnvkaftof, Cesvs em 'pohvtes,

42 Klist estomēn oh vkerricatske haks? Estimv e'ppucet o haks? kicet. Tewe e'ppuce, kicakvtes.

43 Moman estomēn Tewet Puyvfekev of rn Pucasen kicet ome haks?

44 Cehofvt, cv Pucasen, Vm vkvpervn liketske munken, cen horre ce 'leohhuerickvn hahyvrs, kicvtes, makvte.

45 Monkv Tewet Pucasen kicen omat, estomēn e'ppucet o haks? kicvtes.

46 Momen este estomet punvkv hvmkis em vyoposkeko tayvtes: momen mv nettv vtekat estimvt svnvcomv em 'pohetv fekhvmkekates.

\section{SETENTVCKE XXIII.}

Momof Cesvs este sulkat en kerrepvlke esyomen em punayvtes, hvmmaket,

2 Nakcokv-hayvlke Falvsevlke esyomat Moses em ohliketvn oh vpokes;

3 Monkv, nanvke estomen, Vketecvks, ce kicvkat omvlkvn vketecet, momecvkes; momis em vtotketv vevkvye mohmatskvs; makakisem, momecrkekot omekv. 4 Sapokv honhoye ecukoyēsko tayen wrnawicet, este e foluwvn em oh ocaket, momis e'nkewesakv hvmkusis es vkueyicetv komvkekot omekv. 
5 Momis em vtotketv omvlkvn estet hecvkvren omakes; se en tvkhvketv tvptvhen hahicet, kapv em vteken rakrvkuecaket os,

6 Momet hompetv-rakko omof, liketv hvlhawan, momet senekake take ofv liketv enhomvhhotan,

7 Momet nakwikv vlkan vseket, Lapi, Lapi, kihocvren em vlostvket omes.

8 Momis ceme Lapi ce kihohevkates; hvmket, Klist, ce Mvhayv taket omekv; momen omvlkvt etecakkvte toyatskes.

9 Momet, estimvn yvmv ekvnv Pu'rke kihcatskvs; hvmket Ce'rke taket omekv, mv hvlwe likat.

10 Monkat mvhayvlke ce kihohevkates ; hvmket, Klist, ce Mvhayv taket omekv.

11 Momis ece 'pakvke rakke mahat cem vtotkv tares.

12 Momen estimvt o estomet e hvlwecen omat, kvncrpvres; momen estimvt e kvncvpuecat, hvlwehocvres.

13 Moinis cen holwakvkes, nakcokv-hayvlke momen Falvsevlke, mekusapv-vhayvlke toyatskat! hvlwe em ohmekketv este e homv vkhottatskekv ; cemeu esceyatskekot, esceyateu esceyetv em etektvnecatskekokv.

14 Cen holwakvkes, nakcokv-hayvlke momen Falvsevlke, mekusapv-vhayvlke toyatskat! hoktvke-oyevlke en cuko lokatskekv, momet vhayet crpcrken mekusapatskes; monkv ohfvcceckv senrakket cen heckvkvres.

15 Cen holwakvkes, nakcokv-hayvlke momen Falvsevlke, mekusapv-vhayvlke toyatskat! hvmkuse vkvsvmepuecatskvret; uehvtkv, ekvnvu hvmecicatskes, momen cem vkvsamvkof, estemerkv em estuce centake ome svhokkolen hayatskes.

16 Cen holwakvkes, evpayvlke turhecekvlke toyatskat, Estimvt o estomet cuko vcakat es hocefet opunayen omat, estomv tokos; momis estimvt o estomet, cuko 
vcakat en cvtokunaplanen es hocefet opunayen omat, vhuerēs; makatskat.

17 Hoporrenvkeko momen turhecekvlke toyatskat: cvtokunaplanet, monkat cuko vcakat cvtokunaplane vcake hayat estomat senrakket o haks?

18 Momen, Estimvt o estomet altv es hocefet opunayen omat, estomv tokos; momis estimvt o estomet wiyetv oh ocan es hocefet opunayen omat, vhuerēs.

19. Hoporrenvkeko, momen turhecekvlke toyatskat: wiyetvt, monkat altv $\mathrm{mv}$ wiyetv vcake hayat estomat senrakket o haks?

20 Monkv estimvt altvn es hocefet opunayen omat, mvn momet nake oh ocakat omvlkvn es hocefet opunayet os.

21 Momen estimvt cuko vcakan es hocefet opunayen omat, mvn momet mv ofv likateu es hocefet opunayet os. 22 Momen estimvt hvlwen es hocefet opunayen omat, Hesaketvmese em ohliketv vcakan momet mv ohlikateu es hocefet opunayet os.

23 Cen holwakvkes, nakcokv-hayvlke momen Falvsevlke, mekusapv-vhayvlke toyatskat! kofockv, anese, kvme esyomat tis tekvpvke espalan feket, momet vhakv oce senhonhoyakat, fvecetv, merretv momet vkvsvmkvn wikatskvtet ok; heyv nanvken momecatskvnt os, vpvlwrn wikekot.

24 Evpayvlke turhecekvlke, eckvposwuce en cullicet, momis rvcce-kuphe nokmēlatskat.

25 Cen holwakvkes, nakcokv-hayvlke momen Falvsevlke, mekusapv-vhayvlke toyatskat? sesketv momet pvlvknv onvpvn hvsvthvken hayatskekv; momis ofv svpenkvleckv momet svheremahetv es fvefakes.

26 Turheceko Falvsev toyetskat, sesketv, momet pvlvknv ofv taten hrsvtecvs, onvpvu hvsvthvke taren. 
27 Cen holwakvkes, nakcokv hayvlke momen Falvsevlke, mekusapv-vhayvlke toyatskat! sepvlkv hvthicvkvte, onvpv en heckv mehenwvin herakuset omis, ofv este pvsvtke e fune, momen hvsvtkeko vtekat es fvcfvke omatskekv.

28 Matvpomusen centakeu onvpv fvcce omēt este em e heckuecatskes; momis ofv mekusvpkv vhake momen naorketv es fvcket omatskes.

29 Cen holwakvkes, nakcokv-hayvlke momen Falvsevlke, mekusapv-vhayvlke toyatskat! owalvlke en sepvlkv mvhericet, momet facculke en herickv en nehicecet,

30 Momet, Pu'rkvlke en nettv take omof fulleyvten omat, owalvlke e catv pvlatat vpvkēkvnt os, makatskekv.

31 Monkv owalvlke pvsvtakvte 'eppucetake toyatskat centake a e en kerrvlke toyatskes.

32 Monkv ce'rkvlke es em vkerkv fvcecvkes.

33 Cetto, eketnv cetto honvpse toyatskat, estomēt estemerkv hute ohfvececkv emvnvttatske taye haks?

34 Monkv, hecvkes, owalvlken, este hoporrenvlken nakcokv-hayvlken esyomen ec ohtotvkis; momen vpvlwon vtvrtayet, pvsvtatskvres, momet vpvlwvn cen senekake take ofvn tepket, assecet tvlofv etohtvlhuecatskvres.

35 Este facculke e catv yvmv ekvnv oh pvlatkvte, Epel faccv e catvn Sekelivs, Pelekivs e'ppuce cuko-vcakat altv em etenrvwv elecatskvte e catvn vteket, omvlkvt ec oh ocvkvren.

36 Mehenwvn ce kicvkis, Heyv nanvke omvlkvt heyv honvpsen oh vlvkvres.

37 Celuslvmē, Celuslvmē, owalvlke pvsatetskat, momet mv ec ohtothoyat cvto es naf ketskat, etewole estomusen, 
tottolose e'chustakuce tvrpv elecv vteloyat etvpomen cen hopuetake vteloyvkvye tayvtes, momen cemet komatskekvnks!

38 Hecvkes, cen cuko tvnken cem wihokvkes.

39 Ce kicvkikv, Hvtvm cv hecatskeko munket, Pucase hocefkv ofv vlakat vkvsvmket os, makatskvres, Cesvs kicvtes.

\section{SETENTVCKE XXIV.}

Momen Cesvs osset, cuko-vcakat enkvpakvtes; momen en kerrepvlket cuko-vcakat etohtvrkan hecicvranet en yicvtes.

2 Momen Cesvs, Heyv nanvke omvlkv hecatskeko haks? mehenwvn ce kicvkis, Yvmv cvto etohliket hvmkis alvtkeko wihokekares.

3 Momen ekvnhvlwe Olefvn oh likof, ehkusen en kerrepvlket en yicvtes, Pum onvyvs, estofvn heyv nake momvkvr haks? momen cem vlvketv, momen ekvnv em eyuksvu naket eskerkv tar haks? makaket.

4 Momen Cesvs em vyoposket, Vketecvkes, estimvt ce hosicvkekaren, kicrtes.

5 Sulket, Klist toyis, makaket, cv hocefkv ofvn yicvranet ok, momet sulken ehosvkuecvres.

6 Momen horre, horre ohonvkvu pohatskvres; vketecvkes, ce naorvkekaren; heyv nanvke omvlkvt momvranet omekv, momis yuksv hvtē tēs.

7 Tvlwvt tvlwun vnrvpet, momen tvlofvt tvlofvn vnrvpet vkvwvpkvret ok; momen elaukot, enokketv yekcrke mahat momet ekvnv nekeyvt estomis ocvkvres.

8 Heyv nanvke omvlkvt e feke nokketv vlicecat omakes.

9 Momof cen nokkihocvren ce wiyvkvres, momet ce prsvtvkvres; momen tvlwv omvlkvt cen homecvkvres, cv hocefkv vrahkvn. 
10 Momen momof sulket elentappvkvres; momet ete wiyaket, ete'n homecrkvres.

11 Momen owalv laksvlke sulket akvwapket, sulken ehosicvkvres.

12 Momen holwvyeckvt tetayat omecicen sulket vnokeckv en kvsvppe hakvres.

13 Momis estimvt emunket em eyuksicen omat, matvwrt hesakvres.

14 Momen heyv ohmekketv em opunvkv-hervn tvlwvvlke omvlkv es en kerkvn ekvnv omvllkvn es erkenvkhoyvres; momen momof yuksvt vlvkvres.

15 Monkv enhomeckv trnecicvranat Tanvle owalv opunvyecvte cuko-vcakat ofvn hueren hecatskof ; (estimvt ohonayat kerrekvs ;)

16 Cutev vpokat ekvnhvlhawan oh pefatkekvs:

17 Estimvt cuko onvpr oh likat en cuko ofv nake ocat cawvret ahvtrpikates:

18 Monkat crpofv arat accusvke cawvret afulikates.

19 Momen mv nettv take omof nvrkesakat, momen coyicakat en holwakes!

20 Momis cem pefatketv take rvfon, monkat fekvpkv nettv tateu omekaren mekusvpvkes :

21 Momof estemerkv rakket ocvret okv, mv ome ekvnv vlicecvte vteke hiyomat vlakat ocvte sekot, momekos, momet estofvn ocekares.

22 Momen heyv nettrt koconcokekaren omat, enavpeswr hvmkis hesakekvnt omes; momis enhopokvte vrahkvn heyv nettv koconcokvres.

23 Momof, Heyvn, monkat vsvn Klist likes, estimvt ce kicvken omat, vkvsahmatskvs.

24 Klist laksvlke momen owalv laksvlke vkvwapkvkvret $\mathrm{okv}$, momet nake eskerkv rakrvken, momet nake estoma- 
hake heckuecvkvres; momat, mome tayen omat, enhopokat mahusat tis ehosvkuecvres.

25 Hecvkes, homvn cem onayvkis.

26 Monkv, Hecvkes, vnrvwvn likes; ce kicaken omat, vpehyatskvs; Hecvkes, nvthofv ehkakan likes; ce kicaken omat, vkvsahmatskvs.

27 Este E'chuswv em vlvketv vtoyehvtte hvsossv fvecv a vtoyehattet hrsvklatkv fvecr er a hvyvyicat omē taret ok.

28 Estvmvt o estomen nake elat ocen omat, mvn lvmhe vtelokvret okv.

29 Heyv nettv take estemerkv hoyanan vpaken hvset yomucken, hvresse hvyayvkekares, momen kococumpvu hvlwan a pvlatken, hvlwe en yekcetv take nekehoyvres.

30 Momen momof, hvlwe mimvn este E'chuswv es en kerkv heckvres; momen momof ekvnv este em vliketv omvlkvt wahokvres; momen este E'chuswv yekcetv momet vkvsvmkv rakke vpvken hvlwe em aholoce ofvn vlaken hecvkvres.

31 Momet em encelvlken pofketv en hake rakken es vtotvres, momen enake enhopokvte hotvle ostat aenkvpvken, hvlwe yuksv hvmkat yuksv hvmkan rore aenkvpvken vteloyvkvres.

32 Tokvs ke-rakko esvhopakvn en kerrvkes; e lvcce hvte lowvcket ot esse sossicof, meske vwolicen kerratskes :

33 Monkv matvpomen cemet heyv nanvke omvlkvn hecatskof, vwolicet, vhauken vlaken kerrvkes.

34 Mehenwrn ce kicvkis, Heyv honvpse hoyvneko munken, heyv nanvke omvlkvt esfvcike tvlkvres.

35 Hvlwe yvmv ekvnv esyomat sumkvres, momis vm opunvkv sumikekos. 
36 Momis mv nettv, hvse-vkerkv tepakat estimvt kerrekos, hvlwe encelvlke tisem kerrvkekos; momis Cv'rke tvlket kerres.

37 Momen Nov en nettv tate omēn, esste E'chuswv em vlvketvu matvpomvres.

38 Uelauke ehomv nettv omof hompaket, eskaket, etepryaket, etepryepuece emunken mv nettv Nov perrocuko ceyat orvtes :

39 Momet kerrvkeko munken uelauke vlakvtes, momet omvlkvn cahwet, eshoyvnvtet omekv; mv omēt este E'chuswv em vlvketv tares.

40 Momof hokkolet crpofvn welaken, hvmken eset, hvmkan wihokvres :

41 Hoktvke hokkolet 'semfulotkvn em fulotaket kakvres; hvmken eset, hvmkan wihokvres.

42 Monkv vketecvkes, ce Pucase hvse-vkerkv estoman vlakat kerratskekokv.

43 Momis heyvn kerrvkes, cuko-pucase hvse-vkerkv estoman horkopv vlvkvranat kerrvten omat, vketecet, en cuko hoprnkvre etektvnecekvnt omes.

44 Monkv cemeu ce metetakvkekvs; hvse-vkerkv mohmes komatskekofvn este E'chuswv vlaket omekv.

45 Monkv estimvt vtotkv hoporrene, heromat te, $\mathrm{mv}$ em oketv orof hompetv emvren e pucaset en cuko ohfvnke hayvte?

$46 \mathrm{Mv}$ vtotkv, e pucase rulakof, momēn vfasten eshecen omat, en heres.

47 Mehenwvn ce kicvkis, Enake omvlkv ohfvnken hayvres.

48 Momis mv vtotkv holwakat, $\mathrm{Cv}$ pucase em vlvketvn acewices, e feke ofvn maken omat;

49 Momet em etohkvlketv vtotkvlken nafket, hacvlken vpaket hompet momet esketvn vlicecen omat; 
$50 \mathrm{Mv}$ vtotkv e pucase nettv en nene hecekot, momet hvse-vkerkv kerrekofvn vlvkvres,

51 Momet etetahcet, en liketv mekusapv-vhayvlke vpvken em mellvres; mvn hvkihketv, momen enute setetekketv ocvres.

\section{SETENTVCKE XXV.}

Mомо hvlwe em ohmekketv fvcenvlke pale, mv kulke escahwe hokte epayvranat vnrvpvrane vpeyvte svhopahoyvres.

2 Momen cahkepet hoporrenvket omen, cahkepet hoporrenvkekates.

$3 \mathrm{Mv}$ hoporrenvkekat kulke cahwet, nehen ehopakvkekates.

4 Momis hoporrenakat vcvnkvn nehen kulke svpvken escrwakvtes.

5 Hokte epayvranat acewicof, omvlkvt nocicet nocicepvtes.

6 Momen nere nvrkvpv omof hüehketvt hakvtes, Hecvkes, hokte epayvranat vlakes; esosset vnrvpvkes.

7 Momof heyv fvcenvlke omvlkvt asvpvkilet, kulke emvhericakvtes.

8 Momen hoporrenvkekat hoporrenakan, Nehen pun kvpicvkes, kulke pum aslekv, kicakvtes.

9. Momis hoporrenakat em ryoposkaket, Centake esyomeyat pum oreko wites; momis mv wiyakan oh vpeyet e en nesepvkes, kicakvtes.

10 Momen nesvranet vpeyof, hokte epayvranat vlakvtes; momen emetetakakat vpaket etepvyetvn vevkvpeyvtes: momen vhauke rkhothvtes.

11 Enyupvn fvcenvlke vpvlwvt yicvtes, Pucasē, Pucasē, pun hvwecrs, makaket.

12. Momis em vyoposket, Mehenwrn ce kicvkis, ce kerrvkvkos, kicvtes. 
13 Monkv vketecvkes, hvse-vkerkvt ot, nettv tateu estoman este E'chuswv vlakat kerratskekokv.

14 Hvlwe em ohmekketvt este ekvnv hopvye aye em vtotkvlke enhohike enake em wikvte omekv.

15 Momet hvmken talentv cahkepen, momet hvmken hokkolen, momet hvmken hvmken emvtes; este vtekat en yekce vrahrvkvn vevkayen: momet lvpken ayepvtes.

16 Momof mv talentv cahkepe cawvte ayet 'res neset, talentv etv cahkepen hayvtes.

17 Momen matvpomen mv talentv hokkole cawvte, mvu talentv etv hokkolen heckuecrtes.

18 Momis mv hvmke ēsvte ayet, ckvnvn korvtes, momet e pucase en cvtokunaw.vn ehvtes.

19 Eryupv hofunof, mv vtotkvlke e pucase vlāket, etemfaccrtes.

20 Momen mv talentv cahkepe cawvte vlaket, talentv etv cahkepen svlakvtes, Pucasē, talentv cahkepen a'metskemvts; hecrs, $\mathrm{mv}$ sen hoyanat talentv cahkepen hēckuecis, maket.

21 Heres, vtotkv here, herometskat; nanvke nvcomen oh herōmetskes; nanvke sulke ohfvnken ce hayares; ce pucase em afvcketv ofvn ceyvs; e pucaset kicvtes.

22 Momen mv talentv hokkole cawvte vlaket, Pucasē, talentv hokkolen vm wiketskemvts, hecvs, talentv etv hokkolen hēckuecis, kicvtes.

23 Heres, vtotkv here, herometskat; nanvke nvcomen oh herōmetskes, nanvke sulke ohfvnken ce hayares; ce pucase em afvcketv ofvn ceyvs, e pucaset kicvtes.

24 Momof mv talentv hvmke esvte vlaket, Pucasē, hunvnwv merretv ocekot, fokayetskekatet mamet, vwahecetskekatet vteloyetskat kerrimvts: 
25 Momen ev penkalemvts, momet ayit, talentv cenake ekvnv ofv ehit omimvts: hecvs, cenaken ocetskes, kicvtes.

26 E pucaset em vyoposket, Vtotkv holwvyece, enhorretskat; estvn fokayvkat mamit, vwahecvkat vteloyin kerretskemvts ;

27 Monkv vn cvtokunawv esyoposkvllen em wikvranetskvtes, momen vlakvyof, cvnake ohhontv ocen cawryvnt os, kicrtes.

28 Monkv talentv em esvks, momet talentv pale ocan emvkes.

$29 \mathrm{Mv}$ ocat vtekat emhoyen, tayen ocvret okv; momis ocekat $\mathrm{mv}$ ocat tis en cawhoyvret okv.

30 Momen vtotkv lopicekat yomuckofvn vwilivks ; mvn hvkihketv, enute setetekketvu ocvres.

31 Este E'chusw encelvlke vcacakan vpaket en rakke ofvn vlakof, momofvn en rakke ohliketv vcakan oh likvres.

32 Momen ehomvn tvlwvvlke omvlkvt vtelokvres; momen yvpefikv vhecicv yvpefikv cowatv etekvpicate omēn etekvpicvres;

33 Momet yvpefikvn em vkvpervn vpoyet, momis cowatv em vkvskvnvn vpoyvres.

34 Momof Mekkot em vkvperv vpokan, Vwvkes, Cv'rke ec oh merrvte toyatskat, ohmekketv ekvnv vlicece vteke cem etetakuehocvten epucasvkes ;

$35 \mathrm{Cv}$ 'lvwet on, hompetvn a'met; cv 'wvnhket on, esketvn a'met; momet este kerkekot omin, cv yvpayet; 36 Vccvkon, vc vececet; cv nokket on, ye cv hecet; momet cuko-yekcv 'pikvyof, vn yicatskvnkekv, kicvres. 37 Momof faccvlket em vyoposkaket, Pucasē, estofvn ce 'Ivwe ce hecet, ce hompiceyvt haks? monkat ce 'wrnhke ce hecet, esketv ce'meyvt haks? 
38 Estofvn este kerketskekon ce hecet, ce yvpayeyvt haks? monkat vecetskekon ece'cceceyvt haks?

39 Monkat estofvn ce nokket on, monkat cuko-yekcv 'pikctsken ce hecēt cen yiceyvt. haks ? kicvkvres.

40 Momen Mekkot em vyoposket, Mehenwvn ce kicvkis, Heyv etecakkvyvte lopockus mahat hvmkvteken momecatskvtet, vnen omatskvtes, kicvres.

41 Momof em vkvskvnv vpokateu, Ohhomecke toyatskat, v'nkvpaket, totkv emeyuksv-seko Setvne em encelvlke esyomat em etetakuehocvten esceyvkes :

$42 \mathrm{Cv}$ 'lvwet on, hompetvn v'mekot; cv 'wvnhket on, esketvn v'mekot;

43 Este kerkekot omvyan cv yvpayekot; vecvkon vc vccecekot; cv nokket on, cuko-yekcrn vpikvyan $\mathrm{cv}$ hecatskẻkvnkekv, Mekkot kicvres.

44 Momof mvu em vyoposkaket, Pucasē, estofvn ce 'lvwe, monkat ce 'wvnhke, monkat este kerketskekot, monkat vccetskekot, monkat ce nokket, monkat cukoyekcv 'piketsken ce hecēt cem vnicēkvt haks? kicvkvres.

45 Momof em ryoposket, Mehenwvn ce kicvkis, Heyv lopockus mahat hvmkvteken momecatskekatet, cv momecatskekates, kicvres.

46 Momen heyvt estemerkv yuksv-sekon esceyen ; momis faccvlke hesaketv yuksv-sekon esceyvres, kicrtes.

\section{SETENTVCKE XXVI.}

Momen mome ocvtes, Cesvs, heyv opunvkv omvlkvn poyof,

2 Nettv hokkole hoyanof, ohhoyvnkv taranat kerratskes, momen este E'chuswv tohweklepkvn vtvrkvranen wihoyvres, en kerrepvlken kicvtes.

3 Momof plest enhomvhhotat, momen nakcokv-hayvlke, 
momen este em vculvke esyomet plest enhomahtv hvlwat, Kiefvs kicetv, en cukon nvkaftvtes.

4 Momet Cesvs emvkerre ehset; elecvkvret etempunahoyvtes.

5 Momis, Hompetv-rakko omof tokon, este tekyvmketvt ocekaren, makakvtes.

$6^{r}$ Momen Cesvs Pervne tvlofv, Simv lepv en cukon likof,

7 Hoktet alepastv nakvcrnkuce neha fvmecv vcake mahe vcvnken svlakvtes, momet, hompet likof, e'kvn em oh kalvtes.

8 Momis en kerrepvlket hecakof, cvpvkhokvtes ; Estomaten heyv yvmahke haks?

9 Heyv neha fvmecv enrahkv sulken wiyiyet, estemerrakan emhoye tayvtetok, makaket.

10 Cesvs kerrof, Estomen hokte naoricatske haks? vtotketv heren vc oh wikekv:

11 Estemerrakat ece 'pakvke ocatske munkvt omen, momis vntat ocatske munkv tokot omekv.

12 Heyv neha fvmeev cv'na oh kalat es ev herihocvranan momet omekv.

13 Mehenwvn ce kicvkis, Yvmv ekvnv hvmkvn estvmvt o estomen heyv opunvkv-herv eserkenvkhoyat vtekat, heyv hokte heyv momecvte esvkerrickvn onahoyvres, kicvtes.

14 Momof pale-hokkolohkakat hvmket, Cutvse Eskalev kicetvt, plest enhomvhhotan oh ayvtes,

15 Momet, Naken v'matskvr haks? momen cen huericvkares, kicvtes. Momen cvtokunawuce pale-tutcenen em fekvranet etemfvecakvtes.

16 Momen mvn vteken wiyetv etektvnke hopoyvtes.

17 Momen tvklike-tukseko hompetv-rakko nettv enhvteceskv omof en kerrepvlket Cesv's en yicvtes, Estv- 
mvn ohhoyvnkv hompetskvre cem etetakueçeyvre kometske haks? kicaket.

18 Momen kicvtes, Tvlofvn este hvmken em vpeyet, Mvhayvt, Vm oketv vlakes, vn kerrepvlket ve vpaken ce' huten ohhoyvnkvn vfvstvranēs, makes, kicvkes.

19 Momen en kerrepvlke Cesvs kicaten momakvtes; momet ohhoyvnkv etetakuecakvtes.

20 Momen yvfke orof, pale-hokkolohkakan vpaket likvtes.

21 Momen hompakof, Mehenwvn ce kicvkis, hvmkatskat cv wiyvres, kicvtes.

22 Momen tayen e feke en nokhokvtes, momet omvlkvt, Pucasē, vnet o haks? maketvn vlicecake vlkvtes.

23 Momen em vyoposket, Mv vc vpake e'nke pvlvknv vkpikat, matrwvt cv wiyvres.

24 Este E'chuswv oh onaye cohoyvte omēn ayvranet omes: momis en holwakes, mv este wiye huerat! mv estet heckekaten omat, en hervnt os; makvtes.

25 Momof Cutvse, mv wiyat em vyoposket, Vne te, Mvhaya? makvtes. Mahketskes, kicvtes.

26 Momen hompakof, Cesvs tvkliken ehset, E'rken vkvsamet, kvlkayvtes, momet en kerrepvlken emet, Eset, hompvkes; heyvt cv'na tes, kicvtès.

27 Momet sesketvu sehset, E'rken vkvsamet, e'makvtes, Omvlkvt eskvkes ;

28 Heyvt cv catv setemfvecetv mucvset, holwryeckv 'sen kvpvketvn sulken em pvlatket omekv.

29 Momis ce kicvkis, Mucv ayat heyv prrko en lokce ce'tem eskvkvko munken, mv nettv Cv'rke eme tvlofv oh mucvse ce'tem eskvkvy of tvlkvres, kicvtes.

30 Momen yvhiketvn yvhikahkof, esosset, ekvnhvlwe Olefv kicetvn vpeyvtes.

31 Momof, Omvlkatskatet vne ve vrahkvn heyv nere 
ce'lentaphoyvkvres: Yvpefikv-vhecicv nvfkares, momen yvpefikv vtelokvte vwahvres, maket coket omekv.

32 Momis akvwvpikvye rohyupv ce homv taken Kalvlen vyares, Cesvs kicvtes.

33 Petvt em vyoposket, Omvlkvt ece 'rahkvn elentappvkhoye estomis, estofvn cv 'lentapiyekos, kicvtes.

34 Mehenwrn ce kicis, Heyv nere, tottolose en hunvnwv hakeko munken, vc ohfvecv ehelaksecetske svtutcenvres, Cesvs kicvtes.

35 Petvt okat, Ece 'pakit cv 'Ivrane estomis, ec ohfvecv ehelaksehcvkos, kicvtes. Matvpomen en kerrepvlke omvlkvt makakvtes.

36 Momof Cesvs vpaket Kersemene kicetvn rorvtes, momet en kerrepvlken, Heyvn vpokvkes, vsvn ayit mekusapvyof, kicakvtes.

37 Momet Petvn, Sepete e'ppucetake hokkolan esyomen esayvtes; momen vlicecet, efeke nokkuset, honne mahvtes. 38 Momof, Vm puyvfekcvt tayen nokkes, elkv tis oren : heyvmvn fekhonnvkes, momet vc vpaket vketecrkes, kicakvtes.

39 Momet res hopvyusen ayet, e turofvn oh lvtiket, mekusapvtes, Cv'rke toyetskat, mome tayen omat, heyv sesketv v'nkvpvkekvs; mome estomis vne komvyate tokot, ceme kometskatet, maket.

40 Momet en kerrepvlken em vlakvtes, momet nocicen eshecet, Hvse-vkerkv hvmkusis vc vpaket vketecatskeko tayisa? Petvn kicrtes.

41 Vketecet mekusvpvks, nake ce naorkepnece taye eskerretv esceyatskekaret; mehenwvn puyvfekcv vkvsamus, momis enavpeswrt yekcekos.

42 Hvtvm esvhokkolvn ayet, mekusapvtes, Cv'rke toyetskat, heyv sesketv es eskvko v'nkvpvkeko tayen omat, ceme kometskatet momekvs, maket. 
43 Momen vlaket, hvtvm nocicen eshecrtes; e turwvt honhoyet omekv.

44 Momet enkvpaket, hvtvm ahyet, esvtutcenvn mekusapvtes, opunvkv matvwrn maket.

45 Momof en kerrepvlken em vlaket, Mucv nocicet, fekvpepvkes; hecvkes, hvse-vkerkv vwolices, momen este E'chuswv naorkvlke enke ofvn wihoyes, kicvtes.

46 Asvpvklvkes, vpeyvkēres; hecrkes, mv cv wiyat vwolices.

47 Momen opunaye munkof, hecrs, Cutvse, pale-hokkolohkakat hvmket vlakvtes, momen este sulke mahet eslaf kv-cvpko eto-warke tis cawvket, plest en homvhhotv momen este em vculvke atotet, yicvtes.

48 Momen $\mathrm{mv}$ wiyvte eskerkvn e'makvtes, Estoman vfvnkvyat, mvt mv tares; yekcen hvlvtvkes, kicet.

49 Momen moman vpaken Cesvs em vlakvtes, momet, Heres, Lapi ; kicet, afvnkvtes.

50 Momen Cesvs, $V n$ hessē, estomen vlahketska? kicvtes. Momof yicet, Cesvs hvlvtaket, esakvtes.

51 Momen hecrs, Cesvs vpakvte hvmket, e'nken rvtosecet, em eslafkv-cvpkon a ēsvtes, momet plest enhomahtv hvlwat em vtotkvn casket, e hvckon en tacvtes.

52 Momof, Cem eslafkv-cvpko e huten vpikvs; eslafkvcrpko crwakat omvlkvt eslafkv-cvpkon es pvsvtkvret okv.

53 Hiyomat Cv'rke em 'pohin, lvpkusen encelvlke sulsoke pale-hokkolohkake hoyanen a vn 'tote tayes, kometskeko haks?

54 Momis estomēt Cokv, Hiyomēt omvranēs, makat esohfvckvkvr haks? Cesvs kicvtes.

$55 \mathrm{Mv}$ hvse-vkerkv ofvn Cesvs este sulkan kicvtes, Este horkopvn vnrape omēt, eslaf kv-crpko eto-warke tis cawet, cv 'svranet yicatske haks? Nettv omvlkvn cuko-

Muskokee, Matthew. 
vcakat ofv ece 'pakvkit mvhayit likvyat, cv'satskekvnkes.

56 Momis Cokv owalvlke coyvte esohfvckvkvren heyv omvlkvt momet os. Momof en kerrepvlke omvlkvt wikahket, pefatkvtes.

57 Momen mv Cesvs esakat hvlatet, Kiefvs plest enhomahtv hvlwan 'soh vpeyvtes, mvn nakcokv-hayvlke momen vculvke nvkaftan.

58 Momis Petvt vhopvyen vevkaret, plest enhomahtv hvlwat en cuko-hervn eroret, eceyvtes, momet vtotkvlken vpaket likvtes, yuksvn hecvranet.

59 Momen plest enhomvhhotvt, vculvke momen nvkaftvlke omvlkvt akerkueckv fvecekon Cesvs vnrvpen hopoyakvtes, elecvkvret.

60 Momis eshecvkekates; momes, akerrvlke laksvke sulket yicet omis, hvmkis eshecvkekates.' Espoke akerrvlke laksvke hokkolet vlahoket,

61 Heyv hunvnwvt, Hesaketvmese en cuko-vcakat lekahfit, nettv tutcenofvn hayvye tayes, makvnkes, makakvtes.

62 Momen plest enhomahtv hulwat ahuyiret, Naken vyoposketskeko? Heyv ce'nrape akerkuecat naket o haks? kicvtes.

63 Momis Cesvs opunvyekates. Momen plest enhomahtv hvlwat vyoposket, Hesaketvmese wenake likat vcrkvyen es ce hvlatit, mv Klist, Hesaketvmese E'ppuce. toyetsken omat pum onvyetskvren, ce kicis, kicvtes.

64 Mahketskes; mome estomis ce kicvkis, Heyv ertopvrv este E'chuswv yekcetv em vkvpervn liket, hvlwe aholocen vpaket vlaken hecatskvres, Cesvs kicvtes.

65 Momof plest enhomahtv hvlwat em accvken selahlecvtes, momet, Hesaketvmese tvklecet opunayes, ohvtvlakat akerrvlke pu yace haks? Hecvkes, mucv Hesaketvmese tvklecat pohhatskes. 
66 Estomēn vkerricatske haks? makvtes. Em vyoposkaket, Ele taye oren em mvttes, makakvtes.

67 Momof e turofvu em vtof kaket, nvfkakvtes ; momen vpvlwvt enke-tvpeksen es tepkakvtes,

68 Klist toyetskat, ep oh owalvs, Ce tepikat estimvt o haks? kicet.

69 Momen Yetv cuko-herv ofv fettvn likvtes; momen bokte mvnettet em vlahket, Cemeu Cesvs Kalvlvlke vpaketskvnkes, kicvtes.

70 Momis omvlkv ehomvn ehelaksecet, Naken maketskat kerrvkos, kicvtes.

71 Momet sohvpettrn oh osiyof, hokte mvnette etvt hecvtes, momet mv fullan, Heyv hunvnwvu Cesvs Nasvlvlke vpakvnkes, kicvtes.

72 Momen hrtvm, Hesaketvmesen es hocefet, $\mathrm{Mv}$ hunvnwv kerrvkos, maket, ehelaksecvtes.

73 Momen hofunusof etempe svpaklvtet yicet, Mehenwusan mv hvmkat toyetskes; cem punvkvt ec oh kerkuecekv, Petvn kicakvtes.

74 Momof vlicecet, Hesaketvmese es hocefet, tvhikvtes, Mv hunvnwr kerrvkos, maket. Momen moman vpaken tottolose en hunvnwv hakvtes.

75 Momen Pettr Cesvs em punvkv, Tottolose en hunvnwv hakeko munken, vc ohfvecv ehelaksecetske svtutcenvres, kicvte, vkerricvtes. Momet osiyet, tetayen hvkihkvtes.

\section{SETENTVCKE XXVII.}

Hvyvтiкоғ, plest enhomvhhotat momen este em veulvke omvlkvt, Cesvs elecvret vnrvpet etemopunvyecakvtes.

2 Momet wrnayahkof, hvlvtaket, evpayv Pantvs Pilvten 'sem vpeyet, em wikakvtes. 
3 Momof Cutvse, mv wiyvte, elehocyranen ohfvecen hecof, em vkerrickv eticvtes, momet hvtvm crtokunawuce pale-tutcenat plest enhomvhhotv momen vculvken 'res em vlakvies;

$4 \mathrm{Mv}_{\mathrm{v}}$ em mrttekat catv wiyvyat naorkit omis, maket. Momen, Mvt pum estomet o haks? cemet hecepvs, kicakvtes.

5 Momen crtokunawuce cuko-vcakat ofvn tvk pvlahtet enkvpakvtes, momet ahyet e vtarvtes.

6 Momen plest en homvhhotat crtokunawuce cawvtes, momet, Cvtokunawv hute vtehetv fvccekos, catv enrahkvt omekv, makakvtes:

7 Momen temopunvyecakvtes, momet vrkvshayv eme crpofvn es nesakvtes, tvleporvlke hericetvn.

8 Monkv mv crpofv, Catv crpofvn, kihocvtet on heyv nettv oret omes.

9 Momof Cvlemiv owalv opunayvte sohfackvtes, Momen crtokunawuce pale-tutcenat cawvtes, mv enrahkv hakaten, Eslevl e'ppucetake enrvhecrte;

10 Momet vrkvshayv eme crpofvn es nesvtes, Cehofv cr mellvte omen, makat.

11 Momen Cesvt evpayv ehomvn hueren, evpayst em 'pohvtes, Cemet Cusvlke em mekko toyetske haks? kicet. Momen, Maketskes, Cesvs kicvtes.

12 Momen plest enhomvhhotv momen vculvket vnrvpakof, naken vyoposkekates.

13 Momof, Nanvke nvcomen ec oh kerkuecakat pohetskeko haks? Pilvtet kicrtes.

14 Momen opunvkv hvmkis em vyoposkekates; mv moman evpayv tetayen em estomahvtes.

15 Momen ohhoyvnkv hompetr-rakko omof evpayv cuko-yekcv vtehkvlke hvmken este em enrecopet omvtes, estimvn komakat. 
16 Momof cuko-yekcr vpikv kerkusen licakvtes, Pvlapvse hocefken.

17 Monkv nvkaftof, Pilvtet, Estimun cem enrecopvkaren komatske haks? Pvlapvsen? momekat Cesvs, mv Klist kihocaten? kicvtes.

18 Enhomeckvt omecicen em wikaket omen kerrekv.

19 Ohfrececkv ohliketwn oh likof, $\mathrm{Mr}$ hunvnwv frecat estomecetv ohcetskvs, mv omecicen mucv-nettr vpueckv ofvn nanvke sulket cv 'stemerricekv, maket, e 'hiwvt ohtotvtes.

20 Momis plest enhomvhhotr momen vculvket, Cesvs sumecicet, Pvlapvsen vpohvkvren- este sulkan vkvsvmepuecakvtes

21 Evpayv vyoposket, $\mathrm{Mr}$ hokkolat estoman cem enrecopvkaren komatske haks? kicvtes: Pvlapvse, makakvtes.

22 Monkv, Cesvs, mv Klist kihocat, estomecar haks? Pilvtet makvtes. Etohweklepkvn svrahkekvs, omvlkvt makakvtes.

23 Momen, Estomēn? holwvke estomen momece haks? evpayvt makvtes. Momis, Etohweklepkvn svrahkekvs, maket svheremahen huehkakvtes.

24 Pilvte, naken estomeko tayet, momis yiyetv mimvt haket omen hecof, Heyv este fvecat e catv es vm mvttekos; cent momvket onvres, maket, uewvn cahwet, este sulkat e homvn e'nken okkosites.

25 Momof este omvlkvt vyoposkaket, E catv pumen ep oh ocekvs, pun hopuetake haken, makakvtes.

26 Momof Pvlaprsen em enrecopakvtes; momet Cesvs tepikof, etohweklepkvn svrahkvren wikvtes.

27 Momof evpayv en suletawvlke euko tektvnke tvlemen Cesvs esceyakvtes, momet suletawvlke etohkvlke hvmkat omvlkvn $\mathrm{em}$ oh vteloyakvtes. 
28 Momen ena tvlecahket, kapv cahtusen vececakvtes.

29 Momen etofvske kvpotokvn hotanahkof, e'kvn em ohlicaket, kohvn e'nkvpervn em 'pikakvtes; momet e homvn e torkowvn kunhicaket, nakhayvkekates, Cusvlke em Mekko toyetskate! kicaket.

30 Momet ohtofkaket, mv kohvn esahket, e'kvn 'sen nvfkakvtes.

31 Momen nakhayvkekat renyupvn, kapv en cvwakvtes, momet em accrken vccehcet, etohweklepkvn svrahvranet svpeyvtes.

32 Momet esossof, Silenvlke hunvnwv, Simvn hocefken eshecakvtes; mrn etohweklepkv en karonvren ohyekcicakvtes.

33 Momet Kalkorv kicetvn, mv estekvfune likat maketv tes, mvn yihcof,

34 Uetukse kvlakcowv ctohkvlken esketv emakvtes; momen lahsof, eskeko tayvtes.

35 Momen etohweklepkv svrvhakvtes, momet ein accrke, estimvt omvre eskerraket, tekvpicakvtes, momat, $\mathrm{Vm}$ accvke etekvpicet, vn kapv estit omvre eskerrakvnkes, owalv makvtet sohfvckvren.

36. Momen mvn vpoket, vketecakvtes:

37 Momen e'kv onvprn esvnrvpkv hoccen svlicakvtes, Heyvt Cesvas Cusvlke en mekko tes, maken.

38 Momof horkopvlke hokkolet vpaket etohweklepkvn svrahrakvtes; hvmket vkvpervn svrahken, hvmkat vkvskvnvn surahken.

39 Momen hoyvnecat nakhayvkekates, ekvn norotticaket, 40 Momet, Cemet cuko-vcakat vhopahnet, nettv tutcenuse hayetskat, e hesayecrs. Hesaketvmese E'ppuce toyetsken omat, etohweklepkvn a hrtvpkvs, kicaket.

41 Matvpomen plest enhomvhhotv, momen nakcokvhayvlke, vculvkeu nakhayvkekot, 
42 Etv hesahuecvnkes; emetvwr e hesayeceko tayes. Eslevl em Mekkot on omat, hiyomen etohweklepkvn a hutvpkekvs, momen oh vkvsvmeyvres.

43 Hesaketvmesen vfekrakkvnkes; e yacen omat, hiyomen esepekvs: Hesaketvmese E'ppuce toyis, makvnkekv, makakvtes.

44 Horkopvlke mv vpake etohweklepkv svrahrakateu matvpomen nakhayvkekates.

45 Momen hvse-vkerkv esepakan vteket yomucke ekvnv omvlkvn ohranen hvse-vkerkv esostvpakat orvtes. 46 Momen hvse-vkerkv esostvpakat mahe orof, Cesvs opunvkv yekcen es huehket, Eli, Eli, lama sepakteni? makvtes, mv, Vn Hesaketvmese, Vn Hesaketvmese, estoman ev wihketske haks? maketvt omes.

47 Momen etempe svpaklat pohakof, Heyv este Elivsen enhuehkes, makakat sasvtes.

48 Momen moman vpaken mv hvmket letiket, uepvton uetuksen fveecvtes, momet, kohvn 'sohlihcet, eskvren emvtes.

49 Vpvlwvt, Ocekvs, Elivs vlahket hesayecen omat hecvkēres, maakakvtes.

50 Cesvs, hvtrm en hake yekcen es hohikof, em puyvfekcvn wikvtes.

5 l Momen hecvs, cuko-vcakat setentvckv onvpv elecv yeshvtapket etesetkvtes; momen ekvnv nekeyen, cvtou fvlahlvtes :

52 Momen sepvlkv hauhakvtes, momen Cesvs a en kvwvpketv ertopvrvn, mekusapvlke nocicvte e'na sulket vkvwvpkaket,

53 Sepvlkv a sosset, tvlofv vcakan esceyet, sulken em e heckuecakvtes.

54 Momen sentulev, momen mv vpvkake Cesvs vketecakat, ekvnr nekeyen, moinen heyr nanvke momakan 
hecakof, tayen penkvlakvtes, Mehenwusen heyv Hesaketvmese E'ppuce tat tis, makaket.

55 Momen hoktvke sulket mvn fullvtes, hopvyen a hecaket, mv Kalvle awe, Cesvs vfrstake vevkvpeyvtet:

$56 \mathrm{M} v$ vprkakat, Mele Maktelv, Mele Cems Cose tepakat e'cke momen Sepete en hopuctake e'cke esyomat tates.

57 Yafke orof, Alemetvlke este enake-ocet, Cose hocefket vlakvtes, mvu Cesvs en kerrepvt omet:

58 Mvt Pilvte oh ayet, Cesvs e'na em 'pohepvtes. Momof Pilvtet ena emhoyvren, makvtes.

59 Momen Cose ena ehsof, hvnvwelv tvrke hvsvtken es ayokkofvtes,

60 Momet en sepvlkv mucvse cvton a kofvte ofvn wrkecvtes; momet sepvlkv em vhauke cvto rakken oh pvlpibcet, ayepvtes.

61 Momen Mele Maktelv Mele hvmkat tepakat sepvlkv homusen kakvtes.

62 Momen etetakueckv nettv em pvkse omof, plest enhomvhhotv Falvsevlke esyomet Pilvten em etoh vteloket,

63 Pucasē, vkerricēs mv ehosicv hesake munkof, Nettv tutcenof hvtvm a kvwvpkares, makvnkes.

64 Monkv maketsken, sepvlkv vcaken nettv tutcenat orekvs, monkon omat neren en kerrepvlket yicet horkohpet, Prsatkan a en kvwapket os, esten kicake wites: monkv ehoskv espoke hvteceskvn semuntvle tares, kicakvtes.

65 Vketecvlke ocatskes; vpehyet, estomusen momecatske tayat vcaken hayvkes, Pilvtet kicvtes.

66 Monkv vpeyvtes, momet sepvlkv veaken hayakvtes, crto sohlokpicet, vketeckv hayet. 


\section{SETENTVCKE XXVIII.}

Fenvpkv-nettr hoyvnvranusen nettrcakuce nettv enhvteceskv hvyvtkvrane oh ayof, Mele Maktelv Mele hvmkat tepakat sepvlkv hecrranet vlahokvtes.

2 Momen hecrs, ekvnv nekeyv rakket ocvtes: Pucase em encelvi hvlwen a hvtapket, crton vhauken em akneyihcet, oh likekv.

3 En heckv vtoyehrtte omēn, em accvke hetute en hvtke omvtes.

4 Momen em penkvlakat vketecvlke fekëkakvtes, momet este prsvtke omake hakvtes.

5 Momen encelv ryoposket, Ce penkahlvkates, hoktvken kicrtes: Cesvs, tohweklepkv svrahohrnken hopoyatskat kerrikv.

6 Yvmv sekos : kvwrpkepekv, nakvnke omēt. Vthohyet, Pucase wakkvten hecvkes.

7 Momet lvpken vhohyet, prsvtkat a en kvwvpkepat en kerrepvlken em onvyvkes hecvkes, ce homv taken Kalvlen ayes; mvn hecatskvres: hecvkes, cem onahyvkis.

8 Momen lvpken cvto-kofke penkvlkv, afvcketv rakken es enkvpvkaket, tokorkvtes, en kerrepvlken opunvkvn sem vthoyvranet.

9 Momen en kerrepvlke em onvyvranet vhoyof, hecvs, Cesvs vnrahpet, Ece 'fvckvkekvs, kicvtes. Momen vthoyet, elen en hvlvtaket, vrakkuecakvtes.

10 Momof, Ce penkahlvkates; vhoyet, Kalvlen vpeyvks, kicet, etecakkvyvten em onvyrkes, momen mvn cv hecrkvres, Cesvs kicvtes.

11 Tokvs vhoyof, vketecvlke vpvlwvt tvlofvn yicet, plest enkomvhhotan nanvke momvte omvlkvn em onvyakvtes. 
12 Momen veulvke vpaket nvkaftet etempunahohyof, crtokunawv sulken suletawvlken emákvtes,

13 En kerrepvlket neren yihcet, pu nocicof ye horkopakvnks, makvkes ;

14 Momen heyvn evpayvt pohen omat, en lopvkuecēt, ce hesahueceyvres, kicaket.

15 Monkv crtokunawv crwakvtes, momet emvhahoyat vcvkvyen momakvtes: momen heyv maketv Cusvlket makvke mahen heyv nettr oret omes.

16 Momof en kerrepvlke pale-hvmkontvlakat Kalvlēn vpeyvtes, ekvnhvlwe Cesvs em mellvten.

17 Momen Cesvs hecakof, vrakkuecakvtes: momis en kerkvkekat sasvtes.

18 Momen Cesvs vlahket, em punvyakvtes, Yekcetv omvlkvn ekvnv ofvt ot, hvlwe ofv tis v'mhoyes.

19 Monkv vpeyet, tvlwv omvlkvn emvhayvkes, Erke, Eppuce, Puyvfekcv Vcakat hocefkv ofvn paptiset;

20 Naket o estomis ce kicvkvyvte omvlkvn vkvstetv emvhayet; momen hecrkes, estofis ece 'pakvkis, ekvnv yuksv vteken, Cesvs kicvtes. Emén. 


\section{OPUNVKV-HERV}

\section{MAK COYVTE.}

THE GOSPEL ACCORDING TO

\section{A R K,}

TRANSLATED FROM

THE ORIGINAL GREEK

INTO THE MUSKOKEE LANGUAGE.

\section{NEW YORK :}

AMERICAN BIBLE SOCIETY, INSTITUTED IN THE YEAR MDCCCXVI. 



\section{OPUNVKV-HERV}

\section{MAK COYVTE.}

\section{SETENTVCKE 1.}

Cesvr Kuist Hesaketvmese E'ppuce em opnnvkv-herv em vliceckv;

2 Owalvlke ofv cohoyvte vevkvyen, Hecvs, vm punvkvsarv ce homvn vtotis, mv ce homvn cen nene etetakuecrranat;

3 Vnrvwvn huehkat em opunvkvt, Pucase em vyetvn etetakuecrks, en nene lvpotlvken hahicvks, makat.

4 Canet vnrvwr ofvn paptiset, holwvyeckv enkvpakickvn vkerrickv etickv paptesvm 'serkenakvtes.

5 Momen Cutev ekvnv este omvlkvt, Celuslvme vpoke esyomat esosset oh vpeyet, omvlkvt en holwvyeckvn e. ohonvyaken, Cotvne hvtcen Canet vk paptisakvtes.

6 Momet Cane, rvtce-kuphe essen accet, cusse sewvnaketvn etetackvn 'sewvnvket omvtes; momet tvffo-noksv momen fo-semvnole en crmpen hompvtes;

7 Momet erkenaket, Vne 'svn yekce cr yupvn vlaket os, cunehkit, mv em estelepikv eswvnakv enrecopvye taye heretv orvkat.

8 Vne mehenwrn uewrn es ce paptisvkit ohmis : momis emet Puyvfekcr Vcakan es ce paptisvkvres, makvtes.

9 Momen mv nettv take omof mome ocvtes, Cesvs Nasvle, Kalvle ofv, vtet vlaken, Canet Cotvnen vk paptisvtes. 
10 Momen moman vpakusen uewvn enkvpaket vcemkof, sutvt hauhken, Puyvfekcvt pvcehowe vhaket oh hvtapken hecvtes.

11 Momen opunvkv sutvn ahvtapkvtes, Cv'ppuce vnokecke, $\mathrm{mv}$ ofv vc vfackusat, toyetskes, maket.

12 Momen moman vpaken Puyvfekcvt vnrvwvn vtohkvtes.

13 Momen Setvnet vnrapen, vnrvwv ofvn nettr pale osten mvn likvtes; momet punvttvn vpakvtes; momen hvlwe estvlket vfvstakvtes.

14 Momen Cane cuko-yekcv vpihohke emohyupvn, Cesvs, Hesaketvmese em ohmekketv opunvkv-hervn erkenaket, Kalvlen vlakvtes,

15 Momet, Oketv esfrcket on, Hesaketvmese em ohmekketv vwolet os; cem/vkerrickvn eticet, opunvkvhervn vkvsvmvks, maket.

16 Momet Kalvle vkhvse afopken yvkapof, Simvn etecakkat Antolu tepakat hoyvn vkhvse vk wikaken hecvtes, rvro-poyvlket omekv.

17 Momet, Vc vevkvhoyatsken, este em vkwiyvlke toyatskvren ce hayvkares, kicakvtes.

18 Momen moman vpaken en hoyv taken wihket, vevkvhoyvtes.

19 Momen, ohvtvlvkusen ayof, Sepete e'ppuce Cems etecakkvte Cane tepakan hecvtes; mvu en hoyvn mvhericaket perrocukon vtehket omvtes.

20 Momen moman vpaken enhuehkakvtes; momen e'rke Sepete perro-cuko ofvn vtotkvlke pvlhoyat vpvkaken wihket, vevkvhoyrtes.

21 Momen Kvpvnevm esceyakvtes; momen nettvcakon lvpken senekaken eceyet, estemvhayvtes.

22 Momen em mvhakv estomahes, komakvtes, cokvhayvlke omekot, yekcetv oce omet emvhayakekv. 
23 Momen este, puyvfekcv hvsvtkekat vpiken, en senekake take ofvn likvtes; momet huehket,

24 Cesvs Nasvlvlkē, $p u$ wikvs; naken pu 'tenrvwv oce, haks? pu sumhuecvranet vlahketske haks? esti toyetskat ce kerris, Hesaketvmese em Vcakat toyetskes, makvtes.

25 Momen Cesvs, Cvyvyahket, aossvs, maket, em vsehvtes.

26 Momen puyvfekev hvsvtkekat selahlecet, yekcicet hohikof, aossvtes.

27 Momen omvlkvt em estomahakvtes, momet ete'm 'pohaket, Heyvt nake te? heyv mvhakv' mucvse nake te? puyvfekev hvsvthrkekat tis, yekcetvn 'sem punayen, em apohicaket omekv, makakvtes.

28 Momen vyvkhvmkusen ohonvkv ekvnv Kalvle vfolotkan hvmecicvtes.

29 Momen senekaken asossahkof, Cems Cane tepakat vcrkvhoyen, lvpkusen Simvn Antolu tepakat en cukon esceyvtes.

30 Momis Simvn e hiwv e'cket hepetket wakkvtes ; momen lvpken mv ohfvecrn em onayakvtes.

31 Momen vlahket, enken en hvlatet, akvwapvtes; momen moman vpakusen hepetketv em wihken, est' vfastvtes.

32 Momen yafkof, hvset rklvtiken, enokhokat omvlkvn, momen puyvfekcv holwvhoke vtehkat es en yicvtes. 33 Momen tvlofv vpokat omvlkvt vhauke tempen vtelokvtes.

34 Momen enokketv cahmelike vlilakat sulken em wicecet, puyvfekev hvlwvhokat sulken sossicvtes; momet kerrvket ok', mv puyvfekcvlke opvnahoyvre em etektvnecekates.

35 Momet nere nvrkvpv hoyane, hvyatkvronkot emunken, alihket, osiyet, awvnen ayet, mvn mekusapvtes. 
36 Momen Simvn mv vpvkakat esyomat yupvn vevkvpeyvtes.

37 Momet eshecahkof, Omvlkvt ce hopoyaket os, kicakvtes.

38 Momen, Tvlofv vwolakan esceyvkeres, $\mathrm{mv}$ ofvn erkenvkaret; mv esvrahkvn vlakvyvtet ok', kicakvtes.

39 Momet Kalvlen hvmecicet en senekake take ofvn erkenaket, puyvfekcr holwvhokan sossicvtes.

40 Momen lepvt em vlabket, em 'pohet vtokkekvtes, Kometsken omat, cv hvsvtecetske tayes, maket.

41 Momen Cesvs em merruset, enken rvtosecet celayvtes, Komis; hvsvtkvs, kicet.

42 Momen kican vpakusen lepvse e'nkvpahken, hvsvtkvtes.

43 Momet yekcen em vsehet, lvpken vyecicvtes;

44 Momet, E vketecet, estimvn em punahyetskvs; momis ahyet, plest em e heckuecvs, momet ce hvsvtkat vrahkvn nanvke emetv Mose makvte es en kerkueckvn sayvs, kicvtes.

45 Momis osiyet, vlicehcet, tayen kerkuecet ohonvkvn hvmecicvtes, momat Cesvs ohvtvlakan hecken tvlofvn ceyeko tayvtes, momis lvpvtken vnrvwv vlkan aren, estv$\mathrm{mv}$ estomis a vwet en yicvtes.

\section{SETENTVCKE 2.}

Momen nettv vnvcomet yvfyaken, hvtvm Kvpvnevm eceyvtes, momen cuko ofvn likvcukat pohkvtes.

2 Momen moman vpakusen sulket nvkaften, svhere mahekv, ohvtvlakat evpaye tayat etektvnkekates, vhauke tempe tis; momen opunvkvn 'sem erkenvkakvtes.

3 Momen palse es enokke hvmke, ostet sete'n kvwapen, es awet, 'sen yicvtes.

4 Momen este cekfat omecicen vwolicvkeko tayof, $\mathrm{mv}$ 
likat onvpvn cuko ohrvnkvn oh vkueyicakvtes; momet tvkohlicahkof, mv pvtakv palse es enokke oh wakkan es hvtrpecicakvtes.

5 Cesvs em vkvsvmkv taken hecof, palse es enokkan em punayet, $C v$ 'ppucē, cen naorketv ce'nkvpvkakes, kicvtes.

6 Momis cokv-hayvlke vpvlwvt, e feke take ofvn vkerricet,

7 Estomaten heyv hunvnwv Hesaketvmese tvklecet opunaye haks? estimvt naorketv kvpakice taye haks, Hesaketvmese tvlkuset mvn momecekon omat? makakat mvn vpokvtes.

8 Momen moman vpakusen eme ofv momēn vkerricaken Cesvs em vkerrickv ofvn kerrof, em punayet, Estomaten heyv nanvken ce feke take ofvn vkerricatske haks?

9 Naorketv ce'nkvpvkakes; palse es enokkat kicetv, monkat, Ahuyiret, cem pvtakvn cahwet, yvkvpvs, kicetv tepakat, estvmit yekcekot ome haks? kicakvtes.

10 Momis este E'chuswvt yvmv ekvnvn naorketv kvpakickv yekcetv ocat kerratskvren, (palse es enokkan,)

11 Ce kicis, Ahuyiret, cem prtakvn cahwet, cen cukon vyepvs, (kicvtes.)

12 Momen moman vpakusen, akvwrpiket, pvtakvn cahwet, omvlkv ehomvn ossvtes; momat omvlkvt em estomahet, Hesaketvmesen vrakkuecakvtes, Hiyomē heceyvte sekos, makaket.

13 Momen hvtvm Cesvs osiyet, vkhvse afopken ayvtes; momen este sulkat omvlkvt oh vpehyen, emvhayakvtes.

14 Momet hoyanof, Lefi Alfers e'ppuce tokunawv vtelokv cukon liken hecrtes, momet, Vc vevkvtes, kicvtes. Momen akvwvpiket, vevkayvtes.

15 Momen mome ocvtes, Cesvs mv en cuko ofvn hom- 
petvn vlikof, crtokunawr vteloyvlke, momet holwvyecrlke Cesvs en kerrepvlke esyoman vpvkaket vpokvtes; sulket omekv, momet vcrkvpeyvtes.

16 Momen crtokunawv vteloyvlke holwvyecvlke esyoman vpaket hompen Falvsevlke cokv-hayvlke esyomat hecakof, en kerrepvlken, Estomaten crtokunawv vteloyvlke holwvyecvlke esyoman vpaket hompet eske haks? kicakvtes.

17 Cesvs pohof, Este cvfencakat vlekcvn eyacvkekos, momis enokhokat eyacakes; este faccvlke enhuehkvranit vlakit omvkates, momis holwvyecvlket vkerrickv eticvkvren, kicakvtes.

18 Momen Cane, Falvsevlke esyomat en kerrepvlke e elvwecaket omvtes: momen yicet, Estomaten Cane; Falvsevlke esyomat en kerrepvlke e elvwecaket omen, cen kerrepvlket e elvwecvkekot ome haks? kicakvtes.

19 Momen, Hokte epayvranat en cuko vtehkvlke hokte epayvranat vpake vtekat e elvwecake taye haks? Hokte epayvranat vpake vtekat e elvwecrkeko tayes.

20 Momis nettv cakkakvres, momof hokte epayvranat em eshoyvres, momen mv netty take omof e elvwecvkvres.

21 Momen nvpvt nake tvcke mucvsen kapv leskan em vlicekos; momen omat, nake mucvse vlihocat leskan en cawet os, mohmen cetakkat svhere mahe hakes.

22 Momen nvpvt pvrko opuswv mucvsen sukcv leslekan vcahnekos; mecen omat, prrko opuswv mucvsat, sukcvn sehtet, pvlatkes, momen sukev yvmahkvkvres: momis prrko opuswr mucvsat sukcv mucvsen vevnhoyvranet os, Cesvs kicakvtes.

23 Momen mome ocvtes, nettvcakon teleko crpofv vlkan - etehoyanvtes; momen en kerrepvlket, vpeyof, teleko en lokcen es mvmetvn vlicecakvtes. 
24 Momen Falvsevlket, Hecvs, estomaten nettrcakon mometv tokan momake haks? kicakvtes.

25 Momen, Tewe enkusapket elawen, vprkakateu elawakof, estomvte,

26 Epivrv plest enhomahtr en nettr take omof, estomet Hesaketvmese en cukon ecehyet, hecetv tvklike, mv hompesko tetayat, momis plestvlke tvlket hompvkvranat, hompvte, momet vpvkakateu emakvte ohonvkv hecatskekat haks? kicakvtes.

27 Momet, Estert nettvcakon en hayet omekot, nettvcakon este en hayvkvtet omes:

28 Monkv este E'chuswv tat nettrcakou e Pucase tes, kicakvtes.

\section{SETENTVCKE 3.}

Mомет senekaken hvtvm eceyvtes; momen este-hunvnwv enke yuknvtet mvn likvtes.

2 Momen, Nettvcakon em wicecrr haks? komet, vketecakvtes; vnrvpvkvret.

3 Momen este enke yuknvten, Nvrkvprn ahuervs, kicvtes.

4 Momet, Nettrcakon here momecetv holwvke momecetv tepakat estvt fvecrt o haks? momen hesaketv hesayecetv sumecicetv tepakat estvt fvccvt o haks? kicakvtes; momis nanvhokekates.

5 Momen e feke yekcakat omecicen efeknokket, crpakket, vfulotken oh vfvnnakahkof, Ce'nken rvtosecvs, estehunvnwvn kicvtes. Momen rvtosehcen, e'nke hvtrm hvmkat ome temvpoke hakvtes.

6 Momen Falvsevlket, sosiyan vpakusen, vnrvpet, estomet elecvke tayat, Helotvlken 'sete'm punahoyakvtes.

7 Momis Cesvs enkvpaket, en kerrepvlken evpayet, vkhvsen ayvtes : momen este sulke mahet Kalvle, Cutev, 
8 Celuslvme, Itumev, momen Cotvne tvpalvn a vwet vcrkvpeyvtes; momen Tilv Sitvne tepake $\mathrm{em}$ vwolakat, este sulke mahet nake rakrvken momecat pohahkof, en yicrtes.

9 Momen este cekfat omecicen, vceklvfkvkekaren, perrocukuce emetetaket em ehake emunkvren en kerrepvlken kicakvtes.

10 Sulken em wicecakvtet ok': monkv enokketv ocakat vtekat celvyetvn komet vyotecvtes.

11 Momen puyvfekcr hvsvthvkekat hecakof, ehomvn tvk pvlatket, Hesaketvmese E'ppuce toyetskes, maket huehkakvtes.

12 Momen oh kerkuecvkvranekon yekcen kicakvtes.

13 Momet rvnen ohcemiket, estimvn komat enhuehkakvtes; momen en yicvtes.

14 Momen vpvkakvren, momet erkenaket,

15 Enokketv wicecetv, momet puyvfekev holwvhokat sossicetv yekcetvn ocaken vtotvret, palen hokkolohkaken mellvtes.

16 Momet Simvn Petv es vhocefvtes.

17 Momet Cems Sepete e'ppuce, momet Cane Cems etecakkat, (momet mvn Povnvces es vhocefhuecrtes, mv tenetke e'ppuce take maketvts,)

18 Momet Antolu, Felvpe, Paralome, Maro, Tamvse esyomat, momet Cems, Alfevs e'ppuce, Ratevs, Simvn Kenvnvlke,

19 Momet Cutvs Eskalev, mv er wiyvte; momen cukon esceyvtes.

20 Momen este sulkat hvtvm vtelokat svhere mahet omen, tvklike hompetv tis umvkeko tayvtes.

21 Momen en hessvtket pohakof, esvranet sossakvtes, Hoporreneko hakes, makaket omekv.

22 Momen cokv-hayvlke Celuslvme ahvtvpecakat, Pelse- 
pvp oces, momet este-nekricvlke semevpayv eteropotten este=nekricvlken sossicet os, makakvtes.

23 Momen enhuehkaket, opunvkv svhopakvn es em punayet, Estomet Setvnet Setvnen ossice taye haks?

24 Momen tvlofv em etvwr vnrvpe tekvpaken omat, $\mathrm{mv}$ tvlofv huereko tayes.

25 Momen cuko em etvwv vnrvpe tekvpaken omat, mv cuko huereko tayes.

26 Momen Setvnet, e vnrvpe ahuyiret, tekvpaken omat, huereko tayes, momis meyuksvn ocet os.

27 Este estomet este yekce en cukon en ceyet, nakockv em vhopvneko tayes, este yekcat taten wvnayet omekon omat; momet momofvt cuko em vhopanvres.

28 Mehenwrn ce kicvkis, Holwryeckv vtekat, momet holwvke opunvyetv es vtvklecakis omvlkvt este en hopuetake enkvpakihocrres ;

29 Momis estimvt Puyvfekcv Vcakat tvklecat enkvpvyecickv estofis sekares, momis ohfvcceckv meyuksv-sekan em penkvlke tares, makvtes:

30 Puyvfekcv hvsvtkekan oces, makakekv.

31 Momof etecakkakat momet e'cket yicet, topvrvn svpaklet, enhuehket ohtotakvtes.

32. Momen este sulkat, em afulotet vpoken, Ce'cke momet tecakkaketskat ce hopoyet, topvrvn svpakles, kicakvtes.

33 Momen em vyoposket, Estimvt cv'cke monkat tecakkakvyat to haks? kicakvtes.

34 Momet em vfolote vpokakan vfolotken oh vfvnnakaket; Hecvs, cv'ckē, momet tecakkakvyat!

35 Estimvt o estomet Hesaketvmese koman momecen omat, matvwvt tecakkvyat, momet cv wrnwvt omet, $c v$ 'cket omekv, makvtes. 


\section{SETENTVCKE 4.}

Momet hvtvm vkhvse afopken mvhayetrn vlicecvtes: momen este cekfe mahet em vteloket omen, mv omecicen perro-cukon ceyet vkhvsen vk likvtes: momen este sulkat omvlkvt vkhvse en lvpvtken svpakle tates.

2 Momen nanvke sulken opunvkv svhopakvn es emvhayakvtes, momet em mvhakv ofvn kicakvtes ;

3 Pohvkes; Hecvs, fokayvt fokayvranet ossvtes.

4 Momen mome ocvtes, fokayof, vpvlwvt nene afopken pvlatke sasvtes, momen hvlwe fuswrt yicet, lokakvtes.

5 Momen cvto vlke, fakke oce mahekan pvlatke sasvtes; momet lvpkusen hontvtes, fakke en sufken ocekokv :

6 Momis hvse kvwapkof, ettopkvtes; momet talvtes, yvlonkvn ocekokv:

7 Momen vpvlwvt etofvske vlkan pvlatkvtes, momen etofvsket hontet, nokcepelen, en lokce hayekates.

8. Momen vpvlwvt ekvnv heran oh pvlatket, em ettet hontet, ohvtvlayet, vpvlwvt pale tutcenen, vpvlwvt pale epaken, vpvlwvt cukpe hvmken hayakvtes.

9 Momen, Estimvt hrcko es pohetv ocat pohekvs, kicakvtes.

10 Momet entwlkuset arof, pale hokkolohkake vpvkake em afolotakat opunvkv svhopakv ohfvccun em 'pohakvtes.

11 Momen kicakvtes, Centake Hesaketvmese em ohmekketv enake ehkat kerrvranatsken ce'mhoyvket os; momis mv lvpvtke svpaklat heyv nanvke omvlkv opunvkv svhopakv ofvn em mehocet os:

12 Vfvnnaket vfvnnakis, hecvkekon, momet pohaket pohakis kerrvkekaren; monkon omat, estofv tis rafulhuehohcen, en naorketv en kvpakihoce wites.

13 Momen kicakvtes, Heyv opunvkv svhopakvn ker- 
ratskeko haks? momof estomen opunvkv svhopakv omvlkvn kerratskvre te?

14 Fokayvt opunvkvn fokayet os.

15 Momen mv opunvkv fokakan heyvt mvt omakes, nene afopken pvlatkat; mv pohahkof, moman vpakusen Setvnet vlaket, opunvkv e feke take ofv fokakat em vkueyet os.

16 Momen matvpomen crto vlkan fokakat heyvt omakes, mv, opunvkvn pohahkof, moman vpakusen es afvcket crwakat tes ;

17 Momet eme ofv yvlonkv ocvkekot, estomusen $m v$ of $v$ vfekhonnaket, $\mathrm{mv}$ renyupvn estemerkv, hvtvm asseckv tateu opunvkv omecicen kiyapkan vpakusen elentappaket os.

18 Momen $\mathrm{mv}$ tofvske vlkan fokakat heyvt omakes; mv, opunvkvn pohakat,

19 Momen heyv ekvnv vkerrickvt, momen nakockv emvkerretv, momen nanvke etv eyackv esceyaket, opunvkvn nokcepelakes, momen etteko hakes.

20 Momen mv ekvnv heran fokakat heyvt omakes; mv, opunvkvn pohaket, cawet, em etten hayet, vpvlwvt pale tutcenen, vpvlwvt pale epaken, vpvlwvt cukpe hvmken hayaket os.

21 Momen kicakvtes, Kulke sohhuerickv sohhuericvranekot, svkerkv elecv, monkat topv elecv eslicvranet kulken esyihoce haks?

.22 Nake ehke heckvranekat sekon; nake ohrvnke vcayehocvte here kerkvranekat sekot omekv.

23 Estimvt ehvcko es pohetv ocen omat, pohekvs. 24 Momen kicakvtes, Naken pohatskat vketecvkes, svkerkv estomen svkerkuecatskat hvtvm es cem vkerkuehocvkvres: momet pohatskat ohvtvlvken ce'mhoyvkvres. 
$25 \mathrm{Mv}$ ocat, e'mhoyvres, momet mv ocekat, ocat tis em eshoyvret ok'.

26 Momen makvtes, Estet nerkvn ekvnv ofvn vwiken,

27 Momet nocet, neren nettvn akvwapken, estomet omat kerrekon, nerkv hontet, mahet omes; Hesaketvmese em ohmekketv $m v$ omet os.

28 Ekvnvt eme aossen em etten, enhvteceskvn esse, mohmen ekv es hakat, momet mv erenyupvn ekv es hakat fvcfakan ettet os.

29 Momis em ette lokcan vpakusen estvcetvn ohceyicet os, hiyo hakekv.

30 Momen makvtes, Hesaketvmese em ohmekketv nake omet os, makvraneya? momen svhopakv estomen es vhopayeyvr haks?

31 Mvstv e nerkv hvmke, mv ekvnv ofvn fokakof, ner$\mathrm{kv}$ ekvnv ofv ocakat omvlkv 'sen cotkuset o estomis;

32 Fokahkof, mahet, vhockuce omvlkv'sen rakke haket, elvcce rakrvken hayet omen, hvlwe fuswr em vpette elecrn vpokes; $m v$ omet $o s$.

33 Momen opunvkv svhopakv heyv omakat sulken opunvkvn 'sem punvyakvtes, 'mapohicetv umake tayat vcvkvyen.

34 Momis opunvkv svhopakvn omekat em punayvkekates; momet entvlkuset vpokof, nanvke omvlkvn en kerrepvlken em ohhvyvyicakvtes.

35 Momet mata nettrn, yafkof, Trpalvn tiyecrkeres, kicakvtes.

36 Momen este sulkat etvn vtotahkof, perrocuko vpikekv, esvkvpeyvtes. Momen perro-cukucvlke etvu vpvkakvtes.

37 Momen hvnvcofelv rakket kvwapkvtes, momen uemelohlvt perro vnafket vcrnkvtes, svhere mahekv perrocukot fackvtes. 
38 Momen perro-cuko ofv yupv hakan oyapkv oh noce tates, momen ahonecicahket, Mvhaya, pu sumecvranat stoskometskeko haks? kicakvtes.

39 Momen akvwrpiket, hotvlen em vsehet, vkhvsen, Cvyayaket, fekhonnvs, kicvtes. Momen hotvlet fekhonnen, lekuthe rakket ocvtes.

40 Momen, Estoman momēn ce penkvlakvke haks? Estomet on vkvsvmkvn ocatskeko haks? kicakvtes.

41 Momen tayen penkvlaket, Heyv hunvnwv estomet ot omen, hotvle tisem, momen vkhvse tisem em apohice haks? ete kicakvtes.

\section{SETENTVCKE 5.}

Momen vkhvse tvpalvn roricet, Katvlenvlke em ekvnvn esceyvtes.

2 Momen perro aossan vpakusen este-hunvnwvt, puyvfekcv hvsvtkekat vpiken, sepvlkv vlkan aosset, vnrapvtes.

3 Sepvlkv vlkan $e$ huten ocet, momen este estomet crto-telacv tis es wrnayeko tayvte:

4 Tewolen crto-telacv es wrnayet, momet ele semvyekcihocet omen, cvto-telacv ete waret, elesemvyekcickvn tvkohlicvtet omekv; momen este estomet yvmvseceko tayvtes.

5 Momen estofis, neren nettvn ekvnv hvlhawan, momet sepvlkv vlkan aret, huehket, cvton 'se warvtes.

6 Momis Cesvn hopvyen hecof, letiket, homvn latkvtes.

7 Momet yekcicet huehket, Naken pu tenrvwr oce haks, Hesaketvmese Hvlwe Mahat E'ppucē? Cv' stemerricetskekaren Hesaketvmese vcvkvyen es ce hvlatit cem 'pohepis, makvtes.

8 (Puyvfekcv hvsvtkeko toyetskat, mv hunvnwvn aossvs, kicvtet omekv.)

9 Momen, Ce hocefkv naket o haks? maket vpohvtes. 
Momen em vyoposket, Lēcrt cv hocefkv tes, sulke toyē$\mathrm{kv}$, kicvtes.

10 Momen mv ekvnvn sossicekaren tayen em 'pohepvtes.

11 Momen sukhv sulke mahet ekvnhvlhvwe vwolen nanopet fullvtes.

12 Momen puyvfekev holwvhokat omvlkvt, Sukhvn pu cehuecvs, mvn esceyeyvren, kicet, em 'pohakvtes.

13 Momen moman vpakusen em etektvnecakvtes. Momen puyvfekcv holwvhokat sosiyet, sukhvn esceyakvtes: momen sukhv fulle hvmkat omvlkvt pvfpvnet pefatket vikeleftan vkhvsen vk tasecet, vkhvsen vk nokcepelkakvtes; (em vhonkvtkv cukpe-rakko hokkolvteket omvtes.) 14 Momen sukhv hompvkuecakvtet pefatiket, tvlofv momet en lvpvtke ofvn onayakvtes. Momen nake momehocvten hecvranet sossakvtes.

15 Momet Cesvs en yicvtes, momet mv hunvnwv, puyvfekcv holwvke vpikvte, momet lēcr vtehkvte, accet, hoporrene haket, liken hecakvtes; momet penkvlakvtes.

16 Momen hecakvtet mv puyvfekcv holwvke vpikvte em estomvten, momet sukhv ohfvecvu em onayakvtes.

i7 Momen em ekvnv em vtekakat enkvpvkvren em 'pohetvn vlicecakvtes.

18 Momen perro-cukon ecehyof, mv puyvfekev holwvke vpikvtet vpvkepetvn em 'pohepvtes.

19 Mome estomis Cesvs em etektvnecekates, momen em punayet, $C e$ huten cen hessvlken oh ayet, heyv nake rakrvken Pucaset cem mómecvten, momet cem merrvten em onvyvkvtcrs, kicvtes.

20 Momen enkvpaket, Tekapoles tvlofv ofvn nake rakrvke momvke Cesvs em momecvten tvlemicetvn vlicecvtes. Momen omvlkvt em estomahakvtes.

21 Momen Cesvs perro-cukon 'pihket, hvtvm tvpalvn 
tikof, este sulke mahet em vtelokvtes: momen vkhvsen vwolice tates.

22 Momen hecvs, senekake semerpayvlke hvmket, Cilvs hocefket, em vlakvtes; momet hecof, e'le vnakvn tvk latkvtes.

23 Momet yekcen em 'pohepet, Cv'chustuce elvranuset wakkes; em wikvren, ahtet ce'nken oh wvkhokicetskvren cem 'pohepis; momen hesakvres, kicvtes.

24 Momen Cesvs vevkayvtes; momen este sulke mahet vevkvpeyet, vceklvfkakvtes.

25 Momen hecvs, hokte hvmke cate fihne ohrolope pale hokkolohkaken es enokkvte,

26 Momet, nanvke sulken vlekcvlke sulket em momecakvte 'sestemerket, en nakockv omvlkv es sumecicet omis, en here hakekot, momis svhere mahvtet,

27 Cesvs ohfvecvn pohhof, este cekfe yupv vpeyat vpvket vlahket, en kapvn celayvtes :

28 En kapv tvlkusis celayvye tayen omat, vm wikvres, makvtet omekv.

29 Momen moman vpakusen e catv fihnvtet kvrpvtes, momen mv enokketv em wikat $e$ 'na ofvn kerrvtes.

30 Momen yekcetv aossat Cesvs eme ofvn lvpkusen kerret, este cekfat ofvn fulotket, Estimvt vm accvken celahye haks? makvtes.

31 Momen este cekfat ce yotecat hecetskes, momat, Estimvt $\mathrm{cv}$ celahye haks? maketske haks? en kerrepvlket kicakvtes.

32 Momen heyv nake momecan hecvranet, vfulotken vfvnnakvtes.

33 Momis hoktet penkalet, fekeket, momet nake eme ofv moman kerret, vlahket, ehomvn tvk latket, mehenwat omvlkvn em onayvtes.

34 Momen, Cv'chustē, cem vkvsvmkvt temvpoken ce 
hahyes; herkv ofvn vyvs, momen cem enokketv cem wikekvs; kicvtes.

35 Opunaye emunkof, senekake semerpayv en cukon a awat, Ce'chuste elēpes; estoman Mvhayvn ohvtvlaken naoricetske haks? makakvtes.

36 Cesvs, opunvkv mahokan pohan vpakusen, senekake semevpayvn, Ce penkahlats, vkvsvmkv tvlkusis ocvs, kicvtes.

37 Momet este estomis vcvkayvre em etektvnecekates, momis Petv, Cems, Cems etecakkat Cane esyomat tvlken. 38 Momet senekake semevpayv en cukon vlaket, estoke mahan, momen hvkihket taye wahokan hecvtes.

39 Momet ecehyof, Estomaten estoke mahet, hvkihhokatske haks? hoktucet ēlekos, momis nocepet omes, kicakvtes.

40 Momen vpelicakvtes. Momis omvlkvn esossihcof, hoktuce e'rke e'cke tepaken momen vprkakan evpayet, hoktuce wakkan esceyvtes.

41 Momet hoktuce enken en hvlatet, Tvlirv-kumi; mv ohyvtekhoyat, Hoktucē ahuervs, (ce kicis,) kicvtes.

42 Momen vyvkhvmkusen hoktucet ahueret, yvkapvtes, en hoktvletv ohrolope palen hokkolohkakat omekv. Momen vhere mahen em estomahakvtes.

43 Momen nvpvt kerrekaren heren em vsehakvtes; momet hompetvn e'mhoyvren makvtes.

\section{SETENTVCKE 6.}

Momet mvn aosiyet, em ekvnvn oh 'ceyvtes; momen en kerrepvlket vcrkvpeyvtes.

2 Momet nettvcako orof, senekake of $v$ mbayetvn vlicecrtes; momen pohakat sulket em estomahakvtes, Estvmin heyv hunvnwv heyv nanvken en heckvt haks? momen heyv hoporrenkv e'mhoyat naket o haks, momat 
vtotketv yekçke mahis e'nke eteropotten momaket onkv?

3 Heyvt Mele e'chuswr, cuko-hayv, Cems, Coses, Cutv Simvn esyomat etecakkat toko? momen e wrntaket yvmvn epu 'pvkaket omeko? makaket. Momet en homecakvtes.

4 Momis, Owalv em ekvnv, momet en nakvlke vpvke, momet en cuko ofv tvlken vrakkuehocekot omes, Cesvs kicvtes.

5 Momet mvn vtotketv yekcat momeceko tayen, momis enokhokat nvcomusen e'nken oh wvkhokicet $e m$ wicecakvtes.

6.Momet vkvsvmvkekat omecicen em estomahvtes. Momet tvlofuce vlkan aret, mvhayvtes.

7 Momet palen hokkolohkakan enhuehket, hokkolaken vtotetvn vlicecrtes; momet puyvfekcv hvsvthvkekat ohfvnken yekcetvn emakvtes.

$8 \mathrm{M}$ omet vpeyvranat naken sehopakvkekot, ehvlewv en sukcv tis, tvklike tis, monkat crtokunawv en sukev cvtokunawv vtehke tis:

9 Momet kapvn 'se hokkolicekot, estelepikvn vtehaket, setekkekv tvlken esvkvren kicakvtes.

10 Momet kicakvtes, Estvmv estomis cukon esceyatskat fekhonnatsket emunken, mvn enkvpaket vpeyatskvres.

11 Momen estimvt o estomet ce yvpayvket cem apohicvkekon omat, mvn enkvpakatskof, vnrvpe ohkerkueckvn ce'le ofv essu vlikan a fekeyvks. Mehenwrn ce kicvkis, Ohfvececkv nettv omof Satvme Komalv tepakatet mv tvlofvn 'sem mvmomvke tares.

12 Mornen sossaket, estet vkerrickv eticvkvren erkenvkakvtes.

13 Momen puyvfekcv holwvhokat sulken sossicet, enokhokat olefv-nehan es en siyet, em wicecakvtes. 
14 Momen Cesvs ohfvccun Helot mekkot pohvtes, (e hocefkvt hvmkvn pohkvtet ok',) momet, Cane paptisvt pvsvtkvten a en kvwapket os, rnomen monkv vtotketv yekcakat eme eteropotten momaket os, makvtes.

15 Elivs tes, vpvlwvt makaken, vpvlwvt, Owalv, monkat owalvlke hvmke omet os, makakvtes.

16 Momis Helot pohhof, Cane mv e'kv en tacvyvnke, mvt omes; pvsvtkvten a en kvwapket os, makvtes.

17 Helot mahusat est' vtoten, Canen eset, cuko-yekcv ofvn svlvfkuecrtet omekv, etecakkat Felvpe e 'hiwv Helotev vrahkvn: Helot epayvtet omekv.

18 Canet Helot em punayet, Tecakketskat e hiwvn epayetske tayat cem etektvnkekot omes, kicvtet omekv.

19 Monkv Helotevt en homecet, elecetvn komvtes; momis umeko tayvtes.

20 Helot, Canet este-hunvnwv fvccet, momet hvsvtket omen kerret, em penkalvtet omekv, momet vcayecvtes: momet pohof, nanvke sulken momecet, afvcket em apohicvtes.

21 Momen nettv momvre here, mv ${ }^{-H e l o t}$ heckvte nettvn yafke hompetv rakken este em wiketv, kvpetvne homvhhotat, Kalvlevlke este en homvhhotv esyomat en hayakof;

22 Momen mata Helotev e'chuswv hoktet eceyet, opanet, Helot momen vprkake vpokan afvckvkuecof, mekkot, Nake estomis kometskat vm 'pohvs, momen mvn ce'mares, hokte mvnettan kicvtes.

23 Momet opunvkv yekcen 'setemfaccet, Naket o estomen vm 'pohetskis, vm ohmekketv en nvrkvpv tis oren ce'mares, kicvtes.

24 Momen aosiyet, e'cken em punayet, Naken vpohvranvya? kicvtes. Momen, Cane paptisv e'kvn, makvtes.

25 Momen moman vpakusen lvpecet mekkon em vlaket, 
Cane paptisv e'kv pvlvknv vpiken lvpken v'metskvren komis, maket em 'pohvtes.

26 Momen mekko e feke tayen en nokke tates, mome estomis opunvkv yekce setemfaccat, momet $\mathrm{mv}$ vpake vpokvte vrahkvn vhepvketvn komekates.

27 Momet moman vpakusen mekkot $\mathrm{mv}$ elecvranat vtotet, e'kvn 'sem vlvkvren, makvtes: momen ayet, cukoyekev ofvn e'kvn en tacvtes.

28 Momet e'kv pvlvknv vpiken hokte mvnettan 'sem vlakvtes ; momen hokte mvnettat e'cken emvtes.

29 Momen en kerrepvlket pohahkof, yihcet, e'nan ehset, sepvlkv ofvn wrkecakvtes.

30 Momen vpastelvlket Cesvs em vtelokvtes, momet nanvke momecakvte, momen nanvke mvhayvkvte omvlkvn em onayakvtes.

31 Momen, Awvnen vnrvwrn esceyet, vkocuknusen fekvpvkes, kicakvtes ; yicat momen vpeyat sulket omen, hompetv tisem em etektynkvkekates.

32 Momen perro-cukon vtehket, entvlkuset svkvpeyet, ekvnv tvnkan resoricites.

33 Momen enkvpake svkvpeyan hecakvtes, momen sulket Cesvs kerraket, tvlofv omvlkv asosset, vpeyvranan pefatket emuntvlaket, ehomvn mvn roricet, em vtelokvtes. 34 Momen Cesvs, osiyof, este sulke mahen hecet, em merrusvtes, yvpefikv vhecicv ocvkeko omakekv : momet nanvke sulken mvhayvketvn vlicecrtes.

35 Momen entis hvse-vkerkv sulket hoyanof, en kerrepvlket en yicet, Heyvt vnrvwrt os, momen hiyomat hvsevkerkv sulket hoyanes;

36 Vtotetsken ekvnv vwolakan momet tvlofuce vlkan vpeyet, hompetvn e en nesvkepekvs; hompetvn ocvkekokv, makakvtes.

37 Em vyoposket, Cemet hompetvn emvks, kicakvtes. 
Momen, Tvklike enrahkv tenelyv cukpe hokkole oren er nehsēt hompvkvren emvraneya? kicakvtes.

38 Tvklike vnore nvcomen ocatska? er hecvks, kicakvtes. Momen kerrahkof, Cahkepen, momen rvro hokkolen, makakvtes.

39 Momen omvlkv tekvpakvken pvhe lanan vpoyvkvren kicakvtes.

40 Momen tekvpakvken vpokvtes, cukpe hvmken, momet pale cahkepake etekvpakvken.

41 Momen tvklike vnore cahkepan, momet rvro hokkolen cahwof, hvlwen vhecet, ohmeriyet, momet tvkliken kvlkahyet, en kerrepvlken emakvtes, ehomvn ocvkvren; momet rvro hokkolen omvlkvn em etekvpicakvtes.

42 Momen omvlkvt hompet, fvcecakvtes.

43 Momen wokockat momet rvrou vhoskan svmpv fvcke palen hokkolohkaken cvwakvtes.

44 Momen tvklike vnore cahkepan hompakat hunvntake cukpe-rakko cahkepat mahe tates.

45 Momen moman vpaken en kerrepvlke perro-cukon vtehkepuecet ehomvn trpalvn Persitv vfvecvn vpeyecicvtes, este sulkat etvn vtotof.

46 Momet etvn vtotahkof, enkvpaket mekusvpvranet, ekvnhvlwen ohcemkvtes.

47 Momen yafkof, perro-cuko vkhvse vknvrkvpvn vk liken, entrlkuset lvpvtken arvtes.

48 Momet kafet vtotkaken hecrtes; hotvlet mvn vnrvpet omekv; momet nere vketeckv esostvtekan vkhvsen oh yvkapet er em orakvtes, momet hoyvnetvn komvtes.

49 Momis vkhvsen oh yvkapen hecakof, Puyvfekcvt omes, komaket, huehkakvtes.

50 (Omvlkvt hecaket, fekhervkekatet ok'.) Momen lvpken ete'm punayet, Ce mahlvpvtkvkekvs, vne tes, ce penkahlvkates, kicakvtes. 
51 Momet oh vyaket perro-cukon ohcemket eceyvtes; momen hotvlet fekhonnvtes; momen eme ofv svhopvyesko tayen em estomahakvtes.

52 Tvklike vnorvke nakeskerkvn oh vkerricvkekatet ok'; e feke take wvnhe hakvtet ok'.

53 Momen tiyecof, Cenesvle ekvnvn yicet, lvpvtken vcayakvtes.

54 Momen perro-cukon asossan vpakusen kerrakvtes,

55 Momet mv ekvnv afulotat omvlkvn hvmecicet pefatket, arvcuken pohakan, enokhokat pvtakv oh lumhen es fulletvn vlicecakvtes.

56 Momen tvlofucvlken, tvlofv vlkan momet en lvpvtke tis, estrmv estomis eceyat, enokhokan tehoyvnkv ofvn lumhicaket, en kapv em vtekat tvlkusis celayvkvren em 'pohakvtes; momen celayakat vtekat cvfencvke hakvtes.

\section{SETENTVCKE 7.}

Mомоғ Falvsevlket momen cokv-hayvlke vpvlwvt Celuslvmen a vwet Cesvs em vtelokvtes.

2 Momen en kerrepvlke vpvlwvt enke svholwvhokan, (mv okkoskvkekan okat,) es hompaken hecakof, ecayakvtes.

3 Falvsevlket momen Cusvlke omvlkvt, tewolen e'nken okkosvkekon omat, hompvkekot omekv, vculvke em punvkvn vcayecaket.

4 Momet nakwikv vlkan a awat, okkosvkekon omat, hompvkekos. Momen nanvke etv sulket ocakes, mv vcryecetv en heckakvte, sesketv, nake vcrnkv, crto lane nake vcrnkv, momen ohhompetv esyomat okkosetv.

5 Mómof Falvsevlket cokv-hayvlke esyomat em 'pohaket, Estoman cen kerrepvlket vculvke em punvkv vevkvyen yvkvpaket omeko haks? momis enke okkoskvkekon es hompaket os. 
6 Momen, Hocce vevkvyat Esivs ec ohfvecv taken heren owalvtes, mekusapv vhayvlke toyatskat, Heyv este $e$ cukhrrpe 'svc vrakkuecaket os, momis e feke take vn hopryeces.

7 Momis ehvperkvn v'mekusvpakes, este em vhakvn 'sest' emvhayet, maket.

8 Hesaketvmese em vhakvn wiket, este em punvkvn ocatsket os, $m v$ sesketv nake vcrnkv tis okkosvketv, momet mv omakat etv sulken momecatsket os; kicakvtes.

9. Momet, Momvre heren Hesaketvmese em vhakvn ehvperkuecatsket os, cem punvkvn ocatskat vevyecatskvret.

10 Ce'rken ce'rkeu vrakkuecvs; momet, Estit erke monkat e'cken ehanen omat, elekvs, Moses makvtet omekv.

11 Momis centake, Estet, e'rket ot e'cke tateu em punayet, Nake cen lopicvye tayat Kapan tes, mv nakemkv maketvt omen, kicen omat, tayes, makatsket os.

12 Momet e'rke e'ckeu ohvtvlaken naken em momece tayat vfekhonnicatsket os.

13 Hiyomen cem punvkv est' ematskvte eteropotten $\mathrm{He}$ saketvmese em opunvkv ehvperkvn hayet, momet nanvke etv mv omakat sulken momecatsket os, kicakvtes.

14 Momet este sulkat omvlkvn vtelohyof, kicakvtes, Omvlkatskat, vm apohicrkes, momet kerrvkes.

15 Nake este ofv tokat ecehyet, vhopane tayat sekos, momis nanvke asossat, mvt esten vhopvnaket omes.

16 Estimvt ehvcko es pohetv ocen omat, pohekvs.

17 Momet este sulkan wihket, cukon ecehyof, en kerrepvlket mv opunvkv svhopakv ohfvccvn em 'pohakvtes.

18 Momen kicakvtes, Cemeu momēn hoporrenvkeko toyatske haks? Nake este ofv tokat eceyat vhopvneko tayat kerratskeko haks? 
$19 \mathrm{E}$ feke tokon, momis nvrken ecehyet, nanvke hompetv omvlkvn hvsvthihcen, vpvlvthoyan vwihoket omekv.

20 Momen makvtes, Nake este aossat, mvt esten vhopanet os. -

$21 \mathrm{Mv}$ ofv tat, este e feke take asosset, vkerrickv holwrhokat, hoktarkv, empvlse seko hoktarkv, esteleckv,

22 Horkopkv, vleskvpkv, holwvyeckv, este mvkerretv, eenyvmahkueckv, eturwv holwakat, Hesaketvmese tvklecckv, ekvsvmkv, hacohaketv a vwet os;

23 Heyv nak holwvhoke omvlkvt ofv asosset, esten Bhopvnaket os.

24 Momet ahuyiret, mvn enkvpaket, Tilv Sitvne tepakat em vtekakat ofvn roret, cukon eceyvtes, momet nvpvt kerrekaren komvtes, momis ehkeko tayvtes.

25 Hokte hvmket, $\mathrm{mv}$ e'chuswv hoktuce puyvfekcv hvsvtkeko vpiket omen, Cesvs ohfvecvn pohhet, vlaket, e'le tempen tvk latkvtet omekv :

26 (Mv hokte Klekvlket, em etvlwv vcrkvyen Silofenesvlke tates,) momet puyvfekcv holwakat e'chuswv ossicvren em 'pohepvtes.

27 Momis Cesvs, Hopuetake tatet fvcfvkekvs; hopuetake en tvklike eset, efv em vwiketv fvccekot omekv, kicvtes.

28 Momen em vyoposket, Momes, Pucasē, momis efvt ohhompetv elecrn hopuetake em oh wokockan hompaket os, kicvtes.

29 Momen, Heyv maketskat omecicen vyepvs, puyvfekcv holwakat ce'chuswrn osiyes, kicrtes.

30 Momen e huten rorof, puyvfekcv holwakat e'chuswv ossen, momen e'chuswvt topvn oh wvkechoyen hecvtes.

31 Momen hvtrm Tilv Sitvne tepakat em vtekakan enkvpaket, Tekapoles em vtekakan ropottet, Kalvle vkhvsen rorvtes. 
32 Momen ehvcko poheko, punvkv em vtvcken 'sen yicvtes; momen e'nken oh wvkecvren em 'poha. kvtes.

33 Momen este sulkat a enkvpvken ehkusen sayet, e'nke wesakvn, e hrckon em 'pikvket, momet tofiket, e tulaswrn celayvtes.

34 Momet hvlwen vhecet, hihket, Eferv, Haukvs, maketv tes, kicvtes.

35 Momen moman vpakusen e hvckot haukahken, e tulasfvkv enrecopkvtes, momen heren opunayvtes.

36 Momen estimvn em onayvkekaren yekcen em vsehakvtes : momis ohvtvlaken em vsehof, ohvtvlvke svhere mahen tvlemicakvtes.

37 Momet svhopvyesko tayen em estomahakvtes, Nanvke omvlkvn heren momēces; pohvkekat pohaken, momen punayvkekat punayake tayen hayaket os, makaket.

\section{SETENTVCKE 8.}

- Mv nettv take omof este sulke vhere mahet ot, momet nake hompvkvren ocvkekon, Cesvs en kerrepvlken enhuehket,

2 Este sulkan em merrusis, vc vprkake munken hiyomat nettv svtutcenat orekv, momet hompetv ocvkekokv.

3 Momen hompvkekon e hute taken vtotakin ornat, vpeyof, hotosvkvres; hopvyen vwakat svset omekv, kicakvtes.

4 Momen en kerrepvlket, Estvmin vte saset yvmvn vnrvwr ofvn heyv este tvklike es fvcecvkuece taye haks? kicakvtes.

5 Momen, Trklike vnore nvcomen ocatske haks? kicet em 'pohakvtes. Momen, Kolvpaken, makakvtes.

6 Momen este sulkat ekvnvn tvk vpokvren makvtes: 
momet tvklike vnore kolvpakan cahwet, Hesaketvmesen .vkvsamet, kvlkayet en kerrepvlken emvtes, homvn ocvkvren; momen este ehomvn ocakvtes.

7 Momen rvrocvlke nvcomusen ocakvtes: momen Erken vkvsamvtes, momet mvu homvn ocvkvren makvtes.

8 Monkv hompaket fvcfakvtes; momen wokocke vhoskat svmpv fucke kolvpaken crwakvtes.

9 Momen mv hompakvte cukpe-rakko ostvteke tates: momen etvn vtotakvtes.

10 Momen lvpkusen, en kerrepvlket vpvkaken, perrocukon 'pihket, Talmvnurv ekvnv vlkan eceyvtes.

11 Momen Falvsevlket asosset, vpohkv en hayetvn vlicecakvtes, eskerret, nakeskerkv sutv vten hopoyaket.

12 Momen em puyvfekev ofvn hihket, Estoman heyv honvpse nakeskerkvn hopoyake haks? Mehenwvn ce kicvkis, Nakeskerkvn heyv honvpsen emhoyekares, makvtes.

13 Momet enkvpaket, hvtvm perro-cukon 'pihket, tvpalvn tikvtes.

14 Momen en kerrepvlket tvklike svpeyetv ehosaket, perro-cuko ofv tvklike vnore hvmke 'sen hoyanat ocvkekates.

15 Momen em vcahnet, Vketecet, Falvsevlke momen Helot enake espakkueckvn em e vketecvkes, makvtes.

16 Momen e ete'm punahoyet, Tvkliken ocēkokv omes, makakvtes.

17 Momen Cesvs kerrof, kicakvtes, Estoman tvklike ocekat omecicen e ete'm punahoyatske haks? hecekot, momet kerratskeko emunke haks? ce feke take wvnhe emunke haks?

$18 \mathrm{E}$ turwv ocet hecatskeko haks? momet ehvcko ocet pohatskeko haks? momet vkerricatskeko haks?

19 Cukpe-rakko cahkepan tvklike vnore cahkepan kvl- 
kayet em etekvpicvyof, wokocke svmpv fvcke nvcomen cawatskvnka? Palen hokkolohkaken, kicakvtes.

20 Momen cukpe-rakko ostan kolvpakan em ete kvpicvyof, wokocke svmpv fvcke nvcomen cawatskvnka? Momen, Kolvpaken, kicakvtes.

21 Momen, Estomet on kerratskeko e haks? kicakvtes.

22 Momen Persitvn vlakvtes; momen este turhecekon 'sen yicet, celayvren em 'pohakvtes.

23 Momen turhecekan enken en hvlahtet, tvlofvn 'sossvtes; momet e turwvn em oh tofket, e'nken oh ocahkof, Naken hecetska? kicet em 'pohvtes.

24 Momen vfvnnaket, Este eto yvkvpake omaken hecis, kicvtes.

25 Renyupvn e turwvn hvtvm e'nken oh ocet, vfvnnakepuecvtes; momen cvfeknihohcen, este omvlkvn heren hecrtes.

26 Momen en cukon vtotvtes, Tvlofvn eceyekot, tvlofv vtehkat hvmkis 'monahyetskvs, kicet.

27 Momet Cesvs, en kerrepvlket vpvkaken, Seselev Felepi em etvlofv vlkan sossvtes, momet vpeyof en kerrepvlken em 'pohet, Estimvt os, estet cv kicake te? kicakvtes.

28 Momen vyoposkakvtes, Cane Paptisvt, momis Elivs makakat sases, vpvlwvt, Owalvlke hvmkat, makakes.

29 Momen, Momis ceme, Estit os, cv kicatske haks? kicakvtes. Momen Petvt vyoposket, Mv Klist toyetskes, kicvtes.

30 Momen eme ohfvccrn este hvmkusis 'monayvkekaren em vsehakvtes.

31 Momen mvhayetvn vlicecet, Este E'chuswvt nanvke sulken 'sestemerkvranet os, momen vculvke, plestvlke enhomvhhotat cokv-hayvlke esyomat etvn vhepvkakvres, 
momen elehohcen, nettv svtutcenan akvwvpkvres, makvtes.

32 Momet mv opunvkvn kerken opunayvtes. Momen Petvt hvlatet em vsehetvn vlicecvtes.

33 Momis fulotket en kerrepvlken oh hecahkof, Petvn em vsehvtes, $\mathrm{Cr}_{\mathrm{r}}$ topvirvn vyvs, Setvnē, Hesaketvmese enanvke tokon, momis este enanvken vpryecetsket ome$\mathrm{kv}$, maket.

34 Momen este sulkat, én kerrepvlke esyoman enhuehkakof, kicakvtes, Estimvt o estomis vc vcrkvyetvn komen omat, e em ehvperkuecet, etohweklepkvn kvwahpet, vc vcrkvtekvs.

35 Estimvt o estomis en hesaketv hesayecetvn komen omat, sumecicen, estimvt o estomet opunvkv-herv momet vne $v c$ vrahkvn en hesaketv sumecicen omat, mvt hesayecrret ok'.

36 Este ekvnv omvlkv heckuecet omis, em puyvfekcvn sumecicen omat, naken en lopice haks?

37 Monkat naken este em puyvfekev es vyoposiket o haks?

38 Monkv estimvt o estomet, heyv honvpse hoktare momet holwvyecat ofvn vm vlset, vm opunvkvn em vlsvket on omat, este E'chuswvt, hvlwe estvlke hvsvthakat vpvkaken, E'rke en rakketr ofvn vlakof, mvu em vlsvret ok'.

\section{SETENTVCKE 9.}

Moмeт, Mehenwvn ce kicvkis, yvmv svpaklat sases, elkv hecvkeko munket, Hesaketvmese em ohmekketv yekcetv 'svlaken hecahke tvlkvres, kicakvtes.

2 Momet nettv epakat hoyanof, Cesvs Petv, Cems, Cane esyoman evpahyet, kvpvken entvlkuset fullen, rvne hvlwe mahen es vcemkvtes; momet ehomvn emvrahkvtes. 
3 Momen em accvke hvjayvke haket, hvtke mahet, hetute omvke hakvtes, mv tvrkv-hvtecv ekvnv vpokat hvmkis hvthiceko tayat oren.

4 Momen Elivs Moses vpaken em e heckuecakvtes; momet Cesvs ete'm punayakvtes.

5 Momen Petvt vyoposket, Mvhaya, yvmv fulleyvre heres; momen cuko vhake tutcenen hayvkeres, hvmket cenaket on, hvmket Moses enaket on, momen hvmket Elivs enaket on, Cesvn kicvtes.

6 Nake maketvn kerrekokv; tayen penkvlakekv.

7 Momen ohvpetticet aholocet ocvtes : momen aholoce aosset opunvkv vlakvtes, Heyvt Cv'ppuce vnokeckat tes, em apohicvkes, maket.

8 Momen vyvkhvmkusen, afulotken vfvnnakahkof, ohvtvlaken estimvn hecvkekot, Cesvs tvlkuset vpaken hecakvtes.

9 Momen ekvnhvlwen ahvtvpecof, nanvke hecakat estimvn em onayvkeko munken este E'chuswv elkv a en kvwvpke tvlken yekcen kicakvtes.

10 Momen mv maketvn entvlkuset ocakvtes, Elkv aenkvwvpketv naket o haks? maket ete'm 'pohaket.

11 Momen em 'pohaket, Estoman cokv-hayvlke Elivs tatet vlvkvranet os, makake haks? kicakvtes.

12 Momen vyoposket, Mehenwusen Elivs tatet vlvkvres, momet nanvke omvlkvn ohvhericvres, momet este E'chuswv ohfvecr hoccat, nanvke sulken 'sestemerket, nakhahoyekares, maket os, kicet em onayakvtes.

13 Momis ce kicvkis, Mehenwvt's Elivs vlakat, momen ohfvecv hoccat vevkvyen naket o estomen komakat em momecakvtes.

14 Momet en kerrepvlken em vlakof, este sulke mahet em vfolotaken, momen cokvhayvlket vpohkv en hayaken hecvtes. 
15 Momen moman vpaken este sulkat omvlkvt, hecakof, tayen em estomahet, pefatket oh vpeyet, vsekakvtes. 16 Momen, Naken em 'pohatske haks? maket, cokvhayvlken em 'pohakvtes.

17 Momen este sulkat hvmket vyoposket, Mvhaya, cv'ppucen es cem vlahkis, mv puyvfekcv opunayeko ocat;

18 Momen estrmv estomis esat selahlecet os, momen svpvkpvkuecet, e nute setetekket, oyoke haket os: momen cen kerrepvlken em punayvyis, ossicvkvren, momis umvkeko tayis, kicvtes.

19 Em vyoposket, Aeha, este vkvsvmkv oceko toyatskat, en hofune estomusen ece 'pakvranvki haks? en hofune estomusen vm muntvlkekot 'sece'yvranvki haks? 'Svm vtes, kicvtes.

20 Momen 'sen yicvtes: momen hecan vpakusen puyvfekcvt selahlecvtes; momen ekvnvn tvk lvtiket, svpvkpvkuecet, pvlpakvtes.

21 Momen e'rken em 'pohepet, Heyvt oh vlakvte vtekat en hofune estomuse te? kicvtes. Momen okat, Estuce vteken :

22 Momen tewolen totkvn tvk wiket, uewvn vk wikvtet os, elecvret; momis naken momecetske tayen omat, pum merret, pum vnicrs, kicvtes.

23 Vkvsvmetske tayen omat, mv vkvsamat nanvke omvlkv em momvike tayes, Cesvs kicvtes.

24 Momen moman vpakusen hopuewv e'rket huehket, eturopuswv pvlatken, Pucasē, vkvsamis ; momet vkvsvmvkan vm vnicvs, makvtes.

25 Momen Cesvs, estet pefatket yetohkvlken hecof, puyvfekcv hvsvtkekan em vsehet, Puyvfekcv opunayeko momet pohetskekat, Aossvs, momet svnvcomvn ecehyetskvs, ce kicis, kicvtes.

26 Momen puyvfekcvt huehket, tayen selahlecet aos- 
svtes; momen ēle omvtes; mont omet sulket, Ēles, makakvtes.

27 Momis, Cesvs enken en hvlatet akvwapen, ahuervtes. 28 Momen cukon acehyof, en kerrepvlket ehkusen em 'pohaket, Estoman pumet ossicēko tayvt haks? kicakvtes. 29 Momen, Heyv omat, mekusvpkv momen eelvweckvt omekon omat, aosseko tayes, kicakvtes.

30 Momen mvn enkvpaket Kalvlen teropotecrtes: momen nvpvt kerrvren komekates.

31 En kerrepvlken emvhayet kicakvtet omekv, Este E'chuswv este e'nke ofvn wihoket os, momen elecvkvres, momen elehohce renyvpvn nettv resvtutcenan akvwvpkvres, maket.

32 Momis mv maketvn kerrvkekates, momet vpohetvn penkvlakvtes.

33 Momen Kvpvnevm vlakvtes; momen cuko ofvn vpoket omen, Awatskof, naken 'setenrapatskisa? kicet em 'pohakvtes.

34 Momis naken makvkekates; awof estimvt rakke mahe taranat 'setenrvpakvtet omekv.

35 Momen lihket, pale hokkolohkakan enhuehket, Estimvt homv haketvn komen omat, omvlkv 'sem poke omet, omvlkv em vtotkv tares, kicakvtes.

36 Momet hopuewucen eset, en nvrkvpvn licvtes; momet e sakpv ofvn ehsof,

37 Estimvl o estomet heyv hopuewuce omake hvmken cv hocef $\mathrm{kv}$ ofv esen omat, vnen cv'set os; momen estimut cr yvpayen omat, vne tokon, mv vc vtotvten evpayet omes, kicakvtes.

38 Momen Cane em vyoposket, Mvhaya, hvmket puyvfekcv holwvhokat ce hocef $\mathrm{kv}$ ofv sossicen heceyvnks, momen epu 'cvkarekos; momet em vseheyvnks, epu 'cvkarekokv, makvtes. 
39 Momis Cesvs makvtes, Em rsehhatskvs, este estome, cv hocefkv ofv nakeskerkvn momecet, lvpken vc ohfvecv holwaken opunaye tayat sahsekot ok'.

$40 \mathrm{Mv}$ pu'nrvpekat pum vpvlhvmken hueret omekv.

41 Mehenwvn ce kicvkit omikv, Estimvt o estomet, Klist enake toyatskekv, cv hocef kv ofvn uewv esketv sesketuce es ce'mvken omat, em feketv en sumikekos.

42 Momen estimvt o estomet heyv lopucke ve ofvn vkvsvmakat hvmken naorkepuecen omat, enfolotkv en cvtot e nokwv vtvriken, uehvtkv vk wihokat mit en heret os.

43 Momen ce'nket ce naorkepuecen omat, tvcrs, vnvttet hesaketv eceyetskat mit cen heret os, enke hokkolen ocet, elkv tvlofv, totkv vsleko tayan eceyekot:

$44 \mathrm{Mv}$ en cuntv elekon, totkv vslekan.

45 Momen ce'let ce naorkepuecen omat, tvcrs, vnvttet hesaketv eceyetskat mit cen heret os, ce'le hokkolen ocet, elkv tvlofv, totkv vsleko tayan ce tvk wihokekon :

$46 \mathrm{Mv}$ en cuntv elekon, totkv vslekan.

4.7 Momen ce turwvt ce naorkepuecen omat, ossicvs : ce turwv hvmkusen ocet Hesaketvmese em ohmekketv eceyetskat mit cen heret os, ce turwv hokkolen ocet elkv tvlofv totkv ce tvk wihokekon:

48 $\mathrm{Mv}$ en cuntv elekon, totkv vslekan.

49 Omvlkvt totkvn es okcrncrmpehocen, nake ohnekrickv vtekat okcrnwv es vpahoyvret ok'.

50 Okcvnwvt heres, momis okcvnwvt en yekcen sumecicen omat, naken es svpvtecatskvr haks? Ec ofvn okcvnwvn ocet, herkvn ete'm ocvkes.

\section{SETENTVCKE 10.}

Momen ahuyiret, mvn enkvpaket, Cotvne tvpalv Cutev em vtekakan eceyvtes; momen este sulsokat hvtvm em 
vtelokvtes; momen mecvnto omet hvtvm emvhayakvtes.

2 Momen Falvsevlket en yicet, Hunvnwv $e$ hiwv wiketv em etektvnke te? kicet eskerraket em 'pohakvtes.

3 Momen em vyoposket, Moses naken ce kicvkvt haks? kicakvtes.

4 Momen, Moses, Nakcokv es wiketvn en hoccihcet wiketv etektvnecvtes, kicakvtes.

5 Momen Cesvs vyoposket, Ce feke take em wrnhe omecicen heyv vhakvn cen hoccicvtes:

6 Momis nake hocvckvte em vliceckv vteken Hesaketvmeset hunvnwv hokte tepakan hahicvtes.

7 Momen heyv omecicen hunvnwv e'rke e'ckeu wihket, e hiwvn hvlvtvres;

8 Momen $\mathrm{mv}$ hokkolat vpeswv hvmkusvres; monkv - mv svnvcomvn hokkolet omeks, momis vpeswr hvmkus.

9 Monkv mv Hesaketvmese tehvlvtepuecrte estet tekvpihcats, kicakvtes.

10 Momen cuko ofvn en kerrepvlket mata nake ohfvecvn hvtvm em 'pohakvtes.

11 Momen, Estimvt o estomet e hiwvn wihket, etvn epayen omat, vnrvpet hoktaret os.

12 Momen hoktet e'hen wihket, etvn en liken omat, hoktaret os, kicakvtes.

13 Momen hopuetakucen 'sen yicakvtes, celayvkvren: momen en kerrepvlket mv esyicakan em vsehakvtes.

14. Momis Cesvs hecof, efeknokket, Hopuetakuce ve oh vwepvre em etektvnecet, em vsehhatskvs; mv omakat Hesaketvmese em ohmekketvt omekv.

15 Mehenwvn ce kicvkis, Estimvt o estomet hopuewuce omet Hesaketvmese em ohmekketvn e enakuecekon omat, ecehyekos, kicakvtes. 
16 Momet e sakpv ofvn cawet, enken oh wvkhokicet, ohmerrakvtes.

17 Momet vyetvn ossof, hvmket lētket vlahket, e vtokkekvtes, momet, Mvhayv heretskat, nake estomvyatet hesaketv yuksv-sekon epoy ar haks? kicet em 'pohvtes.

18 Momen Cesvs, Estoman here cv kicetske haks? herat hvmkusis sekos, Hesaketvmeset omekon omat.

19 Vhakv palan kerretskes, Hoktahretskvs, Elehcetskvs, Horkohpetskvs, Fvccekon akeriyetskvs, Fvccekon vkonahhetskvs, Ce'rken, ce'ckeu vrakkuecrs; kicvtes.

20 Momen vyoposket, Mvhaya, heyv omvlkvn cv mvnettusvte vteke vfaste vtecicvyvtes, makvtes.

21 Momof Cesvs hecet, vnokecvtes, momet, Nake hvmket cem vhoskes : ahyet, nanvke estomen ocetskat omvlkvn wiyet, estemerrakan emvkvs, momet hvlwe ofv nake ocetskvres; momet ahtet, etohweklepkvn kvwahpet, vc vevkvtes, kicvtes.

22 Momen mv maketv omecicen fekherekates, momet efeke nokket ayvtes; nakeockv rakrvken ocet omekv.

23 Momen Cesvs fulotket hecet, en kerrepvlken em punayet, Mvtvwwvt nakeockv ocakat Hesaketvmese em ohmekketv eceyetv en yekcvke tares! kicakvtes.

24 Momen en kerrepvlket em opunvkv omecicvken em estomahakvtes. Momis Cesvs hvtvm vyoposket, Hopuetakē, mvtrwwvt mv nakeockv em enhonrakat Hesaketvmese em ohmekketv eceyetv en yekcrket os!

25 Rvcce-kuphe esropottv e turwv ropotte tayat en yekce mahekon, este enakocv mimvt Hesaketvmese em ohmekketv eceyetvt en yekcet os, kicakvtes.

26 Momen svhopvyesko tayen em estomahakvtes, Monkv estimvt hesake taye haks? ete kicaket.

27. Momen Cesvs oh hecaket, Este em momvkeko tayes, momis Hesaketvmese tat monkot os; nanvke 
omvlkvt Hesaketvmese em momvke tayet omekv, makvtes.

28 Momof Petvt, Hecvs, nake omvlkvn wihket, ece 'cvkfullēs, kicetvn vlicecvtes.

29 Momen Cesvs vyoposket, Mehenwvn ce kicvkis, Estimvt ve vrahkv, momet opunvkv-herv vrahkvn cuko, tecakkakat, ewvntake, erke, ecke, ehiwv, hopuetake, ekvnv esyoman wihket,

30 Hiyome tan cukpe hvmken, cuko, tecakkakat, ewvntake, eckvlke, hopuetake, ekvnv esyoman en hecket, vsseckvu en hecket; momet ekvnv vlvkvranat ofv hesaketv yuksv-seko epoyvranekat sekot os, kicakvtes.

31 Momis enhvteceskv taranat espoke sulket ocakes, momen espoke taranat enhvteceskvt ocakes.

32 Momen Celuslvmen oh vpeyet nene oh fullvtes; momen Cesvs ehomvn ayvtes; momen em estomahakvtes; momet vcrkvpeyof, penkvlakvtes. Momen hvtvm palen hokkolohkakan evpayet, nanvke ohlvtkvranan em onvyvketvn vlicecvtes,

33 Hecvs, vcemecēt Celuslvmen oh vpeyēs, momen este E'chuswv plest enhomvhhotv cokv-hayvlke $e m$ wihokvres ; momen elkvn 'sem fvccecet, Centilvlken em wikvkvres:

34 Momet nakhayvkekot, 1epkvkvres, momet oh tof ket elecvkvres; momen nettv svtutcenan hvtvm akvwvpkvres, maket.

35 Momof Sepete e'ppucetake Cems Cane tepakat em vlahohket, Mvhaya, nake estomen komeyat pum momecetskvren komēs, makakvtes.

36 Momen, Naken cem momecvkaren komatska? kicakvtes.

37 Cem vrakketv ofvn, hvmket cem akvpervn liken, hvmkat cem akvskvnvn liken kakeyvren pu'mvs, kicakvtes. 
38 Momis Cesvs, Naken vpohatskat kerratskekos. Sesketv 'seskvyat 'seskatsken, paptesvm es paptiskvyat es paptiskatske taye haks? kicvtes.

39 Momen, Momeye tayes, kicakvtes. Momen Cesvs, Mehenwvn sesketv es eskvyat 'seskatskvres, momet paptesvm es paptiskvyat es paptiskatskvres:

40 Momis vm akvperv momet vm akvskvnv liketv est' emvranvyat crnake tokot os, momis mv em etetakuehocat emvkhoyvres, kicakvtes.

41 Momen mr palat pohakof, Cems Cane tepakat vnrvpet crpvkketvn vlicecakvtes.

42 Momis Cesvs enhuehkaket, Mv, Centilvlken ohfvnkaket os, komhoyat pucassetvn es ohvfvstaket, momet en rvkrakvt yekcetvn es ohmekkaket omat kerratskes.

43 Momis cem etenrvwv take momvranekot os, momis ece 'pakvkat estimvt rakke haketvn komen omat, cem vtotkv tares :

44 Momen estimvt o estomet rakke mahat haketvn komen omat, omvlkv em vtotkv tares.

45 Este E'chuswr tisem em rtothokvranekon, est' em vtotkvret vlaket, en hesaketvn sulken es nesetvn est' emvranet vlakvtet omekv, kicakvtes.

46 Momen Cvlekon yicvtes; momet mvn enkvpakof, en kerrepvlke este sulke mahe esyomat vpvkaken, Patemevs turheceko, Temevs e'ppucet, nakvpohet nene afopken likvtes.

47 Momet Cesvs Nasvlvlket omat pohof, Cesvs, Tewe E'ppucē, vm merrvs, huehket maketvn vlicecvtes.

48 Momen sulket, cvyayvkvren kicet, em vsehakvtes: momis ohvtvlaken svhere mahen huehket, Tewe E'ppucē, vm merrvs, kicvtes.

49 Momen Cesvs huyiret, enhuehhokvren makvtes; 
momen, Fekhvmket ahuervs ; ce'nhuehkes, kicet, turhecekan enhuehkakvtes.

50 Momen en kapvn vkiyet, ahuyiret, Cesvs em vlakvtes.

51 Momen Cesvs vyoposket, Naken ce 'stomecaren kometske haks? kicvtes. Hunvnwv turhecekat, Pucasē, renakvt vn heckate! kicvtes.

52 Momen Cesvs, Vyepvs, cem vkvsvmkvt temvpoken ce hahyes, kicrtes. Momen moman vpakusen hecet, nenen ayet Cesvs vevkayvtes.

\section{SETENTVCKE 11.}

Momen Celuslvmen vwolicaket, Olefv rvnen, Perfec Pervne tepakat roricet en kerrepvlke hokkolen vtotet,

2 Trlofuce ce homuse ocan cukolvkvks, momet, cukolakatskan vpakusen corakko wakuce este oh likvte sekan wvnvken eshecatskvres; enrecohpet, svm vthoyvks:

3 Momet este estomet, Estoman heyvn momecatske haks? ce kicvken omat, Pucaset eyacet omes; kicatskvres, momen moman vpaken atotvres.

4 Momen vhoyet, corakko-wakuce fettvn, vhauke tempe, nene tohvlacan wrnvken eshecakvtes: momet enrecopakvtes.

5 Momen mvn svpaklat vpvlwvt, Corakko-wakucen enrecopatskat nak estomatske haks? kicakvtes.

6 Momen Cesvs kicakvte etvpomen kicakvtes; momen vpeyecicakvtes.

7 Momen' corakko-wakucen Cesvs 'sem vlahokvtes, momet em accvke taken oh ocakvtes ; momen oh likvtes.

8 Momen sulket em accvken nenen oh prtapicakvtes, momen vpvlwvt etolvccen a waret, nenen oh prtapicakvtes.

9 Momen homvn vpeyat, momen yupv vpeyateu hueh- 
kaket, Hosanv ; Pucase e hocefkv ofvn vlakat oh merrvkvtet omes :

10 Tewe pu'rke em ohmekketv Pucase e hocef kv ofv vlakat ohmerrvket omekvs: Hosanv hvlwe mahat ofvn, makakvtes.

11 Momen Cesvs Celuslvmen ecehyet, cuko-vcakan eceyvtes, momet vfulotken nak omvlkvn oh vfvnnahkof, yafke hvse-vkerkvt omen, palen hokkolohkakat vpvkaken, Pervnen 'sossvtes.

12 Momet rempvksen, Pervnen rafulehcof, elvwe tates.

13 Momet bopvyen ke-rakko em vpe esse ocen hecet, nake oh ocen eshece taye witen omat komet oh vlakvtes; momet oh vlahkof, esse tvlkusen eshecvtes; ke-rakko em oketv hvte orekatet ok'.

14 Momen vyoposket, Mucv vtekat estimvt cem etten pvpekares, meyuksv-sekon, kicvtes. Momen en kerrepvlket pohakvtes.

15 Momen Celuslvmen yicvtes; momen Cesvs, cukovcakan ecehyet, cuko-vcakat ofv wiye momen nesakaten sossicetvn vlicehcet, tokunawv esyoposkvlke em ohhompetv, pvce-howe wiyakate em ohvpoketvu en cektulcecvtes ;

16 Momet nvpvt nake vtehkv monkat nake vcrnkv cuko-vcakan 'setehoyvnvren em etektvnecekates.

17 Momet emvhayvtes, Tvlwvvlke omvlkvt vn cukon mekusvpkv-cukon kicvkvres, maket coket omeko haks? momis centaket horkopvlke em vpoketvn hayatskes, kicet.

18 Momen Falvsevlket plest enhomvhhotv esyomat pohakvtes, momet nak estomecet elecvke tayan hopoyakvtes; este sulkat omvlkvt em mvhakv estomahe 'sem momakat omecicen em penkvlakvtet ok'.

19 . Momen yaf kof, tvlofvn ossvtes. 
20 Momen hvthvyvtke, hoyvnecof, ke-rakko em vpe yvlonkv oren talen hecakvtes.

21 Momen Petvt vkerricet, Mvhaya, hecvs, ke-rakko em vpe ohtvhiketskrnke yuknepes.

22 Momen Cesvs vyoposket kicakvtes, Hesaketvmese ofvn vkvsvmkvn ocvks.

23 Mehenwvn ce kicvkit okikv, Estimvt o estomet heyv ekvnhvlwen, Vkueket, uehvtkvn ce'k wihokekvs, maken omat, momet e feke ofv melohlekot, nake ocvrane makat vkvsamen omat, nake makan ocvres.

24 Monkv ce kicvkis, Emekusapatskof, nak estomvken ce yacvkat ocvranatskat vkvsvmvks, momet ocatskvres.

25 Momet emekusapet svpaklatskof, estimv vnrvpen naken ocatsken omat, enkvpryecicvks ; Ce'rke takeu hvlwe likat cen naorketv taken ce'nkvpryecicvkvren.

26 Momis enkvpvyecicatskekon omat, Ce'rke takeu hvlwe likat cen naorketv taken ce'nkvpvyecihcrkekos.

27 Momen hvtvm Celuslvmen yicvtes; momen cukovcakat ofvn yvkapof, plest enhomvhhotv, cokvhayvlke vculvke esyomat en yihcet,

28 Yekcetv estomen heyv nanvke es momecet ometske haks? momen estimvt heyv nanvke momecetvn yekcetv ce'me haks? kicakvtes.

29 Momen Cesvs vyoposket, Vneu naike hvmken cem 'pohvranvkis, momen vm vyoposkvkes, momen yekcetv estomen heyv nanvke es momecit omvyat cem onayvkares.

30 Cane em paptesvm, hvlwen vte tat haks? monkat este enake tat haks? vm vyoposkvkes, kicakvtes.

31 Momen e ete'm punayaket makakvtes, Hvlwe vten makēn omat, Monkv estoman vkvsvmatskekat haks? pu kicvres.

32 Momis, Este enakets, makēn omat; esten em pen- 
kvlakvtes, este omvlkvt Cane owalv mehenwe tate omecaket omekv.

33 Momen vyoposket, Kerrēkos, Cesvn kicakvtes. Momen Cesvs vyoposket, Vneu yekcetv estomen heyv nanvke es momecit omvyat cem onayvkvkos, kicakvtes.

\section{SETENTVCKE 12.}

Momet opunvkv svhopakv es em punvyvketvn vlicecvtes. Este humket pvrko crpofvn ahocrtes, momet eto lane tohopken afolotecihcet, pvrko opuswv vcrnkv ocvranan en koriyet, cuko-hvlwvn hayet, vfastvlken em wikvtes, momet ekvnv hopvyen ayvtes.

2 Momet oketv orof, vfastvlken vtotkvn vtotakvtes, vfastvlket prrko-cvpofv em etten emvkvren.

3 Momen eset, rukahfet, nakocekon vyecicakvtes.

4 Momen hvtvm vtotkv etvn ohtotakvtes, momen mvn cvton 'svfvllet nvfkaket, ekvn em vnvttecet, vlesketv vpvken celayvkvten vyecicakvtes.

5 Momen hvtvm etvn vtotvtes, momen mvn elecakvtes, momet eti sulken; vpvlwvn rukafet, vpvlwvn pvsvtakvtes.

6 Monkv vtote tayat hvmket em vhoskvtes, e'ppuce vnokeckat, espoken mvu ohtotakvtes, Cv'ppucen vrakkuecvkvres, maket.

7 Momis mv vfastvlket e ete'm punayakvtes, Heyvt pucase mvnettv tes; vwvks, elecvkeres, momen enake taranvtet punake tares, makaket.

8 Momet eset, elehcet, prrko cvpofvn ossicakvtes.

9 Monkv pvrko cvpofv e pucaset naken estomvre te? Vlahket, mv vfastvlken pvsvtvres, momet pvrko crpofvn etin emvkvres.

10 Momen heyv hoccat, mv Cvto tohtarvlke vwikvte vkvnowv em ekv hakes; 
11 Heyvt Pucase nake momecrte tes, momet pun renakv ehomvn estomahet, makat, hecatskvte seko haks?

12 Momen esetvn komakvtes, eme vnrvpen opunvkv svhopakvn opunayen kerraket ok'; momis este sulkan em penkvlakvtes; moinet wiket vpeyvtes.

13 Momet em opunvkv ofvn esvkvren Falvsevlke Helotvlke esyomat vpvlwusen vtotakvtes.

14 Momen yihcof, Mvhaya, mehenwetsket ot, este stoskometskekat kerrēs, Hesaketvmese em vyetv mehenwetv ofvn mvhayet, este en heckv moskometskekot omekv: Ekv feketv Sesv emetv fvccut o haks? monko haks?

15 Emeyvre te? monkat emèkare te? kicakvtes. Momis em mvkerretv taken kerret, Estoman es cv kerratske haks? tenelyvn 'svm vwvks, hecaren, kicakvtes.

16 Momen esyicrtes. Momen, Heyv vhake momen en hoccvt estimv enake te? kicakvtes. Momen Sesv enakē, kicakvtes.

17 Momen Cesvs vyoposket, Sesv enanvken Sesvn emvkes, momet Hesaketvmese enanvken Hesaketvmesen, kicakvtes. Momen eme ohfvecvn em estomahakvtes.

18 Momof Satusevlket, mv, Akvwvpketv sekos, makakat en yicvtes; momen em 'pohet makakvtes,

19 Mvhaya, Moses pun hoccicat, Hunvnwv etecakkvte hopuetaken ocekot elet, $e$ hiwvn wiken omat, etecakkatet e hiwvn epayet, honvpse etecakkvten em mahecvres, makvtes.

20 Monkv etecakkvte kolvpaket fullvtes; momen homahtatet hokten epahyet, elof, honvpsen wikekates.

21 Momen svhokkolat epahyet elvtes, momet invu honvpsen wikekates; momen svtutcenat matvpomen.

22 Momen mv kolvpakat epayakvtes, momet honvpsen wikvkekates; omvlkv'sem poken hokten elvtes.

23 Monkv akvwvpketv omof, akvwvpehcof, mv kolvpa- , 
kan estomat e hiwv tar haks? mv kolvpakat epayakvtet omekv.

24 Momen Cesvs vyoposket kicakvtes, Cokan, monkat Hesaketvmese en yekcen kerratskekat omecicen cem mvttvket omeko haks?

25 Elkv raenkvwvpehcof, etepayaket, monkat etepayepuehocekos; momis hvlwe estvlke hvlwe tvlofvn vpoke omaket omekv.

26 Momen prsatkvte akvwvpecvranat ohfvecr Moses en cokv ofvn ohonayatskekat haks, eto-fvske ofvn Hesaketvmese Moses em punayat, Vnet Eplehame en Hesaketvmese, Iseke en Hesaketvmese, momet Cekvpe en Hesaketrmese toyis, makvte?

27 Prsatkvte en Hesaketvmese tokon, momis wenahokat en Hesaketvmese tes; monkv tayen cem mvttvket os.

28 Momen cokv-hayvlke hvmket vlahket, ete'm punahoyaken pohet, momet heren em vyoposkaken hecet, Vhakv omvlkv enhvteceskv naket o haks? maket em 'pohvtes.

29 Momen Cesvs, Vhakv omvlkv enhvteceskvt heyv tes, Aeha Eslevl toyctskat, apohicvs; Pucase pun Hesaketvmese Pucase hvmkuset os ;

30 Momen Pucase cen Hesaketrmesen ce feke omvlkvn, cem puyvfekev omvlkvn, cem vkerrickv omvlkvn, cen yekce omvlkvn es vnokecetskvres: heyvt vhakv enhvteceskv tes.

31. Momen svhokkolat, $m v$ omet ot, heyv tes; E vnokecetskat etvpomen cem vwolicvn vnokecvs: heyv vhakv hokkole 'sen rakkat sekos, maket vyoposkvtes.

32 Momen cokv-hayvt kicvtes, Heres, Mvhaya, mehenwvn opunahyetskes, Hesaketvmese hvmkuset omen, eti sahsekot ok'.

- 33 Momen efeke omvlkv, vkerrickv omvlkv, puyvfekev 
omvlkv, momet yekce omvlkv es vnokecetv, momet e vnokecat etvpomet em vwolicv vnokecetv nake ohnekric$\mathrm{kv}$ temvpokakat omvlkv, momet nake pvsvthoyvranat senhoyvnet omes.

34 Momen hoporrenet vyoposken Cesvs hecof, Hesaketvmese em ohmekketvn em vhopvyecetskekos, kicvtes. Momen mv vtekat estimvt vpohkv en hayetv fekhvmkekates.

35 Momen cuko-vcakat ofv Cesvs estemvhayof, Estomen cokv-hayvlket, Klist Tewe E'ppucet os, makaket o haks?

36 Puyvfekcr Vcakat eteropotten opunayet, Tewe mahusat, Pucaset cv Pucasen em punayet, Vm akvpervn liketske munken, cen horrvlke ce 'leohsehoyickvn hayares, makvtet os, makvtes.

37 Monkv Tewe mahusat e Pucasen kicet os, momof estornaten e'ppucet o haks? vyoposket kicvtes. Momen este sulkat afvckvket em apohicakvtes.

3s. Momen em mvhakv ofvn, Cokv-hayvlken em e vketecvkes, mv accvke crpcrke es fulletvn, momet nakwikv vlkan vseketvn,

39 Senekake ofv ohliketv svmomvkus mahat em vlostakat:

40 Hoktvke oyevlke en cuko lokakat, momet vhayet, crpcrken mekusvpakat; heyvt senhoyvnen ohfvcceckvn en heckvkvres, kicakvtes.

41 Momet Cesvs crtokunaphute homusen liket, estomen este sulkat cvtokunawvn cotokunaphuten vtehakan hecvtes: momen nakocvlke sulket sulken vtehakvtes.

42 Momen hokte oye nakoceko vlahket cvto-kunawv lopuckus mahat hokkolen vtehvtes, mvt hoporrenkv en nvrkvpvn oret os. 
43 Momen en kerrepvlken enhohiket, Mehenwvn ce kicvkis, Heyv oye estemerrat tokunaphute vtehakat omvlkv senhoyvnen vtehet os.

44 Heyv omvlkvt enake take sulkat asossen vtehahket omen, emet em estemerketv asossen nake ocvte omvlkvn, mv vhesakvte omvlkvn, vtehet os, kicakvtes.

\section{SETENTVCKE 13.}

Momen, cuko-vcakan ossof, en kerrepvlke hvmket, Mvhaya, cvto momet cuko estomakan hecvs; kicvtes.

2 Momen Cesvs vyoposket kicvtes, Heyv cuko rvkrakan hecetska? cvto hvmkusis etohlike vwihokekat vhoskat sekares.

3 Momen, cuko-vcakat ehomusen rvne Olefvn likof, Petv, Cems, Cane Antolu esyomat ehkusen em 'pohakvtes,

4 Estofvn heyv nanvke ocvranat, momet heyv nanvke momvkvranof, naket eskerkv taranat, pum onvyvs.

5 Momen Cesvs em vyoposket, maketvn vlicecrtes, $\mathrm{E}$ vketecvkes, estit ce mvkerrvkekaren.

6 Sulket cv hocefkv ofvn yicet, Klist toyis, maket, sulken ehosvkuecrret ok'.

7 Momen horre ohfvccrn, momet horre ohonvkvn pohatskof, ce naorvkates, mv omakat ocvkvranat tvlkuset omekv, momis meyuksv hvtet omes.

8 Tvlwvt tvlwvn vnrvpet, momen tvlofvt tvlofvn vnrvpet vkvwvpkvranet omekv; momen ekvnv nekekv estomis ocvkvres, momen elaukot momen feknokketvt ocvkvres : heyv nanvke efeke nokketv vlicecakat tes.

9 Momis e vketecvkes, tekueketvn ce wihokvkvres; momet senekake vlkan ce tephokvkvres : momet cv hocef kv vrahkvn evpayvlke momet mekkvlke ehomvn es ce yihocvkvres, mv vnrvpe kerkueckvn. 
10 Momen heyv opunvkv-herv tawvn tvlwvvlke omvlkv en tvlemihocvranet os.

11 Momis ce yvpayet, ce wihokof, naken makvranatskat enhomvn vkerrihcatskvs, momet homvn ohvkerrihcatskvs: momis mv hrse-vkerkv ofvn nake ce'mhoyat mvn makvranatsket os: cemet opunayatskekon, Puyvfekev Vcakat opunayet omekv.

12 Momen tecakkvtet tecakkaten elkvn em wikvres, momen erket eppucen; momen hopuetaket eckvlke vnrvpen vkvwvpecet, pvsvthoyvren omecicvkvres.

13 Momen cv hocef kv vrahkvn este omvlkvt cen homecrkvres; momis estimvt emunket emeyuksicat, mvt hesakvres.

14 Momis enhomeckv trnecicvranat Tanvle owalv opunvyecvte huervranekaten hueran hecatskof, (estimvt ohonayat kerrekvs,) momof Cutevn vpokat ekvnhvlhawan oh pefatkekvs :

15 Momen cuko onvpv oh likat hvtvpiket, cukon ecehyats, momet nake cuko ofv ocat cawvret ecehyats.

16 Momen cvpofv arat kapv cawvret afulikates.

17 Momis nvrkesakat momet coyicakat mv nettv take omof en holwakvres.

18 Momen cem pefatketv take rvfon omekaren mekusvpvkes.

$19 \mathrm{Mv}$ nettr take omof estemerkv ocvres, mv ome enhvteceskv nake hocvckvte Hesaketvmese hayvte hiyomat vlakat ocvte sekot, momet ocekares.

20 Momen Hesaketvmeset mv nettv vhonkvtkvn kocuknicekaten omat, vpeswr hvmkis hesakekvnt omes: momis enhopokvte mv enhopoyvkvte vrahkvn mv nettv vhonkvtkv kocuknicet os.

21 Momen momof, Hecvs, yvmvn Klist likes, monkat Hecvs, vswn likes, estimvt ce kicvken omat, vkvsahmatskvs. 
22 Klist laksvlke momen owalv laksvlke akvwvpkvkvret ok', momet nanvke eskerkvn, momet nake estomahake heckuecvkvres; mome tayen omat, mv enhopokat mahusat tis ehosvkuecvkvret.

23 Momis vketecvkes : hecvs, nanvke omvlkvn enhomvn cem onahyvkis.

24 Momis mv nettv take omof, mv estemerkv hoyanof, hvse yomuckvres, momen hvresse hvyvyicekares,

25 Momen hvlwe kococumpv pvlvtkvres, momen yekcetv hvlwe ofv ocakat nekeyihocvres.

26 Momen momof este E'chuswv vkvsvmkv rakke, momet yekcetv vpaket aholoce ofvn vlaken hecvkvres.

27 Momen momof en hvlwe estrlken vtotvres, momet enhopokat hotvle ostat aenkvpvken, ekvnv hopvyvke mahat hvlwe hopvyrke mahat roren vteloyvkvres.

28 Tokvs, ke-rakko esvhopakvn en kerrvkes; e lvcce hvte lowaket ot esse sossicof, meske vwolice tat kerratskes.

29 Centakeu matvpomen heyv nanvke ocaken hecatskof, vwolices, vhauken vlaken kerrvkes.

30 Mehenwvn ce kicvkis, Heyv honvpse hoyvneko munken heyv nake omvlkvt esfvcike tvlkvres.

31 Hvlwe ekvnv tepakat sumhokvres, momis vm opunvkv sumikekos.

32 Momis mv nettv hvse-vkerkv tepakat nvpvt kerrekon, hvlwe estvlke monkat Eppuce tis kerrvkekon, Erke tvlket kerres.

33 Vfekcakhet, vketecet, emekusvpvkes : oketv orvranat kerratskekokv.

34 Este hopvyen ayvranat, en cukon wiket, yekcetv vtotkvlken, momet este vtekaten em vtotketvn emaket, vhauke vhecicv vketecvren, makvtes : este $E$ 'chuswv $m v$ omet os. 
35 Monkv vketecvkes; estofvn, yafkis, nere nvrkvpv tis, tottolose en hunvnwv hahokof tis, monkat hvthvyvtken cuko-pucase vlakat kerratskekokv :

36 Monkon omat, vyvkhvmkusen vlaket, nocicatsken es ce hece wites.

37 Momen nake ce kicvkvyan este omvlkvn kicakis, Vketecvkes.

\section{SETENTVCKE 14.}

Momes nettr hokkolet hoyanof, ohhoyvnkv momet tvklike pakkeko hompetv-rakko ocvtes, momen plest enhomvhhotat momet cokv-hayvlket estomen invkerretv eteropotten eset, elecrke tayaten hopoyakvtes.

2 Momis, Hompetv-rakko nettv omof tokon, este tekyvmketv ocekaren, makakvtes.

3 Momen Pervnen, Simvn lepv en cuko ofv hompetvn vlikof, hoktet alepastv tohawv neha fvmece, vcake mahe, espeknv kihocet, vevnken svlakvtes, momet, tohawvn tvkohcet, e'kvn em oh kalvtes.

4 Momen eme ofvn cvpvkhokat sasvtes, Estomaten neha fvmecv hiyomēn yvmahke haks?

5 Tenelyv cukpe tutcenat 'sen hoyvnen wiyiyet, estemerhoyan emhoye tayvtet ok, makaket. Momet hokte vnrvpen ecayakvtes.

6 Momen Cesvs, Ocepekvs, estoman naoricatske haks? vtotketv heren vm momeces.

7 Estemerrakat ece 'pakvke ocatske emunket, estofvn komatskis, en lopicatske tayet ok'; momis vn tat ocatske munkekot omekv.

8 Nake momece tayvten momeces; enhomvn vlahket, neha frmecv cv'na oh kalat es herihocvranan momeces.

9 Mehenwvn ce kicvkis, Ekvnv omvlkvn estrmvt o estomen heyv opunvkv-herv es erkenvkhoyat vtekat heyv nake momecvteu esvkerrickvn onahoyvres, kicvtes. 
10 Momen Cutvs Eskalevt, mv palen hokkolohkakat hvmket, Cesvs emvkvranet, plest enhomvhhotvn oh ayvtes.

11 Momen pohakof, afvckvket tokunawv emetvn temfvccakvtes. Momen estome etektrnkusen wiye tayat hopoyvtes.

12 Momen tvklike pakkeko hompetv-rakko nettv enhvteceskvn ohhoyvnkv elecakof, en kerrepvlket, Estvmvn ohhoyvnkv hompetskvren etetakueceyvre kometske haks? kicakvtes.

13 Momen en kerrepvlke hokkolen vtotet, Tvlofvn cukolvkvks, momen estet pecv uewv vevnken eshvlatet ece'nrvpvres; mvn vevkvhoyvks.

14 Momen estrmv eceyaten, Mvhayvt okat, Cuko-pericv en nvthofv vn kerrepvlke vpaket ohhoyvnkv hompvye tayat estrmvn oce haks? ce kicet os, cuko-pucasen kicetskvres.

15 Momen onvpv nvthofv rakke, here eteticvkvte, tetaken cem mellvkvres; mvn pum etetakuecvks, kicakvtes.

16 Momen en kerrepvlket awoliket, tvlofvn cukolaket, kicakvte etvpomen eshecakvtes; momet ohhoyvnkvn etetakuecakvtes.

17 Momen yafkof palen hokkolohkakat vprkaken vlakvtes.

18 Momen vpoket hompakof, Cesvs okat, Mehenwrn ce kicvkis, Hvmkatskat, ve vpake hompat, cv wiyvres, makvtes.

19 Momen feknokket, hvmkvnton, Vne te? kicetvn vlicecakvtes ; momen etvt, Vne te? kicvtes.

20 Momen vyoposket, Hokkolohkakat hvmke, mv ve vpake prlvknv vcvnkan svklicat tes; kicakvtes.

21 Este E'chuswr oh onayet cohoyvte etvpomen ayet os, momis $\mathrm{mv}$ este wiye huerat en holwakes! mv este heckekaten omat en hervnt os, kicakvtes. 
22 Momen hompakof, Cesvs, tvkliken ehset, ohmeriyet, kvlkayet, emakvtes, Eset, hompvks; heyvt cv'na tes, maket.

23 Momet sesketucen 'sehset, Erken vkvsahmet, emakvtes, momen omvlkvt eskakvtes.

24 Momen, Heyv cr catv setemfrccetv mucrse tes; mv sulke vrahkv pvlatkat.

25 Mehenwrn ce kicvkis, Ohvtvlaken pvrko en lokce eskvko munken mv nettr Hesaketvmese em ohmekketv ofv oh mucvse eskvyof tvlkvres, kicakvtes.

26 Momen yvhiketvn yvhikahkof, rvne Olefvn sossvtes.

27 Momen, Omvlkatskatet vne vc vrahkvn heyv nere vnvttecvlke toyatskvres; Yvpefikv vhecicv nvfkares, momen yvpefikvt vwahvres, maket coket omekv.

28 Momis akvwvpikvye rohyupv ce homv taken Kalvlen vyares, kicakvtes.

29 Momis Petvt, Omvlkvt vnvttecvlke hakake estomis, vn tat mohmvkos, kicvtes.

30 Momen, Mehenwrn ce kicis, Mucv nettv, heyv neren, tottolose en hunvnwr vhokkolv hakeko munken ve ohfvecv ehelaksecetske svtutcenvres, Cesvs kicrtes.

31 Momis ohvtrlvke swhere mahen opunayet, Ece 'pake cv'lvrane estomis, ec ohfvecv ehelaksehcvkos, makvtes. Matvpomen makake vlkvtes.

32 Momen ekvnv Kersemene hocef kan yicvtes, momen, Yvmvn vpokatsken, emekusvpares, en kerrep vlken kicrtes.

33 Momet Petv, Cems, Cane esyoman evpayet, vlicecet, tayen em estomahet, honne mahvtes.

34 Momet, Vm puyvfekcut tayen nokkes, elkv tis oren; yvmvn fekhonnet, vketecrkes, kicakvtes.

35 Momet hopvyecusekot, ekvnvn tvk latkvtes, momet, 
mome tayen omat, mv hvse-vkerkv e'nkvpvkvren emekusapvtes.

36 Momet, Apv, Cv'rkē, nake omvlkvt cem momvke tayes; heyv sesketvn es vm vkueyvs: mome estomis, vne komvyate tokon, momis ceme kometskat momekvs, makvtes.

37 Momet vlahket, nocicen eshecvtes; momet Petvn em punayet, Simvn, nocetska? hvse-vkerkv hvmkusis vketecetv ec umekisa? kicvtes.

38 Vketecet, emekusvpvks, holwake esceyatskekaret. Mehenwvn puyvfekevt vkvsamus, momis vpeswrt yekcekot os.

39 Momen hvtvm ahyet, mata opunvkvn punayet, emekusapvtes.

40 Momet rafulikof, hvtvm nocicen eshecrtes, (e turwv take honhoyet omekv;) momen naken 'sem vyoposkvke tayat kerrvkekates.

41 Momen esvtutcenat vlahket, momet em punayet, Nocice mahet, fekvpepvkes; tetayes; hvse-vkerkvt vlvkes; hecvs, este Echuswv naorkvlke e'nke ofvn wihoyes. 42 Asvpvkilet, vpeyvkeres; hecvs, mv cv wiyat vwolices, kicakvtes.

43 Momen moman vpakusen, opunaye emunkof, Cutvs, palen hokkolohkakat hvmkat, este sulke mahet, eslafkv-cvpko, eto-warke tis cvwvken, plest enhomvhhotv, cokv-hayvlke vculvke esyomat atotaken vpvkaken, vlakvtes.

44 Momen mv wiyat eskerkvn emvke tates, Estimvn vfvnkvyat, mvt mv tares; mvn eset, vhericet 'svpeyvkes, maket.

45 Momet vlakan vpaken, Ivpkusen oh ayet, Mvhaya, Mvhaya, maket, vfvnkvtes.

46 Momen, e'nke taken oh ocaket, esakvtes. 
47 Momen vsvpaklat hvmket, eslafkv-cvipkon a eset, plest enhomahtv em vtotkvn casket, e hvckon en tacrtes.

48 Momen Cesvs vyoposket, Este horkopvn vnrape omet, eslafkv-crpko eto-warke tis cawet, cr 'svranet yicatske haks?

49 Nettv omvlkvn cuko-vcakat ofvn mvhayit, ece 'pakvkvyat cv'satskekvnkes. Momis cokat esohfvckvkvranet os, kicakvtes.

50 Momen omvlkvt wikahket, pefatkvtes.

51 Momen este-hunvnwv mvnette, hvnvwelv tvrke e'natvlkan oh kayet vevkayvtes; momen este-hunvntake mvnettvlket hvlvtakvtes.

52 Momen hvnvwelv tvrken wihket, enatvlket en letkakvtes.

53 Momen, Cesvs evpayet, plest en homahtvn 'soh vpeyvtes; momen plest enhomvhhotv omvlkvt, vculvke cokv-hayvlke esyomat vpvkaket nvkaftvtes.

54 Momen Petvt plest enhomahtv en cuko-herv ofv roren hopvyen vevkayvtes, momet vtotkvlken vpaket, totkvn vliket, tarrvtes.

55 Momen plest enhomvhhotv nvkaftvlke omvlkv esyomat kerkueckv Cesvs vnrvpen hopoyakvtes, elecvkvret; momet eshecvkekates.

56 Sulket fvccekon vnrvpen kerkuecakvtet ok', momis em ohkerkueckv take etelvcrkekates.

57 Momen fvcceko vnrvpe akerrakat akvwrpece sasvtes, 58 Heyv cuko-vcakat enke es hahoyvten lekahfit, nettv tutcene ofvn etvn hayares, enke es hahoyekat, maken pohēmvts, makaket.

59 Momis hiyomēt o estomis em ohkerkueckvt etelvevkekates.

60 Momen plest enhomahtv nvrkvpvn ahuyiret, Cesvs 
em 'pohet, Naken vyoposketskeko? heyv ce'nrape akerkuecakat naket $o$ haks? makvtes.

61 Momis cryayvket naken vyoposkekates. Hvtvm plest enhomahtvt em 'pohet, Vrakkuehocat E'ppuce, Klist, toyetske haks? kicvtes.

62 Momen Cesvs, $M v$ toyis; momen este E'chuswvt, yekcetv em akvpervn liket, hvlwe aholoce ofvn vlaken hecatskvres, makvtes.

63 Momof plest enhomahtv, em accvken selahlecet, Estoman akerrvlke ohvtvlakat pu yace haks?

64 Hesaketvmese tvklecat pohhatskes; naken vkerricatske haks? makvtes. Momen omvlkvt ele taye em mvtten ohfvccecakvtes.

65 Momen vpvlwvt oh tofkaket, e turofvn ohranet, nafket, momet, Owalvs, kicetvn vlicecakvtes; momen vtotkvlket enke tvpeksen es nvf kakvtes.

66 Momen Petvt cuko-herv ofvn lecvn likof, plest enhomahtv em vtotkv hoktvke hvmket vlakvtes;

67 Momet Petvt tarren hecof, oh hecet, Momen cemeu Cesvs Nasvlvlkèn vpaketskis, kicvtes.

68 Momis ehelaksecet, Kerrvkot, naken maketskat cen kerrvkot os, makvtes. Momet sohvpettvn oh ossvtes; momen tottolose en hunvnwv hakvtes.

69 Momen hokte mvnette etvt hecvtes, momet, Heyv esteu mv hvmkat tes, vsvpaklaten kicetvn vlicecvtes.

70 Momen hvtvm ehelaksecvtes. Momen hrtvm emohyupusan vsvpvklatet, Mehenwrn mv hvmkat toyetskes, Kalvlevlket ometsken, momet cem punvkvt $m v$ omet omekv, Petvn kicakvtes.

71 Momis, Hesaketvmesen es hocefet, trhiketvn vlicecvtes, Heyv hunvnwv opunvyecatskat kerrvkos, maket.

72 Momen svhokkolat tottolose en hunvnwv hakvtes. Momen Petvt Cesvs em punvkv vkerricvtes, mv, Tottolose 
en hunvnwv vhokkolvn hakeko munken vc ohfvecv ehelaksecetske svtutcenvres, kicvte. Momet mvn oh vkerrieof, hvkihkvtes.

\section{SETENTVCKE 15.}

Momes hvyatkan vpakusen plest enhomvhhotv, vculvke; cokv-hayvlke, nvkaftvlkeu omvlkvt vpvkaken, tekueketvn ocahket, Cesvs wrnvyahkof, evpayet svpeyet, Pilvten em wikakvtes.

2 Momen Pilvtet em 'pohet, Cusvlke em Mekko toyetska? kicvtes. Momen vyoposket, Maketskes, kicvtes.

3 Momen plest enhomvhhotv nanvke sulken es vnrvpakvtes; momis naken vyoposkekates.

4 Momen Pilvtet hvtvm em 'pohet, Naken vyoposketskeko haks? Nanvke sulken ce'nrvpen akerrakan hecvs, kicvtes.

5 Momis Cesvs naken vyoposkeko munkvtes; momat Pilvtet em estomahvtes.

6 Tokvs, $m v$ hompetv-rakko omof, cuko-yekcv vtehkvlke hvmken este em wiket omvtes, estimvn komakat.

7 Momen Pvlapvse hocefkat, mv vpaket este tekyvmketv hayakvte, mv tekyvmketv ofvn estelecakvten vpaket svlvf kuehoce tates.

8 Momen este sulkat yekcen huehkaket, nake em momecakvnto omen momecvkvren em 'pohetvn vlicecakvtes.

9 Momis Pilvtet em vyoposket, Cusvlke em Mekkon cem enrecopvkaren komatske haks? makvtes.

10 (Plest enhomvhhotv en homecaket em wikaket omen kerrekv.)

11 Momis plest enhomvhhotvt, este sulkan ohyekcicakvtes, Pvlapvse min em enrecopvkvren.

12 Momen Pilvtet vyoposket, Monkv mv Cusvlke em 
Mekkon kicatskat estomecaren komatske haks? hvtvm kicakvtes.

13 Momen, Tohweklepkvn svrvhvs, maket hvtvm huehkakvtes.

14 Momof Pilvtet, Estoman? nake estome holwvken momece haks? kicakvtes. Momen, Etohweklepkvn svrvhvs, maket, svhere mahen huehkakvtes.

15 Momen Pilvtet, este sulkat mahlvpvtetvn komet, Pvlapvsen em enrecopvtes, momet, Cesvs tepikof, em wikvtes, etohweklepkvn svrahkvren.

16 Momen suletawrlket evpayet, cuko tektvnke tvleme Pletlolevm kicetvn esceyakvtes; momet mv suletawvlke em etohkvlketv omvlkvn nvkvftecakvtes.

17 Momet okholatten es vccecet, etofvske kvpotokvn hotahnet, e'kvn em ohlicakvtes.

18 Momet, Cusvlke em Mekko toyetskate! maket vseketvn vlicecakvtes.

19 Momet e'kvn kohvn 'sen naf ket, vtof kakvtes, momet etorkowvn pvkohlicet, vrakkuecakvtes.

20 Momet nakhayvkekofv rohyupvn kapv okholatten en cawet, em accuken es vccecet, evpayet, esossvtes, tohweklepkvn svrahvret.

21. Momet Vleksantv Lufvse tepakat e'rke, Silenvlke, Simvn hvmke, tvlofv en lvpvtken a vtet hoyanen tohweklepkv karonvren ohyekcicakvtes.

22 Momet Kalkorv ekvnvn esyicvtes, mv ohyvtekhoyat, Estekv fune ekvnv tes.

23 Momet pvrko-opuswv smvnv etohkvlken esketvn emakvtes ; momis 'sesekates.

24 Momet etohweklepkv svrvhahkof, vrahrvkv nake crwvkvranat es oh hecet, em accrken tekvpicakvtes.

25 Momen hvse-vkerkv svtutcenat omvtes, momen tohweklepkv svrvhakvtes. 
26 Momen esvnrvpkv hoccat onvpvn hoccrtes, CUSVLKE EM MEKKOT.

27 Momen horkopvlke hokkolet vpaket etohweklepkvn svrahrakvtes, hvmket akvpervn svrahken, hvmkat vkvskvnvn svrahken.

28 Momen cokvte, Momen vhakv kacvlken vpaket vhonkvtkvtes, makat sohfvckvtes.

29 Momen mv hoyvnecat tvklecakvtes, e'kvn norotticaket, momet, Ceme, cuko-vcakat vhopahnet, nettv tutcenuse ofv hayetskat,

30 E hesayecet, etohweklepkvn ahvtvpkvs, makaket.

31 Matvpomen plest enhomvhhotv, nakhayekot, cokvhayvlket vpvkaken, Etvn hesahuecvnkis, e hesayeceko tayes:

32 Klist Eslevl em Mekkot mucvn etohweklepkvn a hvtvpkekvs, hecēt vkvsvmeyvren, maket ete kicakvtes. Momen mv vpake etohweklepkv svrahrakatet tvklecakvtes.

33 Momen hvse-vkerkv sepakat orof, yomucke ekvnv omvlkvn ohranen hvse-vkerkv esostrpakat orvtes.

34 Momen hvse-vkerkv esostvpakan Cesvs, en hake yekcen es huehket, Eloi, Eloi, lamv sepakteni? mv ohyvtekhoyat, Vn Hesaketvmesē, vn Hesaketvmesē, estoman cv wihketske haks? makvtes.

35 Momen vsvpaklate vpvlwvt, pohahkof, Hecvs, Elivsen enhuehket os, makakvtes.

36 Momen hvmket letiket, uepvton uetuksen es fvcecrtes, momet kohvn oh lihcet, eskvren e'mvtes, Ocepekvs ; Elivs ahvtvpecicvret vlvkvranen omat, hecvkeres, maket. 37 Momen Cesvs, en hake yekcen es hohiket, hesaketvn wikvtes.

38 Momen cuko-vcakat setentvckv onvpv elecv yeshrtapket ete setkvtes. 
39 Momen mv sentulevt, homuse huerat, momēn hohiket, hesaketvn wiken hecof, Mehenwusen heyv hunvnwv Hesaketvmese E'ppuce tates, makvtes.

40 Hoktvkeu hopvyen a hecakat sasvtes, mv vpakat Mele Maktelv tates, momen Mele Cems kocuknat, Coses tepakat e'cket, Selome esyomat;

41 Cesvs Kalvlen arof mv vcrkvpeyet em vtotkakvtet, momen hoktvke eti sulket, mv vpaket Celuslvmen yicvtet, a hecakvtes.

42 Momen yafkof, (tetakueckvt omekv, fekvpkv nettv. renhomv tatet ok',)

43 Cosef Alemvrevlket, nvkaftvlke hvmke vrakkat, mvu Hesaketvmese em ohmekketvn mehakat, vlahket, fekhvmket Pilvten er en ceyet, Cesvs e'nan em 'pohvtes.

44 Momen Cesvs entis elen omat Pilvtet estomahes komvtes; momet sentulevn enhohiket, elvte vcewuse tat em 'pohepvtes.

45 Momet, sentulevt em onahyof, enan Cosen emvtes.

46 Momen hvnvwelv tvrke tvlekmusen nesvtes, momet a ehset, hvnvwelv tvrken es vyokkohfet, sepvlkv cvto a kof kvte ofvn wrkecvtes, momet sepvlkv em vhauke crton $\mathrm{v}$ pvlpicvtes.

47 Momen Mele Maktelv Mele Coses e'cke tepakat estvmvn wvkechoyat hecakvtes.

\section{SETENTVCKE 16.}

Momen Nettvcakot hoyahnof, Mele Maktelv, Mele Cems e'cke, Selome esyomat esfvmecickv fvmecakusen nesakvtet omes, yicet es siyvkvret.

2 Momet nettvcakuce nettv enhvteceskvn, hvthvyvtkusen, hvse vkvwapkusen sepvlkvn roricvtes.

3 Momet, Estimvt sepvlkv em vhauken cvton pum vkueyvr haks? ete kicakvtes. 
4 Rakke mahet omvtet ok. (Momet hecakof, cvto a pvlpihocen eshecakvtes.)

5 Momet sepvlkvn esceyet, este-hunvnwv mvnette, kapv hvtke, crpken accet, akvpervn liken hecakvtes; momet penkvlvke tates.

6 Momen, Penkahlatskvs; Cesvs Nasvlvlke, mv tohweklepkv svrahkvten hopoyatsket os; akvwvpikes; yvmv sekos: mv wvkecakvnken hecvks, kicakvtes.

7 Momis vpehyet, ce homv taken Kalvlen eceyat, en kerrepvlke Petv esyoman em onvyvkvtcrs; ce kicvkvte etvpomen, invn hecatskvres.

8 Momen lvpken asosiyet, sepvlkvn enkvpaket pefatkvtes ; fekeket em estomahaket ok': momet este estomis em punvyvkekates; penkvlvket ok'.

9 Momen nettvcakuce nettv enhvteceskvn, hvten Cesvs akvwvpikof, Mele Maktelv, mv puyvfekev holwvhokat kolvpaken asossicvte, taten em e heckuecvtes.

10 Momen ahyet, mv vpvkakvten, wahoket, hvkihhokof, em onayakvtes.

11 Momen hesaken, Mele hecrten pohahkof, vkvsvmvkekates.

$12 \mathrm{Mv}$ renyvpvn, hokkolet tvlofv en lvpvtken cukolakof, emvrahken em e heckuecvtes.

13 Momen vhoskat 'rem onayakvtes, momis mvu vkvsvmvkekates.

$14 \mathrm{Mv}$ hoyanat palen hvmkontvlakat hompetvn vpokof, em e heckuecet, vkvsvmvkekat, momet e feke take em wvnhat ohfvecrn vnrapet em punayakvtes, mv akvwvpikof, hecakvten vkvsvmvkekatet ok'.

15 Momet, Ekvnv omvlkvn hvmecicet, opunvkv-hervn nake hocackvte vtekat 'sem erkenvkvks.

$16 \mathrm{Mv}$ vkvsahmet paptiskat hesakvres, momis vkvsvmekat ohfvecehocvres. 
OPUNVKV-HERV MAK COYVTE XVI.

59

17 Momen heyv eskerkvt mv vkvsamakat vevkvpeyvres; $\mathrm{Cv}$ hocef kv ofvn puyvfekev holwvhokat sossicet; tulaswv mucrsvke es opunayet;

18 Cetton crwvkvres; momet esketr estelece tayat eskaken omis, en nokkicvkekares; enokhokat enken oh lumbicaken, cvfencihocvres ; kicakvtes.

19 Monkv Pucaset, em punayat hoyahnof, hvlwe ofvn eshoyvtes, momet Hesaketrmese em akvpervn likvtes. 20 Momen esosiyet estrmv estomis erkenvkakvtes, Pucaset vpaket vtotken, momet eskerkv vcrkvpeyaten opunvkrn es yekcicen. Emen. 


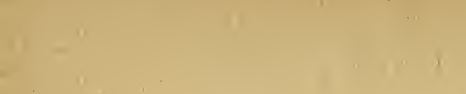

an

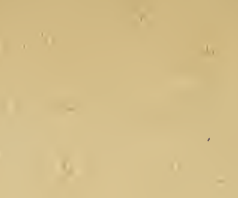

$\Rightarrow \quad 1^{\prime}$

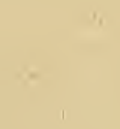




\section{OPUNVKV-HERV}

\section{LUK COYVTE.}

THE GOSPEL ACCORDING TO

\section{U K E,}

TRANSLATED FROM

THE ORIGINAL GREEK

INTO THE MUSKOKEE LANGUAGE.

NEW YORK:

AMERICAN BIBLE SOCIETY, INSTITUTED IN THE YEAR MDCCCXVI. 



\section{OPUNVKV-HERV \\ LUK COYVTE.}

\section{SETENTVCKE 1.}

ESTE sulket vlicecat, pum etenrvwv nanvke fvecehohce vkvsvmkv hakvnke etetvlake heren ohkerkuecvikvtet omekv,

$2 \mathrm{Mv}$ nanvke pum wihokvtet omen, enhvteceskv vteken, eturwv es hece akerrvlke toyēt, momet opunvkv herv vtotkvlke toyēn,

3 Reafelvs here mahe toyetskat, vneu, enhvteceskv mahusat vteken $\mathrm{mv}$ nanvke omvlkvn esfvcken kerrvyet omvte tat, etetvlaken cen hoccicvyat here etetaye es vm momvnks ;

$4 \mathrm{Mv}$ nanvke ofv ce mvhahoyvte em mehenwetvn kerretskvren.

5 Plestvlke hvmket, Vpiv em etohkvlketv hvmkat, Svkelivs hocefket, Elvn e'chustvlke hvmkat, e hiwv, Lesepet hocefken, tepaket, Helot Cutev em mekko en nettv ofvn, kakvtes.

6 Momen hokkolvt, Pucase em vhakv momet nanvke est' em mellvkvte ofvn em mvttekon yvkvpaket, Hesaketvmese ehomvn fvccrket omvtes.

7 Momet hopuewr ocvkekates; Elesepet echussekot omekv; momet hokkolvt vculkv ohcakkevlket omakvtes. 8 Momen mome ocvtes, Hesaketvmese ehomvn em etohkvlketv en nakvfvstetv vevkvyen plest em vtotketvn vfastof, 


\section{OPUNVKV-HERV LUK COYVTE I.}

9 Plestvlke nake momecvkvranvte vcrkvyen Pucase en cuko-vcakan eceyof, esfrmecickv nekricetvt em vtotketvt omvtes.

10 Momen esfvmecickv oketv omof, este sulsokat omvlkvt fettvn emekusvpakvtes.

11 Momen Pucase em este hvlwet, esfvmecickv altv em akvpervn hueret, em e heckuecvtes.

12 Momen Svkelivs hecof, fekherekates, momen penkvlkvt oh latkvtes.

13 Momis hvlwe estet em punayet, Svkelivs toyetskat, ce penkahlats, cem emekusvpkv pohket omekv; momen ce 'hiwv Elesepet ce'ppucen cen heckuecvres, momen Canen hocefetskvres.

14 Momen afvcketv es ece 'fvcke tares, momen en hecketv cakkat sulket es afvckvke tares.

15 Momen Pucase ehomvn rakke tares, momet pvrkoopuswrn monkat esketv yekcrn eskekares, momet e'cke e nvrke vtekat tis Puyvfekcr Vcakat es fvcke tares.

16 Momet Eslevl en hopuetake sulken Pucase en Hesaketvmese taken oh fulhuecvres.

17 Momet Elivs em vkerrickv en yekcetv tepaken ehopaket, Pucase ehomvn ayvres, erkvlke e feken en hopuetaken oh fulhuecet, momet mapohicvkekvlken fvecakat en hoporrenkvn oh fulhuecrranet, momet este Hesaketvmese emetakvken metetakuecvranet ayvres, kicvtes.

18 Momen Svkelivs hvlwe esten em punayet, Naken heyv es kerrare te? vc vculet omin, cv 'hiwvu hoktaluset omekv, kicvtes.

19 Momen hvlwe estet vyoposket okat, Vnet, Keplevl Hesaketvmese ehomv huervyat tot, cem punayet, heyv opunvkv heran es cem vlvkaren ve vtothoyet os.

20. Momen hecrs, opunayko ce haken, punayetskeko tetaye emunken heyv nanvke momvkvranat nettvt vlv- 


\section{OPUNVKV-HERV LUK COYVTE I.}

kvres, vm opunvkv, mv oketv orof esfvcfvkvranat, vkvsvmetskekot omekv, kicvtes.

21 Momen Svkelivs cuko-vcakat ofv momvteken fekhonnat este emehakakat estomahe 'sem momakvtes.

22 Momen mvn aosiyof, em punvyvketv umeko tayvtes, momen enken 'svhopayet, punayekot emunkekv, cukovcakat ofvn nak hecihocet omen kerrakvtes.

23 Momen mome ocvtes, em vtotketv nettv hoyvnakof, e huten ayepvtes.

24 Momen mv nettv momakat hoyahnof, e 'hiwv Elesepet nvrkehset, hvse epaken e vrvnayvtes;

$25 \mathrm{Mv}$ nettv momakvnke ofvn este vpvke vlesketv vm vkueyvranet $v c$ oh hecet, hiyomēn Pucaset cr momecvtet os, maket.

26 Momen hvse esepakof, Hesaketvmese atotet, Keplevl hvlwe estet, Kalvle ekvnv ofv tvlofv Nasvle hocefken ayet,

27 Hokte, hunvnwv kerrvte sekat, este-hunvnwv Tewe en cuko-hvmecvlke hvmkat, Cose hocef ke e 'hiwv tarane etemfvccan oh ayvtes; $\mathrm{mv}$ hokte mvnettat e hocefkv Mele tates.

28 Momen hvlwe estet a en ceyet, Afvckvs, heromkv cem ocat, Hesaketvmeset ece 'pakes; hoktvke vpvke ohmerrvkvte toyetskes.

29 Momen hecof, nak makat omecicen fekherekates, momet heyv vseketv estomēt ot omat oh vkerricvtes.

30 Momen hvlwe estet, Melē, ce penkahlats, Hesaketvmese a vten heromkvt cen hecket omekv.

31 Momen hecvs, nvrkesetskvres, momet ce'chuswv hunvnwv heckuecetskvres; momet Cesvs hocefetskvres.

32 Momen rakke tares, momet Hvlwe Mahat E'ppucen kihocvres, momen Pucase Hesaketvmeset mekko em ohliketv erke Tewe enaken emvres. 
33 Momen Cekvpe en cukon meyuksv-sekon ohmekkvres; momen em ohmekketv em eyuksv sekares, kicvtes.

34 Momof Melet, hvlwe esten em punayet, Hunvnwvn kerrvkot omat, estomēn hiyome tare te? kicvtes.

35 Momen hrlwe estet vyoposket, Puyvfekcv Veakat ec oh vlvkvres, momen Hvlwe Mahat en yekcet ec oh vpettvres; monkv mv nake vcake heckuecvranetskat Hesaketvmese E'ppucen kihocvres.

36 Momen hecvs, cen nake Elesepet, mvu echuswr hunvnwv es nvrkeset os, en hoktvlkv ofv; momen mucvt hvse sepakat tes, mv hokte echusseko kihocvte ohfvecv.

37 Hesaketvmeset on omat nake momeceko tayat naket sekot omekv, kicvtes.

38 Momen Melet, Hecvs, Pucase em vtotkv hokte; cem opunvkv vcrkayat vm momekvs makvtes. Momen hvlwe estet e'nkvpakvtes.

39 Momof mv nettv take omof, Melet ahuyiret, lvpken Cutv em etvilofv hvmken cefahlofvn eceyvtes,

40 Momet Svkelivs en cukon ecehyet, Elesepen vsekvtes.

41 Momen mome ocvtes, Elesepet Mele em vsekaten pohof, estucet e nvrke ofvn taskvtes; momen Elesepet Puyvfekcv Vcakat es fvckvtes.

42 Momet en hake yekcen 'sopunayet, Cemet ohmerrvkvtet hoktvke vpvketskes, momen ce nvrke em ette ohmerrvkvtet os.

43 Momen cv Pucase e'cke vm vlakat, estvmi vtet heyv vm momet ome haks?

44 Hecvs, vc vseketskat opunvkv cv hvcko ofvn hakat vpaken estuce es afvcket cv nvrke ofvn tasikekv.

45 Momen mv hokte vkvsamvte en heres; mv nanvke Hesaketvmese atotet em onahoyvte momvkvranekv, makvtes. 
46 Momen Melet opunayet, Vm vkerrickvt Pucasen vrakkuecet os,

47 Momen vm puyvfekcrt Hesaketvmese cv Hesayecr ofvn afvcikes.

$48 \mathrm{Em}$ vtotkv hokte en hueretv kvncapan oh vkerricvtet omekv, momat, hecrs, mucv ayat este honvpse omvlkvt ohmerrvkvten cv kicvkvres.

$49 \mathrm{Mv}$ yekcat nake rvkrvken cv momecvtet ok' ; momen e hocefkv vcaket $o s$.

50 Momen em penkvlakat honvpse erhonvpse eroret em merkv oh ocet os.

51 Yekcetvn e sakpvn es hecicvtet os; momet ekvsvmakat e feke take em vkerrickv ofvn vwahecvtet os.

52 Yekcakat em ohliketvn a hvtvphuecvtes, momet este en hueretv kvncvpakan hvlhvwecvtet os.

53 Elawakat nake herakan es fvcfihcet, este nake ocakat tvntrken vpeyecicvtet os.

$54 \mathrm{Em}$ merkv vkerrickv ofvn em vtotkv Eslevl em vnicvtet os;

55 Purkvlke, Eplehame momet erhonvpse meyuksvseko em punvyyte vevkvyen, makvtes.

56 Momet Melet hvse tutcenvteken vpaket lihket, e huten fulkvtes.

57 Momen Elesepet estuce heckuecvranvte oketv esfvciken, echuswv hunvnwvn heckuecvtes.

58 Momen em vwolicvlket, momen en nakvlket Hesaketvmeset merkv rakken es ohmerrvten pohakof, vpvkaket afvckakvtes.

59 Momen mome ocvtes, nettv escenvpakan estuce svkvmsisvranet yicvtes, momet erken vhocefet Svkelivs hocefakvtes.

60 Momis e'cket vyoposket, Mohmats, momis Canet hocefkv tares, makvtes. 
61 Momen, Cen nakvlke heyv hocefkvn es hocefkat sekos, kicakvtes.

62 Momen hocefkv estomen es hocefkvren eyacat 'sem vhopayet erken em 'pohakvtes.

63 Momen ohhoccickv tvpeksan em 'pohhet, Canet e hocefkvt omes, maket, hoccicvtes. Momen omvlkvt em estomahakvtes.

64 Momen moman vpakusen e cukwv hvwiken, e tulaswv enrecopke hakvtes, momen Hesaketvmesen vrakkuecet opunayvtes.

65 Momen em afolotvke mv vpokakat penkvlkvt oh latkvtes, momen heyv ohonvkv omvlkvt Cutev cefahlofvn hvmecicvtes.

66 Momen pohakat vtekat e feke take ofvn vtehakvtes, Heyv hopuewv estome tar haks? makaket. Momen $\mathrm{Pu}$ case e'nket vpakvtes.

67 Momen e'rke Svkelivs, Puyvfekev Vcakat es fvciket, owalvtes,

68 Pucase Hesaketrmese Eslevl enaken vrakkuehocekvs, em este em meriyet, pvlken nesvtet omekv;

69 Momet vhesaketv eyvpen a pun huericvtet os, em vtotkv Tewe en cuko ofvn;

$70 \mathrm{Em}$ owalvlke vcacakat ekvnv vliceckv vteke fullvte e cukwv eteropotte opunvyvte omet,

71 Pun horren en hesahoket, pun homecakat omvlkv e'nke en hesahokeyvren;

72 Merkv pu'rkvlke etemfaccvten sohfvcecet, em etemfvccetv vcakan vkerricvret,

$73 \mathrm{Mv}$ etemfvccetv vcake pu'rke Eplehame 'setemfaccvte, 74 Heyon pu'mvret, pumet, pun horre e'nke a 'sepu'ssihohcen, penkvlvkekot,

75 Pun hesaketv en nettv omvlkvn fvecetv momen hvsvtketv ofv ehomvn em vtotkeyvren. 
76 Momen ceme, hopuewv toyetskat, Hvlwe Mahat em owalvn ce kihocvres, Pucase e turofv ehomvn em vyetv vlhekat etetakuecvranet ayetskvret ok'.

77 Momet em este en holwvyeckv enkvpryecickv eteropotten, vhesaketv kerrvkvre emvranet ayetskvret ok',

78 Pun Hesaketvmese em merkv vcakat eteropotten, $\mathrm{mv}$ etehoyvnen hvyatkat hvilwen vtet pum merrvtet os;

$79 \mathrm{Mv}$ yomucke momen elkv em vpette ofv vpokat hvyayvken emet, pu'le herkv vyetv ofv vpeyecicvret.

80 Momen hopuewvt mahet, puyvfekcv ofvn yekce hakvtes, momet vnrvwrn aren, Eslevl em e heckueckv nettvt orvtes.

\section{SETENTVCKE 2.}

Momen mome ocvtes, mv nettv take omof, ekvnv omvlkv este e hocefhokv vhonkvthoyvranet cohoyvren Sesv Vkvstvs makvtes.

2 Sileners Selev ofvn evpayvt huerof, heyv vhonkvtkv enhrteceskvn hahoyvtes.

3 Momen este omvlkvt vhonkvthoyvranen em etvlofv vrahrvkvn vpeyakvtes.

4 Momen Cosef, Kalvle tvlofv Nasvlen aosiyet, Cutevn eceyet, Tewe em etvlofv Perehem ayvtes, (Tewe en cuko momet erhonvpse aosse tatet ok';)

5 Mele ehiwvrane temfaccat nvrkesan vpvken vhonkvthoyvren ;

6 Momen mome ocvtes, mvn welakof heckuecvranvte nettvt fvcfakvtes.

7 Momen e'chuswv hunvnwv enhvteceskvn heckuehcet, vpvllahyet, vpuekv em ohhompetv ofvn wrkecrtes, hompetveuko ofv em etektrnke sekot omekv.

8 Momen mv ekvnv etvwrn yvpefikv-vhecicvlket neren 
en yvpefikv fulle hvmken vhecicet lvpvtken fekhonnvke tates.

9 Momen hecvs, hvlwe estet Pucase enaket oh vlvkakvtes; momen Pucase en hvyayvket em vfoloten hvyvyakvtes; momen tayen penkvlvke tates.

10 Momen hrlwe estet, Ce penkahlvkats, hecvks, afvcketv rakke opunvkv-hervn es cem vlakvkvyet omekv, este omvlkv em ocrranat.

11 Mucv nettvn Tewe em etvlofv ofvn Hesayecv Pucase Klist cen heckvket omekv.

12 Momen heyvt es cen kerkv tares; Estuce vpvllvket vpuekv em ohhompetv ofvn wakken eshecatskvres.

13 Momen vyvkhvmkusen hvlwe este sulkat sulke mahet Hesaketvmesen vkvsvmaket,

14 Momet, Hesaketvmesen vrakkuehocekvs, hvlwe mahaten, momen ekvnvn herkv heromkv tepakat este em ocvkekvs, makaket, hvlwe esten vpvkakvtes.

15 Momen mome ocvtes, hvlwe estet enkvpahket, hvlwen escehyof, yvpefikvhecicvlket ete'm punahoyet, Mucvn Perehem mahusan vpeyet, heyv nake mome Pucase pun kerkuecan er hecvkeres, makakrtes.

16 Momet lvpēcicet yicet, Mele Cosef tepakan, momen estuce vpuekv em ohhompetv ofvn wakken eshecakvtes.

17 Momet hecahkof, heyv estuce ohfvecr nak make em onahoyvten hvmkvn kerkuecakvtes.

18 Momen pohakat omvlkvt nanvke yvpefikvhecicvlke em onvyākat em estomahakvtes.

19 Momis Melet, heyv nanvke omvlkvn veryecet, e feke ofvn oh vkerricvtes.

20 Momen yvpefikvhecicvlket em onahoyvte vevkvye nanvke pohet hecakat omvlkv svrahkvn Hesaketvmesen vkvsamet, momet vrakkuecaket fulecrtes.

21 Momen estuce svkvmsisetv nettv cenvpakat fackof, e 
hocefkv Cesvs kihocvtes, ecke e nvrke ofv hocrckeko emunkof, hvlwe estet momēn hocefvten.

22 Momen Mose em vhakv vcrkvye hvsvteckv nettv Mele es em fvcikof, Celuslvmen svlahokvtes, Pucasen emvkvranet;

23 Hunvnwv ecke e nvrke hvwecat Pucase en nakvcakat kihocvres, Pucase em vhakv ofv make hoccat vevkvyen;

24 Momet, Prce-howe tempvlse hvmken, monkat prce mvnettvke hokkolen, Pucase em vhakv ofv hoccat verkvyen ohnekrickvn emvkvranet.

25 Momen hecrs, este-hunvnwv Semevn hocef ket Celuslvmen likvtes; momet $\mathrm{mv}$ hunvnwvt fvccet, momet emekusapet, Eslevl em emahlvpvtketvn emehaket omvtes; momet Puyvfekev Veakat oh fekhonne tates.

26 Momen elkv heceko emunket Pucase en Klist hecvranat Puyvfekcv Vcakat en kerkuecet omvtes.

27 Momen Puyvfekcvt evpahyen cuko-vcakan ceyvtes, momen e'ckvlket vhakv vfvstetv verkvye estuce Cesvs em momecrranet escukolakof,

28 Momof e sakpv ofvn eset, Hesaketvmesen vkvsamvtes, momet okat,

29 Pucasē, mucvin herkv ofv enkvpvkvre cem vtotkvn em etektvnecetsket os, cem opunvkv verkvyen ;

$30 \mathrm{Cr}$ turwvt cem vhesaketv,

31 Mv este omvlkv ehomv em etetakuecetskvte;

32 Hvyayvke Centilvlke en hvyvyickv, momet cem este Eslevl en rakketvn hecakekv, makvtes.

53 Momen nanvke Cesvs ohfvecv opunvyece makat Cosef momen e'cket em estomahakvtes.

34 Momen Semevñ merkvn oh vpohakvtes, momet e'cke Melen em punayet, Hecrs, heyv hopuewvt Eslevl ofv sulket pvlvtket hvtvm akvwvpkvkvren huerihocet os; momet vnrvpe opunvyehocrranat eskerkv hakvren ; 
35 (Momes, cem puyvfekevu eslafkv-crpkot ropottet sekeyvres ;) momat efeke sulke em vkerrickv enrvmrvkvren, makvtes.

36 Momen Asv em vliketv hvmkat, Fenuel e'chuste, Ane hvmke, owalvt likvtes, hoktvle mahet, hunvnwv kerrekate vteken ohrolope kolvpaken e'he vpakvtet,

37 Momet ohrolope pale cenvpaken ostohkaken oyet omvtes; mvt cuko-vcakan enkvpvkekot, eelaweckv mekusvpkv esyoman nettvn nere tis Hesaketrmesen 'sem vtotkvtes.

38 Momet, mv hvse-vkerkv mahusan ecehyet, emeu Hesaketvmesen vkvsamvtes, momet mv ohfvecvn Celuslvme ofv pucasseko hahokvre en nene hecakat omvlkvn em punayvtes.

39 Momen nak omvlkvn Pucase em vhakv vcvkvyen momecahkof, em etvlofv Nasvlen Kalvle ekvnv ofvn fulhokvtes.

40 Momen hopuewvt mahet, hoporrenkv es fvcket, puyvfekcv ofvn yekce hakvtes, momet Hesaketvmese en herkvt oh fekhonne tates.

41 Momen e'ckvlket ohrolope omvlkvn ohhoyvnkv hompetv-rakko omof Celuslvmen vhoyet omvtes.

42 Momet em vculkv pale hokkolohkicof, hompetvrakko vfvstetv vcrkvyen Celuslvmen vhoyvtes.

43 Momet nettrn fvcecahket, fulhokof, hopuewv Cesvs yupvn Celuslvme ofvn fekhonnvtes; momen e'cke Cosef tepakat kerrvkekates.

44 Momis, Vpeyakat vpakes, komet, nettv hvmke vyetvn vhoyvtes; momet en nakvlke em etekerhoyetv esyomat fullaten hopoyakvtes.

45 Momet eshecrkekof, hopoyaket hvtvm Celuslvmen fulhokvtes.

46 Momen mome ocvtes, nettv svtutcenan cuko-vcakat 
ofv mvhayvlke em apohicet, momet vpohkv en hayet, en nvrkvpvn liken eshecakvtes.

47 Momen en hoporrenkv momet vyoposketv pohakat omvlkvt em estomahakvtes.

48 Momen hecakof, em estomahakvtes, momet, $\mathrm{Cv}$ 'chuswa, estomaten pu hiyomecetske haks? ce'rke tepakeyat pu feknokhoket ce hopoyeyis, e'cket kicvtes.

49 Momen, Estoman cv hopoyatskisa? Cv'rke en nak vfvstetvn vrvranvyat tvlkat kerratskekisa? kicvtes.

50 Momen mv opunvkv em punayan kerrvkekates.

51 Momen vpaket hvtvpiket, Nasvlen vlakvtes, momet. em apohicet likvtes; momis e'cket heyv nak makvte omvlkvn e feke ofvn vcayecrtes.

52 Momen Cesvs hoporrenkv momet ena mahetv ofvn, momet Hesaketvmese esteu em en herat ohvtvlakvtes.

\section{SETENTVCKE 3.}

Tokvs Tipelevs Sesv em mekketv ohrolope es pale cahkepohkakan, Pantvs Pilvt Cutev em evpayvt omof, momen Helot Kalvle en tetlak omof, momen etecakkat Felvpe Itulev momet Tlekonitvs ekvntvckv en tetlak omof, momen Lisenevs Apelene en tetlak omof;

2 Momen Anvs momet Kivfvs plest enhomvhhotvlket on, Hesaketvmese em opunvkv Canen, Svkelivs e'ppuce em vlakvtes, vnrvwv ofvn.

3 Momen vkerrickv etickv paptesvm naorketv es enkvpvketvn erkenaket Cotvne afopke omvlkvn em vlakvtes.

4 Owalv Esiv em opunvkv cokv ofv hoccat vcrkvyen, Haketr hvmket vnrvwr ofvn huehkes, Pucase em vyetv tetakuecvks, en nene lvpotlvken hahicvks;

5 Pvne vtekat fvefvkvres, momet ekvnhvlwe momet rvne vtekat kvncvpuehocvres, momen kunkohat lvpotlvke haken; tenepvkekat tenepvke hakvres. 
6 Momen vpeswv omvlkvt Hesaketvmese vhesaketvn hecvres, maket.

7 Momof este sulket paptiskvre en yicakvten em punayet, Aeha, cetto honvpse toyatskat, estimvt, Cvpvkketv vlvkvranan em pefatkvks, ce kicvkvt haks?

$8 \mathrm{Monkv}$ vkerrickv etickv oren ettvkes; momet vlicecet, Eplehame pu'rken ocēs, mahkatskvs, Hesaketvmese heyv crto asossen hopuetake Eplehame em mahmice tayes, ce kicvkit okikv.

9 Momen hiyomat pucuswv eto eceskvtake entis $\mathbf{v}$ wvkechoyet os; monkv eto em ette heren ettekat vtekat tahcet, totkvn tvk wihoket os.

10 Monkv naken estomvraneya? este sulkat kicet, em 'pohakvtes.

11 Momen, Estimvt kapvn 'se hokkolicat mv ocekan emekvs, momen hompetv ocat matvpomecekvs, em vyoposket kicakvtes.

12 Momen cvtokunawv vteloyvlket paptiskvkvren yicet, Mvhaya, naken estomvraneya? kicakvtes.

13 Momen, Naken cem melhoyvkvte semuntvlen feyihcatskvs, kicakvtes.

14 Momen suletawvlkeu em 'pohakvtes, Pumeu naken estomvraneya? makaket. Momen, Estimvn penkvlehcatskvs, momet est' oh lvkisatskvs, momet cem feketvn 'se mahlvpvtecicvkes, kicakvtes.

15 Momen estet nak en nene hecaket, omvlkvt e feketake ofvn Canen oh vkervicet, Mvt Klist tot omeko haks? komet oh vkerricakof,

16 Cane omvlkvn em vyoposket, Mehenwvn uewvn es ce paptisvkit omis, momis hvmke vne 'svn yekcat vlaket os, mv em estelepikv es wvnakv enrecopetv heretv orvkot os ; mvt Puyvfeker Vcakan momet totkvn es ce paptisvkvres. 
$17 \mathrm{Mv}$ este es-em-weskv e'nke ofv'ts, momen naken en nerkw-en-cesketvn heren hvsvtecvres, momet teleko vtelohyet en tohton vtehvres, momis entrleesse totkv vslekon es nekricvres, kicakvtes.

18 Momet nanvke etv sulken 'sest'. emvcahnet, erkenakvtes.

19 Momis Canet, Helot tetlak etecakkat Felvpe e 'hiwv Helotevs vrahkvn, momet nak holwvke momecvte omvlkvn em vsehhof,

20 Helot, nake omvlkv semuntvlen heyvn ohvpayat, Canen cuko-yekcvn vpikvtes.

21 Tokvs, este omvlkv paptiskahkof, mome ocvtes Cesvs tis paptiskvtes, momet emekusapof, sutvt havkvtes;

22 Momen Puyvfekev Veakat ena oce omet prce-howe omet a oh hvtapkvtes, momen opunvkv hvlwe vtet, Cv'ppuce vm vnokecke toyetskes, ec ofvn vc afackuset os, makvtes.

23 Momen Cesvs em veulkv tat ohrolope pale tutcenat mahen oretv vlicecrtes; Cosef eppucet's komhoyen, momen mvt Heli e'ppucet omen,

24 Mvt Marat e'ppucet omen, mvt Lefi e'ppoucet omen, mvt Melki e'ppucet omen, mvt Cvnv e'ppucet omen, mvt Cosef eppucet omen,

25 Mvt Meterivs e'ppucet omen, mvt Emvs e'ppucet omen, mvt Nevm e'ppucet omen, mvt Esli e'ppucet omen, mvt Nakke e'pprucet omen,

26 Mvt Meare e'ppucet omen, mvt Matvrivs e'ppucet omen, mvt Semei e'ppucet omen, mvt Cosef e'ppucet omen, mvt Cutv e'ppucet omen,

27 Mvt Coanv e'ppucet omen, mvt Lesv e'ppucet omen, mvt Solopepel e'ppucet omen, mvt Svlvriel e'ppucet omen, mvt Neli e'ppucet omen,

28 Mvt Melki e'ppucet omen, mvt Ati e'ppucet omen, mvt Kosv e'ppucet omen, mvt Elmotvm e'ppucet omen, mvt V e'ppucet omen, 
29 Mvt Cose e'ppucet omen, mvt Eliesv e'ppucet omen, mvt Colem e'ppucet omen, mvt Marvt e'ppucet omen, mvt Lefi e'ppucet omen,

30 Mvt Semevn e'ppucet omen, mvt Cutv e'ppucet omen, mvt Cosef e'ppucet omen, mvt. Convn e'ppucet omen, mvt Elivkem e'ppucet omen,

31 Mvt Melev e'ppucet omen, mvt Menv e'ppucet omen, mvt Materv e'ppucet omen, mvit Nervn e'ppucet omen, mvt Tewe e'ppucet omen,

32 Mvt Cvse e'ppucet omen, mvt Opet e'ppucet omen, mvt Pvs e'purcet omen, mvt Salmvn e'ppucet omen, mvt Neasvn e'ppucet omen,

33 Mvt Emenetap e'ppvcet omen, mvt Elvm e'ppucet omen, mvt Eslvm e'ppucet omen, mvt Feles e'ppucet omen, mvt Cutv e'ppucet omen,

34 Mvt Cekvpe e'ppucet omen, mvt Iseke e'ppucet omen, mvit Eplehame e'ppucet omen, mvt Relv e'ppucet omen, mvt Neka e'ppucet omen,

35 Mvt Selvk e'ppucet omen, mvt Sekvn e'ppucet omen, mvt Felek e'ppucet omen, mvt Hepv e'ppucet omen, mvt Selv e'ppucet omen,

36 Mvt Kenvn e'ppucet omen, mvt Afaksvt e'ppucet omen, mvt Sem e'ppucet omen, mvt Noe e'ppucet omen, mvt Lemek e'ppucet omen,

37 Mvt Meruselv e'ppucet omen, mvt Envk e'ppucet omen, mvt Celet e'ppucet omen, mvt Mvlelel e'ppucet omen, mvt Kenvn e'ppucet omen,

38 Mvt Envs e'ppucet omen, mvt Ser e'ppucet omen, mvt Atvme e'ppucet omen, mvt Hesaketvmese e'ppucet omvtes.

\section{SETENTVCKE 4.}

Momen Cesvs Puyvfekcv Vcakat es fvcket, Cotvne 'ratvtes; momen Puyvfekcrt evpayet, vnrvwrn 'res oren, 
2 Este-nekricvt nettv pale osten vnrapvtes. Momet mv nettv take omof, naken hompekates; momen mv nettvt pokof renyupvn elawvtes.

3 Momen este-nekricvt, Hesaketvmese Eppuce toyetsken omat, mahkctsken, heyv cvtot tvklike hakekvs, kicvtes.

4 Momen Cesvs em vyoposket, Estet tvklike tvlken $\nabla$ hesahkekos, momis Hesaketvmese em opunvkv vtekat $v$ hesakvres, maket coket os, kicvtes.

5 Momen este-nekricvt ekvnhvlwe hvlwe mahen es oh 'cemiket, oketv kocuknuse ofvn ekvnv e tvlwv omvlkvn hecicvtes.

6 Momen este-nekricvt, Heyv yekcetv omvlkvn ce'mares, momet en rakketvu, mvn v'mhoyvtet ok, momen estimvt $o$ estomis vne komvyat emis;

7 Monkv v'mekusapetsken omat; omvlkvt cenake tares kicvtes.

8 Momen Cesvs em, vyoposket, $\mathrm{Cr}$ topvrvn vyvs, Setvne toyetskat, Pucase cen Hesaketvmesen emekusapetskvres, momet mv tvlkusen em vtotketskvres, maket coket omekv, kicvtes.

9 Momen Celuslvmen svlakvtes, momet cuko-vcakat sohvpettvn oh huerihcet, Hesaketvmese Eppuce toyetsken omat, heyvn a e tvk wikvs,

10 En hvlwe estvlken ece 'fvstvkvren kicvkvres,

11 Momen cvton ce'le es $\mathbf{v}$ naf ketskekaren $e$ 'nken es ce kvwvpvkvres, maket coket omekv, kicvtes.

12 Momen Cesvs em vyoposket, Pucase cen Hesaketrmese vnrahpetskvs, mahokvtet os, kicvtes.

13 Momen este-nekricvt, Cesvs vnrvpkv omvlkvn emeyuksihcof, vkocuknusen e'nkvpakvtes.

14 Momen Cesvs Puyvfekev en yekcetv ofvn Kalvlen ye fulkvtes, momen ohonvkv mv ekvnv omvlkv eteropottet hvmecicvtes. 
15 Momen senekakvlke enaketake ofvn mvhayvtes, este omvlkvt vrakkuecaken.

16 Momet Nasvlen vlakvtes, $\mathrm{mv}$ are mahvtet, momet momvte vcvkvyen nettvcako omof senekaken ecehyet, ohonayvranet ahuervtes.

17 Momen nakcokv owalv Esivs hoccicvten a e'mhoyvtes; momen nakcokvn enrahmet, cokvte hiyomēn eshecrtes;

18 Pucase em Puyvfekcvt vc oh fekhonnes, punvkvhervn estemerrakat em erkenvkaren 'svm vfeyvtet omekv; efeke kvcēkan em wicecit, svlvfkvlke enrecopkv em ocat, momet turhecvkekat renakv em ocat, 'sem erkenakit, tonufkakat enrecopit,

19 Momet Pucase em ohrolope herat erkenvkaren vc vtotvtet omekv.

- 20 Momen nakcokvn vkhotiyet, senekake vfastvn pvlken a ehmet, likvtes. Momen senekake vtehkat omvlkvt heren hecakvtes.

21 Momen vlicecet, Mucv-nettrn ce hvcko take ofvn heyv cokat esohfvciet os, kicakvtes.

22 Momen omvlkvt a en kerrakvtes, momet opunvkv hervke e cukwv asossat estomahes komakvtes. Momet, Heyvt Cosef e'ppuce toko? makakvtes.

23 Momen, Mehenwvn heyv opunvkv-svhopakvn, Vlekca, e en wicecvs,'svm punayet, momet, Nake Krpvnev ofvn momecetskvte poheyvten heyv cem ekvnv ofvn momecrs, cv kicvranatsket os, kicakvtes.

24 Momet, Mehenwvn ce kicvkis, Owalv estomet em ekvnv ofv evpahoyekot os. .

25 Momis mehenwrn cem onayvkis, Elivs owalv en nettv omof, momet ohrolope tutcene momet hvse epakat oren, hvlwe vkhotken, mv ekvnv omvlkvn elauko rakket fvckof, oyevlke sulke tates, Eslevl ofvn ; 
26 Momis etv hvmkusis Elivs ohtothoyekon, oje hokte Sitvne em etvlofv Seleptrn likvte trlkusen ohtothoyvtes.

27 Momen owalv Elisevs arof, lepvvlke sulke tates, Eslevl ofvn, momis etv hvmkusis hvsvtkekon, Nemvn. Selevlke tvlkuset hvsvtke hakvtes.

28 Momen senekake ofv vtehkat omvlkvt, heyv nanvken pohakof, cvpvkketvt fvcfakvtes ;

29 Momet asvpvkilet, tvlofvn ossicahket, hvlatet, ekvnhvlwe, (mv em etvlofv oh hahoyvte,) em vkeleften 'res oricvtes, a tvk wikvkvranet.

30 Momis nvrkvpvn etehoyanet, enkvpakvtes.

31 Momet hvtvpiket, Kalvle em etvlofv Kvprnevm rorvtes, momet Nettvcako omvlkvn emvhayakvtes.

32 Momen em mvhakv estomahes komakvtes, em opunvkv yekcetv vpvket ok'.

33 Momen este puyvfekev hvsvtkeko vpikat senekake ofvn likvtes, momet en hake yekcen es huehket,

$34 P u$ wikvs, Cesvs Nasvlvlke toyetskat, ceme pume esyomeyat naket etemocake haks? pu sumhuecvranet vlahketska? Estimv toyetskat, ce kerris, Hesaketvmese Enake Vcakat, kicvtes.

35 Momen Cesvs, Cvyvyahket aossvs, kicet, em vsehvtes. Momen puyvfekev holwvket 'metenrvwvn tvk wihkof, en nokkicekot aossvtes.

36 Momen omvlkvt estomahes komakvtes, Heyv opunvkv naket $o$ haks? ohfvnketvn momet yekcetvn puyvfekev hvsvthvkekat es em punayen, esossakekv, maket ete kicakvtes.

37 Momen ohonvkv mv ekvnv vfolotat omvlkvn hvmecicvtes.

38 Momen ahuyiret, senekaken osiyet, Simvn en cukon eceyvtes. Momen Simvn e 'hiwv e'cket hepetketv yek- 
cen es enokket wakkvtes; momen mv ohfvecvn em 'pohakvtes.

39 Momen ohfvnket hueret, hepetketvn em vsehvtes; momen em wikvtes; rnomen moman vpakusen alihket, vfvstakvtes.

40 Momen hvse vklatkof, estimvt este enokketv cahmelike es enokhoke ocakat omvlkvt mvn es en yicvtes, momen enken oh wvkhokjcet, omvlkvn em wicecakvtes.

41 Momen puyvfekev holwvhoket huehkaket, momet, Cemet Klist Hesaketvmese Eppuce toyetskes, makaket, sulken asossvtes. Momen em vsehet, punvyvkvre em etektvnecekates", Klist omaten kerrakekv.

42 Momen hvyatkof enkvpahket, vnrvwrn ayvtes ; momen estet hopoyakvtes, momet en yihcet, fekhonnicakvtes enkvpvkekaren.

43 Momen, Tvlofv etvu Hesaketvmese em ohmekketvn 'sem erkenvkrkvranvyet os, mv esvrahkvn vc vtothoyet ómekv, kicvtes.

44 Momen Kalvle senekakvlkan erkenakvtes.

\section{SETENTVCKE 5 .}

Momen mome ocvtes, este sulkat Hesaketvinese em opunvkv pohetvn komet, ohyekcet em afoloten, vkhvse Ceneselet afopken huervtes.

2 Momet perro-cuko hokkolet vkhvse afopken vkkaken hecvtes, momen rvro mvkwiyvlket asosiyet, hoyvn okkosaket fullvtes.

3 Momen perro-cuko hvmken, Simvn enaken eceyvtes, momet lvpvtke estomusen enkvpvyecvren em 'pohvtes. Momet lihket, perro-cuko aossen este sulkan emvhayvtes.

4 Momet opunvyvte wihkof, Laukan svkvyvs, momet 
rvro svkcvwetv hvmken svkcawatskvren cen hoyv taken vkpvlvtvks, Simvn kicvtes.

5 Momen Simvn vyoposket, Mvhaya, nereise vtotkēt, momis naken esēkon ep oh hvyvtiks, momis cem punvkvt omecicen hoyvn svkwikares, kicvtes.

6 Momen heyvn momecahkof, rvro sulke mahen vkcvwepuecakvtes; momen hoyvt tackvtes.

7 Momen em etohkvlketv perro-cuko hvmkan vtehkat yicet emvnicvkvren 'sem vhopayakvtes. Momen yihcet, perro-cuko hokkolvn fveficakvtes, momet vksumhokvranusvtes.

8 Simvn Petvt heyvn hecnf, Cesvs e torkowv ehomvn tvklvtiket, Aeha, Pucasē, v'nkvpvkvs, este-naorkv toyikv, kicvtes.

9 Fivro sulke cawakat omecicen estomahes komvtet ok', momet vpvkakateu omvlkvt.

10 Momen matvpomen Sepete eppucetake, Cems Cane tepakat, Simvn emvnicvlket omat, mvu em estomahakvtes. Momen Cesvs Simvn kicvtes, Ce penkahlats, mucv ayat esten crwetskvres.

11 Momen perro cuko lvpvtken resvcayahkof, nak omvlkvn wikahket, vevkvpeyvtes.

12 Momen mome ocvtes, tvlofv hvmke ofvn arof, hecvs, este-hunvnwvt lepvse es fvcket arvtes; mvt Cesvs hecof, e turofvn oh lvtiket, Pucasē, kometsken omat, cv hvsvtecetske tayes, kicet em 'pohvtes.

13 Momen, Komis, ce hvsvtkekvs, kicet, enke a rvtosecet celajvtes. Momen moman vpakusen lepvse e'nkvpakvtes.

14 Momen, Estimrn em onayekot, ahyet, plest e hecicvs, momet Moses vhakv emvte vcrkvyen cen hvsvteckv vrahkvn emvtcvs, es en kerkv taken, kicvtes.

15 Momis svheremahen ohonvkv hvmecicvtes; momen 
este sulsoke pohetrn, momet em enanokketv em wicecvren etohvtelokvtes.

16 Momen enkvpaket vnrvwrn cehyet, mekusapvtes.

17 Momen nettv hvmken mome ocvtes, emvhayof, Falvsevlket momet vhakv emvhayvlket Celuslvme tvlofv, momet Kalvle, momet Cutev ekvnv tvlofv vtekat asossvket tempen vpokvtes; momen em wicecvkvre Pucase yekcetv ocvtes.

18 Momen hecvs, este-hunvntake este-hunvnwv palse es enokken pvtakv oh wakken esyicvtes; momet estomen cukon escehyet, Cesvs ehomvn wvkece tayat hopoyakvtes.

19 Momen este sulkat omecicen estomen esceye tayat hecvkekok', cuko onvpvn es ohcemecakvtes, momet fakke-norv tvpestakan vkuehuehcet, em pvtakv svpvken nvrkvpvn Cesvs ehomvn vkhvtvpecicakvtes.

20 Momen Cesvs, em vkrsvmkv taken hecof, Este-hunvnwv toyetskat, cen naorketv ce'nkvpvkakes, kicvtes.

21 Momen cokv-hayvlket momet Falvsevlket vkerricetv vlicecakvtes, Heyv este Hesaketvmese tvklecat estit o haks? Estimvt naorketv enkvpakice taye haks, Hesaketvmese tvlkuset mvn momecekon omat? makaket.

22 Momis Cesvs em vkerrickv taken kerrof, em výoposket, Nake ce feke take ofvn vkerricatske haks?

23 Cen naorketv ce'nkvpvkakes, maketv, monkat, Ahuyiret, yvkvpvs, maketv tepakat, estrmit yekcekot ome haks? kicakvtes.

24 Momis este E'chuswvt yvmv ekvnvn naorketv kvpice taye yekcètr ocat kerratskvren, (palse es enokkat,) Ahuyiret, cem pvtakv cahwet, cen cukon vyvs, (kicvtes.)

25 Momen moman vpakusen ehomv taken ahuyiret, mv ohwakkvte cahwet, Hesaketvmese vrakkuecet, en cukon ayvtes.

26 Momen omvlkvt em estomahakvtes, momet penkvl 
kvn es fvcfaket, Hesaketvmese vrakkuecakvtes, Mucvnettr nake estomahvke heceyis, makaket.

27 Momen heyv nanvke hoyanof, osiyet, crtokunawv vteloyv Lefi hocefket cvtokunawv vtelokv cukon liken hecvtes; momet, Vc vcrkvyvs, kicvtes.

28 Momen ahuyiret, omvlkvn wihket, vcvkayvtes.

29 Momen Lefi hompetv-rakkon en cuko ofvn en hayvtes; momen cvtokunawv vteloyvlke sulke, momet este eti vpvkaket vpokvtes.

30 Momis $m v$ vpokat en cokv-hayvlke, momet Falvsevlket en kerrepvlken em ecayakvtes; Estoman cytokunawv vteloyvlke, momet naorkvlke vpaket hompet momet eskatske haks? kicaket.

31 Momen Cesvs vyoposket, Este cvfencakat vlekcrn eyacvvkekos, momis enokhokat eyacakes, kicakvtes.

32 Este faccvlke enhuehkvranit vlakit omvkates, momis holwvyecvlken vkerrickv eticvkvren.

33 Momen, Estoman Cane en kerrepvlke momet Falvsevlke en kerrepvlked matrpomen etewolen e elvwecet mekusvpkvn hayaket ome haks? momis cenake hompaket eskakes, kicakvtes.

34 Momen, Hopuetake hokte epayvranat en cuko-vtehkvlke hokte epayvranat vpake vtekat elvwvkuecatske taye haks? kicvtes.

35 Momis nettv cakkakvres, momof hokte epayvranat em eshoyvres, momen mv nettv take omof e elvwecvkvres.

36 Momen opunvkv svhopakvn opunayvtes: Este estomet kapv tvcke mucrsen leskan em vlihcekos, momen omat, mucvsat setet os, momet mucvse trckat leskan setenhehrekos.

37 Momen nvpvt pvrko-opuswv mucrsen sukcv leslekan vcahnekos, mecen omat, prrko-opuswr mucrsat sukcvn sehtet, pvlatkes, momen sukev yvmahkvkvres. 
38 Momis prrko-opuswr mucrset suker mucrsen vevnhoyvranet os, momen estvmitis yvmvhikekos.

39 Nvpvt pvrko.opuswv vhassan hvte eskan vpaken mucvsan eyahcekos, Vhassat senheres, makekv, kicakvtes.

\section{SETENTVCKE 6.}

Momen mome ocvtes, nettvcako enhrteceskv resvhokkolan teleko crpofv vlkan ropotecrtes, momen en kerrepvlket telekon mamet, enke ofvn poroyet, hompakvtes.

\& Momen Falvsevlke vpvlwvt, Estomaten nettrcakon mometv tokan momatske haks? kicakvtes.

3 Momen Cesvs vyoposket, Tewe momen vpvkakat elawakof estomvte,

4. Estomet Hesaketrmese en cukon ecehyet, hecetv tvklike, mv hompesko tetayat, momis plestvlke tvlke hompvkvranat, ehset, homipet momet vpvkakateu emakvte, heyv ohonvkv hecatskekat haks?

5 Momet, Este Echuswv tat nettrcakou e Pucase tes, kicakvtes.

6 Momen mome ocvtes, nettvcako eti senekaken ecehyet, mvhayvtes; momen este-hunvnwvt enkvperv yuknvtet likvtes;

7 Momen cokv-hayvlke momet Falvsevlket, Nettvcako wicecvr haks? 'komet vketecakvtes, vnrvpkvn komaket.

8 Momis em vkerrickv taken kerrvtes, momet hunvnwv enke yuknvten, Akvwvpiket, nvrkvpvn huervs, kicvtes. Momen akvwvpiket, huervtes.

9 Mohmen Cesvs, Nak hvmken cem 'pohvranvkis, Nettvcakon here momecetv holwvke momecetv tepakat estrt fvccrt o haks? momen hesaketv hesayecetv sumecicetv tepakat estvt fvccvt o haks? kicakvtes.

10 Momen omvlkvn oh fulotket hecaket, mv este-hunvn- 
wrn, Ce'nken rvtosecvs, kicvtes. Momen momecrtes, momen enke temvpoke hrmkate ome hakvtes.

11 Momen crpvkketvn es fvcfakvtes; momet Cesvs estomece tetayat 'sete'm punahoyvtes.

12 Momen mome ocvtes, mv nettv take omof ekvnhvlwen oh ayvtes, mekusvpvranet; momet Hesaketvmesen emekusape emunken oh hryatkvtes.

13 Momen hvyatkof en kerrepvlken enhuehkakvtes, momet mv asossen pale hokkolohkaken ensvtiket, vpastelvlke hocef huecvtes.

14 Simvn, (mv Petv hocefvtet,) momet etecakkat Vntolu, Cems momen Cane, Felvpe momen Paralome,

15 Maro momen Tamvse, Cems Alfevs e'ppuce, momen Simvn Selotes hocefken,

16 Momen Cutvse Cems e'tecakkat, momen Cutvse Eskalevt, mv Cesvs erwiyv taten.

17 Momen Cesvs vpaket ahvtvpiket, ekvnv tvpeksan huervtes, momen en kerrepvlke etohkvlke sulkat, momet este sulke mahet Cutev omvlkv, momet Celuslvme, momet Tilv Sitvne tepakat em uehvtkv afopke asosse em apohicvret, momet em enokketv em wikvkvren en yicvtet mon svpaklvtes.

18 Momen este puyvfekev hrsvthvkekat es naorakat en yihcen em wikakvtes.

19 Momen este sulkat omilkvt vcelaketvn komakvtes, yekcetv em ossatet omvlkvn em wicecaket omekv.

20 Momen renakv kvwahpet, en kerrepvlken hecet, makvtes, Cen herakes, estemerrvlke, Hesaketvmese em ohmekketv cenaket ok'.

21 Cen herakes, hiyome elawatskat, ce fvcfvkvret ok'. Cen herakes, hiyome hvkihhokatskat, vpelhoyatskvret ok'.

22 Cen herakes, este cen homecrket, vpakatskvte c'enkvpryecicrket, momet ec opunvyecrket, este E'chuswv 
vrahkvn ce hocefhokv holwvhoke omecet sossicake estomis.

$23 \mathrm{Mv}$ nettv omof afvckvket, momet ece 'fackusvket tasecvkvtcvs; hecvkes, feketv rakket hvlwe tvlofv cem ocvket omekv; matvpomen erkvlket owalvlken momecakvtet ok'.

24 Momis nakocvlke toyatskat, cen holwakvkes! ce mahlvpvtketvn cen heckvkvtet ok'.

25 Cen holwakvkes, ce fvcfakat! ce 'lawvkvret ok'. Cen holwakvkes, hiyome vpelhoyatskat! ce feke nokken, hvkihhokatskvret ok'.

26 Cen holwakvkes, este omvlkv heren eo opunvyecakof! momēn erkvlke owalv laksvlke momecakvtet ok'.

27 Momis pohatskat; ce kicvkis, Cen horrvlken vnokecvkes, cen homecakan heren em momecvkes,

28 Ce hanvkan heren opunvyecvkes, momet ece 'holwvyecrkat em emekusvpvkes.

29 Momen ce yvnvwv ce nafkat vpvlhvmkan 'sem fulotkvs; momet kapv-rakko ce 'konahat kapvu cen cvwvre vsehhetskvs.

30 Cem 'pohat vtekat emvs; momet nakockv ece 'konahat pvlken em 'pohhetskvs.

31 Momen este ce momecvkvren komatskat, cemeu em matrpomecvkes.

32 Ece 'nokecakan vnokecatsken omat, feketv estomen ocatske haks? naorkvlke tis este vnokecakat emeu vnokecaket ok'.

33 Momen cen lopicvkan en lopicatsken omat, feketv estomen ocatske haks? estenaorkvlke tis matvpomen momaket ok'.

34 Momen, $\mathrm{Vn}$ heckvres, komatet est' em pvlatsken omat, feketv estomen ocatske haks? naorkvlke tis naorkvlken em pvlakes, matvpomuset en heckvkvren. 
35 Momis cen horrvlken vnokecvkes, momet heren momecvkes, momet pvlvkes, Pvlken pun heckvres, komekot, momen cem feketvt rakke tares; momen Hvlwe Mahat en hopuetake toyatskvres, vrakkuecvkekat momet holwvhokat em merret ok'.

36 Monkv Ce'rke take merkv es fvcke omet merkv es ce fvcfvkekvs.

37 Ohfvecehcatskvs, momet ec ohfvccvkekares; ehvperkuehcatskvs, momen ce 'hvperkvkekares ; enkvpvyecicvks, momen ce'nkvpvyecihocvkvres.

38 Emvks, momen ce'mhoyvkvres; svkerkv heren, vtenkvkvten, esnekeyicvkvten, momen es em pvlatken estet ce hokpen cem vtehvkvres. Svkerkv svkerkuecatskat matan es cem vkerkuehocvkvret ok'.

39 Momen opunvkv svhopakvn es em punvyakvtes; Hecekat hecekan evpaye taye haks? hokkolvt sufkan vk yorkvraneko haks?

40 Enkerrepvt em mvhayvn 'sen hvlwekos ; momis kerretv orat vtekat em mvhayv etvpomvres.

41 Momen estoman tecakketskat e turwv etokvckuce vk ocan hecetske haks? momis tohkakvn ce turwv vk ocat vkerricetskeko haks?

42 Monkat estomēn tohkakv ce turwv vk ocat hecetskekon, tecakketskat, Tecakkvyē, ce turwv etokvckuce cem vk ossihcrrs, kicetskvr haks? momis ceme mahusat tohkakv ce turwv vk ocan hecetskeko haks? Mekusapvvhayv toyetskat, ce turwv taten tohkakvn vk ossicvs, momofvt heren hecetskvres, tecakketskat e turwv etokvckuce em vk esetv.

43 Eto herat em ette holwaken hayekon, eto holwakat em ette heren hayekot omekv.

44 Eto vtekat em etten es kerket omekv; estet eto- 
fvsken ke-rakko em mamvkekot, kvcon pvrko em mamvkekot omekv.

45 Este herat nake here e feke ofv ocat herat aossen, nake heran ossicet omes; momen este holwakat nake holwvke e feke ofv ocat holwakat aossen nake holwakan ossicet omes, efeke en sulke aosse e cukwv opunayet omekv.

46 Momen estoman, Pucasē, Pucasē, cv kicet omofvt, nake ce kicvkvyan momecatskeko haks?

47 Estimvt o estomet a vc oh atet, vm maketvn pohet, momecen omat, estimv omet on omat ce hecicvkares.

48 Este-hunvnwv cuko hayof, sufken korret, en likv cvto oh licate omet os ; momet uelauke kvwapkof, newv fihnat nafkvtes, momis nekeyiceko tayvtes, cvton oh like tatet ok'.

49 Momis pohis momecekat este-hunvnwv cuko en likv sekon ekvnvn oh hayvte omet os; mohmen uewv fihnat yekcen nafkvtes, momen moman vpakusen latkvtes, momen mv cuko em vhopvnketr rakke tates.

\section{SETENTVCKE 7.}

Tokvs, este pohaken, em opunvkv omvlkv emeyuksihcof, Kvprnevm eceyvtes.

2 Momen sentulev hvmke em vtotkv encakat enokket, elvranusvtes.

3 Momen Cesvs ohfvecvn pohhof, vlahket em vtotkv em wicecvren em 'pohepet, Cusvlke em vculvken ohtotvtes.

4 Momen Cesvs en yicat vwicecekot em 'pohakvtes, Em momecetske tetayat heretv oret os ;

5 Pum etvlwv vnokecet, senekaken pun hayvtet ok', makaket.

6 Momen Cesvs vcrkvyakvtes. Momen cuko em 
ahopvyeko erorofvn sentulevt en hessvlken ohtotvtes, Pucasē, e naorihcetskvs, vn cuko eceyetske tetayat heretv orvkokv;

7 Monkv vne tis ec oh atvye tayat heretv oris, komvkis, momis opunvkvn makvs, momen vm vtotkv em wikvres. $8 \mathrm{Vn}$ tis este yekcetv elecv huerihocvte toyikv; suletawvlke cv 'lecvn ocvyet os; momet hrmken, Vyrs, kicin, ayet os, momet etvn, Vtes, kicin, atet os; momet vm vtotkv, Heyvn momecvs, kicin, momecet os; maket.

9 Momen heyv nanvken Cesvs pohof, Estomahet oks, komvtes, momet afulotket, este vcrkfullaten, Ce kicvkis, Vkvsvmkv rakke $\mathrm{mv}$ omat Eslevl ofv eshecvkates, kicvtes.

10 Momen vtothoyvte, cukon ye fulehcof, vtotkv enokkvte em wiken hecakvtes.

11 Momen mome ocvtes, rempvkse tvlofv Nen hocefken eceyen, en kerrepvlke sulket, momet este sulsoket vcrkvpeyvtes.

12 Tokvs, tvlofv en tohopkvhauken vwolicof, hecvs, este-hunvnwv mv e'cke echuswv tvlkusvte èlēn soshoyvtes, momen e'cket oye tates, momen mv tvlofv este vtehkvlke sulket vpvkakvtes.

13 Momen Pucaset hecof, em merrvtes, momet, Hvkihiketskvs, kicvtes.

14 Momen a oh ahtet, este ēlē ohwvkeckvn celayvtes, momen es kvwvpakvte es svpaklvtes. Momen, Este mvnettetskat, akvwvpkvs, ce kicis, kicvtes.

15 Momen elvte alihket, opunvyetvn vlicecvtes. Momen e'cken a en huericvtes.

16 Momen omvlkvn penkvlkvt oh vlakvtes, momen Hesaketvmesen vrakkuecakvtes, Owalv rakket epu 'pake kvwapkes, momet, Hesaketvmeset em estvlke em merret omes, makaket. 
17 Momen heyv ohonvkv Cutev, momet afulotken hvmecicvtes.

18 Momen Cane en kerrepvlke heyv nanvke omvlkvn en kerkuecakvtes.

19 Momen Canet, en kerrepvlke hokkolen enhohiket, Cesvs ohtotakvtes, Vlvkvranvte toyetske haks? monkat etvn en nene hecvranē haks? maket.

20 Hunvntake em vlahokof, Cane paptisvt, Vlvkvranvte toyetske haks? monkat etvn en nene hecvranẽ haks? maket, pumen a ec ohtotes, makakvtes.

21 Momen mata hvse-vkerkv omof enokketv, mornet naknokke en heckakat, momen puyvfekev hvsvthvkeko vtehkakateu sulken em wicecvtes, momet turhecvkekateu renakvn emakvtes.

22 Momen Cesvs em vyoposket, Vhohyet, nanvke hece momet pohatskat, eturwv-hecvkekate hecakat, momen yvkvpvkekate yvkvpakat, lepvvlke en hvsvthakat, pohvkekate pohakat, pvsvtkvte akvwvphoyat, momet opunvkvhervn estemerhoyvlken 'sem erkenvkhoyan Canen em onvyvkes;

23 Momen estimvt o estomet vc vrahkvn elentappet omekon omat, en heret os, kicakvtes.

24 Momen Cane em punvkv-sarvlke vhoyehpof, Cane ohfvccvn este sulsokan 'sem punvyvketvn vlicecet, Naken hecvranet vnrvwvn esossatskvt haks? Kohv hotvle fvmoyicen?

25 Momis naken hecvranet esossatskvt haks? Hunvnwv accvke lowaloke vecen? Hecvs, em accvke herakat, en hompetvu herakat mekkvlke e huten_vpokes.

26 Momis naken hecvranet esossatskvt haks? Owalvn? Mehenwvn ce kicrkis, momen owalv semuntvle estvmahet omes.

27 Heyvt $m v$ tes, opunvyece cohoyvte, Hecrs, vm 
punvkv-sarv ce homvn vtotis, $\mathrm{mv}$ ce homv cen nene etetakuecvranat.

28 Ce kicvkikv, Hokte heckuecvte vpvke owalv Cane paptisv 'sen rakkat sahsekos; momis Hesaketvmese em ohmekketv ofv cotkus mahatet Cane es en rakket os, kicakvtes.

29 Momen este pohakat omvlkvt, momen cvtokunawv vteloyvlke, Cane em paptesvm es paptishoyvtet omen, Hesaketvmesen vrakkuecakvtes.

30 Momis Falvsevlke momet vhakv-hayvlke, Canet paptisvkekatet omen, e vnrvpen Hesaketvmese momekvs komaten vnrvpakvtes.

31 Momen Pucaset makvtes, Heyv este naken es vhopayar haks? momen nake omake haks?

32 Hopuetake nakwikv ocan vpoke, momet 'sete'm vhuehkaket, Cem pof kvkēs, momat opvnatskeks ; cen wahohkēs, momat hvkihhokatskeks, makake omakes.

33 Heyv omecicen makvyet os; Cane paptisvt tvklike hompekot, momet prrko-opuswr eskekot vlakvtes, moman, Puyvfekcr holwvken oces, makatskes.

34 Este E'chuswvt hompet momet esket vlakvtes, momen, Hunvnwv hompetv vnoksan, momet pvrko-opuswr eskvn; cvtokunawv vteloyvlke momen holwvyecvlke en hessen hecvs! makatskes.

35 Momis hoporrenkv echustake omvlkvt fvccecakes. 36 Momen Falvsevlke hvmket en hompvren em 'pohvtes. Momen Falvsevlke en cukon ecehyet, hompvranet vlikvtes.

37 Momen hecvs, hokte, naorkvt omet, mv tvlofv arvtet, Cesvs Falvsevlke en cukon hompet likvcrken kerrof, alvpastr tohawv, neha fvmecv vevnken svlakvtes.

38 Momen topvrv e'le yuksvn vhueret, hvkihkvtes, momet e turopuswv e'len 'sem okkosetvn vlicecet, e kvesse 
es en hrsvtecvtes, momet e'len em afvnket, neha fvmecrn es en siyvtes.

39 Tokvs, mv Falvsevlke enhuehkvte hecof, eme ofvn opunayat, Heyvt owalvt on omat, heyv hokte naorkvt omekv, estimvt ot, momet hokte estomet celayet omat kerrvnt os, makvtes.

40 Momen Cesvs vyoposket, Simvn, nake cem onvyetvn ocis, makvtes. Momen, Mvhaya, makepvs, kicvtes.

41 Este vhuericv hvmket este hokkolen vhuerice tates, hvmken tenelyv cukpe cahkepen vhuerice tates, momen hvmkat pale cahkepen vhuerice tates.

42 Momen nake es feketvn ocvkekof, vkvsamuset hokkolvn em wikakvtes. Monkv vm onvyvs, estvt semuntvlen vnokecvr haks?

43 Simvn vyoposket, 'Sen sulke em wikvte tares, komis, makvtes. Momen, Tayusen ohfvccehcets, kicvtes.

44 Momen hokten a oh fulotket, Simvn em punayet, Heyv hokten hecetska? Cen cukon a ceyvyat uewv cv'le vrahkvn a'metskekis, momis entat e 'turopuswrn. cv'len es vm okkoset, e kvessen 'svn hvsvtehces.

$45 \mathrm{Vc}$ vfvnket sekis, momis heyv hokte a cehyryise vtekat cv'len vfvnketvn fekhonnicekis.

$46 \mathrm{Cv}$ 'kvn olefv nehan 'svm vfeyetskekis, momis heyv hokte tat cv'len neha fvmecvn es vn sihyes.

47 Monkv ce kicis, En naorketv sulkvtet e'nkvpvkakes, tetayen vnokēcekv ; momis estimvn estomusis enkvpaken omat, $\mathrm{mv}$ tat estomusen vnokecet os, kicvtes.

48 Momet, Cen naorketv ce'nkvpvkakes, hokten kicvtes. 49 Momen vpake hompe vpokat, Momen naorketv enkvpryecicat estit o haks? eme ofv maketv vlicecákvtes.

50 Momen, Cem vkvsvmkvt ce hesayehces; herkv ofvn vyepvs, hokten kicvtes. 


\section{SETENTVCKE 8.}

Momen mv erenyupvn mome ocvtes, etvlwv momet etvlofucen hrmecicet erkenaket, Hesaketvmese em ohmekketv ohfvecrn punvkv-hervn kerkuecvtes: momen pale hokkolohkakat,

2 Momet hoktvke puyvfekev hvsvthrkekat, enokketv tis en wicehocvte sasat, Mele Maktelv' kihocat, puyvfekcv holwvhokat kolvpaket asossvte,

3 Momet Coanv, Kusv Helot en cuko-vfastv e hiwvt, momet Susanv, momet vpolwv sulket nake enanvken 'se'mvnicakat vpvkakvtes.

4 Momen este sulke tvlofv vtekat asosset em etohvtelokof, opunvkv swhopakvn 'sopunayvtes:

5 Fokayvt nerkvn fokayvranet ossvtes;-momen fokayof vpvlwvt nene afopken pvlatke sasen, oh svpakholvtes, momet hvlwe fuswv lokakvtes.

6 Momen evton oh pvlatke sasvtes; momet hontan vpakuset yuknvtes, otohicekokv.

7 Mornen etofvske vlkan pvlatke sasvtes; momen etofvske vpaket hontet, nokcepelakvtes.

8 Momen vpvlwvt, ekvnv heran oh pvlatket, hontet, em ette cukpe hrmken hayakytes. Momen heyv nanvken mahkof, Estimvt hvcko es pohetv ocat pohekvs, maket, huehkvtes.

9 Momen en kerrepvlke em 'pohvkepet, Heyv opunvkv svhopakv naket o haks? makakvtes.

10 Momen makvtes, Centake Hesaketvmese em ohmekketv ohfvccv kerretv yekcakat kerrvranatsken ce'mhoyvkes, momis vpvlwv opunvkv svhopakv ofv pohetv emhoyes, momat, hecakis, hecvkekaren, momet pohakis, pohvkekaren.

11 Tokvs, opunvkv svhopakv heyv tes; Enerkvt Hesaketvmese em opunvkv tes.

12 Nene afopke ocan $\mathrm{mv}$ pohakat tes; momof puyv- 
fekcv holwakat vlahket, opunvkv e feke vtehkvte 'sem ossices, vkvsamet hesahokekaren.

13 Cvto oh pvlatkvte mv pohakofvt es afvcket esakat tes ; momet heyvt, yvlonkv ocvkekot; vkocuknusen vkvsvmaket, vnrvpkv ocof, vcekellecet os.

$14 \mathrm{Mv}$ etofvske vlkan pvlatkvte heyv tes, mv pohakofvt, vpeyet, momis mucv hesaketv em vketeckv, nake-ockv momet afvcketvt nokcepelaken, em ette heren lokcicvkekot os.

15 Momis ekvnv heren oh pvlatkvte; mv, opunvkvn pohahkof, efeke mehenwe momet vcayecet, momis komet, em etten hayakat tes.

16 Este estomet, kulken kulehcet, nake vcvnkvn vpvtuecekos; monkat topv' elecvn es lihcekos; momis kulke sohhuerickvn sohhuerices, esceyat vtekat hryayvke hecvkvren.

17 Nake ohrvnke enrvmkvranekat sekon, momen ehke hecket, kerkvranekat sekot omekv.

18 Monkv estomēn pohatskat vketecvkes, estimvt o estomet ocat emhoyvret ok', momen estimvt o estomet ocekat oces komhoyvte tis em eshoyvres.

19 Momof $e$ 'cket momen etecakkakat en yicvtes, momis este cekfat omecicen 'rvsvpakleko tayvtes.

20 Momen, Ce'cke momen tecakkaketskat, ce hecetvn eyacaket, topvrvn svpakles, kicet ern onahoyvtes.

21 Momen em vyoposket, Heyv, Hesaketvmese em opunvkvn pohet, momet momecakat, cv'cket ot, momet vm etecakketvlke tes, kicakvtes.

22 Momen mome ocvtes, nettv hrmken perro-cukon eceyvtes, en kerrepvlke vpvkaken; momet, Vkhvse tvpalvn vpeyvkeres, Cesvs kicakvtes. Momen svkvpeyvtes.

23 Momis svkvpeyof, noce tates; momen hotvle-rakkot 
vkhvsen a oh hvtapkvtes; momen uewvn es fvcfaket, penkvlkv ofvn svpaklvtes.

24 Momen en yicet ahonecicakvtes, Mvhaya; Mvhaya, pu sumecvranēs, makaket. Momof ahuyiret, hotvlen momet uewv melohlan em vsehvtes, momen fekhonnaken, lekuthet ocvtes.

25 Momen, Cem vkvsvmkv take estrmvn oca? kicakvtes. Momen penkvlaket, estomahes komakvtes, Heyv hunvnwv estomet o haks! hotvle tis momen uewv tis nak kican em apohicaket omekv, ete kicaket.

26 Momen Katvlenvlke ekvntvckv Kalvle en tvpalv wakkan yicvtes.

27 Momen aosiyet, lvpvtken huerof, este-hunvnwv hvmket, hofunen puyvfekcv holwvhoke vtehkvtet, accvken accekot, momet cukon likekot, momis este herickv vlkan likvtet, tvlofv aosiyet vnrapvtes.

28 Momet Cesvn hecofvt, huehket ehomvn tvk latkvtes, momet, Naken pu tenrvwr ocē haks, Cesvs Hesaketvmese hvlwe mahat Eppucē ? cv 'stemerricetskekaren cem 'pohis, yekcicet makvtes.

29 (Puyvfekev hrsvtkekat unv hunvnwv aossvren kicekv. Tewolen hvlatvtet omen, momen crto-telacv es wrnayet, momen e'le 'sem vyekcihocet omvtes; momis eswvnakv waren, puyvfekcv holwakat vnrvwvn vtohkvtes.)

30 Momen Cesvs, Naket ce hocef kvt o haks? kicet em 'pohvtes. Momen, Lecrn, makvtes; puyvfekev holwvhoke sulke esceyvtet ok'.

31 Momen sufkan vkhvtvpecvre kicekaren em 'pohakvtes.

32 Momen mvn sukhv sulket ekvnhvlwe onvpvn nanopet fullvtes; momen mvn esceye tetayen em etektvnecvren em 'pohakvtes. Momen em etektvnecakvtes.

33 Momen puyvfekcv holwvhokat este-hunvnwv asosset 


\section{OPUNVKV-HERV LUK COYVTE VIII.}

sukhvn esceyakvtes; momen sukhv fulle hvmkat omvlkvt pvfpvnet vkeleftan vkhvsen vk tasecet, nokcepelkakvtes.

34 Momen hompvkuecakat nake moman hecakof, pefatket vpeyet, tvlofv momet en lvpvtke ofvn onayakvtes.

35 Momen nake moman hecvranet esossvtes; momet Cesvs en yihcet, este-hunvnwv puyvfekcr holwvhokat enkvprkakvte accet, hoporrene hahket, Cesvs e'le vliken hecakvtes; momet penkvlakvtes.

36 Momen hecakvtet puyvfekcr holwvhokan ocvte estomet em wikvte em onayakvtes.

37 Momen Katvlenvlke em ekvntvckv afulotke este sulke vtehkat omvlkvt e'nkvpvkvren em 'pohakvtes; penkvlkv rakket oh cakkvket omekv. Momen perrocukon ohcemiket, hvtrm ye fulkepvtes.

38 Momen hunvnwv puyvfekcv holwrboke asossvte vpvkepetvn em 'pohvtes.

39 Momis Cesvs, Cen cukon ye fulkepvs, momet nake rakrvken Hesaketvmese ce momecvten onvyvtcrs, kicet, etvn vtotvtes. Momen ayet, nake rakrvke Cesvs em momecvten tvlofvn hvmecicet kerkuecrtes.

40 Momen mome ocvtes, Cesvs ra fulikof, este sulkat omvlkvt, e'mehakakvtet ok', evpryakvtes.

41 Momen hecvs, este-hunvnwv Cilvs hocefket, senekake evpayvt, vlahket, Cesvs e'le ehomvn tvk lvtiket, en cuko en 'ceyvren em 'pohvtes :

42 E'chuste hvmkusen, en hoktvletv ohrolope pale hokkolohkakat mahen ocet omen, mvt ēlēt wakkvtet omekv. Momis aye arof este cekfat vceklvfkakvtes.

- 43 Momen hokte, catv-fihne ohrolope pale hokkolohkake es enokkvte, en nakockv omvlkvn vlekcvlke oh sumecicet omis, hvmkis em wiceceko tayvte, 
44 Era topvrvn vlahket, en kapv em vteken celayvtes; momen moman vpakusen e catv fihnvtet fekhonnvtes.

45 Momen Cesvs, Estimvt cv celaye haks? makvtes. Momen omvlkvt, Monkos, makakvlkof, Petvt momen mv vpvkakat, Mvhaya, este cekfat ece 'ceklafket, ece 'yotecet os, moman, Estimvt cr celaye haks? maketske haks? makakvtes.

46 Momen, $\mathrm{Cr}$ celaye saset os, yekcetv ve ossat kerrikv, Cesvs makvtes.

47 Momof hokte ehkekot omat hecof, fekeket a oh atet, ehomvn tvk lvtiket, naket omecicen celayet omat, momen estomen vyvkhvmkusen em wiket omat este omvlkv ehomvn em onayvtes.

48 Momen, $C v^{\prime}$ chustē, heren ce mahlvpvtkekvs; cem vkvsvmkvt temvpoken ce hayes; herkv ofvn vyepvs, kicvtes.

49 Opunaye emunkof, hvmket, senekake evpayv en cukon vtet, Ce'chuste elēpes: Mvhayvn naorihcetskvs, kicvtes.

50 Momis Cesvs pohof, em vyoposket, Ce penkahlats; vkvsvmkv tvlkusis ocetsken omat, cvfeknihocvres, makvtes.

51 Momen cukon eceyof, este estomis eceyvre em etektrnecekot, hoktuce e'rke e'cke tepakat, momen Petv, Cems Cane esyomat $e m$ etektrnecakvtes.

52 Momen omvlkvt hvkihhoket, oh wahokvtes; momis, Hvkihhohkatskvs; ēlekos, momis nocepet omes, kicvtes. 53 Momen, elēpet omen kerrakekv, vpelicakvtes. 54 Momen omvlkvn esossicvtes, momet enken en hvlahtet, Hoktucē, akvwvpkvs, maket huehkvtes.

55 Momen em puyvfekev hvtvm a oh fulken, vyvkhvmkusen akvwapkvtes; momen hompetv e'mhoyvren makvtes. 
56 Momen e'ckvlke estomahes komakvtes; momis nake momvten este estomen em onvyvkekaren, kicvtes.

\section{SETENTVCKE 9.}

Mомо en kerrepvlke pale hokkolohkake enhohiket etohkalet, em wiketvn hayet, puyvfekev holwvhoke omvlkv ohfvnken, momet enokketv wicecvkvren yekcetvn e'makvtes.

2 Momet, Hesaketvmese em ohmekketvn erkenaket, enokhokat em wicecvkvren vtotvtes.

3 Momet, Vpeyvranatskat naken cvwekot, setekkekv tis, ehvlewv en sukcv tis, tvklike tis, cvtokunawv tis cahwatskvs, momet kapv'se hokkkolihcatskvs.

4 Momen cuko estomen esceyatskat, mvn fekhonnvtet, matan enkppaket vpeyet omatskvres.

5 Momen estimvt o estomet ce 'vpayekon omat, $\mathrm{mv}$ tvlofv esossatskof, vnrvpe ohkerkueckvn, ce'le essu vlikat tis a tusiyet omatskvres, kicakvtes.

6 Momen vpehyet, opunvkv-hervn 'serkenaket, momet estvmv estomis omvlkvn em wicecet, tvlofv vlkan teropotecvtes.

7 Tokvs Helot tetlak nake Cesvs momecvten pohvtes; momet, Cane elkvn a en kvwapkes, makakat sasen,

8 Momen, Elivs e heckueces, makakat sasen, Owalvlke vculvke tate hvmkat hvtrm akvwapket omes, vpvlwvt makakan, mv omecicen fekherekates.

9 Momen, Cane tat ekv en tacryvnks; momis nanvke yomakat ohfvecv pohvyat estimvt o haks? Helot makvtes. Momet hecetvn komvtes.

10 Momen vpastelvlket er yicof, nake momecakvte omvlkvn Cesvs em onayakvtes. Momen ehkusen evpahyet, vnrvwr tvlofv Persitv hocefke enake ocan esceyvtes. 
11 Momen este sulkat mvn kerrahkof, vcrkvpeyvtes: momen evpayet, Hesaketvmese em ohmekketv ohfvccrn es ete'm punayakvtes, momet em wicecvre eyacakat em wicecvtes.

12 Momen mv nettv yafkusof pale hokkolohkakat a oh awet, Este sulkat etvn vtotetsken, tvlofucvlke momet ekvnv vwolakan oh vpeyet, momet fekhoniyet, hompetvn heckuecvkepekvs; yvmv fulleyat vnrvwvt ok', kicakvtes. 13 Momis, Cemet hompetvn e'mvkes, kicakvtes. Momen, Tvklike vnore cahkepe momen rvro hokkole sensulkat ocēks, vpehyet, hompetv heyv este omvlkvn en nesēkon omat, makakvtes.

14 (Este-hunvntake cukpe-rakko cahkepat mahe tatet omekv.) Momen, Vpoyvkes, pale cahlsepake etekvpakvken, kicakvtes.

15 Momen mvn momecet, omvlkvn vpoyakvtes.

16 Momof tvklike vnore cahkepat, rvro hokkolateu cawvtes, momet hvlwen vhecet, ohmeriyet, momet kvlkayet, en kerrepvlken emvtes, este sulkat ehomv 'sem ocvkvren.

17 Momen omvlkvt hompaket, fvcfakvtes; momen wokocke em vhoskat svmpv palen hokkolohkaken crwhoyvtes.

18 Momen mome ocvtes, enhvmkuset emekusapof, en kerrepvlke vprkakvtes ; momen, Estit os, estet cv kicake te ? kicet em 'pohvtes.

19 Vyoposkaket, Cane paptisvt, momis, Elivs, makakat sases, momen vpvlwvt, $\mathrm{Mv}$ owalvlke veulvke tate hvmkat akvwapkes, makakes, kicakvtes.

20 Momis ceme, Estit os, cv kicatske haks? kicakvtes. Petvt vyoposket, Klist Hesaketvmese enake, makvtes. 21 Momen em punayet, hunvnwv estomen mvn em onvyvkekaren yekcen em vsehakvtes; 
22 Este Echuswvt nanvke sulken 'sestemerkvranet os, momen veulvke, plestvlke enhomvhhotat, cokv-hayvlke esyomat etvn vhepvkakvres, momen elehohcen, nettr svtutcenan akvwvpkvres, maket.

23 Momet, Estimvt vc vevkvyetvn kumen omat, e em ehvperknecet, nettv omvlkvn em etohwelepkv kvwapet, vc vevkvtekvs, omvlkvn kicakvtes.

24 Estimvt o estomet en hesaketr hesayecetvn komen omat, sumecicen, estimvt o estomet vc vrahkvn en hesaketv sumecicen omat, matat hesayecrranet ok'.

25 Este ekvnv omvlkv heckuecet omis, e sumecicen, monkat vwihoken omat, naken en lopice haks?

26 Estimvt o estomet vm vlset, vm punvkvu em vlset on omat, este Echuswrt, en rakketv, Erkeu momen em estvlke vcacakat en rakketv ofvn vlakof, $\mathrm{mv}$ esten em vlse taret ok'.

27 Momis mehenwvn cem onayvkis, Yvmv svpakle sasat, elkv hecvkeko munket, Hesaketvmese em ohmekketv hecahke tvlkvres.

28 Momen mome ocvtes, heyv opunvkv renyupv nettv cenvpakvtekat Petv, Cane Cems esyoman evpahyet, ekvnhvlwen es ohcemkvtes, emekusvprranet.

29 Momen emekusapof, e turofv en heckv emvrahke haken, em accvke hvtke haket, hvyvyạvtes.

30 Momen hecvs, hunvntake hokkolet Cesvs ete'm punvyakvtes, mv Moses momen Elivs tates.

31 Mvt hvyayvke ofvn e heckuecaket, Celuslvmen en hesaketvn espoyvranat opunvyecakvtes.

32 Momis Petvt momet mv vpvkakat nockv honnet oh wetenkakvtes; momen ahonecakof, en hvyayvken, momen hunvntake hokkole vpvkake sehokaten hecakvtes.

33 Momen mome ocvtes, enkvpvlakaf, Petvt naken makat kerrekot, Cesvs em punayet, Mvhaya, yvmv fulleyat 
heres : momen cuko-vhake tutcenen hayvkeres; hvmket cenaket on, hvmket Moses enaket on, hvmket Elivs enaket on, kicvtes.

34 Hiyomēn opunayof, aholoce vlahket, ohvpetticakvtes, momen aholocen esceyakof, penkvlakvtes.

35 Momen opunvkv en hake aholoce aosset, Heyvt Cv'ppuce vnokeckat's ; em apohicvkes, makvtes.-

36 Momen mv en hake hoyanof, Cesvs enhvmkusen hechoyvtes. Momen entvlkuset ocakvtes, momet nanvke hecakvte ohranet, mv nettv take omof hunvnwv estomen em onayvkekates.

37 Momen mome ocvtes, remprksen ekvnhvlwen ahvtvpehcof, este sulket vnrvpakvtes.

38 Momen hecrs, hunvnwv este sulkat hvmket huehket, Mvhaya, cv'ppuce $v n$ hecetskvren cem 'pohepis, hopuewv mv tvlkusen omikv;

39 Momen hecvs, puyvfekcvt esen, vyvkhvmikusen huehket os; momen selahlecen, svpvkpvkuece haken, tonufet enkvpvketv en yekcen enkvpaket os.

40 Momen cen kerrepvlke ossicvkvren em 'pohepvyan, umvkeko tayis.

41 Momen Cesvs vyoposket, Aeha vkvsvmkv oceko, este yenahatskat, en hofune estomusen ece 'paket vm muntvlkekot'sece'yvranvki haks? Ce'ppucen yvmvin svtes, makvtes. 42 Momen a oh atof, puyvfekev holwakat tewihket, selahlecvtes. Momen Cesvs puyvfekcv hrsvtkekan em vsehvtes, momet hopuewvn cvfeknihcet, pvlket e'rken ohfulecicvtes.

43 Momen omvlkvt Hesaketvmese en yekce estomahes komakvtes, momis, omvlkvt Cesvs nanvke momecvte estomahes komakof, en kerrepvlken kicvtes ;

44 Heyv opunvkv ce hvcko take esceyekvs; este 'E'chuswvt est' enke ofvn wihoyvret ok'. 
45 Momis heyv maketv kerrvkekates, momen hecrkeko tayen em ehkvke tates, momet heyv maketv ohfvecv em 'pohetvn penkvlvke tates.

46 Momof estimvt rakke mahat taranat ohfvccun etenrvpkv kvwapkvtes.

47 Momen Cesvs e feke take em vkerrickvn kerret, hopuewucen ehset, vnakvn licvtes;

48 Momet, Estimvt o estomet heyv hopuewucen cv hocefkv ofvn evpayen omat, cr yvpayet os; momen estimvt o estomet $\mathrm{cv}$ yvpayen omat, ma a'c vtotvten evpayet os; omvlkatskan mv cotkus mahe ece 'pakvkat matat rakke tares, kicakvtes.

49 Momen Cane vyoposket, Mrhaya, hvmket puyvfekcv holwvhokat ce hocefkv ofvn sossicen heceyvnks; momet em vseheyvnks, epu 'crkarekokv, makvtes.

50 Momen Cesvs okat, $E m$ vsehhatskvs, mv pu'nrvpekat pum vpvlhvmken hueret omekv, kicvtes.

51 Momen mome ocvtes, hvlwen erpahoyvranat oketvt vlakof, Celuslvme vyetvn komet, fvccrn vhece munkvtes.

52 Momen punvkv es fullvlken ehomvn vtotakvtes; momen vpehyet Semelevlke tvlofucen esceyakvtes, em etetakuecvranet.

53 Momen evpayvkekates, en heckvt Celuslvmen ayv. rane omet omvtet ok'.

54 Momen en kerrepvlke Cems Cane tepakat hecakof, Pucasē, Elivs momecvte etvpomen totkv hvlwe ahvtapket sumecicvre kiceyvren kometske haks? makakvtes.

55 Momis oh fulotket, em vsehakvtes, Vkerrickv estomen ocatskat kerratskekos ;

56 Este Echuswvt este en hesaketv sumhuecrranet vlaket omekot, momis hesahuecrranet vlakvtet ok', maket. Momen tvlofuce etvn vpeyvtes.

57 Momen mome ocvtes, vpeye nene oh fullof, hunvn- 
wv hvmket, Pucasē, estrmv estomen ayetskis, ece 'cvkvyares, kicvtes.

58 Momen, Culv ekvnhauke ocvkepes, momen hvlwe fuswv puknvkv ocvkepes, momis este E'chuswv estrmv e'kv, licvranat tis ocekos, Cesvs kicrtes.

59 Momet este etvn em punayet, Vc vcrkvyvs, kicvtes. Momis, Púcasē, vm etektvnecetsken, cv'rke tan er hericvranis, makvtes.

60 Prsvtkat em prsvtkan hopelvkekvs, momis cen tat ahyet, Hesaketvmese em ohmekketvn erkenvkvs, Cesvs kicvtes.

61 Momen, Pucasē, ece 'cvkvyares, momis vm etektvnecetsken, cv hute vpokan er vsekvkvranis, este etvt kicvtes.

62 Momen, Este estomet escrpayeckvn enke es hvlahtof, eyupvn y'vhecen omat, Hesaketvmese em ohmekketv enakuecetv heretv orekot os, Cesvs kicvtes.

\section{SETENTVCKE 10.}

Heyv nanvke renyupvn Pucase eti pale kolvpaken mellvtes, momet hokkolaken ehomvn tvlofv omvlkv momen estrmvt o estomis eme ayvranat vpeyvren.

2 Monkv kicakvtes, Mehenwvn hvyo rakket os, momis vtotkvlke nvcomus; monkv hvyo e Pucase vtotkvlke en naklokce a ohtotvren emēkusvpvkes.

3 Vpeyvkes; hecrks, yvhv sulke em etenrvwr yvpefikucvlke omvken ece 'totvkis.

4 Crtokunawv en sukcvn, momen ehvlewv en sukcvn, monkat estelepikv tis svpehyatskvs: momen vpeyatskof este estomen vsehkatskvs.

5 Momet cuko estomen esceyatskat,- Herkvt heyv cukon ocekvs, enhvteceskvn makatskvres.

6 Momen herkv echushunvnwvt mvn liken omat, cen 
herkv take oh fekhonnvres; monkon omat, pvlken a ec oh fulkvkvres.

7 Momet naken ce'mhoyvkat hompet, esket, mv cuko etvwrn vfekhonnvkvtcrs, vtotkvt feketr en hecke tetaye oret omekv. Cukon etohtvlecet vpehyatskvs.

8 Momen tvlofv estomen esceyatskat, ce 'vpahoyvken omat, nanvke ce homvn ohocat hompatskvres.

9 Momen enokhoke vtehkat em wicecet, Hesaketvmese em ohmekketv ece 'wolicvkes, kicvkvtcrs.

10 Momis tvlofv estomen esceyatskat ce'vpahoyvkekon omat, mv etehoyvnkv vlkan esosset,

11 Cem etvlofv take em essu epu 'likat ece'nrvpen hvsvtecēs; mont omisem heyvn kerrepvkes, Hesaketvmese em ohmekketv cem vwolicvkes, makvkvtcvs.

12 Momis ce kicvkis, Mv nettv omof Satvme mv tvlofvn 'sen here tares.

13 Aeha, cen holwakes, Kolesa! Aeha Persita, cen holwakes! vtotketv yekcvke ec ofv take momakvte Tilv Sitvne tepakat ofvn momakvten omat, hofunen sukcrhakvn vchoyet essun tvk vpoket em vkerrickvn eticakvnt omekv.

14 Momis ohfvececkv omof Tilv Sitvne tepakat es cen hervke tares.

15 Momen Kvpvnev toyetskat, sutv oren kvwapketskat, totkv-rakkon ce'khvtvpecihocvres.

$16 \mathrm{Mv}$ cem apohicat $\mathrm{vm}$ apohicet os; momen $\mathrm{mv}$ ece'nrapat vne $v c$ vnrapet os; momen vne $v c$ vnrapat vc vtotvten vnrapet os.

17 Momen pale kolvpakat afvckvket er yicet, Pucasē, ce hocefkv eteropotten puyvfekcv holwvhokat tis pum apohicaket os, makakvtes.

18 Momen, Setvne vtoyehvtte omet sutv a latken hecryvnks. 
19 Hecrs, cetto momen hvcesesterahv oh svpvkletv, momen vnrapv en yekcetv omvlkv ohfvnken yekcetvn ce'mvkis, momen naket o estomis cen nokkihcrkekos.

20 Momet o estomis puyvfekevlke cem apohicakat, heyv es afvcikatskvs; momis ce hocefkv take hvlwe tvlofv ofvn cohoyat min es afvckvkes, kicakvtes.

$21 \mathrm{Mv}$ hvse-vkerkv ofvn Cesvs puyvfekcv ofvn afvckvtes, momet, Cv'rkē, sutv ekvnvu e Pucase toyetskat, ecc 'kvsamis, heyv nanvke nakkerrat momet' hoporrenakat em ehhet, hopuetakucen em enrametskekv; mehenwvt's, Cvrke, momẽt ce homv heret omvtet okv ;

22 Nanvke omvlkv Cv'rke vm wikvtes, momen Eppuce estimvt ot omat hunvnwv estomet kerrekon, Erke tvlket kerres, momen Erke estimv tat estimvt kerrekon, Eppuce momet $\mathrm{mv}$ Eppuce kerrepuecat tvlket kerraket os; makvtes.

23 Momet, en kerrepvlke oh fulotket, ehkusen kicakvtes; $\mathrm{E}$ turwv nanvke hecatskat hecakat en herakes;

24 Owalvlke mekkvlke esyomat sulket heyv nanvke hecatskat hecetv eyacakis, hecrkekates; momen heyv nanvke pohatskat pohetvn eyacakis, pohvkekates, ce kicvkvyet omekv.

25 Momen hecrs, vhakv-hayv hvmket ahueret, Mvhaya, naken estomvyatet hesaketv emunkv ocvye taye haks? eskerret makvtes.

26 Vhakv ofv cokat nake te? estomēn ohonayetske haks? kicvtes.

27 Momen vyoposket, Pucase cen Hesaketrmesen ce feke omvlkvn, cem puyvfekev omvlkvn, cen yekcetv omvlkvn momet cem vkerrickv omvlkvn es vnokecet, cem vwolicv e vnokecetskat etvpomen vnokecvs, makvtes.

28 Momen, Fvccrn vyoposikets; heyvn momecvs, momet ce hesakvres, kicvtes. 
29 Momis e fvccecetvn komet, Monkv estimvt vm awolicvt o haks? Cesvn kicvtes.

30 Cesvs vyoposket, Este hvmket Celuslvme enkvpaket Cvlekon vkhvtapkvtes, momet horkopvlke em etenrvwvn latkvtes, mvt accvke en tefet, momet vnvttet, elvranusen wihket, enkvpvkakvtes.

31 Momen momet ohcen, plest hvmket mv nene vkhvtapkvtes; momet hecof, fekhonnekot hoyanvtes.

32 Momen Lefitvlke matvpomet mvn arof, vlaket hecvtes; momet fekhonnekot hoyanvtes.

33 Momis Semelevlke hvmket, estvn aye arof vnakvn vlakvtes; momet hecof, em merrusvtes.

34 Momen oh ahyet, vnattat, olefv neha momet pvrkoopuswvn es em ohcanet, em wvnayvtes; momet vpuekv enaken oh licet, cuko tvlemen svlahket, vfastvtes.

35 Momen hvyvtiken enkvpakof, tenelyv hokkolen a cahwet, cuko-pucasen ehmet, Vfvstrs, momen nake ohvtvlaken sumecicetskat, rvlakvy of, cem fekares, kicvtes.

36 Monkv mv horkopvlke em etenrvwv latkvte heyv tutcenat estvt em vwolicv tates, kometske haks? kicvtes. 37 Momen, Em merrvtet mv tes, makvtes. Momof Cesvs, Cemeu ahyet, matvpomecrs, kicvtes.

' 38 Momen mome ocvtes, vpeye fullof, tvlofuce hrmken ecehyen, hokte hrmket, Mare hocefket, en cuko ofvn evpayvtes.

39 Momet $\mathrm{mv}$ hokte tecaklkat Mele hocefken ocvtes, mv Cesvs e'le tempen liket, em punvkvn pohvtes.

40 Momis Mare este en yvmvse vtotketv tayen 'se naoricvtes, momet a oh ahtet, Pucasē, tecakkvyat vnhvmkuse vtotkaren $\mathrm{cr}$ wikat stoskometskeko haks? mon$\mathrm{kv} v \mathrm{vm}$ vnicvren kicvs, makvtes.

41 Momen Cesvs vyoposket, Marē, Marē, nanvke sulken vketecet 'se naoricetskes; 
42 Momis nake hvmket kusvpkes; momen Mele mv - nake here vkonahoyeko tayan enhopoyepet os, kicvtes.

\section{SETENTVCKE 11.}

Momen mome ocvtes, estrn emekusapvtet wikof, en kerrepvlke hvmket okat, Pucasē, Cane en kerrepvlke mekusvpkv emvhayvte omen pu mvhayvs, kicvtes.

2 Momen, Emekusapatskof, hiyomēn makrkes; Pu'rke hvlwe liketskat, Ce hocef kvt vcakekvs. Cem ohmekketvt vlvkekvs. Nake kometske hvlwe momat etvpomen yvmv ekvnvn oh momekvs.

3 Nettv vrahkv tvkliken nettv omvlkvn pu'mvtcrs.

4 Momet naorketvn pu'nkvpryecicvs, mv vhuericeyvte enkvpryecicēkv. Momet nake holwake pu yace tetayat 'sep oh ahyetskvs, momis holwakan a 'sepu'ssicvs, kicakvtes.

5 Momet kicakvtes, Ece 'pakvkat estimvt, en hessen ocet, momet nere nvrkvpv oh ayet, $\mathrm{Vn}$ hessē, tvklike vnore tutcenen vm prlvs;

6 Vn hesset aye aret vm vlakan, nake homv licvye tayat ocvkokv, kicatsken ;

$7 \mathrm{Mv}$ ofvn a vyoposket, $\mathrm{Cv}$ naorihcetskvs, vhauke hiyomat vkhotken, vn hopuetake vc vpaket topv lumhēkv, akvwrpikit ce'mvko tayes; maken omat;

8 Ce kicvkis, En hesset omat omecicekon, momis tayen em 'pohepat omecicen ahuyiret, en sulke estomomuse eyacat e'mvres.

9 Momen ce kicvkis, Vpohvkes, momen ce'mhoyvkvres; hopoyvkes, momen eshecatskvres; vnafkvkes, momen cen haukvkvres.

10 Vpohat vtekat en hecket omekv, momen hopoyat eshecet os; momen vnafkat en haukvres.

11 Ece 'pakvkat hvmket hopuetake erket omen, eppucet 
tvkliken em 'pohen omat, cvton e'mvr haks? monkat rvron vpohen omat, rvro vrahkvn cetton e'mvr haks?

12 Monkat echustaken vpohen omat, hvcesesterahvn e'mvr haks?

13 Monkv ceme holwrhokvket omof emetv herakat cen hopuetake emetv kerratsken omat, Erke hvlwe likat mahvkvu em 'pohakat Puyvfekcr Vcakat emvkvres.

14 Momen puyvfeker holwvke, momet punayeko tayat ossicof, mome ocvtes, puyvfekcr holwakat osiyof, punayekate opunayvtes; momen este sulkat estomahes komakvtes.

15 Momis vpvlwvt, Pelsepvp puyvfekev holwvhokat em mekko eteropotten puyvfekev holwvhokat sossicet os, makakvtes.

16 Momen vpvlwyt eskerraket, hvlwe vten eskerkvn em 'pohakvtes.

17 Momis em vkerrickv taken en kerraket, em punayet, Tvlofv em etvwr vnrvpe tekvpakat vtekat hopvnketvn eroret os, momen cuko tat cuko vnrvpe tekvpakat latket os.

18 Momen Setvne ern etvwr e vnrvpet tekvpaken omat, estomet em ohmekketv huervr haks? Puyvfekev holwvhokan Pelsepvp eteropotten sossices, cv kicatskekv.

19 Momen vnet Pelsepvp eteropotten puyvfekev holwvhokan sossicin omat, estimv eteropotten ce'ppuce take esossicvke haks? monkv mvt cem fvccecvlke tares.

20 Momis vnet Hesaketvinese enke wesakv teropotten puyvfekcv holwvhokan esossicin omat, mehenwrn Hesaketvmese em ohmekketv ec oh vlakvkes.

21 Hunvnwv yekcat naksevnickvn ocet, en cuko vhecicof, en nakockv herkv ofvn ocakes.

22 Momis es en yekcat oh vlakof, ohfvniket, naksev- 
nickv mv enhonrvten vkonahet, nanvke svpenkvlecvten tekvpices.

23 Ve vprkekat ve vnrapes; momen ve vpake vteloyekat vwahecet os.

24 Puyvfekev hvsvtkekat este osiyof, ekvnv kvrkapan ares, fekvpkvn hopoyet, momet eshecekot, $\mathrm{Cr}$ hute aosiyvten oh fulkares, maket.

25 Momet vlakof, pasket momet mvherihocen esheces. 26 Momof ayes, momet puyvfekev holwvhoke holwvyeckv eme semuntvlvke kolvpaken evpayet, esceyet, mvn vpokes; momen mv este en hueretv espoket hvteceskvn 'sen holwakes.

27 Momen mome ocvtes, heyv nanvke opunayof, hokte este sulkat hvmket en hake kvwapet, Hopuetakhute ce heckuecvte, momet epese coketskvte en herakes, kicvtes. 28 Momis, Momes, momis Hesaketvmese em opunvkvn pohet vfvstakat mit en herakes, makvtes.

29 Momen este sulsokat vtelokof, maketv vlicecvtes; Heyv honvpse holwvyecvket omes; nake eskerretvn hopoyakes; momis nake eskerretv etv emhoyekon, eskerretv owalv Convse enake tvlkusen emhoyvres.

30 Convse Nenefitvlke nake es en kerretv tate etvpomet este Echuswrt heyv honvpse nake es en kerretv taret ok'.

31 Wahvlv em mekko hokte heyv hunvntake vpaket ohfvececkv omof akvwvpkvres; momet ohfvccecvkvres; ekvnv vtekakat vtet Salomv en hoporrenkv pohvranet vlakvtet ok'; momen hecvs, Salomv es en rakket heyvn likes.

32 Nenefv hunvntake heyv este honvpse vpaket ohfvececkv omof akvwrpiket, ohfvccecvkvres; Convse em erkenvketvn em vkerrickv eticakvtet ok', momen hecvs, Convse es en rakket heyvn likes. 
33 Este estomet kulken kulehcof, ehken monkat svkerkv elecvn eslihcekos, momis a'sceyatet hryayvke hecvkvren kulke sohhuerickvn sohhuerices.

34 Ena en kulke eturwv tes, monkv ce turwvt heren omat, ce'na omvlkvn hvyayvket fvcket os, momis holwaket on omat, ce'na omvlkv yomucket fvcket os.

35 Monkv vketecrkes, hryayvke ece 'pikat yomucket omekaren.

36 Monkv, yomucket estrmv sekon, ce'na omvlkvn hryayvket fvcket on omat, omvlkvn hvyayvket fvcke tares, kulke here hvyvyakat cen hvyvyicat etvpomen.

37 Momen opunayof, Falvsevlke hvmket en hompvren em 'pohvtes; momen ecehyet, hompetvn vlikvtes.

38 Momen hompetvn vlikeko munket e okkosekan Falvsevlket hecof, estomahes komvtes.

39 Momen Pucaset kicvtes, Hiyomat, Falvsevlke toyatskat, halo momet pvlvknv onvprn hvsvthvken hayatskes, momis ec ofv svpenkvleckv momet holwvyeckv es fvckes.

40 Hoporrenvkeko toyatskat, onvpv ocat hayvte, matat ofv ocateu hayekat haks?

41 Momis nake ocatskat asossen heromkv min est' emvkes; momen hecvkes, nak omvlkv cen hvsvthvket os. 42 Momis cen holwakvkes, Falvserlke toyatskat! kofockv, momen lutv momen vhockv omvlkv tekvprke espalan fekatsket, ohfvcceckv momet Hesaketvmese em vnokeckv wikatskekv; heyv nanvken momecatskvnt os, momet vpvlwrn wikekot.

43 Cen holwakvkes, Falvsevlke toyatskat! senekake ofv ohliketv svmomvkus mahan, momet nakwikv vlkan vseketv cem vlostvket ok'.

44 Cen holwakvkes, Falvsevlke momet cokv-hayvlke, mekusapv-vhayvlke toyatskat! este-herickv heckvkeko, estet kerrekot oh yvkvpakan, mv omakatsket ok.' 
45 Mvhaya, hvmmaketskat pumeu vlesketrn pun hayet oketsks, vhakv-hayvlke hvmket momof em vyoposket kicvtes.

46 Momen, Cemeu cen holwakvkes! vhakv-hayvlke toyatskat, sapokv acukoyetv yekcvken est' oh wvkecet, cemet mv sapokv cenke wesakv take hvmkusis es celayatskekot omekv, kicvtes.

47 Cen holwakvkes! ce'rkvlke take owalvlken pvsvtakvtet omen, cemet en sepvlkv hahicatsket omekv.

48 Mehenwrn cemet ce'rkvlke take em vtotketv em vkvsamatskat kerkuecatskes; mvt pvsvtakvtet omen, cemet sepvlkvn en hahicatsket omekv.

49 Monkv, Owalvlken momen vpastelvlken ohtotvkares, momen vpvlwvn pvsatet assecvkvres, Hesaketvmese en hoporrenkvt makvtet os.

50 Heyv ekvnv vliceckv vteken owalvlke e catv pvlatkvte omvlkv heyv honvpsen em 'pohohvren;

51 Epel e catvn Svkelivs, mv cuko vcakat altv em oh etenrvwr elehocvte e catvn vteken. Mehenwrn, ce kicvkis, Heyv honvpsen em 'pohohvres.

52 Cen holwakvkes, vhakv-hayvlke toyatskat! nakkerretv eshaweckv vkonahatskvtet ok'; cemet esceyekot, esceyakateu emvnvttecatskvtes.

53 Momen heyv nanvken kicakof, cokv-hayvlke momen Falvsevlke ohyekcicet, nanvke sulken opunvyecicetvn vlicecakvtes ;

54 Mvkerretvn 'semehaket, momet nake e cukwv aossen 'sehset es vnrvpvre hopoyaket.

\section{SETENTVCKE 12.}

Mомоғ, este en sulke vhonkvtesko tayat vteloken, svhere mahekv etohsvpaklof, Falvsevlke espakkueckv, mv mekusvpkv vhayetvt os, em e vketecvkes, en kerrepvlken taten kicetv vlicecrtes. 
2 Nake ohrvnke enrvmkvranekat sekon, momen ehke kerkvranekat sekot omekv.

3 Monkv naken yomucke ofvn opunayatskvte hryayvke ofvn pohkvres; momen $\mathrm{mv}$ nvthofuce ofv ehvcko ofvn opunayatskvte cuko onvpvn kerkuehocvres.

4 Momen ce kicvkis, vn hessvlke toyatskat, Eña elecaket, momis $\mathrm{mv}$ hoyanat naken momecvke tayan ocvkekat em penkvlahkatskvs.

5 Momis estimvn em penkvlvranatskat homvn cem onayvranvkis; $\mathrm{Mr}$ elehcof, totkv-rakkon vk wike taye yekcetv ocan em penkvlvkes; mehenwvn ce kicvkis, Mvn em penkvlvkes.

6 Cutkucvlke cahkepat hoporrenkv hvmken wihoyet omeko haks? momen hvmkusis Hesaketvmese ehomvn ehoskekos.

7 Momis ce kvesse tis omvlkv ahonkvtkvket os. Monkv ce penkahlvkates, cutkucvlke sulken'sem vcakatsket os.

8 Momen ce kicvkis, Estimvt o estomet este ehomvn cv kerkuecen omat, emeu este Echuswvt Hesaketvmese en hvlwe estvlke ehomvn kerkuecvres.

9 Momis este ehomv vm evtuecat emeu Hesaketvmese en hviwe estrlke ehomvn em ertuehocvres.

10 Momen estimvt 'o estomet este Echuswr vurvpen opunvkvn 'sopunvyecen omat, enkvpvyecihocvres; momis Puyvfekcv Vcakan tvklecat enkvpvyecihohcekos.

11 Momen senekakevlkan, momet fvececvlke momet yekcetv ocakan es ce yihocof, Naken vyoposkeyvr haks? monkat, Estomēn, hvtvm naken opunahoyeyvr haks? komet vkerrihcatskvs :

12 Puyvfekcv Vcakat mata hvse-vkerkv ofvn makvranatskat ce mvhayvkvret ok'.

13 Momen este sulkat hvmket, Mvhaya, tecakkvyan nake ocvraneyvte vn kvpicvren kicvs, kicvtes. 
14 Momen, Este hunvnwv toyetskat, Estimvt cem fvececrn, monkat cem etekvpicv taken ev hayvt haks? kicvtes.

15 Momet, Vketecrkes, momet nak sulecetv kometvn e vfekcakhvkes; este en hesaketv nak sulke epucasat tvlkekot omekv, kicakvtes.

16 Momen opunvkv svhopakvn es em punayaket makvtes; Este nakocv hvmket em ekvnv nake sulket oh lokcvtes;

17 Momen e vkerricet, Naken estomar haks? estvmv vn nak lokcvke hericvye tayat ocvkokv; makvtes.

18 Momet, Heyvn momecares; vn tohton lekaflehcit, senrakrvken hayares, momet nake vn lokcakat, momen vn nakockv omvlkvn mvn vtehares.

19 Momet, Puyvfekca, nakockv sulke, ohrolope sulke em etetayen hericetskes; fekapet, hompet, esket momet afvckepvs, vm puyvfekcrn kicares, makvtes.

20 Momis Hesaketvmeset, Hoporreneko toyetskat, mucr neren cem puyvfekcr cem 'pohohvres: momof nanvke ehopaketskvte estimv enake tar haks? kicvtes.

21 Hiyomēt os, nakockv e en hericis, Hesaketvmese ohfvecv nake ocekat.

22 Momen en kerrepvlken kicakvtes, Monkv ce kicvkis, Cen hesaketv ohfvecv vkerricet 'se naòrihcatskvs, nake hompvranatskat, monkat ena nake oh kayvranatskat.

23 Hesaketvt hompetvn muntvlet os, momen enat accvken muntvlet os.

24 Kakken vkerricvkes, vhocvkekot, momet vteloyvkekot omes; herickv cukon momet tohton ocvkekos; momis Hesaketvmeset hompvkueces. Ceme mahvkvu fuswv'sem vcakatsket os.

25 Momen estomatskat vkerricatskatet cem mahe estekuce hvmke e ohvtvlayatske taye haks? 
26 Monkv nake cotkus mahaten momecatskeko tayer omat, estomēn vpvlwvn 'se naoricatske haks?

27 Pvkpvke lele kihocan estomet mahat vketecvkes; vtotkvkekot, momet fullecvkekot os; mome estomis ce kicvkis, Salomv en rakketv omvlkv ofv heyv hvmkvtekat omet vccekates.

28 Monkv Hesaketvmese pvhe vnrvwv ocat, mv mucv nettv ocet, momet pvkse tvklike esnorickv ofv vpvlvthoyat tis momēn vccecen omat, ceme semuntvle mahen ce'ccecvkvres, vkvsvmkv estomuse ocatskat!

29 Momen naken homprranatskat, monkat eskvranatskat hopohyatskvs; momen cem vkerrickv melohlvkates. 30 Heyv nanvke omvlkvn ekvnv em etvlwvvlket hopoyaket omekv; momen heyv nanvke ce yacvkat Cerke take kerres.

31 Momis Hesaketvmese em etvlofv min hopoyvkes, momen heyv nanvke omvlkvt cem ohvpvkvkvres.

32 Nvcomuse tohkvlkatskat, ce penkahlvkates; Cerke take ohmekketv ce'metvn es afvcket omekv.

33 Nake ocatskat wiyet, heromkvn estemvkes; sukcv lesleke hakekat, momen hvlhawat ofvn pokeko tayat, horkopv vwolicet, monkat sukehtv vyvmahkekan ehopakvkepvkes.

34 Estvmv cenake ocen omat, ce feke taken mvn ocvret ok'.

$35 \mathrm{Ce}$ tetackv sewrnvket omen, cen kulke taket fenkvkekvs.

36 Momen cemet, este e pucase etepvyetv ervlvkvranat emehakakat, momat vlaket vnafkof, vyvkhvmkusen en hvwecvke tayvres, $m v$ omaket omvkes.

$37 \mathrm{Mv}$ vtotkvlke, epucase vlakof, vketecaken eshecat en herakes ; mehenwrn ce kicvkis, Esewvnaket, hompvkvra; nan vpoyet, momet a oh atet em afvstvkvres. 
38 Momen vketeckv svhokkolan vlaket, monkat vketeckv svtutcenan vlaket, mornvkēn eshecen omat, mv vtotkvlke en herakes.

39 Momen heyvn kerrvkes, cuko-pucase hvse-vkerkv estomen horkopv vlvkvranan kerrvten omat, vketecet, en cuko hopvnkvre etektvnecekvnt omes.

40 Monkv cemeu ce metetakvket omekvs; este Echuswvt, hvse vkerkv mohmes komatskekofvn vlaket omekv.

41 Momen Petvt kicvtes, Pucasē, heyv opunvkv svhopakv pumen pum punayetske haks? monkat omvlkv tis ?

42 Momen Pucaset kicvtes, Cuko vfastv herome momet hoporrene, mv epucaset en cuko vtehkvlke ohfvnke bayvte, em oketv orof hompetv emvren; estimvt mv te?

$43 \mathrm{Mv}$ vtotkv momēn vfasten, e pucaset vlaket eshecat, en heres.

44 Mehenwvn ce kicvkis, Nake ocat omvlkv ohvfastvn hayvres.

45 Momis $\mathrm{mv}$ vtotkvt, $\mathrm{Cr}$ pucase vlvketv vcewices, e feke ofvn maket, vtotkv hunvntake, momen vtotkv hoktvke naf ketvn, momet hompet momet esket, hacetvn vlicecen omat;

$46 \mathrm{Mv}$ vtotkv e pucase nettr en nene hecekot, momet hvse vkerkv kerrekofvn vlvkvres, momet etetahcet, en liketv vkvsvmvkekat vpvken em mellvres.

47 Momen mv vtotkv, epncase em eyackvn kerret, $e$ etetakuecet, em eyackv vcrkvyen momecekat, tayen rukaf hoyvres.

48 Momis $\mathrm{mv}$ kerrekate rukafkv oricen momecvtet nvcomusen rukaf hoyvres. Estimvt o estomet sulke emhoyat sulken em 'pohohvres; momen estimvt o estomen sulken em wihokvte, matan sensulken em 'pohohvret ok'. 
49 Totkv ekvnvn ohtotvranit vlakit omis, momet entis vhetket on omat, naken komi haks?

50 Momis paptesvm es paptiskaren ocis, momet, pokeko vtekat, tayen vc ohyekcihocet omes!

51 Ekvnvn herkvn oh est' emvranet vlaket os, cv komatske haks? Monkos, ce kicvkis, momis etenkvpvketv mimvn;

$52 \mathrm{Mucv}$ vtekat cahkepat cuko hvmken vtehket etekvpvkvret ok', tutcenet hokkolen vnrvpaken, hokkolet tutcenen vnrvpaken.

53 Erket eppucen vnrvpet tekvpvkvres, momen eppucet erken vnrvpen; ecket echuswvn vnrvpen, momet echuswvt ecken vnrvpen; ecket emehiwvn vnrvpvres, momet emehiwvt ehe ecken vnrvpvres.

54 Momen este sulkan heyvn kicakvtes; Aholocet hvse-vklatkv aosset kvwapken hecatskof vpakuset, Osket svlakes, makatsket os; momen momēt os.

55 Momen wahvlv hotvle hotalen hecatskof, Hiyet ocvres, makatsken, momēt omes.

56 Mekusapv-vhayvlke toyatskat, sutv ekvnv tepakat en heckvn kerratske tayes, momis estoman heyv oketvn kerratskeko haks?

57 Momes, momen estoman ceme mahusat nake frecan eskerratskeko haks?

58 Ce'nrapi vpaket fvececv oh ayetskof, vhoyatskof heren vketecetskvres, em vnvttetske tetayvren; monkon omat, frccecv ehomvn ce huericen, fvccecvt este-wvnayv ehomvn ce wihken, momen este-wvnayvt cuko-yekevn ce 'pikvres.

59 Cem onayis, mvn enkvpaketskeko emunket, tokunawuce cotkus mahat espoke mahen fehketske tvikusvres. 


\section{SETENTVCKE 13.}

Mv omof Kalvlevlke e catvn Pilvtet em ohnekrickvn svpayvte ohfvecv em onayakat vpvkakvtes.

2 Momen Cesvs ryoposket, Mv Kalvlevlke nanvke hiyomvken es estemerrakvtet ok', naorkvlke Kalvlevlke omvlkv semuntvlvke tates, komatske haks?

3 Monkos, ce kicvkis; momis cemeu vkerrickv eticatskekon omat, omvlkatskat matvpomuset ce 'stemerkvkvres.

4 Monkat pale cenvpohkakat cuko hvlwe Silovm ofvn oh latke pvsatvte naorkvlke Celuslvme tvlofv fullat omvlkv semuntvlvke tates, komatske haks?

5 Monkos, ce kicvkis; momis cemeu vkerrickv eticatskekon omat, omvlkatskat matvpomuset ce 'stemerkvkvres, kicakvtes.

6 Momen heyv opunvkv svhopakvn opunayvtes; Estehunvnwv hvmket ke-rakko vpe eme pvrko cvpofv ofvn vhocken ocvtes; momet vlahket, em etten oh hopoyet, eshecekates.

7 Momof pvrko-cvpofv vfastvn, Hecvs, ohrolope tutcenen vlakit, heyv ke-rakko vpe em etten vhopoyit, momis eshecvkos; tvcrs, estoman ekvnvn ehvperkvn yvmahkuece haks?

8 Momen em vyoposket, Pucasē, mucv ohrolopen hrtvm wiketsket emunken, vnakvn korrit em vlekwihcvrs ;

9 Momen em ette hayen omat, heres; momis monkon omat, mv eryupvn tvcetskvres, kicvtes.

10 Momen nettvcakon senekake hvmke ofvn estemvhayvtes.

11 Momen hecvs, hokte yekcetr sumecice enokketv ohrolope palen cenvpohkaken es enokkvte, momet tepvkockvtet ot, momet estomet alopotkeko tayet likvtes. 
12 Momen Cesvs hecof, enhuehkvtes, momet, Hokte toyetskat, cem enokketv enrecopketskes, kicvtes.

13 Momen enke oh wvkhokicvtes; momen moman vpakusen lvpotke hahket, Hesaketvmesen vrakkúecvtes.

14 Momen Cesvs nettvcako omof wicecvtet ok', senekake semerpayvt cvpvkket vyoposket, Nettr epakat mv ofv este vtotke tayat ocet os ; mv ofvn a vwatsken, cem wicehocvkekvs; momis nettvcako tokon, este sulkan kicakvtes.

15 Pucaset em vyoposket, Mekusapv-vhayv toyetskat, omvlkatskat nettvcakon wakv monkat corakko-wakv ehuten vwvnakvten enrecopet, hvlahtet eskuecetvn svpeyatskeko haks?

16 Monkv heyv hokte, Eplehame e'chuste, hecrs, heyv ohrolope palen cenvpohkake oren Setvne wvnayvte, nettrcakon heyv wvnakv enrecopkeko taye haks? kicvtes.

17 Momen heyv nanvken mahkof, vnrapvlke omvlkv vlsvke hakvtes, momen este omvlkvt afvckakvtes, nake rakrvke eme eteropotten momakvten.

18 Momof makvtes, Hesaketvmese em ohmekketv nake ome haks? momen nake es vhopayar haks?

19 Mvstr enerkvn estet ehset, eme crpofuce ofvn vwihken, momen hontet eto rakke haken, hvlwe fuswv elvecen oh vpokēs; $m v$ omet os.

20 Momen hvtvm makvtes, Naken Hesaketvmese em ohmekketv es vhopayar haks?

21 Espakkueckv hoktet cahwet, hockvte vkerkv tutcene ofvn 'ehhen, momen oce munken omvlkvt pakkvtes; $m v$ omet os.

22 Momen estemvhayet tvlofv tolofuce tis eteropottet, Celuslvmre fvccun ayvtes.

23 Momof hvmket kicvtes, Pucasē, hesahokvranat nvcomuset ome haks? Momen kicakvtes; 
24 Vhauke cotkan esceyetvn vcevllvkes; ce kicvkis, Sulket esceyetvn hopoyis, umvkekaret ok'.

25 Cuko-pucase akvwrpiket, vhauken vkhottehpof, fettvn svpaklet vhauken vnvfketvn vlicecet, Pucasē, $\mathrm{Pu}$ casē, pun hvweçvs; makatsken omat, momen vyoposket, Ce kerrvkot, estrmun awatskat kerrvkos, ce kicvken omat;

26 Momof, Ce homvn hompēt, eskeyvtes, momen pum etvlofv etehoyvnkv ofvn mvhayetskvtes, maketvn vlicecatskvres.

27 Momis makvres, Ce kicrkis, ce kerrvkot, estvmvn awatskat kerrvkos; v'nkvpvkvkes, holwvyeckv vtotkvlke omvlkatskat.

28 Hvkihketv momet enute setekketvt ocvres, Eplehame, Iseke, Cekvpe momet owalvlke omvlkv Hesaketvmese em etvlofv vpoken hecatsken, centake 'sec ossihocof.

29 Momen hvsossv-fvecv, momen hvsaklatkv-fvecv, momen honerv-fvecv, momen wahvlv-fvecru a vwet, Hesaketvmese em etvlofvn vpokvres.

30 Momen hecrs, enhvteceskv taranat espoket ocakes, momen espoke taranat enhvteceskvt ocakes.

$31 \mathrm{Mv}$ nettv etvwvn Falvsevlke vpvlwvt en yicet, Osiyet, yvmvn enkvpvkvs; Helot ce 'lecetvn komekv; kicakvtes.

32 Momen, Vpehyet, mv culvn em onvyvkes, Hecrs, mucv-nettvn momet pvksen puyvfekcv holwvhokan sossicit, enokketvn wicecis, momen nettv svtutcenan es vm pokvres.

33 Momis mucv nettv, pvkse momet rempvksen yvkvpvranvyet os; Celuslvme en lvpvtken owalvt sumkeko tayet omekv.

34 Celuslvmē, Celuslvmē, owalvlke pvsatetskat, momet 
mv ec ohtothoyat cvto es nafketskat; etewole estomusen cen hopuetake tottolose echustakuce trrpv elecv vteloyat omen ce mecvkvye tayvte tat, momis cemet komatskekates!

35 Hecvs, cen cuko tvnken cem wihokes. Momen mehenwvn ce kicvkis, Mucv vtekat cv hecatskekon, $\mathrm{Pu}$ case hocef $\mathrm{kv}$ ofv vlakat vrakkuehocet os, makatske nettv vlahke tvlkvres.

\section{SETENTVCKE 14.}

MoMEn mome ocvtes, nettvcakon tvkliken hompvranet, Falvsevlke en homvhhotvlke hvmkat en cukon eceyof, vketerakvtes.

2 Momen hecrs, hunvnwv hvmket, ena pakketv es enokket ehomvn likvtes.

3 Momen Cesvs vyoposket, vhakv-hayvlke momen Falvsevlken, Nettrcako omof wicecetv fvecvt o haks? kicakvtes.

4 Momen naken makvkekates. Momen ehset, em wicehcet, vyecicvtes ;

5 Momet em vyoposket, Ece 'pakvkat estimvt, corakkowakv monkat wakv tis vksuf kan cem vk latkvken omat, moman vpakusen, nettrcako omof tis, ossihcetskeko te? kicakvtes.

6 Momen heyv nanvke ohfvccvn em vyoposkvkeko tayvtes.

7 Momen enhuehhokan opunvkv svhopakvn punayakvtes, estomēn tektvnke herakan enhopoyaken hecof; kicaket,

8 Etepvyetvn este estomet ce'nhuehken omat, tektvnke svmomus mahaten lihketskvs, hunvnwv es cen hvlwe tis enhuehke witvtet ok';

9 Momen mv tepakatske ce'nhuehkvkvte vlaket, Tek- 
tvnke heyv hunvnwvn emvs, ce kicen, vlsuset tektvnke kvncvpe mahan esetvn vlicecetskvres.

10 Momis ce'nhuehhohkof, ayet, tektvnke kvncvpe mahan likvs, mv ce'nhuehkvtet vlakof, $V n$ hessē, senhvlwen vcemkrs, ce kicvren; momof ece 'paket hompakat ehomvn vkvsvmkv ocetskvres.

11 Estimvt o estomet e hvlwecat kvnevpuehocen, mv e kvncvpuecat hvlwehocvres.

12 Momof e'nhuehkv'teu em punayet, Fvccv-like hompetv, monkat yafke hompetvn hayetskof, cen hessvlke, monkat ce tecakkakat, monkat ce'nahvmkvlke, monkat ce 'wolicv nakocvlke enhohihketskvs; mometsken omat, mvt ce'nhuehkaken, feketvt cem oce witvres.

13 Momis hompetv-rakkon hayetskof, estemerrvlke, pvlvlakat, yvkvpvkekat hecvkekat esyoman enhuehketskvres ;

14 Momen cen hervres; cem fekvkeko tayekv; momis faccvlke aenkvwvpketv omof, cem fehokvret ok', kicvtes.

15 Momen vpvke hompakat hvmket, heyv nanvken pohof, En heres, Hesaketvmese em etvlofv ofvn tvklike hompvranat, kicvtes.

16 Momen kicvtes, Hunvnwv hvmket, hompetv-rakkon hayet, sulken enhuehkvtes;

17 Momen, Vwvkes, hiyomat nak omvlkv tetakes, yafke hompetv orof, em vtotkvt enhuehhokan kicvkvren vtotvtes.

18 Momen omvlkvt temvpvlwvn seokepkv hayetrn vlicecakvtes. Ekvnv tvcken nehsis, momet erhecvranvyet os; vm etektvnecetskvren cem 'pohepis, enhvteceskat kicvtes.

19 Momen hvmkat, Wakv mohwakke cahkepen nesvyet ot, ahyit eskerrvranis; vm etektvnecetskvren cem 'pohepis, makvtes. 
20 Momen etvt, Hokten epayikv; ayvko tayes, makvtes.

21 Momen mv vtotkv vlahket, heyv nanvken e pucasen kerkuecrtes. Monkv cuko-pucase cvpakket, Lvpken tvlofv etehoyvnkv tvptahan momet lopuckusan osiyet, estemerrvlke, pvlvlakat, yvkvpvkekat hecvkekat esyoman yvmvn svtes, kicvtes.

22 Mornet em vtotkvt, Pucasē, naken momekvs, maketskat, mohmes, momen tektvnke emunkes, kicvtes.

23 Momen pucaset, Nene tvleme momet eto-lane tohopke vlkan osiyet, a'scehuecvs, vn cuko fvcke taren, vtotkvn kicvtes.

24 Ce kicvkikv, Mv hunvntake enhuehhokvte hvmkusis vn hompetv-rakkon lahsekos.

25 Momen este sulsoke mahet vcrkvpeyvtes; momen ohfulotiket, kicakvtes,

26 Hunvnwr estomet e'rke, momet e'cke, momet e 'hiwv, momet hopuetake, momet etecakkat, momet ohvtvlakat en hesaketv tis enhomecekot, vc oh aten omat, vn kerrepv haketv umeko tayes.

27 Momen estimvt o estomet, em etohwelepkvn karonet, ve vcrkatekon omat, vn kerrepv haketv umeko tayes.

28 Estit ece 'pakvkat cuko-hvlwen hayetvn komat nanvke espoye tetayen ocen omat, enhvteceskvn lihket, enrahkvn vhonkvtekare te?

29 Monkon omat, em wakkv wvkecof, poyeko tayen omat, hecakat vtekat vpelicetvn vlicecahke wites,

30 Heyv hunvnwv cuko hayetv vlicehcet, poyeko tayvtes, makaket.

31 Monkat mekko estvt, mekko etimvn horre en hayvranen omat, cukpe-rakko palen evpayet, mv cukpe-rakko pale hokkolen evpayet vlvkvranan, en yekcepe tetayat, enhvteceskvn lihket, vkerricekat haks? 
32 Momet monkon omat, hvmkat hopvye heren are emunkof, opunvkv-es-fullvlken vtotet, herkvn hayetvn em 'pohes.

33 Matvpomen ece 'pakvkat estimvt o estomet nake omvlkvn ocat wikekon omat, vn kerrepv haketv umeko tayes.

34 Okcrnwv heret omes, momis okcvnwrt en yekce en sumkvten omat, naken es svpvtecvr haks?

35 Ekvnv monkat svlekwicetv vtelokat tis en lopicekos, momis etvn vpvlvthoyes. Ehvcko es pohetvn ocat pohekvs.

\section{SETENTVCKE 15.}

Momen crtokunawv vteloyvlke naorkvlke esyomat omvlkvt em apohicvranet vnakuecakvtes.

2 Momen Falvsevlke momet cokv-hayvlke vnrvpen opunvyecet, Heyv hunvnwv naorkvlke evpayet, vpaket hompes, makakvtes.

3 Momen heyv opunvkv svhopakvn es opunayet kicakvtes,

4 Ece 'pakvkat este-hunvnwy estomet yvpefikv cukpe hvmken ocet, hrmken sumecicen omat, pale ostrpake ostrpohkakan vnrvwv ofv wihket, mv sumkan er hopoyat hopoye emunket eshecekar haks?

5 Momen eshehcof, es afvcket e foluwrn oh wrkeces.

6 Momet e hute vlahkof, en hessvlke em vwolicvlke esyoman enhohiket vteloyes; Vc vpaket afvckepvkes, vn yvpefikv sumkvte eshecit omikv, kicaket.

7 Ce kicvkis, Matvpomen este naorkv hvmke em vkerrickv eticat hvlwe tvlofv ofv este facculke pale ostvpake ostvpohkake em vkerrickv etickv en sekar tayan senhoyvnen oh afvchokvres.

8 Monkat hokte estvt, tenelyv palen ocat, hvmken 
sumecicen omat, kulken kulehcet, cukon paset, momet vwicecekot hopoyet emunket eshecekar haks?

9 Momet eshehcof, en hessvlke momet vwolicvlke enhohiket vteloyet, Vc vpaket afvckvkes, tenelyv sumecicvyvten eshehcikv, maket os.

10 Matvpomen ce kicvkis, Hesaketvmese en hvlwe estvlke ehomvn naorkv hvmke em vkerrickv eticat oh afvchokes.

11 Momen makvtes, Hunvnwv hvmket eppuce take hokkolen ocvtes.

12 Momen esmunettat $e^{\prime}$ rken kicvtes, $C v^{\prime}$ rkē, nake cvnake taranat v'mes. Momen em vhesaketvn em etekvpicvtes.

13 Momen nettv vnvcomekot hoyanof, eppuce esmvnettatet omvlkvi vtelohyet, ekvnv hopryen ayvtes, momet mvn yvmahket hueret, en nakockv sumecicrtes.

14 Mornen omvlkvn yvmahkuehcof, elauko rakke $\mathrm{mv}$ ekvnv ofv kvwapkvtes; momen enkusvpketvn vlicecvtes. 15 Momen ahyet, mv etvlwv en trsekiyv hvmken er vpakvtes; momen eme crpofv ofvn vtotvtes, sukhvn hompvkuecvren.

16 Momen tvlakohake sukhv hompakvten e nvrke es fvcecetvn eyacvtes; momen estimvt emekates.

17 Momen e vkerricof, Cvrket vtotkvlke sulket tvklike em etetaye semuntvlen ocake tat, vnet elaukot cv 'lvranus!

18 Akvwvpikit, cv'rken oh vyares, momet kicares, $C v^{\prime}$ rkē, hvlwan vnaorkit, momet ce homvn naorkis,

19 Momen ce'ppuce ohvtvlakat ev kihoce tayat orvkos, cem vtotkvlke pvlaketskvte hvmke omen cv hayvs, kicvtes.

20 Momen akvwrpiket, e'rken em vlakvtes. Momis hopvye here emunken e'rket hecet, em merrvtes, momet letiket, e nokwvn oh latket, afvnkvtes. 
21 Momen eppucet, Curkē, hvlwan vnaorkit, momet ce homvn naorkis, momet ohvtvlakat ce'ppuce cv kihoce tetayat orvkos, kicvtes.

22 Momis erket em vtotkvlken em punayet, Kapv here mahan esawet, vccecvkes; momet estenkewesakpikvn e'nken em 'piket, estelepikv em vtehvkes;

23 Momet wakuce nehicvkvten yvmvn esyicet, elecvkes; momet hompet, afvckvkeres ;

24 Heyv cv'ppuce elvnket, hvtvm ahesaket; sumkvnkis, hvtvm hecket omekv, kicakvtes. Momen afvcketvn vlicecakvtes.

25. Momen e'ppuce veulicat cvpofvn arvtes; momet cukon vwolicof, hayeckv momen opvnkvn pohvtes.

26 Momen vtotkv hrmken enhohiket, Heyv nanvket maken oke haks? licet em 'pohvtes.

27 Momen kicvtes, Tecakketskat vlvkes; momen ce'rket wakuce nehicvkvten elehces, cvfeknet momet temvpoken evpayekv.

28 Momen crpakket, eceyetvn eyacekates; monkv e'rket aosiyet, eceyvren em 'pohepvtes.

29 Momen vyoposket, e'rken kicvtes, Hecvs, heyv ohrolope sulken cem vtotkis; cem vhakv tis tohwvlvpkvyvte sekos; mont omis, vn hessvlke vpake afveketv hayvye tetayat cowatuce tis v'metskvt seks;

30 Momis heyv ce'ppuce, hoktvke hoktarvke vpaket cem vhesaketr cen lokvte, er vlahkof, wakuce nehicvkvten em elehcetskes.

31 Momen kicvtes, $C v$ 'ppucē, estofis ve vpaketskes; momen cvnake omvlkv cenake tes.

32 Afvcketv hayet, momet afvckvkeyvre herises, heyv etecakketskat elvnket, momet ahesakes; momet sumkvnkis, hēckes. 


\section{SETENTVCKE 16.}

Mомет en kerrepvlken em punayet, Este nakoce hvmket cuko-vfastvn oce tates; momen mvt, Cen nakockv cen yrmahkuecrtes, kicet em opunvyehocvtes.

2 Momen enhohiket, Naket estomen heyv nake ec ohfvecv pohit omi haks? estomen cuko vfastetskyten e ohonvyvs; ohvtvlakat cuko-vfastv hueretskeko tayekv, kicvtes.

3 Momof cuko-vfastv eme ofvn opunayet, Naken estomvranvya? cv pucase cuko-vfvstetv vc vkonahekv; korrvko tay'es; momet nak vpohetv tat vc vlset os.

4 Cuko vfvstetv vc ossihocof naken estomvranvyat kerris, en cuko take ofvn ev yvpayvkvren, makvtes.

5 Monkv e pucase vhuericakat vtekat enhuehkvtes, momet enhvteceskvn, Nvcomen cv pucase ece 'huericet o haks? kicvtes.

6 Momen, Olefv neha vkerkv cukpe hvmken, makvtes. Momen, Cen nakcokv eskerkvn ehset, lvpken lihket, pale cahkepen coyvs, kicvtes.

7 Momet etvn kicvtes, Nvcomen e vhuericetske haks? Momen, Teleko vkerkv cukpe hvmken, makvtes. Momen, Cen nakcokv eskerkvn ehset, pale cenvpaken coyvs, kicvtes.

8 Momen epucaset cuko-vfastv fvccẹan vkvsamvtes, hoporrenuset momecvtet ok'; yvmv ekvnv hopuetake en hesaketv ofvn hvyayvke en hopuetake 'sen hoporrenvket omekv.

9 Momen ce kicvkis, Nakockv fvcceko ocakan aossen cen hessvlke taken hayvkes; momat meyuksicatskof, vpoketv yuksv-seko ofvn ce yvpayvkvren.

10 Estimvt mv cotkus mahat ohfvecv heromet on omat, sulkat tis ohfvecv heromet os; momen cotkus mahat ohfvecv estit fvecekon omat, sulkate ohfvecvu fvecekot os. 
11 Monkv nakockv fvecekat ohfvecv ce heromvkekaten omat, estimvt nakockv mehenwan cem wikvkvr haks?

12 Momen este-hunvnwv etv enake ohfvecv ce heromvkekaten omat, estimvt cenaken ce'mvkvr haks?

13 Vtotkv estomet epucase hokkolen em vtotikekos; hvmken en homecet, momet hvmkan vnokecet; monkat hvmken hvlatet, momet hvmkan vnrapet ok'. Hesaketrmese nake-ockv eshokkolen em vtotketv umatskeko tayes.

14 Momen Falvsevlket, nake ockv eyacrket omet, heyv nanvke omvlkvn pohaket, vpelicakvtes.

15 Momen kicakvtes, Cemetake este ehomvn e fvececakat toyatskes; momis Hesaketvmeset ce feketaken kerres; nake este vpvke vrakkuehocat Hesaketvmese ehomv enhomeckvt omekv.

16 Vhakv momen owalvlke emunken Canet vlakvtes; mv vtekat Hesaketvmese em ohmekketv 'serkenvkhoyen, este omvlkvt vyekcet esceyet os.

17 Momen sutv ekvnv tepakat sumhoke tayat yekcekon, heyv vhakv ofv satkv cotkusat hvmke tis sumkat mit yekcet os.

$18 \mathrm{E}$ 'hiwvn wihket, etvn epayat vtekat hoktaret os; momen ehe wikvten epayat vtekat hoktaret os.

19 Este-hunvnw̧v nakoce hvmke tates, accvke okholatte hvnrwelv telekmuse tepakan accet, momet nettv omvlkvn hompetv-rakkon ocet, here mahen likvtes;

20 Momen nakvpohv hvmket, hvmkv lekhowet, Lisvle hocefke tates,

21 Momet hompetv wokucke este nakeoce em ohhompetv a pvlatkat hompihocvre eyacen, en tohopkvhauken vwvkechoyvtes; momen ohvtvlakat efvt yicet, lekhowan en lasakvtes.

22 Momen mome ocvtes, nakvpohv ehlen, hvlwe estet 
Eplehame e hokpe ofvn svpeyvtes. Momen nakeocvt ehlen, herihocvtes.

23 Momet totkv-rakko ofv estemerkēt, en renakv kvwapet, Eplehame hopvyen hecvtes, momet Lisvlet e hokpe ofvt on.

24 Momet huehket, Eplehame cv'rkē, vc oh merrvs, momet Lisvle vtotetsken, enke-wesakv eyuksvn uewvn vk cakhehcet, cv tulaswrn es vn kvsvppueekvs; heyv fenke ofvn $\mathrm{cv}$ 'stemerrekv, makvtes.

25 Momisem Eplehamet kicvtes, $C v^{\prime}$ ppucē, cen hesaketv ofv nake herake cen hecken, momen matvpomet Lisvle nake holwvhokat en heckvten vkerricvs; momis hiyomat emahlvpvtken, cemet ce 'stemerkes.

26 Momen heyv nake omvlkv ohvpakat, pume ceme esyomeyat $p u$ tenrvwr sufke rakke vkueyihoceko tayet oces, momat yvmvn enkvpaket tohtiyehcet, ec oricetvn komakat momvkeko tayes; momen ce'nkrpaket a ep oh vwetv komat tis momvkeko tayes.

27 Momen makvtes, $C v^{\prime}$ rkēe, monkv cv'rke huten vtotetskvren cem 'pohepis;

28 Tecakkvyat cahkepen ocikv; heyvn en kerkuecen, heyv estemerketv ocan yesceyekaren.

29 Eplehamet, Moses owalvlke esyoman ocakes; mvn em apohicvkekvs, kicvtes.

30 Momen, Monkos, Eplehame cv'rke toyetskat; momis hvmket elkv a en kvwvpket oh ayen omat, vkerrickvn eticvkvres, makvtes.

31 Momen, Moses owalvlke esyoman em apohicvkekon omat, elkv a en kvwapkat tis vkvsvmepuecrkeko tayet omes, kicvtes.

\section{SETENTVCKE 17.}

Momen en kerrepvlken kicakvtes, Naorkepueckv yicvkekare monko tayet omes, momis en holwakes, mv este omecicen yicat! 
2 Heyv lopucke hvmkat naorkepuecekon, emfolotkv en cvtot e nokwv vtvriken uehvtkv vk wihokat mit en heret os.

3 E vketecrkes; Tecakketskat ece 'naorken omat, em vsehvs; momet vkerrickv eticen omat, em merrvs. .

4 Momen nettv hvmken kolvpaken ece 'naorken omat, momet kolvpaken nettv hvmken a ec oh fulotket, Vkerrickvn eticis, maken omat, em merrvs.

5 Momen vpastelvlket, Vkvsvmkvn pum ohvtvlvyis, Pucasen kicakvtes.

6 Momen Pucaset makvtes, Vkvsvinkv mvstv enerkv hvmke omen ocatsken omat, heyv eto sekvmin hocefkan, Yvlonkv es cupeniket, uehvtkvn vk vhockvs, kicatsken, cem apohicvres.

7 Momis estimvt ece 'pakvkat, vtotkv crpayeckv monkat wakv-hompvkueckvn vrecicvten, cvpofvn ra atan vpakusen, Ahtet hompvs, kicvr haks?

8 Momis, Nake hompvye tayat tetakuehcet, sewvnaket, hompet eskvyof vm vtotketsken, mv hoyanat, hompet esketskvres, min kicekare te?

$9 \quad E$ pucaset, mv vtotkv nake kicvte mornecvte svrahkvn, Ce 'kvsamis, kicvres, komatske haks? Monkos, komis.

10 Cemeu matvpomen, nake ce kicvkvte omvlkvn momehcatskof, Vtotkvlke lopicvkeko toyes; nake momecvraneyvten momēces, makvkvtcvs.

11 Momen mome ocvtes, Celuslvmen ayof, Semelev momen Kalvlen teropottvtes.

12 Momen tvlofuce hvmken eceyof, este-hunvntake lepvvlke palet a vnrvipet awet hopvyen svpaklvtes;

13 Momet en haken kvwrpaket, Cesvs, Mvhaya, pum merrvs, makakvtes.

14 Momen hecakof, Vpehyet, plestvlken e hecicvkes, 
kicakvtes. Momen mome ocvtes, vpeyof, en hvsvthakvtes.

15 Momen hvmket, em wiken hecof, er afuliket, en hake yekcen Hesaketvmesen 'svrakknecvtes,

16 Momet e'le vnakvn $e$ turofvn oh lvtiket, vkvsamvtes; momet Semelevlket omvtes.

17 Momen Cesvs vyoposket kicvtes, Palet en hvsvthaket omekisa? momis ostvpakat ta?

18 Hesaketvmese vrakkuecvrane er afulecat heckvkekon, heyv tvleporv tvlket vrakkueces, kicvtes.

19 Momet, Akvwvpiket, vyepvs; cem vkvsvmkvt temvpoken ce hahyes, kicvtes.

20 Momen Falvsevlket Hesaketvmese em ohmekketv estofvn vlvkvranet omat em 'pohakof, em vyoposket, Hesaketvmese em ohmekketv heckv heret vlvkekot os;

21 Momen, Yvmvn hecvs! monkat, Vsvn hecvs! makvkekares, hecvkes, Hesaketvmese em ohmekketv ece 'pikvket omekv', kicakvtes.

22 Momet en kerrepvlken kicakvtes, Nettvt ocakvres, este E'chuswr en nettv hvmken hecetvn komatskis, hecatskekares.

23 Momen, Yvmvn hecvkes! monkat, Vsvn hecvkes! ce kicvkvres; oh vpehyatskvs, momet vcvkvpehyatskvs.

24 Vtoyehvtte sutv elecv a vtoyehattet etvn er ahvyvyices, mv etvpomen este E'chuswv tares, en nettv omof.

25 Momis heyv oreko munken nanvke sulken es estemerkvres, momet heyv este honvpse etvn vhepvkvkvres.

26 Momen Noe en nettv take tate etvpomvres, este E'chuswv en nettv take omof.

27 Hompaket, eskaket, etepayaket, etepvyepuecaket 
emunken Noe perro-cuko eceyvte nettv orvtes, momen uelauket vlahket, omvlkvn sumecicvtes.

28 Momen Lat en nettv take tate etvpome tares; hompaket, eskaket, nesaket, wiyaket, vhocaket, cukon hayakvtes;

29 Momis mv nettv Lat Satvmen ossvte etvwvn totkv momet tohottoplane hvlwen a osket omvlkvn sumecicvtes ;

$30 \mathrm{Mv}$ ome tares, este E'chuswv e heckuece nettv omof.

$31 \mathrm{Mv}$ nettv omof cuko onvpv oh liket, enake cuko ofv ocen omat, cvwrranet ahvtrpikats; momen matvpomen cvpofv arat fulikats.

32 Lat e hiwvn vkerricvkes.

33 Estimvt en hesaketvn hesayecetvn hopoyen omat, sumecicvres; momen estit en hesaketv sumecicen omat, hesayecvres.

34 Cem onayvkis, $\mathrm{Mv}$ neren hunvntake hokkolet topv hvmken ete'n wvkhoken, hvmken eset, hvmkan wihokvres.

.35 Hoktvke hokkolet tepvket emfolotvkvres; hvmken eset, hvmkan wihokvres. -

36 Hunvntake hokkolet cvpofv ofvn welakvres; hvmken eset, hvmkan wihokvres.

37 Momen em vyoposket, Estvmv tar haks?' Pucasē, kicakvtes. Momen, Estvmv ena ocen omat 'mvn lvmhet vtelokvres, kicakvtes.

\section{SETENTVCKE 18.}

Momen este estofis emekusapet hotosvkekaren opunvkv svhopakvn 'sem punvyakvtes;

2 Hrmmaket, Tvlofv hvmken fvececv hvmket, Hesaketvmesen em penkvlekot, este tis vrakkuece sekat likvtes. 
3 Momen mata tvlofvn oye hoktet arvtes; momet em vlaket, Vc vnrapvn vm ohfvececvs, makvtes.

4 Momen 'mapohicetvn komekat oketvt hoyanvtes; momis renyupvn em vkerrickv ofvt, Hesaketvmese en cv penkvlekot, este tis vrakkuecvkot o estomis,

5 Heyv oyen em ohfvececares, cr naoricekv, momvkon. omat, tewole vlakat $\mathrm{cv}$ hotosihce witet omekv, makvtes. 6 Momen Pucaset makvtes, Fvccecv fvcceko nake makan pohvkes.

7 Momen Hesaketvmeset, hofunen 'mehaket o estomis, enake enhopoke neren nettvn oh huehkakat em ohfvccecvkekare te?

8 Ce kicvkis, Lvpken em ohfvececvkvres. Mome estomais este E'chuswvt vlakof, vkvsvmkvt ekvnvn oh ocen eshecvre te?

9 Momen este, $\mathrm{Pu}$ fvecvket omēs, komet, eme ofvn vfekrakket, vpvltaken enhomecakat fullaten heyv opunvkv svhopakvn 'sem punvyakvtes;

10 Este hokkolet cuko-vcakan cukolakvtes, emekusvpvranet; hvmket Falvsevlket omen, hvmkat tokunawvvteloyvt omvtes.

11 Falvsevlket hueret, hvmmaket eme ofvt emekusapvtes, Hesaketvmese toyetskat, este vpvltake, fveceko vkonah vlke, fvccrkekat, hoktarvlke momen heyv tokunawv-vteloyv tis omvkat svrahkvn ece 'kvsamis.

12 Nettrcakuce hvmkat em ofv vhokkolvn e elvwecit, nake ocvyat vtekat tekvpvke espalan fekvyet os.

13 Momis tokunawv-vteloyv, hopvyen hueret, hvlwen vnvtvksetv taten komekot, e hokpen oh naf ket, Hesaketrmesē, naorkv toyvyat vm merrvs, makvtes.

14 Ce kicvkis, Hvmkat fvccekon, heyv hunvnwv mit frecet en cukon vk hrtapkvtes; estimvt e hvlwecat kvncvpuehocen, estit e kvncvpuecat hvlwehocvret ok'. 
15 Momen momof hopuetakucen celayvren 'sen yicakvtes ; momen en kerrepvlket hecakof, em vsehakvtes.

16 Momis Cesvs enhuehkakvtes, hvmmaket, Hopuetake vc oh vwepvre em etektvnecet, em vsehhatskvs; mv omakat Hesaketvmese em ohmekketvt omekv.

17 Mehenwvn ce kicvkis, Estimvt o estomet hopuewuce omet Hesaketvmese em ohmekketv e enakuecekon omat, estomet ecehyekos.

18 Momen semevpayv hvmket em 'pohet, Mvhayv heretskat, naken estomvyatet hesaketv yuksv-sekon epoyar haks? kicvtes.

19 Momen, Estoman here ev kicetske haks? herat hvmkusis sekos, Hesaketvmeset omekon omat, Cesvs kicvtes.

20 Vhakv palan kerretskes, Hoktahretskvs, Elehcetskvs, Horkohpetskvs, Fveceko akeriyetskvs, Ce'rke ce'ckeu vrakkuecvs, Cesvs kicvtes.

21 Momen, Heyv omvlkvn ev mvnettusvte vteke vfaste vtecicryvtes, makvtes.

22 Momen Cesvs, heyv nanvken pohof, Nake hvmket cem ahoske emunkes; nanvke ocetskat omvlkvn wiyet, estemerrakan ernvkvs, momet hvlwe ofv rake ocetskvres; momet ahtet, vc vevkvyvs, kicvtes.

23. Momen heyvn pohof, efekenokke hervtes; nakeoce mahet omvtet ok'.

24 Momen Cesvs, efekenokke mahat hecof, Mvtvwwvt nakeockv ocakat Hesaketvmese ohmekketv esceyetv en yekcvke tares!

25. Rvcce-kuphe esropottv e turwv ropottat en yekce mahekon, este enakocv mimvt Hesaketvmese em ohmekketv eceyetvt en yekcet ok', makvtes.

26. Momen pohakat, Monkv estimvt hesake taye haks? makakvtes. 
27 Momen, Nanvke este em momvkeko tayat Hesaketvmese em momvke tayes, makvtes.

28 Momof Petvt, Hecvs, nanvke omvlkvn wihket, ece 'cvkfullēs, makvtes.

29 Momen, Mehenwvn ce kicvkis, Estimvt Hesaketvmese em ohmekketv vrahkvn cuko, eckvlke, etecakkakvte, ehiwv hopuetake esyoman wihket,

30 Hiyome tan sulke erohvtvlvken, momet ekvnv vlvkvranat ofv hesaketv yuksv-sekon epoyvranekat sekot os, kicakvtes.

31 Momof pale hokkolohkakan evpahyet, Hecvks, Celuslvmen vpeyês, momen este E'chuswv opunvyece owalvlke coyakvte omvlkvt sohfvckvkvres;

32 Centilvlken em wihohken, mvt nakhayekot, enhomecken celayet, ohtofket,

33 Tepket, elecvkvret ok'; momen nettv svtutcenan hvtvm akvwvpkvres, kicakvtes.

34 Momen heyv nanvke hvmkusis kerrvkekates; heyv maketv em ehkvket omen, nanvke 'sem punahoyat kerrvkekates.

35 Momen mome ocvtes, Cvleko tvlofvn vwolihcof, este-hunvnwv turhecekat nak vpohet, nene afopken likvtes ;

36 Momet, este sulkat hoyvnecen pohof, Naken oka? maket vpohvtes.

37 Momen, Cesvs Nasvlvlket hoyanet os, kicakvtes.

38 Momen huehket, Cesvs, Tewe e'ppucē, vm merrvs, makvtes.

39 Momen homvn vpeyakat em vsehakvtes, cryayvkvren ; momis svhere mahen huehket, Vm merrvs, Tewe e'ppucē, makvtes.

40 Momen Cesvs a 'soh vwhoyvren maket huervtes; momet, vwolihcof, em 'pohet, 
41 Naken ce 'stomecaren kometske haks? makvtes. Momen, Pucasē, renakvt vn heckate! komis, makvtes.

42 Momen Cesvs, Renvyvs; cem vkvsvmkvt ce hesayeces, kicvtes.

43 Momen moman vpakusen renayet, Hesaketvmesen vkvsamet, vcrkayvtes; momen este omvlkvt, 'hecakof, Hesaketvmesen vkvsvmakvtes.

\section{SETENTVCKE 19.}

Momen Cesvs, Cvlekon ecehyet, tehoyanvtes.

2 Momen hecvs, este-hunvnwv Sakevs hocef ket, tokunawv vteloyvlke enhomahtvt, nakeocvt omet,

3 Estit o haks? komet, Cesvs hecetvn komvtes; momis este cekfat omecicen heceko tayvtes, kocoknuset omekv.

4 Momen homvn v letiket, hecvranet, sekvmin vcemkvtes; mvn Cesvs hoyrnvranekv.

5 Momen Cesvs mvn rorof, vnvtrkiset, hecvtes, momet em punayet, Sakevs, lvpecicet ahvtvpkvs, mucv-nettvn cen cukon vfekhonnvranvyat trlket omekv, kicvtes.

6 Momen lvpecicet ahvtapket, afvcket evpayvtes.

7 Momen hecakof, omvlkvt ecayet'setempunahoyaket, Este holwvyecv em oh hvyvtkvranet ayes, makakvtes.

8 Momen Sakevs hueret, Pucasen em punayet, Hecrs, Pucasē, nake ocvyat en nvrkvpvn estemerrakaten emvyet os; momet este estomen oh laksit, naken vkonabvyvten omat, osten ohfulhuecis, makvtes.

9 Momen Cesvs em punayet, Mucv-nettvn vhesaketvt heyv cukon vlvket os, emeu Eplehame e'ppucet omekv :

10 Este E'chuswvt mv sumkvten hopoyet, hesayecvranet vlvket omekv, makvtes.

11 Momet heyv nanrken pohakof, ohvtvlaken opunayet, punvkv svhopakvn opunayvtes, Celuslvmen vwolicekv, 
momen Hesaketvmese em ohmekketvt lvpken heckvres, komakat svrahkvn.

12 Monkv hvmmakvtes, Este vrakke hvmket ekvnv hopvyen ceyvtes, ohmekketvn en heciken, rvlvkvranet.

13 Momet em vtotkvlke palan enhohiket, tokunawv vtarkv palen emakvtes, Es vtotkatske emunken, rvlvkares, kicaket.

14 Momis em etvlofvlket, enhomecaket, punvkvn yupvn ohtotet, Heyv hunvnwv ep ohmekkvren komēkot os, makakvtes.

15 Momen mome ocvtes, ohmekketvt en heciken, rvlahkof, heyv vtotkvlke tokunawrn emakvte este vrahrvkv esyoposke estomuse ohvtvlvyakvte kerrvren, em enhuehhokvren makvtes.

16 Momof enhvteceskat vlahket, Pucasē, cem vtarkvt vtarkv palen ohvtvlayet os, makvtes.

17 Momen, Heres, vtotkv heretskat, nake nvcomuse ohfvecv herometskvtet omekv, tvlofv palen ohfvnket yekcetvn ocetskvres, kicvtes.

18 Momen svhokkolat vlahket, Pucasē, cem vtarkvt vtarkv cahkepen ohvitvlayet os, makvtes.

19 Momen matvpomen, Cemeu tvlofv cahkepen ohmekkvs, kicvtes.

20 Momen etvt vlahket, Pucasē, cen cr penkalat cem vtarkv sehvsvteckv es vyokofke ocvyvten hecvs;

21 Este-hunvnwv merretv ocekot, mv wvkecetskekate akvwapet, momet $\mathrm{mv}$ fokayetskekate vteloyetsket omekv.

22 Momen kicutes, Vtotkv holwryece toyetskat, ce cukwr mahusat aossen ec ohfvececares. Este-hunvnwv merretv ocvkat, mv wvkecvkate akvwapit, momet fokayvkate vteloyit omat kerretskemvts ;

23 Monkv rvlakvyof, crnake ohhontv ocen cawvye 
tetayaten, estoman vn cvtokunawv tokunawv esyoposketvcukon vpiketskekat haks?

24 Momen asvpáklaten kicakvtes, Vtarkvn em esvks, momet vtarkv pale ocan emvkes.

25 (Momen, Pucasē, vtarkv palen oces, kicakvtes.)

26 Ce kicvkvyet omekv, Mv ocat vtekat emhoyvres, momen mv ocekat, mv ocat tis em eshoyvres.

27 Momis vn horrvlke tat, ohmekkaren eyacvkekatet, yvmvn esyicet, cv homvn prsvtvks.

28 Momen hvmmahkof, Celuslvmen vcemket, homvn ayvtes.

29 Momen mome ocvtes, rvne Olefv kihocan, Perpec Pervne tepakat em vnakvn vlakof, en kerrepvlke hokkolen vtotet,

30 Tvlofuce ce homuse ocan vhoyvks, mvn cukolakusatskat corakko-wakuce, este ohlikvte sekan wvnvken eshecatskvres; enrecohpet, svthoyvks.

31 Momet, este estomet cem 'pohet, Estomēn enrecopatske haks? maken omat, heyvn kicvranatsket os; $\mathrm{Pu}$ caset eyacet omekv; kicakvtes.

32 Momen vtothoyat vhoyvtes, momet em onayat etvpomen eshecakvtes.

33 Momen, corakko-wakucen enrecopakof, e pucasvlket, Estoman corakko-wakucen enrecopatske haks? kicakvtes. 34 Momen, Pucaset eyaces, makakvtes.

35 Momet Cesvs es em vlahokvtes; momet em accvken corakko-wakucen ohocaket, Cesvn oh licakvtes.

36 Momen ayepof, em accvken nenen oh pvtapicakvtes.

37 Momen awolihcet, entis rvne Olefvn hvtvpkvranusof, en kerrepvlke sulkat omvlkvt vlicehcet, afvckaket punvkv en hake yekcen Hesaketvmesen es vkvsvmakvtes, vtotketv yekcvke hecakvte omvlkv vrahkvn; 
38 Mekko Hesaketrmese e hōcefkv ofv vlakat ohmerrvkvtet os; Hvlwe ofv herkvt, momet hvlwe mahaten vkvsvmkvt ocekvs, makaket.

39 Momen Falvsevlke este sulke vpvkakat vpvlwvt okakat, Mvhaya, cen kerrepvlken em vsehvkvs, kicakvtes.

40 Momen vyoposket, Ce kicvkis, heyvt cryayvken omat, moman vpakusen cvtot abuehkvkvranet os, kicakvtes.

41 Momet vwolihcof, tvlofvn hecet, oh hvkihkvtes,

42 Cen tis, ceme mahusat, mucv cen nettv ofv tis, nanvke cen herkv vpvkakat kerretskvten omat! momis hiyomat ce turwv em ohrvnket omes;

43 Nettv cem ocvkvret ok', mv omof cen horrvlket sufke ece 'folotken korrvkvres, momet cem afolotvkvres, momet estvmivlkis cem vkhottvkvres.

44 Momet cen hopuetake ece 'tehke ocat ece 'pakvken lekahfet, ce tvpeksicvkvres, momet ec ofv cvto hvmkusis etohliken wikvkekares, cem ohfvcceckv oketvn kerratskekatet omekv, maket.

45 Momet cuko-vcakan eceyet, $\mathrm{mv}$ ofv nake wiye, momen nesakaten sossicetvn vlicecvtes,

46 Coket os, Vn cuko mekusvpetv cuko tes; momis centake horkopvlke em vpoketvn hayatskes, kicet.

47 Momet nettv omvlkvn cuko-vcakat ofvn estemvhayvtes. Mamis plest en homvhhotat, cokv-hayvlke este en homvhhotvlke esyomat elecetvn komakvtes.

48 Momet nak estomecvke tayan eshecvkeko tayvtes; este omvlkvt heren em apohicakekv.

\section{SETENTVCKE 20.}

Momen mome ocvtes, mv nettv take hvmken cuko vcakat ofv estemvhayet, opunvkvhervn erkenakof, plest enhomvhhotat, cokv-hayvlke este em vculvke esyomat oh yicvtes, 
2 Momet, Pum onvyvs, yekcetv estomēn heyv nanvken es momecetske haks? monkat heyv yekcetv ce'mvtet estit o haks? em punayet makakrtes.

3 Momen vyoposket, Vneu nake hrmken cem 'pohvranvkis ; momen vm vyoposkvkes;

4 Cane em paptesvm, hvlwen vte tat haks? monkat este enake tat haks? kicakvtes.

5 Momen 'sete'm punahoyaket, Hvlwe vten, makēn omat, Moman estoman vkvsvmatskekat haks? makvres.

6 Momis, Este enakets, makēn omat, este omvlkvt cvton 'sepu 'fvllet $p u$ nvfkvkvres, Canet owalv tates, kometrn vkvsvmepuehocvtet omekv, makakvtes.

7 Momen vyoposkaket, Estvmvn vte tat kerrēkos, makakvtes.

8 Momen Cèsvs, Vneu yekcetv estomēn heyv nanvke es momecit omvyat cem onayvkvkos, kicakvtes.

9 Momof heyv opunvkv svhopakvn este 'sem punayvketvn vlicecvtes; Este-hunvnwv hvmket, pvrko cvpofvn ahohcet, vfastvlken em wikvtes, momet vhofunen ekvnv hopryen ayvtes.

10 Momet oketv omóf, vtotkvn vfastvlken ohtotakvtes, pvrko crpofv em etten emvkvren; momis vfastvlket, rukahfet, nakocekon vyecicakvtes.

11 Momen hvtvm vtotkv etvn vtotvtes; momen mvu rukahfet, vlesketv vpvken celahyet, nakocekon vyecicakvtes.

12 Momen hvtvm svtutcenat vtotvtes; momen mvu vnvttehcet, ossicakvtes.

13 Momof pvrko crpofv e pucaset okat, Naken estomvranvya? Crppuce vnokeckan vtotares; hecakof, mvtan vrakkuecake wites, makvtes.

14 Momis vfastvlket hecakof, 'sete'm punahoyaket, Heyvt pucase mvnettv tes; vhwet, elecrkeres, enake taranvtet punake hakvren, makakvtes. 
15 Monkv prrko crpofvn ossihcet, elecakvtes. Monkv pvrko crpofv e pucaset naken estomecvkvre te?

16 Vlahket, $\mathrm{mv}$ vfastvlken pvsvtvres, momet pvrko crpofvn vfastvlke etvn em wikvres. Momen pohakof, Mohrnates, makakvtes.

17 Momen heren hecaket, Heyv hoccat naket o haks? Cvto tohtarvlke vwikvte, matvwvt vkvnowv em ekv hakes, makat? makvtes.

$18 \mathrm{Mv}$ cvton estimvt oh latken omat, kvckvres; momis estimvn oh latken omat, lopotvres, makvtes.

19 Momen plest enhomvhhotat momen cokvhayvlke mata hvse-vkerkvn enke oh wvkecetvn komakvtes; emen opunvyecet, heyv opunvkv svhopakvn punayvtet on kerrakvtet onkv, momis este sulkat em penkvlakvtes.

20 Momet vketecaket, mvkerre vketecvlken vtotakvtes, este fvccrke e omecaket, em opunvkvn eset, momat semevpayv en yekcetv momen ohfvnketv ofv wikvkvren.

21 Momen Mvhaya, fvccrn opunayet, momet estemvhayet, estimv en heckv etemetuecekot, momis Hesaketvmese em vyetvn mehenwrn mvhayetsket omat kerrēs;

22 Sesv ekv-feketv emeyvre fvccut o haks? monko haks? maket em 'pohakvtes.

23 Momis emvkerretvn hecet, kicakvtes, Estoman es cv kerratske haks?

24 Tenelyvn cv hecicvkes. Estimv vhake momet en hoccvn oce haks? $E m$ vyoposket, Sesv enakets, makakvtes.

25 Momen, Monkv Sesv enanvken Sesvn emvks, momet Hesaketvmese enanvken Hesaketvmesen emvkes, kicakvtes.

26 Momen este sulkat ehomvn em opunvkvn esvkeko tayvtes, momet em vyoposkat em estomahen, cryayvhokvtes. 
27 Momof Satusevlke, (mv Akvwrpketv sekos, makakat,) vnvcomet en yicvtes, momet em 'pohakvtes,

28 Makaket, Mvhaya, Moses pun hoccicat, Hunvnwv etecakkvtet, ehiwrn ocet, hopuetaken ocekot elen omat, etecakkatet e hiwvn epayet, honvpse etecakkvten em mahecrres, makvtes.

29 Monkv etecakkakat kolvpaket fullvtes: momen, homahtatet hokten epahyet, hopuetaken ocekot elvtes.

30 Momen svhokkolat mata hokten epahyet, hopuetaken ocekot elvtes.

31 Momen svtutcenat epayvtes; momen kolvpakat matvpomen; momet hopuetaken ocvkekot prsatkvtes. 32 Omvlkv 'sem poken hokteu elvtes.

33 Monkv akvwvpketv omof, mv kolvpakan estomat e hiwv tar haks? kolvpakat epayakvtet omekv.

34 Momen Cesvs em vyoposket kicakvtes, Yvmv ekvnv hopuetake etepayaket, etepayepuehocet os:

35 Momis mimv ekvnv, momet pvsatkvte akvwvpketv epoyetv heretv orieakes, komhoyat etepayvkekos, momet etepayepuehocekos :

36 Momen hvtvm svnvcomvn prsvtkeko tayes, hvlwe este in hueretv oricet ok'; momen akvwvpketv hopuetaket omet, Hesaketvmese en hopuetaket omakes.

37 Tokvs, prsatkvtet akvwvphoyat Moses tisem etofvske ocan kerkuecvtet os, Pucasen oket, Eplehame en Hesaketvmese, Iseke en Hesaketvmese, momet Cekvpe en Hesaketvmese makatet.

38 Pvsatkat tokon, momis wenahokat en Hesaketvmeset omekv : momet omvlkvt ehomvn wenahoket omekv. 39 Momof cokv-hayvlke vpvlwvt vyoposket, Mvhaya, heren opunahyetskes, makakvtes.

40 Momen mv renyupv ohvtrlakat nak em 'pohetvn fekhvmkvkekates. 
41 Momen em punayet, Klist Tewe e'ppuce tes, estomen makake te?

42 Momen Tewe mahusat, Sams cokv ofvn, Pucaset ov Pucasen em punayet, Ceme vm akvpervn liketske munken,

43 Cen horrvlke ce 'leohhuerickvn hayares, makvtes.

44 Monkv Tewet Pucasen kicet os, momof estomaten eppucet o haks? kicakvtes.

45 Momof, este omvlkvt pohaken, en kerrepvlken em punayet,

46 Cokv-hayvlken em e vketecvkes, mv accvke cvpcrken es fulletvn komakat, momet nakwikv vlkan vseketvn, momet senekake ofv ohliketv svmomvkus mahat, momet hompetv-rakkon etektrnke svmomvkus mahat em vlostakat;

47 Hoktvke oyevlke en cuko lokakat, momet vhayet crpcrken mekusvpakat; matat ohfvececkv senrakkat en heckvkvres.

\section{SETENTVCKE 21.}

Momet vfvnnaket, este nakocvlke en nakemkv cvtokunaphuten vtehaken hecvtes.

2 Momet hvtrm oye hokte hvmke tokunawv lopuckus mahat hokkolen mvn vtehen hecrtes.

3 Momet, Mehenwvn ce kicvkis, Heyv oye estemerrat omvlkv senhoyvnen vtehet os;

4 Heyv omvlkvt enaketake sulkat asossen nake Hesaketvmese emhoyat vpvyahket omekv; momis emet em estemerketv aossen vhesakvte ocvte omvlkvn vtēhet os, makvtes.

5 Momen vpvlwvt cuko vcakat, $\mathrm{mv}$ cvto hervke momet nakemkv es nehihocat opunvyecakof, emet okatet,

6 Heyv nanvke hecatskat ton omat, nettrt ocv- 
kvres, mv omof cvto etohlike hvmkusis awihokekat sekares, makvtes.

7 Momen em 'pohaket, Momis, Mvhaya, estofvn heyv nanvke ocakvr haks? momen heyv nanvke momvranof, naket eskerkv tar haks? kicakvtes.

8 Momen makvtes, E vketecvkes, ce mvkerhoyvkekaren; Klist toyis, maket sulket $\mathrm{cv}$ hocefkv ofvn yicvret ok', momen oketvt vwolices : monkv mvn vcrkvpehyatskvs.

9 Momis horre momen este tekyvmketv ocakvcuken pohatskof, penkvlahkatskvs; heyv nanvket tatet hoyvnvkvranet omekv: momis meyuksvt moman vpakuse tokot os.

10 Momof kicakvtes, Tvlwvt tvlwvn vnrvpet, momen tvlofvt tvlofvn vnrvpet akvwvpkvkvres :

11 Momen ekvnv nekekv rakrvket estomis ocvkvres, momen elaukot ocvkvres, momen enokketv holwvhokat; momen nakheckv senhoneckvket, momen nake eskerkv rakrvket sutv a vwet ocvkvres.

12 Momis heyv omvlkv enhomvn enken ec oh ocaket, ece'ssecvkvres,' momet senekake vlkan, momet cuko-yekcvn ce wikaket, ev hocef $\mathrm{kv}$ vrahkvn mekkvlke momet evpayvlke ehomvn es ce yihocvkvres.

13 Momen mvt a cen kerre tayat ec oh fulkvkvres.

14 Monkv ce feke take ofvn ivccecvkes, naken est'em vyoposkvranatskat enhomvn vkerricatskekaret.

15 Ecukwv momet hoporrenkv, mv ce'nrapvlke omvlkvt pvlken vyoposketv, monkat vnrvpetv umvkeko tayaten ce'mvkaret ok'.

16 Momen ce'ckvlket, tecakkakat tis, cen nakvlke momet cen hessvlket ce wiyvkvres; momen ec omecicaken ce prsvthoyvranvkat ce sasvket os.

17 Momen cv hocef kv vrahkvn este omvlkvt cen homecrkvres. 
18 Momis ce'kv esse take hvmkis sumkekares.

19 Cem momis kometvn cem puyvfekcr taken es vevyecrkes.

20 Momen suletawvlke etohkvlke sulket Celuslvme em afolotaken hecatskof, momusen em vhopvnketvt vwolican kerrvkes.

21 Momof Cutev vpokat ekvnhvlhawan oh pefatkekvs, momen en nvrkvpv vpokat sossvkekvs, momen ekviv vwolicvke vpokakat escehyates.

22 Heyvt estemerrickv nettvt omakekv, nake hoccakat omvlkvt esfrcfvkvren.

23 Momis nvrkesakat momet coyicakat en holwakes! mv nettv take omof; heyv ekvnvn estemerkv rakket, momen crpvkketvt heyv estrlken oh ocvkvret ok'.

24 Momen eslafkv-crpko em fvske omecicen pvlvtkvres, momet svlvfkvket etvlwv omvlkvn escehoyvres, momen Centilvlket Celuslvmen oh svpvklvres, Centilvlke em oketv esfvcfukvranat oren.

25 Momen hvse, hvresse kococumpv esyomat ofv eskerkv ocaken; ekvnv onvpv tvlwvvlke estemerkv feksumketv vpvket ocvres, uehvtkv uemelohlv tepakat wohkaken;

26 Penkvlkvt momet nanvke ekvnvn oh ocvranat en nene hecakat omecicen este prsvtkv omake haket omen; hvlwe en yekcetv take nekeyihocvret ok'.

27 Momen momof este Echuswvt, yekcetv momet hryayvke rakken ocet, vholoce ofvn vlaken hecvkvres. 28 Momen heyv nanvke mometvn vlicecakof, acvmekset vfvnnakvkvtcvs; cem vhesaketvt vwolicet omekv.

29 Momen opunvkv svhopakvn 'sem punayakvtes; Kerakko em vpe, momen eto omvlkvn hehcrkes;

30 Hvte esse hayakof, ceme mahusat meske mucv vwolicat es kerratskes. 
31 Matvpomen cemet, heyv nanvke momaken hecatskof, Hesaketrmese em ohmekketv vwolicat kerrvkvtcrs. s2 Mehenwrn ce kicvkis, heyv honvpse hoyvneko munken heyv nanvke omvlkvt esfvcike tvlkvres.

33 Sutv ekvnv tepakat sumhokvres; momis vm opunvkv sumikekos.

34 Momen e vketecrkes, estofv tis hompetv vnoksetv, momen hackv, momen heyv hesaketv senaorickv svhere mahen ce feke taken oh wetenken, mv nettvt vyvkhvmkusen ec oh vlvkekaren.

35 Nak senkvnhetv omet ekvnv omvlkv onvpv oh vpokan oh vlvkvret ok'.

36 Monkv vketecrkes, momet estofis emekusa pvkvtcrs, heyv nanvke momvranat omvlkv mvnvttepetv, momet este E'chuswv ehomvn svpvkle tayat heretv oricvkēs, ce komhoyvkvren.

37 Momen nettvn cuko vcakat ofvn estemvhayvtes, momet neren osiyet, rvne Olefv kicetvn likvtes.

38 Momen cuko vcakat ofvn em apohicvranet este omvlkvt hvthvyvtkusen en yicvtes.

\section{SETENTVCKE 22.}

Torvs, tvklike pakkeko hompetv-rakkot vwolicvtes, mv Ohhoyvnkv kihocat.

2 Momen plest enhomvhhotat momen cokv-hayvlket estomen elecvke tayat vhopoyakvtes: este sulkan em penkvlvket omekv.

3 Momof Setvnet, Cutvse Eskalev kicetv, vhonkvtkv pale hokkolohkakat hvmken eceyvtes.

4 Momen ayet, plest enhomvhhotan momen kvpetvnvlken estomēn Cesvs em wiye tayat em opunvyecvtes.

5 Momen afvckvket, cvtokunawv emetvn temfvccakvtes. 
6 Momen temfaccvtes, momet este sulkat fullekon em wiyetv etektvnke hopoyvtes.

7 Momof tvklike pakkeko nettvt, ohhoyvnkv elehocvranat, vlakvtes.

8 Momen, Vhohyet, ohhoyvnkvn pum etetakuecvks, hompeyvren, kicet, Petv Cane tepakan vtotvtes.

9 Momen, Estvmvn etetakueceyvren kometske haks? kicakvtes.

10 Momen, Hecvkes, tvlofvn cukolakatskof, estet, pecv uewv vcrnken es hvlatet, ece'nrvpvres, mvn vcvkvhoyet, cuko eceyaten cukolvkvks.

11 Momet, Mvhayvt okat, Cukopericv en nvthofv, vn kerrepvlke vpaket ohhoyvnkv hompvye tayat estvmvn oce haks? ce kicet os, cuko-pucasen kicatskvres.

12 Momen onvpv nvthofv rakke etetakuehocen cem mellvkvres; mvn etetakuecvks, kicakvtes.

13 Momen vhohyet, kicakvte etvpomen eshecakvtes; momet ohhoyvnkvn etetakuecakvtes.

14 Momen hrse-vkerkv vlahkof, vpastelvlke palen hokkolohkakat vpvkaken, likvtes.

15 Momet, $\mathrm{Cv}$ yacet, cv 'stemerkeko emunket, ece 'pakvket heyv ohhoyvnkv hompetvn komvyvtet os.'

16 Ce kicvkvyet omekv, Hvtvm hompvko emunken Hesaketvmese em etvlofv ofvn esfvcike tvlkvres, kicakvtes.

17 Momet sesketucen 'sehset, Hesaketvmesen vkvsamvtes, momet, Heyvn 'sehset, etekvpicvkes;

18 Ce kicvkvyet omekv, Pvrko en lokce eskvko emunken, Hesaketvmese em ohmekketv vlahke tvlkvres, kicakvtes.

19 Momet tvkliken ehset, Erken vkvsamet, kvlkahyet, e'makvtes, Heyvt cv'na ece 'rahkvn este'mhoyvtet omes; vnen es vc vkerrickvn heyvn momecvkes, maket. 
20 Matvpomen hompetv hoyanat sesketucen sehset, Heyv sesketuce cr catv setemfvccetv mucvset omes, mv ece 'rahkv pvlatkat;

21 Momis hecvkes, $\mathrm{mv} \mathrm{cv}$ wiyat enke ve vpaket ohbompetvn oh ocet os;

22 Momen mehenwvn este E'chuswv em mellvkvte omen ayvranet omes, momis en holwakes, mv este wiye huerat! makvtes.

23 Momen vpvkakat estvt heyvn momecvranet omat ete'm 'pohetvn vlicecakvtes.

24 Momen momof .vnrvpkv ete'm ocakvtes, estit rakke mahat os, komhoyvranat ohfvecrn.

25 Momen, Centilvlke em mekkvlke ohfvnkaket os; momen yekcetvn es ohfvnkakat est' en heromakan kihocet os.

26 Momis cemet $\mathrm{mv}$ ohmatskvs; momis ece 'pakvke rakke mahat esmvnettat omet omekvs; momen ece 'pakvke homahtat est' em vtotkat omet omekvs.

27 Momen hompetv vlikat, monkat mv vfastat, estvt senrakket o haks? hompetv vlikat $\mathrm{mv}$ toko? momis vn tat vtotkv omit ece 'pakvkit omis.

28 Vc vpaket ve vnrvpkv ofvn fekhonnakvte toyatskes. 29 Momen Cv'rket ohmekketv vm mellvte etvpomen ohmekketvn cem mellvkvyet os;

$30 \mathrm{Vm}$ ohmekketv ofvn vm ohhompetvn vh vpoket, hompet eskatskvren, momet mekkvlke em ohliketvn oh vpoket Eslevl em vliketv pale hokkolohkakan ohfvececatskvren.

31 Momen Pucaset okat, Simvn, Simvn, hecrs, teleko etvpomen ce'nyoyrret Setvnet ce yacrtet os. 32 Momis cem emekusapvyvtes, cem vkvsvmkvt sumkekaren; momen rafuliketskof, etecakketskan yekcrkuecrs, kicvtes. 
33 Momen, Pucasē, ece 'crkvyetv vm etetakes, cukoyekev momet elkv vteken, kicvtes.

34 Momen, Ce kicis, Petv toyetskat, mucv-nettv tottolose en hunvnwv hakeko munken cv kerretskat ehelaksecetske svtutcenvres, kicvtes.

35 Momet, Cvtokunawv en sukcv, ehvlewv en sukcv momet estelepikv tis ocekon ece 'totakvyof, naket cen sepekvt haks? kicakvtes. Momen, Naket sepekates, makakvtes.

36 Momof, Momis hiyomat crtokunawv en sukev ocat sayekvs, momen ehvlewv en sukev matvpomen; momen estit eslafkv-crpkon ocekat, en kapvn wiyet, nesekvs.

37 Ce kicvkvyet omekv, Nake vc ohfvecv hoccat sohfvckvranat tvlkuset os, Vhakv kacvlken vpaket vhonkvtkvtes, makat; nake ve ohfvecv ocakat emeyuksvn ocvkvranet omekv, kicakvtes.

38 Momen, Pucasē, eslafkv-cvpko hokkolet yvmvn ocakes, kicakvtes. Momen, Tetayes, kicakvtes.

39 Momet osiyet, Olefv rvnen oh ayvtes, momvnto omet; momen en kerrepvlkeu vcrkvpeyvtes.

40 Momen mvn rohrof, Mekusvpvks, momat holwake 'sec oh ceyvkekaret, kicakvtes.

41 Momet crto awiketv hvmke en hopvyen em akueket omvtes, momet tokkehket, emekușapvtes,

42 Crrkē, kometsken omat, heyv sesketucen es vm vkueyicvs; mome estomis vne komvyate tokot, ceme kometskatet momekvs, maket.

43 Momen hvlwe este hvlwen a hvtapket em e heckuecet, yekcic vtes.

44 Momen em estemerkv rakket omen, ohvtvlaken vyekcet emekusapvtes; momen' em meskat catv cvfke rakrvke ekvnvn tvk pvlatke omet omvtes. 
45 Momen mekusapvtet ahuyiret, en kerrepvlken em vlahkof, feknokketv omecicen nocicaken hecvtes,

46 Momet, Estoman nocicatske halss? asvprkilet, emekusvpvks, holwake 'sec oh ceyvkekaret, kicakvtes.

47 Momen opunaye munkof, hecrs, este sulke mahet, momen mv Cutvs kihocat, pale hokkolohkakat hvmket, homvn ayet, Cesvis vfvnkvranet vwolicvtes.

48 Momis Cesvs okat, Cutvsē, este E'chuswv vfvnketvn es wiyetske te? kicrtes.

$49 \mathrm{Em}$ afolotakat nake ocvranat hecakof, Pucasē, eslafkvcrpkon es caskeyvr haks? kicakvtes.

50 Momen mv hvmkat plest enhomahtv em vtotkvn casket, e hvcko akvpervn en tacrtes.

51 Momen Cesvs vyoposket, Mucv vteken etektvnecvks, makvtes. Momen e hrckon en celayet, em wicecrtes.

52 Momof Cesvs, plest enhomvhhotvlke, cuko-vcakat en kvpetvnvlke vculvke esyome en yicakat, Este horkopvn vnrvpe omet eslafkv-cvpko etowarke tis cawet yicatske haks?

53 Nettv omvlkvn ece 'pakvkit cuko-vcakan arvyof, vc vnrvpet ce'nke taken rvtosecatskekates; momis heyv hvse-vkerkv cenaket's, momet yomucke en yekcetv tes, kicakvtes.

54 Momof esahket, evpayet, plest enhomahtv en cukon esceyakvtes. Momen Petvt hopvyen a vcrkarvtes.

55 Momen cuko en nvrkvpv etektvnkat nvrkvpvn totkvn etecahket, tohkvlket vpokof, Petvt vpaket likvtes.

56 Momis, totkv tempen likof, hokte mvnette hvmket hericet hecet, Momen heyv hunvnwvt vpakvtes, makvtes.

57 Momen Cesvs ohfvccv ehelaksecrtes, Hokte toyetskat, mv esten kerrvkos, maket.

58 Momen $\mathrm{mv}$ hoyanat hofunekon etit hecvtes, momet, 
Cemeu mv hvmkat toyetskes, kicvtes. Momen Petvt, Mv toyvks, kicvtes.

59 Momen $\mathrm{mv}$ hoyanat hvse-vkerkv hvmkvteken, etit yekcen kerkuecvtes, Mehenwvn heyv esteu vpakrtes; Kalvlevlket omekv, maket.

60 Momen Petvt, Este-hunvnwv toyetskat, nak maketskat kerrvkos, makvtes. Momen moman vpakusen punaye emunkof tottolose en hunvnwv hakvtes.

61 Momen Pucaset a oh fulotiket, Petvn hecvtes. Momen Petvt Pucase em opunvkvn vkerricvtes, mv, Tottolose en hunvnwv hakeko munken, ve ohfvecv ehelaksecetske svtutcenvres, kicvte.

62 Momet Petvt osiyet, tayen hvkihkvtes.

63 Momen hunvntake Cesvs hvlvtakat, nakhayekot, nvfkakvtes.

64 Momet e turwvn 'mvrvnayahkof, e turofvn en nafket, Owalvs, ce nvfikat estit o haks? maket, em 'pohakvtes.

65 Momet nanvke etv sulken tvklecaket vnrvpet punayakvtes.

66 Momen hvyatkan vpakusen este em rculvke, plest enhomvhhotat, momet cokv-hayvlke etohkvliket en nvkvftetvn ceyicakvtes,

$67 \mathrm{Mv}$ Klist tat toyetska? pum onvyvs, makaket. Momen, Cem onayvkvyis, vkvsahmatskekos, kicakvtes.

68 Momen vneu cem 'pohin omat, vm vyoposket, monkat $v c$ vyecihcatskekos.

69 Heyv ertopvrv este Echuswvt Hesaketvmese en yekcetv em akvpervn likvres, kicakvtes.

70 Momof omvlikvt, Monkv cemet Hesaketvmese Eppuce toyetske haks? makakvtes. Momen, $M v t$ omes, cv kicatskes, kicakvtes.

71 Momen, Sohkerkv ohvtvlvken estomen pu yace haks? e cukwv aossen pohhēkv, makakvtes. 


\section{SETENTVCKE 23.}

Momen mv este sulkat omvlkvt asvpvkilet, Cesvs evpayet, Pilvten 'sem vpeyvtes.

2 Momen vlicecet vnrvpakvtes, Heyv estet etvlwvn fvyecicet, ekv-feketv Sesvn emetvn vsehen esheceyvnks, Vne mahusat Klist, Mekko, toyis, maken.

3 Momen Pilvtet em 'pohet, Ceme Cusvlke em Mekko toyetske haks? kicvtes. Momen, Maketskes, kicet, em vyoposkvtes.

4. Momof Pilvtet, Heyv hunvnwv nake em mvttat eshecrkos, plest enhomvhhotvlken momet este sulkan kicrtes.

5 Momen svhere mahen ohyekcvke tates, Este eteyamet os, Kalvlen vlicecet momet yvmvn oret, Cutev hvmecicat mvhayet os, makaket.

6 Pilvtet Kalvle ohfvccun pohof; mv este Kalvlevlket omat vpohvtes.

7 Momet Helot em ohmekketv elecvn hueret omat kerran vpakusen Helon ohtotvtes, Helot mv omof Celuslvmen aret omen.

8 Momen Helot Cesvs hecof, afvcke estrmahet omvtes, hofuren hecetvn eyacrtet omekv; momet nake eskerkv momecen hecetvn komvtes.

9 Momof opunvkv sulken em 'pohvtes; momis.naken em ryoposkekates.

10 Momen plest enhomvhhotvlke momet cokv-hayvlke svpaklet tetayen vnrvpakvtes.

11 Momen Helot en suletawvlken vpaket nakhayekot vpelicakvtes, momet kapv hololoccen accecet, hvtvm Pilvten ohtotakvtes.

12 Momen mata nettvn Helot Pilvte tepakat tenhessvlke hahoyet omvtes; mv enhomv enhomeckv te'm ocakvtet omekv. 
13 Momen Pilvtet, plest enhomvhhotvlken, momen semevpayvlken momen este sulkan etoh nvkvftecahkof, 14 Este fiyvthuecr omecatsket, heyv hunvnwvn es vn yicatsket os ; momen hecrs, ce homvn em 'pohhvyat, $\mathrm{mv}$ nanvke es vnrapatske ocat heyv hunvnwv ern mvttat eshecvkis ;

15 Momet os ; momen Helot eshece sekis; centaken ohtotvyisekv, momen nake elkv oren em momehoce sekos; 16 Monkv rukahfit, enrecopares, kicakvtes.

17 (Hompetv-rakko omof hvmken em enrecopvranat tvlkuset omvtet omekv.)

18 Momen etemvprlwusen huehkaket, Vkueyvs heyv esten, momet Palapvsen pum enrecopvs, makakvtes.

$19(\mathrm{Mv}$, este tekyvmketv tvlofv ofv hakvten, momet esteleckv omecicen cuko-yekcvn pihokvtet omat.)

20 Pilvtet, monkv, Cesvs enrecopetvn komet, hvtvm em punayakvtes.

21 Momis huehkaket, Etohwelepkvn svrvhvs; etohwelepkvn svrvhvs, makakvtes.

22 Momen svtutcenvn em punayet, Estoman? holwvke naken momece haks? èlē taye em muttat eshecvkis; monkv rukahfit, enrecopares, makvtes.

23 Momen fekhonnekot en hake yekcrken 'sopunayet, tohweklepkvn svrahkvren inakakvtes; momen mv em opunvkv, plest enhomvhhotat 'mopunvkv esyomat muntalv tes. 24. Momen Pilvtet, makakat vevkvyen momvres, maket ohfvececrtes.

25 Momen mv este tekyvmkat momet esteleckv omecicen cuko-yekcvn pihokvte mv en komakvte em enrecopvtes; momis komakan Cesvn em wikvtes.

26 Momen es vpeyof, Silenvlke tvlofv en lvpvtke aosse, Simvn hvmken hvlahtet, etohwelepkvn oh wrkecakvtes, karonet Cesvs 'svevkayvren. 
27 Momen este etohkvlkat sulke mahet, hoktvkeu vcrkvpeyvtes, mvt en wahoket hvkihhokvtes.

28 Momis Cesvs oh fulotket, Celuslvme echuswrhoktvke toyatskat, vne $v c$ vrahkvn hvkihkekot, momis ceme momet ce'chustake vrahkvn hvkihkvkes, makvtes.

29 Nettvt yicvranet omekv, mv omof, Echussvkekat momet nvrke hopuewv heckuecvkvte sekat, momet epese coyicvkekatet en herakes, mahokvres.

30 Momof vlicecet, ekvnhvlhawan, Ep oh pvlvtkvks; momet rvnen, Ep ohrvnvks, kicvkvres.

31 Momen eto lanofv heyv nanvken momecaken omat, eto talofv naken momecvkhoyvr haks? kicakvtes.

32 Momen etv, holwvyecvlke hokkolen, pvsvtvranat vpvken svpehoyvtes.

33 Momen ekvnv Kalfvle kihocan yicof, mvn svrvhakvtes, momet holwvyecvlkeu; hvmket akvpervn svrahken, hvmkat vkvskvnvn svrahken.

34 Momof Cesvs, Crrkē, em merrvkvs; naken estomakat kerrvkekot omekv, makvtes. Momen em accrke tekvpicaket, estit omvranat eshecakvtes.

35 Momen este sulkat hecet svpaklvtes. Momen semevpayvlkeu vpvkaket nakhayvkekates, Vpvlwvn hesahuecvnkes: e hesayecekvs, Klist, Hesaketvmese enhopoyvtet, on omat.

36 Momen suletawvlken, en yicet, uetuksen 'sem wiyet, nakhayvkekates,

37 Momet, Cusvlke em Mekko toyetsken omat, e hesayecrs, maket.

38 Momen es hocefetv hoccat onvpvn ocat, Klekvlke, Lomvlke Hepluvlke esyomat em punvkv ofvn hoccet omvtes, HEYVT CUSVLKE EM MEKKO TES, maket.

39 Momen holwvyecv, vtvrtakat hvmket, Klist toyetsken omat, e hesayecet pumeu $p u$ hesahuecrs, maket trhikvtes. 
40 Momis hvmkat vyoposket, Hesaketvmesen en ce penkvleko haks? mata ohfvececkv ofvn hueretsket omat, maket em vsehvtes.

41 Momet, Momen pume frccrn ep ohfvccehoces, pum vtotketv vevkvyen feketv esohfvcken pun hecket omekv; momis heyv hunvnwvt nake em mvtte sekos, maket.

42 Momet, Pucasē, cem ohmekketvn eceyetskof, vc vkerricvtcvs, Cesvn kicvtes.

43 Momen Cesvs, Mehenwrn ce kicis, mucv-nettrn palvtis ofvn ve vpaketskvres, kicvtes.

44 Momen hvse-vkerkv sepakvteket omvtes; momen hvse-vkerkv esostvpakat oren yomucket ekvnvn hvmecicrtes.

45 Momen hvse yomuckvtes, momen cuko-vcakat setentrckv tesetkvtes.

46 Momen Cesvs opunvkv yekcen es hohikof, Cv'rkē, ce'nke ofvn vm puyvfekcvn wikis, makvtes. Momet heyvn mahkof, hesakvte wikvtes.

47 Momen sentulevt nak momehocan hecof, Hesaketrmesen vrakkuecrtes, Mehenwvn heyvt hunvnwv fvccet omvtes, maket.

48 Momen este mv hecetv vnvkaftat omvlkvt, nanvke momehocan hecakof, e hokpen oh nafket, fulecrtes.

49 Momen em etekerhoyetv omvlkvt, momen hoktvke Kalvle asosset vcrkvpeyvtet, hopvyen a svpaklet, heyv nanvken hecakvtes.

50 Momen hecrs, hunvnwv semevpayv, Cose hocefke tates; hunvnwv here momet fvccet:

51 (Mvt em vkerrickv take- momen momecakat em vkvsvmekates.) Cusvlke em etvlofv Alemvrev avtet omvtes, emeu Hesaketvmese em ohmekketvn emehaket. 52 Heyvt Pilvtet oh ayet, Cesvs e'nan em 'pohepvtes. 53 Momet a ehset, hvnvwelv tvrken es ayokkohfet, 
OPUNVKV-HERV LUK COYVTE XXIV. 95

sepvlkv, crto a kofkvten, este wvkechoyvte sekat ofvn wrkecrtes.

54 Momen mv nettvt etetakueckvt omvtes, momen nettrcako vwolicvtes.

55 Momen mv hoktvkeu vpaket Kalvlen asossvtet vcrkvpeyet, sepvlkvn, momen estomen ena wrkechoyaten hecakvtes.

56 Momet fulehcet, neha-fvmecv momen esfvmecickvn etetakuehcet, nettvcakon fekvpakvtes, vhakv vcrkvyen.

\section{SETENTVCKE 24.}

Momeт nettvcakuce nettv enhvteceskvn hvthvyvtkusen, emet, momen hoktvke eti vpvkaken, esfvmecickv etetakuecvkvten sepvlkvn esyicvtes.

2 Momet crto sepvlkv em vkueyihocen hecakvtes.

3 Momet esceyet, Pucase Cesvs e'nan hecrkekates.

4 Momen mome ocvtes, mv ohfvecv en kerkvkeko emunkof, hecrs, hunvntake hokkolet, em accvke hvyayvhoken, etempen sehokvtes.

5 Momet hoktvke penkvlaket, potopohokof, Estoman pvsvtkat em etenrvwvn wenakat hopoyatska? kicakvtes.

6 Yvmv sekos; momis akvwvpkēs. Kalvlen are emunke cem punayvkvten vkerricvks,

7 Este E'chuswv, este holwvyecakat e'nke ofvn wihohken, svrahkvres; momen nettv svtutcenan akvwvpkvres, maket.

8 Momen em opunvkvn vkerricakvtes,

9 Momet sepvlkv ocan rafulehcet, heyv nanvke omvlkvn palen hvmkontvlakan momet vpvltake omvlkvn em onayakvtes.

$10 \mathrm{Mr}$ heyv nanvke vpastelvlken em onayakat Mele Maktelv, Coanv, Mele Cems e'cke esyomat, momen hok. tvke eti vprkvkè tates. 
11 Momen em opunvkvt nanvkoketv ome 'sem momakvtes, momet vkvsvmvkekates.

12 Momof Petvt ahuyiret, sepvlkvn oh letkvtes, momet oh cunehket, hvnvwelv tvrke accvke entvlket kaken hecrtes, momet, nak momehocat em estomahet omen, enkvpakvtes.

13 Momen hecvs, hokkolet mv nettrn tvlofuce Emevs hocefken, Celuslvme em vhopvye vkerkv kolvpakvteket omen, vhoyvtes.

14 Momet heyv nanvke ocakvte omvlkvn 'sete'm punahoyvtes.

15 Momen mome ocvtes, ete'm punahoyet ete'm 'pohakof, Cesvs mahusat vlaket, vpahket ayvtes.

16 Momis en renakv mvnvttehocvtes, kerrvkekaren.

17 Momen Cesvs, Yvkvpake, heyv opunvkv es ete'm punahoyatskat estomvket o haks? ce feknokhoket omat, kicakvtes.

18 Momen hvmket, Kleopvs hocefket, vyoposket, Celuslvme ofv cen tvlkuset tvleporvt omet, heyv nanvke heyv nettv take ofvn momehocat kerretskeko haks? kicrtes.

19 Momen, Nanvket o haks? kicakvtes. Momen em vyoposket, Nanvke Cesvs Nasvlvlke, owalv Hesaketvmese momet este omvlkv ehomv opunvkv nake momecetv tepakat ofvn yekcet omvte ohfvecrn:

20 Momen plest enhomvhhotat momet pum evpayvlket elkvn ohfvecehocvranen wikahken, svrvhakvte:

21 Momis mvt Eslevl enrecopvranvte tes, komet enhonrēmvts, momen heyv nanvke ornvlkv ohvpakat, heyv nanvke momehohcvte vtekat mucvt nettr sututcenat tes;

22 Momen ohvtvlakat hoktvke pum etohkvlketv sasat pu feksumhuecakis, sepvlkv hrten fuliyet.

23 Momet e'nan hecvkekot, eryicet, Nak hecihocan, 
hvlwe estrlket, Wenaket omes, makaken heceyis, makaket :

24 Momen epu 'pvkakat vpvlwvt sepvlkvn vpeyet, hoktvke kicakvte etvpomen hecakis: momis emen hecvkekis, kicakvtes.

25 Momen, Aeha ce hoporrenvkeko, momet nak omvlkv owalvlke opunayakvte vkvsvmetv efeke yulohusatskat!

26 Klist, heyv nanvken es estemeriket, em vrakketv eceyvre fvecvt omekat haks?

27 Momen Moses owalvlke omvlkv esyoman es vlicehcet, hoccat omvlkv ofvn mv nanvke eme ohfvecv ocakan em ohhvyayicvtes.

28 Momen mv tvlofuce vpeyvte 'rvwolicakvtes, momen ohvtvlake ayvrane omecvtes.

29 Momen ohyekcicet, Epu 'paket fekhonnvs, yaf ket omen, nettvt hoyvnvranuset omekv, makakvtes. Momen vpaket fekhonnvranet eceyvtes.

30 Momen mome ocvtes, vpaket hompetrn vlikof, tvkliken ehset, ohmeriyet, kṿlkayvtes, momet emakvtes.

31 Momen e turwvt enrvmrakvtes, momen kerrakvtes : momen heckeko hakvtes.

32 Momen ete'm punahoyet, Hoccat pum enramof, pu feke ep ofv vhetkvke omekat haks? makakvtes.

33 Momet mv hvse-vkerkv mahusan asehoket, Celuslvmen fulhoket, palen hvmkontvlakat vpvkakat esyomat etohnvkaftan,

34 Mehenwvn Pucaset akvwvpkes, momet Simvn em e heckuehcvt hakis, makaken eshecakvtes.

35 Momen nenen vhoyof nanvke momehocan, momen tvklike kvlkayan es kerrakvten onvyakvtes.

36 Momen momē opunayakof, Cesvs mahusat en nvrkvpvn hueret, Herkvt cem ocvkekvs, kicakvtes. 
37 Momis em estomahen, feksumkakvtes, momet Puyvfekcvn' hecēt omēs, komakvtes.

38 Momen, Estoman ce fekhervkeko haks? momen estoman ce feke ofv vkerrickvt kvwvpkaket o haks?

39 Hecrks cv'nke momen cv'le, vne mahuset omat; cv celayet, hecrks: vne vpeswr fune esyome ocryat omet puyvfekcvt ocekot omekv, kicakvtes.

40 Momet momēn opunahyof, $e$ 'nke $e$ 'leu hecicakvtes.

41 Momen em estomahet, afvcketv omecicen vkvsvmvkeko emunkof, em punayet, Hompetvn yvmvn ocatske haks? kicakvtes.

42 Momen rvro hotopke tvcken, momen fo-en-crmpen emakvtes.

43 Momen cahwet, ehomvn hompvtes.

44 Momet, Heyvt, opunvkv ece 'pakvkvye emunkof ce kicvkvyvtet omakes, mv nanvke Moses em vhakv, momen owalvlke, momen Sams cokv ofv ve ohfvecv cohoyvte ocakat omvlkvt esfvckvranat tvlkuset omat, kicakvtes.

45 Momof em vkerrickv taken enrvmricvtes, hoccakan kerrvkvren,

46 Momet, Hiyomën coket os, momen Klist momēn ostemeriket, nettv sutcenan akvwvpkvre,

47 Momen e hocefkv ofv vkerrickv etickv, momet holwryeckv enkvpvketv Celuslvmen vlicecet tvlwvvlke omvlkv em erkenvkhoyvre fvccut omemvts.

48 Momen heyv nanvke ohfvecr cemet akerrvlke toyatskes.

49 Momen hecrs, Cr'rke em etemfvecetvn a ec ohtotvkares. Monkv Celuslvme tvlofvn fekhonnatsket emunken, yekcetv hvlwe vten 'sece'cce ohcrkvres, kicakvtes.

50 Momet evpayet Pervne vteken esossvtes, momet e'nken kvwahpet, em merrakvtes. 


\section{OPUNVKV.HERV LUK COYVTE XXIV. 99}

51 Momen mome ocvtes, em merrakof, em eshohyen, hvlwan escehoyvtes.

52 Momen vrakkuecaket, afvcketv rakken es afvckvket Celuslvmen oh fulecvtes:

53 Momet Hesaketvmesen vkvsamet, momet vrakkuecaket, cuko-vcakan fulle emunkv tates. Emen. 



\section{OP U N V V HERA,}

\section{CANE COYVTE.}

THE GOSPEL ACCORDING TO

\section{J OH N,}

TRANSLATED

FROM THE ORIGINAL GREEK

INTO THE MUSKOKEE LANGUAGE.

NEW YORK:

AMERICAN BIBLE SOCIETY,

INSTITUTED IN THE YEAR MDCCCXVI.

1875. 


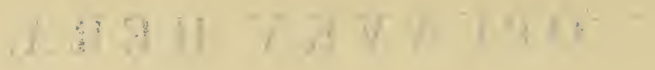

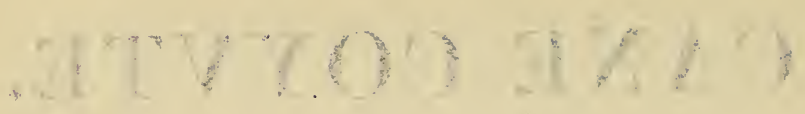$$
\text { Wh of }
$$

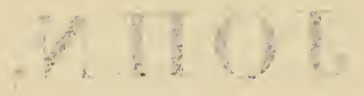

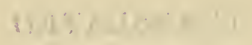

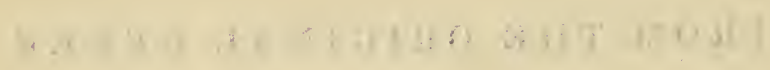

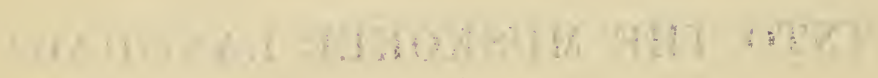$$
\text { SIIS. }
$$

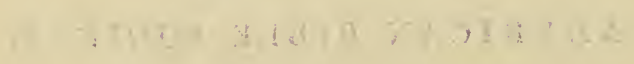

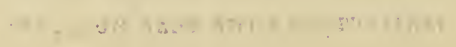




\section{OPUNVKV HERA, \\ CANE COYVTE.}

\section{SETENTVCKE I.}

Hvteceskv omof Opunvkv tates, momen mv Opunvkvt Hesaketvmesen vpakvtes, momen mv Opunvkvt Hesaketrinese tates.

2 Hrteceskv omof matrwvt Hesaketvmesen vpakvtes.

3 Nake omvlkvn mvt hayvte tates, momen mvt sekate mv hakvte hakekates.

$4 \mathrm{Mv}$ ofvn hesaketv ocvtes, momen mv hesaketvt este en hiyayvke tates.

5 Momen hvyayvket yomuckofvn hvyayakes; momen yomuckatet kerrekates.

6 Hesaketrmese atotēt hunvnwvt Cane hocef ket huervtes,

7 Matrwrt eskerkvt vlaket omvtes, Hryayvke oh, kerkuecrranatet, momat mv eteropotten este omvlkvt vkvsvmvkvren.

$8 \mathrm{Mv}$ Hryayvke tokatēs, momis mv Hvyayvke oh kerkuecvranaten atothoye tates.

9 Mvt Hvyayvke mehenwat tates, mvt ekvnvn vlakateste omvlkvn oh hryayicet os.

10 Ekvnvn arvtes, momen ekvnv mut hayvtet omvtes, momen ekvnvt kerrekates.

11 Enaken em vlakvtes, momen enaket evpayvkekates.

12 Momis erpayakat vtekat, mv e hocefkv oh vkvsv, 
makat, Hesaketvmese eppucetake hakvkepvren yekcetvn e'mvtes :

$13 \mathrm{Mv}$ catv es heckat tokon, monkat vpeswr komat tokon, monkat este komat tokon, momis Hesaketvmese es heckaten.

14 Momen Opunvkvt vpeswv hakvtes, momet epu'pakvtes, (momen en rakke heceyat, hvmkuse Erke heckuecvte en rakke omes,) herkv mehenwvu es fvcket.

15 Canet mvn oh kerkuecet, huehkvtes, Heyvt mv tvnkes opunvyecryvnke, $\mathrm{Cr}$ yupv vlakat vn homahtes, ev homv tvnkekv.

16 Momen es em fvcketv, herkvn hvtvm herkvn, omvlkeyatet eseyvtes.

17 Vhakv Moseskv etehoyvnen este emhoyvtes, momis herkv mehenwv esyomat Cesvs Klist etehoyvnen vlakvtes.

18 Este estomet estofvn Hesaketvmese hecvte sekos; Eppuce hvmkuse heckuecvte tvlket, mv Erke e hokpe vpikat, mvt oh kerkuecvtes.

19 Momen heyvt Cane ohonvkv em ohkerkueckv tes, Celuslvme a en kvpvke Plestvlke Lefitvlke esyome, Estimv toyetske haks? kicake em'pohvkvrane Cusvlke ohtotvte.

20 Momen ehelaksecekot, e oh onayvtes, mornet e oh onayet, Klist toyvkos, makvtes.

21 Momen, Nake tohaks monkv? kicaket em'pohakvtes. Elivs toyetskvh? Mv toyvkos, makvtes. Mv Owalv toyetskvh? kicakvtes. Momen hekos maket em ayoposkvtes. 22 Momof, Estimv toyetske haks? kicakvtes, a pu'totakan em onvyeyvren; Estimvn e kicetske haks?

$23 \mathrm{Mr}$ vnrvwr ofv huehke, Hesaketvmese em vyetv lvputke hayvkes, Owalv Hesia makvte omen makat toyis, kicvtes. 
24 Momen mv vtotvkhoyat Falvsevlke tates.

25 Momen, Monkv Klist, monkat Elivs, monkat mv Owalv toyetskekon omat, estomen paptisetske haks? kicaket, em'pohakvtes.

26 Canet em ayoposket, newvn es paptisit omis: momis hvmket kerratskekot ece'pakvket hueres ;

27 Mrt mv tes ev yupv vlakat, ev homv tes, mv em estelepikv sohwrnakv en recopvye taye heretv orvkos.

28 Heyv nanvke momvte Cotvne tvpalv Perepēlvn, Cane paptisan omvtes.

29 'Rem prkse, Cesvs em vlaken Canet hecet, Hecrs, Hesaketrmese en Yvpefiknce, holwvyeckv elivnv en kvpicat makvtes.

30. Heypt okvyvnke, $\mathrm{Cv}$ yupvn hunvnww vlaket vn homahtes makvyvnke; cv homv tatet okv.

31 Momen kerrvkvnkes: momis Eslevl em oh kerkvren newrn es paptisit vlakit omis.

32 Momen oh kerkuecet, Puyvfekcvt pvee-howe omet Hvlwen a hutapket, oh liken heevyrnkes;

33 Momen kerrvkvnkes; momis miv uewv es paptisvs cr kice vc vtotatet, Estimvn Puyvfekcvt hrtapket oh fekhonnen hecetskat, mvt mv tes Puyvekcr Veakat es paptisat ev kicvnkes;

34 Momen hecryvnket heyvt Hesaketrmese Eppuce tes makit kerkuecis, Canet makvtes.

35 Hvtvm 'rèm pvkse Cane en kerrepvlke hokkolen esyomet svpaklvtes:

36 Momet Cesvs yvkapen hecet, Hecvs, Hesaketrmese en Yrpefikuce makvtes.

37. Momen en kerrepvlke hokkolat opunayat pohakvtes, momet Cesvs verkvhoyvtes.

38 Momof Cesvs a fulotiket, vevkvhoyen hecvtes, momet, Naken hopoyatske haks? kicvtes. Momen Lapi! 
(mv oh yvtekhoyat Mvhayv! maketvt omen), Estvmvn liketska? makakvtes.

39 Vthoyet hecvkes, kicvtes. Vthohyet estvn likat hecakvtes, momet mv nettv em fekhonnvlkepvtes, hvsevkerkv espalat mahe tatet okv.

40 Cane opunaye pohahke vevkvhoyvte hvmkat, Simv Petv etecakkat Vntolo tates.

41 Hvteceskv etecakkat mahusat Simv taten eshecvtes, momen, Mesia eshēcēs, kicvtes; mv oh yvtekhoyat Klist tes.

42 Momet Cesvs 'sem vlakvtes: momen Cesvs hecof, Simv Convse eppuce toyetskes! Sefvs ce kihocvres, kicvtes, mv oh yvtekhoyat cvto maketvt omes.

$43^{\prime}$ Rem pvkse Cesvs Kalvlen ayetvn komvtes, momet Felvpen eshecrtes, momet, Ve vevkvyvs, kicvtes. -

44 Tokvs Felvpe Persitv, Vntolo Petv tepaket eme tvlofv, aosse tates.

45 Momen Felvpe Neranen eshecvtes, momet, Vhakv ofv Moseskv, owalvlke esyome, Cesvis Nasvlevlke, Cose eppuce es coyvte eshēcēs, kicvtes.

46 Momen Neranet; Nake heret Nasvlen aosse taye hakes? makvtes. Vhtet hecvs, Felvpet kicvtes.

47 Cesvs Neranet em vlaken hecvtes, momet, Hecvs, mehenwvn Eslevlke tes, mv ofv mukerretv sekot! makwtes.

48 Neranet, Estvmv vten cv kerretska? kicvtes. Cesvs em ayoposket, Felvpe cen huehkeko munkof, ke-rakko em vpe elecv liketskof, ce hecvyrnkes, kicvtes.

49 Neranet em ayoposket, Mvhaya, Hesaketvmese Eppuce toyetskes! Eslevl em Mekko toyetskes! kicvites.

50 Cesvs em ayoposket, Ke-rakko em vpe elecvin ce hecvyvnkes, ce kihcikv vkvsametska? Heyv nake 'sen rakrvken hecetskvres, kicvtes. 
51 Mehenwvn! Mehenwrn! ce kicis, heyv er yupv hvlwat en hauken hecetskvres, momen este Eppuce onvprn Hesaketvmese em estvlket acemecet momet hvtvpecen hecetskvres, kicvtes.

\section{SETENTVCKE II.}

1 Momen nettv resvtutcenan etepryetvt ocvtes Kene tvlofucen Kalvle ofvn. Momen Cesvs ecket mvn arvtes.

2 Momen Cesvs, en kerrepvlke esyoman mv etepvyetvn en huehhokvtes.

3 Momen pvrko-opuswvt orekon, Pvrko-opuswvi ocvkekos Cesvn ecket kicrtes.

4 Momen, Hokte, ceme vne tepakeyat naket pum estoma? Hvse-vkerkv crnaket hvte vlvkekos, Cesvs kicvtes. 5 Naketo estomen ce kicvkis, momecrks ecket vtotkvlken kicvtes.

6 Momen Cusvlke es en hrsvteckv vcrkvyet cvto neemvrkvswr faken hokkole monkat tutcene vcvnke vlken mvn vpohoyvtes.

7 Ueemvrkvswvn newvn fveficvks, Cesvs kicakvtes. Momen cukfvlvwr vteken fvcficakvtes.

8 Tokvs, svkcahwet hompetv-rakko em evpayvn es em vpeyvkes, kicakvtes; mohmen es em vpeyakvtes.

9 Hompetv-rakko em evpayv mv newv prrko-opuswv hahoyat lahsof, momis estrmvn vtetat kerrekates, (momis vtotkvlke newv cvwakat kerrakvtes), hompetv-rakko em evpayvt hokte epayvranan enhuehkvtes.

10 Hvte vlicecof, este vtekat pvrko-opuswv heren a'slicen, momen em etetayen eskakof 'sen holwvkusen aslicrnts; momis centawit prrko-opuswi heran vcayecetsken, hiyomat ores, kicvtes.

11 Heyv enhrteceskv este nake momeceko taye Cesvs 
momecat Kenv tvlofucen Kalvle ofvn en rakke heckuecvtes, momen en kerrepvlke oh vkvsvmakvtes.

12 Heyv eryupv Kepanevn rkhvtapkvtes eme, ecke, etecakkakat, momen en kerrepvlken, momet nettv sulkekon mvn fekhonnakvtes.

13 Momen Cusvlke em ohhoyvnkvt awolice tates; momen Cesvs Celuslvmen ayvtes.

14 Momen Cuko-vcakat ofvn mv wakv yvpefikv pvce esyome wiyakat, momen tokunawv esyoposkvlke vpoken eshecvtes.

15 Momen emet eswrnakvn estepketrn hahyof omvlkvn cuko-vcakan sossicrtes, momen yvpefikv wakv svpvken, momen esyoposkvlke en tokunawr a em prlatvtes, momet ohliompetr cektolcecrtes.

16 Momen pvce wiyakan, Yv nanvken :vkuehuecvkes Crrke en cuko neskvcuko hahyatskvs kicakvtes.

17 Momen, Cen cuko ayvkhvmketv cv lokes, make cokat, en kerrepvlket vkerricakvtes.

18 Momof Cusvlket em ayoposket, Heyv nanvke momecetskat eskerkv estome pu hecvkuecetske haks? kicakvtes.

19 Cesvs em ayoposket, Heyv cuko-acakat sumecicvkes, momen nettv tutcenen a kvwvpares kicvtes.

20 Ohrolope pale-osten epohkaken heyv cuko-vcakat etohtvrhoyvtes, moman cemet, nettv tutcenen a kwwapets kvr haks? Cusvlket makakvtes.

21 Momis ena en cuko-vcakat opunvyecet okvtes.

22 Momekv eme elkv rakvw.vpikof en kerrepvlke heyv kicvte vkerricakvtes, momet cokv-rakko momet opunvkv Cesvs makvte vkvsvmakvtes.

23 Momen eme Celuslvme ofv ohhoyvnkv hompetvrakkon aret, este nake momeceko tayen momecen hecakof, en hocef kvn sulket vkvsvmakvtes. 
24 Momis Cesvs em, wikekates, este omvlkvn kerrvtet omekv.

25 Momen estimvt este ohkerkuecrre enkusvpkekates, este nake vpikat kerrvtet omekv.

\section{SETENTVCKE III.}

Falvagulket este-hunvnwv Nekvtemvse hocef ket Cusvlke semerpayvt huervtes.

2 Mvt neren Cesvs em vlakvtes, momet, Lapi, Mvhiyv Hesaketvmese a en kvprke toyetskat kerrēs, kicvtes, heyv nanvke este momeceko tayat momecetskat este momeceko tayet okv, Hesaketvmeset vprket omekon omat.

3 Cesvs em vyoposket, Mehenwrn, mehenwrn ce kicis, Estimvt svnvcomvn heckekon omat, Hesaketvmese eme tvlofv hehcekos, kicvtes.

4 Este vculehpof, estomet hecke taye haks? Nekvtemvset kicrtes.

5 Cesvs vyoposket, Mehenwvn, mehenwwn ce kicis, Este uewv momen Puyvfekcan tepakan es hecket omekon omat, Hesaketvmese eme tvlofv eceyeko tayet os.

$6 \mathrm{Mv}$ vpeswr es heckat vpeswvt omes, momen mv puyvfekcv es heckat puyvfekcvt omes.

7 Estomahes kohmetskvs, mv, Svnvcomv heckvranatsket os, ce kicryat.

8 Hotvle estrmvn komat hotales, momen en hake pohetskes, momis estrmvn vtetut, estrmvn ayet omat kerretskekos. Mv omet os Puyvfekcv es heckat vtekat.

9 Nekvtemvset em vyoposket, Heyv nanvke estomet mome taye haks? kicvtes.

10 Cesvs em vyoposket, Eslevlke em mvhayv toyetskes, momat heyv nanvke kerretskeko haks? kicvtes. 
11 Mehenwvm, mehenwvn ce kicis, mv nake kerreyat opunvhoyes, momen mv nake heceyvtet kerkuecēs, momen kerkueceyan pum esatskekos.

12 Ekvnv nanvken cem onvyvkvyvten vkvsamatskekon omat, hvlwe nanvken cem onvyrkvyat estomet vkvsamatskvr haks?

13 Momen este estomet hvlwe tvlofv vkvwvpkvte sekos, mv hvlwe tvlofv ahvtvpkvte este Echuswv hvlwe tvlofv likat vkvwvpkekon omat,

14 Momen Moseskv vnrvwr ofv cetto kvwapvte etvpomet este Echuswv kvwrpkvranet os.

$15 \mathrm{Mv}$ oh vkvsamat vtekat estemerkekot, momis hesaketv yvksv-sekon ocvren.

16. Hesaketvmese ekvnv vnokece mahet omekv, Eppuce hvmkuse heckuecvte emvtes, mvn estimvt oh vkvsamat estemerkekot, momis hesaketv yuksvsekon ocvren.

17 Hesaketvmese Eppuce ekvnv ohfv́ccecvren ekvnvn a oh 'totet omekates, momis mv eteropotten ekvnvt hesakvren.

$18 \mathrm{Mv}$ oh vkvsamat ohfvecekot os; momis $\mathrm{mv}$ oh vkvsvmekat ohfvece hoyvnet os, Eppuce hvmkuse heckuecvte hocefkvn oh vkvsvmèkokv.

19 Momen heyvt ohfvcceckv tes; hvy ayvket ekvnvn vlukes, momis estet yomucken hryayvke emuntvlen vnokecvkvtet omes, en nakvfrstetv holwvhoket omekv.

20 Holwake moruecat vtekat hvyayvke en homecet os, momekv hvyayvke oh vtekot os, en nakvfvstetv oh kerkekaren.

21. Momis mv fvecetv momecat hryayvke oh atēs, mv en nakevfvstetv Hesaketvmese ofvn vfvstvkvte tat kerke hakvren.

22 Heyv nanvke 'renyupv Cesvs en kerrepvlken esyomet Cutev ekvnun yicvtes, momen vpaket mvn fekhonnet paptisvtes. 
23 Momen Caneu paptisytes Envn, Silome tempen, mvn uewvt tayet omekv, momen en yicet paptiskakvtes. 24 Cane cuko-yekcv hvte vpihokeko emunkekv.

25 Momof nake vpohkvt kawvpkvtes hvsvteckv ohfvccun Cane en kerrepvlke momet Cusvlke em etenrvwvn. 26 Momen Canen en yicet, Lapi, mv Cotvne tvpalv ece'pakvnke, mv ceme oh kerkuecetskat, heevs, mvt paptisen este omvlkvt en yices.

27 Cane vyoposket, Este naket en hecikekos hvlwe tvlofv a e'mhoyeko vtekat, makvtes.

28 Ceme etvwvt avnkerrvlke toyatskes, Klist toyvkos, momis ehoinv ve vtothoyes, makvyvte.

$29 \mathrm{Mr}$ ehiwv taranat ocat hokte-epayvranat tes, momis. mv hokte epayvranat en hesset hueret pohof vfvcketvn es afvckes hokte epayvranat em punvkv en hake svrahkvn, momet omekv heyv vm afvcketv esfvckes.

30 Eme tat ohvtvlvkvranes, momis vne tat cr yokopvres.

31 Eme hvlwe atatet omvlkvn ohfvnket os, estimvt ekv. nvn atetat ekvnv enakets, momet ekvnv opunvyecet omes; eme hulwe tvlofv atatet omvlkvn ohfvnket os.

32 Momen nake hecvtet momet pohvtet mvn emet kerkuecet os; momis mv kerkuecat estimvt em esekos.

33 Mv kerkueckv em esvte vtekat Hesaketvmese mehenwe tat em eskerkv vlicvtet os.

34 Eme Hesaketvmese vtotvte Hesaketvmése em punvkvn opunvyet omes, Hesaketvmese puyvfekcv svkerkekon e'met omekv.

35 Erke Eppuce vnokecetut nanvke omvlkvn enke ofvn emvtet os:

36. Eppuce oh vkvsamat hesaketv yuksv-sekon ocet os, momen Eppuce oh vkvsvmekat hesaketv hehcekos, momis Hesaketvmese en cvpvkket oh fekhonnes. 


\section{SETENTVCKE IV.}

Cane paptisat 'sensulken Cesvs en kerrepvlken hayet paptisen Falvsevlket pohan Pucaset kerret okv,

2 Cesvs eme mahusat paptisekates momis en kerrepvlket omakvtes.

3 Cutev en kvpaket, hvtvm Kalvlen ayvtes.

4 Momen Semelev ekvnvn 'reteropottvranet omvtes.

5 Momof Semelev tvlofv vlakvtes mv Sikv kihocan, Cekvpe eppuce Cose ekvnv e'mvte vnakv tates.

6 Mohmen Cekvpe em ueenkorkvt mvn likvtes, monkv Cesvs ayvtet hotoset omekv, ueenkorkvn oh likvtes, momen hvse-vkerkv esepakat mahe tates.

7 Semelevlke hoktet uewrn ascrwvranet vlakvtes, Esketvn v'mes, Cesvs kicvtes.

8 En kerrepvlke hompetv nesvranet etvlwvin vpeyvkēpet omekv:

9 Estometon Cusvlke toyetskatet esketvn vm 'pohetske haks? vne Semelevlke hokte toyvyan, Semelevlke hoktet monkv kicvtes. Cusvlke Semelevlke etohkvlketv etem ocekot omekv.

10 Cesvs em ayoposket, Hesaketvmese esteman kerretskvtet on omat momen estimvt Esketv v'mes ce kicet okat kerretsken omat, em'pohetskvint omes, momen uewv hesaken ce'mvnt omes, kicvtes.

11 Naken u'escrwetv ocetskekós, momen ueenkorkv sufken, momen estvmv vten mv newv hesake ocetske haks? hoktet kicvtes.

12 Purke Cekvpe sen rakketsket o haks? mv ueenkorkv pu'mvte, momet eme en hopuetake, momen en wakvlkeu eskakvtes.

13 Cesvs em ayoposket, Heyv uewv eskat vtekat hvtrin ewvnhkvres, kicvtes. 


\section{CANE COYVTE.}

14 Momis mv newv vne emvyat eskat vtekat, estofis ewvnhikekos, momis mv uewv e'mvyat uekiwvt eme ofvn hesaketv yuksv seko vfihne hakvres.

15 Pucase, heyv'uewvn v'mes ev wrnhkekaren, momet ascvwvranet vlvkvkaren, hoktet kicvtes.

16 Ahyet cehen 'renhohiket, momet 'res vlvkvs, Cesvs kicvtes.

17 Hokte ayoposket, Cvhe sahsekos, makvtes. Heren opunvhyetskes, Cvhe sahsekos maketskat, Cesvs kicvtes.

18 Cehetake cahkepen ocetskvtet omes, momis hvmke hiyome ocetskat cehe tokos, momekv mv tat mehenwrn ohketskes, Cesvs kicvtes.

19 Pucase, owalv toyetskat hecis, hoktet kicvtes.

20 Purkvlke heyv ekvnhvlwe ofvn mekusvpakvtet os. Moman Celuslvme mimvn este emekusvpe tayet os, makatsket os.

21 Hokte, heyvn vm vkvsvmvs, heyv ekvnhvlwe ofv tokon, momet Celuslvme ofv tokon, Erkeu mekusvpvranatskat hvse-vkerkv vlakes, Cesvs kicvtes.

22 Ceme naken mekusapal kerrekot mekusapatskes, pume naken mekusapeyat kerres, vhesaketv Cusvlke aosset omekv.

23 Momis hvse-vkerkv vlvkvranet os, momen hiyomat $m v$ tes, momof emekusapvlke mehenwakat Erken emekusupvkvres puyvfekev momet mehenwetv ofvn, Erke mv omakat mekusvpvren hopoyet omekv.

24 Hesaketvmese puyvfekcvt omes, momen mv emekusvpakat puyvfekcv momet mehenwv ofvn mekusvpvkvranet omes.

¿5 Mesiv Klist kihocat vlvkvranet omen kerris, mvt vlakof nanvke omvlkvn pum onayvres, hoktet kicvtes.

$26 \mathrm{Vne}$, cem punvyvyat, $m v$ toyis, Cesvs kicvtes.

27 Momof en kerrepvlket yicvtes, momet hokte ete'm 
punayan estomahes komakvtes, momis, Naken hopoyetske haks, monkat Estomen ete'm punayetske haks? estimvt makekates.

28 Hokte monkv uescawkv wihket tvlofvn ayepvtes, momet,

29 Vhwet, este hunvnwv nake momvyvte omvlkv vm onayan hecvkes heyvt Klist toko haks? hunvntaken kicvtes.

30 Monkv tvlofv asosset en yicakvtes.

31 Heyv em etenrvwrn en kerrepvlket en kusvpaket, Pucase, hompvs, kicakvtes.

32 Momis nake hompetv ocepis, ceme kerratskekon, kicvtes.

33 Momekv en kerrepvlket ete'm'pohaket, Estimvt hompetv 'sem vlahke haks? makakvtes.

$34 \mathrm{Vn}$ hompetvt on omat mv a'crtotvten em eyackvn momecit, em vtotketrn es yukpoyvranvyat tes, Cesvs kicakvtes.

35 Hrse ostet ahoske munkes, hvyo vlvkvranat makatskeko haks? Hecrs, ce kicvkis, vfvnnaket crpofvn oh hecvkes, entis nak lokce es hathakes.

36 Momen nak lokce vteloyat feketv en hecket os, momen em ette hesaketv yuksv sekon em vteloyet os, momen $m v$ fekayvte momen vteloyat etepvket vfvckvkepvren.

37 Momen heyv ofvn heyv maketv mehenwes, Hvinket fekayen momen etvt vteloyes.

$38 \mathrm{Oh}$ vtotkatskekatet] vteloyatskvren ce 'totvkis; este etvt ytotkvtet on em vtotketvn 'sohceyatskēs.

39 Momis Semelevlke mv tvlofv vtehkvte sulket ohvkvsvmakvtes, nake momvyvte omvlkvn vm onahyes, hokte make kerkuecat omecicen.

40 Momen Semelevlket en yicet vpvkvket fekhonnvren em 'pohakvtes, momen nettv hokkolen fekhonnvtes. 
41. Momen 'sen sulket oh vkvsvmakvtes eme em punv$\mathrm{kv}$ mahusat omecicen,

42 Tokvs oh vkvsamēs nake onvyetskvte omecice tokon, momis pume mahusat opunvyat pohēpēkv, momet, mehenwv tes, heyvt Klist ekvnv hesayecvt on kerres, hokten kicakvtes.

43 Momis nettv hokkole 'ren yvpvn mvn en kvpaket Kalvlen oh ayvtes.

44 Cesvs eme mahusat, Owalv em ekvnv ofv hvlwetv ocekos, maket kerkuecrtet omekv.

45 Momekv Kalvlen vlakof, Kalvlevlket, Celuslvme ofv hompetv-rakkon aret nake momecat omvlkvn hecaken, evpvyakvtes, emen hompetv-rakko oh vpeyakvtet okv.

46 Momen Cesvs Kene Kalvle ekvnvn hvtvm 'rvlakvtes, mv uewv pvrko-opuswr hayvtet, Momen este hunvwr hvmket mṿn arvtes, eppucet enokket Kepvnevn, wakken.

47 Cesvs Cutev ekvnvn a osiyet, Kalvlen vlakan pohof, oh ayvtes, momet a vk hvtapket, eppucen em wicecvren em'pohvtes, elvranuset omekv.

48 Momof, Nake eskerkvn, momet nake estomahvken hecatskekon omat, vkvsahmatskekos, Cesvt kicvtes.

49 Pucase, a vk hvtvpkvs, hopuewv vm eleko munken, evpayvt kicvtes.

50 Vyeprs, ceppuce hesakes, Cesvs kicvtes. Momen hunvnwv opunvkv Cesvs em punayat ykvsamvtes, momet vyepvtes.

51 Momen vk hvtapkof em vtotkvlket virvpakvtes, Ceppuce hesakepes, makaket em onvyakvtes.

52 Momof hvse-vkerkv estofv mahen en here hake vyecicvten em vpohakvtes, Vpvksvnke, hvse-vkerkv eskolopakofvn hepetketv en kvpakvnkes, kicakvtes.

53 Momekv hvse-vkerkv matvwv mvketeken Ceppuce 
hesakes Cesvs kicet okvte erket kerrvtes. Momen eme mahusat vkvsamvtes, momen en cuko somvlkvt.

54 Cesvs Cutev ekvnv en kvpahke Kalvle vlake nake este momeceko taye momecat heyvt esvhokkolat tates.

\section{SETENTVCKE V.}

Hiуoнмо Cusvlke en hompetv-rakko ocvtes, momen Cesvs Celuslvme ceyvtes.

2 Momen Celuslvme yvpefikv hute tempen vkhvse $\mathrm{Cu}$ svlke Persitv kicaket ocvtes, sohvpettv chahkepen ocēt.

3 Heyv ofvn enokhokvlke sulke mahet lumhvtes, turwv hecvkekat, en yvkvpkv vnvttakat ena talakat newv nekeyvren em ehakaket.

4 Hesaketvmese-em-este $\mathrm{mv}$ uewv vk hvtapket oketv ocen, momet uewv melohlicet omekv. Uewv melohlof estit homvn vk ceyen omat, enokketv estomen ocvte tis cvfekne haket omvtes.

j Momen este hunvnwr hvmket wakkvtes, enokkvte ohrolvpe pale tutcenen cahkepohkaken.

6 Wakken Cesvs hecof, hofune mahet omen kerrvtes, momet, Ce cvfeknvre ce yace haks? kicvtes.

7 Enokkat ayoposket, Pucase, uewv melohlof vkhvse a'c vkceyicvre este hunvnwv ocvks; momis ayvyof, etvt $\mathrm{cv}$ homvn vk ceyepet os, kicvtes.

8 Ahuyiret, cem pvtakv cahwet, yvkvpvs, Cesvs kicvtes.

9 Momat lvpkusen este hunvnwv cvfeknvtes, momet em pvtakv cahwet, yvkapvtes; Mohmen mv nettv fekepkvnettv tates.

10 Fekepkv-nettr tes, pvtakv 'sayetskvre cem etektrnkekot os, cvfeknan Cusvlket monkv kicakvtes.

$11 \mathrm{Em}$ ayoposkaket, $\mathrm{Cr}$ cvfeknicatet, Cem prtakv cahwet, yvkvpvs cv kihces, kicakvtes. 
12 Momof em'pohakat, Hunvinw estomèt Cem prtakv cahwet, momet yvkvpvs, ce kihce haks? kicakvtes.

13 Momen mv cvfeknihocat estitat kerrekvtes, mvn este tetayet omen, Cesvs etin ayepet omekv.

14 En yupvn cuko-vcalsat ofvn Cesvs eshecrtes, Hecvs, ce crfeknihoces, vnvcomv naoriketskvs nake 'sen holwake ec oh vlvkekaren, Cesvs kicvtes.

15 Este hunvnwv en kvpaket, mohmet Cusvlken, Cesvt ev crfeknicet os, maket em onvyakvtes.

16 Momen monkv Cusvlke Cesvs assecakvtes, mohmet elecetv komakvtes, fekvpkv-nettv ofvn yv nanvke momecekv.

17 Momen Cesvis em ayoposkaket, Cvrke vtotke munkvt os, momen vnet vtotkis, kicakvies.

18 Monkv Cusvlke ohvtvlaken elecetvn komakvtes, fekvpkv-nettv kacat tvlkekot, momis, Hesaketvmeset Crrke tes, maket, Hesaketrmese etetayen e hayet omekv. 19 Monkv Cesvt em ayoposkat em punayat, Mehenwrn, mehenwrn ce kicvkis, Eppuce entvlkat naken estomeko tayes, momis Erke nake momen hecatet momecet os, naken momecat vtekat mvn Eppuceu matvpomen momecet omekv.

20 Erke eppucen vnokecet omekv, momet nake momecat omvlkvn en kerkuecèt omes; momet vtotketv heyv sen rakrvken en kerkuecvres estomahes komatskvren.

21 Erke prsatkvte a kvwapet wenahuecat etrpomen, Eppuce estimvn komat wenahuecet omekv.

22 Erke nvprn ohfvececekot omekv, momis Eppucen ohfvececkv omvlkvn em wikvtet omēs.

23 Erke vrakkuecakat etvpomen omvlkvt Eppuce vrakkuecvkvren. Estimvt Eppucen vrakkuecekon omat, Erke vtotateu vrakkuecekot omes.

24 Mehenwrn, Mehenwrn ce kicvkis, Estit vm punvCroek. 
kvn pohet, mohmet a cv 'totvten oh vkvsamat hesaketv emunkvn oces; Momet ohfvececkv hvlatkekos, momis elkvn en kvpaket hesaketrn tohtvlkes.

25 Mehenwvn, mehenwvn ce kicvkis hvse-vkerkv vlakes, momet hiyomat vlvkes, prsatkvte Hesaketvmese Eppuce en haken pohof, momen pohakat hesahokvres.

26 Erke, hesaketv eme ofv ocat etvpomen Eppucen hesaketv emeu ofv ocvren, e'mvtet omekv.

27 Mohme ohfvececkv hayvranateu yekcetvn emvtet omes ; emet este Echuswvt omekv.

28 Heyv estomahes kohmatskvs, hvse-vkerkv vlaket omekv, mv ofv hopelkv vtehkat omvlkvt en hake pohvkvres.

29 Momet a sossvres, here afvstakvte hesaketv ofvn akvwvpkvkvres, momen naorkakvte ohfvececkv ofvn akvwrpkvkvres.

30 Vntvlkusat naken momecvko tayes; pohvyatet ohfvececryet os; momen vm ohfvececkv fvccrts, nake komepvyat hopoyekot omvkok, momis Erke a cv'totvte koman,

31 Vnet a en kerkuecepin omat, vn kerkueckv fvecekot omes.

32 Hvmket a kerkuecat hueret os, momen mv kerkueckv vc oh kerkuecat mehenwvt on kerris.

33 Cane oh 'totatsken, momen mehenwvn ohkerkuecvtes.

34 Momis hunvnwv kerkuecat esvkot omes, momis hesahokatskvrēn heyv nanvken okis.

35 Kulket hvyayvke momet hvsotet omvnkes, momen alikusen en hvyayvke ofv afvcketv vkvsvmatske tvnks. 36 Momis Cane en kerkueckv sen rakken ocis ; vtotketv poyvranvye Erke v'mvte momecvyatet ve oh kerkuecet omekv, Erket vc vtotvtetat. 
37 Momen Erke vc vtotvtet emet vc oh kerkuecutet omes. En hake pohatskvte sekot omet, en heckvu hecatskvte sekot omes.

38 Momen em punvkv ece'pike ocatskekot omes, mv vtotvten oh vkvsamatskekokv.

39 Cokan vketecvks, mv ofvn hesaketv yuksv sekot oces komatskekv, momen mvt vc oh kerkuecaket omes.

40 A'c oh vwetv komatskekot omes hesaketv ocatskvren.

41 Hunvntake vrakkuecat em esvkot omes.

42 Momis ce kerrvkis Hesaketvmese em vnokeckv ec ofv ocatskekos.

43 Cvrke hocef kv ofvn vlakis, momen cv 'satskekos; etvt eme ehocef $\mathrm{kv}$ ofvn vlaken omat mvn esatskvres.

44 Estomet vkvsamatske taye haks? vrakkueckv eten hopoyepet, momet vrakkueckv Hesaketvmese tvlkuse a en kvpakat hopoyatskekat.

45 Erke ehomv epu'nrvpvres cv kohmatskvs, hvmket ece'nrapvket omes, Moseskr vfekrakkatske mahusatet.

46 Moseskvn vkvsamatskvten omat, vc vkvsamatskvnt os, ve opunvyecet hoccicvtet omekv.

47 Momis hoccican vkvsamatskekon omat, estohmet vm opunvkvh vkvsvmatskvr haks?

\section{SETENTVCKE VI.}

Hexv nanvke hoyanof, Cesvs Kalvle vkhvsen etohtikvtes, $m v$ vkhvse Tipelevs tates.

2 Momen este sulsuket vevkvpeyvtes, enokhokan vtotketv sehoneckan ohcelayan hecakekv.

3 Mohmen Cesvs ekvnhvlwe vcemkvtes, momet mvn en kerrepvlke vpaket likvtes.

4 Momen ohhoyvnkv, Cusvlke en hompetv-rakko, vwolice tates. 
5 Momof Cesvs vfvnnakvtes, momet este sulke mahet a oh awen hecet, Trklike hompvkvranet estvmvn neseyvr haks? Felvpen kicvtes.

6 Momen heyv kicat oh kerretvn komat okvte, eme tat estomvranat kerrepvtet ok.

7 Felvpe em vyoposket, Trklike crto-kunawv pale-hokkole enrahkv tis em etetahyvkekos, cōtkuse vlken ehset o estomis, kicvtes.

8 En kerrepvlke hvmket, Antolo, Simv Petv etecakkatet, kicrtes,

9 Cepanet likes, pale trklike-vnore cahkepen, momet rvro lopockuse hokkolen ocet; momis heyv naket o haks? este sulke momat em etenrvwv?

10 Momen, Este tvk vpoyekvs, Cesvs kicvtes. Mv mahusat pvhe tayet omekv. Momen monkv este tvk vpokvtes, em ahonkvtkv cukpe-rakko cahkepat mahet.

11 Momen Cesrs tvklike cawvtes, momet ohvkrsamof en kerrepulke vwahlvtes, momen en kerreprlket tvk vpokan emakvtes, momet rvrou matvpomecvtes.

12 Momen frcecahkehpof, En wokucke ahoskan vteloyvkes, naket sumikates, en kerrepvlken kicvtes.

13 Monkv etoh vteloyakvtes, momet pale trklike cahkeprte hompahkvten ahoskat em wokucke svmpv palenhokkolohkaken fveficakvtes.

14 Momen mv estet Cesvs vtotketv sehoneckvken momecen hecakofvt, Heyv fvecr mehenwusen mv owalv ekvnv vlvkvranvtet os, makakvtes.

15 Momen yicet, en yekcen es ehset, mekkon hahoye tayen Cesvs keriyet, entvlkuset ekvnhvlwe hvtvm vcem. kvtes.

16 Momen yafkof en kerrepvlkeh vkhvsen hvtvpecvtes.

17 Momen perron vtehiket, momet vkhvse tvpalv $\mathrm{K}_{\mathrm{r}}$ - 
panevm afvecrn vpeyutes, momen yomuckepen, momen Cesvs em vlvkekates.

18 Momen hotvle rakkat hotalet omecicen, vkhvse melohlvtes.

19 Momen vkerkv tutcenat, monkat ostat mahen kafahkof, Cesvs vkhvse oh yvkapet, momet perron vwolicen hecaket, momet penkvlake tates.

20 Momis, Vne tes, penkahlatskvs, kicvtes.

21 Momen vkvsvmēt, perro a oh ceyicakvtes; momen perro lvpkusen ekvnv vpeyvte er vcakvtes.

22 Enhvyvtke este vkhvse tvpalv svpaklvtet, perro etv sekon, hvmke en kerrepvlke vtehkat tvlken, hecakvtes, momen Cesvs perro vpiket en kerrepvlke vcrkayekates, momis en kerrepvlke entvlkuset vpeyepvtes.

23 Momis perro etvt Tipelers awet, mv Pucase ohvkvsame tvklike prphoyvte ocat vnakvn yicvtes.

24 Momen Cesvs momet en kerrepvlkeu sekon este hecakof, emeu perro vtehiket, momet Cesvs hopoyet Kvpanevm er oricvtes

25 Mohmet vkhvse tvpalv er eshecakat, Mvhaya? estofvn vlaketskat haks? kicakvtes.

26 Cesvs em vyoposket, Mehenwvn, mehenwvn ce kicrkis, Nake sehonecke vtotketv hecatskvte omecicen $\mathrm{cv}$ hopoyet omatskekos, momis tvklike hompatske, momet ce cvporvkate omecicen omatskes, kicvtes.

27 Hompetv yvmahke tayat em vtotikatskvs, momis mv hompetv hesaketv emeyuksv-sekat 'roran omvkes, mvn este Echuswvt ce'mvkvren, emen Erke Hesaketvmeset eskerkuecrtet omekv.

28 Mohmen, Naken estomeyatet Hesaketvmese em vtotketv vtotkeye tetaye haks? kicakvtes.

29 Cesvs em vyoposket, Heyv Hesaketvmese em vtotketvt os, mv atotvten oh vkrsamatskvren, kicvtes. 
30 Momekv eskerkv naken heckuecetska, hecēt, oh vkvsvmeye tayat? Naken vtotketske haks? kicakvtes.

31 Purkvlke vnrvwv ofv manvn hompakvtes, Trklike hompvke tayat hvlwe vten e'makvtes, maket os, cokat vevkvyen omekv.

32 Momen Cesvs kicat, Mehenwrn, mehenwvn ce kievkis, mv tvklike hvlwe atat, Moseskv ce'mvkekatet os; momis Cvrke tvklike mehenwrn hvlwe vten ce'mvket os, kicakvtes.

33 Hvlwe a hvtapkatet, en hesaketv ekvnvn ematet Hesaketvmese en tvkliket omekv.

34 Momen, Pucase, mv tvkliken estofis pu'mutcrs, kicakvtes.

35 Momen Cesvs opunvyatet, Vnet hesaketv tvklike toyis; este a 'coh atatet, estofvn elahwekos, momen este ve oh vkvsamatet estofvn ewvnhikekos.

36 Momis Cemeu cv hecatskvnks, momat vkvsamatskekos, ce kicrkis.

37 Cvrke a'mvte omvlkvt a 'coh awvres, momen este a 'coh atat, estomaten ossihcrkos.

38 Hvlwe a hvtapkvyate vne komvyat vfvstvranvyatet tokot os, a 'cvtotate komaten vfvstvranvyatet omvtes.

39 Momen heyvt Crrke a 'cvtotate komatet os; este Cvrke a'mvte omvlkvn hvmkusis sumecicvkot, nettv espoken a huericvranvyat omes.

40 Momen heyvt a 'crtotate komatet os; Eppuce hecet, oh vkvsamat vtekat hesaketv emeyuksv-sekon ocvranet os; momen nettr espoken a huericares.

41 Momen Cusvlke ecayakvtes Trklike hvlwe a hvtapkate toyis, makaten.

42 Momen, Heyv Cesvs Cose eppuce toko haks? erke, ecke tepakat kerreyat, momaten estomen Hvlwe a hvtapkvyat tes maket oke haks, makakvtes. 
43 Momekv Cesvs em vyoposket, Ecayet 'setem punahohyatskvs, kicakvtes.

44 Este estomet ve oh vteko tayes, Cvrke a 'crtotatet vhvlvtekon omate, momen nettv espoken a huericares.

45 Omvlkvn Hesaketvmeset emvhayvres make owalv en nakcokv ofvn hoccet omes. Monkv este vtekat pohhet, Cvrke en kerrate sasat a ve oh atet omes.

46 Nvpvt Erke hecrte sekot omes, momis Hesaketvmese a en kvpvkat tvlket, mvt Erke hecvtet omes.

47 Mehenwusen, mehenwusen ce kicvkis, Este vc oh vkvsamate, hesaketv emeyuksv-sekon ocet omes.

48 Vnet $\mathrm{mv}$ hesaketv trklike toyis.

49 Cerkvlke vnrvwr ofvn manvn hompakvtes, momet pvsvtkvtes.

50 Heyvt tvkliket, hvlwe a hvtapket omes, este mvn hompat elekaranet omes.

51 Vnet trklike hesake hvlwe a hvtrpkate toyis; este estomet heyv tvklike hompen omat, estofvn ehlekos; momen tvklike emvranvyat crna-vpeswr mv ekvnv en hesaketv vrahkv emvranvyat omes.

52 Monkv Cusvlke 'sete'nrvpaket, Estomet heyv este ena-vpeswv pu'men, papeye taye hakes? makakytes.

53 Momen Cesvs em punayatet, Mehenwusen, mehenwusen ce kicrkis, Este Echuswr ena-vpeswr prpekot, e catv eskatskekon omat, hesaketv ece 'pahkvkekos, kicvtes. 54 Estimvt crna-vpeswv papet, cv catv esken omate, hesaketv emeyuksv-sekon ocet omes, momen nettv espoken a huericares.

55 Crna mehenwrn hompetvts, ev catv mehenwrn esketvts.

56 Estet crna-vpeswr papet, cr catv esken omat, vc ofvn liket omen, vneu ofvn likvyet omes.

57 Erke hesake likatet a 'cvtotvte omen, Erken $v$ he- 
sakvyet omes, matvpomen este cv hompate mvu vc $\mathrm{v}$ hesakvres.

58 Heyv tvkliket hvlwe ahvtapkatet omes. Cerkvlke manv hompakat momet prsrtkat omekot omes: este heyv tvklike hompen omate, hesake emunkv tares.

59 Heyv nanvke, Kvpanerm mekusvpkv-cuko ofvn este emvhayofvt, opunayet okvtes.

60 En kerrepvlke vnvcomet heyvn pohvkvtet, Opunvkv yekcet omes; estimvt pohe taye haks? makakvtes.

61 En kerrepvlket heyvn es ecahoyen, Cesvs emet kerrof, Heyvt ce muttecicvk haks? kicakvtes.

62 Enhomv likvtet este Echuswv v kvwapken hecatsken omat, estome taye haks? kicvtes.

63 Puyvfekcvt este hesayecet omes; este ena vpeswv naken lopicekos. Opunvkv cem punayvkvyatet puyvfekcrts, momet hesaketvts.

64 Momis vkvsvmeko ce sasvket os kicakvtes. Cesvs enhvteceskvn vteken este estomvket vkvsvmekat kerrvtes, momen estimvt wiyvranate kerre tatet okv.

65 Momekv, Cvrket emvtet omekon omat, este ve oh vte tetayate sahsekos, ce kicvkit okvyis, kicakvtes.

$66 \mathrm{Mv}$ vtekate en kerrepvlke vnvcomet fulecvtes, mont svnveomv vevkfullekates.

67 Mohmen Cesvs pale hokkolohkakaten oket; Cemeu vpeyepvranatska? kicakvtes.

68 Mohmen Simv Petvt vyvposket, Pucase, estimvn oh vpeyeyvr haks? Cemet hesaketv yuksv-seko opunvkv ocetsket omes, kicvtes.

69 Momen vkvsamēt, kerrēs, mv Klist, Hesaketvimese hesake Eppuce toyetskate.

70 Cesvs em ryoposket, Palen hokkolohkakatskan ce'nhopoyvkvyvtet omeko haks? momen hvmkatskat puyvfekev holwvkets, kicakvtes. 
71 Cutvs Eskalevt, Simv eppucen okvtes, mvt wiyvranet omet, mvt palen es hokkolohkakvtes.

\section{SETENTVCKE VII.}

Heyv nanvke hoyanofv, Cesvs Kalvlen arvtes, Cutev ofv vretvn komekatet okv, Cusvlket elecetvn komet hopoyaket omekv.

2 Momis eto-esse-vpette-es-haye hompetv-rakkot Cusvlke em vwolice tates.

3 Momekv em etecvkkvtevlket, Yvmv en kvpaket, Cutevn vyvs, cen kerrepvlkeu vtotketv celayetskate hecvkvren, kicakvtes.

4 Este nake ehke celayate ohkerkekare eyace sahsekos. Heyv nake vfastetsken omat, ekvnvn em e heckuecrs, kicakvtes.

5 Etecvkkvte tisem oh vkvsvmekates.

6 Momen Cesvs, Vm oketv vlvkeko munkes, momisem cem oketv take tetake emunkvt omes, kicakvtes.

7 Ekvnv cen homecvkeko tetayet omes, momis vnen vn homecet omes, em vtotketvt holwahoket omen oh kerkuecryet omekv.

8 Heyv hompetv-rakko oh vpeyvkes; heyv hompetvrakko oh ayvko munkes, vm oketvt oreko munkekv, kicakvtes.

9 Heyv opunvkv kicakof, Kalvle ofvn likvtes.

10 Momis etecvkkvte vpeyofvn, emeu hompetv-rakko oh ayvtes, kerhoyekon, momis ehke omet.

11 Momen Cusvlke, hompetv-rakko ofvn hopoyet, Estvn like haks? makakvtes.

12 Momen este tetayen opunvkv es ete'm ecakvt ocvtes, Este heret omes maket sasan, vpvlwvt, Monks, este ehosicet os, makakvtes. 
13 Momisem, estet, Cusvlken em penkvlaket omekv, pohaken opunvyecekates.

14 Hompetv-rakko en nvrkvpv orofvn, Cesvs cuko-vcakat ceyet, este emvhayvtes.

15 Mohmen Cusvlke em estomahet, E'mvhahoyvte sekot omat, estomet heyv este cokv kerre haks? makakvtes.

16 Cesvs em vyoposkatet, $\mathrm{Vm}$ muhakv cvnake tokot omes, a 'crtotate enaket omes, kicakvtes.

17 Estimvt mv komaten celayen omate, mvhakv kerrvres, Hesaketvmeset ome tate, monkat vnet e opunvyecit okin omate.

18 Estet e opunvyecen omate e vrakkueckvn hopoyet omes, momisem este atotate vrakkuecetvn hopoyen omat, mvt mehenwvt omes; momen fvecetv-tokot vpikekot omes.

19 Mose vhakv ce'mvkekat haks? Momaten hvmkusatskis vfastatskekot omes. Estomen cv'lecvranet es fullatske haks? kicakvtes.

20 Este em vyoposkaket, Puyvfekcvholwvken ocetskes. Estimvt ce'lecetvn komet es are haks? makakvtes. 21 Cesvs em vyoposket, Vtotketv hvmken mehcin, omvlkvt estomahes komatsket omes, kicakvtes.

22 Monkv Mose svkvmsesvn ce'mvkvtet omes. (Mose enake tokot, Erkvlke enaket omvtes.) Momaten Nettvfekvpkv hunvnwv svkvmsisatsket omes.

23 Mose 'mvhakv kvckekaren, Fekepkv-nettv ofvn svkrmsesvn hunvnwvt en hecken omate, hunvnvwvt Fekepkv-nettvn temvpoken cvfeknihcryaten estomen vn homecatske haks?

24 Nake-en heckv vcrkvye ohfvecehcatskvs momis fvccen ohfrececvkes, kicakvtes.

25 Momen Celuslvme este vpokvte, Mv elecvrane hopohoyate heyv toko haks? make sasvtes. 
26 Momis fekhvmket opunayēs, hecvkes, momen naken kihocekot omes; 'mvpvkvlke heyvt Klist mehenwvt on kerrvkepet ome haks?

27 Momis heyv este estrn vte tate kerrēs, momis Klist vlakofv, nvpvt estrmimvn vte tate kerrēkares.

28 Mohmen Cesvs cuko-vcako ofv este mvhayofvt, $\mathrm{Cv}$ kerratskes, momet estrmvn vtvye tate kerratskes, momen vne komvyatet vlaket omvkot omes, momisem a 'crtotate mehenwvt omes, mvn kerratskekot omes, maket huehkvtes.

29 Momis vne kerris, mvn a en kvpvket omikv, momen mvt a 'crtotet omes, kicakvtes.

30 Mohmof esetvn komakvtes, momis nvpvt enke es hvlvtekates, em oketvt vlvkeko munket omekv.

31 Momen este sulket oh este vkvsvmakat, Klist vlakofvn nake momeceko tetayate heyv este momecat es en hoyvnen momecrr haks? makakvtes.

32 Falvsevlke este sulket ehkuse nake mv omaken es ete'm punahoyen pohakof, Falvsevlke momen plestrlke en homvhhotvlket este-en-wiketvn vtotvtes esvkvren.

33 Mohmen Cesvs e'te'm punayet hofunusekon ece'pakvkvyvtet a 'cvtotvten oh ayvranvyet omes, kicakvtes.

$34 \mathrm{Cv}$ hopoyatskvres, momet es cr hecatskekares, momen estrn likvyate awatskeko tetayes, kicakvtes,

35 Momen Cusvlke ete'm punahoyet, Estvmvn ayet omen, eshecēkar haks? Tvlwvvlke awahaten oh ayvr haks, momet, Trlwvvlken emvhayvr haks?

$36 \mathrm{Heyv}$ nak' maketvt ome haks? Cr hopoyatskis, es cv hehcatskekos, momet estrmvn likvyat ahwatskekos, makat, makakvtes.

37 Hompetv-rakko nettv espoke, mv nettv rakkate ofvn Cesvs hueret, Estimvt ewrnhket on omat, emet vc oh atet eskepekvs, maket, huehkvtes. 
38 Estimvt escokate omen ve oh vkvsamen omat, e nvrken uerakkot uew hesaket a filınvres.

$39^{\circ}$ (Momis heyv, oh vkvsvmakat, Puyvfekcv en heckvranen opunvyecet okvtes, Puyvfekcr Veakat seko munkekv ; Cesvs vrakkueckv seko munkekv.)

$40 \mathrm{Monkv}$ heyv opunvkv pohakof, este sulket, Heyv mv owalv tat mehenwvts, makakvtes.

41 Vpvlwvt, Heyv Klist tis, makakvtes. Momis vpvlwvt, Kut, Klist Kalvlen aossvr haks? makakvtes.

42 Kut, Cokvt, Klist Tewe honvpsen osset, Tewe likvte, Pervme tvlofvn ossvres, makekat haks? makakvtes.

43 Monkv eme vrahkvn este etekvpvketvt em ocvtes.

44 Momen vpvlwvt esetvn komakvtes, momis estit enke oh ocekates.

45 Momof este-em-wiketv plest en homvhhotv, momet Falvsevlken en yicet, Estomen esawatskekisa? kicakvtes.

46 Este-em-wiketv vyoposket, Hunvnwvt, heyv hunvnwv opunvyate ome, opunvyvte seks, kicakvtes.

47 Momof Falvsevlke em vyoposkaket, Cemeu cem etehoskvke haks? kicakvtes,

48 Este 'mvpvke, monkat Falvsevlke oh vkvsamvke sase haks?

49 Momis heyv este vhakv kerrvkekot oh tvhikvket os, makakvtes.

50 Nekvtemvs, ( $\mathrm{mv}$ nerre Cesvs em vlakvtet hvmkat ot,) okat,

51 Kut, pum vhakv, em poheko munket, momet naken estomat kerret, hunvnwv ohfaccecet ome haks? kicakvtes.

52 Em vyosposkaket, Cemeu Kalvlen atetske te? Vketecet, momet hecvs; owalv Kalvle a ossekot omekv, kicakvtes.

53 Momen este omvlkvt e huten vpcyakvtes. 


\section{SETENTVCKE VIII.}

Cesvs Olefvt ekvnhvlwe ayvtes.

2 Momen hvthvyvtkusen hvtvm cuko-vcakon vlakvtes. Momen este omvlkvt en yicrtes, momen liket e'mvhayakvtes.

3 Momen cokv-hayvlke, Falvsevlke esyomat hokte hoktaren eshoyen 'sen yicvtes, momen en nvrkvpvir licof,

4 Mvhaya, heyv hokte hoktare mvketeken eshēcēs.

5 Tokvs, Mose vhakv ofv, mv omat cvton es nvfhokekvs, pu kicvtes. Momis ceme estomēn maketske haks? kicakvtes.

6 Yv makakat, vnrvpuecet, esvnrvpkvn hopoyaket, kicakvtes. Momis Cesvs cunehket enkewesakvn ekvnvn 'soh coyvtes, nak' makakat poheko omet.

7 Momen em'pohakat awicecvkekof, a lvpotiket, $\mathrm{Mv}$ naorketv oceko ece'pakvkat, mv tatet cvto 'svfvllekvs, kicakvtes.

8 Momen hrtvm cunehket, ekvnvn oh coyvtes.

9 Momen pohakat em vkerrickvt ohfvececen, vcule mahat vlicecet, hvmkvnitot osset, pokvtes, momen Cesvs entvlket ahoskvtes, momen hokte nvrkvpvn tvk huervtes.

10 Momof Cesvs, a lvpotiket, este sekon, momis hokte tvlkusen hecof, Holkte mv ece'nrapvlke estvmvn fulla? Este hvmkis ec ohfrececeko? kicvtes.

11 Pucase, nvpvt vc ohfvececeks, makvtes. Momen, Vneu ec ohfvececvks; vyvs, momet vnvcomv naoriketskvs, Cesvs kicvtes.

12 Momof Cesvs hvtvm em punvyakvtes, maket, Vnet ekvnv en hvyayvke toyis, mv a'c vevk ayat yomuckofvn yvkvpekares, momis hesaketv en hvyayvken ocvres. 
13 Momekv, Ceme tawvt e oh kerkuecetskēs, cen kerkueckv mehenweks, Falsevlke kicvtes.

14. Cesvs em vyoposket, kicakvtes, Vnet e oh kerkuecryisem, momis vn kerkueckv mehenwet os, estrmvn vtvyvte tat kerrit, momet estrmvn ayvyat kerrikv, momis centake estrmvn atvyat, momet estrmvn ayvyat onvyatskekot os.

15 Centake vpeswv vevkayen ohfvececatskēs, vne estimvn ohfvececvks.

16 Momis ohfrccecin omat, vm ohfvececkv mehenwet os, vne vntvlkekos, momis vne, momen Erkeu a 'cvtotvten tepakes.

17 Hvtvm, Este hokkole kerkuecat mehenwvt os $m a-$ ket, cem vhakv take ofv coket os.

18 Vnet e oh kerkuecryat hvmkat toyis, momen Elke mv a'crtotvtet vc oh kerkuecet os.

19 Momof, Cerke ta? kicakvtes, Cesvs vyoposket, Centake, vne tis, Curke tis kerratskeks, vne cv kerratskvten omat, Cvrkeu kerratskvnt omekv.

20 Heyv opunvkv Cesvs cvto-kunawv hute ofvn opunayet okvtes, cuko-vcako ofv emvhayof, momen estimvt enken oh wrkecekates, en hvse-vkerkvt hvte vlvkeko munkekv.

21 Ayis; cv hopoyatskvres, momet cen naorketv ofvn pvsatkatskvres, estvn ayvyat, awatskeko tayes, hvtvm Cesvs kicakvtes.

22 Estvmvn ayvyat awatskekot os makekv, e elecvr haks? momof Cusvlket makakvtes.

$23 \mathrm{Kvncvpr}$ a en kvpvke toyatskes, hvlwe a enkvpvke toyis, ekvnv a en kvpvke toyatskes ekvnv a en kvpvke toyvkos, Cesvs kicakvtes.

24 Monkv Cen naorketv ofvn ce prsvtkvkvres ce kicvkvyis, mv toyvyat vkvsvmatskekon omat, cen naorketv ofvn ce prsvtkvkvret okv. 
25 Monkv, Esti toyetska? kicakvtes. Momen, $\mathrm{Mv}$ enhvteceskv vteke ce kicvkvyvte, Cesvs kicakvtes.

26 Nake sulken ec opunvyeckv take, momet ohfvececetv take ocis, momis a 'cvtotatet mehenwes, momen nanvke em pohvyvtet mvn ekvnvn 'sem punvyis, kicakvtes.

27 Erken em opunvyecat kerrvkekates.

28 Este Echuswr kvwahpatskof, momof $\mathrm{mv}$ toyvyat, momet vntvlkusat naken momecvkat, momis Cvrke v'mvhayvten, mvn opunvyvyat kerratskvres, Cesvs kicakvtes.

29 Momen eme a 'crtotatet ve vpaket os, Erket vntvlkusen $\mathrm{cv}$ wikekos, mv nanvke afvcecicat mecvye munkvt omekv.

30 Heyvn makof, sulket oh vkvsvmakvtes.

31 Monkv Cusvlke oh vkvsvmakan em punayet, Vm punvkv ofvn fekhonnatsken omat, mehenwusen vn kerrepvlke toyatskvres.

32 Momet mehenwvn kerratskvres, momen mehenwvt cen recapvkvres, Cesvs kicakvtes.

$33 \mathrm{Em}$ vyoposkaket, Eplvme honvpse toyẽs, momet nvpvn en svlvfkeyvte seks; estomen cen recvpkvkvres, maketske haks? kicakvtes.

34 Cesvs em vyoposket, Mehenwusen mehenwusen ce kicvkis, naorketv vfastat vtekat naorketv en svlvfke tes. 35 Momen svlvfke yuksv-sekon cukon liket omeks, momis Eppucet yuksv-sekon liket os.

$36 \mathrm{Monkv}$, Eppucet cen recapvken omat, mehenwusen cen recvpkvke tares.

37 Eplvme honvpse toyatskat kerris, momis cv'lecetvn hopoyatsks, vm punvkv ce 'pikvkekot omekv.

38 Cvrke vpake hecvyvtet, mvn opunayis, momen cemeu cerke vpake hecatskvtet momecatskes, Cesvs kicakvtes. 
39 Em vyoposkaket, Eplvmet purke tes, kicakvtes. Eplvme en hopuetake toyatsken omat, Eplvme em vtotketvn momecatskēs.

40 Momis hiyomat mehenwr Hesaketvmese em pohvyvtet estet cem onvyvkvyaten cv' lecetvn hopoyatskes, Eplvme heyvn momecekates.

41 Cerke em vtotketrn momecatskes, Cesvs kicakvtes. Monkv, Hoktarkv ofvn pu heckvte tokot os. Erke hvmkusen ocēs, Hesaketvmẹse, kicakvtes

42 Hesaketvmese Cerke taket on omat, vc vnokecatskēs. Hesaketvmese a en kvpahkēt, momat vtvyvtet omekv; cv fekkomat vtryvte tokot omekv, momis ēmet a 'crtotvtes.

43 Estomēton vn nakonvkvn kerratskeko? Vm punvkvn pohatskeko tayekv.

44 Cerke, puyvfekcv-holwakat a en kvpvke toyatskes, momet cerke em eyackvn momecetvn komatskes. Enhvteceskv vteken estelecvt omvtes, momet mehenwv ofvn huerekates, eme ofv mehenwv sekot omekv; lvksetvn punayof, enake aossen opunayet os; laksvt omet, lvksetv erket omekv.

45 Momis vm vkvsamatskekos, mehenwrn cem punayet okv.

46 Estomatskat naorketvn vc ohfvececatske haks? momen mehenwvn opunvyvyet omaten, estomen vm vkvsamatskeko haks?

47 Hesaketrmese v hesakat, Hesaketvmese em punvkvn pohet os. Monkv pohatskekos, Hesaketvmese $\mathrm{v}$ hesahokatskepekokv, Cesvs kicakvtes.

48 Monkv Cusvlket em vyoposket, Semelevlke toyetskes, momet puyvfekcv-holwvken ocetsks makeyat tetayen okēkat haks? kicakvtes.

49 Cesvs em vyoposket, Puyvfekcr-holwvken ocvkos, 
momis Cvrken vrakkuecis, momen cemet cv tvklecatskes, kicvtes.

50 Momis vrakkueckv e en hopoyvkos, hopoye momet ohfvececate hvmkes.

51 Mehenwusen, mehenwusen ce kicvkis, Estimvt vm punvkvn vfasten omat, estofvn elkv hehcekos,

52 Monkv Cusvlket, Hiyomat, puyvfekev holwvken ocetskat kerrēs ; Eplvme elet os, momen owalvlkeu, moman estimvt vm punvkv vfasten omat, estofvn elkvn lahsekos, maketskes,

53 Eplvme purke 'sen rakke toyetska? mv elvtes momen owalvlke pvsvtkes, Estĩmvn e hayetske haks? kicakvtes.

54 Cesvs em vyoposket, Vnet e vrakkuecin omat, vme vrakkueckv nake toks, Vc vrakkuecat Cvrkets; mv pun Hesaketvmeset makatskat.

55 Momen emen kerratskekates, momis kerris, momen kerrvks makin omat, laksv centake omares, momis kerrit em punvkvn vfastĩs.

56 Eplvme Cerke vn nettv hecetv afvcke tates, momet hecet afvckvtes, kicakvtes

57 Monkv Cusvlke, Ohrolope palecahkepat oretskeko munkat, Eplvme hecetskvt haks? kicakvtes

58 Mehenwusen ce kicvkis, Eplvme seko munkof, toyis. Cesvs kicakvtes.

59 Monkv cvton ayocet svfullvkvranvrtes. Momis Cesvs ehket, momet cuko-vcakan osiyet, ete'n nvrkvpvn eteropottet vyepvtes.

\section{SETENTVCKE IX.}

Mомеn Cesvs, hoyanof, hecrtes, este-hunvnwv turheceko em munkv heckaten.

2 Momen en kerrepvlket em'pohet, Pucase, estimvt naCreek. 
orkvtet on ome haks? heyv hunvnwvt, monkat eckvlke naorkvkvtet on, turhecekot heckvtet ome haks? makakvtes.

3 Cesvs em vyoposket, Heyv hunvnwv naorkvtet, momen eckvlket naorkakvten omekos, momis Cehofv 'mvtotketv eme es kerkvren omes.

4 A'crtotate em vtotketvn rtotkvranvyat tvlkus nettv emunkofvn, neret vlaket os, momof nvpvt vtotikekos.

5 Ekvnvn arvye vtekat, ekvnv en hvyavvke toyis.

6 Hvmmake opunayofvt, ekvnvn oh tofiket, tofketvn fakken es hayet, hunvnwy hecekat turwvn es en siyvtes.

7 Momen kicvtes, Ayet, vkhvse Silovm likan er es okkosvs. Momen ayet, er es okkoset, hece haket er vlakvtes. (Silovm maketv Vtothoye maketrts ohyvtekhoyen omat.)

8 Monkv em rwolicvlke, momen turhecekat homv hecakvte, Kut, heyv este nakvpohet, likvnke toko haks? makakvites.

9 Vpvlwvt, Heyvt mv tes, makaken, vpvlwvt $\mathrm{Mv}$ en heckv mahus, makakvtes. Emet, $\mathrm{Mv}$ toyis, makvtes.

10 Momen, Estomen ce turwv renayakvt haks? kicakvtes.

11 Em vyoposket, Hunvnwv, Cesvs kihocat, fakken hayet, ov turwvn'svn siyiyet, Ayet, vkhvse Silovm likan er es okkosvs, cv kicen, ahyit, rokkohsit, momet cv hece hakes.

12. Monkv, Estvn are haks? kicakvtes. Kerrvks, kicvtes.

13 Momen, mv turwv-hecekaten Falvsevlke 'sen yicvtes.

14 Momen, nettrn Cesvs fakke hayet, turwr en hauhicat, Fekvpkv-nettr tates.

15 Momen hvtvm Falvsevlke, Estohmet eturwv hece haket omat em'pohakvtes. Momen, Fakken cv turwvn 'svn siyiyen, oklsosvyvnks, momet hecis, kicakvtes. 
16 Momekv, Heyv hunvnwv Hesaketvmese a en kvpvke toks, Fekvpki-nettv vevyecekokv, Falvsevlke vpvlwvt makakvtes. Momen vpvlwvt, Estomet este naorkv tatet heyv nake este momeceko tayat momece taye haks? makakvtes. Momen estet etekvpakvtes.

17 Este turwv-hecekan hvtrm, Mv ce turwv cen hanhicvte estomes kicetska? kicakvtes? Owalv tes, makvtes.

$18 \mathrm{Mv}$ turwv-hecekate renakv en heckvte ohfvecv Cusvlke vkvsvmvkekot, mv renakv en heckvte eckvlken enhohike tvlkvtes.

19 Momet em'pohaket makakvtes; Heyv ceppuce take, mv turwv-heceko heckvte makatskat te? Momat, Estomet hiyomat hecet ome haks?

20 Eckvlket em vyoposkaket, Heyv puppuce tat kerres, momet eturwv-hecekot heekvtes, makakvtes.

21 Momis nake omecicēn hiyomat hecet omat kerrēkos, momet estimvt eturwv en hauhicat kerrēkos. Em vculkv ores. Emen em'pohvkes. E em opunvyepvres.

22 Heyv opunvkvn eckvlke opunahoyvtes, Cusvlken em penkvlakekv, Estimvt mvt Cesvs tat maken omat, mekusvpkv-cukon ossihocvres, : maket, te'm wkvsvmvkepet omekv.

23 Momet okv, eckvlke, Veulkv ores, empohvkes, makaket okakvtes.

24 Momen hvtvm este eturwv-hecekaten enhohiket, Hesaketvmesen vkrswmkvn emvs; heyv hunvnwv naorkvt omen kerrēs, kicakvtes.

25 Monkv em ryoposket, Este naorkv tat kerrvks, momis nake hvmken kerris, ev turwv-hecekate, hiyomat hecis, kicvtes.

26 Naken ce 'stomecat haks? Estomet ce turwv' cen hauhicat haks? hvtvm kicakvtes.

2.7 Momen em vyoposket. Cem onayvkin hoyvhnes, 
momen pohatskekises. Estomen hvtvm pohvranatska? Ceme takeu en kerrepvlke hakepvranatska?

28 Momen nakhayekot, Ceme mv en kerrepv toyetskes, momis pun tat Mose en kerrepvlke toyepēs, kicakvtes.

29 Hesaketvmeset Mose ete'm punayvtet omen kerrēs, Momis heyv este tat estvmvn vte tat kerrēkos.

30 Momer hunvnwvt em vyoposket kicakvtes, Heyv ofvn nake estomahat oces; estrmvn vte tat kerratskekon, ev turwvn vm en hauhicet omes.

31 Mome estomis, Hesaketvmese este naorkvlke em apohicekot omat kerrēs ; momis este Hesaketvmese emekusapvt ot, nake komaten momecen omat, mvn em apohicet os.

32 Enhrteceskv vtekis este eturwv-heceko heckvtet estehunvnwv eturwv en hauhicat pohohvte sekos.

33 Heyv este-hunvnwv Hesaketvmese a en kvpvke tokon omat, naken momehcekos.

34 Em vyoposkaket, kicakvtes; Naorketv ofv mvkvteken ce heckvtot ot omat, pu'mvhayetske haks? kihcet ossicakvtes.

35 Ossihocvten Cesvs pohet, momet eshecof, Hesaketvmese Eppuce oh vkvsametske te? kicvtes.

36 .Em vyoposket, Pucase estimvt te, oh vkvsvmepvye tayat.

37 Ceme hecetskvtes, momen mvt ce te'm punayet okes, Cesvs kicvtes.

38 Momen, Pucase vkvsamis maket, momet emekusapv tes.

39 Ohfvcceckvn yvmv ekvnv vlvkvyvtet omes, mv hecvkekate hecvkvren, momen $\mathrm{mv}$ hecakvte hecvkeko hakvren, Cesvs makvtes.

40 Momen Falvsevlke mv vpvkvkvtet heyv opunvkv pohakof, Pumeu pu hecvkeko-haks ? kicalsvtes. 
41 Ce hecvkekot omen omat, naorketv ocatskekvntos, momis hiyomat, Hecẽs, makatskekv, cen naorketv ece'pakvkes, Cesvs kicakvtes.

\section{SE'TENTVCKE X.}

Menenwvi, mehenwrn ce kicvkis, estvt yvpefikhute em vhauken eceyekot, momet etvmin vcemket on omat, mvt horkopvt ot, momet nakestrpenkvlecvt os.

2 Momis estet vhauken eceyet on omat, mvt yvpefikvvhecicvt os.

3 Mvn vhaukvhecicvh en hvwecet os, momen em punvkv-en hake yvpefikv pohet os; momen en yvpefikv hocef huecet en huehket os; momet en homahtet es osset os.

4 Momet en yrpefikv vpeyicof ehomvn ayet os, momen yvpefikv vcrkvpeyet os, em punvkv-en-haken kerrvket omekv.

5 Momet este kerrekat vcrkvpehyekos, momis em pefatkes, este kerrekat em punvkv-en-haken kerrvkekot omekv.

6 Heyv svhopakvn Cesvs es em punayet omvtes, momis nake tat kerrvkekates, mv em punayat.

7 Momen Cesvs hvtvm kicat, Mehenwvn, mehenwvn ce kicvkis, vnet yvpefikv 'mvhauke toyis.

$8 \mathrm{Cv}$ homv yicvte omvlkvt horkopvlket ot, momet nakestrpenkvlecvlket os, momis mv yvpefikv em apohicekatet os.

9 Vnet vhauke toyis, vne cv tehoyvnen este estomet eceyet on omat, invt hesakvres, momet eceyet, er a osset nanopkv hecvres.

10 Horkopv vlvkekos, horkopkin, momet eleckvn, momet nak' yvmahketvn komeko vtekat. Mvt hesaketv en heckvkvren vneh vlvkvyvtet os, momet 'res en sulket en heckvkepvren. 
11 Vnet yvpefikvhecicv herat toyis; yvpefikvhecicv herat yvpefikv vrahkv en hesaketv wrkecet os.

12 Momis este hvlwehocat tat, momet yvpefikvhecicv tokot, yvpefikv e pucase tokon omat, yvhv vlaken hecis, yrpefikv wihket, letkes, momen mv yvhv cawes, momet yvpefikvn vwaheces.

13 Este hvlwehocat letkes, hvlwehocet omet, momet yvpefikv vfekcvkhekot omekv.

14 Vnet yvpefikvhecicv herat toyis, momet vn yvpefikv kerris, momen cvnake cr kerret os.

15 Erket ov kerrat etvpomen vneu Erke kerrvyet os. Momen vnet yrpefikv vrahkv vn hesaketr wrkecit omis.

16 Momet yvpefikv etv ocvyet os, heyv hute a 'sossat tokon, mvu 'svlvkvranvyet os, momen mvt vm punvkv pohvkvres, momen fulle hvmkusvres, momet yvpefikvhecicv hvmkusen.

17 Monkv Cvrkeh vc vnokecet os, vn hesaketvn wrkecryet omekv, hvtvm eseparet.

18 Este estomet vm esekot os, momis vne mahusatet wrkecvyet os, wrkecetv yekcetv ocvyet os, momet hvtvm ësetv yekcetv ocvyet os. Heyv vhakv Crrken em esvyvtet os.

19 Monkv hvtrm Cusvlke etekvpakvtes, heyv opunvkv omecicen.

20 Momen sulket Puyvfekcr-holwvken oces, momet haco-hakes; estomen em apohicatske haks? makakvtes.

21 Vpvlwvt, Heyv puyvfekcv-holwvke oce em punvkv tokos. Puyvfekcr-holwvket turwv hecrkekan renahuece taye haks? makakvtes.

22 Momen mekusvpkv hompetv-rakkot Enkinev kihocat, Celuslvmen ocvtes, momet rvfo tates.

23 Momen Cesvs cuko-vcakat ofvn Salomv 'sem ohvpettv kicetvn yvkapvtes. 
24 Momen Cusvlke yicet vnvkaftvtes, momet, En hofune estomen vkvsvmēkon pu hayetske haks? Klist toyetsken omat kerkv heren pum onvyrs, kicakvtes.

25 Cesvs em vyoposkaket, Cem onayvkvyis, vkvsvmatskekos, Cvrke e hocefkv ofv vtotkvyat, mvt ve oh kerkuecet os, kicvtes.

26 Momis vkvsvmatskekos, vn yvpefikv toyatskekokv, ce kicvkryat etrpomen.

27 Vn yrpefikv vm punvkv-en-haken pohvket os, momen kerrakis, momen ve verkvpeyet os.

28 Momen hesaketv yuksv-sekon 'emakvyet os, momet estemerrahkekos, momen nvpvt cvnke 'svm ossiceko tayet omvres.

29 Crrke mv r'mvte tat, omvlkvn'sen rakket os, momen estimvt Crrke enke 'sem ossice tayat yekcetv ocekot os.

30 Vne Crrke tepakeyat hvmkus.

31 Momen Cusvlke, cvton es nvf ketvn komaket, hvtvm crto crwakvtes.

32 Cesvs em vyoposkaket, Vtotketv herake sulken Crrke a en kvpvke ce hecicrkvyes; mv vtotketv estomaten crto es ev nvf katske haks? kicvtes.

33 Cusvlke em vyoposket. Vtotketv herat tat crto es ce nvfkēkos, momis Hesaketvmese tvklecat ornecicen, momet este toyetskatet Hesaketvmese e hayetskekv, kicakvtes.

34 Cesvs em vyoposket, Hesaketvmese toyatskes mahkis, cem vhakv take ofvn es hoccet omeko haks? kicvtes.

35 Hesaketvmese em punvkv em vlakvten hesaketvmesen kicvten omat, momen cokvrakko kvckeko tetayen omat,

36 Erke vcake haye, momet ekvnv atotvte huerat, Hesaketvmesen tvklecetskes, kicatske haks ? vnet Hesaketvmese Eppuce toyis, ce kicvkikv. 
37 Cvrke em vtotketvn vtotkvkot on omat, vm vkvsahmatskvs.

38 Momis momecin omat, vm vkvsamatskeko estomis, vtotketvn vkvsvmvkes, Crrke vnen vc vpvket omen, momet vneu emen vpakvyet omen kerret, momet vkvsvmatskvret.

39 Monkv hvtvm esetrn komakvtes, momis enke em ossakvtes.

40 Momet Cotvne tvpalvn vhyet, enhvteceskv Cane este paptisvte ocan er orvtes, momet mvn fekhonnvtes.

41 Momen sulket $\mathrm{v}$ fullvtes, Cane tat este-nake-momeceko-tayat momecekatet os, momis Cane heyv este opunv. yecrte omvlkvt fvecvt os, makaket.

42 Momen mvn sulket oh vkvsamakvtes.

\section{SETENTVCKE XI.}

Momof Pervne tvlofvn, Mele Mare etecakkat eme tylofv, este-hunvnwv Lisvle hocef ke liket enokke tates.

$2 \mathrm{Mv}$ Melet omvtes, Pucase em vfeye, éle ekv-esse es en hvsvtecrte, mv e cerwvt enokket ot omvtes.

3 Monkv e wrntake oh 'totvtes, Hecrs Pucase, mv vnokecetskat enokkes, makaket.

4 Cesvs pohof, Heyv enokketv elkv orekot os, momis Hesaketvmese vrakkueckv vrahkv tes, mv eteropotten Hesaketvmese eppuce vrakkuehoce tayat os, makvtes.

5 Momen Cesvs, Mare, momen etecakkat, momen Iisvle esyoman vnokecrke tates.

6 Monkv, enokkveuken pohof mata likvtet nettv hokkolen fekhonnvtes.

$7 \mathrm{Mv}$ hoyanof, Cutevn hvtvm vpeyvkveres, en kerrepvlken kicvtes,

8 En kerrepvlket okat, Mvhiya Cusvlket hvte rwole 
cvton es ce nvfketvn komakvnks, moman hutvm mvn vyvranetske haks? kicakvtes.

9 Cesvs ryoposket, Nettr hvmkat hvse-vkerkv palen hokkolohkaket omeko haks? estimvt neitvn yvkapen omat, elentapiyekos, heyv ekvnv en hvyayvken hecekv.

10 Momis estimvt neren yvkapen omat, elentappes, hvyay rket vpikekot omekv, kicvtes.

11 Heyv nanvken mahkof, erenyupv un hesse Lisvle nocet omes, momis mv nocan ahonecicaret ayvyet os, makvtes.

12 Momof en kerrepvlke em punayat, Nocet on omat, en hervies.

13 Momis Cesvs em elkvn opunvyecet okvtes, momen fekapet nocet on okēs, komakvtes.

14 Monkv Cesvs em punayat, kerkusen, Lisvle elet os, kicakvtes.

15 Momen ece 'rahkv taken vrvkate vc afvckes, centaket vkvsamatskvren. Momis oh vpeyvkeres.

16 Momen Tamrse, Tetemvs kihocat, kerrepvlke em etohkvlketrn kicrtes, Pumeu vpeyvkeres, momen vpaket prsvtkeyrres.

17 Cesvs vlakof, entis en hopelkv ofv nettr osten wakkepen hecrtes.

18 Momen Pervne Celuslvme em vwolet omvtes, vkerkv hokkolet mahet.

19 Momen Cusvlke sulket Mare Mele tepakan en yicvtes, e'mahlvpvtvret e cerwv ohfvcevn.

20 Momen Mare Cesvs vlakvevken pohan vpaket, vnrvpvranet ayvtes, momis Mele fekhonnet cuko ofvn likvtes.

21 Momen Maret Cesvs em punayat, Puease yvmvn aretskvten omat, ov cerwv elekvntos.

22 Momen hiyome estomis, Hesaketvmese nak' estomen em'pohetskat, Hesaketvmese ce'mvret on kerris. 
23 Ce cerwv hvtvm akvwrpkvres, Maren Cesvs kicvtes. 24 Maret a em punayet, Nettv-espoke akvwrpketv omof hvtvm akvwvpkvret on kerris, kicvtes.

25 Cesvs Maren em punayat, Vnet akvwrpketv toyit, momet hesaketv toyis, estimvt ve ofvn vkvsamat, elvtet 0 estomis, hesakvres.

26 Momen estimvt o estomis hesaket, ve ofvn vkvsamat, estofis ehlekos. Heyv vkvsvmetske te? kicvtes.

27 Maret em punayat, Henka, Pucase, Klist Hesaketvmese Eppuce yvmv ekvnvn vlvkvranvte toyetsken vkvsamis, kicvtes.

28 Momen, mvn momen mahkof, ayvtes, momet etecakkat Melen ehken en huehkvtes, Pucaset vlakes, momet cen huehkes, kicet.

29 Mvn pohan vpaket, Melet, lvpken ahuyiret, a oh atvtes.

30 Hiyomat, Cesvs tvlofv a eceyeko munket, momis mv Mare er vnrapvte tan fekhonne tates.

31 Cusvlke mv e'mvhlvpvtvkvrane vpake cuko ofv vtehkvte, Melet lvpecicet ahuyiret, ossen hecakof, vcrkvpeyvtes, Este-hopelkvn oh ayet er hvkihkvranet os, kicaket.

32 Momis Mele, mv Cesvs fekhonnan vlaket, hecof, elen v tvk lvtiket, Pucase, yvmvn aretskvten omat, cv cerwv elekvntos, kicvtes.

33 Momen Mele hvkihken, Cusvlke vpvke yicateu hvkihhoken Cesvs hecof, puyvfekcr ofvn hihket, fekherekates.

34 Momen Estvmv wvkecatske haks? kicen, Pucase, ahtet, hecvs, kicakvtes.

35 Cesvs hvkihkvtes.

36 Momen Cusvlket. Hecvkes, vnokece heraten makakvtes.

37 Momen, Heyv hunvnwv eturwv hecrkekat tis en 
hauhicet ont omat, heyv este-hunvnwv elekare tayen hayeko tayvt haks? vpvlwvt makakvtes.

38 Monkv Cesvs hvtrm eme ofvn hihket, hopelkvn vlakvtes. Crto-hauket omvtes, momen crtot oh liket omvtes.

39 Crton akueyicvks, Cesvs kicrtes. Maret, mv elat e wrnwvt, Pucase, hiyomat fvmpepēs, elvte nettv ostekv, kicvtes.

40 Momen Cesvs em prinayat, Vkvsvmetsket on omat, Hesaketvmese en rakke hecetskvres ce kicvkisa? kicvtes.

41 Monkv ele wakkat cvton vkuehoyvtes, Momen Cesvs e turwr hvlwen v kvwapket, Cvrke, ece 'kvsamis, vm apohihcetskekv, makvtes.

42 Momet estofis vm'pohetsket omat, kerrvyes, momis este heyv svpaklat pohet, cent a 'crtotetskvtet omat kerrvkeprren okvyis, makvtes.

43 Momen mv mahkof, yekcen huehket, Lisvle a ossvs, makvtes.

44 Momen elvtē a ossvtes, hopele vtceckv enke eleu es vpvllvket, momen e turofv enockv es vprllvket. Momen En recohpatsken, vyepekvs, Cesvs kicvtes.

45 Monkv Cusvlke sulke Mele en yicat, momen Cesvs nake momecan hecakof, eme oh vkvsvmakvtes.

46 Momis vpvlwvt, Falvsevlken oh vpeyet, nak' estomvken Cesvs momecat en kerkuccakvtes.

47 Momen 'plestvlke en homahtvlken, Falvsevllken esyomat e vteloyet, nvkaftet, okat, Naken estomēn ome haks? Heyv hunvnwv nake-este-momeceko-tetayat sulken momecet os; makakvtes.

48 Hiyomen wikēn omat, este omvlkvt eme ofvn vkvsvmvkvres, momen Lomvnvlket yihcet, pum etvlwv ekvnv tepaken pu'konahvres.

49 Momen hrmket, Kjefvs hocefket, mv ohrolope ofv 
plestvlke en hohmahtvt omvtes. Mvt okat, Naken kerratskekot os ;

50 Momen este hvmket este em elaten, etvlwv omvlkvt yvmahkeko tayet, es pun here tayet omat, vkerricatske- . kot os, kicakvtes.

51 Heyv emet e opunvyecet okekates, momis mv ohrolope ofv plestvlke en homahtvt omekv, Cesvs mv etvlwvn em elvranet omen owalet okvtes.

52 Momet $\mathrm{mv}$ etvlwv tvlkekot, momis Hesaketvmese en hopuetakuce vwahat omvlkvn hvmkusen etoh vteloyvranet os.

53 Momen $\mathrm{mv}$ nettr vteke ayat, Elecrkes maket, et'empunahoyakvtes.

54 Momekv Cesvs Cusvlke hecke vpake vreko hakvtes, momis vnrvwr vnakv ekvnv liken ayet, tvlofv Eflevm kicetvn eceyvtes, momet mvn en kerrepvlke esyomat fekhounvtes.

55 Momen Cusvlke em ohhoyvnkvt vwolice tates, momen este sulket, ohhoyvnkv hvte oreko munken, Celusivme oh vpeyakvtes, e hvsvtecvkvranet.

56 Monkv Cesvs hopoyakvtes, momet cuko vcakat ofv svpaklet Estomen vkerricatske haks? hompetv-rakko vlvkekar haks maket etem punahoyvtes.

57 Momen plestrlke en homahtvlke, Falvsevlke esyomat, ese estvmēt estvmvn hecke tayen kerren omat, onayen, este tayet opunvkv ossicet omvtes.

\section{SETENTVCKE XII.}

Momen ohhvyvnkv oreko nettv epakat vhoske munken, Cesvs Pervnen er orvtes, Lisvle ehle ahesayecvte likan.

2 Mvn hompetv-rakkon en hayakvtes, momen Maret ohvfastvtes, momis Lisvle Cesvs vpvke hompvrane rpokan hvmkat tates. 
3 Momen Mele neha fvmece espeknv kihocet, veake heren, vtarkv hvmken ohmet, Cesvs elen 'sen siyiyet ekvessen 'sen hvsvtecrtes. Momen mv neha en frmece cuko fackvtes.

4 Momen en kerrepvlke hvmket Cutvs, Simv eppucet, wiyvrane hueratet,

5 Estomen heyv neha tokunawr pale ostrteken wiyiyet estemerhoyan emhoyekat haks? makvtes.

6 Heyv makat este estemerhoyan vkerricet okekates, momis horkvpvt omet, sukcvn esarēt omet, momet nake ytehkan esarēt omekv okvtës.

7 Momis Naorihcatskvs, cv hopelkvrane nettv vrahkvn. veayecrtet os, Cesvs kicvtes.

8 Estemerhoyat ece'pakvke ocatske munkvt os. Momis vntat ocatske munkv tokot omekv.

9 Monkv Cusvlke sulket mv fekhonnat tat kerrakvtes, momet a oh vwakvtes. Cesvs vrahkv tvlkekon, momis Lisvle elkv a en kvwapvte hecetv komat omakvtes.

10 Momen plestrvlke en homvhhotat, Lisvleu elecvkes maket, tempunahoyvtes.

$11 \mathrm{Mv}$ omecicen Cusvlke sulket vpehyet Cesvs oh vkvsvmvkepekv.

12 Rempvksen este sulket hompetv-rakko yicet, Cesvs Celuslvmen vlakveuken pohakof,

13 Talv elvecen es crhwet vpeyvtes vnrvprranet, momet huehoket, Hosanv! ohmerrvkvtēs Eslevl em mekko, Hesaketrrnese hocef $\mathrm{kv}$ of $\mathrm{v}$ vlakat, makaket.

14 Momen Cesvis Corakko-wakv mvnettusen eshecof, mvn ohlikvtes escokat omet.

15 Ce penkahlates, Sion echuste toyetskat; hecrs, cem mekko vlakes, corakko-wakv echuswvn obliket.

16 En kerrepvlke heyv nanvke kerrvkekates enhvteceskv; momis, Cesvs vrakkuehohcof, heyv nanvke emen 
opunvyecet cokat omet vkerricakvtes, momen heyv nanvke em momecakvten.

17 Momekv mv este vprkvke Lisvle en hohike en ho. pelkv aossicvte elkv 'rakvwapvte kerkuecakvtes.

18 Este momeceko taye momecrten pohvket ok, heyv omecicen este en yicet omakvtes.

19 Nake estomatskekat hecatska? Falvsevlket ete kicakvtes. Hecvkes, este omvlkvt vcrkvpeyēpes.

20 Momen emekusvprrane hompetv-rakko yican Klekvlket vpvke tates.

21 Matvwvt, Felvpe Kalvle ekvnv Persitv tvlofv aossan en yicet, Pucase, Cesvs hecetvn komēs, kicakvtes.

22 Momen Felvpet vlaket Antulvn em onayvtes, momen hvtvm Antulv Felvpen tepaket Cesvs 'monayvtes.

23 Momen Cesvs em vyoposkaket, Hvse-vkerkv vlakēs, este Echuswv vrvkkuehocvranat, makvtes.

24 Mehenwvn, mehenwvn ce kicvkis, Teleko nerkv ekvnv oh lvtiket lekweko vtekat emunkis, entvlkuset liket os, momis lekwen omat, em ette sulkên hayes.

25 Estimvt en hesaketv vnokecat sumecicvres, momis estimvt en hesaketv en homece yvmv ekvnv huerat, hesaketv yuksv-sekon vcayecepvres.

26 Este vm vtotken omat, vc vcrkvyekvs momen estvmvn likvyat mvn vm vtotkv likvres, momen este vm vtotken omat, mvin Cvrket hvlwecvres.

27 Hiyomat vm puyvfekev fekhereks, momekv naken makar haks? Crrke, heyv hvse-vkerkv enkvpvken cv hesayecrs; momis heyv vrahkvn heyv hrse-vkerkv vlakvyet os.

28 Cvrke, ce hocefkvn vrakkuecrs. Momof opunvkven-hake hvlwe atatet vlakvtes, Vrvkkuecvyvtet omet, momet hvtrm vrvkkuecares maket.

29 Momekv mv este vnakv svpakle pohakat, Tenetikes 
makakvtes, vpvltaket, Hvlwe-estet em punayet os, makakvtes.

30 Cesvs em vyoposket makvtes, Heyv opunvkv-en-hake vne ve vrahkvn vlaket omekos, momis ce 'rahkv taken omès.

31 Hiyomat heyv ekvnv ohfvecet os, hiyomat heyv ekvnv em mekko ossihcet vwihokvres.

32 Momen vnet, ekvnv en kvpvket cv kvwrpken omat, este omvlkvn a e oh hvlvtarēs.

33 Heyv makat elkv estomen elvranat kerkuecat okvtes.

34 Estet em vyoposkakat, Klist hesake emunkvt os, vbakv aossen makat poheyvtet os, momen estomen, Este Echuswr kvwvpkvranet os, maketske haks? Estimvt heyv este Echuswrt o haks?

35 Hvte vhofunusekon hryayvke ece'pakvket os; yvkvpvks, hvyayvke ocatskof, yomucket ec oh vlakvke wites; mv yomucke ofv yvkapat, estvmvn ayat kerrekot omekv, momof Cesvs makvtes.

36 Hvyayvke ocatskof, hvyayvke ofvn vkvsvmepvks, mv momatskat, hvyayvke en hopuetakuce toyatskvren. Heyv nanvken Cesvs opunvyet, momet ayet em ehkakvtes.

37 Nake este momeceko tayat sulken ehomvh momecet omis, oh vkvsvmvkekates.

$38 \mathrm{Mr}$ Esivs owalv nak' makvte es oh fvckvren, mv opunvye, Pucase, estimvt nake kerkueceyat vkvsame haks? Momen estimvn Pucase e sakpv hecihoce haks?

39 Momekv oh vkvsvmvkekates, Esivs hvtvm makvtet omekv,

40 Eturwv hecrkekon hayet, momet efeke en wrnwvheces, momet e turwv es hecrkekot, nomet efeke es kerrvkeko, momet fulotēcekot momen en wicecaren. 
41 Esivs heyv nanvke makat en rakken hecet opunvyecofvt okvtes.

42 Mome estomis evpayvlke rvkrakat sulket oh vkvsvmakvtes, momis Falvsevlke omecicen onvyvkekatēs, mekusvpvkv-cukon sossihoce tayekv.

43 Este vkvsvmvre mimvn Hesaketrmese vkvsvmvre 'semuntvlen vnokecvkvtet omakekv.

44 Cesvs huehket makvtes, Estimvt vc oh vkvsamat, vnen ve oh vkvsamat omekos, momis mv a'c vtotvten oh vkvsamet omes.

45 Momen estimvt cv hecat a'c vtotvten hecet os.

46 Vnet hryayvke ekvnvn vlvkvyēt os, estimvt ve oh vkrsamat yomucke ofv vfekhonnekaren.

47 Momen este vm opunvkv pohet vkvsvmekat ohfvececvkot os; ekvnv ohfvececvranit vlakit omvkos, momis ekvnvn hesayecvranit vlakit omekv.

48 Estimvt cv hvlvtekot, momet vm opunvkv esekon omat, hvmket ohfvecat ocet os; opunvkv opunayvyvtet, mvt ohfvececvres nettv espoken.

49 Vntvlket opunayet okvkos, momis Erke a'c vtotvtet, emet vhakv v'mvtet os, naken makvranvyat, momet naken opunvyvranvyat.

50 Momen em vhakv hesaketv yvksv-sekot omen kerris, momekv naketo estomen opunayvyis, Erke cv kicvte omen opunayvyet os.

\section{SETENTVCKE XIII.}

Tokvs ohhoyvnkv hompetv-rakko oreko munken, heyv ekvnv osiye, Erke oh vyepvranat en hvsē-vkerkv vlaken Cesvs kerret, enake ekvnv vtehkaten vnokecvtet, eyuksv 'roren vnokecvtes.

2 Momen hompetv hoyahnof, Puyvfekev holwakat Cutrse Eskalevt, Simvn eppucen wiyvren e feken en pikvtes. 
3 Cesvs Erket nake omvlkvn enke ofvn en hayen kerret, momet Hesaketvmesen a en kvpvkvtet ot, Hesaketvmesen oh vyepat kerret,

4 Hompvte ahuyiret, kapv etvn kayvtes, momet turofeshrsvteckvn cahwet es e wrnakvtes.

5 Uewrn pvlvknvn a veahnof, en kerrepvlke elen em okkosetvn vlicecrtes, momet turofeshrsvteckv es e wrnakatet es en hvsvtecrtes.

6 Momof Simvn Petvn em vlakvtes, momen Petvt, Pucase, cvle vm okkosetske haks? kicvtes.

7 Cesvs em vyoposket kicvtes. Nake momecryat hiyomat kerretskeks, momis hvteten kerretskvres.

8 Cvle estofis okkohsetskekos, Petvt kicvtes. Cesvs em vyoposket, Ec okkosvkvn omat, ece 'rahkv ve vpvyetskeks, kicvies.

9 Pucase, cvle tvlkekon, momis cvnken, cvkvu, Simvn Petvt kicvtes.

10 Este vklopet on omat, ele tvlket okkosvre vhoskes, momis hvmkvt hvsvtket omes; momen ce hvsvthvkvkes, momis omvlkeks, Cesvs kicvtes.

11 Estimvt wiyvranat kerrekv, Omvlkvt ce hvsvthvkvket ot omeks, maket okvtes.

12 Momekv ele em okkosahkof, en kapv cahwet, momet hvtvm lihket, Naken cem momecvkvyat kerratske haks? kicvtes.

13 Mvhayv, momet Pucase cv kicatskes, momen tetayen okatskes, mv toyikv.

14 Momen vnet, Pucase tat, momet mvhayv toyvyat, cele cem vkkosvkin omat, centakeu cele ete'm okkosatske tayes.

15 Vhayetvn ce'mvkvyet omekv, mv cem momecrkvyat etvpomen momecatskvren.

16 Mehenwvn, mehenwvn ce kicvkis, vtotkv epucasen Creek. 
'sen rakkekot os, momen mv vtothoyat vtotat 'sen rakkekot os.

17 Heyv nanvke kerratsken omat, ece'fackvket os, momecatsken omat.

18 Omvlkatskan ec opunvyecvkit okvks. Estimvn en hopoyepvyvte tat kerris, momis escokat esohfvckvren, mv ve vprke tvklike homprte vc vnrvpen eleceskv kvwapes.

19 Tokvs, hvte vlvkeko munken cem onvyvkis, eshoyanof, vnet mv toyvyat vkvsvmatskvren.

20 Mehenwvn, mehenwrn ce kicvkis, estimvt vtotvyan evpayen omat, vnen $\mathrm{cv}$ 'vpryet os. Momen estimvt vnen cv 'vpayen omat, vc vtotvten evpryet os.

21 Cesvs heyv mahkof, puyvfekev ofvn fekherekot, mo. men kerkuecet makvtes, Mehenwrn, mehenwvn ce kicvkis, hvmkatskat cv wiyvranatskes.

22 Mohmen en kerrepvlket ete hecaket, estimvn opunvyecet okat en kerkvkekates.

23 Tokvs en kerrepvlke hvmke Cesvs vnokecusvtet Cesv e hokpen vccakvtes.

24 Momekv Simv Petvt, estimvn opunvyecet okat em 'pohvren, enken es em vhopayvtes.

25 Momof Cesvs e hokpen rccaket, Pucase, estimvt o haks? kicvtes.

26 Hompetvn esvklihcryof, estimvn e'mvyat, mvt mvtes, Cesvs vyoposket makvtes. Momen svklihcof, Cutvs Eskalevt Simvn eppucen emvtes.

27 Momen pahpof, Puyvfekcv-holwakat eceyvtes, mohmen, Mv momecvranetskat, Ivpken momecvs, Cesvs kicvtes.

28 Vlumhat, nvpvt kerrekates, estoman heyv ete'm punayet okat.

29 Cutvs sukcrn ocet ok, Nake pu yacan hompetv-rakko 
oreko munken nesvs, Cesvs kicet okes, vpvlwvt komaken, monkat naken estemerrakan emvren okes komakvtes.

30 Mohmen hompetv en hecikof, lvpecicet ossvtes. Momen nere tates.

31 Momekv osiyof, Tokvs Este-echuswr vrakket os, momen Hesaketvmeset eme ofvn vrakket os, Cesvs makvtes.

32 Eme ofvn Hesaketrmeset vrakken omat, Hesaketvmesen vrakkuecvres eme ofvn, momet lvpkusen vrakkuecrres.

33 Hopuetakuce, hvte hofunusekon ece'pakvkit omis. Cr hopoyatskvres momen, Estvn ayvyat vwatskeko tayes, Cusvlke kicryvnke omen, hiyomat ce kicvkvyet os.

34 Vhakv mucvsen ce'mvkis, Ete'nokecatskvren, ece'nokēcrkvyvte omen, centakeu ete'nokecatskvren.

35 Vnokeckv etem ocatsken omat, heyvn este omvlkvt es kerrvres vn kerrepvlke toyatskatehkvn.

36 Pucase, estrmvn vyetska, Simvn Petvt kicvtes. Estvmvn ayvyat hiyomat ve verk ayetskeko tayes, momis hvteten ve vcrk ayetskvres, Cesvt em vyoposket kicvtes. 37 Pucase, estomen hiyomat ece'crk ayvlro taye haks? Vn hesaketv ece' rahkv wvkecares, Petrt kicvtes. 38 Cen hesaketv ve vrahkv wvkecetskvr haks? Mehenwvn, mehenwvn ce kicis, tottolose hakeko munken vtutcenv ve vrahkv ehelvksecetskvres, Cesvt vyoposket kievtes.

\section{SETENTVCKE XIV.}

CE feke cen naorvkates; Hesaketvmese oh vkvsamatskes; vneu ve oh vkvsvinvks.

2 Cvrike hute ofv liketv sulket os; monkaten omat, onvyvkvyrnt os. 
3 Momen ayit, liketvn ce metetakuecvkin omat, hvtvm 'rvlakit, ce yvpvyvkares, vnet estrmvn likvyat, cemeu mvn vpokatskvren.

4 Momen estrmvn ayvyat kerratskes, momet ryetv kerratskes.

5 Pucase, estrmvn ayetskat kerrēks, momat estomet vyetv kerreye taye haks? Tamvset kicvtēs.

6 Vnet vyetv toyit, mehenwv toyit, hesaketv toyis; este estomet Erke oh vtekot omes, vnen cr tehoyvn etokon omat, Cesvs kicvtes.

$7 \mathrm{Cv}$ kerratskvten omat, Cvrkeu kerratskvnt os, momen mucv ayat kerratskes, momet hecatskvtes.

s Pucase, Erken pu hecicvs, momen pum etetayvres, Felvpet kicvtes.

9 Felvpe, hofune mahen ece'pakvkit omvyat, hvte cv kerretskeko munke haks? estimvt cr hecat Erken hecvtēs, moman estomen, Erken pu hecicrs, maketske haks? Cesvs kicvtes.

10 Vnet Erke ofvt omin, Erket ve ofvt omat vkvsvmetskeko haks? Opunvkv es ce tem punayvyat, vnet ce tem punayet omvkos, momis Erke ve vpikat, mvt vtotketv momecet os.

11 Vnet Erke ofvt omin, Erket ve ofvt omat vm vkvsvmvks, monkat vtotketv mahusat svrahkvn vm vkvskmvks.

12 Mehenwvn, mehenwvn ce kicrkis, estimvt vc oh vkvsamat, vtotketv momecryat, emeu momecvrēs, momet vtotketv heyv 'sen rvkrvken momecvrēs, Cvrken oh vyepikv.

13 Momen naketo estomen cv hocef kv ofvn vpohatskat, mvn momecares, Eppuce ofvn Erket vrvkkvrēn.

14 Naken cv hocefkv ofvn vpohatskat, momecares.

$15 \mathrm{Vc}$ vnokecatsket on omat, vm vhakvn vfvstvks. 
16 Momen Erken emekusvparēs, momen Emvhlrpatv etvn ce'mvkvrēs, mvt ece'pakvke emunkv tares.

17 Puyvfekcv mehēnwat, ekvnv hvlvteko tayat, hecekot omet, momet kerrekot omekv, momis centake. kerratsket os, ece 'pvkvket omekv, momet ece 'pikvket omvrẹs.

18 Ce mahlvpvtkekon ce wihkvkos; cem vlvkvkarēs.

19 Hvtetusat ekvnv svnvcomvn ev hecekarēs, momis centake tat $\mathrm{cr}$ hecatskēs; hesakvyet omikv, centakeu hesahokatskvres.

$20 \mathrm{Mv}$ nettv omof, vnet Crrke ofvt omin, centaket ve ofvt omatsken, vnet ec ofv taket omvyat kerratskvrēs.

$21 \mathrm{Mv}$ vm vhakv oce, momet vfastat, mvt ve vnokecat tes, momen mv ve vnokecat, Cvrket vnokecvrēs, momen vnet vnokecarēs, momet em e heckuecarēs.

22 Pucase, estomen pum e heckuecat, ekvnvn em e heckuecrranetskeko haks? Cutvse, Eskalevt tokot, kicvtes.

23 Cesvs em vyoposket kicvtes, Este ve vnokecet on omat, vm punvkvn veayecvres, momen Cvrket vnokecvrēs, momen oh vthoyet pum vpoketv en hayeyvres.

24 Mv vc vnokecekat, vn nak' maketv vevyecekot os, momen opunvkv pohatskat crnake tokos, momis Erke vc vtotat omes.

25 Heyv nanvke es ce tem punvyvkvyat, hvte ece 'pakvkvye munkat okvyet os.

26 Momis Emvhlapatv, Puyvfekev Veakat, Erket cv hocef kv ofv vtotvranat, mvt nake omvlkvn ece 'kerricepuecrkrēs.

27 Herkvn cem wikvkis; vn herkvn ce'mvkis; ekvnv estemat omekon ce'mvkit omis, ce feke cen naorvkates, momet cemi penkalvkates.

28 Vyepit cem vlvkvkares, ce kicvkvyat pohatskis. Ve vnokecatsket on omat, ece 'fackvke tayes, Erken oh ayis, makikv, Crke vne 'svn rakket omokv. 
29 Momen, tokvs cem onayvkis hvte eshoyvneko munken, momat eshoyvhnof vkvsvmatskvren.

30 Heyv eryupv sulke ce tem punvyvkvkarēs, heyv ekvny em mekkot vlvkvranekv, momen ve ofv naken ocekot os.

31 Momis mv Erke vnokecrye tat ekvnv kerrvien, Erke vhakv v'mvte verkvye mahusan momecryet os. Asvpvklvks, yvmvn enkvpahket, vpeyvkeres.

\section{SETENTVCKE XV.}

Vnet prrko-em-vpe mehenwv toyis, momen Curket vfìstut omes.

2 Elvece ve ofv ocat ettekat vtekat, vkueyet omes, momet elvece ettat vtekat hvsvtecet omes, ohvtvlaken ettvren.

3 Opunvkr es ce 'tem punayvkvyat eteropotten hiyomat ce hrsvthvkvket omes.

$4 \mathrm{Vc}$ ofvn fekhomnvs, vneu ec ofv take fekhonnin. Elvcce em vpen vlvce emunket omekot, entrlkat, etteko tayat, etvpomen cemeu ve ofvn vfekhonnatskekon omat, ettatskeko tayes.

5 Vnet prrko-em-vpe toyin, cemet elvcce toyatskes. Estimvt ve ofv fekhonnet on, vneu eme ofv fekhonnvyet on omat, matvwrt em etie sulken hayet omes, vnet ece 'pvkvkat naken estohmatskeko tayet omekv.

6 Estimvt ve ofvn fekhonnet omekon omat, lvece omecet rpvlvthoyen, yuknet omes, momen estet vteloyet totkvn tvkpvlaten nokret os.

7 Ve ofvn fekhonnatsken, vm punvkvt ec ofv taken fekhonnet on omat, naken moket, vpohatskis, mvt cem momvkvres.

8 Emette sulken ettakatskaten, heyv ofvn Cvrke vrakkuehocet omes; momatskofvt vn kerrepvlke toyatskvres. 
9 Curke vc vnokecvte omen, vneu ece 'nokecrkvyvtes. Cemet vm vnokeckv ofvn fekhonnvkes.

$10 \mathrm{Vm}$ vhakvn் vcayecatsken omat, vm vnokeckv ofvn fekhonnatskvres, Cvrke em vhakv vcayecryvte, momet em vnokeckv ofv fekhonnvyat omen.

11 Heyv nanvke es ce tem punayvkvyat, vm afvcketvt ec ofv taken fekhonnen, momen cem afvcketv taket esfrckvren okvyis.

12 Ece 'nokecrkvyrte omen, cemeu etenokecatskvret, heyvt vm vhakvt omes.

13 Nvpvt en hessvlke vrahkv en hesaketv wrkecat, vnokeckv heyv es en rakkat nvprt ocekot omes.

14 Nake ce kicvkvyan momecatsket on omat, vn hessvlke toyatskes.

$15 \mathrm{Mucr}$ vtekat vtotkvlken ce kichvkvkos, vtotkv tat epucase nake estomat kerrekot omekv; momis vn hessvlken ce kicvkvyet ohmis, nake Cvrke em'pohvyat vtekat omvlkvn cen kerkuecrkvyet omekv.

16 Centat v'nhopayatskeks, momis vnet ce'nhopoyvkit wiketv ce hayvkis, vpehyet emette sulken ettakatskaten, momen cem ette take oce emunkv taren, momen nake estomis cr hocef kv ofvn Cvrke em'pohatskat ce'mvkvren.

17 Heyv nanvke ce kicvkvyet omes, etenokecatskvren.

18 Ekvnvt cen homecrken omat, cen homecvkeko munket, vntan vn homecvtet omen kerratskes.

19 Ekvnv enake toyatsken omat, ekvnvh enaken vnokecet omes, momis ekvnv a en kvprken ce'nhopoyvkvyet omes, monkv ekvnvh cen homecet omes.

20 Opunvkv es ce tem punayvkvyat ce hohsvkates, Vtotkv e pucase es en rvkkekot omes, monkv vc as. secakvten omat, cemeu ece'ssecvkvres, momet vm opunvkvn vcayecakvten omat, ce $e_{-}^{-}$opunvkv takeu vcayecrkvres. 
21 Heyv nanvke omvlkvn ce momecvkvres, vn hocef $k v$ vrahkvn, mv a'c vtotvten kerrvkekokv.

22 Vlahkit ete'm punvyvkvkaten omat holwvyeckv ocvkekvnt omes, momis hiyomat holwryeckv es ohrvnkv ocvkekos.

23 Estimvt vnen vn homecet on omat, Cvrkeu en ho mecet omes.

24 Vnet vprkakit, vtotketv este-hunvnwr etv momecrte sekaten momecrkaten omat, holwryeckv ocvkekvnt os, momis hiyomat vne Cvrke eshokkoleyan, hokkolvn hecvkvtet ot, momet pun homecvkvtes.

25 Momis hiyomet os, $\mathrm{mv}$ opunvkv em vhakv take of es hocce ocat esohfvckvren, Nake omecice sekon vn homecakvtes.

26 Momis Emahlvprtecicv, mv Erke aenkvpvken a cen 'totvranvkvyat, Puyvfekcv frccat, mv Erke aenkvpakat vlakof, ve ohkerkuecvres.

27 Momen cemeu ohkerkuecatskvres, enhvteceskv vteke ve vpakatskvtet okv.

\section{SETENTVCKE XVI.}

Celentappykekaren heyv nanvke es ce tempunayvket omis.

2 Mekusvpkv-cuko a 'sec ossicvkvres, momes, hvsevkerkv vlvkvres, mv ce'lecvkat vtekat, Hesaketvmese em vtotketvn momecet omis, komvres.

3 Heyv nanvke cem momecvkvres, Erke, monkat vneu kerrvte sekot omakekv.

4 Momis heyv nanvke cem onayvkis, mv hvse-vkerkv vlakof, cem onayvkvyvte vkerricatskvren. Momis enhvteceskv heyv nanvken ce kicvkvkomvts, ce 'pakvkikv.

5 Momis hiyomat $\mathrm{mv}$ a $\mathrm{cv}$ 'totvten oh ayis, momen hvmkatskis, Estrmun ayetska? cv kicatskeks. 
6 Momis heyv nanvke ce kicvkikv, feknokketvt ce feken cen fvefakvks.

7 Momis mehenwrn cem onayvkis, vyepryate min es cem vmomvkēs, ayvkon omat, Mvhlvpatv cem vlahkvkekot okv, momis ayepin omat, a cen 'totvkares.

8 Momen emet vlakof, naorketv, fvccetv, momet ohfvececkv ekvnvn ohkerricvres.

9 Naorketv ohkerricvres ve oh vkvsvmvkekokv.

10 Fvecetv ohkerricures, Crrken oh ayepikv, momen cr hecatskekaret okv.

11 Ohfvececkv ohkerricvres heyv ekvnv em mekkot ohtvecekv.

12 Nanvke ce kicvranvkvyat sulken ocvye munket omis, hiyomat kvwrpatskeko tayes.

13 Mome estomis, Puyvfekcr mehenwat, emet vlakof, mehenwv ofv omvlkvn 'seceyvkvres, eme e opunvyecekaret okv, momis naken pohat, mvn emet opunvyvres, momet nanvke vlvkvranet ce hecicvkvres.

14 Emet ve vrvkkuecvres, cvnaket en hecken, mvn ce hecicvkvret okv.

15 Nanvke Erke ocat omvlkvt cvnakets, momet okv, crnaken ehset, emet ce hecicvkrres makit; okvyis.

16 Hvtetusat cr hecatskekarēs, momen hvtvm hvtetusat cv hecatskvrēs, Erken oh vyepikv.

17 Hvtetusat cv hecatskekarēs, momen hvtvm hvtetusat cr hecatskvres, Erken oh vyepikv, heyv makat, naken pu kicet oke haks? en kerrepvlke vpvlwvt ete kicakvtēs.

18 Momekv heyv Hvtetuse makat, naken maket oke haks? Naken maket okat onvyëko tayes, makakvtes.'? 19 Momen hiyomat em'pohetr eyacvket on, Cesvs kerrekv, Hvtetusat cv hecatskekares, momen hvtvm hvtetusat $\mathrm{cv}$ hecatskvrēs mv vne makvyan, etempohatske haks? kicvtes, 
20 Mehenwvn, mehenwvn ce kicvkis, wahoket, hvkihhokatskvres, momis ekvnv afvckvrēs, momen ce feke cen nokhokvkvres, momis ce feke cen nokhokvkvte afvcketv hakvres.

21 Hokte hopuewv hecknecvranen omat, feke nokket omes, en hvse-vkerkvt vlakekv, momis hopuewv en heckat vpaken, nokke ehoses, estet ekvnvn heckan es vfackekv.

22 Momen centake momet okv ce feknokhokvkes hiyomat, momis hutvm ce hecvkarēs, momen ce feke afvckvrēs, momen cem afvcketv este estomet ce 'konvhhvkekos.

23 Momen mv nettv ofv naken vm'pohatskekarēs. Mehenwvn, mehenwvn ce kicrkis, nake estomen cr hocef kv ofvn Erke em'pohatsken omat, emet ce'mvkvrēs.

24 Naken ev hocefkv ofv vpohatskekon hiyomat oret os: vpohvks, momen cen heckvkvres, cem afvcketv take es frckvren.

25 Heyv nanvke opvnvkv-svhopakvn es ce tempunayvkit okis, momis oketvt vlvkvrēs, momof svnvcomv opunvkv-sṿhopakv es ce 'tempunahyvkvkos, momis Erke kērkusēn ce hecicvkarēs.

$26 \mathrm{Mv}$ nettv omof cr hocef kv ofvn vpohatskvrēs, momen vnet Erken cem emekvsvpvkarēs ce kicvkit okvkos.

27 Erke eme mahusat tis ece'nokecvket okv, vc vnokecatskvtet, momet Hesaketvmesen aenkvpvket vtvyvte tat vkvsamvtskvtet okv.

28 Erken aenkvpahkit, momet yvmv ekvnv vlvkvyet os: momet hvtrm ekvnv wihkit Erken oh ayis.

29 Hecvs hiyomat kērkusen opunayetskes, momet opunvkv-svhopakv tokon opunayetskes, en kerrepvlket kicakvites.

30 Hiyomat nake omvlkvn kerretske tat, momen este es: 
tomet cem poheko tayet omen mucr mehenwrn kerres. Hesaketvmese a en kvpvketskvten heyvn es vkvsamēs.

31 Cesvs vyoposket, Hiyomat vkvsamatska? kicvtes.

32 Hecvs, hvse-vkerkv vlakes, momes, hiyomat vlvkes, mv vwahvranatskat este enake vlken oh ayvres, momet vntvlken ev wikatskvres: montomis vntvlket omekos, Erket ve vpvket okv.

33 Heyv nanvke ce tempunayvkvyat, vne ofvn mvhlvpvtketv ocatskvren okvyet os. Ekvnv ofv estemerkv ocatskvrēs, momis ce mahlvpatkvket omekvs, ekvnv vnet ohfvnkis.

\section{SETENTVCKE XVII.}

Heyv opunvkvn Cesvs opunahyof, e turwrn hvlwen vkvwahpet, Cvrke, hvse-vkerkv vlakes, makvtes. Ceppucen vrakkuecrs, Ceppuceu ece 'rakkuecvren.

2 Nvcomen emetskvtet on omat, hesaketv yuksv-sekon emè tetayen vpeswr omvlkvn ohfvnken yekcetvn emetskvtet etvpomen.

3 Hesaketvmese mehenwv hvmkusetskan ce kerrê, momet Cesvs Klist nur vtotetskvten kerrat, heyvt hesakety. yuksv-sekat tes.

4 Ekvnvn ec oh 'vrakkuecryvtet omes; vtotketv vfvstv ranvye r'metskvte poyis.

5 Momen tokvs, Aeha Crrke, ceme mahusan 'sve vrakkuecrs, ekvnv sekof rakketv ece 'pake ocvyvten.

6 Este ekvnv a sosse v'metskvte ce hocef kv en kerkuecryvtes, cenake tates, momen mvn v'metskvtes, momen cem punvkrn veryecrkvtet omes.

7 Tokvs, nake omvlkvn naketo estomis v'metskvte a cen kvpvkvtet on kerrvkvtet omes.

8 Opunvkv v'metskvte e'makvyvtet onekv, momen e enakuecrkvtet omat, momet a cen kvpakvyvtet omen me- 
henwusen kerrvkvtet omes, momet a'c vtotetskvtet omen vkvsrmvkvtet omes.

9 Em emekusvpakis; ekvnv em emekusvpvkvkos, momis v'metskvte vrahkvn, cenaket omakekv.

10 Momen cvnakeh omvlkvt cenakets, momen cenakeh crnakets, momen mv ofvn ve vrakket omes.

11 Momen tokvs, vnvcomvn ekvnvn vpikvkos, momis heyvt ekvnv vtchkes, momen a ec oh atis. Cvrke vca ketskat, ce hocef kv eteropotten mv v'mvketskvte vcryecvkvs, pume omet hvmkusvren.

12 Ekvnv ofv vprkakvyof, ce hocef kv ofvn veryecrkvyvtes, mv vmvketskvte veryecrkvyvtes, momen hvmkik sumkekos, sumketv eppuce tvlket, cokv esfvckvren.

13 Momen tokvs, a ec oh atis, momet heyv nanvke ekvnv ofvn opunayis, eme take ofvn vm afvcketvn esfveken ocvkvren.

14 Cem punvkv e'mvkvyvtes, momen ekvnvt en homecrkvtes, ekvnv enanvke tokok, vnet ekvnv enake toyvkat etvpomen.

15 Ekvnv esossicetskvien emekusapit omvkos, momis holwakan en kvpryecicetskvren.

16 Ekvnv enake tokot omakes, vne ekvnv enake toyvkat etvpomen.

17 Cem mehenwv eteropotten vcacrken hryvs, cem opunvkvt mehenwv tes.

18 Ceme ekvnv a'c vtotetskvte etrpomen, vneu ekvnvu vtotvkvyet os.

19 Momen emetake vrahkvn vcaken e hayvyet os, mehenwv eteropotten mv emeu vcacvke hakvren.

20 Momet heyv vrahkv tvlken em emekusapit omvkos, momis mv emetake em opunvkv eteropotte ve oh vkvsvmvkvranaten.

21 Cemet, Crrke, ve vpiketsken, momen vne ece'pikv- 
yat etvpomen, ornvlkvt hvmkusvren, entakeu ep'ofvn hvmkusvren, a'c vtotetskvten ekvnvt vkvsvmvren.

22 Momen rakketv v'metskvte e'makvyvtes, hvmkuseyat etvpomet hvmkusvren.

23 Vpikvyetomen, momen vc vpiketskes, hvmkuse etemvpoke hakvkvren, momet ekvnvt a'c vtotetskvte tat kerrvren, momet ve vnokecetskvte etvpomen vnokecvketskvtet omen.

24 Cvrke; mv v'mvketskvte estvmvn likvyat vc vpakvren komis, rakketv v'metskvte hecvkepvren, ekvnv hakeko munken ve vnokecetskvtet omekv.

25 Crrke faccetskat, ekvnvt ce kerrekates, momis vne ce kerrvyvtes, momen heyv a'c vtotetskvte tat kerrvkvtet omes.

26 Momen ce hocef kvn en kerkuecvkvyvtes, momet kerkuecares, mv vnokeckv es vc vnokecetskvtet vpikaken, momen vnet vpikakaren.

\section{SETENTVCKE XVIII.}

Hexv opunvkvn Cesvs opunahyof, en kerrepvlke vpaket ayet Ketlvn hvtcucen tikvtes; mvn evpofucet ocet on, eme en kerrepvlke esyont esceyvtes.

2 Momen Cutvse mv wiyvte mv crpofuce kerre tates, Cesvs, en kerrepvlken vpaket etohkvlke mahet omvtet okv. 3 Momen Cutrse, suletawv, momet este-en-wiketv, plest en hómvhhotat, momet Falvsevlket en heckuecof, kulken, momet etō-kulecvkrten, momet nake fvske ocvken, 'res orvtes.

4 Cesvs, heyv nanvke eme oh vlvkvranat omvlkvn kerret, oh ayet, Estimvn hopoyatska? kicakvtes.

5 Em vyoposkaket, Cesvs, Nasvlevlkē, kicakvtes. Vnet $m v$ toyis, Cesvs kicakvtes. Momen mv Cesvs wiyat vpvkaket huervtes. 
6 Mohmen, Vnet mv toyis kicakat vpakusen, vcekelehcet, ekvnvn tvkpvlatkvtes.

7 Mohmen hvtvm em 'pohakat, Estimvn hopoyatske haks? kicakvtes. Momen Cesvs Nasvlevlkē, makakvtes. 8 Cesvs vyoposket, vnet $m v$ toyit cem onahyvkis; momekv cv hopoyatsken omat, heyvt vpeyepekvs, makvtes.

$9 \mathrm{Mv}$ v'metskvte hvmkis sumecicvks, make eme opunayvte esfvckvren omvtes.

10 Mohmof Simvn Petvt, eslafkv-cvpkon ocet ot, a esvtes, momet plest enhomahtv em vtotkv hrmken e hrcko vkvpervn en tacites. Vtotkv, Malkvs hocefkv tates.

11 Cem eslafkv-crpkon e huten vpikvs. Mv halo Cvrke v'mvte eskvranvko haks? Cesvs Petvn kicvtes.

12 Momekv kvpetvne, suletawv, momen Cusvlke esteen-wiketv esyomat, Cesvs esahket, wrnayakvtes.

13 Momet Anvse tāten 'soh vpeyvtes Kivfvse em mahet omvtet okv, mv Kivfvset mv ohrolopen plestvlke en homahtv tates.

14 Tokvs, Kivfvset, Este-hunvnwv hvmket este em ele tayet ome omecet, Cusvlken 'sem mvhayet omvtes.

15 Momen Simvn Petv Cesvs vcrkayvtes; momen en kerrepv etvt matvpomvtes. Mv enkerrepv plest enhomahtv en kerke tates, momen Cesvs vpaket plest enhomahtv en liketv eceyvtes.

16 Momis Petr fettrn vhauken vhuervtes, momen $\mathrm{mv}$ enkerrepv hvmkat ossvtes, plest enhomahtv en kerkat, momet hoktuce vhauke vhecican ete'm punahyet, momet Petvn a ceyicvtes.

17 Mohmof mv hoktuce vhauke vhecicatet, Cemeu heyv hunvnwv en kerrepvlke toyetskeko haks? Petvn kicvtes. Mv toyvks, makvtes.

18 Momen vtotkvlke momen este-en-wiketr, kvsvppet 
okv, torawvin etecet, vsvpvklvtes tvrraket. Momen Petv mvn vpaket tarret huervtes.

19 Momof plest enhomahtv Cesvs, em mvhakvn, momet en kerrepulken em vpohvtes.

20 Cesvs em vyoposket, Ekvnv kērkusen etempunvyvyvtes; mekusvpkv-cuko ofvn, momet cuko vcakat ofvn, mv Cusvlke nvkaftan mvhayvye munkvts; momet ēhkuse naken makvyvtē sekos.

21 Estomen vm 'pohetske haks. Vm apohicakvten em 'pohvs, naken kicakvyvte; hecvs, nake makvyvte kerrakēs.

22 Momen hiyome opunahyof, este-en-wiketv vnakv svpaklat hvmket enke-tvpeksen Cesvs es naf kvtes, Plest en homahtv momēn em vyoposketske haks? maket.

23 Cesvs em vyoposket, Holwvken opunāyin omat, holwakan kerkuecvs, momis heren omat, estomen $\mathrm{cv}$ naf ketske haks? kicvtes.

24. Tokvs, Anvset Cesvs wrnvken Kivfrse plest enhomahtvn ohtotvtes.

25 Momen Simvn Petvt tarret, huervtes. Momen Cemeu en kerrepvlke hvmkat toyetskeko haks? kicakvtes. Momen e helaksecet, Mr toyvks, makvtes.

26 Plest enhomahtv em vtotkvlke hvmket, e hvcko Petv en tacvte ennaket omet, Vpvketsken crpofuce ofv ce hecvkat haks? kicvtes.

27 Monkv Petv hvtvm e helaksecvtes, momat vpakusen tottoloset hakvtes.

28. Momof Kivfvse en kvprken Cesvs fvcceckv-cukon 'soh vpeyvtes; momen hvten tates; momen emetake fvcceckv-cuko esceyekates svholwvhokekot, momis ohhoyvnkv hompetv hompvkēpvret.

29. Momekv Pilvtet osiyet, oh vyakvtes, momet Vnrvpueckv estomen heyv este-hunvnwv vnrvpe es yicatske haks? makvtes. 
30 Holwvyeckv-vfastv tokon omat, ye cen huericẽkwnt os, em vyoposket kicakrtes.

31 Ehset, cem vhakv take vcrkayen ohfvececvks, Pilvtet kicakvtes. Momekv, Este eleceyvre vhakv toks, Cusvlket kicakvtes.

32 Cesvs em opunvkv, elkv estomēn elvranat kerkuecet makat, es ohfvckvren omvtes.

33 Mohmof Pilvtet fvcceckv-cukon hvtvm ceyet, momet Cesvs hocefet, Cusvlke em mekko toyetska? kicvtes.

34 Heyv nake ceme mahusat maket oketske haks? monkat etimvt vc opunvyecet cem onahoye haks? Cesvs em ryoposket kicvtes.

35 Pilvte vyoposket, Cusvlke toyiya? Cem etvlwv, momen plest en homvhhotat ye ce huericaket os. Naken estometska? kicrtes.

36 Cesvs vyoposket, $\mathrm{Vm}$ etvlofv ekvnv enake tokot omes. Vm etvlofv ekvnv enake taten omat, vm vtotkvlke tepoyakvnt os, Cusvlket cv'sekaren ; momis hiyomat vm etvlofv yvmv vte tokot omes, kicvtes.

37 Momekv mekko toyetska? Pilvtet momof kicvtes. Cesvs vyoposket, Mekko toyvyat, maketskes; heyv 'sv rahkvn cv heckvtet omes, momet heyv omecicen ekvnv vlvkvyvtet omes, mehenwv vfvccecaret. Mehenwv enake vtekat vn hake vpohicet omes, makvtes.

38 Naket mehenwvt o haks? Pilvtet kicvtes. Momet heyv mahkof, hvtvm osiyet, Cusvlken oh ayet, Naket em mvtte tat eshecrkos, kicakvtes.

39 Momis vhakvn ocatsket os, ohhoyvnkv omof, hvmken cem en recopvkaren. Momekv Cusvlke em mekkon cem en recopvranvke haks? kicvtes.

40 Mohmof omvlkvt hrtvm huehkaket, Heyv hunvnwv tokon, momis Pvlapvsē, makakvtes. Hiyomat Prlapvse sestrpenkvlecvt omvtes. 


\section{CA NE COPVTE.}

\section{SETENTVCKE XIX.}

Момекv momof Pilvtet, Cesvs ehset, tepkvtes.

2 Momen suletawvlket, eto-fvske kvpotokvn hotanaket ekvn em oh licakvtes, momet kapv okholatten atcecakvtes.

3 Momet, Cusvlke em mekko toyetskate! makaket, enken es nvf kakvtes.

4 Momekv, Pilvte hvtvm a osiyet, Hecvks, a's cem ossvkis, em mvtte eshecvkat kerratskvren, kicakvtes.

5 Momof Cesvs, eto-fvske kvpotokvn es kvtopoyet, momet kapv okholatten atcet, a ossvtes. Momen Pilvtet Este-hunvnwvn hecvks, kicakvtes.

6 Momen plest enhomvhhotat, momen este-en-wiketv emen hecakof, huehkaket, Etohwelepkvn svrvhvs, etohwelepkvn svrvhrs, makakvtes. Pilvtet, Centaket ehset, etohwelepkvn svrvhvks; vne em mvtten eshecrkot omekv, kicakvtes.

7 Cusvlke em ryoposkaket, Vhakvn ocēs, momen pum vhakv vcrkayen ele tayet omes, Hesaketvmese Eppuce e hāyet omekv, makakvtes.

8 Momekv Pilvtet, $\mathrm{mv}$ opunvkvn pohof, svheremahen penkalvtes.

9 Momet, hvtvm fvececkv-cukon cehyet, Estvmvn atetska? Cesvn kicvtes. Momis Cesvs em vyoposkekates. 10 Momekv Pilvtet, Vm punayetskeko haks? Etohwelepkvn 'sece 'rvhetv yekcetv ocit, momet cen recope:v yekcetv ocvyat, kerretskeko haks? kicvtes.

11. Cesvis vyoposket, Vc vnrve yekeetv ocetskekvnt os, hvlwe vten ce'mhoyekaten omat, monkv ce homv cv huericat naorketv sen rakken oces, makvtes.

12 Momen mv vteken, Pilvtet en recopetvn ohvtvlaken komvtes. Momis Cusvlke huehkaket, Heyv hunvnwon en recopetsken omat, Sesv en hesse toyetskekos ; estimvt,

Creek. 
mekkon e hayat, Sesv vnrvpet opunayet omekv, makakvtes.

13 Momekv mv opunvkvn pohof, Cesvs es ossvtes, momet fvcceckv-ohliketvn oh likvtes, Cvto-pvtihocvte kihocan, momis Hepluvlke em punvkv Kvparvt's.

14 Momen Ohhoyvnkv etetakueckvt omet, momet hrsevkerkv esepakat mahe tates. Momen Pilvtet, Cem mekkon hecvks, Cusvlken kicakvtes.

15 Momis huehkaket, Esvyepvs, esvyepvs, etohwelepkvn svrvhvs, etohwelepkvn svrvhrs, makakvtes. Pilvtet, Cem mekkon etohwelepkvn svrahvrani haks? kicakvtes. Plest en homvhhotat vyoposket, Mekko ocēkos, Sesvt mv tokon omat, makakvtes.

16 Momof em wikvtes, monkv, etohwelepkvn svrahkvren. Momen Cesvs ehset 'svpeyvtes.

17 Momen osiyet, em etohwelepkvn karonet, Estekvfune kicetvn oh ayrtes, Hepluvlke em punvkv Kalkorvt's.

$18 \mathrm{Mvn}$ etohwelepkvn svrvhakvtes, momet etv hokkolet vpaken, hvmkevlket etempalvsen, Cesvs en nvrkvpv tates.

19 Momen Pilvtet es-hocefat hoccihcet, etohwelepkvn 'svlicvtes. Momen hoccat, CESVS NASVLEVLKE, CUSVLKE EM MEKKO, maket omvtes.

20 Momekv heyv es-hocefat Cusvlke sulket hecakvtes, Cesvs etohwelepkv svrahohat tvlofv em vwoluset omvtet okv; momen Hepluvlke, Klekvlke, Lomvnvlke esyomat em punvkin hoccet omvtes.

21 Momof Cusvlke, plestvlke en homvhhotat, Cusvlke em Mekko makekon, momis Cusvlke em Mekko toyis, makvtes, maken coyvs, Pilvten kicakvtes.

22 Pilvte vyoposket, Nake hoccicvyat hoccicit omis, makvtes.

23 Momof suletawvlket, Cesvs etohwelepkv svrvhahkof, em atcvken crwaket, tekvpvke osten hayakvtes, suletawv 
esvrahkvn tekvpvle hvmkevlken, momet kapvn cvwakvtes. Tokvs, kapv etehorkv sekot, onvpv hvmkv yeshvtapket tarket omvtes.

24 Momekv, Selahlecēkot, momis estimvt omvre eshecvkeres, ete kicakvtes ; momat, $\mathrm{Vm}$ atcusvke etekvpicvnkes, momet vn kapv estit omvre eshecakvnkes, Cokv makat sohfvckvren. Momekv heyv nanvke mahusan suletawvlket momecakvtes.

25 Tokvs, Cesvs ecke, momen ecke etecakkat, Mele Tleofvse e hiwv, momen Mele Maktēlv esyomat Cesvs em etohwelepkv tempen svpaklvtes.

26 Momekv Cesvs, ecke momen enkerrepv Cesvs vnokecat $\mathrm{v}$ hueren hecof, Hoktē, cechuswrn hecrs, ecken kicvtes.

27 Mohmof enkerreprn, Cecken hecvs, kicvtes. Momen $\mathrm{mv}$ hvse-vkerkv vteken mv enkerrepvt evpvyepvtes, en liketv min.

28 Heyv eryupvn, Cesvs, nake omvlkv hiyomat esyukpokat kerrekv, Cv wvnhkes, makvtes, Cokv esfvckvren.

29 Momekv uetukse vcrnkv fvcket es likvtes; momen uepvton uetuksen fvcehcet, momet hesvpen oh licet, e cukwrn em vcelicakvtes.

30 Momekv Cesvs, uetukse en hecikof, Esyukpokes, makvtes. Momet ekvn potoyicet, puyvfekcvn wikvtes.

31 Cusvlket monkv, Tetakueckvt omen, ena pvsvtkat etohwelepkvn oh vtvrtake munkekaren, (mv nettvcako nettr rakket omekv,) ele kvcehken, ena vkuehoyvren Pilvten em 'pohakvtes.

32 Momof suletawvlke yihcet, enhvteceskv vtarkan elen en kvcehyet, hvmkateu vpake etohwelepkv svrahkat ele en kvceyakvtes.

33 Momis Cesvs en yicen, entis elepvtet on hecakof, elen en kvceyvkekates. 
34 Momis suletawv hvmket cufunfvsken envcen 'sen sekeyvtes, momen moman vpaken uewv catv esyomet a fihnvtes.

35 Momen mv hecrte ohkerkueces, momen em ohkerkueckv mehenwe tes; momen mehenwvn opunayat kerret omes, vkvsamatskvren.

36 E fune kvckekares, Cokv makat, esfvckvren, heyv nanvken momechoyvtet okv.

37 Momen, Mv sekehoyan hecvkvres, maket, hvtvm etvt coket omes.

38 Heyv 'renyupvn, Cose Alemvtvlket, (Cesvs enkerrepvt omen, momis ehken, Cusvlke em penkalat omecicen,) Cesvs ena akueyvret Pilvten 'em 'pohvtes; momen Pilvtet em vkvsamvtes. Momekv vlahket, Cesvs enan 'sayvtes.

39 Momen Nekotemvseu, (mv enhvteceskv nere Cesvs em vlakvtet,) $\mathrm{mv}$, alo tohkvlke vtarkv cukpe hvmkat mahen 'svlakvtes.

40 Momekv Cesvs enan esaket, hvnvwelv tvrken, esfvmecickv vpvken es ayokk fakvtes, Cusvlke en hopeletv vevkayen.

41 Momen etohwelepkv svrahkan crpofuce tates, momen crpofuce ofvn crtokofke mucrset, mvn este wrkechoyvte sekot, ocvtes.

42 Momekv mvn Cesvs wrkecakvtes, etetakueckvt omen, momen crto-kof ke vwoluset omekv.

\section{SETENTVCKE XX.}

Netrv cakucE nettv enhrteceskvn, hvten, yomuckuse emunken, Mele Maktēlv cvto-kofken vlaket, momet cvto cvto-kof ke vkueyihocen hecvtes.

2 Monkv letket, Simvn Petvn, momet enkerrepv etv, Cesvs vnokecan em vlaket, Pucasen cvto-kofken a ossicet 
es sumechos, momet estvmvn wvkechoyat kerrēkos, kicakvtes.

3 Momekv Petvt, momen enkerrepv hvmkeu tepaket wolket, cvto kofken vlahokvtes.

4 Momof hokkolvt tokorkvtes, enkerrepv hvmkat Petv letkat emuntalet en homvn cvto-kof ken 'rorvtes.

5 Momet, oh cunehket, hvnvwelv tvrke atcvke tvk kaken hecvtes, momis ceyekates.

6 Momof Simvn Petvt vevkayet, vlakvtes, momet cvtokofken cehyet, hvnvwelv tvrke atcvke tvk kaken hecvtes.

7 Momet, enockuce ekv es ayokkof kvte hvnvwelv tvrke atcvke vpakekot, momis tulpuket entvlket etin kaken hecvtes.

8 Momekv momof mv enkerrepv hvmkat, mv tate cvtokof ke 'rorat, ecehyet, hecvtes, momet vkvsamvtes.

9 Cokv, Elkv hvtvm 'ra en kvwvpkvranet os, makat hvte kerrvkekatet omekv.

10 Momof enkerrepvlket hvtvm e hute take mahusan vhoyvtes.

11 Momis Melet hvkihket, cvto-kof ken fettvn v huervtes, momet hvkihkof, oh cunehket, cvto-kof ken fettrn v huervtes, momet hvkihkof, oh cunehket, cvto-kof ke ofvn vfvnnakvtes.

12 Momet Hesaketvmese-em-este hokkolet, hvtke, atcvket, Cesvs ena wakkvten kaken, hvmket ekv likvten liken, hvmkatet ele kakvten liken hecof,

13 Momen, Hokte, Estomen hvkihketska? kicaken, $\mathrm{Cv}$ Pucasen es sumechoyen, estrmvn wvkechoyaten kerrvkat omecices, kicakvtes.

14 Momen hiyomen opunahyof, fulotkat, Cesvs heuren hecrtes, momis Cesvs tat kerrekates.

15 Hoktē, estomen hvkihketska? Estimvn hopo- 
yetska? Cesvs kicen; emet, Cvpofuce-vfastvtes, komet, Pucasē, cemet etimvn 'sayetskvten omat, estvmvn wvkecetskat, vm onvyvs, momen vkueyares, kicvtes.

16 Cesvs, Melë, kicen, a oh fulotiket, Laponi, mvt Mvhayv maketvts, Melet kicvtes.

$17 \mathrm{Cv}$ celahyetskvs, hvte Cvrke v kvwvpkvkot emunkekv. Momis vm etecakketvlken oh vyvs, momet Cvrken, Cerke take, momet vn Hesaketvmesen, cen Hesaketvmese take $\mathrm{v}$ kvwvpkvyan, em onvyvks.

18 Momekv Mele Maktëlv vlaket, Pucasen hecvyis, momen heyv nanvken es cv tempunayis, en kerrepvlken kicakvtes.

19 Momen mv nettv etvwrn, nettvcakuce nettv enhvteceskvt omen, yafkof, enkerrepvlke, Cusvlke em penkvlvket, vhauke vkhothoken, nvkaften, Cesvs vlakvtes, momet, nvrkvpvn hueret, Herkvt cem ocvkekvs, kicakvtes.

20 Momet, heyvn punahyof, enken, envcen tepaket hecvkuecrtes. Momekv enkerrepvlket, Pucasen hecakan afvekakvtes.

21 Monkv hvtvm, Herkvt cem ocvkekvs: Cvrke ve vtotvte etvpomen ece 'totvkis, Cesvs kicakvtes.

22 Momet heyv mahkof, oh hesaket, Puyvfekcv : Vcakan cen heckvkekvs, kicakvtes.

23 Estimv en naorketv en kvpvyecicatskat, en kvpvkaket omēs, momet estimv en naorketv vfekhonnicatskat, vfekhonnaket omēs.

24 Momis Tamvse, Tetemvse kihocat, pale hokkolohkakat hvmkatet vpvkvkekon, Cesvs vlaket omvtes.

25 Momekv enkerrepvlke vpvltaket, Pucasen heceyvnks, kicakvtes. Momis, Enke ofvn vcopv svrahkvten hecit, vcopv svrahkvten evnke-wesakvn vpikvkon omat, momet envcen crnken vpikvkon omat, vkvsahmvkos, Tamvset kicakvtes. 
26 Momen nettv cenvpake renyupvn hvtvm enkerrpvlket ofvn vpoken, Tamvseu vpvkakvtes. Cesvs vlaket, vhauke vkhothokepen, nvrkvprin hueret, Herkvt cem ocvkekvs, makvtes.

27 Momet Tamvsen, Cenke-wesakvn rvtosecet, crnken hecrkvs, momet, cenken rvtosecet, crnvcen vpikvs, momet vkvsvmkv ocekot huyiretskvs, momis vkvsvmet, kicrtes.

28 Momen Tamvse vyoposket, $\mathrm{Cr}$ Pucase, momet vn Hesaketvmese, kicvtes.

29 Tamvsē, cv hehcetsket okv, vkvsahmetskes : hecvkekis, vkvsvmakvte; en herakes, Cesvs kicvtes.

30 Momen mehenwusen, nake-eskerkv etv sulke, heyv nakcokv ofv hoccvkekat, Cesvs momecakvtes, en kerrepvlke en renakv ehomvn.

31 Momis, Cesvs, mvt Klist tot, Hesaketvmese Eppuce tat, vkvsvmatskvren, heyv nanvke coket ot os, momet vkvsamatskat, e hocef $\mathrm{kv}$ eteropotten hesaketv ocatskvren.

\section{SETENTVCKE XXI.}

Heyv nanvke 'renyupv, Cesvs hvtvm enkerrepvlken em e heckuecrtes, vkhrse Tipelevs hocef kan. Momet hiyomēn em e heckuecrtes.

2 Simvn Petvt, Tamvse Tetemvse hocefkat, Neranevl, Kenv, Kalvle e tvlwv, aossvtet, momen Sepete eppuce take, momen en kerrepvlke etv hokkole tohkvlket vpokvtes.

3 Momen Simvn Petvt, Rvro-pokvn ayis, kicaken, Pumen ece 'crkvpēyẽs, kicakvtes. Vpehyet, lvpken perron esceyvtes, momet mv neren naken esvkekates.

4 Momen hiyvtikof, Cesvs lvpvtken huervtes, momis enkerrepvlke Cesvs tat kerrvkekates.

5 Momekv, Hopuetakucē, hompetvn ocatske haks? kicaken, Monkos, em vyoposket kicakvtes. 
6 Momen, Perro em akvpervn hoyv vk wikvks, momen eshecatskvres, kicakvtes. Momekv vk wikakvtes, momet, tokvs, rvro sulke mahat omecicen, asossiceko tayvtes.

7 Momekv mv enkerrepv Cesvs vnokecat, Pucase tes, Petvn kicvtes. Tokvs, Petvt Pucaset omaten pohof, enatvlket omekv, rvro-poyv en kapvn vciyet e wrnahket, vkhvsen e vk wikvtes.

8 Momis en kerrepvlkev pvlwvt, perrucen vtehket, 'svk yicvtes; lvprtke en hopvyekot, estekuce cukpe hokkolvteke en hopvyetvt omekv, hoyv rvron es vk sopotecakvtes.

9 Momen lvpvtken es yicat vpakusen, totkv-torawvn hecakvtet, momet rvrot oh ocen, momet trklike.

10 Rvro hvte poyatskat es vwvke Cesvs kicakvtes.

11 Momen Simvn Petvt ahyet, hoyv rakrvke fvcken, lvprtken 'res vcayvtes, cukpe hvmken pale cahkepen tutcenohkaken, momet, en sulke momis, hoyv vhopvnkekates.

12 Ahwet, hompvks, Cesvs kicakvtes. Momen enkerrepvlke hvmkis, Esti toyetska? kice em'pohetvn eyacvkekates, Pucaset on kerrakekv. .

13 Momekv Cesvs vlaket, tvkliken chset, momet e'makvtes, rvro matvpomen.

14 Tokvs, Cesvs elkv'raenkvwvpkvte 'renyvpv enkerrepvlke em e heckuecat, heyvt 'svtutcenat tes.

15 Momekv hompahkof, Simvn, Convse eppucē, Heyv 'sen hoyanen, ve vnokecetske haks? Cesvs Simvn Petvn kicen, Petvt, Henka, Pucase, ece'nokecvyat kerretskes, kicvtes. Vn yvpefikucen homprkuecrs, Cesvs kicvtes.

16 Hvtvm svhokkolvn, Simvn, Convse eppucē, vc vnokecetske haks? kicen, Henka, Pucase, ece'nokecryat kerretskes, kicrtes. Vn yvpefikvn hompvkuecrs, Cesvs kicrtes.

17 Svtutcenvn, Simvn, Convse eppucē, vc vnokecetske 
haks? kicen, Petvt, e feke en nokkvtes, Vc vnokecetske haks? svtutcenvn kicvtet okv, momet, Pucasẽ, nak' omvlkvn kerretskes. Ece'nokecryat kerrusetskes, kicvtes. Vn yvpefikvn homprkuecrs, Cesvs kicvtes.

18 Mehenwusen, mehenwusen ce kicis, ce mvnnettusof, e wrnaket, estvmvn kometskat yvkvpetskvtes, momis ece 'cule hakof, cenken rvtosecetsken, este etvt ce wrnayvres, momet estvmvn kometskekat ce 'sayvres.

$19 \mathrm{Elkv}$ estomēn Hesaketvmese 'svrakkuecvranat oket, heyvn makvtes. Momen hiyomen punahyof, Vc vcrkvtes, kicvtes.

20 Momof Petvt afolotkat, mv enkerrepv Cesvs vnokecat vcrkayen hecrtes, mv, hompakof, Cesvs e hokpen vccaket, Pucasē, mv ce wiyat, estit o haks? makvtet.

21 Petvt mvn hecet, Pucase, momen heyv este-hunvnwv naken estomvr haks? Cesvn kicvtes.

22 Fekhonne munken vlvkaret komin omat, mvt cem estoma? Cemet ve vevkvtes, Cesvs kicrtes.

23 Momekv heyv opunvkv, Mv enkerrepv elekares, makat, etecakkvten 'rem orvtes. Momis Cesvs, Elekares, makekot, momis, Fekhunne munken vlvkaret komin omat, mvt cem estoma? makvtes.

24 Enkerrepv, heyvt mv tes, heyv nanvken kerkuecat, momet heyv nanvken hoccicat, momen em ohkerkueckv mehenwet on kerrēs.

25 Momen nanvke etv sulket ocakes, Cesvs momecakvtet, momat, omvlkvn cohoyvten omat, ekvnv tis nakcokv cohoyan caweko tayes, komis. Emen. 


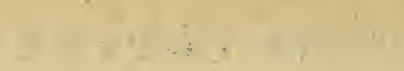

1

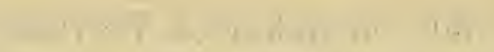

1,

Y

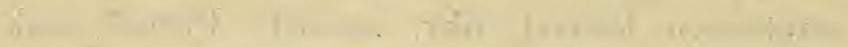

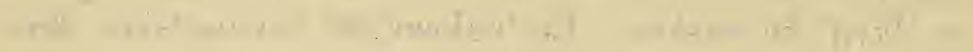

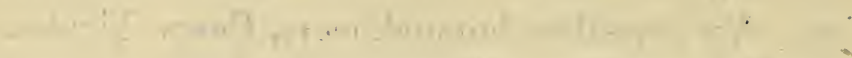

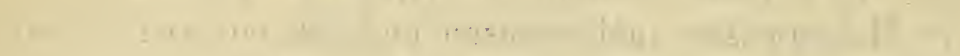

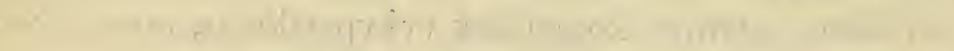

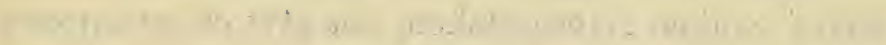

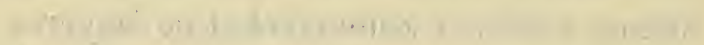

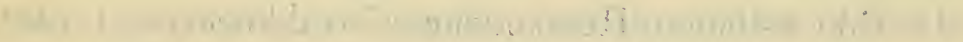

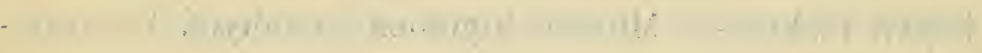

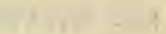

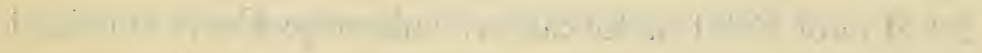

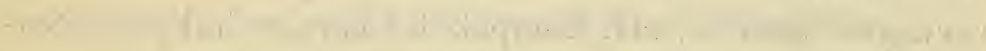

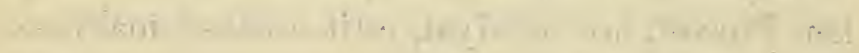

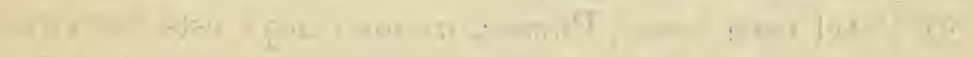

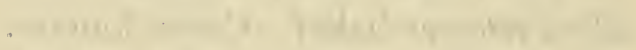

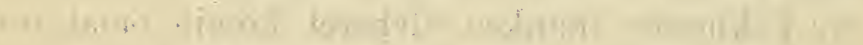

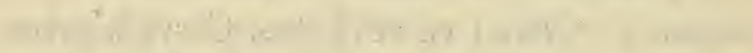

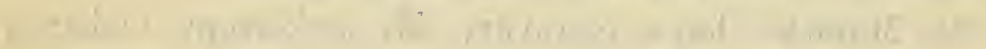

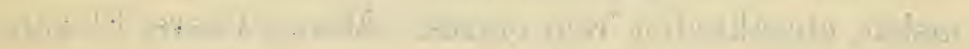

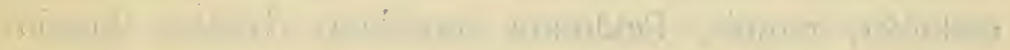

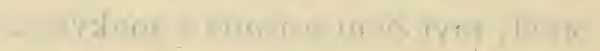
$7+$ i the

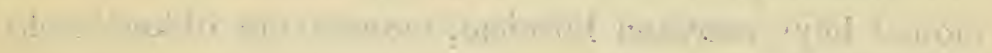

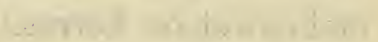

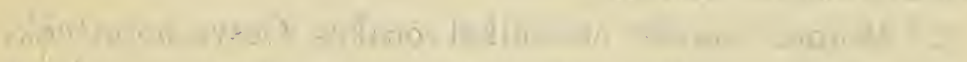

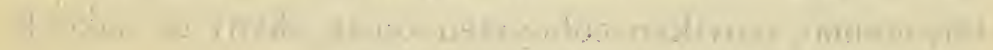

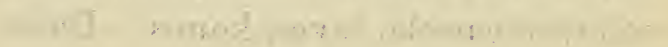




\section{VPASTELVLKE EM FULLETY.}

\section{THE ACTS OF THE APOSTLES,}

TRANGTATED FROM

\section{THE ORIGINAL GREEK}

INTO

\section{THE MUSKOKEE LANGUAGE.}

NEW YORK:

AMERICAN BIBLE SOCIETY, INSTITUTED IN THE YEAR MDCCCXVI. 


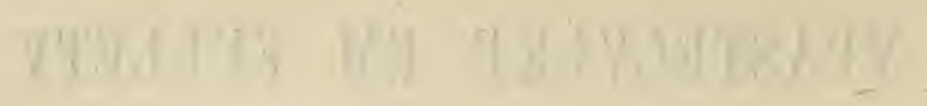

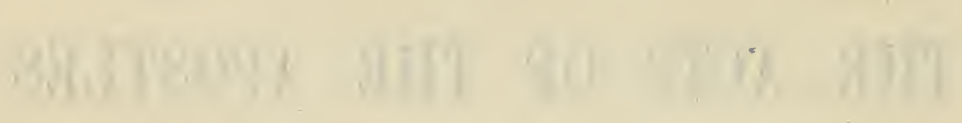




\section{VPASTELVLKE EM FULLETV.}

\section{SETENTVCKE I.}

Reafelvs toyetskat, enhomvn cokvn hayvyvtet os, Cesvs vlicehce nake momecrte, momet este 'mvhayvte omvlkv ohfvecvn,

$2 \mathrm{M} v$ nettr Puyvfekev Vcakat eteropotten vpastelvlke enhopoyvtet, vhakv e'hmet, kvwvphoyvte oren.

3 Mvn, estemerike enyupvt, wenaket, eskerkv kerkvke hēre sulke etehoyvnen em e heckuecrtes, nettr pale osten mvt hecaken, momet Hesaketvmese em etvlofv ohfvecv nanvke ocakan opunvyecrtes.

4 Mvn em etenvkaftet, Celuslvmen enkvprkekot, momis Erke temfaccrten mehakvkvren kicvtes, Mvn vnet cem onayvkin pohatskvtet os, makvtes.

5 Mehenwrn Cane uewrn es paptiset omvtes: momis centake tat, nettr sulke hoyvnekon, Puyvfekev Vcakan es ce paptishoyvkvres.

6 Monkv ètohkvlikof, em 'pohakvtes, Pucasē, mucv hiyomat tvlwv Eslevl ohfulecicetskvr haks? makvtes.

7 Momen, Estofv monkat oketv Erke eme en yekce ofv vtehat kerratskvre cenake tokot os, emet kicakvtes.

8 Momis Puyvfekcv Vcakat ec oh vlvhkvke min yekcetv cen heckvkvres; momet centaket Celuslvme ofv Cutev omvlkvn, momet Semelev ofvn, momet ekvnv hopvye mahat vteken vc ohkerkuecrlke toyatskvres, kicvtes. 
9 Momen heyvn opunahyen, mv hecaken vkvwvphoyvtes; momet aholocet ehset, en renakvn 'sem vrvnakvtes. 10 Momen ayepen hvlwe-fvecvn hericet vhecakof, hecvs, hunvntake hokkolet, em accvke hvthvket, etempen sehokvtes ;

11 Momen mvt okakat, Kalvle hunvntake toyatskat, estoman hvlwen vhecaket svpaklatske haks? Heyv Cesvs, hvlwe ofv a eshoyat, mata hvlwe receye hecatskat, matvpomet hvtvm vlvkvres, makakvtes.

12 Momof ekvnhvlwe Olefv kicetvn enkvpaket, Celuslvmen oh fulecrtes, mv Celuslvme em vwole Fekvpkvnettv nettv hvmke vyetv tes.

13 Momet min ye 'secehyof, ohonvpv nvthofvn ohcemecrtes ; mvn Petv, Cems, Cane, Vntolu, Felvpe, Tamvse, Paralome, Maro, Cems Alfevs eppuce, Simv Selotes, Cutrse Cems etecakkvte esyomat fekhonnakvtes.

14 Heyv omvlkvt hoktvken, momet Mele, Cesvs ecken, momet Cesvs etecakkakan esyomvket mekusvpkv momet empohetv ofvn em vkerrickv hvmkusen vpeye mahvtes.

15 Momen mv nettv take ofvn, (etohkvlkat ehocefhokv cukpe hvmken pale hokkolvteket omen,) Petvt enkerrepvlke en nvrkvpvn ahuyiret, makvtes,

16 Hunvntake tecakkvte toyatskat, heyv Hoccat sohfvckvranet omvtes, mv Tewe e cukwv aosse Puyvfekcv. Vcakat Cutvse, Cesvs esakat em fvyatv hakvte, opunvyecat 17. Es epu 'honkvtket omet, momet heyv vtotketv akvpvken en hecket omvtet okv.

18 Tokvs, heyv hunvnwv holwvyeckv esenfeketvn cvpofvn es nesvtes, momet hakcelopotket lvtiken, nvrkvpvt etetvkociken, e fekce omvlkvt esossvtes.

19 Momen Celuslvme vpokat omvlkvt en kerkvke tates; momat mv crpofv eme em punvkv tat Eseltemv kicaket omes, mv Catv crpofv maketvt omes. 
20 En liketvt tvlvhassekvs, momen mv ofv este estomet lihkates; momen em wiketv etvt esekvs, maket, Sams cokv ofvn hoccet omekv.

21 Monkv Cane paptisvte vlicehce aye nettr Cesvs a pum eshoyvte rorat omof, Cesvs pu homv eceye aosse arof,

22 Heyv hunvntake epu "pake emunkv tatet hvmket epu 'paket Cesvs raenkvwrpketv akerrv hakat heret os.

23 Momen hokkolen, Cose, Paseprs kicetvn, mv hocefkv mahhe Crstrs taten, Merivs tepaken asehoyakvtes.

24 Momet, Pucase, este omvlkv e feke ohkerrv toyetskat, heyv hokkolat estoman enhopoyetske tat, pun kerkuecrs, maket, emekuisvpakvtes ;

25 Momet Heyv vtotketr, momet vpastelvlke en wiketv akvpvke en heckekvs, mv Cutvse naoriket en kvpvkvtet omekv, liketv enaken ayepvret, makaket.

26 Momen eskerretvn hayakvtes; momen eskerretv Merivsen oh latkvtes, momen vpastelvlke pale hvmkontvlakan es vhonkatkvtes.

\section{SETENTVCKE II.}

Momen Pentekast nettr mahusat orof, omvlkvt em vkerrickv hvmkuset vpokvtes.

2 Momen vyvkhvmkusen hvlwe vtet hotvle yekce pvfne en hake omet mv cuko vpokat omvlkvn fvcecrtes.

3 Momen tulaswr eteyakyvpe totkv omet heckaket, setetayen oh vpokvtes.

4. Momen omvlkvt Puyvfekev Vcakat fvcfaket, Puyvfekcrt opunvyetv e'makat vevkvyen tulaswr etv es opunvyetrn vlicecakvtes.

5 Momen Cusvlke hunvntake mekusapvlke sutv elecv etvlwv like tayat asossevlket Celuslvmen vpoke tates.

6 Monkv heyv nake hvmkvn pohkof, este sulsoket etoh- 
kvlkakvtes, momet em estomahakvtes, este omvlkvt em punvkvn 'sopunahoyen pohakekv.

7 Momen omvlkvt en kerkvkekot em estomahakvtes, Hehcvkes, heyv opunahoyat omvlkvt Kalvlevlket omvkeko haks?

8 Momat estomen pum punvkv, mv ofv pu heckvten, opunahoyen pohēt ome haks? ete kicaket.

9 Parenvlke, Metvlke, Elemitvlke, momet Mesopotemev, Cutev, Kapvtosev, Pantrs, Esev,

10 Flecev, Pamfelev, Ecepsen, momet Lepev ekvnv Silene vwolakan vpokat, Lome tvleporvlkeu, Cusvlke momet vkvsamvlke hahoyvte,

11 Kletvlke momet Elepvlke toyeyat, Hesaketvmese em vtotketv estomahakat pum punvkvn es opunahoyen pohēs.

12 Momen omvlkvt, em estomahaket, momet en kerkvkekot, Heyvh nak' eston ome haks ? ete kicakvtes.

13 Vpvlwvt nakhayekot, Heyv hunvntake prrko-opuswv mucvset fvcfvket ot os, makakvtes.

14 Momis Petvt, pale-hvmkontvlakan vpaket hueret, yekcen opunvyvtes, Cutev hunvntake toyatskat, momen Celuslvme vpokatskat omvlkvt, heyvt cen kerkvkekvs, momet rm punvkvn apohicrkes;

15 Heyv hacvkes, komatskat, monkot os, nettv hrsevkerkv svtutcenusat tokv.

16 Momis heyv mv owalv Coel opunvyvte tes;

17 Momen nettv espokakat ofvn momet ocvres, enavpeswv omvlkvn vm Puyvfekev aossen a oh kvlares; momen ceppuce take, momen cechustvlke owalvkvres, momen cen hunvntake mvnettakat nake en heckuehocan hecvkvres, momen cen hunvntake vculakat vpueckvn vpuecvkvres.

18 Momen mv nettv omof vm vtotkvlke, momet vm 
vtotkv hoktvke tis vm Puyrfeker aossen a oh kvlares, momen owalvkvres.

19 Momen hvlwe sutv ofv nake estomahaken, momet kvncrpv ekvnvu nake eskerkvken, catv, totkv momen ekkocen heckuecares.

20 Hvse yomucke haken, momen hvresse catv hakvres, mv Pucase en nettv rakke, kerkat vlvkeko emunken.

21 Momen momet ocrres, estimvto estomet Pucase e hocefkvn oh huehkat hesakvres, Hesaketvmeset maket os, kicvtes.

22 Eslevl hunvntake toyatskat, heyv opunvkvn pohvkes; Cesvs Nasvlevlke, hunvnwv Hesaketvmese es kerkuecat ece 'pvkvke vtotketv este momeceko taye, nake senhonechoke, momet eskerkv Hesaketvmese mv eteropotte cem etenruw take momecrte ceme tis kerratskes;

23 Hesaketvmese hrteta kerrvte, momet momvres komvte eteropotte wihokvten, mvn ehset, enke holwvyecrke es vtahret, elecatskvtet os.

24 Mvn Hesaketrmese elkv en nokken en recahpet, akvwapvtes; mvn es hvlvtket omeko tayvtet omekv.

$25 \mathrm{Mv}$ ohfrecvn Tewe makvte ocet omekv; Pucase vn renakv ehomvh enhomvn hecrye munkv tates; vm vkvpervn likekv, ev nekeyihohcekos ;

26 Monkv ev feke 'safvcken, cv tulaswvu 'safackvnkes; momen ohhvtrlakat $c v$ 'na enhonrkv ofvn fekvprres.

$27 \mathrm{Vm}$ puyvfekev pvsvtkat e hute ofvn wiketskekot, momet cenake Vcakat lekwen hecvren kometskekaret okv.

28 Hesaketv vyetvn vn kerkvkuecetskvtes; afvcketvn ce turof heckvn es ov fveecetskvres, maket.

29 Hunvntake etecakkvte toyatskat, cuko-hvmecvlke em ekv Tewe etektvnkusen cem opunvyehcrkvres, momat elepvtet ot, momet herickvtet on, en herickvhkvm epu 'paken mucv-nettv oret os. 
30 Monkv owalvt omet, momet Hesaketvmeset setemfvccetv-vcakan 'setemfaccat, vpeswv vcrkvyen etetackv em ette ofvn em ohliketv oh likvranat Klist akvwvprranen kerrekv;

31 Heyv hvtẹtvwrn hecet, Klist akvwrpketv opunvyecet, eme em puyvfekev prsvtkat e hute ofvn wihokekon, momet em vpeswr lekwen hecekates, maket okvtes.

32 Heyv Cesvs Hesaketvmese akvwvprtet omen omvlkeyat akerrvlke toyes.

33 Monkv hvlwehocvtet, Hesaketvmese em vkvpervn liket, momet Erke etemfaccat Puyvfekcr Vcakat en heckvtet, heyv hiyome hece, momet pohatskaten oh kalvtes.

34 Tewe hvlwe kvwvpkeko emunkekv, momis, Cehofvt cr Pucase tempunayet, Ceme vm vkvpervn liketske munken,

35 Cen horrvlke ce 'leohhuerickvn hayares, emet makvtes.

36 Monkv Eslevl en cuko-homecvlke omvlkvt mehenw $; n$ kerrvkekvs, heyv Cesvs mahusat vtaratskvte, Klist momet Pucase tepaken Hesaketvmeset hayvtes.

37 Tokvs, heyvn pohakat, e feken en sekehoye ohmen, Hunvntake tecakkvte toyatskat, naken estomvrane haks? Petvn momet vpastelvlke vpvlwvn kicakvtes.

38 Momof Petvt, Cem vkerrickvn eticvkes, momet omvlkvt naorketv cen kvpvkvkvren Cesvs Klist hocefkv ofvn es paptiskvkes, momen Puyvfekcv Vcakat est'emhoye tayat cen heckvkvres.

39 Temfrccetv ceme cenaket's, momet cen hopuetake enaket's, momet hoprye vpokakateu, estohmomusen Pucase pun Hesaketrmeset enhuehkvranat enaket omepekv, kicakvtes.

40 Momet ohvtvlakat opunvkv sulken en kerkuecakv-. 
tes, momet em vcahnet, Heyv honvpse yenahakat enkvpvket e hesahuecvkes, maket.

41 Momen em opunvkv 'safvckvke e enakuecepat paptiskakvtes, momen mv nettv mahusat estempuyvfekcv cukperakko tutcenvteket ohvpvkakvtes.

42 Momet vpastelvlke em vhakvn momet etohkvlketv, momet tvklike kvlkvn, momet mekusvpkv ofvn vpeye māhvtes,

43 Momen feksumketvt estempuyvfekev omvlkvn oh vlakvtes; momen vpastelvlket nake senhoneckvken, momet eskerkv tis momecakvtes.

44 Momen vkvsvmakat etohkvlket, momet nak' omvlkvn tvleme omen ete'm ocakvtes.

45 Momet nakocakat nakuce tis wiyaket, este omvlkvn en kvpicakvtes, enkusvpkakat vcrkvyen.

46 Momet nettr omvlkvn em vkerrickv hvmkusen cuko-vcakat ofvn fullet, momet cuko vlkat tvkliken kalet, e feke em mehenwv afvcketv tepakat ofvn hompaket, vpeye māhvtes,

47 Hesaketvmese vkvsamet, momet este omvlkvt heromkv em ocaken. Momen Pucaset nettv omvlkvn hesahokvranan mekusvpkv-cukon ohvpryvtes.

\section{SETENTVCKE III.}

Momen mekusvpkv hvse-vkerkv omof, esostvpakat tok, Petv Cane tepakat cuko-vcakat vcemhokvtes.

2 Momen este-hunvnwv ecke e nvrke ofv vteke yvkvpvte sekon 'svpehoyet, cuko-vcakat en tohopkvhauke Heruse kicetvn nettv omvlkvn er wrkechoyet omvtes, cuko-vcakat esceyan heromkvn em 'pohepvren.

3 Monkv Petv Cane tepakat cuko-vcakan cukolvkvranen hecat, heromkvn em 'pohvtes. 
4 Momen Petv hericet hecrtes, Canen tepaket, momet, Ep oh hecrs, kicvtes.

5 Momof vketecvtes, Nak' tis v'mvkvranes, komet.

6 Momen Petvt, Cvto-kunaplane momet cvto-kunaphrtke ocvkos; momis nake ocvyat ce'mis; Cesvs Klist Nasvlevlke hocefkv ofvn, ahuyiret, yvkvpvs, kicvtes.

7 Momet enkvpervn en hvlahtet a kvwapvtes; momen vyvkhvmkusen ele eletoktuswr esyomat en yekcakvtes.

8 Momen a kvwvpket a huervtes, momet yvkapet, tasket momet Hesaketvmese vkvsamet, mvn vpaket cukovcakan eceyvtes.

9 Momen yvkape Hesaketvmese vkvsamat este omvlkvt hecakvtes;

10 Momet mvt cuko-vcakat en tohopkvhauke Herusat heromkv vpohepe vlikvtet omen kerrakvtes, momet mv nake em momat vrahkvn tayen em estomahaket, feksumketv es fvefvke tates.

11 Momen mv hunvnwv yvkvpeko en wicehocat Petv Cane tepakan hvlatof, este omvlkvt em estomahaket mv sohvpettv Salvmvn enake kicetvn etoh pefatkvtes.

12 Momis mvn Petv hecat este em vyoposket, Eslevl hunvntake toyatskat, estomen estomahe es cem momvke haks? monkat estomen hericet pu hecatske haks? pun yekce momet pun heretv tis yv este yvkvpeceye omecet.

13 Eplvme, Iseke, Cekvpe esyomat en Hesaketvmese, momet purkvlke en Hesaketrmese Eppuce Cesvs vrakkuecvtes, momet ceme est' em wibket, Pilvte ehomv em ehelaksecatskvtes, enrecrpetvn komof.

14 Momis centat $\mathrm{Mv}$ Veake momet Fvecat belaksecatskvtet os, momet este-elecv min ce'mhoyvkvren vpohatskvtet os ;

15 Momet hesaketv Epucase elecatskvtes, mun Hesaketvmeset ra en kvwapvtet on; pumet a en kerrvlke toyes. 
16 Momen eme en hocefkvt, $m v$ en hocefkv vkvsvmkv eteropotten, heyv hunvnwv, hece kerratskat, yekcen hayet os; momes, mv eme etehoyvne vkvsvmkv ocat omvlkatskat ce homv take heyv etemvpoketv esfvckan e'met os.

17 Momen hiyomat, tecakkvte toyatskat, nakkerratskekat omecicen omatskvten kerris, momen es cem evpryvlkeu.

18 Momis mv nanvke ehomv etvwv em owalvlke omvlkv e cukwv eteropotte, Cesvs estemerkvres, Hesaketvmese make kerkuecrtet, hiyomen sohfvcecvtet os.

19 Monkv cein vkerrickvn etieatsken, mvrahkv ce hahoyvkekvs, mahlvpvtketv oketv Pucase ehomv akvpvket vlakof, cen holwvyeckv take vsholvren;

20 Momen Cesvs Klist enhomv es cen erkenvkhoyvten atohten ;

21 Mvn hvlwet esepvranet os, oketv nak' omvlkv mvherickv Hesaketvmese em owalvlke vcacakat omvlkv e cukwv eteropotte, ekvnv vlicecrte vteken opunvyecvte eroren.

22 Mehenwrn Moses erkvlken em punvyvtet omekv, Owalvn Pucase cen Hesaketvmeset ahuericvres, etecakkatskat etvwvn, vne omen; mvt naket o vtekat omvlkvn ce kicvkat pohatskvres, kicet.

23 Momen momēt ocvres, puyvfekcr mv Owalv em apohicekat vtekat em estvlke enkvpvken sumecihocvres.

24 Momēt os, momen owalvlke Samyvl huervte vteke, momet renyupv vyecicat, $\mathrm{mv}$ opunahoyvte vtekat hiyōmēn heyv nettr ocakan hvte etvwrn onvyakvtet omes.

25 Owalvlke en hopuetake toyatskes, momet Hesaketvmese purkvlke esetemfvecetv en haye Eplvme kicat, Ec ohhonvpse eteropotten tvlwvvlke ekvnv oh fullat omvlkvt en herakvres.

26 Centake taten Hesaketrmeset Eppuce Cesvs akvwah- 
pet, setetayatsken cen naorketv take cem meriyet cen kvpyecicvkvren atotvtet os, kicakvtes.

\section{SETENTVCKE IV.}

Momen esten em punvyakof, plestrlket, momet cukovcakat en kvpetvneu, momet Satusevlke esyomat oh yicrtes,

2 Este emvhayakat, momet Cesvs eteropotte elkv aenkvwvpketv eserkenvkakat svrahkvn efeknokhoket omet.

3 Momet enke taken oh ohcet, svlvfkuecakvtes, vhecihocen hvyvtkvren, yvfkepekv.

4 Mome estomis opunvkv pohakat sulket ohvkvsvmakvtes, momen este-hunvntake em vhonkvtkv cukpe-rakko cahkepat mahe tates.

5 Mome ocvtes erenprkse em evpayvlke, momen este vculvke, momen cokv-hay vlke,

6 Momen Anvs plest enhomahtv, momen Kivfrs, momen Cane, momen Vleksantv, momen plest enhomahtv en nakvlke en sulke estomusen omat Celuslvmen nvkaftvtes.

7 Momet en nvrkvpvn ye kahyof, em 'pohakvtes, Yekcetv estomen, monkat hocefkv estome eteropotten heyv momecet omatske haks? makaket.

8 Momof Petvt, Puyvfekcv Veakat es fvcket, kicakvtes, Este em evpayvlke, momet Eslevl em vculvke toyatskat, 9 Este yekceko en lopihocat ohfvccun, Nake omecicen en wiket ome haks? maket, mucv nettv ep ohvketehocen omat,

10 Omvlkatskat, momen Eslevl estvlke omvlkvt, cen kerkvket omekvs, mv Cesvs Klist Nasvlevlke mv vtare elecatskvte, mvn Hesaketvmeset elkvn rakvwvpvtet omen, momen $\mathrm{mv}$ e hocefkv eteropotten heyv este en wiket ce homv take hueret omes. 
11 Tohtarvlke toyatskat, heyv cvto, Nake toks, komatskvtet omen, mvt vkvnowv em ekv hakes.

12 Momet etv ofv tat vhesaketv sekot os, hocefkv etv sutv elecv este vprke vhesahokvraneyat este e'mhoyvte sekot omekv.

13 Momen tokvs, Petv. Cane tepakat en fekhvmketvn hecakof, momet cokv kerrvkekot, momet nakkerrvkekot omen kerrakof, em estomahakvtes; momet Cesvs vpvkakvtet omaken kerrakvtes.

14 Momen mv este en wicehocat vpvkaket hueren hecakof, vnrvpuece nake makvkeko tayvtes.

15 Nvkvftetv wolhuehcet etvn vhoyecihcof, emet ete'm punahoyaket,

16 Heyv este naken estomecrkvraneya? nake-eskerkv kerken momecakekv, momen Celuslvme vpokakat omvlkvt en kerket omes, momen pumeu helaksecēko tayes.

17 Momis ohvtvlaken este em etenrvwv hvmecicekaren yekcen naken es penkvlvkuecvkeres, svnvcomv este estomen heyv hocefkv ofv 'sem punvyvkekaren, makakvtes.

18. Momen enhuehkakvtes, momet Cesvs hocefkv ofv opunahoyekaren, monkat mvhayvkeko tayuset omvren yekcetv 'sem punayakvtes.

19 Momis Petv Cane tepakat em vyoposkaket, Hesaketvmese en renakv ehomv centake mimvn Hesaketvmese. 'semuntvlen cem apohicvkeyat fvcevt on omat, cemet fvccecvkes, kicakvtes.

20 Pume nake poheye momet heceyvte opunvyecēkare: ep omekot omekv.

21 Momen naken 'sestemerrvkuece tayat eshecakekot, ohvtvlaken naken es penkvlvkuecet meheof, wikakvtes, este svrahkvn; mv nake mohme ocat svrahkvn omvlkvt Hrsaketvmesen vrakkuecaket omekv.

22. Mv este nake-eskerkv estemwiceckv kerkat em mo- 
mehocat em vculkv ohrolope pale ostat hoyvnet omvtet omekv.

23 Momen wihokat, vhoyehpet, em etohkvlketvn e vpvkvkepvtes, momet plest enhomvhhotvlke, momen este em rculvke esyomat nake kicakan omvlkvn kerkuecakvtes.

24 Momen mvn pohakof, em vkerrickv hvmkusen en hake Hesaketvmesen vkvwrpakvtes, momet, Pucase, cemet, Hesaketvmese, hvlwat tis, ekvnv tis, momet uehvtkv tis, momet nake vtehkakat omvlkvn hayvtet toyetskes, makakvtes.

25 Estomen tvlwvvlket crpvkhokvt haks? momen este nake ehvperkvken vkerricvke haks? Tewe cem vtotkv e cukwv eteropotten maketskvtet omekv.

26 Ekvnv mekkvlkeh asvpvklvtes, momen evpryvlke etenvkaftvtes, Pucasen vnrvpet, momet $\mathrm{mv}$ enake Klist, mvu vnrvpet.

27 Mehenwvn Ceppuce vcake Cesvs, mv em vfeyetskvte, Helot, Pantevs Pilvtet, Centilvlke Eslevlke vprkvket vnrvpet etenvkaftvtet omekv;

28 Cenke momet kometskvte naken hrtetvwrn momvres komakvte momecvkvret etenvkaftet.

29 Momen hiyomat, Pucase, em opunvkr es penkvleckvn hecrs, momet cem vtotkvlke cem opunvkvn fekhvmketv omvlkvn 'sopunahoyvren emvkvs;

30 Estemwiceckvn, momet nake-eskerkv, momet nake senhonechoke Ceppuce vcake Cesvs e hocefkv eteropotten momehocvren cenken a rvtosecvs.

31 Momen mekusvpakat hoyahnof, mv etohvtelokat nekeyvtes; momen omvlkvt Puyvfekev Veakat es fvcfvket, Hesaketvmese em opunvkvn fekhrmketvn es opuna. hoyvtes.

32 Momen vkvsamvlke sulkat e feke, em puyvfekcvu hvmkuse tates, momen este estomet, Nake ocvyat ov- 
nake tes, makekates, momis nake omvlkvn tvleme omecakvtes.

33 Momen Pucase Cesvs Klist raenkvwrpketv vpastelvlket yekcetv rakken kerkueckvn 'semakvtes; momen heromkv rakket omvlkvn oh ocakvtes.

34 Momen mv vpvket nake enkusvpkat hvmkis sekates; ekvnv cuko tis ocakat estohmomusat wiyaket, nanvke wihoyat enrahkvn esyicakvtet omekv;

35 Momet vpastelvlke ele ehomvn yvpoyakvtes, momen este nake enikusvpkat vcrkvyen hvmkvnton - vwahlephoyvtes.

36 Momen Coses, mv vpastelvlke Panepvs, (mv ohyvtekhoyat, Mahlvpvtketv eppuce maketvts,) hocefakvte, Lefitvlke, Siplrs tvlofvlket,

37 Ekvnvn ocet, mvn wiyvtes, momet enrahkvn 'svla. ket, vpastelvlke ele ehomvn yvpoyvtes.

\section{SETENTVCKE V.}

Momis este-hunvnwv hvmket, Anvnivs hocefket, e hiwv Svfilv etepvket nake ocakat wiyakvtes;

2 Momet enrahkv akvpvken fekhonnicvtes, (e hiwvu en kerren,) momet kvpvkusen esvlahket, vpastelvlke ele tempen yvpoyvtes.

3 Momis Petvt, Anvnivs toyetskat, estomen Puyvfekcv Holwakat ce feken fvcece haks, ekvnv enrahkv kvpvken fekhonnicet, momet Puyvfekcv Vcakat en laksetskvren? kicvtes.

4 Hvte ocof, cenake tokvt haks? momen neshohyof, cen yekce ofv tokvt haks? estomaten heyv naken ce feke ofvn hocacetske haks? este tokon, momis Hesaketvmesen en lakset ometskes.

5 Momen Anvnivs, heyv opunvkvn pohat, tvklvtiket, 
hesaketvn wikvtes. Momen heyv nanvke pohakat omvlkvn penkvlkv rakket oh vlakvtes.

6 Momen hunvntake mvnettakat asvpvkilet, vpvllahyet, momet esvpehyet er hericakvtes.

7 Momen momet ocvtes, hvse-vkerkv tutcenat hoyahnof, e hiwvt, naken momehocat kerrekot, yeceyvtes.

8 Momen Petvt em vyoposkvtes, Vm onvyrs, hiyomusen ekvnvn wiyatskvt haks? Emet, Momes, mvomusen, kicvtes.

9 Momis Petvt, Naket omecicen Pucase em Puyvfekcvn eskerretvn temfvccatskvt haks? hecvs, ce he hericakat ele vhauken $\mathrm{v}$ svpakles, momet 'sec ossvkvres, kicvtes.

10 Momen vyvkhvmkusen Petv ele ehomvn tvklvtiket, hesaketvn wikvtes. Momen hunvntake mvnettakat escehyet, elepen hecakvtes; momet esossahket, e he tempen er hericakvtes.

11 Momen mekusvpkv cuko omvlkvn, momet heyv nanvke pohakat omvlkvn penkvlkv rakket oh vlakvtes.

12 Momen vpastelvlke enke eteropotten nake-eskerkv momet nake senhonechoke sulken momehocvtes, este em etenrvwrn; (momen Salvmv en sohvpettrn omvlkvt em vkerrickv hvmkusen vpokvtes.

13 Momen vpvltaket este estomis enhotet e oh vpryekates ; momis estet vrakkuecakvtes.

14 Momen vkvsamvlke, hunvntake hoktvkeu, sulsoket ohvtvlaken Pucasen oh vpvkakvtes;)

15 Momat enokhokat tehoyvnkvn esyicet, topv topuce tis oh lumhicakvtes, Petv hoyanat em vpette tis este sasat oh vpettrren.

16 Momen hvtvm tvlofv Celuslvme afolotat este asosset Celuslvmen yicakvtes, enokhokat, momet este puyvfekev holwvhokat es naorvkat esyicen; mohmen omvlkvn em wicehocvtes. 
17 Momof plest enhomahtvt, momet vprkakat omvlkvt, (mvt Satusevlke em etohkvlketvt omet,) asvpvkilet, cvprkketv es fvefahlet,

18 Momet enken vpastelvlken oh ocet, cuko-yekev tvlemen vtehakvtes. -

19 Momis neren Pucase em estet cuko-yekev vhauken hvwehcet, esossihcet,

20 Vpehyet, cuko-vcakat ofvn svpaklet, heyv hesaketv opunvkv omvlkvn este 'monvyvks, kicvtes.

21. Momen mvn pohakat, hvthvyvtkusen cuko-vcakan esceyet, muhayakvtes. Momis plest enhomahtv vlakat momet vpvkakat nvkvftetvn, momet Eslevl en hopuetake em vculvken senetvlken kihocat omvlkvn nvkaftecakvtes, momet cuko-yekcvn ohtotakvtes, mv vpastelvlken a'syihocvren.

22 Momis este-em-wiketv er yicakat, cuko-yekev ofvn eshecvkekot, afulehcet, kerkuecaket,

23 Cuko-yekcv vevyece heren vkhotken, momet vhecicrlke fettvn whauke ehomvn svpaklen hecet, momis hvweceyat, ofv estimvn hecēkis, makakvtes.

24 Momen heyv opunvkvn pohakat, plest enhomahtv hvlwat, momet cuko-veakat en kvpetvne, momet plest enhomvhhotvlke heyv nanvke ohfvccrn, heyvt naken bakvranat en kerkvkekates.

25 Momof este hvmket vlahket, Hecvs, mv hunvntake cuko-yekcvn vtehatskvnke cuko-vcakat ofvn svpaklet, este emvhayaket $o s$, em onvyakvtes.

26 Momen kvpetvne, este-em-wiketv esyomat vpeyet, mvn sosssicakvtes, ohyekcicvkekot, (esten em penkvlaket, crton es pu nvf kake wites, komaket.)

27 Mornet esyicaket, nvkvf tetv chomvn svpvklecakvtes; momet plest enhomahtvt em 'pohet,

28 Heyv hocef kv ofv mvhayetvn yekcen cem vseheye- 
kvnka? momen hecvs, cem mvhakvn Celusvlmen es fvcecatskct os, momet heyv este e catvn ep oh vlvkuecetvn komatsket os, kicakvtes.

29 Momof Petv, vpastelvlke vpvlwv esyomat em vyoposkaket, Este em apohiceko munket, Hesaketvmese min em apohicvraneyet os;

30 Purkvlke en Hesaketvmeset, Cesvs, mv eto vtahre, elecatskvten a kvwapvtet os.

31. Mvn Hesaketvmeset enkvpervn es hvlwecvtes, Evpayv momet Hesayecr taranen, vkerrickv-etickv momet holwvyeckv enkvpvyecickvn Eslevl emvren.

32 Momen heyv opunvkv ohfrcern, pumet, Puyvfekev Vcakat tis, mv Hesaketvmese em apohicakat emvten, a en kerrvlke toyes, em onvyakvtes.

33 Pohakat, efeketake etetacke omen, pvsvtetvn komet tem opunvyecakvtes.

34 Momof nvkvftetv ofvn hvmket, Kemelevl hocefket, Falvsevlket, vhakv-muhayvt, este omvlkv vrakkuecet, ahuyiret, Vpastelvlken sossihcet, estomusen fekhonnicvks, maket,

35 Eslevlke hunvntake toyatskat, e vketecvkes, heyv este ohfvecr estomecrranatskat;

36 Heyv nettvt yiceko munkof, Rutvs, este tetaye e omecen, mvn hunvntake em vhonkvtkv cukpe ostvteket oh vpvkakvtes, mvn elehocen, em apohicakvte estomomusat omvlkvt vwahhet, seko hakvtet omekv.

37 Momen heyv hunvuwv renyupvn Cutvse, Kalvlevlket, este nake ocakat hocefhokv tepakat vhonkvtkv nettr ofvn ahuyiret, e vcrkvpeyecicvtes, momen em apohicakvte estomomusat omvlkvt vwahvtet omekv. 38 Momen hiyomat ce kicrkis, Heyv esten wihkatsken, fullekvs; heyv mvhakv, momet heyv vtotketv este avtet on omat, sumkviret okv; 
39 Momis Hesaketvmese avtet on omat, sumecicatskeko tetayes; wikatskekon omat, Hesaketvmese etepoyatsken es ce kerhohyvke wites, kicakvtes.

40 Momen em vkvsvmakvtes, momet vpastelvlken enhuehkahket, rukafahket, Cesvs e hocefkv ofv opunvyetvn em vsehakvtes; momet vpeyecicakvtes.

41. Momen nvkvftetv ehomvn enkrpahket, Cesvs e hocefkv svrahkvn vlesketv en hahoyvre oricvken eskerhoyat svrahkvn afrckvket vpeyvtes.

42 Momen nettr omvlkwn, cuko-veakat momet cuko omvlkv ofvn, Cesvs Klist es emvhayetvn, momet es erkenvketvn wikvkekates.

\section{SETENTVCKE VI.}

Mv nettv ofvn, enkerrepvlke sulke mahe hakof, Klekvlke asosset vurvpe-opunvyeckvt kvwrpkvtes, Hepluvlken vnrvpet, nettv omvlkv vfvstetv ofvn em oyevlken fvecrn em afvstvkekat svrahkvn.

2 Momof pale hokkolohkakat enkerrepvlke sulsokat enhohiket, Hesaketvmese em opunvkvn wikèt, ohhompetỵn vfvstvraneyat herekot os, kicakvtes.

3 Monkv teeakkvte toyatskat, ece 'pakvkat hunvntake kolvpaken, here opunvyehocan, Puyvfekcv Vcakat hoporrenkvu es fvcfakan, heyv nakvfvistetvn ohvfrstvkueceyvren enhopoyvkes.

4 Momis pumet mekusvpkvn, momet opunvkv vfvstetvn e emeye emunkvres.

5 Momen mv opunvkv sulsokat omvlkvn afveecihcen, Stefen, hunvnwr Puyvfekev Veakat vkvsvmkru es fvckan, momet Felvpe, Plokolvs, Nikenv, Timvne, Pamenvs, momet Anteokvlke vkvsvmepuehocvte Nekolas hocefken enhopoyakvtes. 
6 Mvn vpastelvlke ehomrn svpvklehcen, mekusvpahket, enken oh wvkecakvtes.

7 Momen Hesaketvmese em opunvkv ohvtvlaket ayen; enkerrepvlke em vhonkvtkv Celuslvme ofv tayen ohvtvlakvtes; momen plestrlke sulke mahet vkvsvmkvn em vkvsvmakvtes.

8 Momen Stefen, vkrsvmkv yekcetv tepakat es fvckat, este vlkat ofv nake eskerkv, momet vtotketv estomahaken ruomecvtes.

9 Momof senekake Lepvtinvlke enake kihocat ohvprkakat vnvcomet, momet Silenvlke, Vleksantvlke, momet Seleser Esev tepakat asosset, asvprkilet, Stefen vnrvpinecakvtes.

10 Momet mv hoporrenkv puyvfeker tepvke 'sopunayat vnrvpetv umvkeko tayusvtes.

11 Momet Moses, momet Hesaketvmese vtvkleckvn es opunayen pohes, este.hunvntake makaken emvkerret heckuecakvtes.

12 Momet este, em vculvke, nakcoki-hayvlke esyomakan teyamakvtes; momet oh yihcet, hvlahtet, tekueketvn esyicakvtes ;

13 Momet vkerrv laksvlke, heyv este opunvkv heyv tvlofv vcakat vhakv tepakat vtvkleckv 'sopunayen pohes, makaken asvpvklecakvtes.

14 Momet, Heyv Cesvs Nasvlevlke heyv tvlofvn sumecihcet, semvhakv Moses pu'mvte mvrahkuecrres, maken pohekv, makakan.

15 Momen tekueketv ofv vpokat omvlkvt hericet hecakat, e turofv hvlwe-este e turofv tis ome omen hecakvtes. 


\section{SETENTVCKE VII.}

Момо plest enhomahtv hvlwat, Heyv nanvke momet o haks? makvtes.

2 Momen makvtes, Hunvntake, teeakkvte, erkvlke esyomatskat, pohvkes; Hesaketvmese vrakkat, Eplehame purken, Kelvn er likeko munken, Mesopotemevu liken, em e heckuecrtes,

3 Momet, Cem etvlofvn osiyet, cen nakvlkeu en kvpvkvs, momet ekvnv cem onvyvranvyan a oh vtes, kicvtes.

4 Momen Kaltevlke em ekvnvn osiyet, Kelvn er likvtes, momen, erke elehpof, yv ekvnv vpokatskan oh cunecknecrtes.

5 Momet mv ofvn cutkusis e 'le oh huericetv tis pueasetvn e'mekates; mome estomis, emen erhonrpse yupv yicvranat tis, mvn pucasetvn emetvn temfaccrtes, eppuce oceko emunken.

6 Momen Hesaketrmeset hiyomen em punayvtes, Ec ohhonvpse tvleporvlke em ekvnvn ohcuneckvlket omvres, momen svlvfkuehcet, meske cukpe ostat estemerrvkuehocvres.

7 Momen etvlwv en svlvfkvkvranaten ohfvececares; momen renyupv asosiyet, yvmv ekvnvn ve vfvstvkvres, Hesaketvmeset makvtes.

8 Momet svkvmsesvn setemfvecetvn e'mvtes; monkv Eplehamet Iseken en heckuehohcen, nettv escenvpakan svkvmsisvtes; momen Iseket Cekvpen en heekuehohcen, Cekvpet cuko-hvmecvlke-em-ekv pale hokkolohkakan en heckuehocvtes.

9 Momen cuko-hvmecvlke-em-ekv-take em vholvcahket, Cosef Ecepse escehoyvren wiyakvtes; momis Hesaketvmeset vpakvtes,

10 Momet estemerkv omvlkvn aossihcet, hoporrenkv he- 
romkv tepakan Felo Ecepse em mekko ehomvn emvtes; momen Ecepsen en cuko omvlkvu ohfvnken huericvtes.

11 Momis elaukot momet estemerkv rakket Ecepse Kenvn tepakat em ekvnv omvlkvn oh vlakvtes, momen" purkvlke hompetvn eshecvkekates.

12 Momis Cekvpe Ecepse ofvn teleko ocvcuken pohat, enhvteceskvn purkvlken vtotvtes.

13 Momen svhokkolv vpeyan Cosef e rahulken e cuseu em e kerkuecrtes; momen Cosef en nakvlken Felon en kerkuehocvtes.

14 Momen Cosef vtohtet, erke Cekvpe enhuehkvtes, en nakvlkeu omvlkvn, puyvfekcv pale kolvpaken cahkepohkaken.

15 Monkv Cekvpe Ecepsen vkhvtvpiket, elvtes, emet momet purkvlkeu.

16 Momen Sekem 'sohvpehohyen, cvto-kof ke, mv Eplehame Emv, Sekem erke, eppucetaken nesvte ocat ofvn wrkechoyvtes.

17 Momis Hesaketvmese Eplehame temfaccrte oketvt vwolicof, este Ecepse ofvn sulke hahket, ohitvlaket vyecicet

18 Emunken, mekko etvt Cosef kerrekot akvwrpkvtes.

19 Mvtat pun nakvlken vkerret, purkvlken holwvken svfvistakvtes, en hopuetakuce hesahokekaren vpvlvtakat oren.

$20 \mathrm{Mr}$ omof Moses heckvtes, momet en heckv heruset omvtes: momet erke e hute ofvn vhecihoce heren hrse tutcenat orvtes.

21 Momen ossihohcof, Felo echustet esehpet, echuswv omecet mahecvtes.

22 Momen Moses Ecepsvlke en nakkerretv omvlkv ofvn heren mvhahoyet omvtes, momet nakcelakv opunvkv tepakat ofvn yekce mahet omvtes. 
23 Momen ohrolope em pale ostat ore herof, etecakkakat Eslevl en hopuetaken en cukoperickv kometvt e feken en ceyvtes.

24 Momen hvmken fvccekon estemerrihocen hecat, emvnicvtes, momet $\mathrm{mv}$ en nokkihocan em en prlecet, mv Ecepsvlken elecvtes.

25 Momis etecakkakat, Hesaketvmeset crnken sossvkuecvranat kerrakes, komvtes, momis kerrvkekates.

26 Momen renhvyvtken, tepoyakof, em e heckuecvtes, momet, eten herkepueckvn komet, Hunvntake toyatskat, tecakkvte toyatskes, estoman nak' te'm vhopanatske haks? kicakvtes.

27 Momis mv vwolicvn nak' em vhopanat etvn vyecicrtes, Estimvt ep ohfvnke, momet ep ohfvececr ce hue-" ricvt haks? kicet,

28 Prksvnke Ecepsvlke elecetskvnke omen cv 'lecetvn kometske haks? kicvtes.

29 Momen Moses, yv opunvkvt omecicen letkvtes, momet Metevn ekvnvn tvleporv tates; mvn eppucetake hokkolen en heckuehocvtes.

30 Momen ohrolope pale ostat hoyahnof, Sinv ekvnhvlwe vnrvwv ofvn Pucase em estet eto-pokhe ofvn totkv fenken em e heckuecrtes.

31. Momof Moses hecat, em estomahvtes mv nak' hecat; momet vketecetvn kohmet oh vwolicof, Pucase em opunv$\mathrm{kv}$ en haket em vlakvtes,

32 Makat, Cerkvlke en Hesaketvmese toyit, Eplehame en Hesaketvmese, momet. Iseke en Hesaketvmese, momet Cekvpe en Hesaketvmese toyis. Momof Moses fekekvtes; momct vketecetv umekates.

33 Momof Pucaset em punayat, Cele em estelepikvn tefvs ; $m v$ oh hueretskat ekvnv vcaket omekv.

34 Ecepse ofvn vm estrlke em estemerkvn hecit, hecv: 
yvtes, momet en hihketvn pohit, sossvkuecvranit ahvtvpkit omis. Monkv hiyomat vtes, Ecepsen ece 'totares.

35 Heyv Moses, eyacvkekvnna, Estimvt ohfvnke momet ohfvececr ce huericvt haks ? kihocvte, mv mahusan Hesaketvmeset cm este eto-pokhe ofv em e heckuecrte enke aetehoyanen vtotvtes, ohfvnke momet sossicv taramen.

36 Heyv estet, Ecepse ekvnv ofv, uehatkv Cate ofvu, momet vnrvwv ofvn nake sehoneckvken, momet nake eskerkvn ohrolope pale ostat ofv heckuecof, mvn asossicvtes.

37 Heyvt mv Moseskvt, Owalv vne omen Pucase cen Hesaketvmese tecakkatskvte aossen ahuericrres, mvn 'mapohicatskvres, Eslevl en hopuetaken kicakvte tes. 38 Heyvt, mekusvpkv-cuko vnrvwv ocat ofvn huervte, hvlwe-este Sinv ekvnhvlwe ofv em punayvte, momet purkvlkeu vpakvte, momet opunvkv hesahoke pu'mvranat en heckvtet, mv tes.

39 Mvn purkvlke, mapohicetrn komvkekot, vhepvkakvtes; momet e feke ofvn Ecepse hvtvm yohfulecet,

40 Elvn okakat, Hesaketvmese pun hahievs, pum vhomahtvkvren; heyv Moses, Ecepse ekvnv a 'sepu'ssicvnke, estvn estomat kerrēkos, kicakvtes.

41 Momet mv nettv take omof wakucen hayakvtes, momet nake pvsvthoyvranan mv nakvhaken e'makvtes, momet enke em vtotketvn es afvckakvtes.

42 Momof Hesaketvmeset folotiket, sutv ofv nake sulke oce tayan emekusvpvkvren wikvtes, owalvlke en nakcokvn es hoccat verkvyen, Eslevl en cuko toyatskat, ohrolope pale ostat ofv nake prsvthoyvranan, momet nake pvsvthoyan vnrvwv ofvn v'matskvt haks?

43 Momen Molvk em escukohakv, momet cen hesaketvmese Lemfan en kococumpvu, nakvhake mekusvpvrane 
hayatskvte, esatskvtes, momen Papelvn rvsimvn seceyvkares.

44 Purkvlke vnrvwv ofvn escuko-hakvn ocakvtes; eskerkvn Moses em punayat mellvte omen, vhake hecvte etvpomen hayvren.

45 Mvn purkvlke en heckvkvtet, Caswe vpvkakat Centilvlke epucasakan esyicakvtes, mvn Hesaketvmeset purkvlke ehomvn vtohkvtes, Tewe en nettv eroren.

46 Mvt IHesaketvmese ehomvn heromkvn eshecvtes, momet Cekvpe en Hesaketvmese escuko hakvn es en hecetvn komvtes.

47 Momis Salornvn cukon en hayvtes.

48 Momis mv Hvlwe Mahat cuko este enke es hakvte likekot omes, owalv makat,

49 Hvlwet vm oh́liketvt omes, momen ekvnvt cvle vm ohhuerickvt omes: cuko estomen vn hayvranatske haks? monkat estvn fekapvyat naket $o$ haks? Pucase makvtet os:

50 Momet, Heyv nake omvlkvn evnket hahicvtet omeko haks?

51 Enokwv yekcvke, ce feke, momet hvcko ofv svkvmsishoyvte seko toyatskat, Puyvfekev Veakat svnrapatske munkvt omes; cerkvlke momecvte omen cemeu momecatskes.

52 Owalvlke estoman cerkvlke assecvkekat haks? momet Fvecat vlvkvranat homv kerkuecakan pvsvtakvtes; mvn ceme hiyomat wiyvlke, momet elecvlke hakatskvtet omes.

53 Cemet, hvlwe-este wiyakat vhakv cen heckvtet omis, veayecatskekos.

54 Momen heyv nanvken pohakat, e feke oren warkvtes, momet e nuten oh etetekkakvtes.

55 Momis eme, Puyvfekcv Vcakat es fvcket, hvlwe vnvtaksvtes, momet, Hesaketvmese en rakke, momet Cesvs Hesaketvmese em vkvpervn hueren hecrtes, 
56 Momet, Hehcrkes, sutvt hauhahken, Este Echuswv Hesaketvmese em vkvpervn hueren hecis, makvtes.

57 Momof huehketv yekcen huehkakvtes, momet e hrckon cekkottaket, em vkerrickv hvmkusen oh pefatkvtes.

58 Momet tvlofv ossicaket, cvton vfvllakvtes, momet akerrvlke em accusvke este-hunvnwv mvnette, Sal hocefke, ele tempen rvpoyakvtes.

59 Momen Stefen, Hesaketvmesen oh huehket, Pucase Cesvs toyetskat, vm puyvfekcvn esvs, maken, crton es vfvllakvtes.

60 Momen tvk tokkehket, yekcen huehkvtes, Pucase toyetskat, heyv naorketv em en rvhecahketskvs, makvtes. Momet heyvn makat, nocepvtes.

\section{SETENTVCKE VIII.}

Momen em elkv Sal vkvsvme tates. Momen mv nettv omof, emekusvpkv-cuko Celuslvme ofv vtehkvte asseckv rakket em ocvtes: momen vpastelvlke tokvkekat omvlkvt Cuter Semelev tepakat em ekvntvckv eteropotten vwahvtes.

2 Momen este-hunvntake fvccuket Stefen hericuranet sohvpeyvtes, momet waketv rakken oh hayakvtes.

3 Moinis Sal emekusvpkv-cuko yvmahkuecrtes, cuko vtekat eceyet, hunvntake hoktvkeu esyomakat rasofothuecet, cuko yekcrn vtehvtes.

4 Monkv mv vwahat, hvmkvn vpeyet, opunvkvn erkenakvtes.

5 Momof Felvpe, Semeler tvlofvn vkhvtvpiket, Klist em erkenvkakvtes.

6 Momen nanvke eskerkv Felvpe momecat este pohakat, momet hecakat, em vkerrickv hvmkusen mv nanvke opunayat em apohicakvtes. 
7 Puyvfekev hvsvthvkekat, yekeen huehkaket, sulken vtehkvte asossekv; momen sulket, fekeketr en heckakat, momet yvkvpvkekate en wicehocvtes.

8 Momen afvcketv rakket mv tvlofvn ocvtes.

9 Momis este-hunvnwv hvmket, Simvn hocefket, nake estomahakan mvkerretvn es momecat, Semelevlke em vkerrickvn en hvlvtepuecet, este rakke e omecet opunayat.

10 Mvn omvlkvt em apohicakvtes, cutkus mahat tot, rakke mahan vteket, Heyv hunvnwv Hesaketvmese en yekce rakkat tot omes, makaket.

11 Momet mvn em apohicakvtes, hofunen emvkerretv vtotketv es hacohayvkvtet omekv.

12 Momis Felvpe Hesaketvmese em ohmekketv, momet Cesvs Klist hocefkv. nanvke ohfvecvn erkenakat vkvsvmakat, paptiskakvtes, hunvntake hoktvke esyomvken.

13 Momof Simvn mahusat vkvsamvtes, momet paptishohyof, Felvpen vpake munkvtes, momet nak' sehoneckvke, momet nake eskerkv momehocen hecat, em estomahvtes.

14 Momis Semelev Hesaketvmese em opunvkvn e enakuecakvcuken vpastelvlke Celuslvme vpokat pohakof, Petvn Canen tepaken ohtotakvtes.

15 Mvt rvkhvtvphohkof, Puyvfekev Veakat en heckvkvren em emekusvpakvtes.

16 (Hrte estomis oh lvtkeko munkvtes, Pucase Cesvs hocefkv es paptishoyat tvlkusekv.)

17 Momof enken oh wrkhokicahken, Puyvfeker Vcakat en heckakvtes.

18 Momen Simvn, vpastelvlke enke est' oh wvkecakan Puyvfekev Vcakat en heckaken hecat, evto-kunawrn emetvn kicvtes, 
19 Heyv yekcetv vneu v'mvkes, este estomen cvnke oh wrkhokicvyat Puyvfekcv Vcakat en heckvkvren, maket.

20 Momis Petvt okat, Cen evto-kunawr ece 'paket sumkekvs, Hesaketvmese em estemkvn cvto-kunawvn es nesvke tetayes, kometskekv.

21 Heyv opunvkv ofvn nake hvmkusis, momet nake eutkusis ocetskekos, ce feket Hesaketvmese ehomv fvccekot omekv.

22 Monkv heyr cen naorketvn ce feken es nokkicvs, momen, ce feke em vkerrickv cen kvpvyecihoce witvren, Hesaketvmesen emekusvpvs.

23 Kvlakcuwr en home, momet naorketv eswrnakv ofvn hueretskveuken hecikv, kicvtes.

24: Momof Simvn vyoposkat, Pucasen vm emekusvpvks, yv nanvke opunvyecatskat hvmkusis ve oh vlvkekaren, kicvtes.

25 Momen mv este Pucase em opunvkvn kerkuecahket, erkenvkahkofvt, Celuslvmen yoh fulecrtes : momet Semelevlke em etvlofuevlke sulken opunvkv-hervn erkenvkakvtes.

26 Momen Pucase en hvlwe-estet Felvpen em punayvtes, hvmmaket, Ahuervs, momet wahvlv fvecvn, mv vyetv Celuslvme vte Kesv vkhvtapken vyvs, mvt vnrvwv tes.

27 Momen ahuyiret, ayvtes, momen hecvs, hunvnwv, yunvke, Ereopvlket, yekcetv rakken ocet, Kantese Ereopvlke hokte mekko em eleevn hueret, mv enake veacakat omvl vfastvt, emekusvpvranet Celusivmen vlakvtet,

28 Rafulket, momet em calev ofvn likat, Esivs owalv hoccicuten, ohonvyvtes.

29 Momof, Heyv calevn vwolicet, ohvpvkvs, Puyvfekevt Felvpen kicvtes.

30 Momen Felvpe oh letiket, Esivs owalvn ohonayen 
pohvtes, momet, $\mathrm{Mr}$ nak' ohonayetskat kerretske haks? kicvtes.

31 Momen, Estoman vc ume taye haks, hunvnwr hvmkvteket vm fvyatet omekon omat? makvtes. Momet Felvpe ohcemiket, en likepvren izomvtes.

32 Nakcokv ofv opunvkv ohonayat, heyv tates, Yvpe fikv omen eleckvn sohvpehoyvtes, momet yrpefikuce en tonv ehomv cryayvke omet e cukwrn hrwvklecekates.

33. En kvnerpetv ofv em ohfvececkv em eshoyvtes, momen estinvt en honvpsetv kerkuecvr haks? en hesaketv ekvnv enkvpvyecihocekv.

34 Momen yunvket em vyoposkat, Cem 'pohis, estimvn opunvyecet owalv heyv maket oke haks? eme etrwrn, monkat este hunvnwv etvn oke haks? Felvpen kicvtes.

35 Momof Felvpe e cukwvn hvwvklehcet, momet mv hoccat etan svlicecet, Cesvn em erkenakrtes.

36 Momen em vyetv vhoyof, uewv ocen rorhoyvtes ; momen yunvket, Hecrs, heyvmon uewv oces; cv paptishoyvre naket 'svn tacke haks? makvtes.

37 Momen Felvpet, Ce feke 'somvlkvn vkvsametsken omat, mometske tayes, kicvtes. Momen em vyoposkat, Cesvs Klist Hesaketrmese Eppucet on vkvsamis, makvtes.

38 Momen calevt huervren kicvtes; momen hokkolvt uewvn vk hvtvphokvtes, Felvpe yunvke tepakat, momen paptisvtes.

39 Momen newv er a enkvpvkahkof, Pucase em Puyvfekcvt Felvpen sayepvtes, momen yunvke svnvcomvn hecekates ; momet em vyetv afvcket ayepvtes.

40 Momis Felvpe Esotvn hechoyvtes; momet tehoyanat, tvlofv omvlkvn em erkenaket emunket, Seselevn vlakvtes. 


\section{SETENTVCKE IX.}

Momen Sal Pucase en kerrepvlken punvkv-es-penkvleckvn momet pvsvtetv eyackvn es hesaket, plest enhomahtv hulwan oh ayet,

2 Momet nakcokvn Temaskvs senekake ocakan ohtothoyvranan em 'pohvtes, momat heyv vyetv hunvntake, hoktvkeu eshecaken omat, wvnawvken Celuslvmen svlvkvren.

3 Momet ayat Temaskvs vwolicvtes; momen vyvkhvmkusen hvyayvket hvlwe vtet vfolotet hvyvyakvtes.

4 Momen ekvnvn oh latkvtes, momet opunvkv, Sal, Sal, estoman vc assecetske haks? kicen polivtes.

5 Momen, Pucase toyetskat, estimv toyetske haks? makvtes. Momen, Vne, Cesvs, mv assecetskat toyis, Pucaset makvtes. Nak' fvsfvke ohrefketv cen yekcet os.

6 Fekeket, momet em estomahet, Pucase toyetskat, naken momecare kometske haks? kicvtes. Momen Pucaset, Ahuervs, momet tvlofv eceyvs, momen nak' momecvranetskat cem onahoyvres, kicvtes.

7 Momen hunvntake vevkfullat punayvkeko haket svpaklvtes, enhake pohaket omis, esten hecvkekot.

8 Momen Sal ekvnv oh wakkvte ahuervtes, momen e turwv en hauhahkof, este hecekates, momis enken en hvlvtahket, Temaskvs ofvn svpeyvtes.

9 Momen nettv tutcenat hecekates, momet hompekot, eskekates.

10 Momen enkerrepvlke hvmket, Anvnivs hocefket, Temaskvs likvtes, momen mvn Pucaset, nakhecihocat ofvn, Anvnivs, kicvtes. Momen, Pucase, hecvs, vnet likis, makvtes.

11 Momen Pucaset okat, Ahuyiret, tehoyvnkv Lvpotke 
kihocan oh ayet, Tasvlke, Sal hocefkan, Cutvse en cuko ofvn rvpohvs, hecvs, emekusapekv;

12 Momet nakhecihocat ofvn, este-hunvnwv, Anvnivs hocefket, aceyet, hece hakvren enken oh wrkecen hecrtes, kicvtes.

13 Momen Anvnivs em vyoposkat, Pucase, heyv bunvnwv ohfvecv nak holwvhoke estrmahen ce mekusapvlke Celuslvme vpokat momecvten este sulke etehoyvnen pohvyvnks.

14 Momet heyvmvn plest enhomvhhotvlke a vten yekcetvn ocet os, ce hocefkv oh huehkakat omvlkvn wvnawicvret, kicvtes.

15 Momis Pucaset okat, Vyvs, mvt svtotketv enhopoyvyvte Centilvlke, mekkvlkeu, momet Eslevl en ho puetake ehomv cv hocefkv 'svrvranat omekv;

16 Nake rakrvke estvmahe cv hocefkv svrahkv estemerkvranan hecicaret ok, kicvtes.

17 Momen Anvnivs ahyet, cuko receyvtes momet enken oh wvkhokicet, Tecakkvte Sal toyetskat, Pucase, (Cesvs, atetske cem e heckuecvnke,) vc vtotes, cen renakv cen heciket, Puyvfekev Vcakat es fvcketskvren, kicvtes.

18 Momen vyvkhvmkusen nakcelkv omet e turwvn a em pvlatkvtes, momen momat vpakusen renakv en heckvtes, momet ahuyiren paptiskvtes.

19 Mohmen, hompetv en hecikof, yekce hakvtes. Momof Sal nettv vnvcomen enkerrepvlke vpakvtes, Temaskvn.

20 Momen senekakvlke ofvn, Klist Hesaketvmese Eppuce tat em erkenakvtes.

21 Momis pohakat omvlkvt em estomahakvtes, momet, Heyv este Celuslvme of heyv hocefkv oh huehkakat sumhuecvnket, momet heyv esvrahkvn wrnawvken plest 
enhomahhotvlke'sem vyvret vlaket omekat haks? makakvtes.

22 Momis Sal en yekce em ohvtvlaket emunkvtes, momet heyvt Klist o herat kerkuecet, Cusvlke Temaskvs vpokakat fekhervkekon hahicvtes.

23 Momen nettv sulket sohfvcikof, Cusvlke elecetvnkomet 'setem punahoyvtes.

24 Momis mv emehakakat Sal en kerkvtes. Momen nettr nere tepakan elecetvn komet tohopke vhauken vhecicakvtes.

25 Momof enkerrepvlket neren ehset, svmpvn vpihket, tvlofr em atohopke-rakko a's tvk hvtvpecicakvtes.

26 Momen Sal, Celuslvme vlakat, enkerrepvlke vpvketvn komvtes, momis omvlkvt em penkvlvke tates, momet enkerrepv tat vkvsvmvkekates.

27 Momis Panepvs evpahyet, vpastelvlke 'sem vlakv-

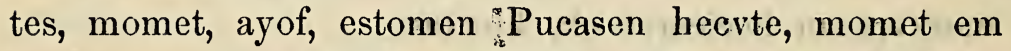
punayvten, momet fekhvmke heret Temaskv ofv Cesvs hocefkv erkenakvten en kerkuecakvtes.

28 Momen Celuslvme ofvn vpvkakvtes, aosset, raceyet.

29 Momet Pucase Cesvs hocefkv ofvn fekhvmke heret opunayvtes, momet Klekvlken vnrapvtes; momis elecetvn komet etefullakvtes.

30 Momis tecakkakat kerrahkof, Seselev 'svk hvtvpehcet, Tasvn ohtotakvtes.

31. Momof mekusvpkv-cuko Cutev, Kalvle Semelev esyomat ocakat herkvn ocakvtes, momet yekcvkuehocvtes; momet Pucase em penkvlkv, momet Puyvfekev Veakat mahlvpvtketv ofvn vpeyet, ohvtvlakvtes.

32 Momen mome ocvtes, Petv mv ekvnv omvlkvn eteropottet aret, Letv mekusapvlke vpokan; mvu vk hvtapkvtes.

33 Momen mvn hunvawv hvmken, Enevs hocefken 
hecvtes, fekeketv enokketvn enokket ot, ohrolope cenvpaken en topv oh wakket omvtes.

34 Momen Petv kicat, Fnevs toyetskat, Cesvs Klist temvpoken ce hayes; ahuyiret, cem pvtakvn pvticrs. Momen vyvkhvmkusen ahuervtes.

35 Momen Letv momet Selvn ofv este vpokat omvlkvt hecaket, Pucasen oh fulotecrtes.

36 Tokvs, enkerrepv Taperv hocefket, ( $\mathrm{mv}$ oh yvteketv Takvs kihocat,) Capvn likvtes; heyv hokte vtotketv herakat, momet merkv vtotketv mecrtet es fvcket omvtes.

37 Momen mome ocvtes, mv nettv take omof, enokiket, elvtes; momen vklopecahkof, ohonvpv nvthofvn wvkecakvtes.

38 Momen Letv Capv vwolicet ok, enkerrepvlke Petvt mvn likvcuken pohakat, hunvntake hokkolen ohtotakvtes, oh vtetv vcewicekären en komaket.

39 Momen Petv ahuyiret, verkayvtes, momen, erohrof, ohonvpr nvthofvn es oh 'cemecakvtes, momen oyevlke omvlkvt hvkihhoket, vnakvn svpaklvtes, Takvs vpake munkof, kapv accvke tis hahicvten hecicaket.

40 Momis Petv omvlkvn sossicvtes, momet tvk tokkehket, emekusapvtes, momet enan oh fulotket, Taperv toyetskat, ahuervs, kicvtes... Momen e turwv arvmricet, Petvn hecat, alikvtes.

41 Momen enken a em wiyiyet, akvwapvtes, momet mekusapvlke momet oyevlke enhohiket, wenaken hecepuecvtes.

42. Momen Capv omvlkv eteropotten kerkvtes; momen sulket Pucase ofvn vkvsvmakvtes.

43. Momen mome ocvites, Capv ofvn, Simvn nakhrrpa. catecrn nettr sulken en fekhonnepvtes. 


\section{SETENTVCKE $\mathrm{X}$.}

Este hvmket, Kvnelevs kihocet, sentulev, metohkvlketv Etvlvlke kihocat en kvpetvnvlke hvmkat, Seselev ofvn likvtes.

2 Hunvnwv fvccet, momet en cuko 'somvlkvt; Hesaketimese em penkvlvket, heromkv sulken este emet, momet estofis Hesaketvmese emekusapet omvtes.

3 Nettv hvse-vkerkv esostvpakof nake hecihocat ofvn hvlwe-estet, Kvnelevs toyetskat, kicet, a en ceyen heckusen hecrtes.

4 Momet mvn hecat, penkvle tates, momet, Pucase toyetskat, naket o haks? kicvtes. Momen, Cem emekusvpkv, momet cen heromkv Hesaketvmese ehomv esvkerrickvn kvwapket os, kicvtes.

5 Momen hiyomat hunvntake Capvn vtotvs, momet Simvn, Petv kihocan, enhuehkvs;

6 Simvn, este nakhvrpe-catecvn, en cuko uehatkv afopke ocan, fekhonnes; mvt nake estometske tetayat cem onvyvres, kicvtes.

7 Momen $\mathrm{mv}$ hvlwe-este Kvnelevs em punayvte enkvpahkof, en cuko vfastvlke hokkolen, momet suletawv eme vfvstake emunkv tate hvmken, emekusapvt omen, enhohiket,

8 Momet heyv nanvke omvlkvn en kerkuecahket, Capvn vtotakvtes.

9 Momen rempvkse vpeyet, momet tvlofv vwolicakof, hvse-vkerkv sepakat mahe orof, Petvt cuko-onvprn emekusvpvranet oh 'cemkvtes.

10 Momet elawnse hakvtes, momet hompetvn komvtes; momis etetakuecakof, nocekon vpueckv omet oh latkvtes ;"

11. Momet hvlwat enrvmken, nakvtehkv hvmket, vecetwhake rakke omet, kvnowv ostat etelvcet, a oh hvtapken, momet ekvnvn a oh hvtepecihocen hecvtes. 
$12 \mathrm{Mv}$ ofv ele-ostakat ekvnv oh fullat mvrahrvkv omvlkvt, momet punvttv, momet nak hvllecusat, momet fuswv hvlwe fullateu vtehket omvtes.

13 Momen opunvkv en haket em vlakvtes, Petv toyetskat, ahuyiret, pvsatet, hompvs.

14 Momis, Mohmekos, Pucase, nake vcakekat, momet: hvsvtkekat hompvyvte sekot omekv, Petvt kicvtes.

15 Momen svhokkolv opunvkv en haket em vlakvtes, Nake Hesaketvmese hvsvtecrte, mvn vcakeko kihcetskvs.

16 Heyv svtutcenv momvtes; momen mv nakvtehkv' hvtvm sutvn vkvwrphoyvtes.

17 Tokvs Petv heyv nak hecihocat hecat nak estoman omat en kerkekof, hehers, hunvntake Krnelevs a ohtotakat Simvn e huten vpohahket, vhauken vsvpaklvtes.

18 Momet huehkat, Simvn, Petv kihocat, mvn fekhon-: nat vpohakvtes.

19 Momen Petv nak hecihocan oh vkerricof, Hehevs,' hunvntake tutcenet ce hopoyakes, Puyvfekcvt kicvtes. 20 Monkv ahuyiret, ahvtvpkvs, momet vevkvyvs, Naken vn kerkekos, komekot, vnet vtotakit omikv, kicvtes.

21 Momof Petv hvtvpiket, mv hunvntake Kvnelevs a ohtotakat er em orvtes, momet, Hehcvkes, vnet mv hopoyatskat toyis; naket omecicen yicatske haks? kicvtes.

22 Momen, Krnelevs sentulev, hunvnwv fvece, momet: Hesaketvmese em penkvle, momet Cusvlke trlwvvlke omvlkv este fvece omecvke likaten, Hesaketvmeset hvlwe-: este vcakan atoten, en cukon ce'nhuehket, opunvkv cem. pohvren 'mveahnes, makakvtes.

23 Momof a 'sceyihcet fekhonnicakvtes. Momen rempvksen Petv vpaket ayvtes, momen etecakkvte etvt, Capv' a vwet, vhorkvsakvtes.

24 Momen rempvksen Seselev seceyvtes. Momen" 
Kunelevs en nakvlken, momet en hessvlke vwolicakat nvkvftecahket, e'mehakakvtes.

25 Momen Petv yeceyof, Kvnelevs tenrapet, ele tempen tvk lvtiket; 'emekusapvtes.

26 Momis Petv, Ahuervs, vneu este toyis, kicet, ahuericrtes.

27 Momen em punayof, ecehyet, nvkaftat sulken hecvtes. 28 Momet, Hunvnwv Cusvlket on omat tvleporv ohvpvkrre, monkat oh vyvre vhakv tokot omen kerratskes; momis Hesaketvmeset este estomen veakeko, monkat hvsvtkeko kicvranvkon vn kerkuecvnks.

29 Monkv ec oh atit omis, seokepkvn hayvkot, v'nhuehhokat vpakusen; monkv cem 'pohis, Nake svrahkvn v'nhuehkatskvt haks? kicvtes.

30 Momen Kvnelevs okat, Nettv ostof e elvwecen, hvsevkerkv hiyomat orvnkes, momet hvse-vkerkv sostvpakof vn cuko ofvn mekusapvyvnks; momen hecrs, hunvnwv em accvke hryayvhoket ev homvn huervnks.

31 Momet, Kvnelevs toyetskat, cem emekusvpkv pohkes, momet cen heromkv Hesaketvmese ehomvn vkerrihocet os.

32 Monkv Capvn vtotvs, momet Simvn, Petv kihocen enhuehkvs, Simvn nakhvrpe-catecv uehatkv vfopke likat en cuko ofvn fekhonnet omes; mvt vlakof, cem punvyvres, makvtes.

33 Monkv moman vpakusen ec ohtotvyvnkes, momen vlaketskat heren ometskes; Monkv hiyomat Hesaketvmese ehomvn omvlkeyat vpokes, Hesaketvmese nak cem onayat omvlkvn pohetvn cen komēt, makvtes.

34 Momof Petv ecukwv hvwvklehcet, Mehenwvn Hesaketvmese este etemetuecekot omen hecis ;

35 Momis tvlwv vtekat ofv estimvt em penkalet, fvecetv vtotkat emen afrcecicet os, makvtes. 
36 Opunvkv Hesaketvmese Eslevl en hopuetake ohtotakvte, Cesvs Klist eteropotte herkvn erkenakvtet; ( $\mathrm{mvt}$ omvlkv e Pucase tes;)

37 Mv opunvkvn kerratskes, mv Cutev ofv omvlkvn kerkuehocvtes, momet mv paptesvm Cane erkenakvte enyupvn Kalvlen vlicecrten;

38 Momat Cesvs, Nasvle aossat, Hesaketvmeset Puyvfẹcv Vcakan, momet yekcetvn es vfeyvtet omes; mvt nak herakan momecet arvtes, momet Puyvfekcv Holwakat svlvfkuecat omvlkvn em wicecvtet os; Hesaketvmese vpakvtet ok. 39 Momen mv Cesvs eto vtahret elehocvtet Cusvlke em ekvnv ofv, momet Celuslvme ofv nak omvlkvn momecvte, pumet a en kerrvlke toyes.

40 Mvn Hesaketvmeset nettv svtutcenof ra kvwapvtes, momet heckv-heren,

41 Este-omvlkv tokon, momis akerrvlke Hesaketvmese enhomv enhopoyvten en heckuecrtes, pumet mv akerrvlke toyen, pvsatkvte aenkvpvken akvwvpkvte renyupvin ete'n hompe ete'm eskeyvtet.

42 Momet, este em erkenvkaket; Hesaketvmese hesahokat momet prsvtkat Ohfvececv taranen ohmellvtet omen, ohkerkueceyvren, mvt pu kicvtet os.

43 Mvn owalvlke omvlkvt a en kerkuecaket os, e hocef kv eteropotten estimvt o estomet oh vkvsamen omat, naorketv enkvpvketv en heckvranet os, makaket.

44 Momen Petv heyv opunvkvn opunaye emunkof, Puyvfekcv Vcakat opunvkv pohakat omvlkvn oh latkvtes.

45 Momen vkvsamvlke svkvmsisakat, Petv vevkawvte en sulke estomomusat, em estomahakvtes, Centilvlkeu estemkv Puyvfekcv Vcakat a oh kvlkekv.

46 Momet estempunvkv mvrahrvkvn es opunahoyet, Hesaketvmesen vrakkuecaken pohakekv. Momof Petr vyoposkat, 
47. Heyv, pume etvpomuse Puyvfekcv Vcakat en heckakat paptishoyvkekare, estimvt uewv sentvcke tetaye haks? makvtes.

48 Momet Pucase e hocefkv ofvn paptisvkhoyvren kicvtes. Momen nettv vnvcomusekon vpvkvren e'nkusvpakvtes.

\section{SETENTVCKE XI.}

Momen vpastelvlke, momet etecakkvte Cutev ofv vpokat Centilvke Hesaketvmese em opunvkv en heckvcuken pohakvtes.

2 Momen Petv Celuslvmen vlahkof, svkvmsisakat vnrvpakvtes,

3 Hunvntake svkvmsisvkhoyekan oh ayet, ete'n hompetskvnks, makaket.

4 Momis Petvt vlicecet, etetayusen en kerkuecet, maket,

5 Capv tvlofv ofvn emekusapvyvnks, momet nockv-seko vpueckv ome nake hecihocan hecryvnks, nakvtehkv vecetv hake rakke omet hvtapkan, vkvnowv osten hvlwen a's hvtvpecihocen, vne mahusan vm vlakvnks.

6 Momen mvn hericet hecet, oh vkerricvyvnks, momet ele-ostvke ekvnv oh fullat, momet punvttvn, momet nak hyvllecusat, momet hvlwe fuswv fullat hecryvnks.

7 Momen opunvkv en haket, Petv toyetskat, ahuyiret, pvsatet hompvs, ev kicen pohvyvnks.

8 Momis, Mohmekos, Pucase, nake vcakekat, monkat hysvtkekat cv cukwv eceyvte sekot os, makvyvnks.

9 Momis opunvkv en hake hvlwe vtet hvtvm vm vyoposkvnks, Nake Hesaketvmese hvsvtecvten vcakekon kihcetskvs.

10 Momen heyv nake svtutcenv momvnkes, momen hvtvm omvlkvn sutv ofvn a hvlvthoyvnks. 
11 Momen hehevs, moman vpakusen este-hunvntake tutcenet cuko likvyan vsvpvklvt hakvnks, Sesvlev awe a ve ohtothoyen.

12 Momen Puyvfekcvt, Mvn vcvkvyvs, naken cen kerkeko omekot, cv kicvnkes. Momen heyv etecakkvte epakat ve vpaken, hunvnwv en cuko esceyeyrnks.

13. Momen estomen en cuko ofv hvlwe-este hueren, momet, Huuvntaken Capvn vtotetsken, Simvn, Petv hocefkan, enhuehkvkekvs ;

14 Mvt, opunvkv mv ceme momen cen cuko omvlkv es hesahoke tayat cern onvyvres, kicen hecvtet pu hecicvnkes. 15 Momen opunvyetv vlicecvyof, Puyvfekev Vcakat enhvteceskv ep oh latkvte omet oh lvtkakvnks.

16 Momof Pucase em opunvkv vkerricvyvnks, opunayvte, Mehenwv Cane uewrn es paptisvtet os, momis ceme Puyvfekcr Vcakan es ce paptishoyvkvres.

17 Momekv Hesaketvmese, Pucase Cesvs Klist oh vkvsvmeyvte pu'mvte etvpomen estemkv emakof, nake toyit, vne Hesaketvmese vnrvpvye tayvt haks?

18 Heyv nanvicen pohakof, cryayvkakvtes, momet Hesaketvmese vkvsvmakvtes, Momekv Centilvlkeu vkerrickvetickv hesaketv oren Hesaketvmese emakveuks, makaket.

19 Momof Stefen ohfvecv asseckv hakat svrahkvn vwahvkvtet Fenese, Siplvs, Anteak vteken vpeyvtes, Cusvlke tvlkusen opunvkvn erkenvkaket.

20 Momen vpvlwvt Siplvlke, momet Silenvlke hunvntaket omvtes; mvt Anteak yicof, Klekvlken em punayet, Pucase Cesvn erkenvkakvtes.

21 Momen Pucase enket vpaken, sulke mahet vkrsamet, Pucasen oh fulecvtes.

22 Momof heyv nanvke opunvyeckv mekusvpkv-cuko Celuslvme rpokat e hvckot pohakvtes, momen Paneprs Anteak vteken vyvren vtotakvtes. 
23 Mvt vlakof, Hesaketvmese en heromkvn hecet, afvcke tates; momet omvlkvn efeke fekhvmken Pucasen es hvlvtvkvren mvcahnakvtes.

24 Este-hunvnwv heret omepekv, momen Puyvfekcv Vcakat, vkvsvmkvu es fvcket omvtes; momen este sulke mahet Pucasen ohvprkakvtes.

25 Momof Panepvs enkvpahket, Sal hopoyvranet Tasvn ayvtes.

26 Momet eshehcof, Anteak svlakvtes. Momen mome ocvtes, ohrolope hvmken mekusvpkv-cuko vpaket etohnvkaftet este sulke mahen mvhayakvtes. Momen enkerrepvlke Anteak ofvn enhvteceskv Klistvlke kihocvtes.

27 Momen my netty take omof, owalvlke Celuslvmen vwet Anteak yicvtes.

28 Momen hvmket, Akepvs hocefket, ahuyiret, Puyvfekcvn es kerkuecrtes, Elauko rakket ekvnv omvl' tehoyvnvres, maket; mvt Klaters Sesv en nettv take omof ocvtes. 29. Momof enkerrepvlke omvlkvt nake ocakat vcrkvyen, Cutev ofv etecakkat vpokan emvnickvn vtotetvn komakvtes.

30 Momet mvn mecakvtes, momet Panepvs Sal tepakat enke ofvn mekusvpkv-cuko em fvyatrlken es ohtotakvtes.

\section{SETENTVCKE XII.}

Mоміs mv oketv omof, Helot mekko mekusvpkv-cuko vpvlwvn estemerrvkuecetvn enken arvtosecvtes.

2 Momen Cems, Cane etecakkat, eslaf kv-cvpkon es elecrtes.

3 Momen Cusrlken afveecicen hecekv, Petvu esetvn komvtes. Momof tvklike pakkeko en nettv tates.

4 Momen ehsof, cuko-yekevn vpikvtes, momet suletawvlke oste eslike osten es en wikvtes, vketecvkvren, ohhoyvnkv hoyanof este 'sem vlvketvn komet. 
5 Monkv Petv cuko-yekev vpihoke munkvtes; momis mekusvpkv-cuko wikekot Hesaketvmesen em emekusvpakvtes.

6 Momen Helot svlvketv metetakvranusof, mv nere Petv cvto-tetvkkakv hokkolen es wrnvken suletawvlke hokkole etenrvwvn nocvtes; momen vketecvlke vhauke ehomvn cuko-yekcrn vhecicakvtes.

7 Momen hecrs, Pucase em estet a oh vlahken, hvyayvket cuko-yekev ofvn hryvyakvtes, momen Petr e nvcen en nafkvtes, momet ahuericvtes, Lvpken ahuervs, kicet. Momof cvto-tetvkkakvt enken en yorkvtes.

8 Momen hvlwe-estet, 'Se wrnvkvs, momet cem estelepikvn vtehvs, kicvtes; momen mecrtes. Momet, Cen kapvn vciyet, vnen ve vevkvyvs, kicrtes.

9 Momen osiyet vcvkayvtes, momet hvlwe-este mv nak momecat mehenwv tat kerrekates, momis, Nak hecihocan hecis, komvtes.

10 Vketeckv enhvteceskv momet svhokkolen tehoyvnhohyof, cvto vhauke tvlofv ayan vlahokvtes, mv emet a en hvwiken, woliket, tehoyvnkv hvmken ropothoyvtes, momen lvpken hvlwe-este enkvpakvtes.

11 Momen Petv er hoporrenof, Hiyomat mehenwrn, Pucase em esten atohtet, Helot enke, momet Cusvlke nak komvten omvlkvn a vc ossicet on kerris, makvtes.

12 Momen oh vkerrihcof, Cane, Mak hocefkat, ecke Mele en cukon vlakvtes, mv sulke tohvteloke mekusvpake vpokan.

13 Momen Petv vhauke svkhotkvn vnafkof, hokte mvnettet, Lote hocef ket, pohvranet vlakvtes.

14 Momen Petv em punvkvn keriyet, es afvcket vhauke hvwecekates, momis letket ecehyet, Petv vhauke ehomv hueran onayvtes.

15 Momen, Ce haco-hakes, kicakvtes. Momis, Momet 
o heret os, make munkvtes. Momof, Hvlwe-este Petv enaket omes, kicakvtes.

16 Momis Petv vnafke munkvtes; momen hvwehcet, hecakof, em estomahakvtes.

17 Momis cryayvkvren enken 'sem vhopvyahket, estomen Pucase cuko-yekcv aossican em onvyakvtes. Momet, Heyv nanvke Cems, momet etecakkateu 'ren kerkuecvks, makvtes. Momet enkvpahket etrn ayvtes.

18 Tokvs hvyatkat vpaken Petv estvn estomat ohfvecv suletawvlke em eteyvmketv cutkekates.

19 Momen Helot hopoyet, eshecekof, vhecicvlken ohvketece poyat, pvsvthoyvren makvtes. Momet Cuter enkvpahket, Sesvlevn vk hvtapkvtes, momet mvn fekhonnvtes.

20 Momen Helot Tilvlke Sitvnvlke tepakat ohfvecv cvpvkke tates. Momis em vkerrickv hvmkuset en yicvtes, momet Plastrs mekko en nockv-cuko vhecicv en hessen hayahket, herkvn vpohakvtes, em ekvnv mekko enaket 'mvnicet ok.

21 Momen Helot, nettv mellet, mekko accvken accet, em ohliketvn oh lihket, em opunvkv crpkuecvtes.

22 Momen, Hesaketrmese em opunvkv en hake tes, hunvnwv enake toks, estet makaket yekcen huehkakvtes.

23 Momen vyvkhvmkusen Pucase em estet enokketvn vlicvtes, vkvsvmkv Hesaketvmesen emekokv, momen, cuntvt lokaken, elvtes.

24 Momis Hesaketvmose em opunvkv kvwapket, ohvtvlaket aye mahvtes.

25 Momen Panepvs Sal tepakat nak em vfvstetv sohfvcecahkof, Celuslvmen enkvpaket, fulhokvtes, momet Cane, Mak hocefkan, evpryakvtes. 


\section{SETENTVCKE XIII.}

Momen Anteak mekusvpkv-cuko ohvtehkat owalvlke momen muhayvlke fullvtes, Panepvs momet Semevn Nicv kihocet, momet Lusevs Silenvlke, momet Menern, Helot tetlakv vpake mahvtet, Sal esyomvket.

2 Pucase em vtotkaket, e elvwecakof, Puyvfekcv Veakat okat, Panepvs Sal tepakan a vn sehoyvks, mv vtotketv enhuehkvyat svrahkvn, makvtes.

3 Momen e elvwecaket, mekusvpahket, momet enken oh lumbicahkof, vtotakvtes.

4 Monkv Puyvfekev Viakat vtoten, Selusevn vhoyvtes; momet mvn enkvpakat, perron ohtehiket Siplvn vhoyvtes.

5 Momen Salvmes kakof, Cusvlke en senekakvlke ofvn Hesaketvmese em opunvkvn erkenvkakvtes. Momet Cane en vtotkv ocakvtes.

6 Momen ote ropotecet, Pefas rorihcof, este-mvkerrvn, owalv laksvn, Cusvlke Pacesvs hocef ken, eshecakvtes ;

7 Mv ekvnv semevpayv, Svcevs Palvs, hunvnwv hoporrene vpvken ; mvt Hesaketvmese em opunvkvn pohetvn komet, Panepvs Sal tepakan enhuehkvtes.

8 Momis este-mvkerrv Elemvs, (mvt hocef kvt ok, ohyvtekhoyat,) vnrvpakvtes, vkvsvmkvn sevpayvn sentvcketvn komet.

9 Momof Sal, (mv Pal kihocat,) Puyvfekcr Vcakat es fvcket, heren hecvtes,

10 Momet, Aeha vkerretv omvl' momet naorketv omvl' es fvcketskat, Puyvfekev Holwakat eppuce toyetskat, fvccetv omvl' vnrapv toyetskat, Pucase em vyetv fvecat fvyecicetskat wiketskeko taya? kicvtes.

11 Momen hiyomat hecvs, Pucase enket ec oh wakkes, momen ce turhecekon, hvse hecetskekon oketvt ocvres. 
Momen vyvkhvmkusen hoperet momet yomucket oh latkvtes, momen enken en hvlatet es vre sasvren hopoyet arvtes.

12 Momof sevpayv nake mehocan hecat, Pucase em mvhakv em estomahet, vkvsamvtes.

13 Momen Pal, momet vpvkakvte Pefas es enkvpahket, Pvkv Pamfelev ofvn roricvtes. Momis Cane enkvpahket, Celuslvmen yoh fulkvtes.

14. Momis Pvkv enkvpvkahkof, Anteak Piseter ofvn yicvtes, momet Nettv-cako omof senekaken secehyet, vpokvtes.

15 Momen vhakv momet owalvlken ohonvyetv hoyahnof, senekake sernevpayvlket ohtotakvtes, Hunvntake tecakkvte toyatskat, opunvkv es este mvcahnetv ocatsken omat, makvkes, maket.

16 Momof Pal ahuyiret, enken svhopayet, Eslevlke hunvntake toyatskat, momet Hesaketvmese em penkvlakatskat, apohicvkes, makvtes.

17 Heyv Eslevl em este en Hesaketvmese Purkvlke enhopoyet, momet este Ecepse ofv tvleporvlke omet vpokof, hvlhvwecet, e sakpv hvlwen es asossicvtes.

18 Momet ohrolope pale ostat oren vnrvwv of vn semvpeyetvn en kvsvmkekates.

19 Momen Kenvn em ekvnv ofv etvlwv kolvpaken sumhuehcof, em ekvnv estimvt omvre eskerretv eteropotten em etekvpicakvtes.

20 Momen renyupv ohfvccecvlken emakvtes, ohrolope cukpe oste pale cahkepe oren, Samyvl owalv eroren.

21 Momen eryupv mekkon komakvtes, momen Hesaketvmese, Sal Kes eppucen emakvtes, hunvnwv Pencemen etvlwv aossen, ohrolope pale oste oren.

22 Momen mvn vkueyihcof, em mekko Tewen a en huericvtes, momet mvn opunvyecet kerkuecrtes, makat, Tewe, 
Crse eppucen eshecryvtes, hunvnwv ev feke vcrkvyen, mvt nanvke komvyat omvlkvn momecrres.

23 Heyv hunvnwv en honvpse avten Hesaketvmeset setemfvecetv vcrkvyen Hesayecrn Eslevl a en huericvtes, Cesvs ;

24 Enhomv, vlvkeko munken, Cane efeke-ctickv paptisetv Eslevl em este omvlkvn 'sem erkenahkof.

25 Momen Cane em vyetv sohfvcecof, Estit ot os, cv komatske haks? Vne $m v$ toyvks, momis hecrkes, hvmket cv yupvn vlakes, mv em estelepikv enrecopetv heretv orvkot os, makvtes.

26 Hunvntake tecakkvtē, Epleham en honvpse en hopuetake toyatskat, momet estimvt Hesaketvmese em penkvlet ece 'pakvkat, cemen heyv vhesaketv opunvkv a cen 'tothoyet os.

27 Celuslvme vpokakat semevpaylkeu emen kerrvkekot, owalvlke em opunvkvn Nettvcako omvlkv ohonahoyat tis kerrvkekokv, emen ohfrccecakat, sohfvcecakvtes.

28 Momen ele tayat ohkerkuecat eshecvkeko estomis, elehocvren Pilvten em 'pohakvtes.

29 Momen nak es cokvte omvl' sohfveecahkof, eto a tvk hvtvpecicakvtes, momet crto-kof ke ofvn wrkecakvtes. 30 Momis Hesaketvmeset pvsatkvten aenkvpvken kvwaputes.

31 Momen Kalvle enkvpake Celuslvme atvte vpvkakvtet nettv sulken hecakvtes, mvt a en kerrvlket omes, este ehomvn.

32 Momen opunvkv herakat cen kerkuecvkeyet os; heyvt ot os ; nake erkvlke setemfaccute ats.

33 Hesaketvmese pume en hopuetake toyeyat, matan 'sep ohfvceces, mv Cesvs akvwapvtet; Sams setentrcke svhokkolat ofvn, Crppuce toyetskes, mucv-nettvn ce heckuehcis, maket coket omekv. 
34 Momen Cesvs svnvcomvn lekwe ohfulkvranekon pvsatkvten aenkvprken akvwapat ohfvecvn hrmmakvtes; Tewe em merretv mehenwakan ce'mares, maket.

35 Momen Sams setentvcke etv ofvn, Cenake Vcakat lekwetvn hecrre em etektrnecatskekares, maket os.

36 Tewe vpvke hesahokat, Hesaketvmese komat vcrkvyen, vfvstehpof, nocvtes, momen erkvlke taten $\mathbf{v}$ wvkechohyen lekwetvn hecrtet omekv.

37 Momis mv Hesaketvmese akvwapat lekwetvn hecekates.

38 Monkv hunvntake, tecakkvte toyatskat, cen kerkvkekvs, heyv hunvnwv eteropotten naorketv en kvpvyecickv cem erkenvkhoyes;

39 Momen mv eteropotten, ohvkvsamat omvl' nanvke omvl' enkvpvket fvccrkes, mv Moseskv em vhakv vcrkvye fvcceko tayvte.

40 Monkv vketecrkes, owalvlke opunvyecakvtet ec oh vlahke wites.

41 Hehcvkes, nakhayvkekvlke toyatskat, momet estomahes komvkes, momet estemerkvkes, cen nettv take ofv vtotketvn vtotkvyisem, este estomet cen kerkuecis, vkvsvmatskekares.

42 Momen Cusvlke senekake sosiyof, Centilvlket, Nettvcako hvmke orof, heyv opunvkvn em erkenvkhoyvren vpohakvtes.

43 Tokvs tohnvkaftvte vwahhof, Cusvlke sulket, momen enkerrepvlke hahoyvte Pal Panepvs tepakan vcrkvpeyvtes ; momen mvt em punvyakat Hesaketvmese en herkv. ofv emomecicakvren emvcahnakvtes.

44 Momen Nettrcako hvmket orof, tvlofv vpokat omvlkvranuset Hesaketvmese em opunrkv pohvranet yicvtes.

45 Momis Cusvlket este sulsokan hecakof, vleskvpkrn es. 
fvefakvtes, momet nake Pal opunayan vnrvpet opunvyakvtes, vnrvpaket, tvhikaket.

46 Momof Pal Panepvs tepakat fekhvmkvke haket, Hesaketvmese em opunvkv estonkon enhvteceskv cemen es cem punahoyvranvtes, momis etvn vkueyatsken, hesaketv yuksv-seko oricatskeko tayat e ohfvececatsken, hecvkes, Centilvlken ohfuleces ;

47 Centilvlket en hvyayvket ot, ekvnyuksv em vhesaketv toyetskvren a ce huericvyvtet os, hiyomën maket Pucaset pu mvhayvtet omekv, kicakvtes.

48 Momen Centilvlke heyvn pohakof, afvckvke tates, momet Pucase em opunvkv vrakkuecakvtes, momen en sulke estomuset hesaketv yuksv seko em melhoyvte tat vkvsvmakvtes.

49 Momen Pucase em opunvkv mv ekvnv omvlkv eteropotten kerkuehocvtes.

50 Momis Cusvlket, hoktvke fvccvke momet vrakkuehocvte, momet este momvrherake etvlwv vtehkan teyvmakvtes, momet Pal Panepvs tepakat assecetvn vlicecakvtes, momet em ekvnv wolhuecakvtes.

51 Momis e le em essun vfekahyet, Ikonevn rorhoyvtes. 52 Momen enkerrepvlke afveketv, momet Puyvfekcv Vcakan es fvcfakvtes.

\section{SETENTVCKE XIV.}

Momen mome ocvtes, Ikonev ofvn hokkolvt etepvket Cusvlke en senekaken cukolakvtes, momen mome opunvyakat, Cusvlke Klekvlkeu“sulke mahet vkvsvinakvtes.

2 Momis Cusvlke vkvsvmvkekat Centilvlken teyvmakvtes, momet em vkerrickv tecakkakat vnrvpen en hahicakvtes.

3. Monkv hofunen fekhvmkvket Pucase ofvn opunaho. yet, fekhonnakvtes, momen Pucaset en heromkv opunvky 
a en kerkuecvtes, momet nake eskerkr, momet nake senhoneckvken eme enke take es momecvkvren e'mvtes.

4 Momis tvlofv este sulkat tekvpakvtes, momen vpvlwvt Cusvlken vpaken, vpvlwvt vpastelvlken vpakvtes.

5 Momen Centilvlke, Cusvlke em vculvke esyomat cvton es vfvllaket estemerrvkuecetvn es vnrvpakvtes.

6 Kerrahket, Lestv Tvpe tepakan, Likeonev em etvlofvlken, momet mv ekvnv vfolotan oh tokorkvtes.

7 Momet mvn opunvkv-herv erkenvkakvtes.

8 Momen hunvnwv hvmket, ele yekcrkekot, mv e'cke e nvrke ofv vteke yvkvpekot em emunkvt, yvkvpvte sekot, Lestvn likvtes.

9. Mvt Pal-opunayat pohvtes, momen emet hericet hecat, em wikvre vkvsvmkv ocet on hecet,

10 Opunvkv yekcen 'sopunayet, Lvpotket ce len oh huervs, kicvtes. Momen tasket, yvkapvtes.

11 Momen este Pal nak momecan hecakof, em opunvkv kvwvpakvtes, Likeonev em punvkv ofvn, Hesaketvmeset este-hunvntake omvket a pun hvtvphokes, makaket.

12 Momen Panepvs Cupetvn kicakvtes, momet Pal, opunaye mahat tok, Mvkulevn kicakvtes.

13 Momen Cupetv em plest mv em etvlofv ehomv tate, wakvn momet eskvpotoyetv herakusan en tohopkvhauken svlaket, este sulket vpvkaken, es emekusvpkvn pvsvtetvn komvtes.

14. Momof mv moman vpastelvlke Panepvs Pal tepakat pohakof em accvken setakvtes, momet este vlkan tokor-i ket, huehkakat,

15 Hunvntakè, estoman heyv nanvke momecatske haks? Pumeu este hunvntake, vkerrickv ocatskat omen oceyet os, momet heyv nake ehvperkakan enkvpaket, Hesaketrmese sutv, ekvnv, uehatkvn, momet nake vtehkakat omvl' hayvten ohfulecatskvren cem erkenakvkes, makakvtes. 
$16 \mathrm{Mv}$ oketv eshoyvnvte ofv etvlwvvlke vtekat em vpeyetv ofv yvkvpvkvre em etektrnece tates.

17 Mome estomis, nak herakat momecet, momet sutv aenkvpvke oske pu'met, momet meske naklokce pu'met, momet pu feke hompetv afveketv tis es pum fvcficet, eme a en kerrv seko wikekates.

18 Momen heyv opunvkvn makake estomis, este nak prsate es emekusvprkvranat vfekhonnicetr en yekcrke tates.

19 Momen Cusvlket Anteak Ikonev tepaken a vwet yicvtes, momet este en lopvkuecen, Pal, cvton es vfvllahkof, tvlofvn ossicakvtes, Elepes, komaket.

20 Momis, enkerrepvlke em vfolotet svpaklen, ahuyiret, tvlofvn eceyvtes, momen remprksen en kvpahket, Paneprs tepaket Trpen vhoyvtes.

21 Momen mv etvlwv opunvkv-herv em erkenvkahkof, momet sulken emvhayahkof, hvtvm Lestv, Ikonev, Anteak esyoman yoh fulhokvtes,

22 Enkerrepvlke em vkerrickvn en yekcvkuecaket, vkvsvmkv ofvn vpeye mahvren emvcahnet, momet estemerkv sulke eteropotten Hesaketvmese em etvlofv esceyvranejet os, kicaket.

23 Momen mekusvpkv-cuko ofv omvl' emwiketv hahicahkof, momet e elvwecet emekusvpahkof, Pucasen em wikakvtes, mvn oh vkvsvmakekv.

24 Momen Pisetev eteropothohyof, Pamfelevn vlahokvtes.

25 Momen Pvkv ofvn opunvkv erkenvkahkof, Atelevn vk hvtvphokvtes;

26 Momen perron vtehket, Anteak vhoyvtes, mv tvlofvn vtotketv sohfveecakat vrahkvn Hesaketvmese em merkvn em wihokvtes.

27 Momen vlahohket, mekusvpkv-cukon etohvtelohyof, Hesaketrmese nake em momecat omvl', momet vkvsvmkv 
em vhauke Centilvlke en hvwecrte tis omvl' en kerkuecakivtes.

28 Momen mvn enkerrepvlken vpaket hofunen kakvtes.

\section{SETENTVCKE XV.}

Momen hunvntaket, Cnter vwet, etecakkakan emvhayakvtes, momet, Moses em vhakv vcrkvyen svkvmsishoyekon omat, ce hesahohkvkekos, kicakvtes.

2 Momof Pal Paneprs tepakat opunvyeckv, momet opunvkv 'setenrvpetr sulken est' vnrvpakof, Pal, Panepvs, vpvlwv vprkakat esyomat Celuslvmen vpehyet, vpastelvlke mekusvpkv-cuko vfastvlke esyoman ehomvn heyv vpohkvn em wvkecvkvren vkerrickv hayakvtes.

3 Momet mekusvpkv-cuko vtehkvlke en heromkv eteropotten Fenes Semelev tepakan teropotecvtes, Centilvlke fulotecvten kerkuecet, momet etecakkakat omvlkvn tayen afrckvkuecrtes.

4 Momet Celuslvmen yihcof, emekusvpkv-cuko, vfastrlke vpastelvlke esyomat evpayakvtes, momen Hesaketvmese eme eteropotten nake momecvte omvlkvn kerkuecakvtes.

5 Momis Falvserlke em etohkvlketv vkvsvmakat vpvlwvt asvpaklet, Svkvmsiset, Moses em vhakvn veayeckvn emvhayvke tetayes, kicakvtes.

6 Momen vpastelvlke makusvpkv-cuko vfastvlke esyomat heyv nake oh vketecvkvret etohvtelokvtes.

7 Momen tenrvpetv sulket hoyahnof, Petvt ahuyiret, Hunvntake etecakkvte toyatskat, hofunen Hesaketrmese pu'nhopohyet Centilvlke cr cukwv aossen opunvkv-herv em opunvkv pohaket, vkvsvmvkvren makvtet on, kerratskes, makvtes.

8 Momen Hesaketrmese este efeke take en kerrat Puyvfekcv Vcakat pu'mvte etvpomen e'maket eskerkuecvtes. 
9 Momet eme pume esyomat pu temeticekot omen, vkvsvmkv eteropotten e feken en hvsvthicvtes.

10 Monkv hiyomat estomen Hesaketvmese eskerratske haks? enkerrepvlke nokwv-ohwakkv oh wvkecatskat purkvlke momet pume tis karoneko tayvte.

11 Momis pume, Cesvs Klist Pucase en heromkv eteropotten, mv etvpomen, pu hesahoke tayat vkvsamēs.

12 Momof este sulsokat omvl' evyayvhokvtes, momet Panepvs Pal tepakan em apohicakvtes, nake eskerkv momet nake senhoneckvke Hesaketvmese eme etehoyvne Centilvlke em momecvten kerkuecakof.

13 Momen cvyayvbohkof, Cems em vyoposket, Hunvntake tecakkvte toyatskat, vm apohicvks.

14 Enhvteceskvn Hesaketvmeset e hocef kv vrahkvn Centilvlke asossen esten crwvranet omet oh ayvten Semevn kerkuehces.

15 Momen owalvlke em opunvkv heyvn tentvkhaket os, 16 Heyv enyupvn rvlvkares, momet Tewe em escukohakv latkvte hvtvm hayares, momet yvmahkvkvte hvtvm en hayares, momet ahuericares,

17 Este vpvlwv Pucasen hopoyvkvren, momet Centilvlke es cv hocef hoyvte vtekateu, Pucaset, heyv nanvke omvlkv mecat maket os, make cokat vcvkvyen.

18 Ekvnv em vliceckv vteke Hesaketvmese em vtotketv omvlkvt en kerket omes.

19 Monkv Centilvlke enkvpvke Hesaketvmese oh fulotecat naorihceyats, komis ;

20 Momis, nake-vhake vfvstetv, momet empvlse seko hoktarkvn, momet nake e catv pvlvtkeko pvsatkat, momet catvn e en kvpvyecicvren kicet en hoccicet, ohtotvkeres. 21. Moses, hofunvlke tate, tvlofv omvlkv ofv mv eme es erkenakat ocet omekv, Nettvcako omvl' en senekake ofv ohonahoyet omekv, makvtes. 
22 Momof vpastelvlke, mekusvpkv-cuko vfastvlke, momet mekusvpkv-cuko somvlkvt eme a enkvpvke hunvntake enhopoken, Cutvs Pasepvs hocefkan, Silvs tepakan, etecakkakat semevpayvlken, Pal Panepvs vevkvhoyvren, Anteak ohtotakat here 'sem momakvtes.

23 Momen en hoccihcet mvn es vtotakvtes, hiyōmēn; Vpastelvlke, mekusvpkv-cuko vfastvlke etecakkakat esyomat Centilvlke etecakkakat Anteak, Selev, Selese vtehkan vsekaket os ;

24 Este a pu'nkvpvket vpeyet opunvkvn es ce naoricvten poheyvtet os, cem vkerrickv naoricet, Svkvmsiset, vhakvn vcayecvks, maket, mv vhakv mome emeyvte sekon;

25 Here es pum momvnks, vkerrickv hvmkusen etohnvkafteyof, hunvntake enhopoke, Pal Paneprs tepake, pum vnokeckakat, vpvke ec ohtoteyat,

26 Heyv hunvntaket Pucase Cesvs Klist en hocefkv esvrahkv en hesaketv esten wikvkvtes.

27 Monkv Cutvs Silvs tepakan vtotēs, mv nake etvwvn e cukwvn es cem onvyvkvres.

28 Heyv nanvke momvre herakat tokat nake honne ec oh wikëkot omat es epu fvcket omen, Puyvfekcv Vcakateu es afvcke trnks.

$29 \mathrm{Mr}$ vpeswr nake vhake emetr, momet catv, momet nake e catv pvlvtkeko prsatkat, momet em pvlse seko hoktarkvn e en hvlatet, mvn e en kvpryecicatsken omat, heren fullatskvres. Ece sekēs.

30 Monkv vtothohyof, Anteak yicrtes, momet este sulsokat etohvteloyahkof, cokvn a e'makvtes.

31 Momen ohonayakof, mahlvpvtketvn es afvckakvtes.

32 Momen Cutvs Silvs tepakat emeu owalvlket omepekv, etecakkakat opunvkv sulken 'semvcahnaket, yekcvkuecakvtes.

33 Momen mvn estomusen fekhonnahkof, etecakkakan 
enkvpahket vpastelvlke oh vhoyetvn herkv ofvn em etektvnkvtes.

34 Mome estomis Silvs mata fekhonnetvn es afvcke tates.

35 Pal Panepvs tepakat Anteak ofvn fekhonnakvtes, mvhayet, momet Pucase em opunvkvn erkenvkaket, etv sulket vpaken.

36 Momen nettr hoyanet vyecicof, Etecakkeyat tvlofv vtekat vtehkan Pucase em opunvkvn em erkenakeyvtet 'ren hecet, estomakat er hecvkeres, Pal Panepvsen kicrtes.

37 Momen Panepvs Cane Mak hocef kan evpryetvn oh yekce tates.

38 Momis Pal vkerricat, Evpryetv herekos, komvtes, Pemfelev tvlofon emen enkvpahket, vpaket vtotketvn vevkvyekatet ok'.

39 Momen etenrvpetv yekce mahet omekv, ete'nkvpakvtes; monkv Panepvs, Mak evpayet, perron vtehikat, Siplvs esayvtes.

40 Momen Pal, Silvs evpahyet enkvpakvtes, etecakkakat Hesaketvmese en heromkvn em wikaken.

41 Momen Selev Selesev tepakan eteropottvtes, mekusvpkv-cukon yekcrkuecet.

\section{SETENTVCKE XVI.}

Momof Trpe Lestr tepakan en vlakvtes; momen hecvs, enkerrepv hvmket mvn likvtes, Temorevs hocef ket, Cusvlke hokte vkvsamv echuswrt, momis erke Klekvlke tates.

2 Momen etecakkakat Lestv Ikonev tepake vpokat mv este here omecvket omvtes.

3 Mvn Pal, Ve vevkvyekvs, komvtes, momet Cusvlke mv fullakat svrahkvn ehset svkvmsisvtes, erke Klekvlket on omvlkvt kerrvket omekv. 
4 Momen tvlofv vlkan ropothoyofv, vpastelvlke momet mekusvpkv-cuko vfastvlke Celuslvme vpoke vhakv hayvkvten vcayecvkvren em wikakvtes.

5 Monkv mekusvpkv-cuko vkvsvmkv ofvn yekcvke hahken, nettv omvl' em vhonkvtkv ohvtvlakvtes.

6 Monkv Flecev momet Kelesev ekvnvn etehoyvnhohyof, momen Esev ofv opunvkv erkenvketv Puyvfekev V cakat em rsehaken,

7 Mesevn rorhohyof, Pirenevn vpeyetvn komvtes, momis Puyrfekcvt em etektrnecvkekates.

8 Momen Mesev hoyvnhohyof, Tlovs vk hvtvphokvtes.

9 Momen neren Pal nak hecihocen hecvtes; estehunvnwv Masetonevlket hueret, em 'pohepet, Masetonevn a oh cehyet, pum vnicys, maken.

10 Momen nake hecihocen hehcof, lvpken Masetonev esceyetvn komēmvts, Mvn opunvkv-herv em erkenvkeyvren Pucase pu'nhuehkvcuks, komēt.

11 Monkv Tlovs 'senkvpaket Semoresern vyetv fvccun vpeyet, yicēmvts, momet rempvkse Neapoles roricemvts.

12 Momet mvn enkvpahket, Felepi, mv Masetonev ekvnv mvn wakkat em etvlofv rakkat, momet kalvnet omen, yicet, mv tvlofv nettv vnvcomusekon fekhonnēmvts.

13 Momen Nettvcako omof, tvlofv sosiyet, uerakko afopken vpeyēmvts, emekusvpkv ocvnton, momet tvk vpohket, hoktvke yican em punayēmvts.

14 Momen hokte hvmket, Leter hocefket, nak okholatte wiyvt, Rivtilv tvlofv aosset, Hesaketvmesen emekusapet, $p u$ pohemvts, e feke Pucaset en hvwecen, Pal nak makan vfastemvts.

15 Momen eme, momet en cuko-vtehkvlkeu paptishohyof, pum 'pohepet, Pucase em vtotkv fveces, cv kohmatsken omat, vn cukon escehyet, fekhonnvkes, $p u$ kicemvts. Momet ep ohyekcicemvts. 
16 Momen mome ocemvts, emekusvpkvn vpeyeyof, hoktuce hvmket, puyvfekev holwvken ocet, em owaletv vevkvyen e pucasvlke sulken ern ohvtvlayvtet pu'nrapemots.

17 Matat Pal pumen $\mu^{2}$ 'cvkvyemrts, momet huehket, Heyv hunvntake Hesaketvmese hvlwe mahat em vtotkvlket, mv vhesaketr vyetr pun kerkuecat omakes, makemuts.

18 Momen nettv sulken heyvn mecemvts. Momis Pal, efeke nokket, oh fulotiket, Cesvs Klist en hocefkv ofvn, mv hokte aossvs, ce kicis, puyvfekcvn kicemvts. Momen mata hvse-vkerkv omof aossemvts.

19 Momen e pucasvlke em ohvtvlvkvranvte em enhonrkv sumkepen hecakof, Pal Silrs tepakat crwahket, nakwikv ocan esemerpayvlken es en yicemvts.

20 Momet ohfvececvlken 'sen yicemvts, Heyv estehunvntake, Cusvlke tat, tayen pum etvlofvn naoricakes, makaket,

21 Momet, Lomvnvlke toyeyat, semvpeyetv, mv omat e enakueceye tayat, monkat vfasteye tayat pum etektvnkekot oman, muhayaket os.

22 Momen este sulsokat vnrvpet asvpaklvtes, momen ohfvececvlke em accvke en rokroyahkof, tephokvren makakemvts.

23 Momen tepketv sulken oh.lumhicahkof, eukoyekcrn vtehakvtes, cuko-yekev vhecicv vevyecrren opunvkv en wiket.

$24 \mathrm{M}_{v}$ opunvkv mome en hecikof, cuko-yekcr ofv mahhan vtehvtes, momet eton e len 'sem ryekcrkuecrtes.

25 Mornen nere en nvrkvpv omof, Pal Silvs tepakat emekusvpaket, Hesaketvmesen vyvhikakvtes; momen svlvfkvlke pohakvtes. 
26 Momen vyvkhvmkasen ekvnv-nekeyv rakke mahet ocvtes, mv momat cuko-yekcv en likv nekeyvtes, momen vyvkhvmkusen vhauke omvlkvt hauhakvtes, momen eswrnakv omvlkvt enrecvprehokvtes.

27 Momen cuko-yekcv vhecicv nocepvte vhonecet, cukoyekcv vhauke hauhaken hehcet, em eslaf kv-crpkon a esvtes, momet, Svlvfkvlke pefatkepes, komet, e elecvranvtes.

28 Momis Pal, yekcen huehket, Omvlkvt vpokēkv, e en nokkihcetskvs, kicvtes.

29 Momof kulken vpohvtes, momet letket ecehyet, Pal Silvs tepakat ehomvn fekeket er tvk latkvtes ;

30 Momet, awolhuehcet, Pucasvlke, naken estomvyat cv hesakvr haks? kicet.

31 Momen Pucase Cesvs Klist oh vkvsvmvs, momen ce hesakvres, cen cuko-vtehkvlkeu, kicakvtes.

32 Momen emet on, cuko vtehkakat omvlkvn Pucase em opunvkvn em punvyakvtes.

33 Momen cahwet nere en hvse-vkerkv mata omof rukaf hoyan em okkosvtes, momen vyvkhvmkusen eme, momet cuko-vtehkvlke enake omvlkvn paptishoyvtes.

34 Momen en cukon a secehyof, hompetvn ehomvn 'sem ocvtes, momet afvckvtes, Hesaketrmesen oh vkvsamet, en cuko 'somvlkvt.

35 Momen hvyvtikof ohfvececvlket sacvlken ohtotakvtes, Mv hunvntake enrecopvkes, kicet.

36 Momen cuko-yekcv vhecicv heyv maketv Pal 'monayvtes, Ohfvccecvlket ce'nrecopvkvren atotakes; monkv hiyomat wolket, herkv ofvn vhoyvks, kicet.

37 Momis Pal em punayet, Ep ohfvccekon pu rukafakvnkes, Lomvnvlke toyeyan, momet cuko-yekcvn epu 'tehakvnks, momen hiyomat ehken 'sepu'ssicvkvrane haks? monko tayes; momis emet awet 'sepu'ssicvkekvs, makvtes. 
38 Momen sacvlke heyv opunvkv ohfvececvlken em onvyakvtes, momen Lomvnvlke tacuken pohakof penkvlakvtes,

39 Momen yicet, enkusvpaket, awolhuehcet, tvlofv wolkvren en komakvtes.

40 Momen cuko-yekev woliket, Letev en cukon er crkolakvtes, momet etecakkakat hecahkof, emahlvpvtahket, enkvpvkakvtes.

\section{SETENTVCKE XVII.}

Tokvs, Amfepoles Apolonev tepaken eteropothohyof, Reselonikvn, Cusvlke en senekake likan rorhoyvtes.

2 Momen Pal, em vretv vcrkvyen, est' em ecehyet, Nettvcako tutcenen Cokat aossen em punayakvtes.

3 Klist estemeriyet, hvtvm elkv ra en kvwvpkvranet omvten em enramet, en kerkuecet, momet, Heyv Cesvs, mv cem erkenakvkvyat, Klist tes, kicet.

4 Momen vpvlwvt Klist oh vkvsvmahket, Pal Silvs tepakan ohvpvkakvtes, momet Klekvlke fvccakat sulke mahet, momet hoktvke enhomvhhotv vhonkvtkv cutkekot. 5 Momis Cusvlke vkvsvmvkekat, vleskvpkv es nekeyet, hunvntake en hueretv fvccrkeko evpryaket, este sulken etoh-vteloyahket, tvlofv omvlkvn eteyvmakvtes, momet Cesvne en cukon oh pefatket, esten a 'sem ossickvn en komakvtes.

6 Momen eshecvkekat, Cesvne momen etecakkakat vpvlwvn tvlofv semevpayvlken es oh vpeyvtes, huehket, Heyv este, ekvnv rvkpvlakvtet, yvmv tis yicet os, makakvtes.

$7 \mathrm{Mv}$ Cesvne evpayat, momen heyv omvlkvt Sesv em vhakv vnrvpakes, Mekko etvt oces, Cesvt, makaket.

8 Momet esten naoricakvtes, tvlofv semevpayvlkeu, heyv nanvke pohakofv. 
9 Momen Cesvne, vpvltakeu temfvccetvn es hvlvtepuecahket, vpeyecicakvtes.

10 Momen etecakkakat lvpken Pal Silvs tepakat neren Pelevn ohtotakvtes; mv rorhohyof, Cusvlke en senekaken cukolakvtes.

11 Heyv estet Resvlonikvlke esenhervke tates, mv em vkerrickv vkvsvmakuset opunvkv e enakuecakvtes, nettv omvlkvn Cokan oh vketecaket, heyv nanvke mome tot omat eskerraket.

12 Monkv sulket vkvsvmakvtes, Klekvlke hoktvke vrakkuehoce sasat, momet hunvntake vhonkrtkv cutkekot.

13 Momis Cusvlke Reselonikv vtehkvte Hesaketvmese em opunvkvn Pelev ofvn Pal erkenakvcuken kerrakofv, mvn rorihcet, este eteyvmakvtes.

14 Momof vyvkhvmkusen etecakkakat Pal uehatkvn vyvrane omen vtotakvtes, momis Silvs Temorevs tepakat matan fekhonnakvtes.

15 Momen mv Pal evpayakvte Arens roricvtes, momet Silvs Temorevs tepakat lvpken em vlahokvren opunvkv en heckahkof, enkvprkakvtes.

16 Momen Pal Arens ofvn mehaket likof, em puyvfekcvt ofvn en nekeyvtes, tvlofv nakvhake es fvcket on hecof.

17 Monkv en senekake ofvn Cusvlken etenrvpuecvtes, momet este fvecakateu, momet nakwikv ofv tis yicakat nettv omvlkvn etenrvpuecrtes.

18 Momof Epekulevlke Stoekvlke esyomat felasvfvlket omet vnrvpuecakvtes. Momen vpvlwvt, Heyv nanvkokv naken makvrane haks? makakvtes; momen vpvlwvt, Hesaketvmese kerkvkekat kerkuecv tacuks; makakvtes, Cesvs momen akvwvpketvn em erkenakekv.

19 Momen esahket, Eleapvkvn esvpeyvtes, Heyv mvhakv mucvse opunvyecetskat nake tat kerreye taye haks? makaket. 
20 Nanvke kerkvkekon pu hvcko es em vlaketskekv; monkv heyv nanvke naken okat kerretvn pu yaces.

21 ( $\mathrm{Mv}$ Arensvlke, momet tvleporvlke mv vpokat omvlkvt nake mucvse onvyetv monkat pohetv tvlkusen em oketv es yvmahkuecaket omakekv.)

22 Momof Pal Eleapvkvn en nvrkvpvn hueret, Arensvlkē, nanvke vtekat ofv nanvke emekusvpkvn vheremahecatsket on hecis, makvtes.

23 Etehoyanit nanvke emekusapatskan hecvyate, altvn hvmket vhoccet HESAKETVMESE KERKEKO ENAKE vcoken hecryvnks. Monkv kerkekon emekusapatskat, mvn cen kerkuecvkis.

24 Hesaketvmese, ekvnv momet nanvke vtehkat omvlkvn hayvte, hvlwe momet ekvnv e Pucaset on, emekusvpkv-cuko enke es hakvten vpiket likekot os.

25 Momet nake enkusvpke omen este enken 'sem emekusvphoyet omekot os, nake omvlkvn en hesaketv, momet hesakateu, momet nake omvlkvn emet.

26 Momet etvlwv omvlkvn ekvnv onvpvn oh vpokvren catv hvmken es hayvtet omes, momet oketv homv melhoyvten, momet em vpoketv em vfolotke em mellvtes.

27 Pucasen hopoyvkvren, mvyaket hopoyet eshecake witen omat; mome estomis omvlkeyis pum vhopryece. kot omes.

$28 \mathrm{Mv}$ Hesaketvmese ofvn hesahoket, nekehoyet, momet pu hesaket omekv, momen centake cen yvhiketv hayvlket vpvlwvt okat, Pumeu mata vhonvpse toyekv, makakvtet omekv.

29 Monkv pume Hesaketrmese en honvpse toyēkv, mvtat cvto kunaplanen monkat crto-kunaphvtken, monkat cvton este vtotketv, momet este em vkerrickv eteropotten kvlvfhoyvte omet omes, komvranēkot os.

30 Momen heyv oketv nakkerretv sekat Hesaketvinese 
oh mesettvtes, momis hiyomat este omvlkvn estrmit o estomis em vkerrickvn eticvkvren kicet os.

$31 \mathrm{Mv}$ hunvnwv Cesvs wiketv hayat ekvnv fvecetv etehoyvnen ohfvececvranat nettvn mellvtet ok, este omvlkvn 'sen kerkuecat elkvn rakvwapvtet os.

32 Momen elkv raenkvwvpketvn pohakof, vpvlwvt nakhayvkekates; momen vpvlwvt, Heyv nake ohfvecv hrtvm cem poheyvres, kicakvtes.

33 Monkv Pal en nvrkvpv huervte enkvpakvtes.

34 Mome estomis este vpvlwvt vyokcaket, momet vkvsvmakvtes, Tionesevs Eleapvkvlke, hokte Temele hocefket, momet este vpvlwv esyomat mv vprkvke tates.

\section{SETENTVCKE XVIII.}

Heyv nanvke hoyanof, Pal Arens enkvpahket, Kolenrvn ayvtes.

2 Momet Cusvlke hvmken, Akwelv hocefke, Pantvs ofv heckvte, hvtuse Etvle vtet, e hiwv Pleselv tepaken eshecet, em vlakvtes, (Klatevs Cusvlke omvlkvt Lome enkvpvkvkvren kicvtet okv.)

3 Momen eme em vtotketv matat omekv, vpvkehpet vtotkvtes, mv em vtotketv escukohakv hayvlke tatet okv.

4 Momen Nettrcako omvl' senekake ofvn etempunayet, Cusvlke momet Klekvlken ohyekcrkuecrtes.

5 Momen Silvs Temorevs tepakat Masetonev enkvpahket, vlahokat, $\mathrm{Pal}$ em puyvfekev em vwetenket, Cesvs $m v$ Klist tes, maket, Cusvlken en kerkuecrtes.

6 Momen emet vnrvpaket, Hesaketvmese tvklecakof, èm accvken fekeyet, Ce catv take ceme cekv onvprn oh ocekvs; vne cv hvsvtkes; mucv Centilvlken oh vyares, kicakvtes.

7 Momen mvn enkvpahket, hunvnwv hvmke Custvs 
hocefke, Hesaketvmese emekusapv, en cukon eceyvtes, mv en cuko senekake tewolicuset omen.

8 Momen Klesprs, senekake semevpayv hylwe mahat, en cuko 'somvlkvt Pucasen oh vkvsvmakvtes; momen Kvlenrvlkeu sulket pohaket, vkvsvmaket, paptisvkhoyvtes.

9 Momof Pucaset neren nake hecihocat ofvn Pal em punayvtes, $\mathrm{Ce}$ penkahlates, momis opunvyrs, momet cryvyahketskvs;

10 Vnet ece 'pakikv, momen este estomet ce tohlatket, cen nokkihcekos; heyv tvlofv ofvn este sulken ocikv, kicvtes.

11 Momen mvn vpaket, Hesaketvmese em opunvkv emvhayet, ohrolope hrmken hvse epaket hoyanen likvtes.

12 Momen Kaleo Ekiv ekvnvn mekko vyoposkvt huerof, Cusvlke vkerrickv hvmkusen Pal oh pefatket ohfvececkv ohliketvn resoricvtes.

13 Heyv este vhakv vnrvpe Hesaketvmese emekusvpvkvren esten em vlopvkuecet os, makakvtes.

14 Momen Pal hrte ecukwrn hawvklecrranof, Kaleot okat, Cusvlke toyatskat, naket frecekot, monkat naorketv holwvket on omat, cem apohicvkvyat hoporrenkvt vpvket ont os ;

15 Momis vpohetv opunvkv, momet hocefhokv, momet cem vhiakv take ohfvecvt on omat, cemet oh hecrkes; vntat nake momakat ohfvececv toyvranvkot omekv, Cusvlken kicvtes.

16 Momen Kaleot ohfvececkv-ohliketv enkvpvken vtohkvtes.

17 Momof Klekvlke omvlkvt, Sasrenes, senekake semevpayvn esahket, ohfvceeckv ohliketv ehómvn tepkakvtes. Momen Kaleo heyv nanvke ohfvecv stoskomekates.

18 Momen óhvtvlakat nettv sulken mvn Pal fekhoniyof, 
tecakkakat vsehket, perron pihket, Selevn ayvtes, Akwelv Pleselv tepakat vpaket vhoyen, esetemfvecetvn ocet ome$\mathrm{kv}$, Senklev ofvn ekv-esse tonepvtet omen.

19 Momet Efesvn vlahket, mvn wikvtes, momis emet senekaken ecehyet, Cusvlken em ohhvyvyicvtes.

20 Momen ohvtvlaken vpaket likvren komakof, em vkvsvmekates.

21 Momis vsekahket enkvpakvtes, Estome estomis heyv hompetv-rakko Celuslvme ocvranat vfvstvranvyet os ; momis Hesaketrmeset komen omat, ra ec oh fulkvkares, kicvtes. Momet, perron pihket, Efesvn enkvpakvtes.

22 Momet Sesvlevn yvcahket, erosiyet, emekusvpkvcukon ervsekahkof, Anteak vk hvtapkvtes.

23 Momen oketv hofunen mvn yvmahkuehcof, enkvpahket, Kelesev ekvnv, Flecev ekvuv tepakat etehoyane poyvtes, enkerrepvlke omvlkvn yekcrkuecet.

24 Momen Cusvlke hvmket, Vpalvs hocefket, Vleksantlev ofvn heckvtet, hunvnwv punvkv-crpkueckv vkvsvmket, momet nakcokv-rakko ofv yekce mahet, Efesvn vlakvtes.

25 Heyv hunvnwr Pucase em vyetv ofv emvhahoyet omvtes, momen puyvfekcv ofv yekcet okv, Pucase en nanvken vketece ore heren opunayet, este mvhayvtes, Cane en paptesvm tvlkusen kerret.

26 Momen senekake ofvn fekhvmket opunvyetv vlicecrtes. Momen Pleselv Akwelv tepakat pohakof, evpvyahket, Hesaketvmese em vyetv senheren em ohkerkuecakvtes.

27 Momen Ekivn ceyetvn komof, etecakkakat enkerrepvlken evpayvkvren nakcokvn ohtotakvtes; mv vlahkofv, heromkv eteropotte vkvsvmakvte tayen emvnicvtes. 28 Este ehomvn, nakcokv-rakko eteropotten, Cesvs mv Klist tot omat, heckv heren hecrkuecet, Cusvlke tayen vkvsvmepuecekv. 


\section{SETENTVCKE XIX.}

Momen mome ocvtes, Vpalvs Kvlenrvn likof, Pal, ekvnv hvlhawan etehoyahnet, Efesvn rorvtes, momet enkerrepvlke vnvcomen eshecof,

2 Vkvsahmatskvte vtekat Puyvfekcv Vcakat cen heckvkvt haks? kicrtes. Momen, Puyvfekcv Vcakat oce tat tis pohēks, kicakvtes.

3 Monkv naken es ec oh paptishoyvkvt haks? kicvtes. Momen, Cane em paptesvm omeyvtes, kicakvtes.

4 Momof Pal okat, Mehenwrn Cane vkerrickv etickv paptesvm es paptisvtes, esten em onayat yupv vlvkvranan oh vkvsvmvkvren, mvt Cesvs Klist tokv, kicvtes.

5 Momen heyvn pohakat, Pucase Cesvs en hocefkv ofvn paptiskakvtes.

6 Momen Pal enke oh wvkhokihcof, Puyvfekev Vcakat oh vlakvtes, momen tulaswrn es opunahoyvtes, momet owalakvtes.

7 Momen hunvntake omvlkat palen hokkolohkakat mahe tates.

8 Momen senekaken ecehyet, hvse tutcenen fekhvmket opunayvtes, est' vnrapet nanvke Hesaketvmese em etvilofv ohfvecr ocakan em ohyekcicet.

9 Momis vpvlwvt, efeket wvnwahen, vkvsvmvkekot, momis mv vyetv ohfvecv este sulsokat ehomvn holwvken 'sopunahohyof; Pal enkvpakvtes, momet enkerrepvlke etekvpicrtes, Tilanvs en cokv-mvhakv ofvn nettv omvlkvn est' vnrapet.

10 Momen heyv ohrolope hokkolen emomecicvtes, momat Esev ofv Cusvlke momet Klekvlke vpokakat omvlkvt Pucase Cesvs em opunvkvn pohakvtes.

11 Momen Pal enke etehoyvnen Hesaketvmeset vtotketv senhoneckvke mahen momecrtes. 
12 Momat $P a l$ e na enkvpvken enockv tis, monkat homvohpackv tis enokhokat es en yihocvtes, momen enokketv enkvprkaken puyvfekev holwahokat esossvtes.

13 Momof Cusvlke etefullvlke eksasisvlke mv puyvfekcv holwvke ocakan Pucase Cesvs en hocefkvn 'svhocefet, Cesvs, Pal erkenakat, vcvkvyen es ce hvlvthicēs, kicakvtes.

14 Momen Cusvlke, Sefv hocefket, plestvlke en homahtv eppucetake kolvpaket heyvn momecakvtes.

15 Momen puyvfekev holwakat vyoposket, Cesvs kerrit, momet Pal kerris, momis centake, esti toyatska? makvtes.

16 Momen hunvnwv, mv puyvfekev holwvke vpikvtet oh tasiket, emuntahlet; ohfvniken, enatvlkvkvranuset, vnvttvket $\mathrm{mv}$ cuko em pefatkvtes.

17 Momen heyv Cusvlke Klekvlkeu Efesvn vpokakat omvlkvt kerrakvtes, momen omvlkvn penkvlkvt oh vlaken, Pucase Cesvs en hocefkrn vrakkuehocvtes.

18 Momen vkvsvmakat sulket yihcet, e ohkerkuecet, en nakcelakvn ohonvyakvtes.

19. Vtotketv estomahakan celayvkvtet nakcokvn esyicet, este omvlkv ehomvn nekricakvtes ; momen enrahkv vhonkvthoyat, cvto-kunaphvtke cukpe-rakko pale cahkepvtes. 20 Tokvs, Hesaketvmese em opunvkv yekce heret vkueket ruticvtes.

21 Heyv nanvke eshoyahnof, Pal, em vkerrickv hayat, Masetonev Ekiv tepakan tehoyanvyof, Celuslvmen oh vyares, komvtes, momet, Mvn likvyvtet Lomen hecvranvyet omes, makvtes.

22 Monkv em vtotkakvte hokkolen, Temorevs Elastvs tepaken vtotvtes, momis emet Esev ofvn vhofunusen fekhonnvtes. 
23 Momen mv omó mv vyetv ohfvecv teyvmketv cutke tokot ocvtes. Hiyonton omvtes.

24 Hunvnwv, Temetlevs hocefket, crtokunaphvtke nak es hayvt omet, Tianv vhakvkusen cvto-kunaphvtke Tianv es en hayet, nake 'se en hopokv cutkekon hayvlken 'sen heckuecet omet;

25 Heyv mvn momet mv omv vtotkvlken heyv nakhayv etohvtelohyof, okat, Hunvntake toyatskat, heyv pum vtotketv etehoyvnen pun nakockv oceyet on kerratsket os, makvtes.

26 Momen mv Efesvs ofv tvlkekon, momis Esev omvlkvranusen, heyv Pal este sulken em vlvpvkuecet folothuecen hecet momet pohatsket os, Nake est' enke es hakat hesaketvmese tokvkekot os, maket omekv.

27 Monkv heyv pum vtotketv pum enhvperke taye penkvlkat trlkekot os, momis Tianv hesaketvmese hokte rakkat en cuko tis enhvperkuehoce tayet os, momen en rakke tis sumecihoce tayet os; mvn Esev omvlkv, momen ekvnv este oh vpoke ocakat omvlkvt emekusvpaket ont omaten, makvtes.

28 Momen heyv pohahkof, cvprkketvn es frcfakvtes, momet huehkaket okakat, Efesvlke en Tianv rakket os, makakvtes.

29 Momen tvlofv omvlkvt teyvmketvn es fackvtes, momen Masetonevlke Pal en horkasvlke, Kivs Elestakvs tepakan cvwahkof, vkerrickv hvmkusen pefatiket, rēvtvn esceyvtes.

30 Momen Pal mv este em eceyetvn komen, enkerreprlket em etektrnecrkekates.

31 Momen en hessvlke Esev semevpayvlke vpvltaket eceyet e yvmahkuecekaren en komvket ohtotakvtes.

32 Monkv vpvlwvt nak hvmken es huehken, momen vpvlwvt mvrahkvn huehkakvtes, nvkaftat eteyvmkepekv, 
momen sulke mahat kerrvkekates, náken en nvkaftet omat.

33 Momen Vleksantvn este sulkan aossicakvtes, Cusvlket homvn vyecicaken. Momen Vleksantv, enken es vhopayet, seokepkv este en hayetv eyace tates.

34 Momis Cusvlket on kerrakof, omvlkvt, erı opunvkv hvmkusen, hvse-vkerkv hokkolvteken huehkaket, Efesvlke en Tianv rakket os, makakvtes.

35 Momof tvlofv en cokv-hayv este cryayvkuehcof, okat, Efesvs hunvntake toyatskat, Tianv nake-vhake hokte Cupetv a en Ivtkvte likan Efesvlke etvlwr vrakkuecv tat, este estomet kerrekat haks? kicvtes.

36 Monkv heyv nanvke vnrvpe opunvyehoceko tayet omekv, cryayvket ot nake helvpke momecatskekan heres. 37 Heyv hunvntake, mekusvpkv-cuko en horkopvlke tokot, momet mv cen nake-vhake hesaketvmese en hokteu vtvklecvkekot oman esyicatskekv.

38 Monkv Temetlevs, momen vtotkvlke vpvkakat nake es est' vnrvpkv estomvken ocvket on omat, vhakvt ocet os, momen este em wiketv sehoket os, $m v$ ofvn etemfvccrkepekvs.

39 Momen nanvke etv ohfvecrn vpohatsken omat, vhakv vcrkvye nvkvftetv ofvn cem fvccrkvres.

40 Momen heyv mucv nettr tekyvmketv omecicen vpohkv es pun hahoye tayet penkvlket os, nake omecicat sekot omen heyv nvkvftetv ohfvecrn ohkerkuecvraneyat seokepkv sekot os, kicvtes.

41: Momen momēn opunahyof, mv nvkaftat vwahecvtes.

\section{SETENTVCKE XX.}

Momen mv tekyvmketv fekhoniyof, Pal enkerrepvlken enhuehket, momet vsehket, Masetonevn ayrranet enkvpakvtes. 
2 Momet mv ekvnvn etehoyane pohyof, momet vcahnetv sulken e'mahkof, Klesen vlakvtes;

3 Momet mvn hvse tutcenen fekhonnvtes. Momet Selevn vyvranof, Cusvlket vnrvpet emehakaket omen, yohfulket, Masetonevn eteropottetvu komvtes.

4 Momen Sopetv Pelevlket, momen Elastvs Sekvntrs tepakat Resvlonikvlket, momen Kivs Trpevlket, momen Temorevs, momen Tekekvs Tlofimvs tepakat Esevlket, Esev vteken vhorkaskemvts.

5 Heyvt homvn vpeyaket, Tlovn pu mehakakemvts.

6 Momen tvklike pakkeko en nettrtake hoyahnof, perron vtehiket, Felepin enkvpahket, netts svcahkepan mv Tlovs tvlofvn vpokvten vlahohker, mvn nettv kolvpaken fekhonnēmvts.

7 Momen nettvcakucen nettv enhvteceskvn tvklike kvlkicvkvranet enkerrepvlke nvkaften, Pal, (rempvksen enkvpvketvn emetetakekv, em erkenakemvts, momet opunvkv emomecicen nerenvrkvpv oremvts.

8 Momet onvpv nvthofv etohvtelokat kulket tayemvts.

9 Momen vhauknce ofvn hunvnwv mvnette hvmket, Yutekvs hocefket, nockv sufket ohlvtiken, nocepet oblikemvts, momen Pal hofunen erkenakekv, nockvt ohfvniken, ohtihe svtutcenen ra tvk lvtiket, elēpen ra eshoyemuts.

10 Momen Pal ahvtvpiket, oh latkemvts, momet otaket, E naorihcatskvs, en hesaketv vpikekv, makemvts.

11. Monkv hvtvm yohcemiket, tvklike kvlkahyet, homipet, hofunen, hvyatke vteken, opunahyof, enkvpakemvts.

12 Momen hunvnwv mvnettat hesaken esyicemvts, momet tayen mahlvpvtkakemvts.

13 Momen homvn perro-cukon ob vpehyet, vtehiket, Asvs tvlofvn vpeyēmvts, mvn Pal a oh piketvn komēt, emet yvkapet vyvranet, mōmēn eskerkuecrnkękv. 
14 Momen Asvn ye pu 'pahkofv, a oh pihket, Metelenen roricēmvts.

15 Momet mvn enkvpaket, rempvksen Kivs vnakvn roricēmvts, momet rempvksen, Semvs rorihcēt, Tlokelevm fekhonnëmvts, momet rempvksen Miletvs roricēmvts.

16 Pal, Efesvs fekhonnekot hoyvnetvn komemvts, Esev ofv oketvn yvmahketvn eyacekokv, mome tayen omat, Pentekast nettvn Celuslvmen vretvn eyacet en helvpket omekv.

17 Momet Efesvn vtohtet, mekusvpkv-cuko vfastvlken Miletvs tvlofvn ra enhuehkemvts.

18 Momen en yihcof, makat, Nettv enhvteceskv Esev eceyvyvte vtekat estomen oketv vtekat ece 'pakvkvyvte kerratskes,

19 Momet Cusvlke v'mehakakvte omecicen vnrvpkv vc oh vlakvte; momet turopuswr sulken, momet ekvncvpueckv omvlkvn ehopakit, Pucasen em vtotkit,

20 Momet nak cen lopicvke tayat ohranvkot, omvlkvn cen kerkuecvkit, momet estvlemicit, momet fekhonnicvkct es cen cukopehlit, ce mvhayvkit,

21 Cusvlke momen Klekvlkeu Hesaketvmese ohfvecr efeke-etickv, momet Pucase Cesvs Klist ohfvecv vkvsvmkvn cem ohkerkuecvkin, kerratskes.

22 Monkv hiyomat hehcrkes, vn yvfekev ofv es cv hvlvtket Celuslvmen ayis, mimv nake estomvket ve oh lvtkvranat kerrvkot,

23 Momis eswrnakv momet feknokketv v'mehakakat Puyvfekev Vcakat tvlofv lihkomvlkvn akerkuecat kerris.

24 Momis heyv nanvke estomakis 'se naoricvkos, momen, vn hesaketv v'ncakes, komvkot os, vm vyetv momet vtotketv Pucase Cesvs a enkvpvken vn heckvte, mv Hesaketvmese en heromkv em opunvkv-herv ohkerkuecan afvckety vpvken respoyvye tayen omat. 
25 Monkv hiyomat hecvkes, cemetake omvlkvt, ece 'pvke ayvye Ḧesaketvmese em ohmekketv es em erkenakvkvyvte, svnvcomvn cv turofv hehcatskeko tat, kerris. 26 Monkv mucv-nettvn, este omvlkv e catv enkvpvken cv hvsvtket on, cen kerkuecvkis.

27 Hesaketvmese nake komat vtekat cen kerkuecetv take vfolotkvkatet omekv.

28 Monkv e vketecvks, momet etohvtelokat omvlkvn, Puyvfekcv Vcakat ohvketecvlke ce hayvkvten, Hesaketvmese em emekusvpkv-cuko e catv mahusat es nesvte, hompicetvn vketecvkes.

29 Heyvn kerrikv, ce' nkvpakvkvyof renyupvn yvhv este ohyekcrket es cem eceyvkvres, etohvtelokat tis wikvkekot.

30 Cemetake mahusat asosse tis este hunvntake akvwapket, nanvke fvccekon opunahoyvres, kerrepvlke e oh vwecicvkvret.

31 Monkv e vketecet, vkerricvkvtcrs, ohrolope tutcenat, netta nereu fekhonnvkot, ev turopuswr oce, huyiromvlkv emvcahnvyvten.

32 Monkv hiyomat, etecakkvte toyatskat, Hesaketvmese, momet en herkv opunvkvn, mv ec ohvtvlaye taye, momet mv hvsvthakate omvlkv vpvkepatskvren ce'mvke taye yekcetv ocan ce wikvkis.

33 Este estome en cvto-kunaphvtke, en cvtokunaplane tis, em accrke tis em vleskvpryvte sekos.

34 Momen heyv enke vne vn kusvpkakat em vtotkaket, momet vc vpvkakvteu em vtotkakvten ceme tis kerratskes.

35 Nanvke omvlkvn ce hecicvkvyvtes, estomen mv omen vtotket, yekcrkekan mvnicvranatskat, momet $\mathrm{Pu}$ case Cesvs em opunvkv, Est' emetvt esetvn senheres, makvten vkerricaket, kicakvtes. 
36 Momen heyv nanvken mahkof, tokkehket, omvlkvn vpaket mekusapemvts.

37 Momen omvlkvt tayen hvkihhokvtes, momet Pal e nokwvn oh pvlatket, vfvnkakemvts,

38 Svnvcomv cv turofv hehcatskekos, make opunvkv opunayat omvlkv semuntvlen e feke en nokhokicen. Momen perro-cuko vteken vpvkakemvts.

\section{SETENTVCKE XXI.}

Momen mome ocemvts, enkvpahket, perron vtehikeyof, fvecvn vpeyet, Koos roricēmvts, momen rempvkse Lotes rorihcet, mvn enkvpakeyat, Petelvn roricēmvts.

2 Momen perro-cuko Fenesev ayen eshehcet, ohtehiket, vpeyēmvts.

3 Momet Siplvs eshehceyof, vkvskvnvn wihket, Selevn vpeyēmvts, momet Tilvn esvcakēmvts, mvn perro-cuko nake vtehkvten asossicvranekv.

4 Momet enkerrepvlken eshehcet, nettvkolvpaken fekhonnēmvts, mvt Puyvfekev eteropotte Celuslvme oh ayekaren Pal kicaken.

5 Momen mv nettv taken esohfvcehceyof, enkvpahket, pum vpeyetvn vpeyemvts, momen emetake omvlkv, ehitake, hopuetake epu 'pvkake emunken, tvlofv sossemvts, momet afopken tokkehket, emekusapēmvts.

6 Momen etesekahkeyof, perro-cukon pu 'tehiken, e huten yohfulecemvts.

7 Momen Tilvn awet, perro vtehketvn espoyeyat, Tolemis yicēmvts, momet etecakkakan rsehket, nettv hvmken fekhonnēmvts.

8 Momen rempvkse Pal vpvkakeyvtet vpeyet, Seselevn roricēmvts; momet kolvpakate hvmkat, Felvpe erkenvkv, en cukon escehyet, mvn vpaket fekhonnēmvts. 
9 Momen mv hunvnwv etvwvt echustvlke osten ocemvts, hoktvke mvnettvke, owalakēn.

10 Momen nettv sulken vpokēkv, owalv hvmket, Ekepvs hocefket, Cutevn vtet vlakemvts.

11 Momet pum vlahkof, Pal semewvnaketvn cahwet, eme enke ele tepakvn es wvnahyet, Hiyomen Puyvfekcv Vcakatet maket omes, Celuslvme Cusvlke vpokatet hiyomēn mv hunvnwv heyv sewvnaketv epucasen wrnvyvkvres, momet Centilvlke enke ofvn em wikvkvres, makvtes. 12 Momen heyv nanvke pohheyof, pume momet mata vpokakat esyomet Celuslvme oh vyekaren em 'pohepēmvts. 13 Momof Pal vyoposket, Hvkihhoke, cv feke vn nokkicatskat, naken omatske haks? Celuslvme ofv Pucase Cesvs e hocefkv vrahkv ev wvnvkvre tvlkekot, momis elkvn vm etetaket omekv, kicemvts.

14 Momen vkvsvmepuehoceko tayof, Pucase komatet momekvs, mahkēt, wikēmvts.

15 Momen mv nettv take enyupvn nanvke ehopakeyvten crwehpet, Celuslvmen oh vpeyëmvts.

16 Momen Seselev enkerrepvlket pu 'cvk vpeyēmvts, momet hvmke Nesvn, Siplvsvlken, enkerrepv vculen en fekhonepeyvren esyicēmvts.

17 Momen Celuslvme yihceyof, etecakkakat afvckvket epu vpvyakemvts.

18 Momen rempvkse Cems oh vpeyet, Pal epu 'paken, seceyēmvts, momen mekusvpkv-cuko vfastvlke omvlkvt vpaket vpokēmvts.

19 Momet emet vsekahkof, eme em vtotketv eteropotte Centilvlke em etenrvwr Hesaketvmese nanvke momecvten svrvbecuset en kerkuecemvts.

20 Momen pohakof, Hesaketvmesen vkvsvmaket, Etecakkvte toyetskat, Cusvlke cukpe-rakko nvcomet vkvsvmvke tat hecetskes, momen omvlkvt vhakv es fekcakhvkes, kicakemvts. 
21 Momen, Cusvlke Centilvlke vpvke vpokat omvlkvn, Mosen enkvpaket, en hopuetakucen svkvmsisvkekot, semvpeyetv vevkvyen yvkvpvkekare mvhayetskvten em onahoyen ce pohakvtet os.

22 Monkv naket o haks? este sulsokat, vlahketskat pohvkvret okv, etohvteloke tvlkvres.

23 Monkv heyv ce kiceyaten momecrs. Este-hunvntake Hesaketvmese ohfvccv temfvecetv e oh ocvken osten ocēs.

24 Mvn cahwet, vpaket e hvsvtecrs, momet ekvn vkasvkvren, en yvmahkuecrs, momen omvlkvt, ec ohfvecv nanvke en kerkuehocvte nake tokot omen, momis cemeu tetayusen aret, vhakv vcayecetsket on, kerrvkvres.

25 Centilvlke vkvsvmakat ohfvecv heyvn komēt, hoccicẽmvts, mv omakat hvmkusis vfvstvkekares, nanvke nakevhake emhoyan, momet catv, momet nake ecatv pvlvtkeko pvsvtkat, momet empvlse-seko hoktarkvn e en kvpvyecicvken omat, kicakēmvts.

26 Momof Pal, hunvntake cahwet, rempvkse vpaket e hvsvtehcet, ehvsvtecetv en nettvtake esfackan kerkuecvret, cuko-vcakan eceyvtes, momen vrahrvkv nakemetv Hesaketvmese em emhoyvre vteken.

27 Momen nettv kolvpakat pokvranusof, Cusvlke Esev ofv vpokatet cuko-vcakat ofvn liken hecakof, este sulkat omvlkvn tekyamahket, enken oh ocakemvts,

28 Huehkaket, Hunvntake Eslevlke toyatskat, vnicvks! Heyv este, este, vhakv, heyv liketv esyome vnrvpen este omvlkvn estvmv estomis emvhayet, momet ohvtvlakat Klekvlken cuko-vcakan escehyet, heyv liketv hrsvtkat svholwvyecat tes.

29 Enhomvn heyv tvlofv ofvn Tlofimvs Efesvlken eme vpaken hecakvnkekv, momet vkerricaket, $\mathrm{Pal}$ mvn cukovcakan asceyet omvtes, komakvtes.

30 Momen tvlofv omvlkvt nekeyemvts, momen este 
etohpefatkemvts, momet Pal esahket, cuko-vcakat ossicakemvts. Momen moman vpaken vhauke vkhothokemvts.

31 Momen elecvkvranof, este-es-likat en kvpetvne en homahtvn opunvkvt em vlakemvts, Celuslvme omvlkvt tekyvmkes, maket.

32 Lvpken suletawvlken kvpetvnvlkeu cahwet, oh letkvtes. Momen suletawvlke kvpetvne en homahtv esyomen hecakof, Pal nvfkakvte wikakvtes.

33 Momof kvpetvne enhomahtv vwolihcet, esvtes, momet cvto-tetvkkakvn hokkolen es wvnvyvkvren kicvtes, momet estimvt ot, nake momecat est' em 'pohemvts.

34 Momen este sulsokat, vpvlwvt nake hvmken, vpvlwvt nake etvn es huehkakvtes; momen eteyvmkat omecicen mehenwetvn kerreko tayof, tohopken secehoyvren kicemvts.

35 Momen vccakvn vcemkof, mome ocvtes, este en yekcetvt omecicen suletawvlket svpeyemvts:

36 Este sulsokat svevkvpeyet, huehkaket, Mv esten etvn sayvs, makakekv.

37 Momen Pal, tohopke seceyvranof, Cem punvyetv vm etektvnke te? kvpetvne enhomahtvn kicemvts. Momis em vyoposket, Klekvlke em punvkvn kerretske te? kicemvts.

$38 \mathrm{Mv}$ Ecepsvlke heyv nettrtake ehomv tekyvmketvn hayet, momet este-pvsatvlke cukpe-rakko osten sosiyet, vnrvwvn esceyvte toyetskeko? kicemvts.

39 Momis Pal, Vne Selesev ofv Tasvs tvlofv Cusvlket omit, tvlofv holwvkuse toko tvlofvlke toyis, momen este etempunvyetv vm etektvnecetskvren cem 'pohis.

40 Momen em etektvnehcof, Pal, vccakvn oh hueret, enken esten es em vhopayemvts. Momen cryvyvketv rakke hakof, Hepluvlke em punvkvn 'sem punayemvts, maket, 


\section{SETENTVCKE XXII.}

Hunvisiake, etecakkvte, momet erkvlke esyomatskat, hiyomat opunvkv seokepkv cen hayvyan vm apohicvkes.

2 (Momen Hepluvlke em punvkvn 'sem punayen pohakof, senhoyvnen cryayvhokemvts; momen makemvts,)

3 Mehenwrn Cusvlke hunvnwv toyis, Seleser etvlofv Tasvn cv heckvtet, momet heyv tvlofv ofvn Kemelevl ele tempen ev mahvtet, momen erkvlke em vhakv vevkvyen esfvcken v'mvhahoyet, momet cemetake omvlkvt mucvnettvn Hesaketvmese ohfvecr ce fekcakhvkat omit huerit omvyvtet os.

4 Momet heyv vyetv elkv vteken assecit, hunvntake, hoktvkeu wrnawicit, cuko-yekcvn vtehvyvtet omes.

5 Momen plest enhomahtvt a vn kerrvt omes, momet vculvke etohkvlkat omvlkvt, mvt cokv etecakkakat ohtothoyat v'mvkvtet on, Temaskvn oh ayimvts, mv vpokat estemerrvkuehocvren wvnawvken Celuslvme resvlvkaret.

6 Momen mome ocemvts, fvccv-likat mahen Temaskvn vwolicvyof, hvyayvke rakke hvlwe vtet vm vfolotet hvyvyakemvts.

7 Momen ekvnvn tvk latkimvts, momen, Sal, Sal, estoman vc assecetske haks? opunvkv cv kicen pohimvts.

8 Momen, Pucase, estimv toyetska? kicit em, vyoposkimvts; momen, Cesvs Nasvlevlke, mv assecetskat toyis, cv kicemvts.

9 Momen ve vprkakvte, mehenwrn hryayvke hecet, penkvlakemvts, momis $\mathrm{mv} v \mathrm{vm}$ punayat em punvkv en hake pohvkekomvts.

10 Momen, Naken estomvranvya? Pucasē, kicimvts. Momen, Ahuyiret, Temaskvn eceyvs, momen mvn nanvke momecvranetske cem melhoyvte omvlkv ohfvecv cem onahoyvres, Pucase cr kicemvts. 
11 Momen mv hvyayvke en rakket omecicen cv heceko tayof, mv ve vpakvtet enken es cv yvpayen, Temaskvn eceyimvts.

12 Momen Anvnivs hvmket, vhakv vevkvye hunvnwv fvecet, Cusvlke $m v$ vpokat omvlkvt heren ohkerkuecaken, $13 \mathrm{Vm}$ vlahket, hueret, Sal etecakkvte toyetskat, renv$\because v s$, cv kicemvts. Momen mv en hvse-vkerkv etan emen oh hecimvts.

14 Momen, Purkvlke en Hesaketvmeset, eme nake koman kerre, momet mv Hvmke Frccat hece, momet e cukwv en hake pohetskvren ce mellvtes.

15 Nake hece, momet pohetskvte este omvlkvn aenkerrv toyetskvret okv.

16 Momen hiyomat estomen acewicetske haks? Ahuervs, momen ce paptishoyekvs, momen Pucase e hocefkvn oh huehket, cen naorketv ce'n kvpvken okkosvs, cv kicemots.

17 Momen mome ocvtes, Celuslvme yecehyvyof, cukovcakat ofvn emekusapvyof tot omis, cv nocekon vpueckv omet ve oh latkemvts.

18 Momen, Lvpecet lvpken Celuslvmen ossvs, vne $v c$ ohfvecv cem ohkerkueckv esahkekot ok, cv kicen hecimvts.

19 Momen, Pucase ec oh vkvsvmakat cuko-yekcv vtehakvyvte, senekake omvlkv ofv nvfnakvyvten kerrakes.

20 Momen a cen kerrv Stefen e catv pvlatkof, vhuerit, elvre estemvkvsamit, momet $m v$ elecakvte em accvken vhecicimvts, makimvts.

21 Momen, Ayvs, hopvyen Centilvlken ec ohtotaret okv, cr kicemvts.

22 Momen heyv opunvkv vteken mapohicakemvts, momet en haken kvwvpaket, Este momat ekvnv enkvpvken sayvs, hesake taye orekokv, makakemvts. 
23 Momet huehkaket, em accvke vkayaket, momet fakken hvlwen vpvlvtakofvn,

24 Kvpetvne enhomahtvt, Tohopken asceyvks, kicet, momet tepket ohkerhoyvren, makemvts, estoman momvteken es huehkaket omat kerretvn komet.

25 Momen eswrnakvn es wvnayahkof, Este Lomvnvlket omat ohfvcceko munke tepkatskvre vhakv vcvkaye te? Pal sentulev vhueran kicemvts.

26 Mvn sentulev pohhof, kvpetvne enhomahtvn rem onayet, Nake momecetskat vketecvs, heyv hunvnwv Lomvnvlket ok, kicemvts.

27 Momof kvpetvne enhomahtvt vlaket, Lomvnvlke toyetsken omat, vm onvyvs, kicemvts. Henka, makemvts. 28 Momen kvpetvne enhomahtvt em vyoposket, Enrahkv rakken heyv Lomvnvlke en hueretv es heckuecvyvtes, makemvts. Momis vne matat cv heckvtet omes, Pal kicemvts.

29 Momof mv ohkerrvkvranvte vyvkhvmkvn enkvpvkakvtes; momen kvpetvne enhomahtvt, Lomvnvlket on kerrof, momet mv wvnayate svrahkv penkalemvts.

30 Remprkse omof, estomaten Cusvlke vnrvpaket omat mehenwvn kerretvn eyacet ok, svlvfkvte enrecapemvts, momet plest enhomahhotvlke, momet en nvkaftvlkeu omvlkvt yicvren kicemvts, momet Pal es hvtvpiket, ehomvn licemvts.

\section{SETENTVCKE XXIII.}

Momen Pal, tekueketv hericet oh hecat, Hunvntake etecakkvte toyatskat, mucv-nettv vteken Hesaketvmese ehomv vm vkerrickv omvlkv heren esarvyvtet omes.

2 Momen Anvnivs plest enhomahtvt mv etempe svpaklvten e cukwv em vtepkvkvren kicemvts.

3 Momof Pal okat, Hesaketvmeset cemen ce nvfkvres, cvto-tohopke hrtecvkvte toyetskat, vhakv vevkvyen vc 
ohfvececrranet liket ometskat, momet vhakv vnrvpen $\mathrm{cv}$ nvfhokvren maketske haks? kicemvts.

4 Momen vsvpvklatet okakal, Hesaketvmese em plest enhomahtv vtvklecetske haks? makakemvts.

5 Momof Pal, Etecakkvte toyatskat, plest enhomahtv tat kerrvkis, Cem este semevpayv holwvke opunvyehcetskvs, maket coket omekv; kicemvts.

6 Momis Pal, vpvlwvt Satusevlket on, vpvlwvt Falvsevlket on, kerrof, tekueketv ofvn huehket, Hunvntake etecakkvte toyatskat, Falvsevlke toyit, Falvsevlke eppuce toyis, prsatkvte a en kvwvpketr, momet em enhonrkv vrahkvn ve ohfvecehocet omes, makemvts.

7 Momen heyvn opunahyof, Falvsevlke Satusevlkeu etenrvpkvt emetenrvwr ocemvts, momen sulsokat etekvpakemvts.

8 Satusevlke akvwrpketv, hvlwe-esteu, puyvfekcvlkeu sahsekos, makaket omekv; momis Falvsevlket hokkolvn kerkuecaket omes.

9 Momen huehketv rakket ocemvts, momen cokvhayvlke Falvsevlke vpvkakvte vpvlwvt akvwrpkaket, Heyv hunvnwv ofv naorketv hecèkot omes, momis puyvfekcrt, monkat hvlwe-estet em punayvten omat, Hesaketrmese etepohyeyats, makakemvts.

10 Momen tenrvpkr rakket ocof, kvpetvne enhomahtvt, Hvlatet, telatahlece wites, komet, penkalet, suletawvlken ohtotemvts, hvtrpehcet, ryvkhvmkusen em esahket, tohopken asceyvkvren.

11 Momen my 'ren nere omof, Pucaset vhueret, Pal toyetskat, ce 'fvckekvs, Celuslvme ofv a vn kerretskvte etvpomen Lome ofvn a vn kerretskvret okv, kicemvts.

12 Momen hryatkof, Cusvlke vnvcomet $\mathrm{em}$ etohkvlketvn hayet, ohtvhiketv, elecvn e hahyet, Pal elecēko vtekat hompēkot, eskēkares, makakvtes. 
13 Momen heyv etemfvccetv hayakat pale ostat hoyvne tates.

14 Momen plest enhomahhotvlke momen mvculvke en yihcet, Ohtvhiketv rakke elecvn, Pal elecēko vtekat naken homipēkos, maket etemfvccēs, kicakvtes.

15 Monkv hiyomat centaket nvkaftvlke vpvket kvpetvne enhomahtvn en kerkuecrks, nake esohfvcken em 'pohvranatske omen, pvksen ce homv taken huericvren; momen pume, hvte vwoliceko munken, elecetv pu metctakes, makakvtes.

16 Momen Pal e wvnwv e'chuswvt emehakakvcuken pohof, ahyet, tohopke ecehyet, Pal rem onayvtes.

17 Momof Pal, sentulevlke hvmken huehket, Heyv hunvnwv mvnettan kvpetvne enhomahtvn 'sem vyvs, nake hvmken 'monvyetvn ocet omekv, makvtes.

18 Monkv evpahyet, kvpetvne enhomahtvn 'sem vlakvtes, momet, Pal svlvfkat vn huehket, Heyv hunvnwv mvnettv, nake ce kicetvn ocet omen, es cem vyaren vm 'pohhes, makvtes.

19 Momof kvpetvne enhomahtvt enken en hvlahtet, vrvnakusen vcrkahyet, Nake vm onvyetv ocetskat, nake te? kicet em 'pohvtes.

20 Momen, .Cusvlket pvksen Pal tekueketvn esceyetskvren cem 'pohetvn temvkvsvmaket omes, esohfvcken naken em 'pohvkvrane omet, kicvtes.

21 Momis em apohihcetskvs, hunvntake pale ostat hoyvnet e'mehakaket, ohtvhiketv elecvn etemfvccaket, Elecēko vtekat, hompēkot, esikēkos, makaket os, momen hiyomat em vkvsvmvketskvre en nene hecet emetetakvkes, kicvtes.

22 Monkv kvpetvne enhomahtv hunvnwv mvnettat vyecicvtes, momet, Estimvn monahyetskvs, heyv nanvke vn kerkuecetskat, kicvtes. 
23 Momen, sentulevlke hokkolen en hohiket, Suletawrlken cukpe hokkolen, momen corakko-ohvpokvlket pale kolvpaken, cufunfvske-cawvlket cukpe-hokkolen nere en hvse-vkerkv tuteenof Seselevn vpeyvren metetakuecvs, kicvtes.

24 Momet vpuekvn, Pal oh licahket, vcayecaket Felekse evpayvn res em oricvren etetakuecrs, kicvtes.

25 Momen cokv hiyomēn hoccicvtes;

26 Klatevs Lesevs Felekse evpays here mahat vseket os ;

27 Heyv hunvnwv Cusvlket esakvtes, momet elecakvnt os, momof suletawvlken es vlahkit, em esakvyvnks, Lomvnvlket on kerrvyof.

28 Momet naket omecicen vnrvpakat kerretvn komvyof, em etekueketrn a's ceyvyvnks;

29 Eme em vhakv em vpohkvn 'svnrvpaken, momis ele taye, monkat svlvfke taye ore em muttekon, kerrvyvnks.

30 Momen Cusvlke mv hunvnwv e'mehakakvenken vm onahoyof, lvpken ec ohtotit, momet vnrapvlken nake 'svnrvpkvn ocvke tat ce homvn onvyvkvren, kicakvyvnks. Ece 'sekis.

31 Momof suletawvlket, kihocat vcvkvyen, Pal esahket, neren Antepetlvn es yicvtes.

32 Rempvksen corakko-ohvpokvlket es vevkvpeyvren wikahket, tohopken yefulecvtes.

$33 \mathrm{Mv}$ Seselevn yihcet, cokvn evpayvn a emahkof, Pal mv ehomvn en licakvtes.

34 Momen evpayv ohonahyof, em etvlwv estomat tat vpohvtes; momen Selesevn vte tan kerrof,

35 Ce'nrapvlket yicof, cem apohicares, kicvtes. Momen Helot em ohfvcceckv-cukon vpihokvren, makvtes. 


\section{SETENTVCKE XXIV.}

Momen nettv cahkepet hoyahnof, Anvnivs plest enhomahtvt, mvculvke es hvtapket, momet opunayv hvmke, Tvtvlvs hocefket, Pal vnrvpen evpayvn en kerkuecakvtes.

2 Momen enhuehohkof, Tvtvlvs vnrvpetvn vlicecvtes, maket, Ceme ce tehoyvnen cryayvketv rakken es epu 'fvcket omen, momen cem fvyatkv herat eteropotten nake herakat heyv etvlwv em momehocet omen,

3 Estofis, momet estrmvt o estomis, vkvsvmkv emetv 'somvlkvn punaken hayeyet omes, Felekse here mahets. kat.

4 Mome estomis ohvtvlaken ce naoricvkaret, cen heromkvn opunvkv nvcomusen es pum apohicetskvren cen kusapis.

5 Heyv hunvnwv este naoricrt ot, ekvnv eteropotte Cusvlke omvlkv etenrvpkv en hayvt ot, momet Nasvlevlke esemevpayvt on esheceyet omekv;

6 Momet ohvtrlakat cuko-vcakat svholwvyecetvn komen; mvn ehsēt, pum vhakv vcvkvyen ohfvccecetvn pu yacrnks ;

7 Momis kvpetvne enhomahtv, Lesevt, vlahket, yekcetv rakken punke ossen es pu 'konahrnks.

8 Vnrvpuecvlke cemen ec oh vpeyvren kicet, mvn vketecetskat, heyv nanvke es vnrapeyat omvlkvn kerretske tayes.

9 Momen Cusvlke em vkvsvmakvtes, Heyv nanvke mo: mēs, makaket.

10 Momof evpayvt opunvyvren es em vhopahyof, Pal em vyoposket, makvtes, Heyv etvlwv ohrolope sulken ohfvececv toyetskvten kerrvyet okv, 'sve afvcke mahet es ve vrahkv vyoposkis; 
11 Celuslvme emekusvpvrane ohvyvyvte hvte netta palen hokkolohkaket omat kerretsket omekv.

12 Momen cuko-vcakat ofv, estimv vnrvpe estempunayin, monkat este tenrvpkv en hayin, senekake ofv tis, tvlofv ofv tis, es cv hecvkekvnks;

13 Momet nanvke es ve vnrvpakat ohfvececvkeko tayes. 14 Momis heyv tan cem e ohkerkuecis; nake vhakv ofv, momet owalvlke ofv hoccvke ocat omvlkvn vkvsamit, mv vyetv tekvpvketv kicakat verkvyen purkvlke en Hesaketvmesen em vtotkvyet os ;

15 Momet Hesaketvmese ohfvecv enhonrkvn, mv emetakeu vkvsvmakat, ocit, pvsatkvte fvecakat fvccrkekateu a en kvwvpketv ocvranat vkvsvmvyet omes.

16 Momet heyv ofvn vtotkvyet omes, estofis Hesaketvmese ohfvecv, momet este ohfvccvin vkerrickv mahlvpvtke ocaren.

17 Tokvs, ohrolope sulket hoyanof, heromkvn momet Hesaketvmese nake emetvn vm etvlwv 'sem vtaret vlakvyet omvnks.

18 Momof Cusvlke vpvlwuset, Esev vwet, cuko-vcakat ofvn $c v$ hvsvtken es cv hecakvnks, este sulkat vpvkvkot, estoke mahateu sekon.

19 Mvt, nake es ve vnrvpkv ocvken omat, ce homvn svpaklet, kerkuecvkvranvtes.

20 Monkat heyv eta tis, naorketv estomen ve ofvn eshecakvten omat, onvyvkekvs, tekueketv ofvn huervyof,

21 Pvsvtketv aenkvwrpketv ohfvecvn mucv-nettvn ve ohvketecatsket os, vpvkakvye huervye huehkvyvte, -heyv opunvkv hvmkat tawv.

22 Momen Felekse, heyv nanvken pohof, mv vyetv senfvccen kerrekv, em acewicvtes, momet, Lesevs kvpetvne enhomahtvt vlakof, esfvcken cem ohvketecvkares, kicvtes.

23 Momet sentulevn, Pal etektrnken e'met, momet em 
etekerhoyetv em afvstvkepvre, momet a oh vwvkepvre sentackekon, vhecicvs, kicvtes.

24 Momen nettv nvcomuse enyupvn Felekset e hiwv Tluselv, Cusvlke hoktet omen, mvn evpayet vlahkof, Pal em vlvkvren ohtohtet, Klist ofv vkvsvmkv ocat ohfvecvn em apohicvtes.

25 Momen fvccetv, eemfvyatkv, momet ohfvececkv vlvkvranan opunvyecof, Felekse fekeket, Mucvn cem vyetvn vyvs, oketv etektvnket vn heckofvn, cen huehkares, kicvtes.

26 Monkv, Enrecvparen Pal cvto-kunawvn v'mvres, komekv, etewolusen enhuehket, etempunayvtes.

27 Momis ohrolope hokkolet hoyanof, Pasevs Festrs Felekse vyoposkvtes, momen Felekset Cusvlke afveecicetvn komet, Pal svlvfkēn wikvtēs.

\section{SETENTVCKE XXV.}

Monkv Festrs kvntvckv acehyof, nettv tutcenet hoyanof, Sesvlev enkvpaket Celuslvmen oh ayvtes.

2 Momof plest enhomahtvt, momet Cusvlke enhomahhotvlke Pal vorvpet en kerkuecakvtes, em 'pohaket,

3 Pal vnrvpen en heromvkvren komaket, mvn Celuslvmen ra enhuehkvren, vyetv ofvn mehakaket elecvkvren.

4 Momis, Pal Sesvlevn fekhonnihocekvs, momen vne hofunusekon ayvranis, Festrs maket vyoposkvtes.

5 Monkv mv yekcetv ocvke ece 'pakvkat, vc vcrkvpehyet, heyv hunvnwr vnrvpvkekvs, eme ofv naorketv ocet on omat, kicvtes.

6 Momen nettr pale esenhoyvnen vpahkofv, Sesvlevn vk hvtapkvtes, momet rempvkse omof, ohfvececkv ohliketvn oh liket, Pal esyihocvren makvtes.

7 Momen vlahkof, Cusvlke Celuslvmen vwakvtet em vfoloten svpaklet, opunvkv yekcvke, momet sulken, ohfvccecvkeko tayat, Pal 'svnrvpakvtes ; 
8 Momen emet e em vyoposket, Cusvlke em vhakv, cukovcakateu, momet Sesvn vnaorkvkvyvte sekos, makvtes.

9 Momis Festrs, Cusvlken afvcecicetvn komet, Pal em vyoposket, Celuslvmen oh ayet, mvn cr homvn heyv nanvke ohfvecv cem fvecvren kometske haks? kicvtes.

10 Momof Pal okat, Sesv em ohfvcceckv ohliketvn vhueris, mvn vm fvccvre tayet os; Cusvlke naken em vhopanvyvte sekos, momat kerrusetsket omes.

11 Naken vhopanvyvten omat, monkat elkv oren naken momecryvten omat, elkv e vsehvkot os, momis heyv nanvke es vc vnrvpakat sekon omat, estimvt vnen em wikvkeko tayes; Sesvn oh huehkis, makvtes.

12 Momof Festrs, nvkaftvlken setempunahyof, Sesvn oh huehket ohmetska? Sesvn oh ayetskvres, kicet em vyoposkvtes.

13 Momen nettr nvcomet eshoyahnof, mekko Vklepvt Pvnese tepakat Festrsen vsekvkvranet Sesvlevn vlahokvtes.

14. Momen nettv sulken mvn kahkof, Festvs mekkon Pal em en kerkuecvtes, Felekset wikvten, hunvnwv hvmket svlvfkēt likes, maket.

$15 \mathrm{M}_{v}$ ohfvecvn, Celuslvmen likvyof, plest enhomahhotvlket momet Cusvlke em vculvket ohfvcceckvn komaket oh kerkuecakemvts.

16 Mvn em vyoposkit, Lomvnvlket este vnrvphoyat elkvn em wikvranet omeks, vnrapvlket tehecaken, momet nake 'svnrvphoyat emeu e em vyoposke taye em etektvnkeko emunken omat, kicakimvts.

17 Monkv yvmvn vtelohkof, acewicvkot, rempvkse omof, ohfvececkv ohliketvn oh likit, hunvnwv esyihocvren makimuts.

18 Vnrapvlke vsvpaklof, nake momvke tares komvkaten es vnrvpakemvts. 
19 Momis es vnrvpakat em emekusvpkv ohfvccrn, momet Cesvs hvmke mv elvten, Hesakes, Pal kicat, vpohkv ocakemvts.

20 Momen vpohkv mv omakat vn kerkekokv, Celuslvmen oh ayet, mv nanvke mvn em fvccrren kome tat em 'pohimvts.

21 Momis Pal, Vkvstvt pohvren oh hohikof, Vhecihoce emunken Sesvn ohtotares, kicimvts.

22 Momof Vklepvt, Vntat hunvnwrn em apohicetvn cv yaces, Festrn kicvtes. Momen, Pvksen pöhetskvres, kicvtes.

23 Momen rempvkse, Vklepvt Pvnese tepaket en heckv rakken es vlahohkof, momet kvpetvnvlke enhomahhotvlke, momet mv tvlofv en rvkrakvn pohvkvranet pohetv-cukon escehyof, Festrs mahken, Pal esyihocvtes.

24 Momen Festrs, Vklepv toyetskat, momet este hunvntake heyv epu 'pakatskat omvlkvt hecatsket os, heyv hunvnwv mv ohfvecr Cusvlke sulsoke omvlkvt Celuslvmen, momet yvmv tis, vc oh huehkaket, Ohvtvlaken hesakekan tayes, makaket os.

25 Momis elkv ore naorkekot, eme tis Vkvstvs oh huehken hecryat ohtotetvn ohfvececryet omemvts.

$26 \mathrm{Mv}$ ohfvecr cv pucase naken en hoccicetv ocvks. Monkv ce homv taken esvlakis, momet mekko Vklepv toyetskat, ce ce rvhecit, ohvketeckv ohcof, nanvke hoccicetv ocaret.

27 Svlvfke est' ohtote nanvke es vnrvpkv kerkueceskat hoporrenkv toko es vm momet okv, makvtes.

\section{SETENTVCKE XXVI.}

Momen Vklepvt, Cem etektvnkes ece 'rahkv opunvyetskvre, Pal kicvtes. Momof Pal enken a rvtosecet, e em vyoposkvtes; 
2 Vklepv mekko toyetskat, vc afvckes, komis, mucvnettv Cusvlke narvke es ve vnrvpakat omvlkvn ce homvn e em vyoposkvranikv;

$3 \mathrm{Mr}$ omecice mahat, cemet Cusvlke em vpohkvn, momet semvpeyetv omvlkvn kerretsket omes, momekv cen heromkvn es vm apohicetskvren cen kusapis.

4 Enhvteceskvn vm etvlwvn tvlofvn, Celuslvmen likit, cr mvnettusvte vteken, vm vretvn Cusvlke omvlkvt kerrvket os;

5 Enhvteceskv vteken cv kerrakvtet, (kerkuecetv komvken omat,) pum emekusvpkv em etekvpvketv vfekcakhe mahat em vyetv vevkvyen Falvsevlket arvyvtet omat, kerkuecvke tayes.

6 Momen hiyomat setemfvecetv herat, Hesaketvmese purkvlke en hayvte enhonrkv vrahkvn ve ohfvecet huerit omis.

7 Mvn oricetvn komaket pum etvlwv pale hokkolohkakat, Hesaketvmese nettv nereu emomecicet em vtotkaket, enhonrvket omes. Mv enhonrkv vrahkvn, mekko toyetskat, Cusvlke ve vnrvpaket omes.

8 Hesaketvmese prsvtkvte akvwapat estoman nake vkvsvmhoyeko taye es cem momet o haks?

9 Mehenwvn vntat Cesvs Nasvlevlke e hocefkv vnrvpe nanvke sulken momecvranvyat omes, komvyvtet os.

10 Momet $\mathrm{mv}$ naken Celuslvme ofvn momecryvtet omes, momet plest enhomvhhotvlke enkvpvket yekcetv vn hecken, mekusapvlke sulken cuko-yekcv ofvn em vkhothuecryvtet omes, momet, pvsvthoyof, pvsvthoyvren opunvkv vpryecryvtet os.

11 Momet senekake omvlkv ofvn tewolen estemerrvkuecit, opunvkv yekcvken opunvyepuecit, momet en cv homece mahet, tvleporvlke em etvlofv vteken assecakvyvtet omes. 
12 Momen plest enhomahhotvlket $v m$ wiketvn hayet, momet yekcetvn v'maken, Temaskvn ayvyof,

13 Mekko toyetskat, fvccv-likof, vyetv ofvn hvyayvke hvse en hvyayvke emuntvlen, vne momet ve vprkakvte pu folotken hvyayvken hecimvts.

14 Momen omvlkeyat ekvnvn tvk pvlvtikeyof, punvkv en hake vm punayet, momet Hepluvlke em punvkv ofvn, Sal, Sal, estoman vc assecetske haks? Nak fvsfvke oh refketv cen yekcet os, maken pohimvts.

15 Momet, Pucase, estimv toyetska? makin, Cesvs, mv assecetskat toyis, makemvts.

16 Momis ahuyiret, ce'len oh huervs, heyv vrahkvn cem e heckuecikv, heyv nanvke hecetskvte, momet nanvke mv ofv cem e heckuecvranvyat vtotkv, momet a en kerrv ce hayaret;

17 Este momet Centilvlke hiyomat ec oh 'totvyat cen kvpvyecicit;

18 Mvn ec oh 'totakis, e turwv take en hvwecet, momet yomucke enkvpvken hvyayvken oh fulothuecet, momet Setvne en yekce enkvpvken Hesaketvmesen oh fulothuecet, naorketv enkvpvketvt, momet hvsvthvke hahoyvte vpvke epucasvke haketv en heckvkvren, vkvsvmkv vc ofv ocat eteropotten, makemvts.

19 Monkv, mekko Vklepv toyetskat, heyv nake hvlwe vtē hecke ocat em vkvsmvkot omvkomvts ;

20 Momis Temaskvn vpokat taten, momet Celuslvmen, momet Cutev ekvnv omvlkvn, momet Centilvlken, em vkerrickvn eticaket Hesaketvmesen oh fulotecet, momet vtotketv vkerrickv etickv orvken momecvkvren en kerkuecakimvts.

21 Heyv nanvke omecicen Cusvlke cuko-vcakat ofvn $\mathrm{cv}$ 'sahket, cv 'lecetvn komakemvts.

22 Monkv Hesaketvmese a enkvpvken vnickv vn hecken, 
rvkrakat lopuckateu a en kerrakit, nanvke owalvlke, moinen Moses mome ocvkvres, makakvte tvlkusen punayet, mucv-nettv oren huerit omis ;

23 Klist estemerkvrane, momet elkv raenkvwvpketv enhvteceskv taranat, este momet Centilvlken hvyayvke hecvknecvranat tat kerkuecis.

24 Momen hiyomen e em punayepof, Festrs, yekcicet huehket, Pal toyetskat, ce hacohakes, cokvkerretv tayat ce hacohayes.

25 Momis, Festrs here mahetskat, ov hacohakeks, momis mehenwr momet mahlvprtketr opunvkvn opunayis, kicemvts.

26 Mekkot heyv nanvken kerret omekv, mv ehomvn etektvnkusen opunvyvyat. Heyv nanvke hvmkis em ehkekat komikv; heyv nake vkvnowv ofvn momehocekatet omekv.

27 Mekko Vklepv toyetskat, owalvlke vkvsametske te? Vkvsvmetske tat kerris.

28 Momof Vklepvt, Klistvlke hakaren 'sve oricvranusetskes, Pal kicvtes.

29 Momen Pal, Cen tvlkusekon, momis mucv-nettv cv pohakat omvlkvt vne ome orvkvranusat tvlkekot, momis orvke tate! komit, Hesaketvmesen es oh hecis, heyv eswrnakv tvlkuset momekon.

30 Momen heyv nanvken opunahyof, mekko, evpayv, Pvnese esyomat, momet mv vpvkakvte asvpaklvtes;

31. Momet etvn vpeyahkof, etempunahoyet, Heyv hunvnwv elvre taye, monkat wvnvkvre tetaye naken momeceks, makakvtes.

32 Momof Vklepvt, Heyv este Sesvn oh huehkekaten omat, en recvpke tayvnt os, Festrs kicvtes. 


\section{SETENTVCKE XXVII.}

Momen Etvlen perron vtehkēt vpeyvranēn pum frciyof, Pal momen svlvfkvlke vpvlwvn, sentulev, Culevs hocefken, Vkvstvs en suletawvlke etohkvlkat en kvpetvnvlke hvmkan em wikakemvts.

2 Momen Etlvmeter em perro-cukon ohtehket Esev ekvnv afopken svkvpeyvranet, esenkvpakemvts, Elestakvs, Masetonevlket, Resvlonikvn vtet, epu 'paken.

3 Momen rempvkse Sitvn rvcakēmvts, momen Culevs Pal heromen vfastet, en hessvlken oh vyepet, e emahlvpvtvren em etektvnecvtes.

4 Momen mvn esenkvpahkeyof, Siplvs afopken svkvpeyēmvts, hotvlet vurvpet okv.

5 Momen uehatkv Selesev momet Pamfelev vnakv svktiyehcēyof, Lesev ekvnv Milv tvlofvn roricēmvts.

6 Momen mvn Aleksantlev tvlofv perro-cukot Etvlen ayen sentulevt eshehcet, ep ohtehemvts.

7 Momen nettv sulken yulohusen svkvpeyet, Nitvs afopken, roricvranuseyof, hotvlet pu mvnvttecen, Klete afopken, Salmrn vnakvn roricēmvts.

8 Momet hoyvnece mahekot, Svcaketv Herakusat kicetvn, tvlofv Lesev vnakvn, roricemvts.

9 Momen oketv hofunet yvmahkepen, svkvyetvt entis penkvlke hakof, momen eelvweckv hoyvnepekv, Pal emvcahnemvts ;

10 Hunvntake toyatskat, heyv svkvpeyeyat perro-cuko momet nanvke vtehkat tvlkekot, pun hesaketvu yvmahketvt, momet vhopvnketv tayet ocvranen hecis, kicet.

11 Momis sentulev Pal nanvke makat esemuntvlen perro epucase, momet vfastv min vkvsamemvts.

12 Momen perrvcakv rvfo yvmahketv herekokv, Mvn enkvpaket, estomet perrvcakv Klete, Fenesen roricē tayen 
omat, mvn rvfon yvmahkeyvres, este sulkat makakemvts, mv perrvcakv Hrsaklatkv-enhonerv fvecvn momet Hvsaklatkv-em-wahvlv fvccrn wakket os.

13 Momen wahvlv hotvlet yulohusen hotalof, Mv komeyvte pun heckes, komet, en recvpahket, Klete afopken svkvpeyemvts. .

14 Momis renyupv hofune mahekon hotvle rakket, Yulakletvn kicetvt, perron naf kemvts.

15 Momen perro-cuko vkēsen, hotvle vnrvpen vkayeko tayof, vkatiyvren wikēmvts.

16 Momen ote hvmke, Klatv kicetvn vnakvn svkvpeyen, perruce pun sumkekaren ep omekaranusēmvts.

17 Mvn a vk esahkof, sevnickvn omaket, perro-cuko wvnayahket, Oktahv vksvmohen es epu'klvtike wites, komet penkvlaket, hvnvwelvn kvncvpuecahken, esvkatiyēmvts.

18 Momen hotvle-rakkot tayen es pum melohlicen, rempvkse perro-cuko tvhoknicakemvts.

19 Momen nettv svtutcenof, perro-cuko nanvke vtehkvten punke mahusaten es apvlatēmvts.

20 Momen hrse kococumpvu nettv sulken heckvkekof, momen hotvle-rakko cotkuse tokot ep oh wakket on, pu hesahoke taye enhonrkv omvlkvt sumkemvts.

21 Momis eelaweckv hofune hoyanof, Pal en nvrkvpvn 'ren huyiret, Hunvntake toyatskat, heyv yvmahketv, momet hopvnketv e en cakkuecatskekaret, Klete senkvpvkekot vm apohicvranatskvtes.

22 Momen hiyomat ece 'fvckvke taren ce mvcahnvkis, ece 'pakvkat hvmkusis en hesaketv sumkvranekok', momis perro-cuko tvlkuset.

23 Hesaketvmese, enake toyvyat, momet em vtotkvyatet, em estet nereise vc vhueret,

24 Pal toyetskat, ce penkahlats, Sesv ehomvn erhuerv- 
ranetsket omekv, momen hecvs, Hesaketvmeset mv esvkfullvlke ece 'pakat omvlkvn cemēs, cv kicis.

25 Monkv ece 'fvckvkekvs, hunvntake toyatskat, mv vm onahoyvte mahusat taranat, Hesaketvmesen vkvsvmvyet omekv, kicemvts.

26 Momis ote hvmken ep oh pvlvtkvranes.

27 Momis nere pale ostohkakat orof, estvmi estomis 'sepu 'tohken, Etlevn svkfullen, nere nvrkvpv mahe orof, perro-cuko vfastvlket, Ekvnvt ocen vwolicēs, komakemvts.

28 Momet vkvhopahyet, estele-vhopakv cukpe hvmken pale hokkolen kerrakemvts; momet ohvtvlvkusen svkvpehyet, hvtvm vkvhopayat, estelevhopakv pale ostvpaken kerrakemvts.

29 Momof Cvton oh pvlvtike witeyes, komet penkvlaket, perro-cuko yupvn esvkhvlatkv osten a vk pvlvtahket, Hvyatkate! komakemvts.

30 Momen perro-cuko vfastvlket, perro-cuko esosset, pefatketvn komaket, perro-cuko-homv esvkhvlatkv a vk pvlvtvrane omecaket, perrucen uehatkvn vk hvtvpecihcof,

$31 \mathrm{Pal}$ sentulevn momet suletawvlken em punayet, Heyvt perro-cukon vtehket fekhonnvkekon omat, ce hesahokeko tetayes, kicemvts.

32 Momof suletawvlket perruce eswrnakvn warahket, enkvpvkvren wikakemvts.

33 Momen hvyvtkvranusof, Pal omvlkvt, hompetvn cahwet, hompvkvren em 'pohet, Fekhonne emomecice e elawece, naken cahwet hompatskekat heyvt nettv pale ostohkakat tes, kicakemvts.

34 Monkv hompetv crwatskvren cem 'pohvkis, heyvt ece 'cayecvranvket omekv, hvmkatskis cekv-esse cekv a Ivtkvranekot omekv.

35 Momen hiyomen opunahyof, tvkliken ehset, omvlkv 
ehomvn vkvsvmkvn Hesaketvmesen emet, kvlkahyof, hompetvn vlicecemvts.

36 Momof omvlkvt, em vkerrickv herake haken, hompetvn crwakemvts.

37 Momen perro-culko vtehkeyat omvlkat este em puyvfekev take cukpe hokkolen pale kolvpaken epohkakemvts. 38 Momen em etetayen hompvkehpof, telekon uehatkvn a vk pvlvtaket, perro-cukon tvhoknicakemvts.

39 Momen hvyatkof, ekvnvn kerrvkekomvts, momis uewv afvske hvmken lvpvtke ocen eshecaket, mrn perrocukon res vceyetvn komakemvts, momecvke tayen omat. 40 Momen svkhvlvtkvn warahket, uehatkvn em wikahket, esfvyatkv eswrnakvn enrecrpakemvts, momet hvnvwelv rakkat hotvlen 'sem wihkēt, lvpvtke fvccrn svkvpeyēmvts.

41 Momen uehatkv hokkole etenrapen 'svk pvlvtiken, perro-cuko erohvtvlahyen, homvt vkyekcehpen, nekeyeko tayet vk lihken, uemelohlv en yekcet e yupvn kacemvts.

42 Momen, Svlvfkvlken pvsvtvkeres, monkon omat, omayet esosset pefatke wites, suletawvlket maket vcahnakemvts.

43 Momis sentulevt, Pal vcayecetvn komet, nake makakat em vsehvtes, momet, Estit omvyvke tayat tatet $e$ vk pvlvtahket, lvpvtken roricvkekvs, makemvts ;

44 Momen vhoskat vpvlwvt toselkvn 'se hvlvtaket, vpvlwvt perro-cuko nake $v k$ wokockan 'se hvlvtaket, lvpvtken roricvkekvs, makemvts. Momen mome ocemvts, omvlkvt hesahoket lvpvtken roricakemvts.

\section{SETENTVCKE XXVIII.}

Momen hesahohkof, mv ote Melitvn kihocet omat kerrakemvts.

2 Momen tvleporvlket heromkv cutkuse tokon pu heci- 
cakemvts, momat omvlkeyan pu evpvyahket, totkvn etecakemvts, kvsvppe momet oskat svrahkvn.

3 Momen Pal, eto wokuckuce sulken vtelohyet, totkvn oh vpohyof, cettot hiyan aosiyet, enken em vyokcemvts.

4 Momen mv tvleporvlket mv nak senhonecke enken em vtarken hecakof, emet etempunahoyet, Heyv estehunvnwv este elecvt o hervcuks, hesaket uehatkv aosset ont omis, fvecetvt ohvtvlvken hesakvre em etektvnecekot omes, makakemvts.

5 Momen mv nak senhonecken totkvn a tvk fekehyet, naket en nokkekomvts.

6 Momis hvsvfkvres, monkat vyvkhvmkvn ehlet, tvk lvtkvres, komaket vketecakemvts; momis hofune mahen hecahkof, holwvke estomet oh vlvkekon, em vkerrickvn mvrahkuecahket, hesaketvmese tes, makakemvts.

7 Ekvnv mv vfolote ocakan $\mathrm{mv}$ ote este en rakkat, Pvplevs hocefket, enaket ocakemvts, momen mvt epu vpayet, heromet nettv tutcenen epu 'fastemvts.

8 Momen mome ocemvts, Prplevs erket hepetkat, momet cate nvrkakvn es enokket wakken; Pal, mvn cehyet, emekusapet, enken oh wrkhokicet, em wicecemvts.

9 Monkv heyvn momehocof, ote ofv enokketv vlilakat vpvitakeu yicet, em wikakemvts.

10 Ohvtvlakat, heyv estet nake es vrakkuecetv sulken es epu 'rakkuecakemvts momet en kvpvkakeyof, nanvke este eyace tayat es ep oh ocakemvts.

11 Momen hvse tutcenet hoyanof, perro-cuko Aleksantlev aosse, mv es en kerkv Kastv Palvks tepakat omen, ote ofvn rvfo yvmahkvten ohtehket, es en kvpakēmvts.

12 Momen Selekyuse resvcahket, mvn nettv tutcenen fekhonnēmvts.

13 Momen mvn es en kvpakēt, vfolotecet Lecevm yicē- 
mvts, momen, nettv hvmke hoyanof, wahvlv hotvlet hotalemvts, momen rempvkse Puteoli yicēmvts.

14 Momet mvn etecakkakan eshehcēt, nettv kolvpaken em fekhonneyvren komakemvts. Momen moman Lome fvccun vpeyēmvts.

15 Momen etecakkakat Lome vpokat ep ohfvecvn pohakof, mvn a awet, Apei Folvm, momet Hompetv-cuko Tutcenat vteken pu'nrvprranet yicrtes, mvn Pal hecof, Hesaketvmesen vkvsamet, fekhvmketvt en heckemvts.

16 Momen Lomen yihceyof, sentulevt svlvfkvlken vketecvlke en kvpetvnen em wikemvts, momis Pal entvlket, suletawv hvmke vhecicen, vprket likvren em etektrnechoyemvts.

17 Momen mome ocemvts, nettv tutcenat hoyanof, Pal Cusvlke en homahhotvlken vteloyvtes, momen etohkvlikof, em punayet, Hunvntake etecakkvte toyatskat, este monkat purkvlke semvpeyetv vnrvpe naken momecvyvte sekot ont oman, svlvfkēn Celuslvme enkvprken Lomvnvlke enke ofvn $\mathrm{cr}$ wihokemvts.

18 Mvt vc ohvketecahkof, vn recvpakvnt omes, cv 'le tetaye ore naken momecvkokv.

19 Momis Cusvlke vn recvphoyvren vnrvpen opunahohyof, Sesvn oh huehkvranvyat tvlkemvts, nake vm etvlofv 'svnrvpetv ocvkis.

20 Monkv heyv vrahkvn cen huehkvkit omis, ce hecit, momet $c e$ tempunayvkaret, Eslevl em enhonrkv vrahkvn heyv cvto-tetvkkakv es cv wrnvket omekv, kicemvts.

21 Momen, Cutev aosse ec ohfrecr nakcokv tis pun heckekon, etecakkakat yicakat estomet ec ohfvecv holwvke opunayvkekot, momet pu hecicvkekvnks, kicakemuts.

22 Momis naken vkerricetske tat cem pohetvn pu 
yaces; heyv emetohkvlketv hrmkat ohfvecv hvmkvn vnrvpen opunvyehocet omat kerrēt omekv.

23 Momen nettv em mellahkof, este sulket en liketvn en yicaken, mvn Hesaketvmese em ohmekketvn em ohkerkuecet, momet ohhvyvyicet Moses em vhakv momet owalvlke aossen hvthvyvtken yafke vteken Cesvs ohfvccvn em vcahnakemvts.

24 Momen nanvke opunayat vkvsvmakat sasen, vkvsvmvkekateu sasemvts.

25 Momen emetvwrt ete'm vkvsvmvkekofvt, en kvpvkakemvts, Pal heyv opunvkv hvmken opunahyof, Esivs eteropotten Puyvfekcv Vcakat purkvlken heren em punayet,

26 Heyv esten oh ayet, Pohatskat pohatskvres, momet kerratskekares, momet hecatskat hecatskekares, momet kerratskekares, kicvkvtcvs, makvtes.

27 Heyv este e feke take wvnwvhes, momen e hvcko take pohetvn tefnes, momen e turwv vkhottakekv; monkon omat, estofis eturwv es hecet, ehvcko es pohet, efekeu es kerret, fulotecen em wicecvkvye tayen, makvtes.

28 Monkv cen kerkvkekvs, Hesaketvmese em vhesaketv Centilvlken ohtothoyet omes, momen em apohicvkvres.

29 Momen heyv opunvkv opunahyof, Cusvlket enkvpvkakemvts, momet opunvkv tayen emetvwvt es etenrvpakvtes.

30 Momen Pal ohrolope etemrpoke hokkolen eme en cuko pvlepat liket, en yicakat omvlkvn evpayemvts.

31 Sentacke sekon, momet fekhvmketv omvlkvn Hesaketvmese em ohmekketvn es erkenaket, momet mv nanvke Cesvs Klist ohfvecv ocakat 'seste'mvhayet. 


\section{Corrigendae.}

\section{V HERICKV.}

MARO IV. 2. Momen nettr pale-osten nere paleosten e elvwehcof, renyupvn elawvtes.

MARO VIII. 28. Momen trpalv Krkesevlke ekvnvn rorof, este hokkolet, este-nekricvlke vtehket, cvpvkhoke mahet, este estomet $\mathrm{mv}$ hoyvneko tayaten, esteherickvn awolket vurvpakvtes.

MARO XVII. 27. Mome estomis vnvttecēkaren, vkhvsen ahyet, cufokunhen vkwikvs, momet rvro enhvteceskv akvwapkan esvs, momet e cukwrn en hvwecetskof, cvto-kunawvn eshecetskvres; mvn ehset, vc vrahkv ece 'rahkvu emvkrs.

MARO XVIII. 14. Matvpomen, heyv lopuckusat hvmkis sumkvre Cerke take hvlwe likat nake komat tokot omes.

MARO XIX. 14. Hopuetakuce em etektrnecet, vc oh vwepvre em vsehhatskvs, mv omaket hvlwe ohmekketvt omekv, Cesvs makvtes.

MARO XXIV. 45. Monkv vtotkv hoporrene, heromat, $\mathrm{mv}$ em oketv orof hompetv emvkvren e pucaset en cuko ohfvinke hayvte estimvt o haks?

MARO XXVI. 11. Estemerrakat ece 'pakvke ocatske munkvt os, momis vntat ocatske munkv tokot omekv.

CANE III. 4. Este vculehpof, estomet hecke taye haks? svhokkolv ecke e nvrken ecehyet, hecke taye haks? Nekotemvset kicrtes. 
CANE VI. 9. Cepanet likes, ketinv tvklike-vnore cahkepen, momet rvro lopuckuse hokkolen ocet; momis heyv naket o haks, este sulke momat em etenrvwv?

CANE VI. 62. Monkv enhomv likvtet este Echuswv $\checkmark$ kvwapken hecatsken omat, estomvre te?

CANE VII. 4. Este hecke kerhoyvren eyacet, ehke naken celayate sahsekot omekv. Heyv nanvke vfástetsken omàt, ekvnvn em e heckuecrs, kicakvtes.

CANE XIV. 2. Crrke hute ofv liketv sulket os; momekaten omat, cem onvyvkvyvnt os. Liketvn ce metetakuecvranit ayis.

CANE XIV. 26. Nomis Emvhlvpatv, Puyvfekev Vcakat, Erket cv hocefkv ofv vtotvranat, mvt nanvke omvlkvn ce mvhayet, nanvke omvlkv ce kicvkvyvten ece 'kerricepuecvkvres.

CANE XX. 11. Momis Melet hvkihket, cvto-kofken fettrn vhuervtes, momet hrkihkof, ohcunehket, cvto-kofke ofvn vfonnakvtes.

CANE I. IV. 10. Momen vnokeckv heyv ofvts, pumet Hesaketvmese vnokeceyvtet tokon, momis emet pumen epu'nokecet ot, Eppucen vtotvte tat pun naorketv esvrahkv nakwikv taren.

CANE I. V. 6. Uewv momet catvu etehoyvne vlakvtet heyv tes, Cesvs Klist mot ot os, momen uewv etehoyvne tvlkekon, momis uewv catv tepakv etehoyvnen. Momen Puyvfekevt akerrv tes, Puyvfekcrt mehenwvt omekv.

EFESVLKE I. 3. Cesvs Klist pu Pucase Erke, momet en Hesaketvmese vrakkuehocekvs, mvt hvlbawat ofv Klist ofvn puyvfekcv merretv omvlkvn es ep ohmerrvtes. 


\section{COKV VPASTEL PAL}

\section{LOMVNVLKE OHTOTVTE.}

THE EPISTLE OF PAUL THE APOSTLE TO THE

\section{ROMANS,}

TRANSLATED

FROM THE ORIGINAL GREEK

INTO THE MUSKQKEE LANGUAGE.

NEW YORK :

AMERICAN BIBLE SOCIETY, INSTITUTED IN THE YEAR MDCCCXVI.

[Muskokee, 16mo.]

1881. 


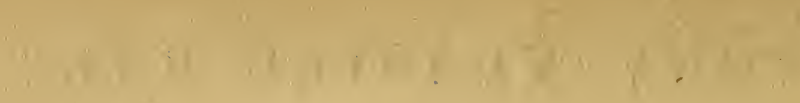
5

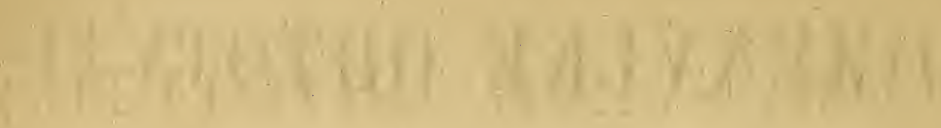
.

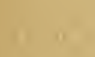

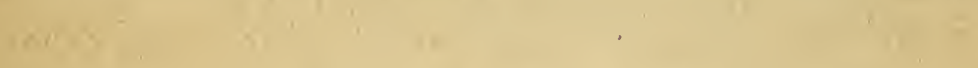

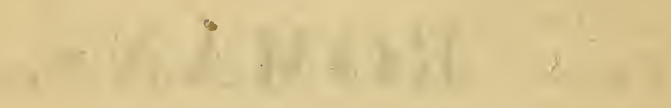

$$
\begin{aligned}
& \text { 1. }
\end{aligned}
$$

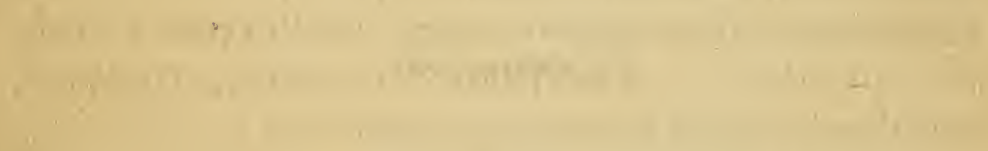

$$
\begin{aligned}
& 1+10+2
\end{aligned}
$$

1.

\section{sins}




\section{COKV VPASTEL PAL}

\section{LOMVNVLKE OHTOTVTE.}

\section{SETENTVCKE I.}

PaL, Cesvs Klist em vtotkv, vpastel enhuehhokvte, Hesaketvmese em opunvkv-hervn oh melhoyvtet,

2 (Mv cokv vcacakat ofv em owalvlke eteropotten enho$\mathrm{mv}$ etemfaccuten;)

3 E'ppuce Cesvs Klist pu Pucase ena-vpeswr vcrkvyen Tewe e nerkv etehoyvnen hahoyvte,

4 Yekcetv eteropotten, hrsvtketv em Puyvfekcr vcvkvyen, prsatkvte aenkvwvpketv eteropotten, Hesaketvmese Eppucet omat kerkuehocvte ohfvecrn;

$5 \mathrm{E}$ hocef $\mathrm{kv}$ vrahkvn tvlwvvlke omvlkv ofv vkvsvmkv vpohickvn, mv eteropotten herkv momet vpastel em wiketvn $\mathrm{mv}$ etehoyvnen pun heckvtet os.

6 Cemeu mvn vpaket Cesvs Klist ce'nhuehkvkvtet os.

7 Hesakefvmese vnokecvkvte, faccvlke hakvren enhuehhokvte Lome vpokat omvlkvn; Heromkv momet herkv Hesaketvmese Pu'rke, Pucase Cesvs Klist tepakat aenkvpvket cem ocvkekvs.

8 Esvliceckvn, omvlkatskat ece 'rahkvn Cesvs Klist eteropotten vn Hesaketvmesen vkvsamis, cem vkvsvmkvt ekvnv omvlkv eteropotten opunvyehocet omekv.

9 Hesaketvmeset, mv vm puyvfekcrn E'ppuce em opunvkv-herv ofvn es em vtotkvyan, vm mekusvpkv ofvn fekhonnvkot centaken estofis hocefvkvyan a vn kerrvt omekv. 
10 Cem vlvkvkaren em 'pohvyet os, estome estomis Hesaketvmese komat vevkvyen hiyomat estonkon heren ayvye tayen omat;

11 Ce hecetv taken cv yacuset onkv, emetv puyvfekev ohfvecv ocat ce'mvkaret, ce yekcvkuehocat vrahkvn.

12 Heyvt omes, ece 'pakvkit v'mahlvpvtkvren vkvsvm$\mathrm{kv}$ centake momet pu tenrvwv ocat eteropotten.

13 Tecakkvte toyatskat, Centilvlke vpvlwv em etenrvwv em ette ocvyat etvpomen cem etenrvwv takeu ocaret, cem vlvketv taken tewolen komvyvtet omis, (hiyomat oren vm mvnvttehocvten,) kerratskekaren komvkot os.

14 Klekvlke momet Papelevlke hokkolvn, hvtvm, hoporrenvke momet hoporrenvkekat hokkolvn em e vhuericvkvyet os.

15 Monkv yekcetv vc ofv ocat oren, ceme takeu, Lome vpokatskat- opunvkv-hervn cem erkenvketv take vm etetakes.

16 Klist ein opunvkv-hervn es vc vlsekokv : mvt vkvsamat vtekat vhesaketvn Hesaketvmese en yekcet omekv, Cusvlke taten, momet Klekvlkeu en heckat.

$17 \mathrm{Mv}$ ofv Hesaketvmese em fvecetv vkvsvmkv avtet vkvsvmkvn oren hecket omekv, Mv fvccat vkvsvmkvn eteropotten hesakvres, make hoccat vcvkvyen.

18 Este, mv mehenwetv fvccetv-tokat ofv hvlvtakat em fvccetv-tokat momet Hesaketvmese vrakkueckv-tokat omvlkv vnrvpen Hesaketvmese en crpvkketvt hvlwen avtet kerkuehocet omekv.

19 Nake Hesaketrmese ohfvecv kerhohoye tayat eme ofv taken kerhohoyet omekv; Hesaketymeset en kerkuecakvtet ok'.

20 Eme ohfvecv nake hechoyekat ekvnv hocvckvte vteken heckusen hechoyet omekv, nake hahihocvte eteropotten kerhohoyet omen, en yekce estofis oce munkat 
momet estofis Hesaketvmeset omat, mvn hechoyet omen ; momat seokepkv ocvkekot os :

21 Hesaketvmesen kerrakis, Hesaketvmese omecet vrakkuecekot, monkat em merkv vrahkvn vkvsvmvkekatet ok'; momis em vkerrickv ofv ehvperkvke haket, e feke take hoporrenekat yomucke hakvtet os.

22 Hoporrenvke e okicaket, hoporrenvkeko hakvtes,

23 Momet Hesaketvmese hopvnkeko tayat en hvyayvken mvrahkuecet, este hopvnke taye, momet fuswv, momet eleostakat, nakhvlecakat esyome vhaken hayakvtes.

24 Momen monkv Hesaketvmeset e feke take em eyackv eteropotten hvsvtketv-tokan em wikakvtes, eme em etenrvwv em ena taken vlesketv en hvyaken;

25 Mvt Hesaketvmese em mehenwetvn mvrahkuecet lvksetvn hayakvtes, momet Hayvten, mv meyuksv-sekon vrakkuehocat senhoyvnen nake hahoyvten vrakkuecet em vtotkakvtes. Emen.

26 Heyv omecicen Hesaketvmeset vkerrickv holwvhokan em wikakvtes; hoktvke enaketake nake em munkv vcrkvye ocan eticet em munkv vnrvpen fullvtet ok'.

27 Hunvntakeu matvpomen este em munkv vcrkvye ocan wikaket, eyackv holwvke ete'm ocakat ofvn vhethokvtes; hunvntaket hunvntaken etohkvlket nake holwakan vtotkat, momet naorkakvten eme ofv feketv momvre tetayat en heckaken.

28 Momen en nakkerretv ofv Hesaketvmese ocetvn eyacvkekate etvpomen, Hesaketvmeset vkerrickv holwakan em wikakvtes, nake momvkvranekaten momecvkvren ;

29 Nakfvccrkekat omvlkv, empvlse seko hoktarkv, holwryeckv, nakockv-vnoksetv momet enhomeckv es fvcfvket omen, momet vleskvpkv, esteleckv, punvkv setenrvpkv, ehosickv, esternrvpkv es fvcfaket; wrswakvlket, 
30 Nanokokvlke, Hesaketrmese enhomecvlke, enhomecakat, ekvsvmakat, punvkvn 'sekvsvmakat, nake holwahoken heckuecakat, eckvlke em apohicvkekat,

31 Hoporrenvkeko, temfvccetv, kacvlke, enahvmkvlke vnokecvkekat, fekhervkuecesko tayat, merrvkekat;

$32 \mathrm{Mv}$, Hesaketvmese em ohfvcceckv, mv nake mv omakan momecakat pvsvtke taye em mvttvket on fuccecat kerrakis, mv nanvken momecakat tvlkekot, momis mvn momecakat em vpvyecvket os.

\section{SETENTVCKE II.}

Monkv este-hunvnẃv est' ohfvececetskat vtekat, seokepkv ocetskekos, mv nake ohfvecv este etin ohfvececetskat e ohfvccecetskekv; cemet ohfvececetskat mata nanvken momecetskekv.

2 Momis Hesaketvmese mv omvken momecakat vnrvpe ohfvececkv ocat mehenwetv revkvyet omat kerrēs.

3 Momen, este-hunvnwv, nake mv omakan momecakat ohfrccecetskat, momet matan momecetskat, Hesaketvmese em ohfvcceckvn mvnvttepares, kometske haks?

4 Monkat Hesaketvmese en heromkvt vkerrickv-etickvn ec ohyekcicat kerrekot, en herkv, en yvmvskv momet momis-kometv vcrpkat esyomet en sulken ehvperkuecetske haks?

5 Momis cem wrnhat, momet ce feke mvrahkekat vcvkvyen crpvkketv nettr momet Hesaketvmese ohfvecec$\mathrm{kv}$ fvccat kerkueckv nettv vlvkvranan cvpvkketvn e en hericetskes ;

$6 \mathrm{Mr}$ estimvt o estomis em vtotketv vcrkvyen pvlken em fekvranat:

$7 \mathrm{Mv}$ heren momecakat ofv momis-komet emunkat ete. ropotten afvcketv, vrakketv aye-munketv esyome hopo. yakat hesaketv yuksv-sekon emvkvres: 
8 Momis mv tepokv em vlostvke, mehenwetvn em apohicvkeko, fvecetv tokat min em apohicakat, enhomeckv, crpvkketv,

9 Estemerkv efeknokketv esyoman, este em puyvfekcv holwvke momecat vtekat emvkvres ; enhvteceskvn Cusvlken, momet Centilvlkeu ;

10 Momis heren momecatvtekat afvcketv, vrakketv, herkv esyoman; enhvteceskvn Cusvlken momet Centilvlkeu:

11 Hesaketvmese ofv este etemetueckv sahsekot ok'.

12 Estomomuset vhakv sekon naorkakvten omat, vhak sekon estemerkaken, estomomuset vhakv ofv 'naorkakvten omat, vhakv vevkvyen ohfvecehocvret ok'.

13 (Hesaketvmese ehomv fvecakat vhakv pohvlke tokon, vhakv momeevlke min fvccvkuehocvret ok'.

14 Centilvlke, vhakv ocvkekat em munkv vcrkvyen nanvke vhakv ofv ocakan momecakof, heyvt vhakvn ocvkekis, e vrahkvn vhakvt omakes.

$15 \mathrm{Mvt}$, vhakv em vtotketv e feke take ofv hoccaken este hecicaket os, eohvkerrickv take akerret omen, momen em vkerrickv take vnrvpueckvn eten hayet, monkat seokepkvn eten hayen.)

$16 \mathrm{Mv}$ nettv Cesvs Klist etehoyvnen Hesaketvmese vm opunvkv-herv vcrkvyen este enake ehkakat ohfvececvranat ofvn.

17 Hecvs, Cusvlke ce hocef ket on, vhakv ofvn fekapet, Hesaketvmesen es ekvsametsket os,

18 Momet naken komat kerret, momet, vhakv aossen ce mvhahoyet omen, nakè hervke mahan vrakkuecetskēs;

19 Momet, Vne turhecrkekan em evpayv, yomucke ofvn vpokat en hryayvke,

20 Hoprrenvkekat nake em ohhvyayicr, momet hopuetakuce em mvhayv toyis, e kometske heres; nakkerretv momet vhakv ofv mehenwetv ocat en heckvn ocetskat. 
21 Monkv etvn mvhayetskat, e emvhayetskeko? Este horkopekares, make erkenaketskat, horkopetske te?

22 Estet holktvrekares, maketskat, hoktaretske te? nakvhakakan enhomecaketskat, mekusvpkv enanvken hoprnetske te?

23 Vhakvn es ekvsametskat, vhakv-kackv eteropotten Hesaketvmesen ehvperkuecetske te?

24 Hoccat vcrkvyen, cemet omecicatsken, Centilvlket Hesaketvmese e hocef kvn tvklecaket omekv.

25 Mehenwvn, vhakvn vfastetsken omat, svkvmsiskvt lopicet os; momis vhakvn kacetsken omat, ce svkvmsishoyvte tis ce svkvmsishoyekate ome haket os.

26 Monkv mv svkvmsishoyekatet vhakv em fvccetvn vfasten omat, svkvmsishoyekat tis svkvmsishoyes komhoyvraneko?

27 Momen svkvmsiskv tokat, mv emunkv vevkvyet omat, vhakv vfasten omat, ceme, letv svkvmsiskv tepakat vcvkvye vhakv-kacvt ometskat, ec ohfvccecekare te?

$28 \mathrm{Mv}$ heckuse tvlkusat Cusvlke tokon; mv svkvmsiskv heckuse vpeswv ofv ocat tvlkusis svkvmsiskv tokot os.

29 Momis ofvt omat Cusvlke tes; momen svkvmsiskv efeke enaket's, letv ofv tokon, puyvfekcv ofvt ocen; mv vkvsvmhoyat, este enake tokon, Hesaketvmese enaket os.

\section{SETENTVCKE III.}

Monkv estomen Cusvlket homahtet o haks? monkat svkvmsiskv naken lopice te?.

2 Estome estomis sulkes; omecice mahat, Hesaketvmese em opunvkvn mvn em wikvkhoyvtet omekv.

3 Vkvsvmvkekat sasvten omat, estome te? mv em vkvsvmkv sekat Hesaketrmese enhonrkv lopiceko tayen hayvrane haks?

4 Mohmats; este estomis laksvt omen, Hesaketvmeset 
mehenwet omekvs; hoccat vevkvyen, Cem opunvkv ofv fvcce ome hueret, ec ohfvccehocof muntvlet hueretskvren. 5 Momis pun holwryeckvt Hesaketvmese em fvecetv heret omen kerkuecen omat, naken makvraneya? Hesaketvmese naorketv vrahkvn estemerricat fvccekot ome te? (este-hunvnwr omēn opunayis ;)

6 Mohmats; hiyomekv, momēn omat, estomēn Hesaketvmeset ekvnrn okfvocecvr haks?

7 Momēt omen laksvyat eteropotten Hesaketvmese em mehenwetv ohvtvlakat ohvtvlaket ayen en rakketvn kerkuecen omat; estomaten monkv este-naorkv omēn ve ohfvecehoce haks?

8 Momen, (fvecekon epu 'punvyehocat omekon,) momen, Herat aossvren, holwakan momecvkeres, makaket $\mathrm{os}$, (pu kice sasat omekon,) $\mathrm{mv}$ ohfvecehocrre frcert omes.

9 Monkv estome te? pumet Centilvlke senherakeya? Estome estomis, monkos; Cusvlke Centilvlke esyomat omvlkvt naorketv elecvn vpoket omat homv etan fvcceceyvtet omekv :

10 Hoccat vevkayat, Frecat sekos, hvmkusis :

11 Heren kerrat sekos: Hesaketvmese hopoyat sekos.

12 Omvlkvt fiyecaket, tohkvlket lopicvkeko taye hakvtet os; heren momecat sekos, hvmkusis.

13 'Sen nokmelkv take sepvlkv hauke tes; e tulaswr taken ehosickvn es vtotkakvtet os; cetto aspetv kihocan en fenenv e cukhrrpe take elecvn oces;

$14 \mathrm{E}$ cukwv taket tvhiketv home opunvkv tepakan es. fveket os ;

15 E 'le take catv-pvlvtkv ohfuccun pvfprnet os.

16 Hopvnketv momet estemerketvt em vpeyetv taken oh ocakes;

17 Momen herkv em vyetvn kerrvkekatet os. . 
18 E turwv take ehomvn Hesaketvmese em penkvlkv sekot omes.

19 Momen naket o estomen vhakv makat vhakv elecv svpaklan kicet os; ecukwv omvlkvt em vkhotken, ekvnv vtekat Hesaketvmese ehomvn em mvttet svpaklvren, kerreyet os.

20 Monkv vhakv vtotketv eteropotten eme ehomvn vpeswr hvmkusis fvccrn hahohyekos; naorketv kerretv vhakv etehoyvnet omekv.

21 Momis hiyomat Hesaketvmese em fvccetv vhakv sekat ofvn hecket os, vhakv owalvlke esyomat akerraken.

22 Hesaketvmese em fvccetv Cesvs Klist vkvsvmkv eteropotten, $\mathrm{mv}$ omvlkv oran, omvlkv oh ocan okis; temvrahketr sekot omekv;

23 Omvlkvt naoriket, Hesaketvmese vrakkueckvn yupvklvtkakvtet omekv;

24 Enrahkv sekon, em merkv eteropotten, neskv Cesvs Klist ofv ocat etehoyvnen pu fvccvke omet pu svpaklehocet omen :

$25 \mathrm{Mvn}$ Hesaketvmeset merkv heckueckvn ahuericvtet os, e catv ofv vkvsvmkv ocat eteropotten em fvccetvn holwvyeckv momehocvte enkvpvyecickv kerkueckvn, Hesaketvmese hofunen mehakat etehoyvnen:

26 Hiyomat em fvccetv kerkueckvn; Cesvs ofvn vkvsamat fvece omēn huerice estomis fvccvt omvren.

27 Monkv sekvsvmkv estrmvn oce te? Semvkhotkes. Vhakv estomet esemvkhotke te? vtotketv em vhakv te? Monkos; vkvsvmkv em vhakv eteropotten.

28 Monkv heyon vkerriceyet os; vhakv em vtotketv sekon, vkvsvmkvt esten fvece omen hayet os.

29 Cusvlke tvlkuse en Hesaketvmese te? Centilvlkeu en Hesaketvmese toko? Momes, Centilvlkeu :

30 Hesaketvmese hvmkuset svkvmsishoyat vkvsvmkv 
etehoyvnen, momet svkvmsishoyekat vkvsvmkv eteropotten fvccuke omēn hayet omekv.

31 Monkv vhakvn vkvsvmkvn es ehrperkuecē haks? mohmates; momis vhakvn yekciceyet os.

\section{SETENTVCKE IV.}

Monkv Eplehame pu'rke vpeswr vevkvye eshecat naken kicvraneya?

2 Hiyomet omekv, Eplehame vtotketv eteropotten fvece. omēn hahoyvten omat, es ekvsvme tayat ocet os; momis Hesaketvmese ehomv tokon.

3 Momat Cokat naken make haks? Eplehame Hesaketvmesen vkvsamet omen, mvt fvccetv vyoposken esvkerrihocvtet omes.

4 Momen mv vtotkat em feketv merkv omecice tokon, momis vhuericat omecices komhoyet os.

5 Momis $\mathrm{mv}$ vtotkekot, momis $\mathrm{mr}$ fvccekan fvece omen hayan oh vkvsamat, em vkvsvmkvt fvecetv vyoposkes, komhoyet os.

$6 \mathrm{Mv}$ hunvnwv, vtotketv sekon, Hesaketvmeset fvece omecat em afvcketvn Tewe opunvyecat etvpomen;

$7 \mathrm{Mv}$ en holwryeckv take enkvpakihocan, momet en naorketv take ohrvnhoyan en herakes;

$8 \mathrm{Mv}$ hunvnwv Hesaketvmeset naorkv omecekat en heres, makat.

9 Heyv afvcketvt svkvmsishoyvte tvlkusen oh vlake haks? Svkvmsishoyekateu oh vlaket omeko haks? Eplehame em vkvsvmkv fvecetv vyoposke omehocvtes; makeyet omekv.

10 Monkv estomēn komhoyvt haks? svkvmsiskvt en hoyanof, monkat svkvmsiskvt en heckeko emunkof komhoyvt haks? Svkvmsiskv en hoyvnekon, momis en heckeko emunkof komet omhoyvtes. 
11 Momen svkvmsiskv eskerkvn, svkvmsiskv en heckeko emunkof, vkvsvmkv etehoyvne fvecetv ocvte eskerkueckvn en heckvtet os; vkvsvmakat omvlkv e'rke take hakvren, svkvmsisvkhoyeko estomis; mvu fvecvkes komvkhoyvren :

12 Momet mv svkvmsisvkhoyat tvlkekot, momis $\mathrm{mv}$ vkvsvmkv Eplehame pu'rke svkvmsiskv en heckeko emunke ocvte ele-vkakv vcrkvye yvkvpakateu, svkvmsisvkhoyat e'rke hakvren.

1\% Setemfvecetv, Ekvnvn epucasetskvres, makat, Eplehamen monkat erhonvpsen vhakv eteropotten e'mhoyekon, vkvsvmkv. em fvccetv eteropotten emhoyvtet omekv.

14 Hiyomet omekv, vhakv este setemfvccetv vcvkvyen pucasvke taranet omaken omat, vkvsvmkvn ehvperkuehocen, setemfvccetv lopiceko taye haket os.

15 Vhakv crpvkiketvn vtotket omekv; estrmvn vhakv sekon omat, vhakv-kackv sekot omekv.

16 Monkv vkvsvmkv eteropotte tes, merkv etehoyvne taren; momat erhonvpse omvlkv setemfvecetv en yekcihocvren; vhakv ofv svpaklat tvlkuse tokon, momis Eplehame, omvlkeyat pu'rket omat, em vkvsvmkv ofv svpaklat, mvu em ocvkvren.

17 (Tvlwvvlke sulke e'rken ce hayis, make cokat vevkvyen ;) mv ohvkvsamvte ehomvn, mvt Hesaketrmeset omat, mv prsatkvten wenahuecet, nanvke ocvkekat nanvke ocake omēn kicat.

$18 \mathrm{Mv}$ enhonrkv vnrvpen enhonrkv ofvn vkvsamvtet os, tvlwvvlke sulke e'rke hakvret; Cen honvpse momē tares, makat vevkvyen.

19 Momet vkvsvmkv ofv yekcet omet, em vculkv ohrolope cukpe hvmkvteket omis, e 'na entis elat tis vkerricekot, monkat Sele en hopuetakhute elateu vkerricekates;

20 Hesaketvmese em etemfvccetvn elentappekates, vkv- 
svmekat omecicen; momis vkvsvmkv ofv yekcet, Hesaketvmesen vkvsvmkvn emvtes ;

21 Momet nake etemfaccrten momece tayes kome hervtes.

22 Monkv fvecetv vyoposken em ohvkerrihocvtet os.

23 Momen em ohvkerrihocvtes, makat eme trlkuse vrahkvn coyet omhoyekates,

24 Momis pumeu epu 'rahkvn, ep oh vkerrihocvranat, Cesvs pu Pucasen pvsatkvte a en kvwapvten oh vkvsamèn omat;

$25 \mathrm{Mv}$ pun naorketv vrahkvn wihokvte, pu fvccvke omet svpakleyvren, hvtvm a kvwvphoyvten.

\section{SETENTVCKE V.}

Monkv, vkvsvmkv eteropotten fvccvke omēn pu svpaklehocen, Hesaketvmese ohfvecvn herkvn ocēs, pu Pucase Cesvs Klist eteropotten.

$2 \mathrm{Mv}$ eteropotte tan heyv herkv, mv ofv svpakleyat, eceye tayat oceyet omēt, Hesaketvmese en hryayvke em enhonrkv ofvn epu 'fvcket omēs.

3 Momen mv tvlkusekon, estemerkvu es epu 'fvcket omēs, estemerkvt momis-kometvn vtotken;

4 Momen momis-kometvt nake es kerretvn vtotken; nake es kerretvt enhonrkvn vtotkan kerrēkv:

5 Momen enhonrkvt vlsecekot os; Hesaketvmese em vnokeckv, Puyvfekcv Vcake pu'mhoyat etehoyvnen, pu feke ofvn hvmecicet omekv.

6 Pun yekce seko emunken, oketv orof, Klist holwvyecvlken em elvtet omekv.

7 Estimvt este faccv em eleko tayuse omet omekv, momis este enheroman em ele taye estet fekhvmke taye sase wites.

8 Momis Hesaketvmeset vnokeckv pum ocat hiyomēn 
kerkuecet os; holwvyecvlke toyeye emunkof, Klist pum elvtes.

9 Monkv mucr mahvkvu, e catv eteropotten pu fvece omet svpaklēn, Cesvs eteropotten crpvkketv enkvpvken pu hesahokvres.

10 Vnrapvlke toyeye emunkof, E'ppuce em elkv eteropotten Hesaketrmese ohfvecvn pu tenherkepuehocvten omat, mucv pu tenherkepuehohcof mahvkvu; en hesaketv eteropotten hesahokeyvret ok'.

11 Momen mv tvlkusekon, momis ohvtvlakat pu Pucase Cesvs Klist, mv etehoyvne etenherkv mucv pun heckvte eteropotten, Hesaketvmese ofvn epu 'fvcket os.

12 Monkv este hrmke etehoyvnen naorketvt yvmv ekvnvn ecehyen, naorketv etehoyvnen elkvu eceyvtet omen, momen momat, omvlkvt naorkakvtet omekv, elkvt omvlkvn oh vlakvtet omen,

13 (Vhakv heckvte vteken naorketvt ekvnv ofvn ocvtet omekv, momis estvmvn vhakv sekon omat, este naorke ohvkerrihocekot os.

14 Mome estomis, Atvme atat Moses vteken Atvme nak' kihocvte etohwvlvpkat omet naorkvkekate tis elkvt ohmekkvtes, Atvmet mv. vilvkvranvte vhaket omen.

15 Momis nakemkv enrahkv sekat naorketv omekot os: hvmke en naorketv etehoyvnen sulket prsatket on omat, Hesaketvmese em merkv, momen estemkv merkv etehoyvne ocat, $\mathrm{mv}$ este hvmke Cesvs Klist etehoyanat mahvkvu sulken em ohvtrlaket os.

16 Momen nakemkv hvmke naorkvte ornekot os. Fvececkvt hvmke eteropotte tates, ohfvcceckvn, momis nakemkv enrahkv sekat, naorketv sulke omecicen, fvcce ome hueretvn omvtes.

17 Monkv este hvmke en naorketv eteropotten hvmke etehoyvnen elkvt mekkvten omat; $\mathrm{mv}$ merkv momet 
fvccetv estemkv sulke en heckvkvte mahvkvu hvmke Cesvs Klist etehoyvnen hesaketv ofvn mekkvke tares.)

18 Monkv hvmke en naorketv eteropotten omvlkv ohfvccecvkhoyvien fvcceckvt oh vlakvte etvpomet, hvmke em fvecetv eteropotten estemkv enrahkv sekat omvlkvn oh vlakvtes, frece ome hueretvn, hesaketv oren.

19 Este hvmke apohicekat omecicen sulket naorkvlke hahoyvte etvpomet hvmke apohicat omecicen sulket fvecrke hahoyvret ok'.

20 Momen naorketv sulke hakvren vhakvt eceyvtet os. Momis estvmvn naorketv sulkvten omat, senhoyvnen heromkvt sulket omvtes:

21 Momat elkv oren naorketvt mekkvte etvpomen heromkvt hesaketv yuksv sekat oren, pu Pucase Cesvs Klist etehoyvnen, fvecetv eteropotten mekkvren.

\section{SETENTVCKE VI.}

Monkv naken makvraneya? Heromkvt sulkvren naorketv ofvn emomecicvranē haks?

2 Mohmates; pumet, naorketv ohfvecvn pu prsatkat, estomēn mv ofvn ohvtvlaken emomeciceyvr haks?

3 Vnvcomēt Cesvs Klist ohfvecvn paptishoyvtet omat em elkv ohfvecvn paptishoyvtet omen kerratskeko haks?

4 Monkv paptesvm eteropotten elkv ohfvecrn mv vpvken pu herihocēt os: Klist Erke en rakke eteropotten prsatkvte aenkvpvken akvwvphoyvte etvpomen pumeu hesaketv mucrse ofvn yvkapeyvren.

5 Em elkv vhake ofv tohkvlkēn pu 'hochoyvten omat, a en kvwvpketv vhakateu of vn matvpomeyvret ok'.

6 Heyvn kerrēt, pum este vculat $m v$ vpvken svrahohvtes, naorketv em ena yvmahkvren, momat ohvtvlaken naorketvn em vtotkēkaren.

$7 \mathrm{Mv}$ elat naorketv ohfvecvn pucasseko haket omekv. 
8 Momen Klist vpvken pu pvsvtkēt on omat, vpaket hesahokvraneyateu vkvsameyet os :

9 Klist pvsatkvten a enkvwapkvtet omat hvtvm elekon kerrêt; elkvt ohvtvlaken ohfvnkekos.

10 Elvte tat vhvmkvn naorketv ohfvvecvn elvtet, momis hesakat tat Hesaketvmese ohfrcern hesaket omekv.

11 Monkv cemeu matvpomen, Mehenwvn naorketv ohfvccun pu pvsvtket, momis Cesvs Klist pu Pucase eteropotten Hesaketvmese ohfvccrn pu hesahoket os, komet, e vkerricvkes.

12 Monkv naorketvt ce 'na take elat ofvn, em eyackv 'mapohickvn, ohmekikats.

13 Momet ce 'nalvcce naorketv es vtotketvn holwvyeckvn em wihkatskvs, momis pvsatkvte aenkvpvken hesahoke omvket, Hesaketvmesen em e wiket, ce 'nalvcce fvccetv es vtotketvn Hesaketvmesen em wikvks.

14 Naorketv ce pucaset omekaret ok': vhakv elecv tokon, heromkv elecvn svpaklatsket omekv.

15 Monkv esto haks? vhakv elecv tokon, heromkv elecvn svpakleyat omecicen naorkvraneya? Mohmates.

16 Estimv em apohickvn vtotkvlken em e wikatskat, mv em apohicatskat em vtotkvlke, naorketvn elkv ohfvccvn, monkat apohickvn fvecetv ohfvecvt svpaklatskat kerratskeko haks?

17 Momis, naorketv em vtotkvlke toyatskvtet, ce feke take vten mv muhakv em etelvckv cem wihokvten em apohicatskvte vrahkvn Hesaketvmesen vrakkuehocekvs.

18 Monkv naorketv ohfvccvn pucasseko hakatskat, fvecetv em vtotkvlke hakatskvtes.

19 Ce 'navpeswr yekcekat omecicen este omit opunvyvyet os ; ce 'nalvcce taken hvsvtketv tokat, momet holwvyeckv em vtotkvlke holwvyeckvn em wikatskvte etv- 
pomat, hiyomat ce 'nalvcce taken fvccetv em vtotkvlken hvsvtketrn em e wikvranatsket omekv.

20 Naorketv em vtotkvlke toyatskof, fvecetv ohfvecv pucasseko toyatskvtet omekv.

21 Monkv mv nanvke hiyomē es vlsatskat ofvn em ette estomēn ocatskvt haks? mv nanvke em eyuksvt elkvt omekv.

22 Momis hiyomat naorketv ohfvecv pucasseko momet Hesaketvmese em vtotkvlke ce hakvkat, cem etten hvsvtketvn, momet em eyuksv hesaketv meyuksv sekon ocatsket os.

23 Naorketv es em feketv clkvt omen, Hesaketvmese em estemkvt, Cesvs Klist pu Pucase eteropotten, hesaketv yuksv sekot omekv.

\section{SETENTVCKE VII.}

Tecakivete toyatskat, (vhakv kerrakan ém punayvlit okikv, ) este hesake vtekan vhakvt ohfvnket omen kerratskeko haks?

2 Hokte ehe ocat, e'he hesake vtekan vhakv vcrkvyen vwrnvket omekv; momis, ehet elen omat, vhakv e'he ohfvccv ocat enkvpvken enrecopket os.

3 Monkv $e$ 'he hesake emunken hunvnwv etin en liken omat, hoktarvn kihocvres; momis ehet elen omat, $\mathrm{mv}$ vhakv ohfvecrn pucasseko haket os; monkv hunvnwv eti en like estomis hoktarv tokot omes.

4 Monkv, tecakkvyvte toyatskat, Klist e 'na eteropotten cemeu vhakv ohfvccrn ce prsatkvket os ; etvn, mv prsatkvte enkvpvken akvwvphoyvten, ce hvlvtepuehocen, $\mathrm{He}$ saketrmesen etten en heckueceyvren.

5 Enavpeswr ofvn fulleyof, holwvyeckv vhakv etehoyvne ocakat en nekeyetvt, ette elkv oren heckuecetvn pu 'nalvece ofvn vtotkakvtet omekv. 
6 Momis hiyomat, $\mathrm{mv}$ of $\mathrm{pn}$ hvlvthoyat elet omen, vhakvn 'sepu'ssihocet os, letv em vculkv ofv tokon, puyvfekcv em mucvskv ofvn vtotkeyvren.

7 Monkv naken makvraneya? Vhakvt holwvyeckv te? Mohmates. Momis vhakv etehoyvne tokon omat, naorketvn kerrvkatet os: vhakvt, Vleskahpetskvs, makekaten omat, vleskvpkvn kerrvkvnt omekv.

8 Momis naorketvt, vhakvt omecicen, vleskvpkv omvlkvn vc ofvn vtotkakvtet os. Vhakv sekat naorketvt elet omvtet ok'.

9 Vhakv sekon hesakvyvte ocet omekv; momis vhakvt vlakof, naorketvt vhesaken, vnet elvyvtes.

10 Momen vhakv hesaketv ohfvecvn hahoyvte elkv eroret omen eshecvyvtet os.

11 Naorketvt, vhakvt omecice omecet, v'mvkerret, vhakvn es $c v$ 'lecvtet omekv.

12 Monkv vhakv hvsvtket omen, nake $p u$ mvhahoyvte hvsvtket, fvecvt momet heret omekv.

13 Monkv vc ohfvecv nake herat elkv hahoyet omvte te? Mohmates. Momis naorketvt, mv nake herat eteropotten elkvn vc ofvn vtotken, naorketvt omat heckvren; momet naorketv vhakv eteropotte holwvke here hakvren omvtes.

14 Vhakv puyvfekev ohfvecvt omat kerreyet omekv; momis vnet vpeswr toyin naorketv elecvn cr wihoyet os.

$15 \mathrm{Mv}$ momecryat em vkvsrmvkot omekv, Mecares, komvyat, mvn momecvkot, naken enhomecvyat mvn momecryet omekv.

16 Monkv, Mecares, komv'kan momecin omat, vhakv heret omat em vkvsamvyet os.

17 Monkv mucv vtekat mvn momecat, vne tokon, naorketv ve ofv likat momecet omes.

$18 \mathrm{Vc}$ ofv, (cv 'navpeswv 'ofvn okis,) nake here likat, 
sekot omen kerrikv, kometv vc vpaket omis, here momecetvn eshecrkot omekv.

19 Nake here komvyaten momecvkon, nak holwvke komvkan momecryet ok'.

20 Momen naken komvkan momecin omat, ohvtvlaken vne tokon, naorketv ve ofv likat mvn momecet os.

21 Monkv heyv vhakvn eshecis, heran mecetvn komvyof, holwakat ve vpaket os.

22 Ofv este vcrkvyat Hesaketrmese em vhakvn es vc afvcket omekv :

23 Momis cv 'nalvcce take ofvn vhakv etv vm vkerrickv em vhakv vnrapet, momet naorketv vhakv cv 'nalvece take ofv ocat en svlvfke cr hayen eshecis.

24 Aeha! hunvnwv estemerkusvyat! heyv elkv em enan estimvt vm enrecopvr haks?

25 Cesvs Klist eteropotten Hesaketvmesen vkvsamis. Monkv mehenwvn Hesaketvmese em vhakvn vkerrickvn es em vtotkvyet os; momis cv 'navpeswrn naorketv vhakvn.

\section{SETENTVCKE VIII.}

Monkv hiyomat mv Klist Cesvs ofvn fulle, enavpeswv vcrkvye tokon, puyvfekcr vcrkvyen fullakat vnrvpe ohfvececkv en sekot omes.

2 Cesvs. Klist ofv hesaketv em Puyvfekev em vhakvt naorketv elkv tepakat em vhakvn vm enrecopvtet ok'.

3 Hiyomet omekv, vhakv, enavpeswv omecicat yekcekot omen, naken momeceko tayan, Hesaketvmeset E'ppuce mahusat, naorketvt omecicen, enavpeswv holwvyece vhake ofvn vtoten, enavpeswr ofvn naorketrn vnrvpen ohfvccecvtes;

4 Pume, enavpeswv verkvye tokon, Puyvfekcv vevkvyen fulleyat ofvn vhakv em fvccetv esfvckvren.

$5 \mathrm{Mv}$ enavpeswv vevkvyen fullat nake enavpeswr ohfvc- 
cv ocakan vkerricaken, $\mathrm{mv}$ puyvfekev vevkvyen fullat' puyvfekcv enanvken vkerricaket omekv.

6 Enavpeswr em eyackvt elkvt omen, puyvfeker em eyackvt hesaketvt omet, herkvt omekv.

7 Enavpeswv em eyackvt Hesaketvmese vnrvpkvt omet, Hesaketvmese em vhakv elecvn huerekos, momet elecv hueretv umeko tayet omekv.

$8 \mathrm{Monkv} \mathrm{mv}$ enavpeswv ofvn svpaklat Hesaketvmesen afveeciceko tayes.

9 Momis cemet, enavpeswv ofv tokon, momis Puyvfekcv ofvn svpaklatskēs, Hesaketvmese em Puyvfekcvt ec ofv taken liken omat. Momis este estomis Klist em Puyvfekcrn ocekon omat, Klist enake tokot os.

10 Momen Klist ec ofv taken liken omat, naorketvt omecicen ena elet os; momis fvecetvt omecicen Puyvfekev hesaketvt omes.

11 Momis mv Cesvs prsatkvte aenkvpvken kvwapvte em Puyvfekcrt ec ofv taken liken omat, ohvtvlakat mv Klist pvsatkvten aenkvpvken kvwapvtet, em Puyvfekcv ec ofv take likat eteropotten ce 'na elv taken wenahuecrres.

12 Monkv, tecakkvte toyatskat, enavpeswv vevkvyen hesahokvraneyat, enavpeswvt epu 'huericvkekot os.

13 Enavpeswv, vcrkvyen hesahokatsken omat, ce prsatkvken, momis Puyvfekcv eteropotten ena em vtotketvn ohfvnkatsken omat, hesahokatskvret ok.

14 Estomomuse Hesaketvmese em Puyvfekcvt evpayat; mvt Hesaketvinese e'ppuce taket omakekv.

15 Hvtvm ce. penkvlvkvre, svlvfketv em puyvfekcvn cen heckvkekon, momis eenakueckv em Puyvfekcv, mv etehoyvnen, Apv, Pu'rken makeyat cen heckvkvtet omekv.

16 Puyvfeker mahusat, pum puyvfekev take vprken, Hesaketvmese en hopuetake toyeyat, akerret os: 
17 Momen hopuetaket on omat, epucasvke taranat; Hesaketvmese enaken epucasvke taranat; momet Klist vpvken epucasvke taranat, vpvken pu 'stemerkēn omat, vpvken epu 'rakkvke taren.

18. Estemerketv hiyome ocakat, $m v$ vrakketv ep ofvn heckvkvranat setehopayesko etetayet os, komvyet omekv. 19 Hesaketvmese eppuce take heckvke hakvranan nake hahoyvte en neneheckv yekcat emehaket omekv.

20 Nakhahoyvte komat tokon, momis mv enhonrkv ofv svlvfkuecrtet omecicen, ehvperketv elecvn huerihocvtet omekv.

21 Nakhahoyvte etvwvt vhopvnkv en svlvf ketv ossihocet omen, Hesaketvmese en hopuctake epucasseko svpvkletv vrakkat of vn svpehoyvret ok'.

22 Nakhocackvte omvlkvt hihket, tohkvlken nak' en nokkat ofvn hiyomat oren nekeyaket kerreyet omekv.

23 Momen $m v$ tvlkusekon, ohvtvlakat pume mahusat, Puyvfekcv em ette enhvteceskvn pun hecket omat, pume mahusat tis, eenakueckvn, $m v$ pu 'na take enrecopkvren emehakēt ep ofvn hihkeyet os.

24 Enhonrkv etehoyrnen hesahokeyet omekv: momis enhonrkv heckat enhonrkv tokot os; hiyomet omekv, estet naken hecen omal, estomaten mvn ennenehece emunke haks?

25 Momis naken hecēkat em enhonrēn omat, momiskometvn es emehakeyet os.

26 Matvpomen Puyvfekcvt pu yekcvkekaten pum vnicet os; nake momvre here emekusvpvraneyat kerrēkot ome$\mathrm{kv}$, momis Puyvfekev mahusat hihketv onahoyeko tayan es pum emekusapet omekv.

27 Momen estefeke taken heren hecat Puyvfeker em eyackvn kerret os, Hesaketvmese komat vevkvyen emekusapvlken em emekusapet omekv. 
28 Momen nanvke omvlkvt, mv Hesaketvmese vnokecake, eme nak komat vcvkvye enhuehhokvte en herakvre tohkvlket em vtotkaket omat, kerrēs.

$29 \mathrm{Mr}$ homv kerrvtet, E'ppuce vhake vhayvkvren homvn mellvtet omekv, tecakkvte sulke vpvke ette enhvteceskv hakvren.

$30 \mathrm{Mv}$ homvn mellvtet, mvu enhuehkvkvtet, mv enhuehkvkvte, mvu fvccvke omēn svpvklecvtet, momet fvccvke omen svpvklecvte mvu vrakkuecvkvtet omekv.

31 Monkv heyv nanvke ohfvccrn naken makvraneya? Hesaketvmeset pum vpvlhvmken hueren omat, estimvt epu'nrvpe taye haks?

$32 \mathrm{Mv}$ E'ppuce mahusan est' em encakekot, momis omvlkeyat epu 'rahkvn wikvtet, estomaten mv vpvken nak omvlkvn vkvsamuset pu'mvraneko haks?

33 Estimvt Hesaketvmese em enhopoyvkvte vnrvpen vrvhecvkvr haks? Hesaketrmeset fvcce omēn huericet os.

34 Ohfvececat estimvt o haks? Klist elvte tes, momes, momet mehenwvn hvtvm akvwvpkvtes; momen ohvtvlakat Hesaketrmese em akvpervn liket, pum emekusapet os.

35 Estimvt Klist em vnokeckvn pu tekvpicvr haks? Estemerkv, monkat fekokketv, monkat asseckv, elauko, monkat enatvlketv, monkat penkvlkv ofv hueretv, monkat eslafkv-crpkot omvr haks?

36 Hoccat vevkayat, Ece 'rahkvn nettv temvpoken pu prsvthyet omen; yvpefikv pvsvthoyvrane omēn ep ohvkerrihocet omes.

37 Momis heyv nanvke omvlkv ofvn, mv epu 'nokecvte eteropotten, ohfvnkvlke semuntvlēn svpakleyet omes. 38 Elkv, monkat hesaketv, monkat hvlwe-estvlke, monkat ohmekketv ocakat, monkat yekcetv ocakat, monkat nanvke hiyome ocakat, monkat nanvke taranat, 
39 Monkat hvlwe, monkat sufke, monkat nakhahoyvte etv estomis, Hesaketrmese em vnokeckv, Cesvs Klist pu Pucase ofv ocan pu tekvpicetv umeko tayes, komvye heret omekv.

\section{SETENTVCKE IX.}

Kurst ofvn mehenwvn opunayit, laksvkon, vm vkerrickvt Puyvfekcv Vcakat ofvn,

$2 \mathrm{Vn}$ honne mahe ocet on, feknokketrn ov feke ofv ocvye munkvt omat, a vn kerret os.

$3 \mathrm{Vm}$ etecakketvlke, enavpeswr vcrkvye cr 'nahvmkvlke vrahkvn, Klist vnaremv cv hayvren komvye taye omet omekv :

4 Mvt Eslevlket omen; eenakueckv, momet hvyayvke, momet nak em wihokvte ocakat, momet vhakv emetv, momet Hesaketvnese em vtotketv, momet etemfvecetv ocakat em ocvket os;

5 Erkvlke tate enaket on, $\mathrm{mv}$ aossen enavpeswr vcrkvyen Klist, omvlkv ohfvnkat, Hesaketvmese meyuksv-seko vrakkuehocet omat vlakvtet os. Emen.

6 Hesaketvmese em opunvkv ehvperkv omvte omekon; Eslevlke omvlkvt Eslevlket omvkekokv:

7 Momen Eplehame vhonvpset o estomis, mvt omvlkvn en hopuetaken hayekot os: momis Iseke ofvn cen honvpsen hocef huehocvres.

8 Heyvt omes; enavpeswr en hopuetake tat Hesaketvmese en hopuetake tokot omes; momis setemfrccetv en hopuetake en honvpse mvt's komhoyet omes.

9 Heyvt setemfvccetv opunvkvt omekv; Heyv oketv omof vlvkares, momen Selet echuswr hunvnwvn ocvres.

10 Momen heyv tvlkusekon, momis ohvtvlakat Lepekv hunvnwv hvmke, pu'rke Iseke en nvrkehsof,

11 (Hopuetakucet hvte heckvkekot, monkat nake here, monkat holwakan momecvkeko emunken, Hesaketvmese 
momvres komvte, vtotketv omecicat tokon, momis $\mathrm{mv}$ enhuehkat omecicen enhopoyetv vcvkvye huervren;)

12 Semvculat semmvnettan em vtotkvres, hokten kihocrtet omekv.

13 Cekvpen vnokecit, Esan enhomecryvtet os, make hoccat vevkvyen.

14 Monkv naken makvraneya? Fvecetv tokat Hesaketrmese ofv oce te? Mohmates.

15 Moses em punayet, $\mathrm{Mv}$ em merrvyat em merrares, momet mv merkv em ocvyat merkvn em ocares, makvtet ok'. 16 Monkv estimvt komat enake tokon, monkat mv letkat enake tokon, momis Hesaketvmese mv em merrat enake tes. 17 Cokat Feleon em punayet, Heyv nake mahusat vrahkvn a ce huericvyvtet omes, vn yekce ec ofvn hecicit, yvmv ekvnv omvlkv ofvn cv hocefkvn kerkuehocvren, kicet omekv.

18 Monkv estimvn em merretvn komat em merret, estimvn komat wvnhecet omes.

19 Monkv vm punayet, Monkv estomaten pum mvtte omecet punaye munke haks? Naken koman estimvt vnrapvtet ome te? cv kicetskvres.

20 Monkos, hunvnwv toyetskat, momis ceme, Hesaketvmese vnrvpen vyoposketskat, estimv toyetske haks? Nake hahoyvtet, mv hayvten em punayet, Estomaten hiyomēn cv hayetskvt haks? makvre te?

21 Vrkvshayvt fakke eskvlke hvmke vrkvswr hvmke vrakkvren es hayet, etv vrakkekaren es hayetv yekcetvn oceko haks?

22 Hesaketvmeset, en crpvkke heckuecetvn, momet en yekce kerkuecetrn komet, momis-kometv hofunen crpvkketv nake-vcrnkv sumecihocvranat es emehakvten omat,

23 Momet merkv nake-vcrnkv hryayvken homv emetetakuecvte ohfvecv em vrakketv en sulke kerkuecvren, 
24 Pume tis, mv enhuehkvtet, Cusvlke tvlkekon, momis Centilvlkeu, pu mehakvten omat, estome te?

25 Ose hoccat ofv opunaye omēt, Este crnake tokvkekaten este crnaken kicares; momet mv hokte vnokeckekaten vnokeckan kicares.

26 Momen mome ocvres, estrmv, Centaket vm este toyatskekos, kihocvten, mvn Hesaketvmese wenakat en hopuetaken kicvkhoyvres.

27 Momen Esivs Eslevl opunvyecet, Eslevl en hopuetake em vhonkvtkv uehvtkv oktahv omēt o estomis, nvcome vhoskat hesahokvres.

28 Vtokketvn poyet, fvecetv ofvn kocoknicrret ok', momet vtotketv kocoknen Pucaset yvmv ekvnvn oh hayvret ok', maket huehkvtes.

29 Momen Esivs homvn makvte omēn Sepeore e Pucaset nerkvn pum wikekaten omat, Sotomv omēt on, Komalv omvken pu hahoyvnt os.

30 Monkv naken makvraneya? Centilvlket, fvecetvn vevkvpeyekatet, fvecetvn epoyvkvtet os, $m v$ fvccetv vkvsvmkv etehoyvne ocat.

31 Momis Eslevl, fvecetv em vhakvn vcvkayvtet, fvecetv em vhakvn epoyekatet os.

32 Estoman? Vkvsvmkv eteropotte tokon, vhakv em vtotketv eteropotten hopoyakvtet omekv. Mv evto elentappueckvn oh elentappakvtet omekv;

33 Hoccat vevkvyat, Hecrs, Sivn ofvn crto elentappueckvn, momet crto esemmvttetvn licares, momen estimvt mvn oh vkvsamat vlsekares.

\section{SETENTVCKE $\mathrm{X}$.}

Tecarkvte toyatskat, Eslevl ohfvecv cv feke em eyackvt, momet Hesaketvmesen emekusapvyat heyv tes, hesahokvren. 
2 Hesaketvmese ohfvecv vkerrickv yekcen ocakat a en kerrakikv, momis kerretv vevkvye tokot os.

3 Mvt, Hesaketvmese em fvccetvn kerrekot, eme mahusat em fvccetvn ahuericetvn hopoyet, Hesaketvmese em fvccetv elecvn svpaklvte sekot omekv.

4 Vkvsamat vtekat, Klist vhakv em eyuksvt en hueret omekv, fvccetvn.

5 Moses vhakv em fvccetvn opunvyecet omekv, Mv este $\mathrm{mv}$ nanvken momecat $\mathrm{mv}$ ofvn hesakvres, makat.

6 Mornis fvecetv vkvsvmkv etehoyvne ocat hiyomēn opunayet os; Estimvt, akvwvpiket hvlwe-tvlofvn eceyvr haks? (Klist ashvtvpketvn oket,) ce feke ofvn mahketskvs. 7 Monkat, Estimvt sufkan vkhvtvpkvr haks? (Klist pvsatkvten aenkvpvken akvwvpetvn oket.)

8 Momis naken make te? Opunvkvt ece 'wolet, ce cukwv ofvn, momet ce feke ofvn oces; vkvsvmkv opunvkv erkenakeyat $\mathrm{mv}$ tes:

9 Heyvn makat, Pucase Cesvs ce cukwrn es kerkuecet, Hesaketvmeset pvsatkvten aenkvpvken kvwapvtet omat ce feken es vkvsametsken omat, ce hesakvres.

10 Estet fvccetv oren efeken es vkvsamen, kerkueckv vhesaketv orat ecukwv eteropotten hahoyet omekv.

11 Estimvt mvn oh vkvsamat vlsekares, Cokat maket omekv.

12 Cusvlke Klekvlke tepakat temvrahketv sekot omekv: Pucase hvmke omvlkv ohfvnkat mv ohhuehkakat omvlkv ohfvecr nake-ocvt omekv.

13 Estimvt o estomet, Pucase e hocefkvn oh huehken omat, hesakvret ok'.

14 Monkv estimv ofv vkvsvmvkekatet estomẽn oh huehkvkvr haks? momet mv ohfvecv pohvkvte sekan 
estomēn ofv vkvsvmvkvr haks? momet, erkenvkv sekon, estomēn pohvkvr haks?

15 Momen vtotvkhoyekon omat, estomēn erkenvkhoyvr haks? cokat vevkvyat, $\mathrm{Mv}$ herkv em opunvkv-herv erkenvkakat, momet nake herake ohfvccvn opunvkv estafvcecicvken esyicat e'le take mvtawwvt herakusa!

16 Momis opunvkv-herv omvlkvt em apohicaket omekot os. Pucasē, estimvt pun kerkueckvn vkvsamvt haks? Esivs. maket omekv.

17. Monkv vkvsvmkvt pohetv eteropotten vlaken, pohetvt Hesaketrmese em opunvkv eteropotten vlaket omekv.

18 Momis pohvkekate te? makis. Mehenwvn momes, en hake take yvmv ekvnv omvlkvn hvmecicen, em opunvkvt ekvnv yuksvlken oricvtet os.

19 Momis makvyet os, Eslevl kerrekate te? Enhvteceskvn, Moses; Este tokvkekan vfekcakhetv oren es ce 'lésecicet, etvlwv hacohakan es ce crpvkhokicvkares, maket os.

20 Momis Esivs, fekhvmke mahet, $\mathrm{Mv}_{\mathrm{v}} \mathrm{cr}$ hopoyvkekatet es $\mathrm{cv}$ hecaken, $\mathrm{mv}$ vc vpohvkekatet em e heckuecrkvyvtes, maket os.

21 Momis Eslevl em punayet, Nettv temvpoken este apohicvkeko, momet pvlken vyoposkakat cv'nken a em wiyvkvyvtes, maket os.

\section{SETENTVCKE XI.}

Monkv makvyet os, Hesaketvmeset em estvlken vhepakvt haks? Mohmates. Vneu, Pencrmen em vliketv, Eplehame en honvpse aosse, Eslevlke toyikv.

2 Hesaketvmeset, em esten, mv homvn kerrvtet, vhepakvke sekates. Cokat Elivs ohfvecv nake makan ker- 
ratskeko haks? Eslevl vnrvpen Hesaketrmesen em punayet hvmmakvtet omat;

3 Pucasē, cem owalvlken pvsatet, cem altvn lekvflecakvtet os; momet, vntvlkuset vc vhosket omen, vn hesaketvn hopoyakes.

4 Momis Hesaketvmeset em vyoposkat naken make haks? Hunvntake cukpe-rakko kolvpake, mv Pevl vhakan ehomv etorkowv taken kunhicvkvte sekan e em vcvyecrkvyvtet os.

5 Monkv hiyomat tis heromkv em enhopoyetv vevkvyen vhoskat ocet os.

6 Momen heromkv etehoyvnet on omat, ohvtvlaken vtotketv etehoyvne tokot os: monkon omat, heromkvt ohvtvlaken heromkv tokot os. Momis vtotketv etehoyvnen omat, ohvtvlaken heromkv tokot os; monkon omat, vtotketvt ohvtvlaken vtotketv tokot os.

7 Monkv estome te? Eslevl naken hopoyvtet epoyekon, momis enhopokvtet epoyet omen, vpvlwvt hecvkeko hahoyvtes, mucv nettr oren:

8 (Hoccat vevkayat, Hesaketvmeset nockv em munkvn, eturwvn hecvkekaren, ehvckon pohvkekaren emakvtes.)

9 Momen Tewe, Em ohhompetvt, naksenkvnhetv, momet nake esepueckv, momet es elentappueckv, momet es em feketvt en hayvkhoyekvs :

$10 \mathrm{E}$ turwv taket ohyomuckvkekvs, hecvkekaren, momen e'ra taken kunkuhicvs, estofis, maket os.

11 Monkv makvyet os, Pvlatkvren elentappakvtet o haks? Mohmates; momis mv pvlatkvte eteropotten vhesaketvt Centilvlke em vlakvtet os, Cusvlket vfekcakhvke hahoyvren.

12 Momēt on, em vhopvnketvt yvmv ekvnv en nakockv, momet em vhonkvtkv kocuknicirvt Centilvlke en nakoekv, haken omat, en sulketvt mahvkvu mvt hakekare te? 
13 Centilvlke toyatskat, ce tempunayvkis, Centilvlke em vpastel toyikv; vm wiketvn vrakkuecis :

14 Estome estomis cv 'navpeswr mahusan vfekcakhetv oren nekeyicrye tayen omat, momet vpvlwvn hesahuecrye tayen omat.

$15 \mathrm{Mv}$ vhepakvkhoyvtet yvmv ekvnv em etenherketv haken omat, evpvyvkhoyat nake tar haks, prsatkvten a en hesaketv tokon omat?

16 Ette erhvteceskv vcaket on omat, eskvlkateu vcakes; momen yvlonkvt vcaken omat, elvcceu vcacvket omekv.

17 Momen elvcce vnvcomet a kvcekvket omen, ceme, olefv semvnole toyetskat, $\mathrm{mv}$ mehenwakat vpvken olefv. en yvlonkv en nehetv tepakan ete'm ocatsken omat,

18 Elvcce vnrvpen ekvsahmetskvs. Momis ekvsametsken omat, yvlonkvn hvlatetskekon, yvlonkvt ce hvlatet os.

19 Monkv, Elvccet a kvcekvtes, oh rvfkaren, maketskvres.

20 Heres; vkvsvmvkekat svrahkvn a kvcekvtes, momen vkvsvmkv eteropotten hueretsket os. Vkerrickv hvlhawan ohcetskvs, momis penkvlvs :

21 Hesaketrmeset elvcee olefv em munkv verkvye ocakat vcryecrkekaten omat, vketecvs, cemeu ece 'cryeceko witet ok'.

22 Monkv Hesaketvmese en heromkv en yekcetv tepakan hecvs; mv pvlatkvte ohfvecvt on omat, yekcetvn; momis ec ohfvecv heromkvn, mv en heromkv ofvn fekhonnetsken omat; monkon omat, cemeu a tvcketskvres.

23 Momen eme takeu vkvsvmkv toko ofvn fekhonnvkeko emunken omat, oh rvfkvkvres; Hesaketvmeset hvtvm oh rvfrice tayet omekv.

24 Momet omen cemet, mv em munkv verkvye olefv semvnolet on, a ce tvchoyen, nake hocackvte em munkv 
vnrvpen olefv heran oh rvfketskvten omat, heyv, elvcce mehenwakat, em munkv vevkvye elvccet omakat mahvkvu em olefv mahusan oh rvfkvkar haks?

25 Tecakkvte toyatskat, Centilvlke etemvpoketv ascehuehoceko emunken, vpvlwusen turhecekat Eslevl oh cakkvtet omat kerrekot svpaklatskuren komvkot os, $\mathrm{Pu}$ hoporrenet os, komet, e oh vkerricatske witet ok'.

26 Momen momēn Eslevl omvlkvt hesakvres, cokat vcrkvyen; Holwvke aossicvt, Sivn aosiyet, Cekvpe en holwvyeckvn em vkueyicvres.

27 Heyvt etemfvecetv em ocvyat omekv, en holwvyec$\mathrm{kv}$ taken em vkuehuehcryof, makat.

28 Opunvkv-herv ohfvccvt on omat, ece 'rahkv taken vnrapvlket omakes; momis enhopoyetv ohfvecv tat, erkvlke vrahkv taken vnokeckvket omes.

29 Hesaketvmese en nakemkv enhuehketv tepakat vkerrickv-etickv vpvkekot omakekv.

30 Oketv hoyvnakvte ofvn Hesaketvmesen vkvsamatskekis, eme take vkvsvmvkekat eteropotten hiyomat mer$\mathrm{kv}$ cen heckvkvte etvpomen;

31 Heyvu, hiyomat, cem merkv eteropotten eme takeu merkv en heckvkvi'en vkvsvmvkekatet ok'.

32 Hesaketvmeset, omvlkvn em merrvkvren, omvlkvn vkvsvmvkekat ofvn em vkhottvtet omekv.

33 Mvtawwrt Hesaketvmese en hoporrenkv en nakkerretv hokkolvt sufket omeko haks! Em ohfvececkv ofv kerrēsko tayen, ēm vyetv take eshecrkhoyeko tayuset os!

34 Momēt omekv, estimvt Hesaketvmese em vkerrickvn kerrvt haks? monkat estimvt etempunayvt omvt haks?

35 Monkat estimvt homvn ehmen, hvtvm em fehokvr haks? 
36 Nanvke omvlkvt eme avtet, eme eteropottet, eme em ocvket omekv; vrakketv mvn em ocekvs, emeyuksv sekon. Emen.

\section{SETENTVCKE XII.}

Monkv, tecakkvte toyatskat, Hesaketvmese em merkv take vcrkvyen, ce'na take nake emhoye wenake, hvsvtke momet Hesaketvmese afvcecican ematskvren ce'nkusapvkvyet os; mvt nake momatskvre tetayet ok'.

2 Momen yvmv ekvnvn vhahyatskvs, momis cem vkerrickv ohmucvseckv eteropotten mvrahkuehocet omvkes, Hesaketvmese nakkomat here momet afvcecice momet temvpokan eskerratskvren.

3 Heromkv v'mhoyat eteropotten este ece 'pakvkat vtekat em punayit heyvn makvyet omekv; Momvre tetaye senhoyvnen $e$ oh vkerricekot, momis mahlvpvtken $e$ oh vkerricvs, Hesaketvmese este vtekaten vkvsvmkv svkerkan emvte vcrkvyen.

4 Ena hvmkat ofv enalvcce sulken ocēt, momen enalvcce omvlkv em vtotketv hvmkuset omekon;

$5 \mathrm{Mv}$ etvpomen, pume, sulke toyeyat, Klist ofv ena hvmket omet, omvlkeyat etelvcvke toyēkv.

6 Monkv nanvke pu'mhoyvte, heromkv pu'mhoyvte vcrkvye temvrahrvken ocēn, owaletvt on omat, vkvsvmkv svkerkat vevkvyen owalvkeres;

7 Monkat vtotketvt, vtotketvn vfastvkeres, monkat mv mvhayat mvhayetvn,

8 Monkat mv estemvcahnat, emvcahnetvn; estit nake estemat mehenwetv ofvn momecekvs ; mv emwiketv huerat vfekcakhetvn es vfvstekvs; $m v$ merkvn heckuecat afvcketvn es em merrekvs.

9 Vnokeckv vhayetv vprkekan ocekvs. Mv holwakat en homecet, mv heran hvlvtvks. 
10 Tecakkvte em rnokeckvn 'setenokecusvkes, rrakkueckv ofvn $e$ oh vkerricetskat senhoyvnen est' vrakkuecet.

11 Vtotketv ofv enhorrvkekot; vkerrickv ofv vfekcakhet; Pucasen em vtotket;

12 Enhonrkv ofvn afvcket; estemerketv ofvn momiskomet; mekusrpkv ofv emomecicet emunket;

13 Vkvsamvlke nake enkusvpketvn vfastet; etenheromkvn vcvkvpeyet;

$14 \mathrm{Mv}$ ece'ssecakan heren opunvyecvkes; heren opunvyecet, ohtvhihkatskvs.

15 Afvckakan vpvken afvcket, hvkihhokan vpvken hvkihkvkes.

16 Vkerrickv hvmkusen ete'm ocvkes. Nake hvlhawan stoskomelsot, este kvncrpakan etenheromvkes. Hoporrenvke toyēs, komet, e ohvkerrihcatskvs.

17 Holwakan holwakan estimvn prlken ehmatskvs. Este omvlkv ehomvn fvccen naken epoyvkes.

18 Mome tayen omat, umatske taye vcrkayat, este omvlkv vpvken herketv ofvn fullvkes.

19 Vnokeckatskat, e ohfvecvn pvlehcatskvs, momis crpvkketvn em etektrnecrkes. Empvlecetv crnaket's; rnet fekares, Pucaset maket os, make cokat vevkvyen.

20 Monkv cen horret elvwet on omat, hompicrs; ewvnhken omat, eskuecrs; hiyomecetskat toraw vhethoken e'kvn em oh rpoyetskrres.

21 Nake holwakat ce muntvlekon, nake heran nake holwakan es ohfvnkvs.

\section{SETENTVCKE XIII.}

Puyvfekcv vtekat yekcetv ohfvnkakat elecrn huerekvs. Yekcetv Hesaketrmese artekat sekot omekv; yekcetv ocakat Hesaketrmeset mellvtet omekv. 
2 Monkv mv yekcetv vnrapat nake Hesaketvmese mellvten vnrapet os; momen mv vnrvpakat ohfvececkvn e en heckuecvkvres.

3 Ohfvnkvlket vtotketv herakat tokon, holwvhokan vpenkvlecet onkv. Monkv yekcetv em penkvletvn ce yaceko haks? mv heran momecetsken, mvt vkvsvmkvn ce'mvres :

4. Hesaketvmese em vtotkvt omekv, es cen hervren. Momis nake holwakan momecetsken omat, penkvlvs; eslafkv-cvpkon ehvperkvn hvlvtekot omekv; Hesaketvmese em vtotkv estemerricvt $\mathrm{mv}$ holwvke momecan crpvkketvn ohvlvkuecvranat hueret omekv.

5 Monkv apohicvket svpvklvranatsket omes, cvpvkketv vrahkv tvlkekon, momis eohvkerrickvu vrahkvn.

6 Heyv vrahkvn ekv-feketvu fekatsket omekv; mvt, heyv nake mahusat vfvstake emunkvt, Hesaketvmese em vtotkvlket omakekv.

7 Monkv nake vhuericakat omvlkvn vhuerakan em fekvkes, nake-oh-feketv ahuericakat nake-oh-feketvn; ekvfeketv ahuericakat ekv-feketvn em feket, em penkvlhoye tayan em penkalet, vrakkuehoce tayan vrakkuecet.

8 Este estomis, etenokeckv tvlken ece 'huericen, nake etvn ece 'huerihcats; mv este etvn vnokecat vhakvn esfvcececvtet omekv.

9 Heyvt omekv, Hoktahretskvs, Elehcetskvs, Horkohpetskvs, Fvccekon akeriyetskvs, Vleskahpetskvs, momen vhakv etv ocen omat, heyv opunvkv ofv etohkvlken ocet omes, E vnokecetskat etvpomen cem vwolicvn vnokecvs.

10 Vnokeckvt em vwolicvn holwakan em vtotkekot os; monkv vnokeckvt vhakv esfvceckvt omes.

11 Momen heyvn, oketvn kerret, nocvte ahonecetv hvsevkerkv momvre heret omen kerret; hiyomat pum vhesaketvt vkvsameyvten sem vwolet omekv. 
12 Neret hoyvnvranuses; nettvt vwolices; monkv yomucke em vtotketvn vpvlatet, hvyayvke setepokvn vccvkeres.

13 Nettv ofv omēn, fvccen yvkvpvkeres; eshvyvtketv hackv tepakat ofv tokon, hoktarkv nake yvmahkueckv tepakat tokon, etepokv vleskvpkv tepakat ofv tokon:

14 Momis Pucase Klist Cesvn vccrkes, momet enavpeswv em eyackvn em ehopahkatskvs.

\section{SETENTVCKE XIV.}

$\mathrm{Mr}$ vkvsvmkv ofv yekcekan evpryvkes, momis punvkv setenrvpkv kerkvkekan ascehuecekot.

2 Hvmket, Nake omvlkvn hompvye tayes, komet vkvsamen, etv, yekcekat, nakvhockucen hompet omekv.

$3 \mathrm{Mv}$ hompat mv hompekan enhomehcats; momen mv hompekat $\mathrm{mv}$ hompaten ohfvccehcats; Hesaketvmeset evpayvtet omekv.

4 Cemet, este etv em vtotkvn ohfvccecetskat, estimv toyetske haks? E pucase mahusat ohfvecrn hueret monkat latket os; momet huervres; Hesaketvmeset huerice tayet omekv.

5 Hvmket nettv hvmken nettv etv senhoyvnen vkerricet os; hvmkat nettv omvlkvn temomvlkusen vkerricet os. Este vtekat em vkerrickv mahusat ofvn esfvcken vkvsvmekvs.

6 Nettv vrakkuecat Pucase ohfvccvn vrakkuecet os; momen mv nettv vrakkuecekat Pucase ohfvecvn vrakkuecekot os. Mv hompat Pucase ohfvccvn hompet os, Hésaketvmesen vrakkuecet omekv; momen mv hompekat Pucase ohfvccvn omes, hompekat, momet Hesaketvmesen vrakkuecet os.

7 Epu 'pakat hvmkusis e vrahkusen wenaket omekon, • nvpvt e vrahkusen elekot os. 
8 Wenahokēn omat, Pucase ohfrecrn wenahokeyet os, monkat prsatkēn omat, Pucase ohfvccrn prsatkeyet omekv; monkv wenahoket, monkat pvsatkeye estomis, Pucase enake toyēs.

9 Heyv vrahkvn Klist ehlet, akvwrpiket, hvtrm hesakvtes, pvsatkvte hesahokat esyomat e Pucase hakvren.

10 Momis estomaten tecakketskan ohfrececetske haks? monkat estomaten tecakketskan ehvperkuecetske haks? omvlkeyat Klist em fvcceckv ohliketv ehomvn svpvklvraneyet omekv.

11 Hesakvyat etvpomen, etorkowv omvlkvt cv homon pekohlen, etulaswv omvlkvt Hesaketvmese ehomvn ohkerkuecvres, Pucaset maket os, make hoccet omekv.

12 Monkv omvlkeyatet Hesaketvmese ehomvn e ohkerkuecvraneyet os.

13 Monkv ohvtvlaken etohfvececet ohmeyates; momis heyv min fvccecet, este estomet elentappueckv, monkat naorkepueckvn etecakkan ehomvn lihcats.'

14. Klist Cesvs ofvn heyvn kerrit, vc vkerricepuehocet omes; eme ofvt on omat nake hvsvtkekat sekot os; momis mv, Hvsvtkeks, komat ehomvn hvsvtkekat tes.

15 Momis cen hompetvt tecakketskān e feken en nokkicen omat, hiyomat vnokeckv vevkvyen aretskekot os. Mv Klist em elvten cen hompetvn es sumecihcetskvs.

16 Monkv cen heretvn holwvken opunvyehohcats ;

17 Hesaketvmese em ohmekketvt hompetv esketv tepakat tokon, momis fvecetvt, herkvt, afvcketv Puyvfekcv Veakat ofv ocat esyomat omes.

$18 \mathrm{Mv}$ heyv nanvke ofvn Pucase em vtotkat, Hesaketvmesen afvcecicet, esteu afveecicet omes.

19 Monkv herkv enanvken vcvkvpeyet, nake etemvnickv ocakan vevkvpeyvkeres. 
20 Hompetv vrahkvn Hesaketvmese em vtotketvn hopahnetskvs. Mehenwvn nanvke omvlkvt hvsvthvket omis, mv este mv omecicen elentappet hompat, mvt eme ohfvecrn holwvket omes.

21 Vpeswrn hompekot, monkat pvrko-opuswrn eskekot, monkat nake estomis $\mathrm{mv}$ omecicen tecakketskat elentappet, monkat naorkepuehocet, monkat en yekcen es sumecihocan hompekan heres.

22 Vkvsvmkvn ocetske haks? Hesaketvmese ehomvn e ofvn ocvs. Mv este nake em etektvnecat ofvn e ohfvccecekat afvcket omes.

23 Momen mv en kerkekat hompen omat, ohfvecehocet os, vkvsvmkvn es hompekot omekv; nake vkvsvmkvn avtekat holwvyeckvt omekv.

\section{SETENTVCKE XV.}

Monkv pume, yekcakeyat, e afvcecicēkot, mv yekcvkekat en yekce sekan kvwvpvraneyet os.

2 Pum vwolicvn en hervren nake vnickv ofvn omvlkeyat afvcecicvkeres.

3 Klist tis e afvcecicekatet ok'; momis, $\mathrm{Mv}$ ce tvklecakat en tvkleckv take vc oh latkvtes, make hoccat vcrkvyen. 4 Nanvke estomis homvn cokakat, pu mvhayat coyvkhoyvtet ok'; cokat momis-kometv emahlvpvtketv tepake omecicat eteropotten enhonrkvn oceyvren.

5 Momen momis-kometv emahlvpvtketv tepakat en Hesaketvmeset, Klist "Cesvs vevkvyen vkerrickv hvmkusen ete'm ocatskvren ce'mvkate!

6 Momat vkerrickv hvmkusen, ecukwvu hvmkusen Hesaketvmese, mv pu Pucase Cesvs Klist Erken vrakkuecatskvren.

7 Monkv Klist Hesaketvmese em vrakketv vrahkvn epu yvpayvte etvpomen ete yvpryvkes. 
8 Monkv Klist, erkvilke nake etemfvecakvte yekcvkuecvien, momet Centilvlket Hesaketvmesen, em merkv vrahkvn vrakkuecvkvren, Hesaketvmese em mehenwetv vrahkvn svkvmsiskv vtotkvt omvtes, makvyet os;

9 Heyv omecicen Centilvlke vprken ec ohkerkuecares, momet ce hocefkvn vyvhikares; make cokat vcrkvyen.

10 Momen hvtvm, Centilvlke toyatskat, em estrlke vpvken afvckvks, maket os.

11 Momen hvtvm, Hesaketvmesen vkvsvmvkes, Centilvlke omvlkatskat, momen, este omvlkatskat, mvn vrakkuecrkes.

12 Momen Esivs hutrm maket os, Crse en yvlonkvt ocvres, momet mv Centilvlke ohmekketvn akvwvpkvranat; mv ofvn Centilvlket enhonrvkvres.

13 Momen enhonrkv en Hesaketvmeset vkvsvmkv ofvn afvcketv herkv tepakat omvlkvn es ce fvcecekvs; Puyvfekcv Veakat en yekcetv eteropotten enhonrkv esfvcken ocatskvren.

14 Momen, tecakkvyvte toyatskat, vne mahusat, ec ohfvecr taken heyvn ve vkvsvmepuehocet os; ceme takeu heretv es fvcket omet, kerretv omvlkv es fvcket, momet ete'm vcahnatske taye svpaklatsket os.

15 Mome estomis, tecakkvte toyatskat, ece 'kerricepuecit, heromkv Hesaketvmese a'mvtet omecicen, fekhvmketv ohvtvlaken kvpvkusen es cen hoccihcrkis.

16 Centilvlke ohfvecv, Hesaketvmese em opunvkvhervn es vtotket, Cesvs Klist em vtotkv taren, Centilvlke nake Hesaketvmese em wihokvtet, Puyvfekcv Vcakat eteropotten vcake haket, afvcecicet omvren.

17 Monkv C'esvs Klist eteropotten naken es ekvsvmvye tayat ocryet os, mv nanvke Hesaketrmese ohfvecv. ocakat ofvn. 
$18 \mathrm{Mv}$ nanvke Klist, Centilvlke apohicvke hayvret, opunvkv vtotketv tepakat etehoyvnen nakeskerkv, nakestomahake esyome yekcakat eteropotten, Hesaketvmese em Puyvfekcv en yekce eteropotten, $\mathrm{cr}$ tehoyvnen momecekaten opunvyecetv fekhvmkvkaret ok';

19 Momat Celuslvme aenkvpvken afolotken Elelekvn oren Klist em opunvkv-hervn esfvcken erkenakvyvtet os.

20 Momes, hiyomen opunvkv-hervn erkenvketvn vcevllvyvtet omes; Klist hocefhoyekan erkenakvyvtes, este eti em enlikvn cukon oh hayvkaret:

21 Momis cokat vevkvyen, $\mathrm{Mv}$ ehomvn hocefhoyekat hecvkvres, momen pohvkekatet kerrvkvres.

22 Momen heyv omecicen cem vlvketv taken komvyis, tayen v'mvnvttehocet omvnks.

23 Momis hiyomat estvmv erkenakvye tayan ohvtvlaken ocvkokv, momet ohrolope sulken cem vlvketv taken $\mathrm{cv}$ yace heret omvtet ok,

24 Estofvn Spene ekvnvn ayin omat, cem vlvkvkares; ayvyof ce hecvkares, komit, momet, Mvn vyaren vm vnicvkvres, ce komit, enhonrkvn ocvyet omekv; mvn rorvyat enhomvn ce hecvkvyat estonkon cr mahlvpatken omat.

25 Momis hiyomat, emekusapvlken heromkvn es em vfvstvkaret, Celuslvmen ayis.

$26 \mathrm{Mv}$ Masetonev Ekiv tepakan vpokat mv mekusapvlke estemerrvke Celuslvme vpokan heromkv vnvcomen em vtotvketvn es afvckvket omvnkekv.

27 Mehenwrn mon es - afvckvket omes; momen eme taken vhuericvket omes. $\mathrm{Mr}$ nake puyvfekev ohfvecr ocakat enaken Centilvlket etencawvlke hakvten omat, mvu nake enavpeswv ohfvecr ocakan es afvstvkvre nake momecvkvranat tes. 
28 Monkv heyvn momecit, heyv etten heren em wikakvyof, Spenen eceyvranit ye cen hoyvnvkares.

29 Momet cem vlakvkvyof, Klist em opunvkv-herv em merkv em fvcketv ofvn vlvkvranvyan kerris.

30 Momen, tecakkvte toyatskat, vm emekusapatskat vc vpaket vcevllatskvren pu Pucase Cesvs Klist vrahkvn, momet Puyvfekev em vnokeckv vevkvyen ce'nkusapvkis ;

31 Vkvsvmvkekat Cutev ofv vpokan enkvpvken or huerihocen, vtotketv Celuslvmen vfastvranvyat emekusaprlket es afvckvke taren;

32 Hesaketvmese komat eteropotten afvcketv ofvn cem vlakit, centake vne esyomeyan pu yekcrkuehocvren.

33 Monkv herkv en Hesaketvmeset omvlkatskan ece 'pakvkekvs. Emen.

\section{SETENTVCKE XVI.}

Fepe tecakkeyvte, mv mekusvpkv-cuko Senklevn ocat em vtotkvn heren cem opunvyecvkis;

2 Momat mekusapvlket momvkvre here omet, Pucase ofvn evpayet, nakvfrstetv estomis $\mathrm{mv}$ ohfvecv em vnicatskvre enkusapkat ofvn em vnicatskvres; este sulken em vnicet, vneu vm vnicvtet ok'.

3 Prselv Akwelv tepake Klist Cesvs ofv vtotkvlke vm etohkvlketv ;

4. Vn hesaketv vrahkvn e'nokwv take mahusan wvkecakvtet ok'; vne tvlkekon, momis Centilvlke em mekusvpkv-cuko omvlkvt vkvsvmakan vsekvkvtcrs.

5 Momet mekusvpkv-cuko ehute take ocan vsekvkvtcvs. Epenetvs vm vnokecke, Klist ofv Ekiv em ette enhvteceskvn vsekvkvtcrs.

6 Mele, mv tayen pum vtotkvten vsekvkvtcrs. 
7 Antlonekvs Cunev tepake, vn nakvlke, svlvfkvlke vm etohkvlketv, mv vpastelvlke vpvken este pohkvke, cv ho$\mathrm{mv}$ Klist ofvt omakvten vsekvkvtcvs.

8 Amplevs, Pucase ofv vm vnokeckan vsekvkvtcrs.

9 Vpene, Klist ofv pum vnicv; Stikes vm vnokeckat tepakan vsekvkvtcvs.

10 Vpeles, Klist ofvn eskerhoyvten vsekvkvtcvs; Alestapulvs en cuko-vtehkvlken vsekvkvtevs.

11 Vn nake Helotevn vsekvkvtevs. Mv Pucase ofvn. svpakle Nasesv en cuko-vtehkvlken vsekvkvtcvs.

12 Tlifenv Tlifosv tepake, Pucase ofv vtotkakan vsekvkvtcvs. Prses vnokecke, hokte Pucase ofvn tayen vtotkvten vsekvkvtevs.

13 Lufvs, Prcase ofvn enhopokat, momet vne tepakeyat pu'cken vsekvkvtcrs.

14 Esenkletvs, Flekvn, Hrmas, Petlopvs, Hvmes, tecakketvlke vpvkakat esyoman vsekvkvtçvs.

15 Filalokvs, Culev tepake, Nelevs e'wrnwr tepake, momet Olempvs, momet mekusapvlke vpvkakat omvlkvn vsekvkvtcrs.

16 Afvnketv vcakan es ete 'sekvkvtcrs. Klist ém mekusvpkv-cukot ece' 'sekakvket os.

17 Momen, tecakkvte toyatskat, mv, mvhakv kerratskvte vnrvpen fullet, etekvprketv elentappetv esyoman omecicakan vketecet, em e fulecatskvren ce'nkusapvkis.

$18 \mathrm{Mv}$ omakat, pu Pucase Cesvs Klist tokon, e'nvrke taken em vtotkaket, opunvkv herakan, momet opunvkv yvmvskvken este nakkerrvkekat e'feke taken es ehosvkuecaket omekv.

19 Cem apohickv take ohonvkv este omvlkvn em vlakvtet omekv. Monkv ece 'rahkv taken vc afvckes; mome estomis nake herat ohfvecvn hoporrenvket, momis nake holwakat ohfveevn nakkerrvkekot omatskvren cv yacet os. 
20 Momen herkv en Hesaketvmeset ce'le take elecvn lvpken Setvnen tonufvres. $\mathrm{Pu}$ Pucase Cesvs Klist en heromkvt ece 'pakvkekvs. Emen.

21 Temorevs vtotkv vm etohkvlketv, momen vn nakvlke Lusevs, Cesvne, Sosepetv esyomat ece 'sekakes.

22 Vne Trsevs, heyv cokv hoccicvt, Pucase ofvn ece 'sekvkis.

23 Kivs vm evpayvt, momet mekusvpkv-cuko omvlkv em evpayvt ece 'sekvkes. Elastvs tvlofv vfastvt, momet Kwatvs tecakkvte hvmket ece 'sekakes.

$24 \mathrm{Pu}$ Pucase Cesvs Klist en heromkvt omvlkatskan ece 'pakvkekvs. Emen.

25 Monkv mv, vm opunvkv-herv Cesvs Klist 'sem erkenvketv tepakat vcrkvyen, mv nake ehkat yvmv ekvnv vliceckv ohrvnkvte,

26 Momis hiyomat hecke haket, owalvlke en Cokat etehoyvnen, momet Hesaketvmese meyuksv seko likat kicrte vcrkvyen, vkvsvmkv apohickv vrahkvn etvlwvvlke omvlkv en kerkuehocvte, $m v$ vcrkvyen, yekcen svpaklatskvre ce yekcvkuece taye yekcetv ocat:

27 Hesaketvmese, mv tvlkuse hoporrenat, Cesvs Klist eteropotten meyuksv sekon vrakkuehocekvs. Emen. 


\title{
COKV ENHVTECESKV
}

\section{SVHOKKALAT TEPAKAT}

\section{VPASTEL PAL}

\section{KVLENRVLKE 0HTOTOLVTE.}

THE EPISTLES OF PAUL THE APOSTLE TO THE

\section{CORINTHIANS,}

\author{
TRANSLATED
}

FROM THE ORIGINAL GREEK

INTO THE MUSKOKEE LANGUAGE.

\section{NEW YORK :}

AMERICAN BIBLE SOCIETY, INSTITUTED IN THE YEAR MDCCCXVI. 


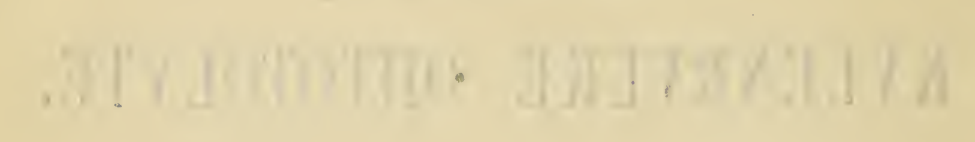




\section{COKV ENHVTECESKV VPASTEL PAL}

\section{KVLENRVLKEN OHTOTVTE.}

\section{SETENTVCKE I.}

PAL, Hesaketvmese komat verkvyen, Cesvs Klist em vpastel hakare v'nhuehkvte, tecakkvte Sosrenes tepakeyat,

2 Mekusvpkv-cuko Kvlenrvn ocat, mv Cesvs Klist ofv vcacvke hakvte, mekusapvlke hakvre enhuehkvkhoyvte, mv estrmv estomis pu Pucase Cesvs Klist e hocefkv ohhuehkakat, mvt entake esyomeyat pu Pucaset ok, es omvlkvn oh 'totakeyet os:

3 Heromkv momet herkv Hesaketvmese Pu'rke momet Pucase Cesvs Klist aenkvpvket ece 'pakvkekvs. 4 Ece 'rahkv take, mv heromkv Cesvs Klist ce'mvkvte vrahkvn, estofis vn Hesaketvmesen vrakkuecis ;

5 Opunvkv omvlkv momet nakkerretv omvlkv ofvn nakeocvlken Cesvs ce hayen, momet nake vtekat ofvn;

6 Cesvs Klist ohkerkueckvt ec ofv take yekcihocat etvpomen;

7 Momat, pu Pucase Cesvs Klist em vlvketv mehakatskat, nakemkv hvmkusis ofv yupvklvtkatskekos :

8 Momen mvu meyuksv oren ce yekcvkuecvres, pu Pucase Cesvs Klist en nettv omof em mvttvkekot fullatskvren.

9 Hesaketvmese mehenwet omes, mvt E'ppuce Cesvs Klist pu Pucase em etohkvlketv toyatskvren ce'nhuehkvkvtet os. 
10 Momen, tecakkvte toyatskat, hvmkusen opunahoyatsken, tekvpvketv cem ofv take sekaren, momis vkerrickv hvmkuse, fvcceckvu hvmkuse ofvn etelvevke herakatskvren, pu Pucase Cesvs Klist e hocefkv ofvn ce'nkusapvkis.

11 Tecakkvyvte toyatskat, Etenrvpkv em etenrvwv taken ocaket os, maket ec ohfvecv taken Kloe en cukovtehkvlket vn kerkuecakvtet omekv.

12 Momen heyvn makvyet os, Ece 'pakvkat hvmket, $\mathrm{Pal}$ enake toyis, maken; hvmkat, Vpalvs enake toyis; momen hvmkat, Vne Sefvs enake toyis; momen hvmkat, Klist enake toyis ; makake vlket omatskes.

13 Klist tekvpvke te? Pal ece 'rahkv taken etohweklepkvn svrahkvte te? monkat Pal e hocefkv ofvn ce paptishoyvkvte te?

14 Klespvs Kivs tepakat tokat hvmkusatskis paptisvkatet omekv, Hesaketvmesen vrakkuecis ;

$15 \mathrm{E}$ hocef $\mathrm{kv}$ ofvn paptisvtes, estimvt cv kicekaren.

16 Momen Stefenvs en cuko-vtehkvlke, mvu paptisvyvtes; vpvlwv ohfvecrn etv hvmkusis paptisvyvten omat, kerrvkot os.

17 Paptisare tokon, opunvkv-hervn erkenvkaren Klist vc vtotvtet omekv; opunvkv en hoporrenkv ofv tokon, Klist em etohweklepkv ehvperke hake witet ok'.

18 Etohweklepkv ohonvkv estemerkvkvranan hacohaketvt omet, momis pume hesahokeyan Hesaketvmese en yekcet omekv.

$19 \mathrm{Mv}$ hoporrenat en hoporrenkvn sumecicit, nakkerrakat em vkerrickvn ehvperkuecares, make coket omekv.

20 Hoporrenat ta? cokv-hayv ta? yvmv ekvnv opunvkv-setenrapv ta? Hesaketrmeset yvmv ekvnv hoporrenkvn haco-haketvn hayekvt haks?

21 Hesaketvmese en hoporrenkv vevkvyen yvmv ekvnvt 
hoporrenkv eteropotten Hesaketvmesen kerrekat enyupvn erkenvketv en hacohaketv eteropotten vkvsvmakan hesahuecetvt Hesaketvmesen afvcecicvtet omekv.

22 Cusvlket nakeskerkvn vpohaken, Klekvlket hoporrenkvn hopoyaket omekv.

23 Momis Klist etohweklepkv svrahkvte, Cusvlken selentappueckvn, momet Klekvlken hacohaketvn;

24 Momis mv enhuehhokvten, Cusvlke Klekvlke esyoman, Klist, Hesaketvmese en yekcen, momet Hesaketvmese en hoporrenkvn 'sem erkenakeyet os.

25 Hesaketvmese en hacohaketv este 'sen hoporrenet omen; Hesaketrmese en yekcetv tokat este 'sen yekcet omekv.

26 Tecakkvte toyatskat, ce'nhuehhokan hecatsket ome$\mathrm{kv}$, enhuehhokat enavpeswv vcrkvye hoporrenakat, monkat yekcakat, monkat vrakkuehocat sulkekot omat hecatskes :

27 Momis Hesaketvmeset hoporrenakan es vlsvkueckvn, yvmv ekvnv nake hoporrenvkekan, momet yekcakan es vlsvkueckvn yvmv ekvnv nake yekcrkekan enhopoyvtet;

28 Momet yvmv ekvnv enanvke vrakkuehocekan, momet enhomeckakan; momet nanvke sekan nake ocakan es sumecickvn, Hesaketvmeset enhopoyvtes;

29 Enavpeswr hvmkusis ehomvn ekvsvmekaren.

30 Momis mv awet omatskes, Klist Cesvs ofvn, mvn Hesaketvmeset hoporrenkvn, momet fvccetvn, momet hvsvtketvn, momet pvlke-neskvn pun hayvtet os.

31 Momat, cokat vevkvyen, Estit ekvsamat Hesaketvmese ofvn ekvsvmekvs.

\section{SETENTVCKE II.}

Momen, tecakkvte toyatskat, Hesaketvmese en kerkueckvn cem onayit, cem vlakvkvyof, opunvkv monkat hoporrenky en hvlwen es cem vlvkvkates. 
2 Ece 'pakvkvyat nake etvn kerrvkon, Cesvs Klist momet etohweklepkv svrahkvte tvlkusen kerretvn komit fvccecryvtet omekv.

3 Momet vn yekce sekon, penkvlkv ofvn, momet fekeketv rakke ofvn ece 'pakvkvjutes.

4 Momen vm opunvkv erkenvketv tepakat este en hoporrenkv em opunvkv eslopicakusat ofv tokon, Puyvfekcv yekcetv tepakat eskerkv vpvket omvtes:

5 Momat cem vkvsvmkvt, este en hoporrenkv ofv tokon, momis Hesaketvmese en yekcetv ofvn huervren.

6 Momis temvpokakat vpvken hoporrenkvn opunayeyet os ; momis yvmv ekvnv, monkat em mekkvlke en hoporrenkv, mv sumkat, tokon:

7 Momis nake ehke, ohrvnkvte ofvn Hesaketvmese en hoporrenkv, mv pum vrakketv vrahkvn, Hesaketvmese ekvnv seko emunkof mellvten es opunahoyeyet os.

8 Mvn yvmv ekvnv mekkvlke hvmkusis kerrekates, kerrakvten omat, rakketv e Pucasen elecvkekvnt omekv.

9 Momis cokat vevkvyat, Hesaketvmeset vnokecakat nanvke emetakuecvkvtet, eturwvt hecekon, ehvckot pohekon, est' e'feken esceyekatet os.

10 Momis Hesaketvmeset, em Puyvfekcr eteropotten, mvn pum enramvtet os; Puyvfekcvt nanvke omvlkvn, Hesaketvmese enake ehkakat tis, vketecet omekv.

11 Hiyomet omekv, estimvt nake este ofv ocalcan kerre te, mv puyvfekcv eme vpikat kerret omekon omat? matvpomen estimvt Hesaketvmese enanvken kerrekon, Hesaketvmese em Puyvfekcv tvlkuset kerret os.

12 Momen yvmv ekvnv em puyvfekev tokon, mv Puyvfekcv Hesaketvmese atan eenakueceyvtet os; momat mv nanvke Hesaketvmeset enrahkv sekon pu'mvten kerreyvres.

13 Opunvkv este en hoporrenkvt este mvhayat ofv 
tokon, momis mv opunvkv Puyvfekcv Vcakat pu mvhayat ofvn $\mathrm{mv}$ nanvken opunayet, puyvfekcr enanvke ocakat puyvfekcv enanvken 'setehopayēs.

14 Momis estet em munkv vcrkvye huerat Hesaketrmese em Puyvfekev enanvken eenakuecekot os; hacohaketvt omake 'sem momet omekv; momet kerreko tayet os, puyvfekcv eteropotten kerhohoyet omekv.

15 Momis mv puyvfekev verkvye arat nake omvlkvn ohfvececet omis, este estomet emen ohfvececekot os.

16 Momen estimvt Pucase em vkerrickvn kerrvt haks, mvhayvret? Momis pumet Klist em vkerrickvn oceyet os.

\section{SETENTVCKE III.}

Momen, tecakkvte toyatskat, puyvfekev vevkvye fullat ec omecrkvko tayet, momis enavpeswr vcrkvye fulle, Klist Cesvs ofv hopuctakuce ec omecet cem punayvkvyvtes.

2 Vpeswr tokon epesen es ce hompvkuecryvtes; hiyome oren kvwrpatskeko tayvtet ok, momet hiyomat kvwopetv umatskeko tayet omekv.

3 Enarpeswr vcrkvyen fullatske emunkekv; hiyomet omekv, vleskvpkv, vnrvpkv, etepvketv esyomat ece 'pakvke ocet omen, enavpeswr vcrkvyen fullet, este fulle omet fullatskeko haks?

4 Vnet, Pal enake toyis ; hvmket makof, hvmkat, Vpalvs enake toyis; makekv, enavpeswv vcvkvyen fullatskeko?

5 Monkv Pal, monkat Vpalvs estit o haks, Pucase este vtekat emvte etvpomen, erkenvkvlke mv eteropotten vkvsvmatskvte tokon omat?

6 Vnet vhocin, Vpalvs em otohicrtes, momis Hesaketvmeset ohvtrlayvtes.

7 Monkv vhocat nake tokon, em otohicateu nake tokot omes, momis Hesaketvmeset mv ohvtrlayat omes. 
8 Momen $\mathrm{mv}$ vhocat, momet em otohicat hvmkuset omes; momen este vtekat em vtotketv mahusat vevkvyen em feketv mahusat en heckvres.

9 Hesaketvmeze etohkvlke vtotkvlke toyēkv; Hesaketvmese eme crpofvt omet, Hesaketrmese en cuko toyatskes.

10 Cuko hayv homahtv hoporrene omēt, Hesaketvmeset heromkv v'mvte vcvkvyen, enlikvn hahyin, este etvt mvn oh hayet os. Momis ohhayat vtekat estomēn mvn ohhayat vketecekvs.

11 Enlikv hahoyvte, mut Cesvs Klist ot omekv, mv likat tokat este estomet hayeko tayekv.

12 Momen este estomet cvtokunaplanen, cvtokunaphvtken, crto vcacakan, eton, pvhe-tvckvn, monkat teleko em vpen heyv enlikvn es ohhayen omat;

13 Este vtekat em vtotketvt heckvres; totkv eteropotten kerke haken, nettvt kerkuecvret ok ; momen totkvt este estome em vtotketvn, estomet omat, eskerrvres.

14 Estimv em vtotketv mvn ohhayvtet oce munken omat, feketvt en heckvres.

15 Estimv em vtotketvt nekren omat, nake em vhopvnkvres, momis emet, totkv eteropotte omēn, hesakvres.

16. Hesaketvmese en cuko-vcakat toyatskat, momen Hesaketvmese em Puyvfekcr Vcakat ec ofv taken like tat kerratskeko haks?

17 Hesaketvmese en cuko-vcakan estimvt vhopanen omat, mvn Hesaketvmeset hopanvres; Hesaketvmese en cuko-vcakat, mv cuko-vcakat ceme toyatskat, vcaket omekv.

18 Este estomet e emvkeriyats. Ece 'pakvkat estimvt yvmv ekvnv ofv hoporrenet on omat, este hoporreneko hakekvs, hoporrene taren.

19 Yvmv ekvnv en hoporrenkvt Hesaketvmese ehomv 
hacohaketv tes; Hoporrenakat em emvkerretv take ofvn hvlvtaket os ; make coket omekv.

20 Momet hvtvm, Hoporrenakat em vkerrickvn Pucaset kerres, ehvperkvket omat.

21 Monkv este estomet este ofvn ekvsahmats; nanvke omvlkvt cenake taket omekv;

22 Pal, monkat Vpalvs, monkat Sefvs, monkat yvmv ekvnv, monkat hesaketv, monkat elkv, monkat hiyome nake ocakat, monkat ocvkvranat; omvlkvt cenake taket on ;

23 Centake Klist enake toyatsken; Klist Hesaketvmese enake tes.

\section{SETENTVCKE IV.}

KLIST em erkenvkvlke, momet Hesaketvmese en nakeehkakat ohvfastvlket omakes, estet pu komekvs.

2 Momen ohvtvlakat, ohvfastvlke tat, estet herome eshechoyvren enhonhoret os.

3 Momis vnet on omat, centaket ohfvececatskat, monkat este ohfvececkv es $v c$ ohfvccehocat nake cotkuset os; momen vne tis e ohfvececrkos.

4 Vnet e ohfvecrn naken kerrvkok'; mome estomis mvt ev fvecvn cv hayekos; momis mv ve ohfvececat Pucase tes.

5 Monkv oketv orekon, Pucaset vlvkeko emunken, naken ohfvecehcatskvs; mvt yomucke en nake ehkakat ohbvyayicet, estefeke em vkerrickv take heckvken hayvres; momen momof este vtekaten Hesaketvmeset vkvsvmvres.

6 Momen, tecakkvte toyatskat, svhopakv ofvn heyv nanvken Vpalvs tepakeyan ohtvlecicvyvtes, ece 'rahkv taken; momat pume eteropotten $\mathrm{mv}$ cokat senhoyvnen est' oh vkerricatskekaret kerratsken, hvmkvtekatskis hvmke vrahkvn etv vnrvpen ete'm ekvsamatskekaren. 
7 Hiyomet omekv, este etv emvrahken estit ce haya? momen ce'mhoyeko heckuecetskvte naken ocetske haks? monkv ce'mhoyen heckuecetskvten omat, estoman ce'mhoyeko heckuecetskvte omet ekvsametske haks?

8 Hiyomat ce fvcfvket os, hiyomat nakeocvke toyatskes, ece 'pvkēkon ohmekkatskvtes; momen ohmekkatskvte! ece 'paket ohmekkeyvren komis.

9 Vpastelvlke toyeyat espoken elkvn oh melhoyvte omēn Hesaketvmeset homvn pu svpaklecvtes, komikv; yvmv ekvnv, hvlwe estrlke momet esteu ehomvn oyupkvn pu hahoyet omekv.

10 Pumet Klist vrahkvn pu hoporreneko haken, momis centaket Klist ofvn hoporrenvke toyatskes ; pu yekcvkekon, cemet ce yekcvkes; centaket vrakkvke toyatskes, pun tat pu'nhomechoyes.

11 Mucv hvse-vkerkv tis oren pu 'lvwet, $p u$ 'wvnhiket, $p u$ 'natvlkvranuset, $p u$ nvfhoken, fekhonnetvn ocekot;

12 Vtotkeyet os, pu'nke mahusan es vtotkēt: holwvken ep opunvyehocis, heren opunayēs; epu'ssehocis, vkvsvmeyet os :

$13 \mathrm{Pu}$ tvklehocis, est' enkusapēs; yvmv ekvnv enake holwvke mahat, momet nake vtekat em vholwvke mahat omvkēn mucv nettv oren pu hahoyet os.

14 Heyv nanvke cen coyvkvyat ec vlsvkuecetv tokot os; momis cv'ppuce take vm vnokeckvlke omatsken cem veahnvkis.

15 Hiyomet omekv, Klist ofv mvhayvlke cukpe-rakko palen ocatsket o estomis, $c e^{\prime}$ rkvlke take sulken ocatskekos, Klist Cesvs ofv opunvkv-herv eteropotten ce heckuecvkvyvtet ok.

16 Monkv ce'nkusapvkvyet 'os, 'svc vhayvlket omvkes.

17 Heyv vrahkvn Temore cv'ppuce vm vnokecke, momet Pucase ofv beroman cem vtotvkvyvtes, mvt vm vretv 
Klist ofv ocakan, estrmv estomis mekusvpkv-cuko omvl$\mathrm{kv}$ ofv mvhayvyat vevkvyen, ece 'kerricepuecvkvres.

18 Momis cem vlvkvranvkvko omēn pakkvket svset os. 19 Momis Hesaketvmeset komen omat, lvpken cem vlvkvkares, momet mv pakkakat em opunvkv tokon, momis en yekce taken kerrares.

20 Hesaketvmese em ohmekketvt opunvkv ofv tokot, yekce ofvt os.

21 Naken komatske haks? Estepketvn es cem vlakit, monkat vnokeckv momet eyasketv em vkerrickv ofvn cem vlvkvkare te?

\section{SETENTVCKE V.}

Hoктавкv ece 'pakvke ocat hvmkvn pohket os, momet hoktarkv mv ome mahat Centilvlke em ofv opunvyehocat sekot oman, estet e'rke e 'hiwv epayat.

2 Momen $\mathrm{mv}$ heyv naken momecvtet cem etenrvwr taken ossihocvren, hvkihhokatskvr tayvte tat momecatskekot, ce pakkvkes.

3 Hiyomet onkv, vne, mehenwvn cv 'na ofvn ece 'pakvkot, momis puyvfekcv ofvn ece 'pakvkit, ece 'pakvkvye omvyet, mv heyv naken momën momecrte ohfvccv entis ohfvecëcis;

$4 \mathrm{Pu}$ Pucase Cesvs Klist e hocefkv ofv, vm puyvfekcv esyomatskat, pu Pucase Cesvs Klist en yekcet vpvkaken, nvkaftatskof,

5 Este momēn, enavpeswr vhopvnkvre vrahkvn, Setrnen em wikatskvres, puyvfekcvt Pucase Cesvs Klist en nettv omof hesakvren.

6 Cem ekvsvmkv taket herekos. Espakkueckv estomusis likhvmkat omvlkvn pakkuecat kerratskeko?

7 Monkv espakkueckv rculan ossicvkes, naklike mucrse hakatskvren, tvklike pakkeko toyatskat etvpomen. Klist 
pum Ohhoyvnkv tis epu 'rahkvn Hesaketvmese es afvcecickun elehócrtet omekv:

8 Monkv ohhoyvnkv hompetvn, espakkueckv vcule tokon, monkat enhomeckv holwryeckv tepakat em espakkueckv tokon, momis vhayetv-tokat mehenwetv tepakat en tvklike pakkekat es vfrstvkeres.

9 Hoktarvlken vpahkatskvs, kicit, cokv ofvn cen coyvyvnks :

10 Momis yvmv ekvnv hoktarvlke, monkat nake este'm vleskapvlke, monkat fvcceko vkonahvlke, monkat nak vhake emekusapvlke vpvkatskvren esfackusen cem vsehvkit omvkos; momen omat, ekvnyn esossvranat tvlkusekv.

11 Momis hiyomat cen coyvkis, Estimvt tecakkvten kihocat, hoktarv, monkat vleskapv, monkat nake vhake mekusapv, monkat tvklecv, monkat este hacr, monkat fveceko vkonahvt on omat, vpahkatskvs; este mv oman vpaket homipatskvs.

12 Hiyomet omekv, naket vm oce haks, mv fettv svpaklateu ohfvccecvranvyat? ofv svpaklan centaket ohfvececatskeko?

13 Momis mv fettr svpaklan Hesaketvmeset ohfvececet os. Monkv mv este holwryecan cem etenrvwr taken ossicvkes.

\section{SETENTVCKE VI.}

HVMkvтEKatskat, este etimv nake vnrvpen ocatskat, mekusapvlke ehomv tokon, fvccrkekvlke ehomv vhakv 'soh vyetv ce fekhrmke taye haks?

2 Mekusapvlket ekvnvn ohfvccecvkvranat kerratskeko? momen centaket ekvnvn ohfvccecvranatsken omat, nake lopuckus mahan ohfvececatske tayat heretv oricatskeko haks?

3 Hvlwe-estvlken ohfvececvraneyat kerratskeko? momet on omat, yvmv hesaketv enanvke mahvkvu ohfvcceceyvres. 
4 Monkv yvmv hesaketv enanvke ohfveevkrranat ocen omat, mekusvpkv-cuko ofv vrakkuecvkhoye mahekan ohfvccecvlken svpvklecvkes.

5 Ec vlsepueckv taken okis. Momët o haks? Ece 'pakvkat este-hunvnwv hoporrene em etecakkakvte em etenrvwv taken ohfviceece tayat hvmkis seko haks?

6 Momis etecakkat etecakkate vnrvpen vhakvn'soh ayet os, vkvsvmekvlke ehomv take tisem.

7 Monkv hiyomat oren cem mvttvket ocet os, nanvke ohfvecehocvranat cem etenrvwr take ocvket omekv. Estoman nake cem vhopvnkvkan momis-kometv min es esatskeko? Estoman fveceko nake ce 'konahvten momis komet esatskeko?

8 Momis centaket esten naken em vhopanet, fvecekon naken est'en vkonahatsket os, momet heyvn etecakkatskvten momecatskes.

9 Momen este frccrkekat Hesaketvmese em ohmekketvt en heckvkeko tayan kerratskeko? Cem etehosikvkates: etepayeko hoktarvlke, monkat nak vhake mekusapvlke, monkat etepayvkvte hoktarvlke, monkat afveketr vnokecvlke, monkat hunvntake hunvntaken etohkvlket nake holwvke vtotkvlke,

10 Monkat horkopvlke, monkat vleskapvlke, monkat hacvlke, monkat tvklecvlke, monkat fveceko vkonahvlke, Hesaketvmese em ohmekketvt en heckvkekares.

11 Momen $\mathrm{mv}$ omakat centaket sasatskvtes: momis okkosket omatskes, momis veake haket omatskes, momi Pucase Cesvs e hocefkv ofv, momet Hesaketvmese em Puyvfekcv eteropotten fvccrke omen ce hahoyvkes.

12 Nanvke omvlkv ohfvecv vm etektrnket ocet os, momis nake omvlkvt lopicvkekot os; nanvke omvlkv ohfvecv vm etektrnket os, momis nake hrmkusis en yekce elecrn e huericvkares. 
13 Nanvke hompetv envrke em ocaken, envrke nanvke hompetr em ocet os; momis heyv envrke momet mv nanvken Hesaketvmeset sumhuecvres. Momen enat hoktarkv em ocat tokon, Pucase em ocet os, momen Pucaset ena em ocet os.

14 Momen Hesaketrmeset Pucasen elkv a en kvwaprtes, momet pumeu en yekce mahusat etehoyvnen a pu kvwrpvres.

15 Ce'na take Klist em enalvccet omakat kerratskeko? Monkv Klist em enalvecvken cahwet, hokte-hoktarv em enalvecvken hayvranvya? Mohmates.

16 Momen estimvt hokte-hoktarv vlvccat ena hvmkuset omat kerratskeko? hokkolet ena-vpeswv hvmkusvres, maket omekv.

17 Momis Pucase vlvecat puyvfekcv hvmkuset os.

18 Hoktarkvn em pefatkvkes. Naorketv estomis omvlkv este-hunrnwr momecat ena vnaorketv tokot os; momis mv hoktarat em ena mahusan vnaorket os.

19 Momen ce'na take, Hesaketvmese ce'mvte, Puyvfekcv Vcakat ece 'pikat en cuko-vcakatet omen, cemet e enake toyatskekot omat kerratskeko?

20 Enrahkvn es ce neshoyvkvtet ok'; monkv œ'na take momet cem puyvfekev taken Hesaketvmesen es vrakkuecrkes, mvt Hesaketvmese enaket omakekv.

\section{SETENTVCKE VII.}

Momen mv nanvke opunvyece vn hoccicatskvte ohfvccrn : Hunvnwvt hokten celayekan heres.

2 Mome estomis, hoktarkv omecicen, hunvnwv vtekat e 'hiwv mahusan ocen, hokteu e 'he mahusan ocekvs.

3 Hunvnwvt ehiwv heromkv enaken emen; hokteu ehen matvpomen emekvs.

4 Ehiwvt e'na mahusat ohfvecrn yekcetvn ocekon, mo- 
mis ehet ocen; momen matvpomen ehet e'na ohfvecvn yekcetvn ocekon, momis ehiwvt oces.

5 Ete'm vkvsvmatskat tvlken, momet oketv vkocoknusen, eelvwece mekusvpkv vrahkv tokon omat, hvmkat enaken ete'n fekhonnihcatskvs, momen hvtvm etohvhoyvkvtcrs, Setvnet eemfvyatkvn ocatskekat omeeicen es ce kerrvkekaren.

6 Momis, Makvs, ev kihoce tokon, vm etektrnechoyet on, heyvn makvyet os.

7 Este hunvntake omvlkvt vne omakate! komryet omekv. Momis, Hesaketvmeset ehmen, omvlkvt emkv em etetaye vlken emvten ocvket os, hvmket hiyomēn, hvmkat momēn.

$8 \mathrm{Monkv}$ etepayvkekvlke oye-hoktvke esyomen heyvn kicvkvyet os; Vne etrpomet emomecicaken omat, heres.

9 Momis e en hvlvtvkeko tayen omat, etepayvkekvs; etepvyetvt vhetketv senheret omekv.

10 Momis vne tokot, Pucaset maken, etepayakan kicakvyet os; Ehiwvt ehen enkvpahkats :

11 Momis enkvpaken omat, ehesekot emunkekvs, monkat ehen etenherkekvs; momen hunvnwvt ehiwvn wihkates.

12 Momis vpvlwrn, Pucase tokot, vnet em punayvkis, Tecakkvte estomet ehiwv Cesvs ohvkvsamv tokon ocet on, vpaket liketv vkvsamet on omat, wihkates.

13 Momen hokteu, ehe Cesvs ohvkvsamv tokon ocen, vpaket liketv vkvsamen omat, hoktet wihkates.

14 Hiyomet omekv, hunvnwv vkvsvmekat ehiwv eteropotten vcake haken, hokte vkvsvmekat ehe eteropotten vcake haket os; monkon omat, cen hopuetake take vcacvkekvnt os; momis hiyomat vcacvket os.

15 Momis vkvsvmekat enkvpaken omat, enkvpvkekvs. Tecakkvte hunvnwv monkat tecakkvte hokte heyv oma- 
kat ofv svlvfketv elecvn huerekot os; momis Hesaketvmeset herkvn pu'nhuehkvtes.

16 Hiyomet omekv, ehiwv toyetskat, $c e^{\prime}$ hen hesayece witetske tayat, estont kerretska? momen hunvnwv toyetskat, ce 'hiwvn hesayece witetske tayen omat, estont kerretska?

17 Momis Hesaketvmeset este vtekate em etekvpicvkvte vcrkvyen, momet Pucaset este vtekate enhuehkvte vevkvye etvpomēn vrekvs. Momen mekusvpkv-cuko omvlkv ofvn momēn mellis.

18 Estimvn svkvmsishoyvten enhuehhokvtet o haks? mvt svkvmsishoyeko hahkates. Svkvmsiskv tokat ofvn estimvn enhuehhokvtet o haks? mvn svkvmsishohyats.

19 Svkvmsiskv nake tokot omen, svkvmsiskv tokat nake toks, momis Hesaketvmese em vhakv take vfvstetvt.

$20 \mathrm{Mv}$ ofv huere enhuehhokvte vtekat mata ofvn emomecicekvs.

21 Vtotkvt ometsken ce'nhuehhokvt haks? mvn stoskohmetskvs; momis pucasseko ce haketske tayen omat, mv min es afvekvs.

22 Estimvt, vtotkvt omen, Pucase ofvn enhuehhokat Pucase em este-pucassekot omen ; mv pucasseko enhuehhokat Klist em vtotkvt omekv.

23 Enrahkvn es ce neshoyvkvtet os ; este em vtotkvlke hahkatskvs.

24 Tecakkvte toyatskat, estimvt o estomis hueretv mv ofv enhuehhokvten emomecicekvs, Hesaketvmesen vpvket.

25 Momen hoktvke epayvkhoyvte sekat ohfvecv Pucase em vhakvn ocvkos ; momis heromvye taren merkv Pucase v'mvte vcrkvyen vm vkerrickvn kerkuecis.

26 Monkv estemerkv hiyome ocat omecicen heyvt heres, komryet os; este momēn hưerat heres, makis. 
27 Hokten etehvlvtetske te? Enrecrpketvn kohmetskvs. Hokten etchvlvtetskeko? hokten hopohyetskvs.

28 Momis hokten epayetskat naorketskekates; momen hokte ehesvte sekat ehesat naorkekates. Mome estomis mv omakat enavpeswr ofvn naorkvn ocvkvres; momis cein merrvkis.

29 Momis, tecakkvte toyatskat, heyvn makis, Oketv kocoknuset os; vhoskat, mv ehîwv ocakat ocvkeko omēt; 30 Momet mv hvkihhokat hvkihkvkeko omēt; afvckakat afvckvkeko omēt; nesakat nake ocvkeko omēt;

31 Momet yvmv ekvnv es e vnicakat yvmahkuecrkeko omēt omvranet os; yvmv ekvnv en heckv hoyvnepet omekv.

32 Momis senaorickv cen svkekaren komvyet os. Ehiwv ocekat Pucase enanvken 'senaoricet os, estomèt Pucasen afvecice tayat vkerricet:

33 Momis ehiwv ocat yvmv ekvnv enanvken 'senaoricet os, estomet ehiwvn afvcecice tayat vkerricet.

34 Momen hokte ehe ocat mv ocekat tepakat temvrahkvt os. Ehe ocekat Pucase enanvken 'senaoricet os, ena $\mathrm{em}$ puyvfekcr tepakat ofvn vcake taren; momis ehe ocat yvmv ekvnv enanvken 'senaoricet os, estomet ehen afvcecice tayat vkerricet.

35 Momen ceme mahusat ece 'rahkv taken cen lopicvkaret, nake-senkvnhetvn ec ob wikvkare tokon, momis nake momvre here vrahkvn, momet fvyvtkekon Pucasen hvlvtatskvren heyvn makvyet os.

36 Momis, Hokte epahoyrte sekat crnake ohfrccrn momvre tetayen momeevkos, estimvt komen omat, momen mv hoktet hoktvle mahet on, momen mv tvlkuset momvre heret on omat, naken komat momekvs, naorkekos; etepayvkekvs.

37 Momis, naket ohyekcicekon, estimvt nake eme ma- 
husat komat ohfvnken yekcetvn ocet, e feke ofv emomecickvn yekcen ocet, Hokte epahoyvte sekat crnaken vevyecares, komet, e feke ofvn frccecrten omat, heren momecet os.

38 Monkv hokte epahoyvren emat heren momecet os, momis epahoyvren emekat senheren momeces.

39 Ehiwvt e'he hesake vtekan vhakvt e'hen vwvnvyet os; momis e'het elen omat, estimvn komat ehesvre em etektrnket os; Pucase ofv tvlkusen.

40 Momis, vne vkerricvyat vcrkvyen, momēn likat mit afvcket omes; momen ohvtvlakat, Hesaketvmese em Puyvfekcrn ocis, komvyet os.

\section{SETENTVCKE VIII.}

Momes nanvke nake vhakakat emvkhoyat ohfvecr tat, omvlkvt kerretvn oceyat kerreyet os. Nakkerretvt pakkuecen, momis vnokeckvt yekcicet os.

2 Este estomis, Naken kerris, komen omat, kerrvre etetaye oren nak hvmkusis kerreko emunkvt os.

3 Momis estimvt o estomet Hesaketrmesen vnokecen omat, emet emen kerres.

4 Monkv hompetr nake vhakakat emvkhoyan hompetv ohfveç tat, nake vhake yvmv ekvnv ofv nake tokot omen, momen Hesaketrmese hvmkuset, etv sekot omen, kerreyet os.

5 Hiyomet omekv, sutv ofv monkat yvmv ekvnv ofvt on omat, hesaketvmese kicvkhoyat sulket o estomis, (hesaketvmese sulket on, pucasvlke sulket omen,)

6 Momis pume kerreyat Erke, Hesaketvmese hvmkuset, mv avtet nanvke omvlkvt ocaken, pumet eme ofv toyeyat liket os ; momet Pucase Cesvs Klist hvmkuset, mv etehoyanat nanvke omvlkvt ocaken, eme etehoyvne pume fulleyat kerres. 
7 Momis omvlkvt heyv kerretvn ocvkekot os; nakvhaken vkerricet, nakvhake emhoyvte omēn, mucv hvsevkerkv oren, mvn hompakat svset omekv, momen em eohvkerrickvt, yekcekot omet, svholwvke haket os.

8 Momis hompetvt Hesaketvmese ehomvn heraken pu hayekot os ; hompën omat, semvmomvkeyet omekot, monkat hompēkon omat, svheremahvkeyet omēkot omekv.

9 Momis e vketecrkes, estome estomis heyv cem etektvnkvke ocat este yekcvkekat ehomvn selentappetvt hakekaren.

10 Hiyomet omekv; este estomet nakvhake en cukovcakat ofvn hompetvn vliketsken hecen omat, mvn hecat $\mathrm{mv}$ este yekceko em vkerrickvn yekcihcen, nake nakvhakvken emvkhoyvten hompekare te?

11 Momen tecakketske yekcekat, mv vrahkvn Klist elvtet omaten, cen nakkerretv eteropotten sumkvr haks?

12 Momis tecakkaketskan vnaorket, em vkerrickv take yekcekan mvnvttecatskof, Klist vnaorkatsket os.

13 Monkv hompetvt tecakkvyvten naorkepuecen omat, vpeswvn estofis hompvkares, tecakkvyvten naorkepuecvkaret.

\section{SETENTVCKE IX.}

VPastel toyvko? pucassekot huervko? Cesvs Klist pu Pucasen hecvkate te? cemetake Pucase ofv vm vtotketr toyatskeko?

2 Este vpvitake ohfvecvn vpastel toyvkon omis, mehenwvn centake ohfvccrn mv toyis; Pucase ofvn vpastel em wiketv crnake tat em eskerkv toyatskekv.

$3 \mathrm{Vm}$ 'pohake em vyoposkvyat heyv tes :

4 Hompetv esketv tepakat yekcetvn ocēko?

5 Vpastelvlke etv, Pucase etecakkakvte, Sefvs esyomat ehiwv monkat ewvnwvn evpayakat omēt evpryetv yekcetvn ocēko? 
6 Monkat vntvlkuset Panepvs tepakeyat vtotkvranēkat yekcetvn ocēko!

7 Estimvt e em fekvranat suletawv hake te? estimvt, pvrko-crpofvn ahohcet, en lokcen pvpeko haks? monkat estimvt vpuekv fulle hvmken hompvkuecet, $m v$ vpuekv e'pesen hompeko haks?

8 Este omēn heyv nanvken maki haks? monkat vhakvt matan makeko te?

9 Wakv-vtotkv telekon cesapan yupopihcetskvs, Mose em vhakv ofvn coket omekv. Hesaketvmeset wakvvtotkvlken vkerricet ome te?

10 Monkat epu 'rahkv trlkusen make te? Mehenwvn epu 'rahkvn coket omes; momat mv crpvyecat enhonrkv ofvn crpvyecen, mv enhonrkv ofvn cesapat mv vpaket em enhonrkvn epoyvren.

11 Centake nake puyvfekev ohfvecv ocakan cem fokayeyvten omat, nake enavpeswr ohfvccv ocatskan vteloyēn omat, nake rakke $t e$ ?

12 Este etvt ec ohfvecv taken heyv yekcetvn tohkvlket ocaken omat, pume mahvkvu ocēko taya? Momis pumet heyv yekcetvn es e vfastēkates; momis nanvke omvlkvn em etektvnecēs, Klist em opunvkv-hervn emvnvttecēkaret.

$13 \mathrm{Mv}$ nake vcacvken vfvstakat cuko-vcakat enanvken vhesahoket omen, mv altvn vfvstakat altv enanvken kvpvken es e vfvstakat kerratskeko haks?

14 Matvpomen Hesaketvmeset mv opunvkv-hervn erkenakat opunvkv-hervn vhesahokvren mellvtet os.

15 Momis vnet heyv nanvke hvmkusis es e vfvstvkates; momen heyv nanvken hoccicvyat mvn vm momehocvre vrahkv tokot os: heyv es e vrakkuecryat este estomis vm ehvperkuecekon, elare mit vn hervret ok'.

16 Opunvkv-hervn erkenakvye estomis, naken es e 
vrakkuecvye tayat ocvkot omekv; momvranat tvlkusat vc oh wakkekv; momen opunvkv-hervn erkenvkvkon omat, vn holwakes!

17 Momet komvyat omecicen heyvn momecin omat, feketvn ocis; momis mvn komvkis, opunvkv-herv vfvstetvn vm wihoket os.

18 Monkv es vm feketvt naket o haks? Opunvkv-hervn erkenakvyof, Klist em opunvkv-hervn enrahkv sekon hayit, yekcetvn opunvkv-herv ofvn ocryan fvccekon es e vfvstvkare tes.

19 Este omvlkv ohfvecv cr pucasseko estomis, sensulken epoyaret, omvlkv em vtotkvn e hayvyvtet ok'.

20 Momet Cusvlke ohfrecrn Cusvlke ome cv hakvtet os, Cusvlken epoyaret; vhakv elecvn svpaklat ohfvecvn vhakv elecvn huervye omet $c v$ hakvtes, vhakv elecv svpvklan epoyaret.

21 Vhakv ocvkekat ohfvecrn vhakv ocvko omet, vhakv ocvkekan epoyaret; (momis Hesaketrmese ohfvecr vhakv ocvko huervkot, Klist ohfvecv vhakv ofvn hueris.)

22. Yekerkekan epoyaret, yekcvkekat ohfvecrn yekceko ome $\mathrm{cr}$ haket, este omvlkv ohfrcern nanvke omvlkv $\mathrm{cv}$ hakvtet os, estome estomis hesahuecvranvyat svsvren.

23 Momen opunvkv-herv vrahkvn heyvn momecryet os, ece 'pakvkit vneu mvn epoyaret.

24 Etemvrkv ofvn pefatkat, omvlkvt pefatkis, hvmkuset ohfvnketv eskerkv en hecket omat kerratskeko? Epoyatskvre tetayet pefatkvkes.

25 Etemuntvlkv vcevllat vtekat nanvke omvlkv ohfvcevn e em fvyatet os; momen mvt kvtvlvkv vhopvnke tayan epoyetvn momecaket os; momis pumet mv vhopvnkeko tayan epoyeyvret.

26 Monkv vnet vn kerkeko omekon letkis; momet hotvle nafke omvkot tepoyvyet os. 
27 Momis cv'nan ohfvnkit, svlvfken hayvyet os ; estome estomis este etvn em erkenvkakvyvtet omis, vne mahusat este vhepvkhoyvte $\mathrm{cr}$ hakekaren.

\section{SETENTVCKE $X$.}

Momen, tecakkvte toyatskat, heyvn kerratskekare komvkot os ; pu'rkvlke omvlkvt vholoce elecvn fullet, omvlkvt uehvtkvn eteropottecrtes,

2 Momet omvlkvt vholoce uehvtkv tepakat ofvn Moses ohfvecrn paptiskakvtes:

3 Momet omvlkvt puyvfekev en hompetv vlken hompakvtes :

4 Momet omvlkvt puyvfekev em esketv vlken eskakvtes ; puyvfekev Crto vcrkayakan es eskakvtet ok, mv Cvtot Klist ot omen.

5 Momis sulken, Hesaketvmeset em afvckekok', vnrvwv ofvn prlatkvtes.

6 Momen heyv nanvke omvlkvt eskerkv pun hakvren momaket omvtes, mvt nak holwvhokan eyacakvte omēt $\mathrm{p}_{\mathrm{u}}$ yacekaren.

\% Momen nakvhake emekusapvlke sasvte mv ohmatskvs; Estet hompet, eskvranet vpokvtet, ahkopvnvranet asvpvklvtes, make cokat vevkvyen.

8 Momen hoktarake saset, nettv hvmkan cukperakko pale hokkolen tutcenolkaket pvlatkvtes; mv omēn hoktvhleyats.

9 Vpvlwvt Klist eskerrakat, cetto holwvhokatet pvsvtakvtes; mv omēn eskeriyeyats.

10 Momen ecayakat sasen, sumecicvt sumhuecrtes; mv omaket ecahyatskvs.

11 Momen heyv nanvke omvlkvt eskerkvn em momaket omvtes, momet pume ekvnv yuksakat pun yicen svpakleyat pum veahnetvn hoccet omvtes. 
12 Monkv, Hueris, komat, lvtike witet ok', e vketecekvs.

13 Eskerkv este eskerhoye munkv tokat ec oh vlakvkekates: momis Hesaketvmeset mehenwet os, kvwvpatske tayat senhoyvnen es ce kerhoyvre etektvnecekot, momis es ce kerhoyat vpvken kvwrpatske tayvren mvnvttepetvn cen hayvkvres.

14 Monkv, tecakkvyvte vm vnokeckatskat, nakvhake mekusvpkvn em pefatkvkes.

15 Este hoporrenakat em punayvye omet opunayis; naken makvyan centaket ohfvccecrkes.

$16 \mathrm{Vkvsvmkv}$ sesketv merkvn oh vpoheyat Klist e catv em etohkvlketvt omeko haks? Mv tvklike kvlkayeyat Klist e'na em etohkvlketvt omeko?

17 Pume sulkeyat tvklike hvmkuset, ena hvmkuse toyē$\mathrm{kv}$; mv tvklike hvmkan omvlkeyat etenhompvlke toyēkv. 18 Eslevl enavpeswr vcrkvyen hecrkes; mv nanvke nekrihocvkvranan hompakat altr enanvken etenhompvlke toko?

19 Monkv naken makvya? Nakvhake naket omen, . monkat nakvhakakat emhoyat nake tes, makvya?

20 Momis nanvke Centilvlket emakat Hesaketvmese tokon, puyvfeker holwrhokan emaket os, makis: momen puyyfeker holwrhokat etohkvlke toyatskvren komukot os.

21 Pucase 'sem esketv puvvfekev holwvhokat'sem esketv hokkolvn es eskatskeko tayes. Pucase em ohhompetv puyvfekcv holwvhokat em ohhompetv tepakan etohkvlken cawatskeko tayes.

22 Fekcakhetv oren Pucasen crpakkueceye te? Eme 'sen yekcrkeye te?

23 Nanvke omvlkvt vm etektvnket os, momis nake omvlkv momvkvre here tokot os: nanvke omvlkvt vm etektrnket os, momis nake omvlkvt lopicvkekot os. 
24 Estimvt o estomet enake tokon, este eti enaken hopoyekvs.

25 Eohvkerrickv vrahkvn vpohkvn hayekot, nake estomis nakwikv vlkan wihoyan hompvkes.

26 Ekvnvt, momet es em fvcketvt Pucase enaket omekv.

27 Vkvsvmekvlke hvmket hompetv-rakkon ce'nhuehkvken, vyetvn komatsken omat, eohvkerrickv vrahkvn vpohkvn hayekot, nake ce homv es cem ohocvkan hompvkes;

28 Momis, Heyvt nakvhakakat emhoyet os, estimvt ce kicvken omat, mv kerkuecvte, momet eohvkerrickv vrahkvn homipatskvs, ekvnr momet es em fvcketv $\mathrm{Pu}$ case enaket ok' :

29 Eohvkerrickv makvyat, cenake tokon, este etv enaken okis; hiyomet omekv, estomaten $\mathrm{cr}$ pucasseko huervyat este etit em eohvkerrickv vcvkvyen $v c$ ohfvecece haks?

30 Heromkv eteropotten etemesv toyin omat, estomaten mv omecicen vkvsamvyat vrahkvn holwaken vc opunvyehoce haks?

31 Monkv hompatskat, monkat eskatskat, momet naket o estomen momecatskat, Hesaketrmese vrakkueckvn momecrkes.

32 Cusvlke, Centilvlke, monkat Hesaketrmese em mekusvpkv-cukon elentappuehcatskvs:

33 Momecvyat etvpomen este omvlkvn nanvke omvlkv ofv afvckvkuecit, nake vn lopicvre tokon, momis este sulke nake en lopicetvn hopoyis, hesahokvren.

\section{SETENTVCKE XI.}

Es vc vhayeprlket omvkes, Klist vhayepv toyvyat etvpomen.

2 Momen, tecakkvte toyatskat, nanvke omvlkv ofvn vc vkerricet, cem wikvkvyvte nanvke pum melhoyvte vfastatskan ece 'kvsamvkis. 
3 Momis Klist este-hunvnwv vtekat e'kvt omen, hunvnwvt hokte e'kvt omen, Hesaketvmeset Klist e'kvt omat kerratskvren komis.

4 Hunvnwv vtekatet $e$ 'kvt ohrvnken emekusapet, monkat owalet on omat, e'kvn vlesketvn en hayet os.

5 Momis hokte vtekat, ekvt ohrvnkekon, emekusapet, monkat owalen omat, e'kvn vlesketvn en hayet os, mvt vkase etvpomuset omekv.

6 Hiyomet omekv, hoktet ohrvnkekon omat, tonet omekvs; momis hoktet tonat monkat vkasat vlesketvt on omat, ohrvnket omekvs.

7 Hiyomet omekv, mehenwvn hunvnwvt ekvn ohranvranekot os, Hesaketvmese vhake momet em vrakketvt omekv : momis hoktet hunvnwv em vrakketvt omes.

8 Hunvnwvt hokte avte tokon, momis hoktet hunvnwv avtet omekv.

9 Momen hunvnwwn hokten en hahoyekon, momis hokten hunvnwrn en hahoyvtes.

10 Heyvt omecicen hoktet ekvn yekcetvn oh ocrranet os, hvlwe-estvlke vrahkvn.

11 Mome estomis hunvnwvt hokte vpvkekot omekot omen, hokteu hunvnwr vprkekot omekot os, Pucase ofv tat.

12 Hokte hunvnwv avte tat etvpomen, hunvnwvt hokte etehoyvnet omes; momis nak omvlkvt Hesaketvmese avte vlket omes.

13 Hoktet ohrvnkekot Hesaketvmesen mekusapen omat, nake momvre heret oma? centake ofvn fvececrkes.

14 Hunvnwr ekvesse crpcrken ocen omat, vlesketv en hayat, nak em munkv mahusat ce mvhayekot oma?

15 Momis hoktet ekvesse crpcrken ocen omat, mvt es em evrakkueckvt omes, ekvesse eseohrvnkvn e'mhoyvtet ok'. 
16 Momis estimvt etehvnkv em vlosten omat, pun tat es pum vpeyetv mv omakat hvmkusis ocēkon, Hesaketvmese em mekusvpkv-cuko takeu ocvkekot os.

17 Momen heyv cen kerkuecrkvyat ofv tat, mv etenvkaftatskat svmomuse hakvre tokon, svheremahecatskekv, ece 'kvsamvkvkos.

18 Enhvteceskat, mekusvpkv-cuko ofvn nvkaftatskof, etekvpvketv cem etenrvwv taken ocakvcuken pohvyet omekv, momet vpvlwvn vkvsvmvyēs.

19 Vkvsvmhoye taye orvke ece 'pakvkat heckvke hakvren, etekvpvketv cem etenrvwv taken ocvkvranet omekv. 20 Monkv hvmkusen etohkvlkatskof, heyvt yvfkehompetv Pucase mellvte hompatskvre tokot os.

21 Hompakat vtekat este etvt hompeko emunken eme mahusat yvfke-hompetvn hompepet os, momen hvmket elvwet on, hvmkat hacet os.

22 Cuko ofv hompet eskatske tayan ocvkatskeko haks? monkat Hesaketvmese em mekusvpkv-cukon ehvperkuecet, ocvkekan vlsvkuecatske haks? Naken ce kicvkvranvya? heyv ofvn ece 'kvsvmvkare te? Ece 'kvsamvkvkos.

23 Pucase v'muten cem wikvkvyvtet omekv, momat Pucase Cesvs, mv nere wihoyvte mahusan, tvkliken ehset, 24 Erken vkvsahmof, kvlkayet, Ehset, hompvkes; heyvt cv'na ece 'rahkv taken kvlkat tes; heyvn es vc vkerrickvn momecvkes, kicrtes.

25 Matvpomen, yvfke hompetvn homipof, sesketvn'seset, Heyv sesketvt cr catv ofv setemfrccetv mucrset os; etewole estomomusen eskatskat, es vc vkerrickvn heyvn momecatskvres, kicrtes.

26 Etewole estomomusen heyv tvkliken hompet, heyv sesketvn 'seskatskat, Pucase em elkvn kerkuecatsket omekv, vlvkvranat oren. 
27 Monkv estimvt mome taye orekot heyv Pucase en tvkliken hompet, es em esketvn 'sesket on omat, Pucase e'na e'catv tepakat ohfrecvn em mvtte tares.

28 Momis estet es e kerrekvs, momet momẽn $m v$ tvkliken hompet, $m v$ sesketvn 'seskekvs.

$29 \mathrm{Mr}$ momvre taye orekot hompet, esken omat, ohfvcceckvn e en hompet, e em esket omekv, Pucase e'na esvhockvpkv tat kerrekot.

30 Heyv omecicen yekerkekat momen pulsakat sulket ece 'prkvkes, momen sulket nocicvkes.

31 E ohfvccecẽn omat, ep ohfvccehocekaret ok'.

32 Momis ep ohfvccehocof, Pucaset pu 'stemerrvkuecet os, ekvnv vpvken ep ohfvccehocekaren.

33 Monkv, tecakkvte toyatskat, hompvranet nvkaftatskof, ete'mehakvkvtevs.

34 Momen estimvt elvwet on omat, e hute min hompekvs; etohkvlkatskat ec ohfvccvkvre tokaren. Momen vlakvyof, nak vhoskan eteticares.

\section{SETENTVCKE XII.}

Momen, tecakkvtet oyatskat, nakemkv puyvfekev ohfvecv ocakat ohfvecvn kerratskekaren komvkot os.

2 Centilvlke toyatsken, ce yvpahoyvkate mahusat vcrkvyet, nakvhake opunahoyekat 'sec ohvpeyvket omhoyvten kerratskes.

$3 \mathrm{Monkv}$ heyvn cen kerkuecrkvyet os; estimvt Hesaketvmese em Puyvfeker eteropotten opunayen omat, Ccsvs ohtvhikvkvten kicekot, momen Puyvfekev Vcakat eteropotte tokon omat, Cesvs Pucasen kiceko tayes.

4 Momen nakemkv cahmelikvket svset os, momis Puyvfekcrt hvmkuses.

5 Momen nakvfvstetv cahmelikvket svset os, momis Pucase hvmkuset os. 
6 Momen vtotketv cahmelikvket ornes, momis Hesaketvmese hvmkat tes, nak omvlkv ofvn nakomvlkvn vtotkat.

7 Momis Puyvfekcr heckueckvn este vtekaten en lopickvn emhoyet omes.

8 Puyvfekcvt este hvmken hoporrenkv em opunvkvn emen; mv Puyvfekcv etvwrt nakkerretv em opunvkvn hvmkan emet omekv :

9 Mata Puyvfekcvt vkvsvmkvn hvmken emen; mata Puyvfekcrt hvmkan estemwiceckv emkvn emaket os:

10 Hrmken nakeskerkv vtotketv taken; hvmkan owvletvn; etvn puyvfekcvlke kerretvn; etvn este etulaswv mvrahrvkvn; etvn etulaswv opunvkv yvteketvn emhoyet os:

11 Momis mata Puyvfekcvt, komat vevkvyen, este vtekaten em etekvpicet, heyv nanvke omvlkvn vtotket os.

12 Ena hvmkuset, enalvcce sulken ocen, $\mathrm{mv}$ ena hvmkusat em enalvcce omvlkv, sulket omat, ena hvmkuse etvpomet Klist omet omekv.

13 Puyvfekev hvinuse eteropotten omvlkeyat ena hvmkuse ofvn pu paptishoyet omekv, Cusvlke monkat Centilvlke, pucase ocvket, monkat pucassvkeko toyeyis; momet omvlkeyat Puyvfekcv hvmkuse ofvn pu'skvkuehocvtet omekv.

14 Enahkvn elvcce hvmkusekot, momis sulket omekv. 15 Enke toyvkok', ena enake toyvkos, elet make estomis, ena enake toko?

16 Momen, Eturwv toyvkok', ena enake toyvkos, ehvckot make estomis, ena enake toko?

17 Ena omvlkvt eturwvt on omat, pohetv estrmvn ocvrana? Omvlkvt pohetvt on omat, vwenvyetv estvmvn oce haks?

18 Momis hiyomat Hesaketvmeset enalvcce vtekat ena ofvn ocvkvtet os, eme komat verkvyen. 
19 Omvlkvt enalvcee hvmkuset on omat, ena estrmvn oce haks?

20 Momis hiyomat enalvece sulket ot, momis ena hvmkuset os.

21 Momen eturwvt, Es ce vnickv ocvkos, enken kiceko tayet on; momen ekvt, Es ce vnickv ocvkos, elen kiceko tayes.

22 Momis mv enalvece yekcvkeko mahe es pum moma. kat mahvkvu sevnickvt omakes.

23 Momen mv pu'nalvcce vrakkuehocvkekvr tetaye es pum momakat, mvn vrakketv senhoyvnen oh ocēs, momen mv pu 'nalvcce hervkeko omakat mit senhoyvnen herake haket os.

$24 \mathrm{Pu}^{\prime}$ nalvcce herakat enkusvpkvkekok', momis Hesaketvmeset, mv enkusvpkan vrakkueckv senhoyvnen emet, enan etelvcrkuecvtet os.

25 Ena ofv enalvcce tekvpvke sekon, momis enalvcce vkerrickv momvlken ete'm ocvkvren.

26 Momen enalvece hvmket estemerken omat, enalvece omvlkvt vpaket estemerkaket os; monkat enalvcce hvmken vrakkuehocen omat, enalvcce omvlkvt vpaket afvckaket os.

27 Momen cemet, Klist enat omet, em enalvcce vlke toyatskes.

28 Momen Hesaketvmese mekusvpkv-cuko ofvn svpvklecvtet svset os; enhvteceskv vpastelvlke, svhokkalan owalvlke, svtutcenan mvhayvlke, vwihekat nakeskerkvlken, mohmen emwiceckv emvkhoyvten, vnickv taken, ohfvnketv taken, etulaswv cahmelikvken svpvklecvtes.

29 Omvlkvt vpastelvlke $t e$ ? omvlkvt owalvlke $t e$ ? omvlkvt mvhayvlke te? omvlkvt nakeskerkvlken momecvlke te?

30 Omvlkvt emwiceckv emvkhoyvten ocaket ome te? 
omvlkvt ctulaswv mvrahrvkvn es opunayaket ome te? omvlkvt yvtekaket ome te?

31 Momis emkv hervke mahan vyekcen komvkes; momen mome estomis vyetv svmomusan cen kerkuecvkis.

\section{SETENTVCKE XIII.}

Este momet hvlwe-estvlke etulaswv taken 'sopunayvye estomis, vnokeckvn ocvkon omat, cvto-lane hake momet sempvl hakat omẽ cv haket os.

2 Momet owaletv yekceton ocit, nake ehkakat momet nakkerretv omvlkvn kerrit, momet ekvnhvlhawan vkuehuecrye tayat vkvsvmkv omvlkvn ocvyet o estomis, vnokeckvn ocvkon omat, nake toyvks.

3 Momet estemerrakan hompvkueckvn vn nakockv omvlkvn emit, cv'nan nekrvren emvyet o estomis, vnokeckvn ocvkon omat, naken vn lopicekos.

4 Vnokeckvt hofunen momiskomet, enheromet os ; vnokeckvt vleskapekot, e vkvsvmekot, pakkekot os:

5 Vlesketvn e en hayekot, enaken hopoyekot, vyrkhvmkvn crpvkkekot, nakholwakan vkerricekot os ;

6 Fvecetv tokat es afvckekot, mehenwetvn es afvcket os.

7 Nanvke omvlkvn kvwapet, nanvke omvlkvn vkvsamet, nanvke omvlkvn em enhonret, nanvke omvlkvn momiskomet kvwapet os.

8 Vnokeckvt estofvn sumkekos; momis owaletvt on omat, sumecvres; etulaswv taket on omat, fekhonnvkvres, nakkerretvt on omat, sumkvres.

9 Kvpvkusen kerrēt, kvpvkusen owaleyet ok'.

10 Momis mv temvpokat vlahkof, mv esfrckekat fekhonnvres.

11 Hopuewvt arvyof, hopuewv omit opunayit, hopuewv omit kerrit, hopuewv omit vkerricvyvtes; momis hunvnwv vc orof, hopuewv enanvken wikvyvtes. 
12 Hiyomat estvkheckv eteropotten, heckusekon, heceyet os; momis momof turofvn tenhēcuset heceyvres; hiyomat kvpvkusen kerrēs; momis momof cv kerhoyat etvpomen kerrares.

13 Momen hiyomat vkvsvmkvt, enhonrkvt, vnokeckvt, heyv tutcenat ocvke emunkvts; momis heyv senrakice mahat vnokeckv tes.

\section{SETENTVCKE XIV.}

VNOKECKVN vevkrpeyvks, momet puyvfekev enanvken komvkes, momis owalatskvre mit komvkes.

2 Estit punvkv kerkekon opunayen omat, este em punayet omekot, momis Hesaketvmesen em punayet omekv; estit en kerrekok'; mome estomis puyvfekev ofv tat nake ehkakan punayet os.

3 Momis mv owalat, yekcvkueckvn, emvcahnetvn momet mahlvpvtetvn esten em punayvket os.

4 Estit punvkv kerkekon opunayat e yekcicet os; momis mv owalat mekusvpkv-cukon yekcicet os.

5 Omvlkvt etulaswv mvrahrvkvn 'sopunayakate! ce komvkis, momis owalatskvre min $c v$ yaces; mv etulaswv mvrahrvkvn 'sopunayat, mekusvpkv-cuko yekcvren' yvtekekon omat, owalat mit rakket omekv.

$6 \mathrm{Monkv}$, tecakkvte toyatskat, naken cen lopicvkvranvya, etulaswv mvrahrvkvn' 'sopunayit, cem vlakvkin, nakhecickv eteropotten, monkat nakkerretv eteropotten, monkat owaletv eteropotten, monkat mvhakv eteropotten cem punayvkit omvkon omat?

7 Momen nake en hake ocvke wenahokekat tis, fehpvt, monkat kervlvt, en hake mvrahrvkvt omekon omat, fehpv hakat, monkat estvt kervlvt okat estomēn kerhoyvre te?

8 Hiyomet omekv, pof ketvt en hake kerkekon haken omat, estit horren e em etetakuecvr haks? 
9 Ceme takeu matvpomen, punvkv heren kerrvke tayan punayatskekon omat, estomēn nake makatskan kerhoyvre te? hotvle ofvn punayatskvret ok'.

$10 \mathrm{Yvmv}$ ekvnv ofvn en hake mvrahrvkv sulket omake wites, momen mv hvmkusis naken okekat sekos.

11 Monkv mv opunvkv en hake naken okan kerrvkon omat, $\mathrm{mv}$ opunayan ehomvn tvleporv toyares, momen mut cr homvn tvleporv tares.

12 Monkv centakeu matvpomen, puyvfekev enanvken tayen vlosticatskekv, ohvtvlvken ocatskvren hopoyvkes, mekusvpkv-cuko yekcickv vrahkvn.

13 Monkv punvkv kerkekon opunayat yvtekrret emekusvpekvs.

14 Punvkv kerkeko ofvn mekusapin omat, vm puyvfekcvt mekusapis, vm vkerrickvt ettekot omekv.

15 Monkv estome te? puyvfekcvn es cmekusapit, vkerrickvu, mvn es emekusvpares; puyvfekcvn es yvhikit, vkerrickvu es yvhikares.

16 Monkon omat, puyvfekevn es vkvsametsken omat; vkvsametskof, mv nakkerrekat en hueretv huerat estomēn, Emen, makvr haks, naken maketskan kerrekot omofvt.

17 Mehenwvn heren vkvsametsket omekv, momis mvt etin yekcicekot os.

18 Omvlkatskan ce muntvlvket etulaswv mvrahrvkvn 'sopunayvyat vn Hesaketvmesen vkvsamis.

19 Momis etulaswv kerkeko ofvn opunvkv cukpe-rakko palen punayvkot, vm vkerrickv eteropotten mekusvpkvcuko ofvn opunvkv cahkepusis opunvyetv min komvyet os, vpvitaken mvhayaret.

20 Tecakkvte toyatskat, hoporrenkv ofvn hopuetakucet omekot, momis enhomeckv ofvn hopuetakucet omvkes; momis hoporrenkv ofvn hunvntaket omivkes. 
21 Tvleporvlke e tulaswv, momet tvleporvlke e cukhvrpe heyv esten 'sete'm punayvkares, momen mome estomis vm apohicvkekares, Pucaset maket os, makat vhakv ofvn coket os.

22 Monkv etulaswr mvrahrvkvt, vkvsvmakat tokon, momis vkvsvmvkekan es en kerkvn ocaket os; momis owaletvt, vkvsvmvkekat tokon, momis vkvsvmakan vrahkvt omes.

23 Monkv mekusvpkv-cuko omvlkvt hvmkusen nvkvftaken, omvlkvt etulaswv mvrahrvkvn es opunahoyen omat, momen mv nakkerrvkekat, monkat vkvsvmekvlket esceyaken omat, Hacohakes, ce kicvkekar haks?

24 Momis omvlkvt owalaken, vkvsvmekat, monkat nakkerrekat yeceyen omat, omvlkvt kerrepuecaket, omvlkvt ohfvccecvkvres;

25 Momen momēn e feke enake ehkakan enrvmhoyen, momof e turofvn oh latket, Hesaketvmesen vkvsamet, Hesaketvmeset mehenwvn vpikvket os, maket kerkuecvres. $26 \mathrm{Monkv}$, tecakkvte toyatskat, estomet ome te? etohvtelokatskof, omvlkatskat esyvhiketvn ocen, mvhakvn ocen, etulaswvn ocen, nakhecickvn ocen, nake oh yvteketvn ocatsket os. Nanvke omvlkvt yekcickv vrahkvn momvkekvs.

27 Estit punvkv kerkekon opunayen omat, hokkolet, monkat tutcenan vtekuset omen, hrmkvntot opunayen, hvmket yvtekekvs.

28 Momis yvtekvt sekon omat, cryayvket omekvs, mekusvpkv-cuko ofvn; momet e em punayet, Hesaketvmesen em punvyekvs.

29 Owalakat hokkolet, monkat tutcenet opunayaken, vpvltaket ohfvccecvkekvs.

30 Este vnakv likan naken hecihocen omat, enhvteceskvt cryayvkekvs. 
31 Omvlkvt kerraken, omvlkvt emahlvpvtkakvren, omvlkatskat hvmkvntot owalatske tayet ok'.

32 Momen owalakat em puyvfekcrtaket $\mathrm{mv}$ owalakat em elecv taket omes.

33 Hesaketvmeset, tekyvmketv tokon, momis mahlvpvtketv hayvt os; mekusapvlke em mekusvpkv-cuko omvlkv ofv etvpomen.

34 Mekusvpkv-cuko ofvn cen hoktvke taket nahnvkekot omvkekvs; opunvyetv em etektvnkvkekot omekv; momis vhakv makat vevkvyen apohicet svpvklvranet os.

35 Momen naken kerretvn komaken omat, ehute e'hetaken em 'pohvkekvs: hoktvket mekusvpkv-cuko ofvn punayaken omat, vlesketvt omekv.

36 Hesaketvmese em opunvkvt centaken aossvt haks? monkat centake tvlkusen cem vlakvt haks?

37 Owalv toyit, monkat Puyvfekcv Vcakan ocis, e kome sasen omat, nanvke cen hoccicvkvyat Pucase em vhakvt omakat kerkuecekvs.

38 Momis estimvt nakkerrekon omat, kerrekot omekvs.

39 Monkv, tecakkvte toyatskat, owaletvn komę, etulaswv mvrahrvkvn 'sopunvyetvn vsehhatskvs.

40 Nanvke omvlkvt momvre heren, momet etewiwvhoke heren momet omekvs.

\section{SETENTVCKE XV.}

Momen, tecakkvte toyatskat, mv opunvkv-hervn cem erkenakvkin, mv eenakuecatskvte, ofv svpaklatskan cen kerkuecvkvyet os.

2 Momen $\mathrm{mv}$ eteropotten hesahokatsket os, nake cem erkenakvkvyvten cem vkerrickv ofvn vcayecatsken omat, momet ehvperkvn vkvsamatskekaten omat.

$3 \mathrm{Mv}$ vne $v n$ heckvten enhvteceskvn cem wikvkvyvtet 
ok', mv Cesvs Klist pun naorketv vrahkvn elvtet omat, Hoccat vcrkvyen;

4 Momet herihohcen, Hoccat vcrkvyen, nettv svtutcenan akvwrpkvtet omat;

5 Momen'Sefvset, mohmet $\mathrm{mv}$ palen hokkolohkakat hecakvtet omat;

6 Momen mv renyupvn tecakkvte cukpe cahkepe hoyvnet vhvmkvn hecakvtet omat; $\mathrm{mv}$ sulke mahat hiyomat fulle munkvts, momis nocicakat svset omes.

$7 \mathrm{Mr}$ renyupvn Cems hehcen, momof vpastelvlke omvlkvt hecakvtes.

8 Momen omvlkv espoke vneu, este em oketv toko heckvte omēt, hecvyvtes.

9.Vpastel kihoce taye orvkot, vpastelvlke sencotkus mahat toyikv; Hesaketvmese em mekusvpkv-cukon assecvyvtet ok'.

10 Momis Hesaketvmese en heromkv eteropotten nake toyvyat $m v$ toyit omis; momen en heromkv ve ohfvecv ocvtet ehvperkv tokates; momis omvlkv senhoyvnen vtotkvyvtet os: momis, vne tokon, Hesaketvmese en herom$\mathrm{kv}$ ve vpakvtet momecvtes.

11 Monkv vnet, monkat entaket, momēn erkenakēn, momēn vkvsamatskvtes.

12 Momen Klist vkvwrpkvte tat cem etenrvwr taken erkenvkhoyet oman, estomēn, Pvsatkvte aenkvwvpketv sekos, makakat ece 'pvkvke sase haks?

13 Momis prsatkvte aenkvwvpketv sekon omat, Klist akvwvpkekaten omēs.

14 Momen Klist akvwvpkekaten omat, pum erkenvketvt ehvperkvt omen, cem vkvsvmkv takeu ehvperkvt os.

15 Momes, momen Hesaketvmese a en kerrvlke fvccvkekon es pu hechoyet os; Hesaketvmese a en kerrēt, 
Klist akvwapvtet os, makeyvtet ok'; moman pvsatkvtet akvwvpkvkekon omat, mvn akvwvpekaten omēs.

16 Klist akvwvpkekatet ok', pvsatkvtet akvwvpkekon omat:

17 Momen Klist akvwrpkekaten omat, cem vkvsvmkv taket ehvperkvt os; cen naorketvtake ofv emunkv toyatskes.

18 Momen momēn omat, mv Klist ofvn nocicakat sumecrtet omes.

19 Heyv hesaketv ofv tvlkusen Klist ofvn enhonrkvn ocēn omat, este omvlkv 'sem estemerkvke mahat toyēs.

20 Momis hiyomat Klist, pvsatkvten a en kvwvpiket, $\mathrm{mv}$ nocicvte em ettetake enhvteceskv haket os.

21 Este etehoyvne elkv vlakvte etvpomen este etehoyvne prsatkvte aenkvwrpketvt vlakvtet ok'.

22 Atvme ofvn omvlkvt prsatkat etvpomen Klist ofvn omvlkvt hesahokvret ok'.

23 Momis este en hueretv mahusat vevkvyevlken, Klist ettetake enhvteceskvt on, momen em vlvketv omof, Klist enaketaket renyupvt on.

24 Momof ohmekketvn Hesaketvmese, Erken em wihkof, ohfvnketv omvlkvn, momet este em wiketv yekcetv tepakat omvlkvn lekahfet, meyuksv tares.

25 Ohmekket emunket en horre omvlkvn e'le elecvn hahye tvlkvranet omekv.

26 En horre espoke sumecihocvranat elkvt's.

27 Nanvke omvlkvn e'le elecvn svpvklecvtet omekv. Momis, Nanvke omvlkvn $m v$ elecvn svpvklehocvtet os, makat, mv nanvke omvlkv elecv svpvklecvtet mv vpvken vhonkvtkekot omat, hecket os.

28 Momen nanvke omvlkvt elecvn en hahkof, momof Eppuceu, mv nanvke omvlkvn elecv en hayvte emeu 
elecvn huervres, mv momat Hesaketrmeset omvlkv ofv omvlkv taren.

29 Monkon omat, mv prsatkvtet akvwvpkvkekon omat, mv pvsatkvte ohfvecun paptiskakvtet estomvkvre te? momen estomaten prsatkvte ohfvecrn paptiskake te?

30 Momen estomaten hrse-vkerkv omvlkvn penkvlkv ofvn svpakle haks?

31 Klist Cesvs pu Pucase ofv afvcketv ec ofv take ocryat vcrkvyen, Nettv omvlkvn elis, makvyet os.

32 Este em fulletv vcrkvyen, Efesv tvlofvn, punvttv sehoneckakan etepoyvyvten omat, naken vn lopice haks, pvsatkvtet akvwvpkvkekon omat? hompēt, eskvkeres; pvksen pu pvsvtkvret ok'.

33 Cem etehohsvkates: etempunahokv holwvhokat fulletv herakan hopanet os.

34 Frecetv ohfvecrn ahonecet, naorikatskvs; Hesaketvmese ohfvecvn kerrvkekat sasekv: heyv opunayvyat ece'lsvkuecvkvyat okis.

35 Momis, Estomen prsatkvten akvwrphoye te? momet ena estoman es yice haks? estet makvres.

36 Ce hoporrenekat, $\mathrm{mv}$ fokayetskat elekon omat, honte taye hakekot os:

37 Momen $\mathrm{mv}$ fokayetskat $\mathrm{mv}$ nake taranan fokayet omekot, nerkv tvlkusen, teleko tateu, monkat nerkv etv tateu fokayetskes.

38 Momis Hesaketvmeset, komat vevkyyen, emvpen emet, nerkv vtekat emvpe vrahkvn emet os.

39 Vpeswv omvlkvt vpeswv hvmkuset omekos: momis hvmket este vpeswvt omen, etvt ele-ostakat vpeswrt on, etvt rvro vpeswrt on, etvt fuswr vpeswrt omes.

40 Momen nanvke hrlwe ofv ocakat, hvtrm ekvnv oh ocakat svset os; momis hvlwe ofv ocakat en hryayvke hvmken, ekvnv oh ocakat en hvyayvke etvt os. 
41 Hvse en hvyayvket hvmken, hvresse en hryayvket etvt omen, kococumpv en hvyayvke etvt os: kococumpv hvmkat kococumpv etv en hvyayvke emvrahket omekv.

42 Momen prsatkvte aenkvwvpketv matvpomet os. Hopvnketv ofvn fokahoyen, hopvnkeko tayat akvwrpket os.

43 Vlesketv ofvn fokahoyen, hvyayvke ofvn akvwvphoyet os; yekcetv sekon fokahoyen, yekcetv ofvn akvwvphoyet os.

44 Ena cmmunkvn fokahoyet omen, puyvfekev enan akvwrphoyet os. Ena emmunkat ocet omen, puyvfekev enat ocet os.

45 Momen momēn hoccet os; Atvme este-hunvnwv enhvteceskvt puyvfekcv hesake haket omen, Atvme svhokkolat puyvfekev hesaketv emat haket omvtes, makat.

46 Mome estomis, puyvfekcv enake tat enhvteceskv tokon, momis ena emmunkv vevkvye mit on, momen puyvfekcv emmunkvt renyupv tates.

47 Este-hunvnwv enhvteceskv ekvnvn akvpvket, ekvnvlket omes: este-hunvnwv svhokkolat Pucase hvlwe ahvtrpkat os.

48 Fakke es hayvkhoyvte tat fakke es hayvkvte hvmkat etvpomvket os, momen hvlwe ofv ocakat hvlwe ofv ocat hvmkat etvpomvket os.

49 Momen fakke es hayvkvte hvmkat en heckvn es fulleyvte etvpomen hvlwe ofv hvmkat en heckvn es fulleyvres.

50 Momen, tecakkvte toyatskat, heyvn makvyet os; Enavpeswr catv tepakat Hesaketvmese em ohmekketv epucaseko tayen; hopvnketvt hopvnketv tokan epucaseko tayes.

51 Hecvs, nake ehken ce hecicvkvyet os; omvlkvt nocicēkares, momis omvlkeyat pu mvrahkv hakvres, 
52 Oketv cotkus mahan, mesettickv hvmkuse ofvn, pofketv espoke hakof; pofketvt hakvret ok', momen pvsatkvtet hopvnkvkeko tayet akvwvpkvkvres, momen pu mvrahkv hakvres.

53 Heyv nake hopvnke tayat hopvnkeko tayan accen, heyv nake ele tayat eleko tayan accvranet omekv.

54 Monkv heyv nake hopvnke tayat hopvnkeko tayan accen, heyv nake ele tayat eleko tayan accof, mv maketv, Ohfvnketv ofvn elkvn nokmelket os, make hoccat hoyvnvres.

55 Elkv toyetskat, cen re estvmvn oce haks? este-herickv toyetskat, cem ohfvnketv estvmvn oca?

56 Elkv en ret holwvyeckvt omen, holwvyeckv en yekcet vhakvt os.

57 Momis Hesaketvmeset vkvsvmket omekvs, mv pu Pucase Cesvs Klist eteropotten ohfvnketvn pu'mat.

$58 \mathrm{Monkv}$, tecakkvyvte vm vnokeckatskat, yekcen svpaklet, vkueko tayaket, Pucase em vtotketv estofis es fvcfvket omvkes, cem vtotketv take Pucase ofv ehvperkv tokot omat kerratsket omekv.

\section{SETENTVCKE XVI.}

Momen mekusapvlke em vtelokv ohfvecvt on omat, mekusvpkv-cuko Kelesev ocakan $m v$ vhakv emvyvte etvpomen momecrkes.

2 Nettvcakuce nettv enhvteceskvn omvlkatskat Hesaketvmeset ohvtvlvyetv em vnicvte vevkvyen naken hericekvs, vlakvyof vtelokv svkekaren.

3 Momen vlakvyof, mv cokv vtotvranatskat ofv vkvsvmakatskan cen heromkv taken Celuslvmen esyicvren vtotvkares.

4 Momen ayvyan heren omateu, ayin ve verkvpeyvres. 
5 Momen Masetonevn eteropotiyof, cem vlvkvkares, Masetonevn eteropottikv.

6 Momet ece 'pakvkit fekhonnin rvfot hoyvne witvres, estrmvn ayvranvyan $v m$ vnicet vkocuknusen ve vevkvpeyatskvren.

7 Mucv ayvyat ce hecrranvkvkok', momis, Hesaketvmeset komen omat, ece 'pakvkit vkocuknusen fekhonnares, komit, enhonrkvn ocvyet os.

S Momis Efesvn fekhonnvye emunken Pentekast orvres.

9 Vhauke rakke momet etektvnke a'mat vm en 'hauken, vnrapvlke sulket ok'.

10 Momen Temorevs vlaken omat, penkvlekot cem etenrvwr taken vrvren vketecvkes; Pucase em vtotketvn vtotket omekv, vne momecvyat etvpomen.

11 Monkv estimvt ehvperkuehcats : momis ce'nkvpakof, herkv ofvn vkocuknusen vevkvpeyvkvtevs, vm vlvkvren; tecakkvte vpvken en nene hecakikv.

12 Tecakkvte Vpalvs ohfvecvt on omat, tecakkakvte vpvket cem vlvkvkvren tayen enkusapvyvnks: momis hiyomat cem vlvketv taken komvronkot omvnks; momis em etektvnkusof, vlvkvres.

13 Vketecvkes; vkvsvmkv ofvn yekcen svpvklvkes; hunvntake omēt fullvkes; yekcrket omvkes.

14 Nanvke omvlkv momecatskan vnokeckvt vpvkekvs.

15 Tecakkvte toyatskat, (Stefenvs en cuko-vtehkvlken kerratsket os, momet, Ekiv em ette enhrteceskvt omet, estomēn mekusapvlke em vtotketvn e wikakvtet omat kerratskes ;)

$16 \mathrm{Mv}$ omakan, momet epu 'paket vnice, vtotkat vtekan em apohicatskvren ce'nkusapvkis.

17 Stefenvs, Fatunetrs, Ekivkvs esyome yican es ve afvcket os: nake momecatskeko cem vhoskvkan esfvcecakekv. 
$18 \mathrm{Vm}$ puyvfekcrn, momet cem puyvfekcv taken afvekvkuecakekv: monkv mv omakan vrakkuecvkes.

19 Mekusvpkv-cuko Esev ocakat ece 'sekakes. Akwelv Pvselv tepakat, momen mekusvpkv-cuko e hute ofv ocat Pucase ofvn tayen ece 'sekakes.

20 Tecakkakvte omvlkvt ece 'sekakes. Vfvnketv vcakēn 'setesekvkes.

21 Pal toyvyat cv'nke es vsekvyat.

22 Estimvt Pucase Cesvs Klist vnokecekon omat, Vnaremvt omekvs; Melvn erv.

$23 \mathrm{Pu}$ Pucase Cesvs Klist en heromkvt ece 'pakvkekvs. 24 Klist Cesvs ofvn vm vnokeckvt omvlkatskan ece 'pakvkekvs. Emen.

Cokv enhvteceskvn Kvlenrvlken ohtothoyvte, Felepi tvlofvn hoccihocvten, Stefenvs, Fatunetrs, Ekivkvs, Temorevs esyomet 'sem vpeyvtes. 


\section{COKV VPASTEL PAL KVLENRVLKEN \\ OHTOTVTE SVHOKKOLAT.}

\section{SETENTVCKE I.}

$\mathrm{P}_{\mathrm{AL}}$, Hesaketrmese komat vcrkvye Cesvs Klist em vpastel, tecakkvte Temorevs tepakat, Hesaketvmese em mekusvpkv-cuko Kvlenrv ocan mekusapvlke Ekiv hvmkv vpokat es omvlkvn ohtotakeyat;

2 Heromkv momet herkv Hesaketvmese Pu'rke, momet Pucase Cesvs Klist aenkvpríet cem ocvkekvs.

3 Hesaketvmese, mv pu Pucase Cesvs Klist E'rke, merkv E'rke, momet emahlvpvtketv omvlkv en Hesaketvmese,

$4 \mathrm{Mv}$ emahlvprtketvn Hesaketvmeset, pume mahusan es pu mahlvpatan estemerkv ofv vpokan es emahlvpvteyvren pu feknokketv omvlkv pu mahlvpatan, vrakkuehocekvs.

5 Klist em estemerketv ep ofv sulket ocake etvpomen Klist eteropotten pum emahlvpvtketv sulket ocakekv.

6 Momen pu 'stemerkēn omat, cem mahlvpvtketv take momet cem vhesaketv take vrahkv tes, mv estemerkvn es pu 'stemerkakat mahusan kvwvpatskvre yekcicet omat; monkat pu mahlvpvtkvken omat, cem mahlvpvtketv take momet cem vhesaketv take vrahkvt os.

7 Momen enhonrkv ec ohfvecr take oceyat yekcet os; estemerkv ofv tohkvlkvlke toyatskat etrpomen emahlvpvtketv ofv tohkvlkvlke toyvranatskat kerrēt.

8 Tecakkvte toyatskat, pu feknokketv Esev ofvn ep oh vlakvte, estomēn svhopayēsko tayan, pun yekce 
senhoyvnen pun honhoye, hesaketv tis enhonrkvn wikeyvte oren kerratskekaren komëkot omekv:

9 Momis elkvn ohfvcceckvn pume ofvn oceyvtes, e em enhonrēkot, momis Hesaketvmese prsatkvten akvwapan enhonreyvren :

10 Mvt elkv en rakke mome a'sepu'ssicvtes, momet a'sepu'ssicet os; a'sepu'ssice emunkvres, komet, $\mathrm{mv}$ ofvn enhonrkvn oceyet os;

11 Mekusvpkv es pum emekusapatskat eteropotten cemetakeu etohkvlket vnicatsken, momat pume epu'rahkvn sulket Erken vkvsvmvkvres, mv nakemkv este sulke eteropotte pu'mhoyvte vrahkvn.

12 Emvkerretv sekon, Hesaketvmese em mehenwetv ofvn, enavpeswv en hoporrenkv ofv tokon, momis Hesaketvmese en heromkv eteropotten yvmv ekvnv ofvn, momet ec ohfrecv take min semuntvlen ocêt etefulleyvtet omat pum eohvkerrickvt akerret os: heyvt pum afvcketv tes.

13 Nanvke etv tokon, nanvke ohonayet, fvccvt os, komet, kerkuecatskvte tvlkusen cen hoccicvkeyet omekv; momen, Eyuksv vteken mvn kerkuecvkvres, ce komet, enhonrkvn ocis ;

14 Fvccvket os, pu komet kvpvkusen kerkuecatskvte etvpomen, ceme taket Pucase Cesvs en nettr ofv pum afvcketv toyatskat etvpomen pumet cem afvcketv taket omeyat kerkuecet.

15 Momen nak cen lopickv svhokkolv ocatskvren heyv enhonrkv ofvn enhomvn cem vlvketvn komvyvtes;

16 Momet ce hoyahnvkit Masetonevn ecehyet, hvtvm Masetonevn aosset cem vlakvkin, cemetake, Cutev fvccvn ayvyof, vkocuknusen vc vevkvpeyatskvren komimvts.

17 Monkv heyvn ohvkerricvyof, tahoknicvye tat haks? monkat henka, henka, monkat hekos, hekos, maketv vc 
vpakvren, mv nanvken ohvkerricryat enavpeswv verkvyen ohvkerricvye te?

18 Momis Hesaketvmese mehenwat etvpomen pum opunvkv ec ohfvecr take opunayeyat henka, momet hekos tokates.

19 Hesaketvmese Eppuce, Cesvs Klist, mv cem etenrvwv take pume, vne, Selfenvs, Temorevs esyomeyat erkenakeyvte, henka momet hekos maketv tokates, momis eme eteropotten henka maketvt omvtes.

20 Pume pu tehoyvnen, Hesaketvmese em vrakketvn omecicen, Hesaketvmese em etemfvccetv omvlkvt eme ofvn henka, momet eme ofvn Emen maketvt omakekv.

21 Momen mv cemetake esyomeyan Klist ofv pu yekcvkuecet, momet pum vfeyvtet Hesaketvmese tes;

22 Momet mvt em eskerkvn epu 'licet, Puyvfekev em eskerkvn pu feke take ofvn pu'mvtet os.

23 Momen Hesaketvmese vm puyvfekcr a en kerrvn enhuehkvyet os, cem etektvnecvkvyat Kvlenrvn vlvkvko munks.

24 Cem vkvsvmkv take ohfvnkeyat tokon, momis cem afvcketvn temvnicvlke toyēs, vkvsvmkv eteropotten svpaklatskekv.

\section{SETENTVCKE II.}

Momis feknokketv ofvn cem vlvkvkvkares, komet, vc vrahkvn vm vkerrickvn hayvyvtes.

2 Hiyomet omekv, vnet ce feke cen nokhokicvkin omat, estimvt vc afvcecice haks, mv este e feke en nokkicvyvte $v c$ afvcecicet omekon omat?

3 Momen, $\mathrm{Vm}$ afvcketv $\mathrm{mv}$ omvlkv em afvcketv tes, ce komvkit, heyv nake mahusan cen hoccicvkvyvtes, vlakvyof, mv vc afvcecicvkvranvtet cv feke vn nokkicvkekaren.

4 Estemerkv rakke momet feknokketv vpvken turopus- 
wv sulket pvlatken, cen hoccicvkvyvtet ok'; ce feknokhokvre tokon, momis $\mathrm{mv}$ vnokeckvn esfackusen cem ocvkvyan kerratskvren.

5 Momis estimvt efeknokketv hayvten omat, kvpvkuse tvlkusen cv feken vn nokkicvtes: omvlkatskan svhere mahen ec oh wetenepuecvkvkaren.

6 Heyv estemerkv sulket 'sestemerricakvtet este $\mathrm{mv}$ omat em etetayes.

7 Monkv pvlhvmken em merre mit emahlvpvtvranatsket os: feknokketv swhere mahatet $\mathrm{mv}$ esten nokmelekaren.

8 Monkv vnokeckv eme ohfrecv ocatskat yekeicatskvren ce'nkusapvkis.

9 Momen heyv vrahkvn cen hoccicvkvyvtes, ec ohfvecv taken, nanvke omvlkv ofv apohicatsken omat, eskerkvn kerretvn komit.

10 Estimvn naken enkvpryecicatsken omat, vneu enkvpvyecicis; hiyomet omekv, naken enkvpvyecicvyv: ten omat, $\mathrm{mv}$ enkvpryeciciyvten Klist vyoposket, ece 'rahkv taken, enkvpvyecicvyvtes:

11 Setvnet pu mvkerrekaren; nanvken ohvkerrican kerreyet omekv.

12 Momen Klist em opunvkv-hervn erkenvkvranit Tlovn vlahkin, Pucaset vhauken vn hvwehcof,

$13 \mathrm{Vm}$ puyvfekcv ofvn fekvpkvn ocvkomvts, Titvs tecakkvyvten eshecvkok'; momis vsekahkit, mvn enkvpaket, Masetonevn eceyimvts.

14 Momen Hesaketvmeset, mv estofis Klist ofvn ohfvnken pu hayet, eme ohkerretv en fvmeckvn pu teropotte estvmv estomis hecken hayat vkvsvmkekvs.

15 Hesahokat momet estemerkvkvranat ofvn Hesaketvmese ehomvn Klist en fvmeckv toyēkv :

16 Hvmken hesaketv en fvmeckv hesaketv orat, momet 
hvmkan elkv en fvmeckv elkv orat toyês. Momen heyv nanvken estit ume taye ore haks?

17 Hesaketvmese em opunvkv hopvnake sasat omëkot, momis mehenwetvn aosse omet, momis Hesaketvmesen aosse omēt, Hesaketvmese ehomvn Klist ofvn opunayeyēt omekv.

\section{SETENTVCKE III.}

$\mathrm{CE}$ homv taken heren e opunvyecetvn hvtvm vlicecē haks? monkat here opunvyeckv cokvn ec ohtothoyan, monkat here opunvyeckv cokvn vtotatskvren vpvltake omēt pu yace haks?

2 Ceme taket pun nakcokv pu feke ofv cohoyvte, este omvlkvt kerret, ohonayakat toyatskes :

3 Ceme taket, Klist en nakcokv pume eteropotte vfvshotat, coyetv en lvste tokon, Hesaketvmese wenake em Puyvfekcvt hoccicat; crto tvpestakan oh hocce tokon, momis efeke vpeswv tvpestakan oh hoccat toyatsket omat kerkusen ec ohkerkuehocvket os.

4 Momen enhonrkv mv omēn Klist eteropotten Hesaketvmese ohfvecrn oceyet os:

5 Puntvlkat naket a ep osset os, komet vkerriceye tayat omēkon, momis Hesaketvmeset nake umetvn pu'met os;

6 Momen mvt setemfvccetv mucrsat vfvstetv umeye tayen pu hayvtet os; letv toko, momis puyvfekev vfastvlke: letvt elecen, momis puyvfekevt wenaken hayet omekv.

7 Momis elkv em vfvstetv cvton oh hoce, momet oh kvlvfhoyat hvyayvket omvten omat, momat Eslevl en hopuetaket Mose e turofvn, en heckv en hvyayvken, mv hoyvnepvranet oman, hericet vhecvkeko tayvte;

8 Puyvfekcv em vfvstetv mahvkvu hvyayvkekar haks?

9 Hiyomet omekv, ohfvcceckv em vfvstetvt hvyay vket on omat, mehenwrn fvccetv em afvstetv en hvyayvke mahvkvu muntvlet os. 
$10 \mathrm{Mv}$ hvyayvken hahoyvtet, mv hvyayvke et muntalat omecicen, heyv ohfvccrn hryayvken ocekot omekv.

$11 \mathrm{Mv}$ sumecihocvranat hryayvket omvtet on omat, mv oce emunkat mahvkvu hryayvket os.

12 Monkv enhonrkv mv oman ocēkv, fekhvmketv rakken es opunayeyet os :

13 Momen Moses Eslevl en hopuetaket mv sumkvranat e yuksvn hericet hecvkekaren sohrvnkvn e turofvn es ohranvte, omēkot os :

14 Momis em vkerrickv taket em vrvnakvtet os; setemfvecetv vculan ohonahoyof, $\mathrm{mv}$ esohrvnkv etat vkueyihocekon, mucv nettr oren oce munkvt omekv; mv sohrvnkv Klist ofvn sumecihocet omen.

15 Momis Mosen ohonahoyof, mv sohrvnkv e feke taken es ohrvnke munken, mucv nettvt ores.

16 Mome estomis mvt Pucasen ohfulket omof, mv esohrvnkvn vkueyihocvres.

17 Momen Pucaset mv Puyvfekcrt os: momen estvmv Pucase em Puyrfekert ocen omat, mvn pucasseko hueretvt oces.

18 Momis omvlkeyat, pu turofv ohrvnrvkeko omēn, Pucase en hvyayvken estakheckv ofv omēn hecēt, hvyayvken enkvpvket hvyayvke oren mata vhaken pu hahoyet os; Pucase em Puyvfekcr eteropotte omēn.

\section{SETENTVCKE IV.}

Monkv heyv vtotketvn oceyat omecicen, merkv pun heckvte vevkvyen pu hotosvkekot os;

2 Momis nakfvccvkeko ehkakan wikeyvtet os ; mvkerretv ofvn fullēkot, momet Hesaketvmese em opunvkvn mvkerretv ofvn celayēkot; momis mehenwetvn kerkueceyat eteropotten Hesaketvmese ehomvn, este omvlkv em vkerrickv takeu ehomvn pum fvccetvn heciceyet os. 
3 Momis pum opunvkv-hervt ehken omat, sumecan em ehket os.

$4 \mathrm{Mv}$ sumecat ofvn yvmv ekvnv en hesaketvmese vkvsvimvkekat em vkerrickv taken oh yomucecicvtes, Klist, Hesaketvmese vhaket omat, em opunvkv-herv hvyayakat en hvyayvket $\mathrm{mv}$ orvken hvyayvkekaren.

5 Pume mahusat tokon, momis Klist Cesvs Pucasen, momet Cesvs vrahkvn pumet cem vtotkvlken erkenakeyet omekv.

6 Hesaketvmeset, mv yomucke aosset hvyayvket hvyayvkvre kicvtet, Cesvs Klist en heckv ofvn Hesaketvmese en hryayvke kerretvn es hvyayvkvren, pu feketake ofvn hvyayvkvtet omekv.

7 Momis heyv nakeockvn vtehkv fakke es hayvkhoyvte vpiken oceyet os, yekcetv en heretv pume avte tokon, Hesaketvmese avte taren.

8 Vfolotken $p u$ naore estomis, $p u$ feknokhokekot os; pum vkerrickvt tekyvmkakis, enhonrkvn wikēkos;

9 Epu'ssehocis $p u$ wihokekot os; $p u$ tvkwihokis, $p u$ sumecekot os;

10 Estofis Pucase Cesvs em elkvn pu 'na take ofvn esfulleyet os, Cesvs en hesaketvu pu 'na ofvn hecke taren. 11 Pume wenahokeyat, Cesvs vrahkvn elkvn em wihoket omekv, Cesvs en hesaketvu pu 'navpeswv elat ofvn hecke taren.

12 Monkv ep ofvn elkvt vtotken, momis ec ofv taken hesaketvt vtotket os.

13 Pumet mata vkvsvmkv vkerrickvn ocēt, Vkvsamvyvnks, momet monkv opunayvyrnks, make cokat vevkvyen, pumeu vkvsamēs, momet monkv opunahoyeyet os ; $14 \mathrm{Mr}$ Pucase Cesvn akvwapvtet, Cesvs etehoyvnen pumeu a $p u$ kvwapet, momet ece 'pakvken $p u$ svpvklecvranan kerret. 
15 Nanvke omvlkvt ece 'rahkv taket omekv, mv heromkv sulkat este sulke em vkvsvmkv take eteropotten sulket omet, Hesaketvmese es vrakkueckv taren.

16 Mvt omecicen pu hotosvkekot os; momis pum ohonvpv este sumket omis, pum ofv este nettv omvlkvn mucrsehocet os.

17 Pum estemerkv tahoknat, oketv kocuknus mahe ocat, hvyayvke en honne $m v$ senhoyvne estrmahen, $m o$ met meyuksv-sekon pum vtotket omekv;

$18 \mathrm{Mv}$ nanvke heckakat tokon, mv nanvke heckvkekan heceyof: mv nanvke heckakat yvmv ekvnv em oketv enanvket omen, momis mv nanvke heckvkekat meyuksvsekat enanvket omekv.

\section{SETENTVCKE V.}

Heyv escukohakv ekvnv cuko oceyat sumken omat, cuko Hesaketvmese aenkvpvken, cuko enke es hahoyekon, meyuksv-sekon hvlhawat ofvn oceyat kerreyet omekv.

2 Pun cuko hvlwe atan oh accrkeye taren $p u$ yace heret, heyv ofvn hihkēkv :

3 Mehenwin accrkeyet on omat, accrkekon es pu hechoyekaren.

4 Heyv escukohakv vtehkeyat pun honhoyet, hihkēkv, accrken pu'konahohvre pu yacat tokon, momis ep oh 'vcehocvren, mv ele tayan hesaketvt nokmelvren pu yacet.

5 Momen mv heyv nake mahusat vrahkvn ep oh vtotkvtet Hesaketvmese tes, mv ohvtvlaken Puyvfeker em eskerkvn pu'mvtet.

6 Monkv enhonrkvn oceye munkvt os, ena ofvn fulleyof, Pucase enkvpvken fulleyat kerret.

7 (Heceyat tokon, vkvsvmkv eteropotten fullēkv:)

8 Mehenwvin enhonrkvn ocēs, momet ena enkvpvken Pucase vpvket fulletv min es epu 'fvcket os. 
9 Monkv vpvket, monkat vpvkēko estomis, emen afvceciceyvren vcevlleyet os.

10 Klist em ohfvececkv ohliketv ehomvn omvlkeyat er heckvraneyat tivlkuset omekv; este vtekat nake momecvte vevkvyen, here monkat holwake estomis, mv nanvke ena ofvn momecakvte en heckvren.

11 Monkv Pucase em penkvlkvn kerrēt, esten ohyekcicakeyet os ; momis Hesaketvmese e homvn pu heckvket os ; momet cem ohvkerrickv take ofvn pu heckvket omat enhonrkvn ocis.

12 Hvtvm ce homv taken heren e opunvyecēkok', momis ep ohfvecv nake seafvcecickvn ce'mvkeyet os, $\mathrm{mv}$ efeke ofv tokon, nake en heckv ofvn'se afvcecicakan nake 'sem vyoposketv ocatskvren.

13 Hiyomet omekv; pu hoporrenvkekis, Hesaketvmese ehomvt os; monkat pu hoporrenvke tis, ece 'rahkv taket os.

14 Hiyomet omekv, Klist em vnokeckvt ep ohyekcicet os; hiyomēn ohfvccecēkv; hvmket omvlkvn em elvten omat, omvlkvt prsatke tates.

15 Momet, omvlkvn em elvtet, mv wenahokat mucv vtekat, e vrahkv take tokon, mv em ehlet, hvtvm akvwrpkvte vrahkvn hesahoket fullvren elet omvtes.

16 Monkv mucv vtekat enavpeswv vevkvyen nvprn kerrēkos; momes, enavpeswv vcvkvyen Klist kerreyvtet o estomis, mucv ayat kerrēkos.

17 Monkv estit Klist ofvt on omat, nake hocvcke mucvse tes; nake vculakat hoyvnepes; hecvs, nake omvlkv muevsvke haket os.

18 Momen mv Cesvs Klist etehoyvnen pu tenherkepuecrtet, etenherketv erkenvketv pu'mvte, Hesaketvmesen nanvke omvlkvt avtet os;

19 Heyvt omen, Hesaketvmeset Klist ofvn en naorketv 
taken vrvhecekot, ekvnvn e etenherkepuecrtes, momet tenherketv opunvkvn pum wikvtes.

20 Monkv Klist opunvkv' 'sem fullvlke toyēs, Hesaketvmeset pu tehoyvnen $c e^{\prime}$ nkusapvke omēn, Hesaketvmesen tenherkvkes, maket, Klist vyoposkēt, ce'nkusapvkeyet os.

21 Pumet eme ofvn Hesaketvmese em fvccetvn pu hahoyvren, mv naorketv kerrvte sekan epu'rahkvn naorketvn hayvtet ok'.

\section{SETENTVCKE VI.}

Monkv em etohkvlke vtotkvlke toyeyat, ohvtvlaken Hesaketvmese en heromkv cen heckvkat ehvperkv tokaren ce'nkusapvkēs.

2 (Oketv momvre herat ofvn cem apohicit, vhesaketv nettv ofvn cem vnicvyvtes; hecvs, hiyomat oketv momvre heret os; hiyomat vhesaketv nettvit omes, maket omekv.)

3 Nake hvmkusis ofvn nak selentappetvn estemekot, erkenvkvlke em wiketvn em mvttes kihocekaren :

4 Momis Hesaketvmese em erkenvkvlke omēt, nanvke omvlkv ofvn e ohkerkuecēt, yvmvsketv sulke ofvn, estemerkv sulke ofvn, enkusvpketv take ofvn, nake pun honhoyat ofvn,

5 Rukafkv ofvn, cuko-yekcetv vpiketv ofvn, tekyvmketv ofvn, vtotketv ofvn, vketeckv ofvn, eelvweckv ofvn;

6 Hvsvtketv ofvn, nakkerretv ofvn, momis-kometv hofunat ofvn, heromkv ofvn, Puyvfekev Vcakat ofvn, vnokeckv mehenwat ofvn,

7 Mehenwetv opunvkv ofvn, Hesaketvmese en yekce ofvn, fvccetv setepokv akvpervn momet vkvskvnvn ocat etehoyvnen ;

8 Vrakketv momet vrakketv tokat etehoyvnen, opu- 
nvyeckv herat momet holwake opunvyeckv etehoyvnen; fvecvket omis, mvkerrvlke omvket ;

9 Kerkvke heret omis, kerkvkeko omvket; pvsatke omēt omis, momen hecvs, hesahokēs; pu 'stemerrvkuehocet omis, pu pvsvthoyēkos :

10 Feknokhoket omis, estofis $p u$ 'fvckvket; nake ocekvlket omis, sulken nake ocvken hayaket; naken ocvkekot omēt, momis nanvke omvlkvn ocēs.

11 Kvlenrvlke toyatskat, pu cukwvt cen 'haukvken, pu 'feken rakkuehocet os.

$12 \mathrm{Ep}$ ofv ce cutecihocvkekos, momis ce nvcofv take mahusan ce cutecihocvket os.

13 Momen matvpomuse vyoposketv, (vn hopuetake em punayvye omit opunayis;) cemetakeu ce rakkuehocvkekvs. 14 Vkvsvmekvlke temomvlkekon nokwv-ohwakkv e em ohwvkikatskvs: hiyomet omekv, fvccetv tokat etohkvlketv estomēn te'm oce haks? monkat hryayvke yomucke etempunahoyetv estomēn te'm oce haks?

15 Monkat Klist Pelyvl tepakat tepvketv estomēn ete'm oce haks? monkat vkvsamat vkvsvmekan naken ete'm oce haks?

16 Monkat Hesaketvmese en cuko-vcakat momet nake vhakakat etohkvlketv estomēn ete'm oce haks ? Cemetake Hesaketvmese wenake likat en cuko-vcakat toyatskekv; Emetake ofvn likit, emetake ofvn vrares, momet vnet en Hesaketvmese take toyin, mvt vm estet omvkvres, Hesaketvmeset makvte vcrkvyen.

17 Monkv em etenrvwv taken asosiyet, kvpvket omvkes, Pucaset maket os, momet mv hvsvtkekan celahyatskvs; momen vnet ce yvpayvkares,

18 Momet Ce'rke take toyares, momen cemetaket cv'ppucetake momet cv'chustrlke toyatskvres, Hesaketvmese yekcetv omvlecat maket os. 


\section{SETENTVCKE VII.}

Monkv, vm vnokēckusatskat, heyv etemfvccetvn ocet, ena puyvfekev tepakat em vholwvketv omvlkvn vkueyet, e hvsvthicvkeres, Hesaketvmese em penkvlkv ofvn hvsvtketvn esfucken hayet.

2 Epu yvpvyvkes; nrpvn naken em vhopanëkot, nvpvn hopanēkot, nvpvn frccekon vkonaheyvte sekos.

3 Ec ohfvececkv taken heyvn makit okvkos, Cemetaket ece 'pvken pvsatket momet wenahokeyvren pu feken vtehkatskes, enhomvn makvyvtet ok'.

4 Ec ohfvecr taken vm opunvkv en fekhvmketv rakket os; ec ohfrecr taken vm ekvsvmkv rakket os; mahlvpvtketvn es cv fvcket os; pu feknokketv omvlkv ofvn ve afveke estrmahet os.

5 Hiyomet omekv, Masetonevn escehyeyof, pu'navpeswvt fekvpkvn ocekates, momis vfolotken pu 'stemerrihocvtes; topvrn tepokvt ocaken, ofvn penkvlkvt ocakvtes.

6 Momis Hesaketvmeset, mv kvnevpakan mahlvpatat, Titvs vlakat eteropotten pu mahlvpatvtes:

7 Momen vlakat eteropotte tvlkekon, momis mv emahlvprtketv ec ofv taken es emahlvpvtkvte eteropotten, cem eyackv take yekcan, ce feknokketr taken, cem vkerrickv take yekce ve ohfvecv ocan pum onayof; momat svhere mahen $v c$ afveket omvts.

8 Hiyomet omekv, cokvn es ce feknokhokicryvtet o estomis, vkerrickvn eticvkos, vkerrickvn eticvyvtet o estomis; mata cokvt vkocuknusen ce feknokhokicvtet ómen hecikv.

9 Hiyomat ce feknokhokvkvte vrahkv tokon, momis vkerrickv etickv oren ce feknokhokvtet omat es vc afvcket os; Hesaketvmese vcrkvyen ce feknokhokihocvtet omekv, pume omeciceye naken ce yvmahkvkekaren. 
10 Hiyomet omekv, feknokketv Hesaketvmese vcrkvye ocat vkerrickv etickv vhesaketv oran, mv ohfvecvn vkerrickv etickv oceko tayen vtotket os: momis yvmv ekvnv efeknokketvt elkvn vtotket os.

11 Hiyomet omekv, heyv nake mahusan hecvkes, Hesaketvmese vcrkvyen ce feknokhokvkvtet, cemetake ofvn vketeckv estomēn hayvten, momet e em fvcceckvn, momet crpvkketrn, momet penkvlkvn, momet kometv yekcan, momet vkerrickv yekcan, momet prleckvn! $N a$ nvke omvlkv ofvn heyv nake ohfvecvn ce hrsvthakvken e kerkuecatskvtet os.

12 Monkv cen hoccivkvyvtet o estomis, mv este nake este em vhopanvte vrahkv tokon, monkat $\mathrm{mv}$ em vhopvnhoyvte vrahkv tokon, momis vketeckv Hesaketvmese ehomvn ec ohfvecv taken oceyat cen kerkvke hakvren, momecvyvtes.

13 Monkv ce mahlvpvtketv take ofvn pu mahlvpvtkvtes; momes, Titvs em afvcketv omecicen svhere mahen epu 'fvcke tates, omvlkatskat em puyvfekcvn afvcecicatskvtet omekv.

14 Hiyomet omekv, ec ohfvecr taken es ekvsamit em punayvyvten omat, ve vlsekot os; nanvke omvlkvn mehenwetv ofvn cem punayvkvyvte etvpomen mv esekvsvmkv Titvs ehomvn hayvyvte mehenwen eshechoyet os.

15 Momen, omvlkatskat cem apohickv taken, estomēn penkvlkv momet fekeketv ofvn evpayatskvten vkerricof, em vnokeckv envcofv cem ocvkat ohvtvlaket os.

$16 \mathrm{Monkv}$ nanvke omvlkv ofvn enhonrkvn cemetake ofvn ocvyat ve afveket os.

\section{SETENTVCKE VIII.}

Momen, tecakkvte toyatskat, Hesaketvmese en heromkvn Masetonev em mekusvpkv-cuko emakvten : 
2 Estomẽn estemerkv rakken eskerhoyakof, em afvcketv take en sulket, momet nakeockv enkusvpketr take rakkat omecicaken, emetv eyackv sulket em ohvtvlvkakvtet oman, cen kerkuecvkeyet os.

3 Umake tayat vevkvyen, momes, umake taye senhoyvnen, eme mahusat emetv vkvsvmaket,

4 Pum 'pohepet, estemetvn ehset, mekusapvlke ohfvecv heromkv etohkvlketvn eseyvren tayen pu'nkusvpakvtet omat akerris.

5 Momen pum enhonrkv verkvye tokon heyvn momecakvtet os, momis enhrteceskvn emc mahusan Pucasen em e wikaket, pumeu pum e wikakvtes, Hesaketvmese komat vevkvyen.

6 Momet on, Titvs mata heromkvn ece 'pakvken vlicecrtet omekv, mvn ec ofv taken espoyvren em 'poheyvtet os.

7 Monkv nak omvlkv ofvn, vkvsvmkvn, momet opunvyetvn, momet nakkerretvn, momet vketcckv omvlkvn, momet epu 'nokeckvn sulecatskat etvpomen heyv heromkvn sulecatskvren vketecvkes.

8 Vhakv omecicat tokon, momis este eti em vketeckvt ornecicen, momet cem vnokeckv take em mehenwetvu eskerraret heyvn opunvyvyet os.

9 Pu Pucase Cesvs Klist, nakeocet omvtet o estomis, ece 'rahkv taken, em estemerkv eteropotten nakeocvke hakatskvren, nakoceko hakvte en heromkvn kerratskekv. 10 Momen heyv ofvn vcahnetvn emis; heyvt cen lopickv taket ok', enhomvn emetv tvlkusekon, momis vkerrickv vkvsvmen ocetvn vlicecatskvte ohrolope hvmke senhojvnet omekv.

11 Monkv mv momeckvn poyvkes, momat kometv vkvsvmkv ocvte etvpomen nake ocatskat aossen momeckvt ocvren. 
12 Enhvteceskvn vkerrickv vkvsamat ocen omat, estet nake ocekat vevkvye tokon, nake ocat vcrkvyen Hesaketvmeset em afvcket omekv.

13 Este etvt en tahoknvken, momet cemetake ec ohwetenkvkvren komvkot omekv.

14 Momis temmomvlkusat ocvren, mucv mahusan cen sulketv taket mv oricrkekan es em fvcecvren, momet en sulketv takeu mv oricatskekan es cem fvcecen, temmomvlketv ocvren komis.

15 Estit sulken vteloyat em etetayat senhoyvnen ocekates, momen nvcomusen vteloyat em etetayen ocvtes, make cokat vevkvyen.

16 Momis Hesaketvmeset, mv ec ohfvecv taken mata vfekcakhetv heran Titvs e feken vpikvtet, vkvsvmkekvs.

17 Mehenwvn emvcahnetvn eenakuecvtet ok'; momis vkvsamusekv, eme komat omecicen ec oh ayvkvtes.

18 Momen mv vpvken, mv etecakkvte opunvkv-herv ofv mekusvpkv-cuko omvlkv ofv vkvsvmhoyan vtoteyvtes.

19 Momen mv tvlkusekon, mekusvplkv-cukot mvn enhopoyakvtes, heyv heromkvn mata Pucase em vrakketvn, momet cem vkerrickv vkvsamat kerkueckv vfasteyan svpeyeyof, epu 'crkayvren :

20 Heyvn e vketecēt, heyv nake sulke vfasteyat ohfvccrn, Em mvttvkēs; estimvt makekaren.

21 Hesaketvmese ehomv tvlkusekon, esteu ehomvn nake fvccakan ehopaket.

22 Momen mv vpvken tecakkeyvte, $\mathrm{mv}$ tewolen nanvke sulke ofvn vketece mahen, momet hiyomat mahvkvu, enhonrkv rakke ec ofv taken ocvyat vrahkvn vketece heren esheceyvten vtoteyvtes.

23 Titvs ohfvecrt on omat, vm etepvketv, momet ec ohfvecv take vtotkv vm etohkvlketv tes; monkat tecak- 
keyvte, mvt mekusvpkv-cuko em vpastelvlke, momet Klist em vrakketvt omakes.

24. Monkv cem vnokeckv take, momet ece 'rahkv take ekvsvme ec opunvyecvkeyvte eskerkvn emetaken, momet mekusvpkv-cuko ehomvn hecicvkes.

\section{SETENTVCKE IX.}

MeKusapvlike vfrstetv ohfvecvt on omat, cen hoccicvkvyat momecvke tayat semuntvlet omekv :

2 Cem vkerrickv take en helvpketvn kerrikv, mv ec ohfvecv taken Masetonev vpokat ehomvn es ekvsamvyvtet oman, Ekiv ohrolope hvmkevnke metetakvtet omen ; momen cem vketeckv heyv ohfvccv ocat este sulke mahe em vkerrickvn nekehuecvtet os.

3 Mome estomis, heyv nake ohfvecr $\mathrm{mv}$ ec ohfvecv taken ekvsamet punahoyeyvtet ehvperkvt omekaren, makvyvte vcvkvyen, ce metetakvkvren, tecakkakvten vtotimvts :

4 Monkon omat, Masetonevlket ve vpaket yicet, ce metetakvkekon es ce hecvken omat, pumet, (centake mit ome tis makëkot,) heyv heren ekvsameyat ohfvecvn vlesketv pun hake wites.

5 Monkv tecakketvlket, homrn ec oh vpeyvket, cen heromkv, mv ohfvecvn homvn cen kerkuehocvten, metetakuecvkvren emvcahnetv momvranat tvlkuse es vm momemvts, estematskat encaketv etehoyvne tokon, heromkv eteropotte taren.

6 Momis heyvn makvyet os; Estit encake omēt fokayat encake ome tat vteloyvres; momen estit encakeko omēt fokayat sulken vteloyvres.

7 Este vtekat e feke ofv vkerricat vcrkvyen emekvs; efeke en nokkicekon, momet ohyekcihocekon, Hesaketvmeset es afvcke esteman vnokecekv. 
8 Momen Hesaketvmeset heromkv omvlkv ec ohfvecv take sulke taren hayetv ume tayet os; momat estofis, nanvke omvlkv ofvn tayecatsket, vtotketv herakat omvlkv es fvcke toyatskvres.

9 (Vwahecet, estemerrakan emvtet os; em fvccetvt oce munkvt os, make cokat vevkvyen.

10 Momen $\mathrm{mv}$ fokayv nerkv emat tvkliken hompetvn ce'mvket, momet nerkv fokayatskan cem ohvtvlayvket cem fvccetv take em etten ohvtvlayakate!)

11 Nak omvlkv ofv heromkv omvlkv oren nakeocvlken ce hahoyvken, mvt omecicen pume pu teropotten vkvsvmkvn Hesaketvmesen emhoyet os.

12 Heyv heromkv vfvstetvt mekusapvlke enkusvpketv ohfvecvn em vnicat tvlkusekon, momis Hesaketvmese vkvsvmkv sulke eteropotten sulecet os;

13 Mvt Hesaketvmese en heromkv rakke mahe ec ofv take ocat omecicen ece 'nokecakuset, cem emekusvpakvkat momet heyv heromkv vfvstetvn eskerret;

14 Cesvs Klist em opunvkv-herv apohicatske e ohkerkuecatskat, momet encakekot emaket, momet este omvlkvn heromkvn em vwahecatskat vrahkvn Hesaketvmesen vkvsvmakof.

15 Hesaketvmeset vkvsvmket omekvs, em emkv onahoyeko tayat vrahkvn.

\section{SETENTVCKE $\mathrm{X}$.}

Momen vne, Pal mahusat, mv ece 'pakvkvyof cem etenrvwv take kvncapuset, momis ece 'pvkvkof ec ohfvecv take fekhvmke toyvyat, Klist en yvmvsketv, momet em merkv vcvkvyen ece kusapvkis :

2 Enavpeswr vevkvyen fulleyat omēn ep ohvkerricvke sasan fekhvmketv mvn es vnrvpares komvyat, ece 'pakvyof, mvn es' fekhvmkvkaren ce'nkusapvkis. 
3 Enavpeswr ofvn fulleyet o estomis, enavpeswr vevkvyen suletawvlke toyēkok' :

4 (Pun horre setepokv enavpeswrvlket omekon, momis Hesaketvmese eteropotten tohopke yekcvke lekvfkvn yekcrket os.)

5 Vkerrickv hahihocan, momet nake hvlwe Hesaketvmese kerretv vnrvpe e hvlwecat omvlkvn lekahfet, momet Klist em apohickvn vkerrickv vtekan en svlvfkuecet;

6 Momet cem apohickv taken esfvckof, apohickv tokat omvlkv empvlecepetv $p u$ metetakes.

7 En heckv take vevkvyen nanvken hecatske haks? Estimvt e em enhonret, Klist enake toyis, vkerricet komen omat, eme mahusat heyvn hvtvm vkerricekvs, emet Klist enake etvpomen pumet Klist enake toyẹs.

8 Hiyomet omekv, pun yekcetv, Pucaset, cen sumketv take tokon, momis yekcvkueckvn pu'mvten, ohvtvlaken 'sekvsamvye witet o estomis, vc vlsekares;

9 Cokvn es ce feksumhuecvranvkvye omēt omvkaren.

10 Nakcokv a pun 'totakat honhoyet, yekcvket omen; momis ena epu 'pakat yekcekon, em opunvkvt ehvperket omekv, makakes.

$11 \mathrm{Mv}$ omat heyvn vkerricekvs; ece 'pvkvkēkot, nakcokv eteropotten opunvkv ofv toyeyat etvpomen ece 'pakvkeyof, nake momeceyat ofv toyeyvres.

$12 \mathrm{Mv}$ eme tat e vkvsvmvke sasat em vhonkvtkv vpvken vhonkvtkeyvre, monkat mvn es e vhopayetv pu fekhvmkeko tayekv; momis mvt eme tat eme vlket e vhopayaket, eme ta vlket etehopayakat hoporrenvket omekot os.

13 Momis nanvke es pum vhopakv en lvpvtken omēkot, momis mv vhopakv Hesaketvmeset pu'mvte verkvyen, vhopakv centake tis ec orvken sekvsvmeyvres.

14 Hiyomet omekv, centake oricēko omēt pum vhopakv 
senhoyvnen e crpkuecēkok'; Klist em opunvkv-hervn erkenakeyat centakeu ec oricēkv.

15 Nake es pum vhopakv en lvpvtke ocakan sekvsvmēkot, vpvlwv em vtotketv ofvn okis; momis cem vkvsvmkv taket ohvtvlakof, pum vhopakv vcvkvyen esfvcken pu 'rakkuecatskvres, komet enhonrkvn ocēs;

16 Momat este etv em vhopahoyat ofv nanvke pu metetakuehocan es ekvsvmēkot, ekvnv cem vsimv ocakat ofv opunvkv-hervn erkenakeyēs.

17 Momis estit ekvsamat Pucase ofvn ekvsvmekvs.

18 Estit e vkvsamat vkvsvmke tokon, momis mv Pucaset vkvsamat vkvsvmket omekv.

\section{SETENTVCKE XI.}

$\mathrm{V}_{\mathrm{N}}$ hacohaketvn estomusis momis komatskate? momen mehenwvn ve ohfvecvn momis komvkes.

2 Ec ohfvecv taken Hesaketvmese verkvyen fekcakhit omikv; hokte mvnette fvece omvken Klist ehomvn ce svpvklecaren $c e$ 'he take taranat hvmken cem etemfaccvkvyvtet ok'.

3 Momis rnv cettot em emvkerretv eteropotten Ef ehosicvtet o etvpomen estome estomis cem vkerrickv taket mv mehenwetv Klist ofv ocat enkvpvken hopvnke wites, komit $\mathrm{cv}$ penkales.

$4 \mathrm{Mv}$ vlakat, mv erkenvkēkaten, Cesvs etvn erkenaken omat, monkat mv evpayatskekatet puyvfekcv etvn evpayatsken omat, monkat $\mathrm{mv}$ eenakuecatskekatet opunvkvherv etvn eenakuecatsken omat, momvre heren em etektvnecatskē tayet omekv.

5 Vpastelvlke hvlhvwe mahat nake hvmkusis ofvn senkvncapet omvkates, komikv.

6 Opunvkv ofv vm mvhahoyeko omvyet o estomis, nak- 
kerretv ofv tokon; momis nanvke omvlkv ofvn ece 'pakvken kerkvke heret pu hakvtet os.

7 Enrahkv sekon Hesaketvmese em opunvkv-hervn cem erkenakvkit, ce hvlwehocvkvre vrahkv e kvncvpuecvyvte naorket omvyvt haks?

8 Es ece 'fvstvkaret mekusvpkv-cuko etvn vkonvhakit, feketvn a en crwvkvyvtes.

9 Momen ece 'pakvkvye, naken cv kusapof, estimvn en honnvkates; nake cv kusapan tecakketvlket Masetonevn awat es vm fvcecakvtet ok'; momen nanvke omvlkv ofvn cen honnvkaret e vketecvyvtes, momet e vketecares.

10 Klist em mehenwetv vc vpikat vcrkvyen Ekiv em ekvnvvlkan estimvt heyv ekvsamvyan vfekhonnicekares.

11 Naket omecica? ece 'nokecrkvkok' omvya? Hesaketvmeset kerres.

12 Momis nake momecryat momecares, mv nake omecickvn komakan nake omecickvn a en tvcrkaret; nake mv ofv ekvsamat ohfvecrn pume omvken eshechoyvren.

$13 \mathrm{Mr}$ omakat, vpastel laksvlke, vtotkv mvkerrvket, Klist em vpastelvlke vhakvken e hayaket os.

14 Momen mvt estomahet omekos; Setvne tis hryayvke hvlwe-esten e vhayekv.

15 Monkv, em vtotkvlket fvccetv em vtotkvlke e vhayaken omat, nake rakke tokot os; $\mathrm{mv}$ em eyuksv taket em vtotketv vcrkvyet omvranet os.

16 Estimvt, Hoporrenekot os, cr kohmates, hvtvm makis ; mome estomis $c v$ hoporreneko omēn cr yvpayvkes, estomusis ekvsvmaren.

17 Naken makvyat Pucase vcrkvyen makvkot os, momis hoporrenkv vpvkeko omēn, heyv ekvsvmkv en fekhvmketv ofvn opunayis. .

18 Sulket enavpeswr vcrkvyen ekvsvmakekv, vneu ekvsvmares. 
19 Cemet ce hoporrenvket omēt, este hoporrenekvlke ece 'fvckvket em etektvnecatskekv.

20 Estimvt ce svlvfkvkuecen, monkat ce lokvken, monkat cem esvken, monkat e hvlwecen, monkat ce turofvn cem vnaf kvken omat, etektvnecatskekv.

21 Vlesketv ohfvecvn, pu yekcrkekate omen opunayis. Mome estomis, nake estomis ofvn estet fekhvmket on omat, $m v$ ofvn vneu fekhvmke toyis ; (hoporreneko omēn opunayis.)

22 Hepluvlket omake haks? vneu mv toyis. Eslevlket omake haks? vneu mv toyis. Eplehame en honvpsvket o haks? vneu mv toyis.

23 Klist em erkenvkvlket omake haks? (este hoporreneko omēn opunayis,) $m v$ senhoyvne toyis; vtotketv ofv sensulken momecit, rukafkv vhonkvtkv sekon, cukoyekcrn vpikvyvte, tewole sulke mahen, elkv tewolen.

24 Cusvlket $\rightsquigarrow$ kafkv pale tutcenen ostvpohkaken vcahkepan cv rukvfakvtes.

25 Vtutcenvn estepketvn es cv tephokvtes, vhvmkvn cvton es cv nvfhokvtes, vtutcenvn perro-eukot vk vhopvnken cv'stemerkvtes; nettv hvmken nere hvmken laukan vk arvyvtes;

26 Vyetv tewolen, uewv em penkvlkv ocakan, naksestvpenkvlecvlke em penkvlkv ofvn, penkvlkv vm etvlofvlke omecicakan, penkvlkv tvlofv ocakan, penkvlkv vnrvwv ocakan, penkvlkv uehatkv vk ocakan, penkvlkv tecakketvlke laksvlke em etenrvwv of vn;

27 Hotoskv enanokke tepakat ofvn, vketeckv tewolakat ofvn, elauko ewvnhketv tepakat ofvn, eelvweckv tewolakat ofvn, kvsvppe momet enatvlkvranusat ofvn arvyvtes.

28 Momen ohvtvlakat $\mathrm{mv}$ nanvke $\mathrm{vc}$ ofv toko nettv omvlkvn $\mathrm{mv}$ ve oh latkat, mekusvpkv-cuko omvlkv ohvketeckvt. 
29 Estimvt yekcekon, vneu cv yekceko haks? estin crpvkhuehocen, momen vnet vc vhetkvko haks?

30 Ekvsvmvranvyat tvlkuset on omat, nanvke cr yekcekat ofvn es ekvsvmares.

$31 \mathrm{Pu}$ Pucase Cesvs Klist E'rket, mv estofis vrakkuehocat, laksvkan kerret os.

32 Temaskvs ofvn evpayv, Eletvs mekko elecv hueratet, cv'setvn komet, Temaskvlke em etvlofvn suletawvlken es vhecicvtes:

33 Momen vhaukuce eteropotten, svmpvn vpikin, tvlofv vtohopke afopken a cv tvkhvtvpecihocen, enketaken em ossvyvtes.

\section{SETENTVCKE XII.}

Ervsamvrat nake momvre here tokot os. Nanvke Pucaset cv hecicet, momet vn kerkuecvten oprunvyecetvn orares.

2 Klist ofvn este hvmken kerrvyvte vtekat ohrolope palen ostohkakat senhoyvnet os, (ena ofvt ot, ena of $v$ tokate kerrvkos ; Hesaketvmeset kerres;) mv esten hvlwe svtutcenan vyvkhvmkusen a eshoyvtes.

3 Momen, (ena ofvt ot, ena ofv tokat kerrvkos; Hesaketrmeset kerres ;)

4 Estomen heyv esten palvtis ofvn a eshoyen, opunvkv opunahoyvkeko tayan, mv opunayvre este em etektvnkekan pohvten kerrvyvtes.

5 Este mv omat ohfvccrn ekvsvmares, momis vne ofvn, nanvke $\mathrm{cr}$ yekcekat ofv tokon omat, ekvsvmvkares.

6 Hiyomet omekv, ekvsvmetvn komvyet o estomis, este hoporreneko toyvkares ; mehenwvn opunvyaret ok'; momis fekhonnis, estimvt, mv cv hecat, monkat vc ohfvecvn pohat hoyvnen vc oh vkerricekaren.

7 Momen nake cv hecihocvte sulkat omecicen, tayen cv hvlwehocekaren, $c v^{\prime}$ navpeswv ofvn nakfvsken v'mhoyv- 
tes, Setvne em opunvkv-sarvt cv nvfkvren, tayen cv hvlwehocekaren.

8 Heyv vrahkvn vtutcenvn Pucasen enkusapvyvtes, v'nkvpvkvren.

9 Momen, $\mathrm{Vn}$ heromkvt cem etetayes: vn yekcetv yekcetv sekat ofvn temvpoken hahoyekv, cv kicvtes. Monkv es vc afvcket nanvke $\mathrm{mv}$ ofv $\mathrm{cr}$ yekcekat ofv min ekvsvmares, Klist en yekcetvt ve oh fekhonnvren.

10 Monkv yekcetv ocvkat, tvkleckv ofvn, enkusvpketv ofvn, asseckv ofvn, efeknokketv Klist vrahkv ocakat ofvn, heyon es ve afvcket os; cr yekcekof min cv yekcet ok'.

11 Ekvsamvyat este hoporreneko cv haket os; centaket vc ohyekcicatskvtes; centaket vc vkvsvmvranatskvtet ok'; nake toyvkot o estomis, nak hvmkusis ofvn vpastelvlke hvlhvwe mahan eyupv toyvkok'.

12 Mehenwvn yvmvsketv omvlkv ofvn, eskerkv, nake estomahakat, momet nakmomeckv yekcakat ofvn vpastel em eskerkvn cem etenrvwr taken momecakvyvtes.

13 Hiyomet omekv, mv ofv mekusvpkv-cuko vpvlwvn eyupv toyatskat naket o haks, vne mahusat cen honnvkvkate tokon omat? heyv vm mvttan vm merrvkes.

14 Hecvs, vtutcenvn cem vlvketvn v'metetakes; momet cen honnvkvkares: cenake tokon, centaken hopoyvkvyet ok' : hopuetaket eckvlke naken en hericvkekon, eckvlket hopuetaken en hericvkvranet omekv.

15 Momen vkvsamuset centaken cen yvmahkuecin, ece 'rahkv taken cv yvmahkuehocvres; svhere mahen ece 'nokecvkin, yupvklatken ve vnokecket o estomis.

16 Momis momekvs, ec oh wetenkvkates, momis mvkerret omit, mvkerretv eteropotten ce cawvkvyvtes, make sase wites.

$17 \mathrm{Mv}$ ec ohtotvkvyvte hvmkusis eteropotten a ce'nkvpakan 'se ohvtvlvyvyvt haks? 
18 Titvs em 'pohhit, mv vpvken tecakkvte hvmken vtotvyvtes. A ce'nkvpvkvken Titvs 'se ohvtrlvyvt haks? vkerrickv hvmkuse ofvn, momet mata elehvpo ofvn yvkapëkvt haks.

19 Hvtvm, Seokepkvn pu homvn hayakes, pu komatske haks? Klist ofvn Hesaketvmese ehomvn opunayis ; momis, vm vnokeckusatskat, ce yekcvkueckv vrahkvn nanvke omvlkvn momecess.

20 Heyv penkvlkv vm ocet omekv; estofvn vlakvyof, $\mathrm{mv}$ komvyat omatsken es ce hecvko witen, monkat cemet $m v$ cy komatskat omen es cv hecatskeko wites: punvkv setenrvpkv, etem vleskvpkv, crpvkketv, etehvnkv, eratopvrv opunvyeckv, wvswahoketv, ekvsvmkv, este tekyvmketvt ocake wites:

21 Momet hvtvm vlakvyof, cem etenrvwv taken vn Hesaketvmeset cv kvncvpuecen, sulke, mv entis naorkahket, mv hvsvtketv tokan, momet hoktarkvn, momet nake svholwvhokan momecakvte ohfvecvn vkerrickv eticvkekaten oh hvkihkakvye witvres.

\section{SETENTVCKE XIII.}

Heyv cem vlakvkvye svtutcenat tares. Opunvkv vtekat akerrvlke hokkole monkat tutcene é cukwv take ofvn yekce tares.

2 Homvn cem onayvkvyvtes, momet svhokkolan, ece 'pakvkvye omēn, homvn cen kerkuecvkis; momet ece 'pakvkvkot, hiyomat $\mathrm{mv}$ heyv enhomvn naorkakvten, momet vpvlwv omvlkvn en hoccicakis; Hvtvm vlakin omat em etektvnecahkvkos.

3 Klist vc ofv opunayat eskerkv, $\mathrm{mv}$ ec ohfvecv taken yekcekot omekon, momis ec ofv taken yekcet oman hopoyatskekv. 
4 Hiyomet omekv, yekcetv toko eteropotten etohweklepkvn svrahkvte estomis, Hesaketvmese en yekce eteropotten hesaket os. Pumeu eme ofvn yekcvkeko toyēkv, momis Hesaketvmese en yekce ec ohfvecr take ocat eteropotten hesahokeyvres.

$5 \mathrm{E}$ oh vketecrkes, vkvsvmkv ofvn svpaklet omatsken omat; ceme mahusat es e kerrvkes. Ece 'hepvkhoyvkekon omat, Cesvs Klist ece 'pikvket oman, ceme mahusat kerratskeko haks?

6 Momis epu 'hepvkhehocēkot omat kerratskvren komit, enhonrkvn ocis.

7 Momen nak holwvke hvmkusis momecatskekaren Hesaketvmesen mekusapis; pumet fvccvkeye este 'sem momvkvre tokon, momis vhepvkhoye omeye este 'sem momvket o estomis, cemetaket nake fvccan momecatskvren.

8 Mehenwetv vnrvpen naken momecēko tayēkv, momis mehenwetvn em momecēs.

9 Pumet pu yekcekon, cemet ce yekcvket omof, epu 'fvcket ok' : momet cem etemvpoketv taken, heyvn mehenwrn komēs.

10 Monkv ece 'pakvkot heyv nanvken cen hoccicvkit omis, ece 'pakvkvyof, mv yekcetv Pucaset, sumecickv tokon, momis yekcickvn a'mvte vcrkvyen fvsken cem punayvkit omvkaren.

11 Vhoskat, tecakkvte toyatskat, ece 'fvckvkekvs. Temvpoket omvkes; heren ce mahlvpvtkekvs; vkerrickv hvmkusen ete'm ocvkes; herkv ofvn fullvkes; momen vnokeckv herkv tepakat en Hesaketvmeset ece 'pakvkvres.

12 Vfvnketv vcakan 'sete 'sekvkes.

13 Mekusapvlke omvlkvt ece 'sekakvkes.

$14 P u$ Pucase Cesvs Klist en heromkvt, momen Hesa- 
ketvmese em vnokeckvt, momen Puyvfekcv Vcakat em etohkvlketvt omvlkatskan ece 'pakvkekvs. Emen.

Cokv svhokkolan Pal Kvlenrvlke ohtotvte, Masetonev ofv, Felepi tvlofvn liket hoccicvtes; momen Tftvs Lukvs tepakat svhoyvtes. 



\section{COKV VPASTEL PAL}

\section{KELESVLKE OHTOTVTE.}

THE EPISTLE OF PAUL THE APOSTLE TO THE

\section{GALATIANS,}

TRANSLATED

FROM THE ORIGINAL GREEK.

INTO THE MUSKOKEE LANGUAGE.

NEW YORK:

AMERICAN BIBLE SOCIETY, INSTITUTED IN THE YEAR MDCCCXVI.

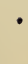

[Muskokee, 16mo.]

$$
1885 .
$$




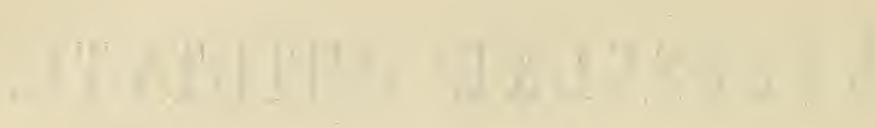

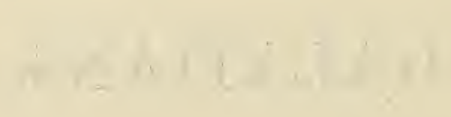




\section{COKV VPASTEL PAL \\ KELESVLKE OHTOTVTE.}

\section{SETENTVCKE I.}

PAL, (este eteropotte tokon, momet este etehoyvne tokon, momis Cesvs Klist eteropotten, momen Erke Hesaketvmese, mv prsatkvten aenkvpvken kvwapvte eteropotten,) vpastel toyvyat,

2 Momet tecakkvte ve vprkakat omvlkvt mekusvpkvcuko Kelesev ofv ocakan ohtoteyat;

3 \& 4 Erke Hesaketvmese, momet pu Pucase Cesvs Klist, mv, Hesaketrmese momet Pu'rke komat vcrkvyen, yvmv ekvnv holwakan a 'sepussicvret pun naorketv vrahkv e wikvte avtet heromkv herkv tepakat cem ocvkekvs.

5 Mvn vrakketv estofis emeyuksv-sekon em ocekvs. Emen.

6 Lvpke mahe Klist en heromkv ofv ce'nhuehkvkvten enkvpake opunvkv-herv etv 'sohceyatskat estomahes, komvyet os.

$7 \mathrm{Mv}$ etv tokot omen; momis mv ce naoricet, Klist em opunvkv-hervn mvrahkuecetv komakat svset omekv.

8 Momis pumet, monkat encelv hvlwe ahvtapket, opunvkv-herv cem erkenakeyvte tokon, etvn cem erkenakvken omat, mvt ohtvhikvkvtet omekvs.

9 Enhomv makeyvte etrpomen hiyomat hvtvm makvyet os; Estimvt opunvkv-herv eenakuecatskvte emvrahken cem erkenaken omat, ohtvhikvkvtet omekvs. 
10 Hiyomet omekv, hiyomat esten monkat Hesaketvmesen afvckvkueci haks? monkat esten afvckvkuecetvn hopoyi haks? esten afrckvkuecin omat, Klist em vtotkv toyvkaret ok'.

11 Momis, tecakkvke toyatskat, mv opunvkv-herv cem erkenakvkvyvte este vcrkvye tokot omat cen kerkuecrkis.

12 V'mvhahoyekon, este eteropotte tokon, momis Cesvs Klist en kerkueckv eteropotten vn heckvtet ok?

13 Cusvlke em mekusvpkv ofvn arvyvte ocan, estomen svkerkuehoceko taye Hesaketvmese em mekusvpkv-cukon assecit, yvmahkuecvyvten,

14 Momet Cusvlke em mekusvpkv ofvn cv kerre mahe hakat, vm etvlofvlke vm vculkv etvpomakan emuntalit, cv'rkvlke tate em opunvkv ohfvecvn svhere mahen vketecvyvtet oman pohatskvtet ok'.

15 Momis Hesaketvmeset, mv cv'cke e nvrken a vc ossicet, momet en heromkv etehoyvnen v'nhuehkvtet; 16 E'ppuce Centilvlke em etenrvwrn erkenvkaren mvn vc ofvn kerkuecetvn es afvcket omof, este e catv, monkat enavpeswrn tempunayvkot,

17 Monkat ev homvn vpastelvlke taten oh ayit, vyvkhvmkusen Celuslvmen vcemkvkates; momis Elepern ecehyit, hvtvm Temaskvn oh fulkimvts.

18 Momen ohrolope tutcenet hoyahnof, Celuslvmen vcemkimvts, Petvn hecvranit, momet mvn vpaket nettv palen cahkepohkaken fekhonnimvts.

19 Momis vpastelvlke etv hvmkusis hecvkon, Pucase etecakkvte Cems tvlkusen hecimvts.

20 Momen heyv nanvken cen hoccicvkvyat, hecvs, Hesaketvmese ehomvn, laksvkos.

$21 \mathrm{Mv}$ renyuprn Selev Selesev tepakat ekvnv ocakan ohceyimvts. 
22 Momen Cutev em mekusvpkv-cuko Klist ofv ocakat cv turofheckvn kerrvkekomvts.

23 Momis heyv tvlkusen pohakvtes, Mv oketv hoyvnakvte ofvn epu'ssecvte tat mv vkvsvmkv yvmahkuecrtet hiyomat erkenaket os.

24 Momet vc ofvn Hesaketvmesen vrakkuecakvtes.

\section{SETENTVCKE II.}

Momen ohrolope palen ostohkakat hoyanof, Titvs evpayit, Panepvs vpakit, hrtvm Celuslvmen vcemkimvts. 2 Momen nake $v n$ kerkuehocvte omecicen vcemiket; mv opunvkv-herv Centilvlke em etenrvwvn erkenakvyan - em ohhvyayicakimvts, momis, estome estomis letkvyat, momet letkvyvtet ehvperkvt omekaren, mv este here kerkakan ehkusen em punayakimvts.

3 Momis Titvs ve vpakatet, Klekvlket ot omis, svkvmsiskvren ohyekcihocekomvts.

4 Momén tecakkvte vhayvlket, $\mathrm{mv}$ pucasseko hueretv Klist Cesvs ofv oceyan vketecvranet, pu svlvfkuecetvn komakat, ehkusen ascehuehocat omecicet omemvts.

5 Mvn hvse-vkerkv hvmkusis apohicet, em etektvnecēkomvts, opunvkv-herv em mehenwetvt ece 'pakvke emunkvren.

6 Momis mv naket os komhoye taye sasat ton omat, naket omakvte estomis, cv homv nake tokot os; este en heckvn Hesaketrmeset es afvckekot os; mv naket os komhoyat, etempunahoyeyof, naken vm ohvpayvnkekv.

7 Momis mv vpvlhvmke ocan; svkvmsiskv opunvkv-herv Petv em wihokvte etvpomen svkvmsiskv-toko opunvkvherv vm wihokvten hecakof;

8 (Mv vpastel em wiketv svkvmsiskv este ohfvccrn Petv ofvn yekcen vtotkat, matat Centilvlke ohfvecrn vc ofvn yekcen vtotkvtet ok ${ }^{2}$;) 
9 Momen Cems, Sefvs Cane esyomat, mv cukelet omvkes, komhoyat, mv heromkv v'mhoyvten hecakof, Panepvs tepakeyan etohkvlketv enkvpervn pu'makemvts, pumet Centilvlken oh vhoyen, ent svkvmsiskv esten oh vpeyvkvret.

10 Estemerrakan vkerriceyvre tvlkusen komakemvts, momen vneu matan ohyekcimvts.

11 Momis Petvt Anteak vlakof cturofvn em vhuerit vnrapimvts, em vsehohvr tetayet omekv.

12 Hiyomet omekv, Cems aenkvpvke yice sasat yiceko emunkof, Centilvlken vpaket hompemvts ; momis, yihcof, enkvpaket em vkuekemvts, svkvmsiskviken em penkalet.

13. Momen Cusvlke vpvltakeu vpaket estemvkerret fullemvts; momat Paneprs tis em mvkerretv taken es fvyecicakemvts.

14 Momis fvecen, opunvkv-herv em mehenwetv vcrkvyen fullvkekon hecryof, omvlkv ehomvn, Cemet, Cusvlket ometskat, Cusvlke onkot, Centilvlke fullat omēn aretsken omat, estomaten Centilvlken Cusvlke omet fullvren ohyekcvkuecetske haks? Petvn kicit em punayimvts.

15 Pumet, pum munkv vevkvyen Cusvlket omèt, Centilvlke naorkvlke sasat toyēkot,

16 Vhakv em vtotketv eteropotte tokon, momis Klist Cesvs oh vkvsvmkv'eteropotten esten fvccehocet omat kerrēt, pume mahusat, vhakv em vtotketv eteropotte tokon, Klist oh vkvsvmkv eteropotten pu fvecehocvren Cesvs Klist ofvn vkvsvmeyvtet os; vhakv em vtotketv eteropotten ena.vpeswv hvmkusis fvccehocekaret ok'.

17 Momis Klișt eteropotten pu fvccehocvren hopoyeyof, pume mahusat holwvyecvlken es pu hechoyen omat, Klist monkv naorketv em vtotkvt ome te? Mohmates. 
18 Hiyomet omekv, mv hopanvyvten hvtvm tohtarin omat, vhakv-kacvn e hayvyet os.

19 Hiyomet omekv; Hesaketvmese ohfvccrn wenakaren, vhakv eteropotten vhakv ohfvecrn cv'let os.

20 Klist vpaken tohweklepkvn ev svrahket os; momis wenakis ; mome estomis, vne tokon, momis Klist ve ofvn wenaket os; momen mv hesaketv enavpeswr ofvn mucrn es wenakvyat Hesaketrmese E'ppuce, mv vc vnokecet vc vrahkvn e wikvte ohvkvsvmkvn es wenakvyet os.

21 Hesaketvmese en heromkvn vhepvkvkos; hiyomet omekv, fvecetv vhakv eteropotten vlaken omat, Klist ehvperkvn elvtes.

\section{SETENTVCKE III.}

Afна Kelesvlke ce hacohakvkat, ce turwv take ehomvn Cesivs Klist opunvyece, cem etenrvwr taken tohweklepkvn svrahkan hecke heren cohoyat, mehenwetvn apohicatskekaren estimvt ce mvkerrvkvt haks?

2 Heyv tvlkusen cen kerretv taken komis. Vhakv em vtotketv eteropotten, monkat vkvsvmkv apohickv eteropotten Puyvfekcv cen heckvkvt haks?

3 Momvteken ce hacohakvke te? Puyvfeker ofvn vlicecatskvtet omen, hiyomat enavpeswr eteropotten temvpoke ce hakvke te?

4 Nanvke en sulke momvteken ehvperkvn es ce 'stemerkvkvt haks? ehvperkv tat mehenwet on omat.

$5 \mathrm{Mr}$ Puyvfekvn es ece 'fastet, cem etenrvwr taken eskerkvn vtotkat, vhakv em vtotketv eteropotten, monkat vkvsvmkv apohickv eteropotten momecet o haks?

6 Eplehamet Hesaketvmesen vkvsaman, fvecetv vyoposkes, en komhoyvte etvpomen.

7 Monkv heyvn kerrvkes, mv vkvsvmkv vevkvyen fullat, matat Eplehame en hopuetaket omakes. 
8 Momen Cokat, vkvsvmkv eteropotten Centilvlke fvccrke omēn Hesaketvmeset hayvranen homvn hecet, enhomvn opunvkv-hervn Eplehamen em erkenakvtes, Ec ofvn Centilvlke omvlkvn ohmerkvke tares, maket.

9 Monkv vkvsvmkv vevkvyen fullat Eplehame vkvsvm$\mathrm{kv}$ vevkvye arvte vpvken ohmerrvkvtet os.

10 Estomomuset vhakv em vtotketv enaket omakat tvhiketv elecvn svpaklet omekv; Vhakv en nakcokv ofvn nanvke hoccat omvlkvn momecet, mv ofvn emomecicet omvkekat ohtvhikvkvtet omes, maket coket omekv.

11 Momis Hesaketvmese ehomvn este estomis vhakv eteropotten este-facev en hueretvn huereko tayat kerket os, Fvccakat vkvsvmkv eteropotten hesakvret ok'.

12 Momen vhakvt vkvsvmkv enake tokot os, momis, Mv este mvn momecat mv ofvn hesakvres.

13 Klist, epu 'yoposken tvhiketv haket, vhakv' en tvhiketv enkvpvken pu nesvtet os, Eton oh vtarkat vtekat ohtvhikvkvtet os, maket coket omekv.

14 Ohmerkv Eplehame ocvtet Cesvs Klist eteropotten Centilvlken oh vlvkvren; momet pumet vkvsvmkv eteropotten Puyvfekev em etemfvecetvt pun heckvren.

15 Tecakkvte toyatskat, este omēn opunayis; este em etemfvccetvt $o$ estomis, yekcihocen omat, este estomet yvmahkuecekot, momet naken ohvpayekot omes.

16 Momen etemfvecetvt Eplehame momet en honvpse tepakvn ete'n hahihocvtet omes. Momen, Cen honvpse taken, sulken opunvyece omēt, makekot, momis hvmkuse omēt, Cen honvpsen, mv Klist omen, maket os.

17 Momen heyvn makvyet os; Etemfvccetv Hesaketvmeset Klist ofv yekcicvte, vhakv, ohrolope cukpe osten pale tutcenen renyupv hakvtet, temfvccetvn elecet, yvmahkueceko tayes.

18 Hiyomet omekv, estet vhakv eteropottet naken puca- 
svranet on omat, ohvtvlakat etemfvecetv eteropotte tokot os; momis etemfvecetv etehoyvnen Hesaketvmeset Eplehamen mvn emvtes.

19 Monkv $m v$ vhakvt estomeckvt o haks! Vhakv kvcekvtet omecicen ohvpakvtes, $\mathrm{mv}$ honvpse etemfvccetv ete'n hahoyvtet vlvkeko emunken, momet etenherkepuecr e'nke ofvn encelvlke etehoyvnen hahoyvtes.

20 Momen etenherkepuecv hvmkuse enake tokot os; momis Hesaketvmeset hvmkuses.

21 Monkv mv vhakvit Hesaketvmese em etemfvccetvn. vnrvpet o haks? Mohmates; hesaketvn eme taye vhakvn est' emhoyvten omat, mehenwvn fvccetvt $m v$ vhakv eteropotten ocvnt omekv.

22 Momis Cokat este omvlkvn naorketv elecvn etohkalvtes, mv etemfvecetv Cesvs Klist ohvkvsvmkv ete- . hoyvne ocan vkvsvmakat omvlkvn emhoyvren.

23 Momis vkvsvmkvt vlvkeko emunkof, $m v$ vhakv elecvn pu svlvfket omvtes, mv vkvsvmkv $\mathrm{mv}$ renyupvn kerkuehocvranan pum vkhotket omvtes.

24 Monkv vkvsvmkv eteropotten fvcce omēn pu hahoyvren Klist 'sep oh ayvret mv vhakvt pu mvhayv tates.

25 Momis vkvsvmkv vlake hoyanat ohvtvlaken mvhayv elecvn vpokēkos.

26 Klist Cesvs ohvkvsvmkv eteropotten omvlkatskat Hesaketrmese en hopuetake toyatskekv.

27 Hiyomet omekv, estomomusatsken Klist ofvn ce paptishoyvten omat, Klist vccatskvtes.

28 Cusvlke monkat Klekvlket sekos; svlvfke monkat pucassekat sekos; hunvnwv monkat hoktet sekos; omvlkatskat Klist Cesvs ofvn hvmkusatskekv.

29 Monkv Klist enake toyatsken omat, Eplehame en honvpse, momet etemfvecetv verkvyen pucasvke taranat toyatskes. 


\section{SETENTVCKE IV.}

Momen heyon makvyet os; Mv epucase taranat, hopuewvt ome vtekat, vtotkv estomēn em mvrahkekot os, omvlkv e pucaset o estomis.

2 Momis oketv erke mellvte oreko vtekat mvhayvlke momet evpayvlke elecrn hueret os.

3 Momen matrpomen pumet, hopuetakuce toyeyof, nanvke yvmv ekvnv es hahoyvte elecrn pu svlvfkvket omvtes.

4 Momis oketv esfackof, Hesaketrmeset E'ppuce mahusan, hokte aossen es haye, vhakv elecvn hahoyvten atotvtes.

5 Vhakv elecv vpokvten pvlken nesetrn Hesaketvmese e'ppucetake haketv pun heckvren.

6 Momen eppucetake toyatskat omecicen E'ppuce em Puyvfekcv, Apv Pu'rke, maket huehken, ce feke take ofvn atotvtes.

7 Monkv ohvtvlaken vtotkv tokot, eppuce toyetskes; momet eppucet on omat, Klist eteropotten Hesaketvmese enake epucasvranat toyetskes.

8. Momis Hesaketvmesen kerratskekof, $\mathrm{mv}$ em munkv vcrkvyen hesaketvmese tokvkekan em vtotkatskvtes.

9 Momis hiyomat, Hesaketvmesen kerrepatskof, momet Hesaketvmeset ce kerrvkvte mahvkvu, estomaten nanvke ekvnv es hahoyvte yekcvkeko, ehvperkakan, mv hvtvm svlvfke haketvn ce yacvket, hvtvm ohfulecatske haks?

10 Nettrn, momet hvsen, momet oketvn, momet ohrolope taken vketecatsket os.

11 Ec olffuccv taken ev penkvies, ehvperkvn ece 'rahkv taken vtotketrn es ov hotose witvtes, komvyat.

12 Tecakkvte toyatskat, cemet vne omēt omatskvren 
ce'nkusapvkis, centake omēt omikv; nak' 'stomusvten vm vhopanatskekates.

13 Enhvteceskusat, enavpeswv yekcusekat eteropotten opunvkv-hervn cem erkenakvkvyvtet oman kerratskes.

14 Momen mv es ev kerretv cv 'navpeswv ofv ocvten ehvperkuecet, monkat vhepvkatskekates, momis Hesaketvmese em encelv, momet. Klist Cesvs omēn cr yrpayatskvtes.

15 Monkv ohmerkv okatskvte estrmvn oce haks? mome tayvten omat, ce turwv taken es vk ossihcet, vmatskvnt omat a cen kerrvkikv.

16 Monkv mehenwetvn cem onvyvkvyat omecicen ce'nrapv take cr haket o haks?

17 Ec ohfvecv taken vfekcakhvket os, momis herekon; a cem vkhottetvn komakes; eme ohfvccrn vfekcakhvkatske taren.

18 Momis nake herat ofvn estofis vfekcakhetv heret os, vne ece 'pakvkvyof tvlkekon.

$19 \mathrm{Vn}$ hopuetakucē, Klist ec ofv taken hahoyeko vtekat, hvtvm ce heckuecetvn vcevllvyet omat,

20 Hiyomat ece 'pakvkit, vin opunvkv en haken mvrahkuecetvn komis; ec ohfvecv taken vn kerkekokv.

21 Vhakv elecv svpvkletvn eyace ce sasvkat, vm onvyvkes, vhakrn pohatskeko?

22 Eplehamet eppucetake hokkolen, hrmket hokte svlvfket en heckuecrten, hvmkat hokte pucassekot heckuecvten ocvtes, maket coket omekv.

23 Momis mv svlvfkat heckuecvtet enavpeswv vcrkvyet omen, mv pucassekat heckuecrtet etemfvecetv vevkvyet omvtes.

$24 \mathrm{Mr}$ nanvket nake svhopakvt omakes; heyvt etemfvecetv hokkolat omakekv; hvmket ekvnhvlwe Sini avtet svlvfketv ohfvcevn heckuecat, mvt Hekv tes. 
25 Hiyomet omekv, Hekvt ekvnhvlwe Sini, Elepev ofv likat tes, momet Celuslvme mv hiyome ocet, svlvfketv ofv en hopuetaken es likat etvpomet os.

26 Momis Celuslvme hvlwe likat, pucassekot omet, omvlkeyan pu'cket omes.

27 Echusseko, heckuecetskekat, afvckvs; heckuecetskekat, vyvkhvmkusen huehkvs; ehe oce, hopuetake ocat sensulke estvmahen $\mathrm{mv}$ entvlkuse hueratet ocet omekv, makel coket omekv.

28 Tokvs, tecakkvte toyatskat, pumet Iseke omvte omeyet, setemfvecetv en hopuetake toyēs.

29 Momis momof, mv enavpeswr vcrkvyen heckvtet mv Puyvfekcr vevkvye heckvten assecvte etvpomen hiyomis momet os.

30 Momis Cokat naken maka? Mv svlvf ke hokten e'chuswv-hunvnwv tepakvn wolhuecrs, mv svlvfke hokte e'chuswvt mv hokte pucassekat e'chuswr vpvken naken epucasvranekok'.

31 Monkv, tecakkvte toyatskat, mv svlvf ke hokte echustake toyēkot, momis pucassekat enake toyēs.

\section{SETENTVCKE V.}

Monkv mv pucasseko hueretv Klist es pu'nrecapvte ofvn yekcen svpaklet, mv svlvfketv en nokwv-ohwakkvn hvtvm cem ohtvkhahkvkats.

2 Hecrs, vne Pal toyvyat heyvn ce kicvkvyet os; Ce svkvmsishoyvken omat, Klist naken cen lopihcrkekos.

3 Hiyomet ornekv, Este svkvmsishoyat vtekat hvtvm en kerkuecis, vhakv omvlkvn momecetvn em e vhuericet os.

4 Ece 'pakvkat estimvt vhakv eteropotten ce fvccehocvken omat, Klist ec ohfvecv taken ehvperkv haket os; heromkv enkvpvken a ce tvkpvlatkvkes. 
5 Pumet Puyvfekcv eteropotten fvecetv em enhonrkv vkvsvmkv etehoyvne heckan mehakeyet omekv.

6 Svkvmsiskvt monkat svkvmsiskv tokat Klist Cesvs ofvn naken lopicekon, momis vkvsvmkv, vnokeckv eteropotten vtotkat, lopicet omekv.

7 Heren pefatkatskvtes; mehenwetvn apohicatskekaren estit ce mvnvttecvkvt haks?

8 Heyv ohyekcickvt mv ce'nhuehkvkan a vtet omekot os. 9 Espakkueckv estomusis cvlvpke hvmkat omvlkvn pakkuecet os.

10 Vkerrickv etvn ocvkekares, ce komvkit, Pucase ofvn cem enhonrvkis; momis ce naoricvkat em ohfvececkvn kvwvpvres, estimvt omet o estomis.

11 Momen vnet on omat, tecakkvte toyatskat, svkvmsiskvn erkenakin omat, estomaten asseckvn es cv 'stemerke haks? Momen omat, tohweklepkv elentappetv hakvte fekhonnet os.

$12 \mathrm{Mv}$ ce naoricakat warhoyate! komis.

13 Hiyomet omekv, tecakkvte toyatskat, pucasseko hueretvn ce'nhuehokvtet os; heyv tvlkusen, mv pucasseko hueretvn enavpeswv em etektvnken hayet omekot, momis vnokeckv eteropotten ete'm vtotkvkes.

14 Vhakv omvlkvt opunvkv hvmkuse ofvn esfrcket omekv, mv, E vnokecetskat etvpomen cem vwolicvn vnokecvs, makat ofvn.

15 Momis ete'kkatsket ete'n lokatsken omat, vketecvkes, ete sumecicatskekaren.

16 Monkv heyvn makvyet os, Puyvfekev ofvn yvkvpvkes, momof enavpeswv em eyackvn esfvcecatskekares.

17 Hiyomet omekv, enavpeswrt Puyvfekcv vnrvpen eyacen, momen Puyvfekcvt enavpeswv vnrvpen eyacet os; momen heyvt ete'nrvpaket os, momat nanvke komatskat momecatskeko tayes. 
18 Momis Puyvfekcvt ce yvpayvken omat, $m v$ vhakv elecvn svpaklatskekot omes.

19 Momen enarpeswv vtotketv heckvket omet, heyvt omakes; hoktarkv, empvlse-seko hoktarkv, hvsvtketv tokat, eemfryatkv tokat,

20 Nake vhakake emekusvpkv, famvkakv, homeckv, etehvnkv, etemvcevlkv, cvpvkketv, etenrvpueckv, estetekyvmketv, estetekvpvketv,

21 Vleskvpkv, esteleckv, hackv, eshvyvtketv, momet nanvke mv omakat; $m v$ nanvke ohfvecrn cem onvyvkvyvte ocat etvpomen hvtrm homvn cem onayvkis, Estimvt nanvke mv omakan momecakat Hesaketvmese em ohmekketvt en heckvkekares.

22 Momis Puyvfeker em ettet, vnokeckv, afvcketv, herkv, momis-kometv hofunat, yvmvskv, heretv, vkvsvmkv,

23 Eyasketv, momet eemfvyatkvt omes; heyv omakat vhakv vnrapat sekot os.

24 Momen mv Klist enake take tat enavpeswr em vnokeckv em eyackv vpvken tohweklepkvn svrahakvtet os.

25 Puyvfekcv ofvn wenahokēn omat, monkv Puyvfekcr ofvn yvkvpvkeres.

26 Ete crpvkhokicekot, ete'm vleskapekot, vrakketv ehvperkan pu yahcats.

\section{SETENTVCKE. VI.}

TeCakkvte toyatskat, naorketvt esten ohcakken omat, ceme, puyvfekev verkvyen fullatskat, eyasketv vkerrickv ofvn mv oman prlken evpryvkes; cemeu es ce kerkekaren e vketecet.

2 Nake hónhoyan ete'n kvwrprkes, momet momên Klist em vhakvn esfrcecvkes. 
3 Hiyomet omekv, estet, nake tokot omof, Nake toyis, komet vkerricen omat, e emvkerret os.

4 Momis este vtekat em vtotketv mahusan es e kerrekvs, momof este etv of tokon, e ofv tvlkusen afvcketvn ocvres.

5 Este vtekat eme mahusat en nakhonnen kvwvpvranet omekv.

6 Opunvkr ofv mvhahoyat, mvt mvhayan nak herakat omvlkv ohfvecrn em onvyekvs.

7 Cem etehosikvkates: Hesaketvmese emvkerhoyekot os; estet naken fokayat mun vteloyvret ok'.

8 Hiyomet omekv, estit em enavpeswv ohfvecvn fokayat enavpeswr vcrkvyen hopvnketvn vteloyvres; momis $\mathrm{mv}$ Puyvfekev ohfvecrn fokayat, Puyvfekcv verkvyen hesaketv yuksvsekon vteloyvres.

9 Momen here momeckvn es pu hotohsates; oketv momvre orof vteloyeyvres, pu hotosaket omēkon omat. 10 Monkv, pum etektvnkof, omvlkwn heran em momecvkeres, momet vkvsvmkv en euko-vtehkvlke mahvkvu.

11 Cokv cy'nke es cen hoccicvkvyat en crpke estomuset omat hecatsket os.

12 Este estohmomuset em enavpeswr ofv heekv here haketvn komakat, mvt, Klist em etohweklepkv vrahkvn assehocekare tvlkusen, ce svkvmsishoyvren ec ohyekcieaket os.

13 Hiyomet omekv, mv svkvmsishoyvte mahusat vhakvn vfvstukekos; momis ce svkvmsishoyvren komakes, ce 'navpeswr take ofvn es ekvsvmvkvret.

14 Momis vn tat naken es ekvsahmvyates, pu Pucase Cesvs Klist em etohweklepkv ofv tokon omat, mv eteropotten ve ohfvecvn ekwnvt etohweklepkvn svrahken, vneu ekvnv ohfvecvn sve vrahket os.

15 Svkvmsiskv, monkat svkvmsiskv tokat Klist Cesvs 
ofvn naken lopicekon, nak hahoyvte mucvsehocat tvlkuset lopicet omekv.

16 Momen estomomuse heyv mvhakv vcrkvye yvkvpakat, mvn, momet Hesaketvmese em Eslevl, herkv merkv tepakat oh ocvkekvs.

17 Mucv vtekat estit cv naorihcats, Pucase Cesvs es en kerkv ev 'na vlumhen sarikv.

18 Tecakkvte toyatskat, pu Pucase Cesvs Klist en heromkvt cem puyvfekev taken vpvkekvs.

Kelesvlken ohtotet Lome ofvn hoccihocrtes. 


\section{COKV VPASTVL PAL \\ EFESVNVLKE OHTOTVTE. \\ SETENTVCKE I.}

PaL, Cesvs Klist em vpastvl toyvyat, Hesaketvmese komat vcrkvyen, emekusapvlke Efesvs tvlofv vpokat, momet Klist Cesvs ofv em enhonrvke tayat oh 'totakis.

2 Heromkv momet herkv Hesaketvmese Purke, momet Pucase Cesvs Klist a enkvpvket ece 'pakvkekvs.

3 Hesaketvmese, momet Cesvs Klist pu Pucase Erken vrakkuehocekvs, mvt puyvfekev merretv hvlwe ocat omvlkvn Klist ofvn es ep oh merrvtes.

$4 \mathrm{Pu}$ hvsvthvke, em mvttvkeko, vnokeckv ofv ehomv fulleyvren, ekvnv hocackeko munken eme ofv pu'nhopoyeprte omen.

5 Em eyackv yvmaskusat rcvkvyen, homvn pu mellvten Cesvs Klist eteropotten en hopuetakuce hakeyvren.

6 En heromkv en rakketv vkvsvmkvren, mv ofvn Vnokeckate ofvn em vlostet pu hayvtes.

$7 \mathrm{Mv}$ ofvn, e catv eteropotten nesketr momet holwryeckv enkvpryecickv oceyet os, en heromkv en sulke vevkvyen.

$8 \mathrm{Mv}$ ofvn sulke mahen pum ocvtes, nakkerretv hoporrenkvu omvlkv ofvn. 
9 Ehke nake komat pun kerkuecvtet, mv eme ofv en yvmvsvkares komvte vevkvyen omvtes.

10 Afvstetv oketv esfvcfakof, hvlwe ocakat, momet ekvnv ocakat omvlkv Klist eme ofvn hvmkusen vteloyvres ;

11 Eme ofv etan punake taranvten heckueceyvtes, homvn pu mellvkvtet omen, momecares komvte vcrkvyen, mvt em eyackv hoporrenkv vcrkvyen nak' omvlkvn vtotket omes ;

12 Pume, enhvteceskv Klist ofv enhonrkv hayeyvtet, en rakke vkvsvmkvren fulleyvren.

$13 \mathrm{Mv}$ ofvn centakeu vfekrakkatskvtes, mehenwv em opunvkv, cem vhesaketv opunvkv-hervn pohatskvte renyupvn, temfvccukvte Puyvfekcv Vcakat vkvsamatskat rohyvpvn es ce kerkuecrkvtes.

14 Mvt punake taranat eskerkvt omes, nake neshoyvte e enakueckv, momet en rakketv vkvsvmkv oren.

15 Monkv vneu, Pucase Cesvs ofvn vkvsvmkv, momet mekusapvlke omvlkv vnokeckv em ocatskat pohvyvte rohyupvn,

$16 \mathrm{Vm}$ emekusvpkv ofvn ec ohfvecv take vkerrickv hayit, momet 'sece 'rahkv take vkvsvmkvn hayvyat wikvkot os ;

$17 \mathrm{Pu}$ Pucase Cesvs Klist en Hesaketvmese, rakketv Erket hoporrenkv, momet kerkueckv puyvfekev ce'mvkvren, eme kerretv ofvn.

18 Cem vkerrickv take e turwvt hvyayihocen, cemet en huehketv enhonrkv nake tat, momet en nakockv mekusapvlke ofv en rakketv en sulke tat,

19 Momet vkvs ımeyat ohfvecv en yekce en rakke estvmahe nake tat kerratskvren, en yekce, rakke em vtotketv vevkvyen.

20 Mvn emet, Klist ofv momecrtes, prsatkvte enkvpv- 
ken a kvwapof, momet hvlhawat ofvn em akvpervn licet,

21 Ohfvnketv, yekcetv, nekeyickv, pucasetv, momet hocef $k v$ yvmv ekvnv ofv tvlkekon, momis mv vlvkvranat tis ofvn hocef hoyat omvlkv onvprn licet;

- 22 Momet nak' omvlkv ele elecrn vpohyet, emen mekusvpkv-cukon emet, ekv nak' omvlkv ofv taranen.

$23 \mathrm{Mv}$ emekusvpkv-cuko enat omet, mv omvlkv ofvn omvlkvn fveficat en sulke tes.

\section{SETENTVCKE II.}

Momes naorketv, holwvyeckv ofvn ce pvsvtkakvte ce wenahuecvket os.

$2 \mathrm{Mr}$ ofvn fullatskvte ocet os, yvmv ekvnv vretv vcrkvyen, hotvle en yekcetv em mekko, puyvfekcr hopuetake apohicvkekat ofvn vtotkat vcrkvyen fullet.

$3 \mathrm{Mv}$ vpvket pumeu omvlkeyat pum vpeswv naken komat ofvn etefulleyvte ocet os, vpeswr momet vkerrickv em eyackvn fveficet, momet pum emunkv vcrkvyen crprkketv en hopuetake vprltake omet omeyvtes.

4 Momis, Hesaketvmese merretv ofv nake oce likat em vnokeckv rakke,

5 Naorketv ofvn pu prsatkvte omof tis, 'sepu 'nokecrte svrahkvt Klist.vpvken a pu hesahuecvtes; (heromkv es ee hesahokes ;)

6 Momet tohkvlken $p u$ wenahuehcet, hvlhawat ofv Klist. Cesvs ofvn tohkvlke vpoken pu hayvtes;

7 Cesvs Klist eteropotten yvmvskv epohfvecr ocat ofvn, en heromkv en sulke estvmahen heckuecvret, oketv yicvranat ofvn.

8 Vkvsvmkv eteropotten, heromkv es ce hesahoket omekv; momen ceme ce teropotte tọkos; Hesaketvmese em estemkvt's. 
9 Vtotketv eteropotte tokon, estimvt 'sekvsvmekaren. 10 Pumet em vtotketv toyekv, Klist Cesvs ofvn hocvekvtet, vtotketv herakat svrahkv, mv Hesaketvmese homv tetakuecvten, mv ofvn fulleyvren.

11 Monkv, vpeswv ofvn Centilvlket omatsken, mv, vpeswv ofvn enke eshoyat Svkvmsesvn kihocat, Svkvmsesvntokon ce kicaken, fullatskvten vkerricvkes,

12 Momet mv omof, Klist acvkekot, Eslevlke em etvlwv ohfvecv tvleporvlket, temfvecetv temfvecakvten kerrvkekot, enhonrkvn ocvkekot, Hesaketvmeseu ocvkekot ekvnv ofv omatskvten vkerricvkes.

13 Momis Klist Cesvs eteropotten, cemet, hopvyen fullatskvte ocan, hiyomat Klist e catv eteropotten vwolicen ce hahoyvket os.

14 Emet pun herkvt omekv; hokkolvn hvmkusen hahyet tohopke setekvpickvn lekafvtes.

15 Em vpeswr ofvn homeckv, nakvfvstetv melhoyvte em vhakvn sumecihcet, eme ofv hokkolat este hvmke mucrsen hayvret, herkvn hayet,

16 Momet hokkolvn ena hrmke ofvn tohweklepkv aeteropotten Hesaketvmese em etenherkepuecvret, mv ofvn homeckvn elehcet,

-17 Momet vlahket, centake hopvye fullatskvten, momet vwolicakaten herkvn em erkenvkakvtes.

18 Momen eme etehoyvnen Puyvfekev hvmke ofvn hokkoleyat Erken oh vhoyeye tetayat ocet os.

19 Monkv hiyomat ohvtvlakat este kerkvkeko, tvleporvlke toyatskekot, momis mekusapvlke vpvke tvlofvlke, Hesaketvmese en cuko-vtehkvlke toyatskes.

20 Momet vpastvlvlke, momet owalvlke en likvn oh hakatsket os, Cesvs Klist mahusat vkvnowv-cvtot omen.

$21 \mathrm{Mv}$ ofvn cuko omvlkvt heren eteliket, Hesaketvmese en cuko vcake hakat oren ohvtvlaket os. 
$22 \mathrm{Mv}$ ofvn cemeu etelike hakatsket os, Puyvfekev eteropotten, Hesaketvmese e hute hakatskvret.

\section{SETENTVCKE III.}

Heyv esvrahkvn, Pal, Cesvs Klist em este svlvf kuehocat toyvyat, Centilvlke toyatskat svrahkv,

2 Hesaketvmese en heromkv vfvstetv cemetake ohfvecv a'muten pohatskvten omat,

3 Kerkueckv eteropotten nake ehkat vn kerkuecvtet omen, opunvkv nvcomuse ofvn hoccicvyvte omet,

$4 \mathrm{Mv}$ eteropotten, mvn hecatskof, Klist en nake ehkat kerrvyat kerratskvres.

$5 \mathrm{Mv}$ hiyomat, Puyvfekev aetehoyvnen vpastvlvlke veacakat, owalvlke esyomat en kerkuehocat omen, honvpse hoyvnakvte ofvn este en hopuetake en kerkuehocekates.

6 Centilvlke vpvkvke pucasvlket ot, ena hvmkuset, momet Klist ofv opunvkv-herv aetehoyvnen em etemfvccety tohkvlket crwvkvren.

7 Hesaketvmese en heromkv em estemkv en yekcetv em vtotketv eteropotten a'mvte vcrkvyen mvn opunvkvherv vfastv es cv hakvtes.

8 Vnen, mekusapvlke lopuckus mahat 'sen cutkusat toyvyat, heyv heromkv a'met os, Centilvlke vpvken Klist en nake-ockv enrvmrvkeko tayat erkenvkaren;

9 Momet nake ehkat em etohkvlkètvn, mv ekvnv enhvteceskv vteken Hesaketvmese, mv Klist etehoyvnen nake omvlkv hahicvte ofvn ehkvten este omvlkvn hecvkuecaren.

10 Hiyomat, mekusvpkv-cuko eteropotten, Hesaketvmese hoporrenkv mvrahrvkv sulke estvmahen ocat, hvlhawat ofvn mekkvlke, ohfvnkakat esyomat en kerrvke taren, 
11 Meyuksv-seko komvte, mr pu Pucase Cesvs Klist ofvn komvte, verkvyen.

12 Eme ohvkvsvmkv eteropotten fekhvmketv momet enhonrkv ofvn oh vpeyeye tetayat oceyet os.

13 Monkv vm estemerketv 'sece 'rahkv take svrahkvn ce hotosvkekaren komis; mv estemerketv cem vrakketv taket omakes.

14 Heyv esvrahkvn pu Pucase Cesvs Klist Erke ehomvn cr torkowvn pekohlicis ;

15 Cuko-hvmecvlke omvlkvn, hvlwe, ekvnv ofvu mvn es hocef huehocet os ;

16 En rakketv en sulsuke vcrkvyen em Puyvfekcv eteropotten, ofv-este ofvn yekcetv es ce yekcvkuehocvren ce'mvkvren emekusapis.

17 Vkvsvmkv eteropotten Klist ce feke take ofvn likvren, momat vnokeckvn likvn oh hayet, $\mathrm{mv}$ ofvn yvlonkvn hayet,

18 Mekusapvlke omvlkv vpvket en tvphe, en crpke, en sufke, en hvlwe kerratske tayvren,

19 Momet Klist em rnokeckv kerresko tayat kerratsket, Hesaketvmese em fvcketv omvlkvn es ce fvcfvke taren.

20 Hiyomat $\mathrm{mv}$ omvlkvn vpoheyat, momet vkerriceyat 'sen hoyvne estvmahen momece tayat, yekcetv ep ofvn vtotkat vcrkvyen,

$21 \mathrm{Mv}$, mekusvpkv-cuko ofvn Cesvs Klist eteropotten, oketr omvlkv etehoyvnen, ekvnv meyuksv-sekon, rakketvt em ocekvs. Emen.

\section{SETENTVCKE IV.}

Monkv vne, Pucase em este svlvf kuehocat toyvyat, huehketv es cen huehhokvkat oren fullatskvren cen kusapvkis,

2 Ekvncrpueckv omvlkv, eyasketv momet momis-ko- 
metv ocet, cem etenrvwr take vnokeckr ofvn e en hvlatet,

3 Puyvfekcv hvmkusetv vevyecetv vcevllvket, herkv semetelvckv ofvn.

4 Ena hvmken, momet puyvfekev hvmket os; cen huehhokvkat enhonrkv hvmkuse ofv cen huehhokvte etvpomen.

5 Pucase hvmken, vkvsvmkv hvmken, paptesvm hvmken.

6 Omvlkv Erke, momet Hesaketvmese hvmket, omvlkv 'sen hvlwe, omvlkv eteropotte, momet omvlkatskat ofvt's.

7 Momis epu'rahrvkvn, Klist em estemkv svhopakv vevkvyen, heromkvn pu'mhoyet os.

8 Monkv hvlwen vkvwapkof, svlvf ketvn svlvf kuehcet, emkv est' emvtes, maket os.

9 Tokvs, vkvwapkat naket o haks? homvn ekvnr sufsoke mahan vk hvtvpkvte tokon omat?

10 Eme hvtapkatet, mv nake omvlkvn fvcficvret, hvlhawat omvlkv'sen hvlwe vkvwvpkvtet, matat omes.

11 Momet emakvte vpastvlvlke sasen, owalvlke sasen, punvkv-herv es fullvlke sasen, erkenvkvlke mekusvpkvcuko vhecicvlke momet mvhayvlke sases.

12 Mekusapvlke mvherickv svrahkvn, momet ohvfvstetv vtotketv, momet Klist em ena here tohtvretv svrahkvn;

13 Momet emunken omvlkeyat vkvsvmkv, momet Hesaketvmese Eppuce kerretv hvmkusetv este-hunvnwv etemvpoken, momet Klist em fvcketv rakketv svhopakvn roriceyvres.

14 Svnvcomvn hopuetake fvllehole, momet mvhayetr en hotvle vtekan es pu fulhoyekaren, momet este emvkerretv, momet en cvfeknetv sehosickv, esenkvnhetvt omekv.

15 Momis vnokeckv ofvn mehenwrn opunahoyet, nake 
omvlkv ofvn, eme esceyet mahvkeres, Klist, mvt ekvt ok'.

$16 \mathrm{Mv}$ aenkvpvken ena hvmkvt etetayusen etelvcvket omet, momet teweteknuset omet, mv eteropotten etetekkekv vtekat emvnicet omes, vrahkv vtekat vhopake vtotkat vevkvyen, mvt, ena ohvtvlayet vnokeckv ofv e etohtvretv svrahkvn.

17 Monkv heyvn makit kerkuecis, Pucase ofvn, Centilvlke vpvltake em vkerrickv ehvperkv ofvn fullakat omet mucv vtekat fullatskekaren.

18 En kerretvn ohyomucecicet, Hesaketvmese vhesaketv en lvpvtket omakes nakkerreko vtehtekat eteropotten, e feke take em wrnhet omecicen.

$19 \mathrm{Mv}$ feke-nokkat kerreko hahket, holwvyeckvn e em e wikaket os, vnoksetv ofvn nake hvsvtkekat omvlkv vfvstvkvret.

20 Momis momēn Klist kerratskvtet omekos.

21 Cesvs pohatskvten, emet ce mvhayvkvten omat, Cesvs ofv mehenwv ocat vcrkvyen.

22 Fullatskvte ocat ohfvecvn este vculat etvn ocvkes, mvt ejackv ehosicat vcrkvyen hopvnkes,

23 Momet mucvsvks cem vkerrickv em puyvfekev ofvn, 24 Momet este mucvsat vccrks, mv Hesaketvmese vcrkvyen fvccetv momet hvsvtketv mehenwat ofvn hocackat.

25 Monkv lvksetvn etvn ocvks; omvlkatskat, cem vwolicvn mehenwvn tempunahoyvkes, etelvcet omvkekv. 26 Ce crpvkhokis, naorikatskvs; ce crpvkhoke emunken hvse vklvtikats.

27 Momet Puyvfekcv Holwakat em etektrnehcatskvs.

$28 \mathrm{Mv}$ horkopvte hvtvm horkohpāts, momis vtotketv min nake heran enken es rtotkekvs, este nake enkusapkusat eme tetayet ocvret. 
29 Opunvkv hvsvtkekat hrmkusis ce cukwrn osiyats; momis mv herat, tohtvrkv emvnickv svrahkv, pohakat heromkv emvret.

30 Momet Hesaketrmese em Puyvfekcr Vcakat elesecihcatskvs, mv neskv nettv oren es ce kerkuehocat.

31 Home-opunvkv, homeckv, crpvkketv, estoke-mahetv, momet vtvkleckv cen kvpakvkekvs, momet holwvyeckv omvlkvn.

32 Momet ete'n heromvket, ce feke lowvcloket, ete'm merrvket omvkes, Klist svrahkv Hesaketvmeset cem merrvkyte etvpomen.

\section{SETENTVCKE V.}

Monkv hopuetakuce vnokeckillke omaket, Hesaketrmese . vevkfullvlket omvkes.

2 Momet vnokeckv ofvn fullvkes, Klist epu 'nokecvte omen, momet e pum e wikvtes, Hiesaketvmese emhoyvre, momet nake ehomv elehocvre fvmecusat svrahkvn.

3 Momis hoktarkv, nake hvsvtkekat omvlkvn, holvckv tis hvmkis ce tenrvwr take hocefikats, mekusapvlke momvret omekv.

4 Vretv svholwvke tis, opunvkv hacohake tis, este' $n$ lopiceko tayat, momis vkvsvmkv emetv min mecvks.

5 Heyvn kerratsket omekv, hoktarv hvmkis, momet este hrsvtkekat, momet nake vholacv, mv nake-vhake mekusapvt omat, Klist momet Hesaketvmese etvlwv ofvn nake epucasvranat ocekot os.

6 Estimvt opunvkv ehvperkvke es ce mvkeriyvkats, hcyv nanvke omecicen Hesaketvmese en crpvkket hopuetake apohicrkekat oh vlaket omekv.

7 Monkv em vpahkatskvs.

8 Yomucke toyatske ocvtet omekv, momis hiyomat 
Pucase ofvn hryayvke toyatskes; hryayvke hopuetake , omet fullvkes.

9 (Puyvfekcv em ette herketv omvlkv, momet fvecetv, momet mehenwv ofvt omekv;)

10 Nake Hesaketrmese afvcecice tayat eskerratsket fullvkes.

11 Momet yomucke em vtotketv ettrkekat ofvn naken temohcatskvs, momis vnrvpvkes.

12 Nake ehken vfvstakat opunvyecetv tis vlesketv vpvket omekv.

13 Momis nake vnrvphoyat omvlkvn hvyayvke, eteropotten heckvket os; naken hecken hayat vtekat hvyayrke tes.

14 Monkv, Nocetskat, vhonehcet, prsvtkvten a en kvwvpkvs, mohmen Klist hryayvken ce'mvres maket os.

15 Monkv vketecvkes, vhericet fullatskvret, hoporrenvkekat omekot, hoporrenakat omet;

16 Oketvn nesaket, nettvt holwahoket omekv.

17 Monkv naken kerrvkekot ohmatskvs, momis Hesaketvmese naken komat kerrvket omvkes.

18 Momet prrko-opuswr es hacahkatskvs, mv ofv yvmahkueckv ocat, momis Puyvfekcrt es ce fvcfvkekvs;

19 Yvhiketv, vkvsvmkv-yvhiketv, momet puyvfekev ohfvecr yvhiketvn 'se em punahoyet, yvhikaket, ce feke ofvn Hesaketvmesen vkvsvmaket,

20 Nake omvlkv svrahkvn vkvsvmkvn emake emunkvt, pu Pucase Cesvs Klist e hocefkv ofvn Hesaketvmesen momet Erken vkvsvmaket;

21 Hesaketvmese em penkvlkv ofvn ete'm apohicrket omvkes.

22 Ehitake, cehetaken em apohicrks, Pucase Cesvs ohfvecrt omen.

23 Ehet ehiwv e'kvt omekv, Klist mekusvpkv-cuko 
e'kvt omat etrpomen; momet emet ena Hesayecvt omes.

24 Monkv mekusvpkv-cuko Klist em apohicat omen, matvpomen ehitake ehetaken nak' omvlkv ofvn em apohicvkelves.

25 Ehetake, cehitaken vnokecvkes, Klist mekusvpkvcukon vnokecet, e em e wikvte etvpomen.

26 Opunvkv eteropotten uewv es okkosetvn es hvsvtecet, vcaken hayvret,

27 Emekusvpkv-cuko vrakkuehocvre tetayen, svholwvke cutkusis, momet yucke hvmkusis, momet mv omakat hvmkusis ocekon e em e heckuecvret, momet svholwvkekot, vcake taren.

28 Ehetake eme ena vnokecakat etvpomen e hitaken vnokecvkvranet os. Estimvt e hiwvn vnokecat e vnokecet os.

29 Estimvt estofvn ena-vpeswv en homecvte sekot ome$\mathrm{kv}$, momis vfastet vcayecet os, Pucase mekusvpkv-cuko em momecat etvpomen.

30 Pucase ena, ena-vpeswv, momet e funen etelvcrket omekv.

31 Heyv esvrahkvn estet erken, ecken wikvres, momet e hiwvn etelvevres, momen $\mathrm{mv}$ hokkolat ena-vpeswv hvmkusvres.

32 Heyvt nake ehke rakket omes; momis Klist mekusvpkv-cuko tepakan opunvyecit okis.

33 Mome estomis omvlkatskat e vnokecat etvpomen omvlkvt e hiwvn vnokecekvs, momen ehiwvt e hen vrakkuecekvs.

\section{SETENTVCKE VI.}

Hopuetake, ce'ckvlken em apohicvks, Pucase ofvn, heyvt fvecvt omekv. 
2 C'erken hvtvm ce'cken vrakkuecrs, mvt vhakv temfvccetv ocat enhvteceskv tes.

3 Cen hervren, momet hofunen ekvnvn oh vretskvret.

4 Momen erkvlke toyatskat, cen hopuetaken crpvkhokihcatskvs, momis Pucase ofvn mahecet, mvhayet, vfvstvkes.

5 Vtotkvlkē, ena-vpeswr vcrkvye. ce pucasvken em apohicvks, penkvlkv fekeketv vprken, ce feke em mehenwetv ofvn, Klist ohfvecrt o omen;

6 Renakv vtotketv tokon, este afvcecicvlke omekot, momis Klist em vtotkvlke omet, efeke vtet Hesaketvmese nake komat momecvket,

7 Vkerrickv heren Pucasen em vtotkvks, momet este tokon.

8 Heyvn kerret, este estomet nake estomis momecen omat, Pucase vtet matan en heckvres, pucase oce tis, pucasseko tis.

9 Momen pucasvlkè, matan em momecvks, ce'stemerricares maketvn e en hvlatet, momet heyvn kerret, cemeu ce Pucase take hvlwe ofvn likes, momet este etemetueckv en sekot os.

10 Espoken, tecrkkvyvte, Pucase ofvn ce yekcrkekvs, momet en yekcetv en yekce ofvn.

11 Setepokv Hesaketvmese est'emat omvlkvn vchoyvks, Puyvfekcv Holwakat em mvkerretv vnrvpen svpaklatske tayvret.

12 Catv, vpeswr tis ete'n cevlhoyet omēkokv, momis ohfvnkvlke vnrvpet, yekcetv ocakat vnrvpet, yvmv ekvnv en yomucke evpryvlken vnrvpet, puyvfekev holwryeckv es hrlhawat vnrvpet, en cevlhoyet os.

13 Monkv setepokv Hesaketvmese est'emat omvlkvn crwepvks, nettv holwvhokat ofvn est' vnrapatske tayvret, momet nak' omvlkvn momecatskof, svpaklatske tayvres. 
14 Monkv svpvklvkes, ce tetackvn mehenwvn es wvnawicet, fvccetv hokpe-svrvnakvn vlumhicet,

15 Momet opunvkv-herv herkv emetetaketvn ce.'letaken vtehvket,

16 Omvlkv emuntalat, vkvsvmkv sevrvnvkvn ocatskofvt, mvn holwvyecv em fvske fenkakat omvlkvn es vslecatske tayvres.

17 Vhesaketv sekvpvtukvn crwvkes, momet Puyvfekcv em eslafkv-cvpkon, mvt Hesaketvmese em opunvkvt omes.

18 Estofis Puyvfekev ofvn kusvpkv omvlkvn, momet mekusvpkvn es emekusvpaket, momet mv ohfvccvn momis-kometv omvlkvn, momet emekusapvlke omvlkvn em emekusapet es vketecaket,

19 Momet vm emekusvpatsket punvkvn v'mhoyvren, fekhvmket cr cukwvn hvwvklecaren, opunvkv-herv en nakeehkat kerkuecaren,

$20 \mathrm{Mv}$ esvrahkvn este-en-wiketv svlvf kuehocat toyvyat, $\mathrm{mv}$ ofvn fekhvmkit, opunvyare tetayat oren opunvyaren.

21 Momen vn nakvfvstetvn, momet estomen arvyat kerratskvre svrahkv, Tekekvs, tecakkvte vnokecke, momet Pucase ofv vtotkv enhonrvke tetayat, nak' omvlkvn cen kerkuecvkvres.

22 Mata svrahkvn mvn ec oh 'totvkvyvtes pun nakvfvstetvn kerratskvren, momen ce feke taken emahlvpvtvkvren.

23 Tecrkkakvte herkvt em ocvkekvs, momet vkvsvmkv vpvke vnokeckvt Hesaketvmese, Erke, momet Pucase Cesvs Klist aenkvpvket.

24 Vnokeckv vhopvnkeko tetayat pu Pucase Cesvs Klist 'svnokecakat omvlkvn heromkvt vpvkvkekvs. Emen.

q Pal Heyv cokv Lom ofvn coyvtes momet Efesvnvlken Tekekvs 'soh 'totvtes. 



\section{COKV VPASTEL PAL}

\section{FELEP V LKE，KOLASVLKE,}

\section{RESVLONIKVLKE I. \& II.,}

\section{TEMORE I.\& II., \& FILEMVN.}

THE EPISTLES OF PAUL THE APOSTLE TO THE

PHILIPPIANS, COLOSSIANS, I. \& II. THESSALONIANS,

I. \& II. TIMOTHY, \& PHILEMON. TRANSLATED

FROM THE ORIGINAL GREEK

INTO THE MUSKOKEE LANGUAG̣E.

\section{NEW YORK :}

AMERICAN BIBLE SOCIETY, INSTITUTED IN THE YEAR MDCCCXVI.

[Muskokee, 16mo.]

$$
1885 .
$$




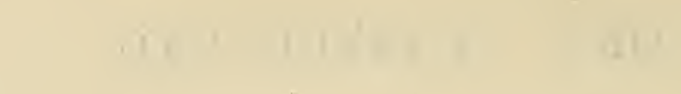

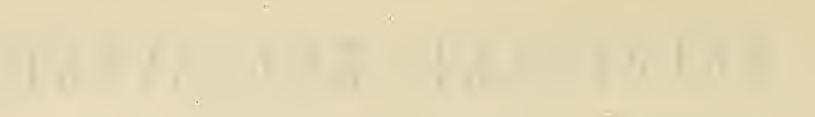

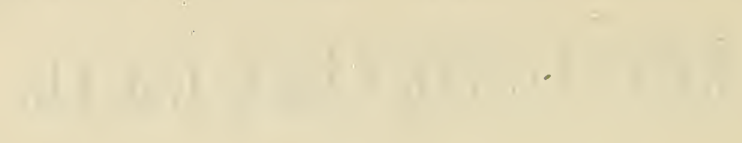

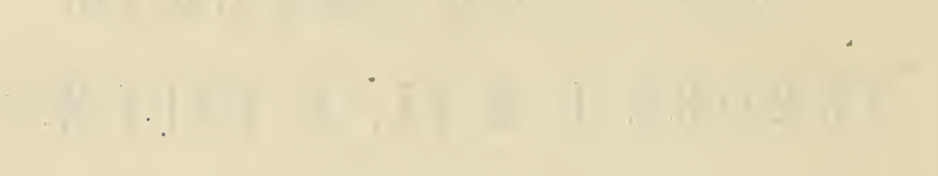

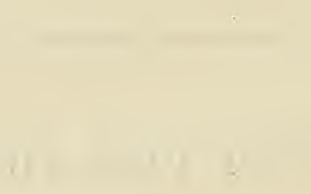

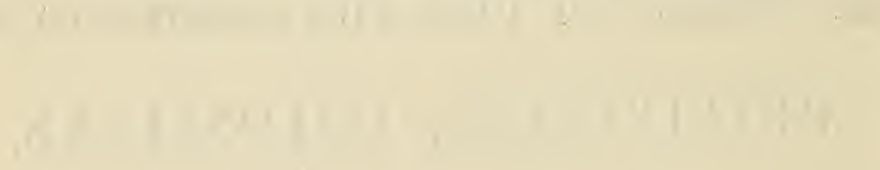

-

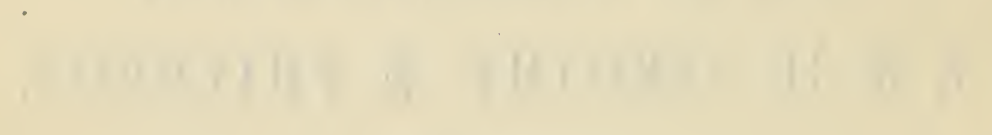

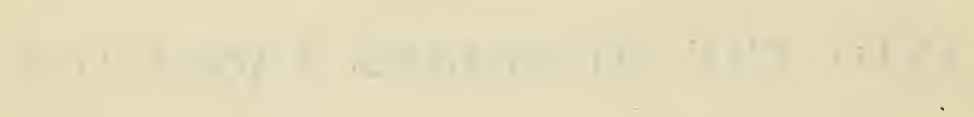




\section{COKV VPASTEL PAL}

\section{FELEPVLKE OHTOTVTE.}

\section{SETENTVCKE I.}

Cesvs Klist em vtotkvlke, Pal Temore tepakat Klist Cesvs ofv mekusapvlke Felepi tvlofv vpokat omvlkvn, momet mekusvpkvcuko vhecicvlke tekvnvlke esyoman ohtotaket os.

2 Heromkv momet herkv Hesaketvmese Pu'rke, momet Cesvs Klist akvprket cem ocvkekvs.

3 Estofv estomis ece 'kerricvkvyof vn Hesaketvmesen rkvsamis,

4 Estofis omvlkatskat cem emekusapvkvyat vtekat vc afvcket vpohkvn hayit,

5 Opunvkv-herv ofv etohkvlketv nettv enhvteceskv vteke hiyome oren ocatskat vrahkvn vc afvcket omekv.

6 Heyr mahusan komvye heret omit, mv vtotketv heren ec ofv take vlicecrtet Cesvs Klist en nettr eroren momecvres ;

7 Momvre heren omvlkatskat heyv ec ohfvecr take vkerricvye tayat etvpomen, cv feke ofvn ce 'tehvkikv; hiyomet omekv, vm eswrnakv, momet opunvkv-herv vpryeckv yekcickv tepakat ofvn ve vpakatsken heromkv etencawvlke toyēs.

8 Cesvs Klist envcofv ofvn estomēn ce yacrkvyat, Hesaketvmeset a vn kerrvt omekv.

9 Momen cem vnokeckv taket nakkerretv momet hoporrenkv omvlkv ofvn ohvtvlake mahvren; 
10 Momet nak' hervke mahan frccecet, mehenwuset, momet cem mvttvkeko emunket,

11 Fvecetv em ette Cesvs Klist eteropotte oce, momet Hesaketvmese vrakketv vkvsvmkv tepakan omecicakat es ce fvcfaket emunken, Klist en nettvt vlvkvren, heyvn mekusapis.

12 Momis, tecakkvte toyatskat, heyvn kerratskvren komis, mv nake vm momakat opunvkv-herv ohvtvlaket ayvre min momakvtes:

13 Momat vn svlvfketv Klist ofv ocat mekko en cukoherv ofv hvmkvn, momet etv estvmv estomis heren kerhoyet os ;

14 Momen Pucase ofv tecakkvte sulket vn svlvfketvt omecicen, enhonrvke hahket, penkvlkv ocekot, Hesaketvmese em opunvkvn punvyetv fekhvmketv em ohvtvlvkvket os.

15 Mehenwvn vleskrpkvt momet etenrvpkvt omecicen Klist es erkenakat sasen; momis vpulwvt kometv herat omecicen erkenvkakes:

16 Hvmket, mehenwetv ofv tokon, etenrvpkvt omecicen, vn svlvfiketvn estemerkvn ohvpryetvn komet, Klist es erkenvkaket os :

17 Momis hvmkat, vnokeckvt omecicen, opunvkv-herv vpvyeckv vrahkvn ev huerihocet omen kerret, erkenvkakes.

18 Monkv estome te? Estomet o estomis, mvkerretvt monkat mehenwetvt omecicet o estomis, Klist es erkenvkhoyet os, momen mv ofvn ve afvckes, momes, momet ve afvcke tares.

19 Hiyomet omekv, cem mekusvpkv take, momet Cesvs Klist em Puyvfekev vm vnicat eteropotten heyvt vm vhesaketv ohfvccun vnicvranat kerris;

20 Neneheckv yekcan, momet enhonrkv ocvyat vcrkvyen, 
nake hvmkusis ohfvecvn vc vlsekon, momis estofis ocvte etvpomen hiyomat fekhvmketv omvlkv ofvn, hesaketv etehoyvnen, monkat elkv etehoyvnen, Klist cv'na ofvn vrakkuehocvren komit enhonris.

$21 \mathrm{Vc}$ vrahkvt on omat, hesaketvt Klist tot omen, eletvt eohvtvlvyetvt omekv.

22 Momis enavpeswv ofvn cv wenaken omat, heyvt vm vtotketv em ettet os, momen naken enhopoyrranvyat kerrvkos.

23 Hiyomet omekv, nake hokkole em etenrvwvn vc ohyekcihocet on, yvmvn enkvpaket, Klist vpvketvn komis; mvt semvmome estvmahet omen:

24 Mome estomis, ece 'rahkv taken, enavpeswr ofvn fekhonnvyat nake momvre here mahat os.

25 Momen heyv enhonrkvn ocvyat, omvlkatskat, cem vnickv take, momet vkvsvmkv afvcketv svrahkvn, ece 'pakvkit likit, fekhonnvranvyat kerris;

26 Momat hvtvin cem vlakvkvyat omecicen, cem afveketv take vc vrahkv ocat, Klist Cesvis ofvn ohvtvlvkvren.

27 Klist em opunvkv-herv vcrkvyen fullatske tvlkusekvs, momat vlakit ce hecvkin omat, monkat vlvkvkis, nake ec ohfvecv take ocakat, estomen puyvfekev hvmkuse ofvn, momet vkerrickv hvmkuse ofvn svpaklet, opunvkv-herv vkvsvmkv ohfvecvn tohkvlket vcevllatskan pohares;

28 Momet ce'nrapvlket omecicen nake estomis ohfvecrn empenkvlatskekan pohares; mvt en sumketv take ohfvecv eskerkvt omen, momis ec ohfvecv taken vhesaketv eskerkvt os, momet Hesaketvmesen avtet os.

29 Hiyomet omekv, mv etepokv ve ofvn hecatskvten, momet hiyomat vc ofvt on pohatskat mahusan ocet,

30 Klist vrahkvn ohvkvsvmvranatskat tvlkekon, momis mv vrahkvn ce 'stemerkvkvren ce'mhoyvket os. 


\section{SETENTVCKE II.}

Monkv Klist ofvn mahlvpvtketv estomet, monkat vnokeckv em afvcketv, monkat Puyvfekcr vpvketv, monkat envcofv yvmasketv, momet merkv ocaken omat,

2 Vkerrickv mv omakan, momet mata vnokeckvn ocet, vkerrickv hvmkusen, momet kometv hvmkusen ocet, vm afvcketvn fvcecvkes.

3 Etenrvpkv, monkat ehvperkv ekvsvmkv eteropotten naken momehohcats, momis eyasketv ofvn este estomis, vpvltaket es vn hervket os, komet, vkerricekvs.

4 Este vtekat, enanvke tokon, momis este eti enanvken mvu ohvkerricekvs.

5 Heyv vkerrickvt, mv Klist Cesvs ofvn ocvtet, ece 'pikvkekvs.

6 Mvt, Hesaketvmese vhake tat, Hesaketvmese etvpometv nake vpenkvleckvt's, komekates :

7 Momis, hocefkv pohkekon e en hayet, vtotkv en heckvn vciyet, este omē hakvtes:

8 Momen este en heckvn ocen eshechoyof, e kvncvpuehcet, elkv, tohweklepkv elkv tis oren, apohice hakvtes.

9 Momen monkv Hesaketvmeset tayer hvlwehcet, hocef kv hocefkv etv vtekat senhvlwen e'mvtet os;

10 Momat Cesvs e hocefkvt pohkof, hvlwe fullat, momet yvmv ekvnv fullat, momet ekvnv elecv fullat e torkowv vtekat pvkorvres;

11 Momet etulaswv vtekat, Erke Hesaketvmese vrakkueckvn, Cesvs Klist Pucase tes, maket kerkuecvres.

12 Monkv, vm vnokeckatskat, ece 'pakvkvyof vm apohicatskvte tvlkekon, momis hiyomat ece 'pakvkvkat min senhoyvne estvmahen $v m$ apohicatske omēn, penkvlkv momet fekeketv vpvken cem vhesaketv mahusan esfvcken vtotkvkes ; 
$13 \mathrm{Mv}$ ec ofv taken, en heromkv vrahkvn, momecetv momet kometv tepake vtotkat Hesaketrmeset omekv.

$14 \mathrm{Ecakv}$ momet punvkv setenrvpkv sekon nanvke omvlkvn momecvkes :

15 Momat cem mvttvkeko momet fvccvket, Hesaketvmese e'ppucetake, Cem muttvkes, ce kihoceko tetayat etvlwv ekvyekce momet yenahat, mv vpvkaken ekvnv ofv hryayvke omvket hryayvhokatskat em etenrvwvn omatskvren ;

16 Momet hesaketv opunvkvn kerkuecet omvkes; momat ehvperkvn letkit, monkat ehvperkvn vtotkit omvkate vrahkvn Klist en nettv ofvn ve afvcke taren.

17 Momen cem vkvsvmkv take em vtotketv momet enake Hesaketvmese emetv onvprn Hesaketvmese ohfvccon cv'lehocen omat, vc afvckes, momet omvlkatskat ece 'pakvikit afvekvyet os.

18 Momen mata vrahkvn ece 'fackvket, vc vpvkaket afackatsket os.

19 Momis cen sehoketv taken kerrit, heren vm mahlvpvtkvren, lvpken Temorevs cem vtotvkares, komit, Pucase Cesvs ofvn enhonrkvn ocis.

20 Vkerrickv em vkerrickv oman ocet, cen sehoketv taken faccusen moskomvren ocvkokv.

21 Omvlkvt Cesvs Klist enanvke tokon, eme mahusat enanvken hopoyakekv.

22 Momis eskerhoyvten, estomēn eppuce erke vpake omẽn vc vpaket opunvkv-herv ofv vtotkvten kerratskes.

23 Monkv lvpken, vm estomvranat kerrvyof vpakusen, mvn vtotares, komit, enhonrkvn ocis.

24 Momis lvpken vne mahusat vlvkares, komit, Pucase ofvn cv'nhonres.

25 Mome estomis Epaflotitvs tecakkvyvte, vtotkv vm etohkvlketv hvmkat, momet suletawv vm etohkvlketv, 
momet mv vm eyackvn vfastvten, momis cem opunvkvsarvn cem vtotvranvkvyat tvlkuset os, komvyvnks.

26 Ce hecetv take eyace heret, en honne mahemvts, enokkvten pohatskvtet ok'.

27 Momen mehenwvn enokkemvts, elvranuset, momis Hesaketrmeset em merremvts; momen eme tvlkekon, momis vneu vm merremvts, feknokketv feknokketvn ohvprken ocvkaren.

$28 \mathrm{Monkv}$ vcayece herit vtotimvts, hvtvm hecatskof, ece 'fackvke taren, momen feknokketvt vn yupvklvtkvren.

29 Monkv Pucase ofvn afvckvke heret evpryvkes, momet este mv omakan vrakkuecrkes :

30 Hiyomet omekv, vm vnickv ohfvccun esfvcken vtotkatskekaten esfvcecetvn komet, Klist em vtotketv vrahkvn, en hesaketvn vcayecekot, elkvn vwolicusemvts.

\section{SETENTVCKE III.}

Espoken, tecakkvyvte toyatskat, Pucase ofvn afvckvkes. Nanvke matan hvtvm cen hoccicvkvyat, mehenwrn vn yekcekos, momet cen hervket os.

2 Efvn em e vketecvkes; holwvyeckv vtotkvlke em e vketecrkes; kvnsiskv vcrkvpeyvlken em e vketecvkes.

3 Pumet ena-vpeswvn enhonrēkot, $m v$ puyvfekev of vin Hesaketvmesen vrakkuecet, momet Klist Cesvs ofvn afvcket, svkvmsiskv toyēkv.

4 Mome estornis vnet enavpeswv ofv tis enhonrkvn ocrye tetayes. Enarpeswr ofvn enhonrkin ocvye tetayes, este etimvt komen omat, vnet semuntvlen komvyes.

5 Nettv escenvpakan svkvmsishoyet, Eslevl en cukohvmecvlket, Pencemen em aliketv aossvyvtet, Hepluvlke mahhet; vhakv ohfrccun, Falvsevlket;

6 Vketeckv ohfvccvn, mekusvpkv-cukon assecit; $\mathrm{mv}$ 
fvecetv vhakv eteropotte ocat ohfrcevn $v m$ muttekot omvyrites.

7 Momis mv nake vn lopicake tayvte, Nake vn lopickv tokos, Klist vrahkvn komvyvtes.

8 Momen mehenwvn mv nakkerretv Klist Cesvs cv Pucase ofv ocat en heretv vrahkvn nak omvlkvt ehvperkvt's, koinvyet os; mv Cesvs vrahkvn nanvke omvlkv vn sumkvten kvwapit, nake vpvlvthoyvte omēn vkerricis, Klist epoyaret,

9 Momet, vm fvecetv mv vhakv vcrkvye ocat tokon, momis mv Klist vkvsvmkv eteropotte, mv fvecetv Hesaketvmesen avtc vkvsvmkv eteropotte ocan ocin es cv hechoyvren;

10 Momet Klist kerrit, momet a en kvwvpketv en yekcen, momet vpakit em estemerketvn kerraren, elvte omēn vc omehocen :

11 Estome estomis elkv a en kvwrpketvn epoyvye tayen omat.

12 Entis eenakuecrye, momet entis temvpoke cv hahoyvte omekon; momis vcrkvyrye emunkvts, mv nake vrahkv Klist Cesvs cv 'svten esaren.

13 Tecakkvte toyatskat, Ehsis, e komvkot os: momis heyv hvmkusan momecis; nake cv'ra topvrv ocakan ehosit, momet nake cv homv ocakan oretvn vcevllit,

14 Satkv vfvecvn ohyekcet ayemahis, Klist Cesvs ofvn Hesaketvmese huehketv ohfvnketv eskerkv vrahkvn.

15 Monkv pumet, estomomuse temvpokvkeye tat hiyomēn vkerricvkeres, momen nake etv ohfvecvn murahken vkerricatsken omat, heyv tisem Hesaketvmeset cen kerkuecvkvres.

16 Mome estomis, mv nake entis eenakueceyvte ohfvecvn vhakv hvmkusen 'se em fvyvtvkeres, nake hvmkusen vkerricvkeres. . 
17 Tecakkvte toyatskat, etohkvlket ve vevkfullvlket omvkes, momet pume svhopakvn ocatskat omvke fullan heren hecvkes.

18 (Hiyomet omekv, sulket, mv ohfvecvn tewolen cem onayvkvyvtet, momet hiyomat $\mathrm{ev}$ turopuswr tis pvlatken, ce kicvkis, Klist em etohweklepkv vnrapvlket omakes.

$19 \mathrm{Mv}$ em eyuksv taket hopvnketvt omen, en hesaketvmeset e nvrke take tes; mv em ekvsvmkvt em vlesketv ocen, yvmv ekvnv enanvken oh vkerricaket os.)

20 Hiyomet omekv, pum etohkvlketvt hvlwe ofvt on, momet mata vten Hesayecr Cesvs Klist Pucasen en neneheceyet os.

21 Mvt pu'na kvncapan em ena vrakkat ome hakvren mvrahkuecvres, vtotketv mv etcropotte nanvke omvlkv eme mahusat elecrn svpvklece tayat vevkvyen.

\section{SETENTVCKE IV.}

Monkv tecakkvyvte $v m$ vnokecke, momet vm eyackv, vm afvcketv momet vn kvpotokv vcake toyatskat, momēn Pucase ofvn yekcen svpvklvkes, $v m$ vnokeckatskat.

2 Yuotevn enkusapis, momet Senteken enkusapis, Pucase ofvn vkerrickv hvmkusen ocvkvren.

3 Momet cemeu, vtotkv vm vpvketv mehenwe toyetskat, ce kusapis, mv hoktvke vc vpake opunvkv-herv ofv vtotkakvten, momet Klementen, momet vtotkvlke vm etohkvlketv vpvltaken, $\mathrm{mv}$ e hocefhokv hesaketv en nakcokv ofv ocakan, emvnicvkes.

4 Pucase ofvn estofis afvckvkes; momen hvtvm makis, Afvckvkes.

5 Cen yvmvsketvn omvlkvt kerrvkekvs; Pucaset vwolices.

6 Naken 'se naorihcatskvs; momis nak omvlkv ofvn, 
mekusvpkv, momet vpohkv, momet vkvsvmkv emetvt vpvkaken, cem vpohkv taken Hesaketvmeset kerrekvs.

7 Momen Hesaketvmese en herkvt, mv hoporrenkv omvlkv ohfvnkat, ce feke take, momet cem vkerrickv taken Klist Cesvs eteropotten vcvyecvkvres.

8 Espoken, tecakkvte toyatskat, nanvke mehenwvke vtekat, nake fvccrke vtekat, nake herake vtekat, nake hvsvthakuse vtekat, nake vnokeckakat vtekat, nake vkvsvmvke taye vtekat, nak herat hvmkusis ocen omat, momet nake vkvsvmvke taye hrmkusis ocen omat, heyv nanvken oh vkerricvkes.

$9 \mathrm{Mv}$ nanvke vc ofv kerret, eenakuecet, pohet momet hecatskvten, mvn momecvkes; momen herkv en Hesaketvmeset ece 'pakvkvres.

10 Momis hiyomat espokan cem vkerrickv take vc ohfvecv ocat oh mucvsehocat vrahkvn Pucase ofvn tayen vc afvckvnks: $m v$ ofvn vkerricatskvtet os, momis cem etektvnkvkekates.

11 Nake kusvpkat opunvyecryat tokon, estomēn arvyis, mv ofv vm mahlvpvtke taren kerrvyvtet ok'.

$12 \mathrm{Cv}$ kvncvpuehocan kerrit, vm ohvtvlakan kerris; estvmv estomis, momet nanvke omvlkv ofvn ev fveecat, momet cv 'lawat, vm ohvtvlakan momen kusvpketv kvwrpetvn v'muhahoyet os.

13 Klist $\mathrm{mv}$ cv yekcicat eteropotten nanvke omvlkvn momecrye tayes.

14 Mome estomis, vm estemerketv ofvn ve vpake kvwapatskvte estonkon momecet omatskvtes.

15 Momen, Felepvlke toyatskat, heyvn kerrvkes, opunvkv-herv vlicecen, Masetonevn enkvpakvyof, mekusvpkvcuko etv hvmkusis emetv esetv tepakat ohfvecrn ev tempunayekon, ceme take tvlkuset $\mathrm{cr}$ temrpunayatskvtes. 
16 Resvlonikv ofv tisem vhvmkvn momet vhokkolvn vn kusvpketv omecicen naken vtotatskvtet ok'.

17 Naken v'mhoyvren komvyat okvkok', momis ette ece 'rahkv taken sulke taren komvyet os.

18 Momis nanvke omvlkvt vn hecken, sulken ocis; nanvke atotatskvte, mv fvmeckv fvmecusat, nake Hesaketvmese esvrakkueckv ehomv here, momet afrcecican Epaflotitvs y'vm ocemvt ok'.

19 Momis vn Hesaketvmeset cen kusvpketv omvlkvn 'sohfvcecvres, en nake-ockv en rakketv ofv ocat vcrkvyen, Klist Cesvs eteropotten.

20 Momen Hesaketvmesen momet Pu'rken vrakkueckvt em ocekvs, meyuksv-sekon. Emen.

21 Mekusapv vtekan Klist Cesvs ofv vsekvkes. Tecakkvte vc vpvkakat ece 'sekakes.

22 Mekusapvlke omvlkvt ece 'sekakes, Sesv en cukovtehkvlke mahvkvu.

$23 \mathrm{Pu}$ Pucase Cesvs Klist en heromkvt omvlkatskan ece 'pakvkekvs. Emen.

I Heyvn Lome ofvn cohoyan, Epaflotitvs Felepvlken 'sem ayvtes. 


\section{COKV VPASTEL PAL \\ KOLASVLKE OHTOTVTE.}

\section{SETENTVCKE I.}

PaL, Hesaketvmese komat vcrkvyen Cesvs Klist em vpastel toyvyat, momet tecakkvte Temorevs,

2 Mekusapvlke momet Klist ofv tecakkvte heromvke Kolasen vpokan ohtoteyet os. Heromkv momet herkv Pu'rke Hesaketvmese, momet Pucase Cesvs Klist avtet ece 'pakvkekvs.

3 Estofis cem emekusapvket, Hesaketvmese momet pu Pucase Cesvs Klist E'rken vkvsvmkvn emeyet os ;

4 Cem vkvsvmkv take Klist Cesvs ofv ocan, momet mekusapvlke omvlkvn vnokeckv em ocatskan poheyof,

$5 \mathrm{Mv}$ enhonrkv hvlwat ofvn cen herihocvkat, mv opunvkv-herv em mehenwetv em opunvkv ofvn enhomv pohatskvte vrahkvn;

6 Mvt ekvnv hvmecicat etvpomen cem vlakvket os; momet $m v$ pohatskvte en nettv, momet mehenwetv ofvn Hesaketvmese en heromkvn kerratskvte vteken ec ofv taken ettvte etvpomen ettet os.

7 Epeflas, vtotkv pum etohkvlketv vnokecket, momet ec ohfvecv taken Klist em vtotkv heromat cem onayvkvte etvpomen ;

8 Momet mvt cem vnokeckv take Puyvfekev ofv ocan pum onayvtet $o s$.

9 Heyv vrahkvn, pumeu, mv nettr poheyvte vteken cem 
emekusapvket, hoporrenkv momet puyvfekev nakkerretv omvlkv ofvn nake eme komat kerretvn es ce fvcfvkvren komeyat, fekhonnēkot cem emekusapvkeyet os;

10 Momat Pucase vcvkayv ore heret, afvcecickv omvlkv oren fullēt, vtotketv herat vtekat ofvn etten hayet, momet Hesaketvmese kerretv ofvn cem ohvtvlvkvren;

11 Yekcetv omvlkvn es ce yekcihocen, en yekcetv rakkat vevkvyen, yvmvsketv omvlkv, momet momis kometv hofunat oren, afvcketvt vpvken;

12 Erke, mv mekusapvlke hvyayvke ofv vpokat nake ocakan etencawvlke toyeyvre orvke pu hayvten vkvsvmaket;

13 Mvt yomucke en yekcetvn a's epu'ssihcet, Eppuce em vnokeckan em ohmekketv ofvn es pu tohwvlvpkvtet os ;

14 Eme ofvn, e catv eteropotten pvlken pu neshoye tayan, $m v t$ holwvyeckv enkvpketvt omen, oceyet os.

15 Mvt Hesaketvmese heckeko taye vhaket, nake hocackvte vtekat em enhvteceskv-heckvte tes;

16 Hiyomet omekv, mvt nak omvlkvn hahicvtet os; hvlwe ofv ocakat, monkat ekvnv oh ocakat, nanvke heckakat, monkat heckvkeko tayat, mekkvlke em ohliketv tis, monkat ohfvnkvlke em wiketv, monkat ohmekketv ocakat, monkat yekcetv ocakat, emet nanvke omvlkvn hahicen, eme vrahkvn omvlkvt hocackvtet os;

17 Momen emet nanvke omvlkv enhomvt omvtet, eme eteropotten nanvke omvlkv ocaket os;

18 Momet emet ena em ekvt ot, $m v$ ena mekusvpkvcukot omen; vliceckvt omet, prsatkvte enkvpvken enhvteceskv heckvte tes; momat omvlkv ofvn homahtetrt enake taren.

19 Fvcketv omvlkv eme ofvt ocvre Erken afvcecicvtet ok; 
20 Momen mvt Eppuce em etohweklepkv e catv eteropotten herkvn hahyet, eme etehoyvnen nanvke omvlkvn e en herkepueckvn es afvckvtes; eme etehoyvnen, makis, nanvke ekvnv oh ocakan, monkat nanvke hvlwe ofv ocakan.

21 Momet centaken, vtotketv holwrhokat eteropotten vkerrickv ofv trleporvlke momet vnrapvlke hakatskvtet o estomis,

22 Hiyomat elkv eteropotten enarpeswv ena ofvn ce hvsvthvken, cem muttvkekon; momet chomv nake'es. cem vsehokvke tayat cen sekon ce svpvklecetvn komet, ce tenherkepuecvtet os ;

23 Vkvsumkv ofvn yekcen, momet nekeyekot svpaklatske munken, opunvkv-herv, mv pohatskvtet, momen nake hocackvte hvlwe elecv ocakat vtekat 'sem erkenvkhoyvte, vne, Pal toyvyat $\mathrm{mv}$ em erkenvkvn cv hahoyvtet em enhonrkvn em vkueyecatskekon omat.

24 Hiyomat vm estemerkv take ece 'rahkv taken es cv 'stemerkan es $v c$ afvckes, momet cv 'navpeswv ofvn Klist em estemerkv $v m$ vhoskakan, em ena vrahkvn, mvt mekusvpkv-cukot omen, fveecryet os.

25 Nake Hesaketvmese em ohmelletv ece 'rahkv take v'mhoyvte vcrkvyen, mv em erkenvkvn cv hahoyvtes, Hesaketvmese em opunvkvn esfvcecaren;

$26 \mathrm{Mv}$ nake chkat oketv hoyvnakvte, momet este honvpse sulke em ehkvtet, momis hiyomat mekusapvlken en kerkuehocet os :

27 Mvn Hesaketvmeset heyv nake ehkat en hryayvke en rakke Centilvlke vpvke ocan en kerkuecetvn komvtes; mvt ec ofv take Klist, hvyayvke em enhonrkvt omes :

28 Mvn es erkenakēt, este omvlkvn emvcahnet, hoporrenkv omvlkv ofvn este vtekan emvhayeyet os, Klist Cesvs ofv temvpoken este vtekat Hesaketvmese ehomvn huericeyvret, 
$29 \mathrm{Mv}$ ohfvecvn vneu, mv ve ofv yekcen vlotkat em vtotketv vcvkvyen, yekcen vcevllit, vtotkvyet os.

\section{SETENTVCKE II.}

Hгомет omekv', ece 'rahkv taken, momet Leotesev vpokat, momet estomomuse enavpeswv ofv ev turofv hecrkekate vrahkvn senaorickv yekce momvteke ocvyan kerratskvren komis ;

2 Efeketake vnokeckv ofv etelvcrket, nake kerretv en kerke here esfvckat oren, Hesaketvmese, momet Erke, momet Klist enake ehkat kerkuecetv oren, mahlvpvtkvke taren komit.

$3 \mathrm{Mv}$ ofvn hoporrenkv nakkerretv tepakat en nakockv omvlkvt ehkaket omes.

4 Momen estimvt cem vlopvkuecet ce mvkerrekaren heyvn makvyet os.

5 Hiyomet omekv, enavpeswv ofv ece 'pakvko estomis, vc afvcket etelilvke heren fullatskan, momet cem vkvsvmkv nekeyihoceko taye Klist ofv ocan hecit, puyvfekev ofvn ece 'pakvkvyet os.

6 Monkv Klist Cesvs Pucasen eenakuecatskvte etvpomen eme ofvn fullvkes;

7 Eme ofvn yvlonkvn hayet, eme ofvn ce hahoyvken, momet ce muhahoyvte vcrkvyen, vkvsvmkv ofvn ce yekcihocen, mvn es ce fvcfaken, Hesaketvmesen vkvsamet fullvkes.

8 Klist vevkvye tokon, este em opunvkv vcrkvye, momet yvmv ekvnv nake es hahoyvte vevkvyen, felasvfe momet vkerretv ehvperkat eteropotten estimvt ce hopvnekaren e vketecvkes.

9 Eme ofvn, Hesaketvmese es em fvcketv omvlkvt ena. ofvn liket omekv.

10 Momen Klist, mv ohfvnketv momet yekcetv omvlkv ekvt omat ofvn temvpoket omatskes; 
11 Momet eme ofvu mv svkvsvmsiskv enke es hahoyvte tokon es ce svkvmsiskvket os, Klist en svkvmsiskv eteropotten enavpeswv en naorketv enan em vkueyatsket omen:

12 Eme vpvken paptesvm ofvn ce herihocen, $m v$ ofvu Hesaketvmese, mv emen elkv a en kvwapvtet em vtotketv vkvsvmkv eteropotten eme vpvken akvwapkatskvtet os.

13 Momen cemet, cen naorketv momet ce 'navpeswv svkvmsiskv tokat ofvn ce prsatkvkvten cen naorketvn cen kvpakihcet, eme vpvken ce wenahuecrkvtet os.

14 Momet nanvke epu'nrvpe melhoyvte ocakvte en hoccickv, mvt epu'nrvpet omvten, vslehcet, mvn vkueyet, em etohweklepkvn svrahvtes.

15 Momet ohfvnketv momet yekcetv ocakan hopahnet, heckv heren nakheckvn es hayet, $\mathrm{mv}$ ofvn ohfvnkvtes.

16 Monkv hompetv, monkat esketv, monkat nettv vcake ohfvecrn, monkat hvresse mucrsat, monkat fekvpkv nettv take ohfvccun estimvt ec ohfvecehcrkats :

17 Mvt nanvke taranat em vpettet os; momis enat Klist enaket's.

18 Estimvt mv nanvke hecekaten eceyet, momet em vkerrickv enavpeswv omat ehvperkvn es ekvsamat,

19 Momet Ekv, mv enkvpvken ena hvmkvt etekkekv momet eswrnakv eteropotten, etelvcet momet yekcihocet omen, Hesaketvmese em ohvtvlvketvn es ohvtvlakat, mv Ekvn hvlvtekot, eme komat ekvncvpueckv momet encelvlke vrakkueckv eteropotten cem feketvn ce 'konahhvkates.

20 Monkv yvmv ekvnv nanvke es hahoyvte aenkvpvken Klist vpvken ce prsatkvtet on omat,

21 Estomaten ekvnvn vpokatske omet, nanvke melhoyvte, $\mathrm{mv}$ 'se vfvshotof, omvlkv hupvnkvkvranat, em apohicet, 
este em vhakv, momet nanvke estet mvhayakan vcrkvyen fullatske haks?

22 Vcelakekot, lasekot, celahyatskvs;

23 Mehenwvn mv nanvke, enavpeswvn afvcecickvn, vrakketv mahhe ofv tokon, eme komat mekusvpkv, ekvncvpueckv, momet ena heren vfvstekat ofvn hoporrenkv en heckvn ocaket os.

\section{SETENTVCKE III.}

Monkv Klist vpvket akvwapkatskvtet on omat, mv nanvke hvlwe min, Klist Hesaketvmese em akvpervn likat ocakan hopoyvkes.

2 Nanvke yvmv ekvnv oh ocakat tokon, mv hvlwe ocakan vnokecrkes.

3 Ce prsatkvket on, cen hesaketvt Hesaketvmese ofvn Klist vpvken ehket ocet omekv.

4 Klist pun hesaketvt e hecicof, momof centakeu hvyayvke ofvn vpakatsken ce hechoyvkvres.

5 Monkv ce'nalvcce yvmv ekvnv ocakan ehvperkuecvkes, hoktarkvn, hvsvtketv-tokan, vkerrickv herekan, eyackv holwakan momet vleskvpkv, mvt nakvhake mekusvpkvt omes.

$6 \mathrm{Mr}$ nanvke vrahkvn Hesaketrmese en crpvkiket apohickv toko en hopuetaken oh vlaket os:

$\eta \mathrm{Mr}$ ofv centakeu yvkapatskvte ocet os, $\mathrm{mv}$ ofvn fullatskof.

8 Momis hiyomat heyv nanvke omvlkvn, cvpvkketvn, homeckvn, holwvyeckvn, Hesaketvmese vtvkleckvn, momet opunvkv holwakan ce cukwv asossen vpvlvtvkes. 9 Ete'n lvkisatskvs, este vculan en nakvfrstetvn vprken etvn ocet,

10 Este mucvsat mv hayvte vhakat vcvkvyen nakkerretv ofvn mucrsehocvten vccatskvtet omekv. 
11 Mvn Klekvlket momet Cusvlket sekon, svkvmsiskv monkat svkvmsiskv tokat sekon, Papelevlke, Serevlke, svlvfke, pucassekat esyomat sekon, momis Klist omvlkv ofvt, omvlkv' $t$.

12 Monkv, Hesaketvmese enhopoyvte herake momet vnokeckvke omēn, envcofv merrakusen, heromkv, vkerrickv kvncapan, eyasketvn, momet momis kometv hofunan vecvkes ;

13 Momis kometvn ete'm ocet, momen, este estimv vnrvpen naken ocen omat, ete'm merret omvkes; Klist cen kvpryecicvkvte etvpomen momecvkes.

14 Momet heyv nanvke omvlkv semuntalat vnokeckvn vccvkes, mvt temvpoketv es em wvnakvt omes.

15 Momen Hesaketvmese en herkvt, mvn ena hvmkuse ofv ec oh huehhokvkat, ce feke take ofvn mekkekvs; momen Hesaketvmesen vkvsvmkvn emvkes.

16 Klist em opunvkvt esfvcken, hoporrenkv omvlkv ofvn, ec ofv taken likekvs; yvhiketv, vkvsvmkv-yvhiketv, momet puyvfekev ohfvecr yvhiketvn 'sete'm muhayet, momet 'sete'm vcahnet, vkvsvmkvt ce feke take vpiken Hesaketvmesen vyvhikvkes.

17 Momen nake estomis, opunvkv monkat nakcelakv ofvn, momecatskat, omvlkvn Pucase Cesvs e hocefkv ofvn momecvkes, mv eteropotten Hesaketvmesen, momet Erken vkvsamet.

18 Hoktvke toyatskat, ce 'hetake em apohicet omvkes, Pucase ofv momvre herat vevkvyen.

19 Hunvntake toyatskat, ce 'biwv taken vnokecet, enhomehcatskvs.

20 Hopuetakē, nanvke omvlkv ofvn ce'ckvlken em apohicvkes, heyvt Pucasen heren afvcecicet ok'.

21 Erkvlkē, cen hopuetaken crpvkhokihcatskvs, enhonrkvt en sumkvkekaren. 
22 Vtotkvlke toyatskat, nanvke omvlkv ofvn enavpeswv vcrkvye ce pucasvlken em apohicrkes; renakv vtotketv tokon, este afvcecicvlke omekot; momis ce feke take em mehenwetv ofvn Hesaketvmesen em penkalet:

23 Momet nake estomis momecatskat, efēke vtet, este tokon, Pucasen em momece omēt momecvkes;

24 Nake epucashoyvranan em feketrn Pucaset ce'mvranvken kerret; Pucase Klist em vtotkatskekv.

25 Momis estimvt naken esten em vhopanat, mv vhopanvte vevkvyen en heckvres; momen este etemetueckvt sekot os.

\section{SETENTVCKE IV.}

Pucasvlke toyatskat, nake fvecan momet momvre heran cem vtotkvlken emvkes; cemetakeu hvlwe ofvn Pucasen ocatskat kerret.

2 Mekusvpkv ofvn vpeye mahvkes, momet mata ofvn vketecet, Hesaketvmiesen vkvsvmvkes;

3 Momen pumeu pum emekusapet, opunvyetv vhauken Hesaketvmeset pun hwwecvren, Klist en nake ehkat, mv vrahkvn svlvf kuehocat toyvyat, kerkuecaren;

4 Opunvyar tayat vevkvyen kerken hayaren.

$5 \mathrm{Mr}$ lvpvtke svpvklat ohfvecvn e hoporrenicet fullvkes, oketvn nesaket.

6 Heromkvt cem opunvkv taken estofis vpvkekvs, okcrnwvn es cvmpet omen, estomēn este estomis momvre heren em vyoposkatske tayan kerratskvren.

7 Momen nake vc ohfvecv ocakat omvlkvn Tekekvs, tecakkvte vnokecke, momet vtotkv herome, mornet $\mathrm{Pu}$ case ofv vtotkv vm etohkvlketvt cen kerkuecvkvres;

$8 \mathrm{Mv}$ vrahkvn, ce 'stomvkat kerrvren, momet ce feke taken mahlvpvtvren, mvn cem vtotvkvyvnks,

9 Momet vpvken Onesemvs, cem etohkvlketv take hvmkat, tecakkvte herome momet vnokecken vtotvyvnks. 
Mvt nanvke yvmv momehocat omvlkvn cen kerkuecvkvres.

10 Elestakvs ve vpake svlvfkat ece 'sekakes, momet Makvs, Panepvs e wvnwv e'chuswrt, (mv ohfvcev nanvke ce kihocvkvtet; cem vlakvken omat, evpryvkes;)

11 Hvtvm Cesvs, mv Cvstvs kihocat; heyrt svkvmsiskvlket omakes. Heyv tvlkuset, Hesaketvmese em ohmekketv ohfvecv vtotkvlke vm etohkvlketvt, mv v'mahlvpvtketvt omakvtet omakes.

12 Cem etohkvlketv take hvmkat, Klist em vtotkv, Epeflas, estofis ece 'rahkv taken mekusvpkv ofvn yekcen cem vtotkvket, Hesaketvmese nake komat omvlkv ofvn temvpoke momet heren hahoyet svpaklatskvren emekusapet ece 'sekaket os.

13 Hiyomet omekv, a en kerris, centake cem ocvket, momet Leotesev vpokat, momet Hielapoles vpokat vnokeckv rakken $\mathrm{em}$ ocvket os.

14 Luk heleshayv rnokeckat, Temvs tepakat ece 'sekaket os.

15 Tecakkvte Leotesev vpokan, momet Nemfan, momet mekusvpkv cuko en cuko ofv ocan vsekvkes.

16 Momet heyv cokvn cem. etenrvwv taken ohonahohyof, Leotesevlke em mekusvpkv-cuko ofvn onahoyvren, momet cokv Leotesev atan cemeu ohonayatskvren vhecvkvtevs.

17 Momet Akepvn, Mv vtotketv Pucase ofv cen heckvten vketecrs, freecetskvret, kicvkvtcrs.

18 Pal toyvyat cv'nke eteropotten vseketvt's. Vn svlvfketvn vkerricvkes. Heromkvt ece 'pakvkekvs. Emen.

I Pal Lomen liket hoecihcen, Onesemvs, Tekekvs tepakat Kolasvlken 'sem vhoyvtes. 


\section{COKV ENHVTECESKV VPASTEL PAL}

\section{RESVLONIKVLKE OHTOTVTE.}

\section{SETEN'TVCKE I.}

Pal, Selfenvs, Temorevs esyomeyat Resvlonikvlke em mekusppkv-cuko, mv Erke Hesaketrmese momet Pucase Cesvs Klist ofv ocan ohtoteyet os; Heromkv momet herkv Hesaketvmese Pu'rke momet Pucase Cesvs Klist aenkvpvket ece 'pakvkekvs.

2 Pum mekusvpkv ofvn ce hocefhuecet, omvlkatskat ece 'rahkv taken estofis Hesaketrmesen vkrsameyet os;

3: Momet fekhonnēkot cem vkvsvmkv em vtotketvn, momet senaorickv vnokeckvt omecicen, momet pu $\mathrm{Pu}$ case Cesvs Klist ofv enhonrkv em momis-kometvn, Hesaketvmese momet Pu'rke ehomv ocatskan vkerricēt:

4 Momet, tecakkvte vnokeckatskat, Hesaketvmeset ce'nhopoyvkvten kerrēt.

5 Pum opunvkv-hervt, opunvkv ofv tvlkekon, momis yekcetv ofvu, momet Puyvfekev Veakat ofvn momen enhonrkv esfrckat ofvn cem vlakvkvtet ok', pumet ece 'rahkv taken hunvntake estomvket ece 'pakvket omeyvten kerratskekv.

6 Momen centaket, estemerkv rakke, momet Puyrfekev Vcakat em afvcketv ofvn opunvkvn eset, epu 'crkfullvlke, momet Pucase vcvikfullvlke hakatskvtes:

7 Momat Masetonev Ekiv tepakat ofv vpoke vkvsamvlke omvlkvn em evhopakvt omatskvtes. 
8 Hiyomel omekv, centake avtet Pucase em opunvkvt Masetonev Ekiv tepakat ofv tvlken pohkekates; momis estvmv estomis vkvsvmkv Hesaketvmese ohfvecv ocatskat hrmecicvtet os; momat naken makēkis tayes.

9 Hiyomet omekv, ep ohfvecr ye's cen ceyetv take estomēn oceyvten, momet estomen nak vhakakat enkvpahket, Hesaketrmesen a oh fulotecet, Hesaketrmese wenake momet mehenwat em vtotketvn komet,

10 Momet E'ppuce hvlwe vten, mv prsatkvte aenkvpvken kvwapvie, mvt Cesvt omen, mv crpvkketv vlvkvranat enkvpvken pu hesahuecrten mehaketvn komatsken eme mahusat ep oh kerkuecaket os.

\section{SETENTVCKE II.}

Tecakkvte toyatskat, ye's cen ceyeyvkeyvte ehvperkvt omekaten ceme mahusat kerratskekv.

2 Momis kerratskes, mv 'renhomvn Felepi tvlofvn pu 'stemerrihocet, vlesketv vpvken pu celahoyvte hoyanof, Hesaketvmese em opunvkv-hervn, etenrvpkv sulken es ce tempunayvkeyvret, pun Hesaketvmese ofvn pu fekhvmke tates.

3 Vcahneyvtet mvkerretv ofv, monkat husvtketv tokat ofv, monkat ehosickv ofv tokatet ok'.

4 Momis, Opunvkv-hervn em wihokvre heretv oricakes, Hesaketvmeset pu komvte vcrkvyen opunahoyeyet os; esten afvckvkueceyat omēkon, momis Hesaketvmese, pu. feke take eskerran afvcecicet.

5 Hiyomet omekv, cemet kerratskes, ece 'kvsamēt mvkerret cem punayvkeyvte sekos, momet vleskvpkv seokepkvn hayet omékates; Hesaketvmeset akerrvt omes. 6 Momet Klist em vpastelvlket omet, este en honnicvkeyvre pum etektrnkvtet o estomis, estet, centake, monkat este vpvlwvt vkvsvmkvn pu'mvkvren hopoyëkates, 
7 Momis hopuetakuce vhecicv en hopuetake vhecice etvpomen cem etenrvwr taken yrmaskuseyrtes.

8 Monkv ece 'nokecēt ce yacēkv, Hesaketrmese em opunvkv-herv tvlket omekon, momis pum puyvfekev mahusat tis ec'metv pu metetakvket omvtes, pum vnokeckat omatskekv.

9 Tecakkvte toyatskat, pum vtotketv hotoskv tepakan vkerricatskekv; netty neren vtotkēt, hrmkusatskis cen honnicvkēkaret, Hesaketvmese em opunvkv-hervn cem erkenakvkeyvtet ok'.

10 Vkvsamatskat cem etenrvwv taken estomēn hvsvtken, momet fvccen, momet pum mvttekot fulleyvte ohfvccrn centake akerrvlket omatskes, momen Hesaketvmeseu.

11 Momen estomēn Hesaketrmese mv em ohmekkety momet hryayvke ohfvecun ce'nhuehkvkvte ehomvn heren fullatskvren,

12 Erket en hopuetake momecat omēn omvlkatskan cem vcahnet ce mahlvpatet, akerreyvten kerratsket os.

13 Momen heyv vrahkvn fekhonnēkot Hesaketvmesen vkvsamvyet os, Hesaketvmese em opunvkvn cen kerkuecrkeyvten eenakuecatskof, este em opunvkv omekon, momis mehenwusen Hesaketvmese em opnnvkvt omen, mv yekcen ec ofv taken vtotkat omēn eenakuecatskvtet ok.

14 Hiyomet omekv, tecakkvte toyatskat, mekusvpkvcuko Cesvs Klist ofv Cutev ocakan vevkfullvlke hakatskvtes; mv mekusvpkv-cuko Cusvlket estemerrvknecvte omēn cem etvlofvlket nake matvpomakan es ce 'stemerrvkuecakvtet ok.

$15 \mathrm{Mv}$ Cusvlket Pucase Cesvn, momet eme mahusat em owalvlken pvsvtaket, epu'ssecakvtes; momet Hesaketvrnesen afvcecicekot, este omvlkvn vnrvpaket os.

16 Centilvlke, hesahokvren, em punayeyvren pum vse- 
haket os, momat estofis en holwvyeckvn fvcecvkvres; crpvkketv esfrckuset oh vlvkaket omekv.

17 Momis pume, tecakkvte toyatskat, efeke ofv tokon, ena tvlkuse ofvn oketv kocuknusen ce'nkvpvken epu 'kuehoyvte tis, ce turofv taken hecetvn pu yace heret, yekcen vcevlleyvtes.

18 Monkv vhvmkvn momet vhokkolvn cem vlvketvn komeyvtes, vne, Pal okis; momis Setvnet pu invnvttecrtes.

19 Hiyomet omekv, pum enhonrkv, monkat afvcketv, monkat afvcketv en kvpotokv vcakat naket $\mathrm{mv}$ te? $\mathrm{Pu}$ Pucase Cesvs Klist ehomv svpaklat, em vlvketv omof, centake mv toyatskeko haks?

20 Centake pum vrakketv momet afvcketv toyatskekv.

\section{SETENTVCKE' III.}

Monkv ohvtvlaken kvwapēko tayof, Puntvlkusen Arens tvlofon pu wihokan heres, komeyvtes;

2 Momet Temorevs tecakkeyvte, Hesaketvmese em erkenvkv, momet pum etohkvlketv Klist em opunvkvherv ofv vtotkvn vtoteyvtes, ce yekcvkuecet, cem vkvsvmkv take ohfvecrn ce mahlvpvtvkvren:

3 Heyv estemerkvt estimvn nekeyicvkekaren, heyv nanvken pum wihokvtet omen ceme mahusat kerratskekv.

4 Mehenwusen, ece 'pvkakeyof, $\mathrm{Pu}$ 'stemerrihocvres, homvn ce kicvkeyvtet ok; momvte mahusan, momen mon kerratskes.

5 Heyvt omecicen, ohvtvlaken kvwvpvko tayof, cem vkvsvmkv taken kerretvn komit vtotvyvtes, Estome witat, vnrapvt es ce keriyvken, pum vtotketvt ehvperke wites, komit.

6 Momis hiyomat Temorevs vpokatskan vtet pum vla- 
ket, cem vkvsvmkv vnokeckv tepakat ohfvecvn, momet estofis heren epu 'kerricet, ce hecetv take pu yace herat etvpomen pu hecetvn komatskat, heyv opunvkv heran es pum vlakof;

$7 \mathrm{Mv}$ vrahkvn, tecakkvte toyatskat, cem vkvsvmkv taket omecicen, pum estemerkv enkusvpketv tepakat omvlkv ofvn ec ohfvecv taken pu mahlvpvtkvtes:

8 Hiyomet omekv, Pucase ofvn yekcen svpaklatsken omat, hiyomat hesahokeyet os.

9 Hiyomet omekv, afvcketv pun Hesaketvmese ehomvn ece 'rahkv taken es epu 'fackat vrahkvn vkvsvmkv estomēn Hesaketvmesen hvtvm emeye taye haks?

10 Momet ce turofv taken hecēt, cem vkrsvmkv take orice mahekan esfvceceyvren neren nettrn tayen cem emekusapeyet os.

11 Tokvs, Hesaketrmese mahusat, momet Purke, momet pu Pucase Cesvs Klist pum vyetr ec orvken mellekvs.

12 Momen Pucaset vnokeckv ete'm ocet, momet este eti em ocatskat ohvtvlaket mvn es fvefvket omatskvren ce hayvkekvs, pumet ec ohfvecr take omeyat etvpomen:

13 Momat, pu Pucase Cesvs Klist, em mekusapvlke omvlkvt vpvkaken, em vlvketv omof, Hesaketvmese Pu'rke tat ehomvn ce feke taken hysvtketv ofvn em muttvkekon yekcvkuecrres.

\section{. SETENTVCKE IV:}

Monkv ohvtvlakat, tecakkvte toyatskat, estomēn fullet, Hesaketvmesen afveecicvranatskat cen kerkuecvkeyvte etvpomen ohvtvlaket vpeyatskvren cem 'pohepet, Pucase Cesvs ofvn cem vcahneyet os.

$2 \mathrm{Mv}$ vhakv Pucase Cesvs eteropotten ce'mvkeyvten kerratskekv. 
3 Hesaketvmese nake komat heyvt omekv, hvsvtketvn ocet, hoktarkvn e enkvpryecicatskvren:

4. Momet omvlkatskat estomēu en nakvcvnkv hvsvtketv vrakketv tepakat ofvn ocatske tayat kerratskvren,

5 Centilvlke Hesaketvmesen kerrvkekat en nakvcvnkv enarpeswr em eyackv ofvn ocakat omekot;

6 Estimvt estemvkerret, nake estomis ohfrccrn etecakkvten fvecekon naken vkonahekaren; mv omakat omvlkv Pucase em prlecrt omekr, homavn cem onayet, cen kerkuecvkeyvte etvpomen.

7 Hesaketrmeset hrsvtketv tokan pu'nhuehkekatet ok, momis hrsvtketv min.

8 Monkv estit vhepakat, este tokon, momis Hesaketvmese, mv em Puyvfekev Vcakat pu'mvten vhepaket os.

9 Momis vnokeckv etecakkakvte ete'm ocvranat ohfvecrn cen hoccicvkvkis tetayes, centake mahusat ete'nokecvranatsken Hesaketvmeset ce mvhayvket omekv.

10 Momen mehenwvn tecakkakvte Masetonev vpokat ohfvecrn min momecatsket os; momis tecakkvte toyatskat, ohvtvlaket vpeyatskvren;

11 Momet mahlvpvtket fulletvn komet, cen nakcelakv mabusan vfastet, momet ce kicvkeyvte vcrkvyen ce'nke take mahusan es vtotkatskvren ce'nkusapvkeyet os.

12 Momat mv en lvpvtke sehokat ohfvcevn fvccun fullèt, naken cen kusvpkvkekares.

13 Momis, tecakkvte toyatskat, mv nocicat ohfvecrn naken kerrekot, rpvltake mv enhonrkvn ocrkekat omēn feknokket omatskvren komvkos.

14 Hiyomet omekv, Cesvs ehlet akvwrpkvten vkvsamēn omat, matvpomen mv Cesvs ofvn nocicat Hesaketvmeset eme vpvken svlvkvres.

15 Hiyomet omekv, Pucase em opunvkv eteropotten heyvn ce kicvkeyet os, Pumet Pucase vlvkvranan vteken 
hesahoket fekhonnē emunkat mv nocicat ehomvn vpehyēkos.

16 Pucase mahusat, huehketvt pohken, momet akencelv en hake, momet Hesaketvmese em pofketvt haken, hvlwan ahvtvpkvret ok; momen Klist ofvn prsatkvtet homvn akvwrpkvkvres.

17 Momof pumet, wenahokēt vpokeye emunkat, mv vpvken vholoce ofvn a pu crwhoyvres, hotvle ofvn Pucasen vnrapeyvren, momet momēn Pucasen estofis vpakeyvres.

18 Monkv heyv opunvkvn 'setemahlvpvtvkes.

\section{SETENTVCKE V.}

Momis, tecakkvte toyatskat, oketv momet pum etektvnke ocakat ohfvecvn cen hoccicvkvkis tetayes.

2 Nere horkopv vlakat omēn Pucase en nettvt vlaket omat centake mahusat kerratskekv.

3 Hiyomet omekv, Mahlvpvtketv momet herkvt oces, makakof, hokte estuce heckuecrranat en nokket vyvkhvmkin em vlakat etvpomen sumketvt oh vlvkvres, momen mvnvttvkepekares.

4 Momis, tecakkvte toyatskat, centaket yomuckofvn vpokatskekos, mv'nettvt horkopv omēt ce cakkvren.

5 Omvlkatskat hvyayvke en hopuetake, momet nettv en hopuetake toyatskes: pumet nere, monkat yomucke enake toyēkos.

6 Monkv eti nocicakat omēkot, vketecet, pu mahlvpatkuset omekvs.

$7 \mathrm{Mv}$ nocicakat neren nocicaken, $\mathrm{mv}$ hacakat neren hacaket omekv.

8 Momis pumet, nettv enake toyeyat, $p u$ mahlvpatkuset, vkvsvmkv vnokeckv tepakat sevrvnakvn, momet sekvpvtokvn vhesaketv enhonrkvn accet omvkeres. 
9 Hesaketvmeset cvpvkketv tokon, pu Pucase Cesvs Klist eteropotten vhesaketvn heckueceyvren pum mellvtet ok'.

10 Mvt epu 'rahkvn elvtes, vhonecet monkat nocicet o estomis, wenahoket tohkvlket vpakeyvren.

11 Monkv etemahlvpatet, teyekcvkuecvkes, mv momatskat etvpomen.

12 Momen, tecakkvte toyatskat, mv ece 'pvkaket vtotket, Pucase ofvn ec ohfvnket cem vcahnakan kerret,

$13 \mathrm{Em}$ vtotketv vrahkvn vnokeckv ofvn heren vrakknecatskvren ce'nkusapvkeyet os. Momen tenherket vpokvkes.

14 Momen, tecakkvte toyatskat, ekvyekcakan em vsehet, vkerrickv ofv yekcvkekan mahlvpatet, yekcvkekan yekcen svpaklecet, este omvlkv ohfvccrn hofunen momis komvkes.

15 Estimut holwakan holwakat vyoposken este em pvlecekaren vketecvkes, momet ceme mahusat cem etenrvwv taken este 'omvlkv esyomat ohfvccrn nake heran estofis vcvkvpeyvkvtcvs.

16 Estofis afvckvkvters.

17 Fekhonnekot emekusapvkvtevs.

18 Nake omvlkv ofvn Hesaketvmesen vkvsvmvkes, Klist Cesvs ofvn Hesaketvmeset nake ec ohfvecv take komat heyvt omekv.

19 Puyvfekcv Heran vslehcatskvs.

20 Nake owalvkhoyvten ehvperkuehcatskvs.

21 Nanvke omvlkvn eskerret, nake heran yekcen hvlvtvkes.

$22 N a k$ holwvke en heckv omvlkvn e em vsehvkes.

23 Momen herkv en Hesaketvmese mahusat esfrcken ce hvsvthicvkekvs; momen pu Pucase Cesvs Klist em vlvketv vteken, cem puyvfekev omvlkv, cem vkerrickv 
take, momet ce'na emmvttvkekon vcryehocate! komit, Hesaketomesen emekusapis.

$24 \mathrm{Mv}$ ce'nhuehkvkat heromet os, $\mathrm{mv}$ momecvrane tvlket omat.

25 Tecakkvte toyatskat, pum emekusvprkes. 26 Tecakkvte omvlkvn vfvnketv vcakan es vsekvkes.

27 Heyv cokvn tecakkvte vcacakat omvlkv em ohonayvkhoyvren, Pucase ofvn yekcen ce kicvkis. 28 Pucase Cesvs Klist en heromkvt ece 'pakvkekvs. Emen.

I Cokv Resvlonikvlke enhvteceskvn em vtotet Arens tvlofvn hoccihocvtet omes. 


\section{COKV PAL}

\section{RESVLONIKVLKE OHTOTVTE SVHOKKOLAT.}

\section{SETENTVCKE I.}

PaL, Selfenvs, Temorevs esyomat Resvlonikvlke em mekusvpkv-cuko Hesaketrmese Pu'rke momet Pucase Cesvs Klist ofv ocan ohtoteyat:

2 Heromkv momet herkv Hesaketvmese Pu'rke momet Pucase Cesvs Klist aenkvprket ece 'pakvkekvs.

3 Tecakkvte toyatskat, momvre heret omen, ece 'rahkv taken estofis Hesaketvmesen vkvsvmvrancyat trlkuses, cem vkvsvmkv taket tayen ohvtvlaket omen, omvlkatskat vnokeckv esfvcken ete'm ocatsket omekv:

4 Momet on, pume mahusat, ece'ssehocan momet estemerketv kvwapatskat omvlkv ofvn cem momis kometv vkvsvmkv tepakat vrahkvn Hesaketvmese em mekusvpkvcuko ocakat ofvn ec ohfvecr taken 'sekvsameyet os:

5 Mvt Hesaketvmese em ohfvececkv fvccat eskerkv hēckuset os, momat Hesaketvmese em ohmekketv, mv vrahkv ce'stemerkakat en heckvkvre heretv orices, ce komhoyvkvres:

6 Hiyomet. omekv, Hesaketvmese mv ece'ssecakan estemerkvn 'sem pvlecvkepvret fvecvt omes:

7 Momet, Pucase Cesvs, sutvn ahvtapket, em encelvike yekcakat vpvkaken,

$8 \mathrm{Mr}$ Hesaketvmesen kerrvkekot, pu Pucase Cesvs Klist 
em opunvkv-herv vpohicvkekat totkv fenke ofvn em pvlecvkepof, ceme ce naorihocan vyoposken fekvpkvn epu 'pvkaken ce'mvkat fvccvt os.

$9 \mathrm{Mv}$ nettv Pucaset vlakof, em mekusapvlke ofvn vrakkuehocvren, momen vkvsvmakat omvlkvt, estomahes, komvkvren, (pun kerkueckv cem etenrvwv taken vkvsvmhoyvtet ok',)

$10 \mathrm{Mv}$ apohicvkekaten eme likan enkvpvken, momet en yekce en hryayvke enkvpvken estemerkv meyuksv-sekon es estemerrvkuehocvres.

11 Monkv estofis cem emekusapvket pun Hesaketvmeset, heyv enhuehhokan heretv orices ce komet, en heretv en kometv herat omvlkvn, momet vkvsvmkv em vtotketvn yekcetvi esfveecvren emekusapeyet os.

12 Momat pun Hesaketrmese momet Pucase Cesvs Klist en heromkv vcrkvyen ec ofv taken pu Pucase Cesvs Klist e hocefkvn vrakkuehocen, eme ofvn centakeu ece 'rakkuehocvkvren.

\section{SETENTVCKE II.}

Momen, tecakkvte toyatskat, pu Pucase Cesvs em vlvketv omecicen, momet mvn em vtelokeyat omecicen,

2 Puyvfeker eteropotten, monkat vm opunvkv eteropotten, monkat cokv vtoteyvte oman eteropotten, Klist en nettvt vwoluses, komet, cem vkerrickv ofvn ce naorihocen, ce feknokhokekaren ce'nkusapvkeyet os.

3 Estome estomis estimvt ce mvkeriyvkats; ehomvn yupvklvtketv ocen, mv naorketv hunvnwv, estemerketv e'ppucet heckeko vtekat, mv netivt vlvkekaret ok'.

4 Mvt nake omvlkv Hesaketvmese kihocan, monkat vrakkuehocan vnrapet, senhvlwen e hvlwecet os; momat Hesaketvmese omēt, Hesaketvmese en cuko-vcakat ofvn liket, Hesaketvmese toyis, make omēt e heckuecet os. 
5 Ece 'pakvkvye emunkof heyv nanvken cem onvyvkvyvte vkerricatskeko haks?

6 Momen, em oketv omof heckvren, naket mvnvttecet omat hiyomat kerratskes.

7 Holwvyeckv enake ehkat entis vtotkekv; momis hiyome vnvttecat vnvttece emunken vkueke tvlkvres.

8 Momen momof mv Holwvyecat heckvres, mv Pucaset e cukwr en hesaketv eteropotten elecet, em vlvketv en hvyayvke eteropotten sumecicvranat:

$9 \mathrm{Mv}$ Holwvyecat em vlvketv, Setvne em vtotketv vcrkvyet omet, yekcetv omvlkv momet eskerkv omvlkv, momet nake estomahe laksakat omvlkv,

$10 \mathrm{Mv}$ estemerkvkvranat ofv fvecetv tokat ehoskv omvlkvn vpvket os; hesahokvren, mehenwetv vnokeckvn eenakuecvkekatet ok.

11 Momen heyvt omecicen, lvksetvn vkvsvmvkvren, Hesaketvmeset ehoskv rakken ohtotvkvres :

12 Momat mv mehenwetvn vkvsvmekot, momis fvecetv tokan es afvckakat omvlkvn ohfvecehocvren.

13 Momis tecakkvte, Pucase em vnokeckvlke toyatskat, Hesaketvmeset, enhvteceskv vteken Puyvfekev ce hvsvthicat, momet mehenwetvn vkvsamatskat eteropotten vhesaketvn cen heckvren ce'nsathoyvtet omecicen, ece 'rahkv taken Hesaketwmesen estofis vkvsvmvraneyat tvlkuses :

14 Pum opunvkv-herv eteropotten, pu Pucase Cesvs Klist em vrakketvn eenakuecatskıren, mvn ce'nhuehkvkvtet os.

15 Monkv, tecakkvte toyatskat, mv vculvke em punvkv cokv hocciceyvte, monkat opunvkv eteropotte ce mvhahoyvten hvlatet, yekcen sxpvklvkes.

16 Momen pu Pucase Cesvs Klist mahusat, momet Hesaketvmese, mv Pu'rket omat, mv epu 'nokecet, 
heromkv enhonrkv here tepakat eteropotten mahlvpvtketv meyuksv sekon pu'mvtet,

17 Ce feketaken mahlvpatet, opunvkv momet vtotketv herakat vtekat ofvn ce yekevkuecekvs.

\section{SETENTVCKE III.}

Espokat, tecakkvte toyatskat, pum emekusvpvkes, Hesaketvmese em opunvkvt, pvfnet ayen, vrakkuehocvren, cem etenrvwr take vrakkuehocat etvpomen:

2 Momet este ekv-yekcakat, momet holwryecvlke enkvpvken epu 'cayehocvren; omvlkvt vkvsvmkvn ocvkekokv.

3 Momis Pucaset heromet os; mv ce yekcrkuehcet, nak holwakat enkvpvken ece 'cayecvkvres.

4 Momen nanvke ce kicvkeyan momecaket omet, momet, momecrkvres, ce komèt, ec ohfvecv taken Pucase ofvn enhonrkvn oceyet os.

5 Momen Pucaset, Hesaketvmese em vnokeckv oren, momet momis komet Klist enneneheckvn ce 'feke taken mv mellekvs.

6 Momen, tecakkvte toyatskat, tecakkvte opunvkv emeyvte vcrkvye tokon, fvcceko arat vtekan e enkvpvyecicatskvren, pu Pucase Cesvs Klist e hocefkv ofvn ce kicvkeyet os.

7 Hiyomet omekv, cemetake mahusat, estomēn epu 'crkvpeyvranatskat kerratsket os, pumet cem etenrvwv taken frccekon fullēkatet ok;

8 Momet estit o estomis ehvperkvn tvkliken en hompēkates; momis nettrn momet neren vtotketv hotoskv tepakat ofvn vtotkeyvtes, estimv toyatske estomis, cen honnicukēkaren.

9 Yekcetvn ocēko omecicat tokon, momis epu 'cvlfullatskvre vrahkvn svhopakvn e hayetvn komeyat omecicet os. 
10 Hiyomet omekv, ece 'pakvkeye emunkof, heyvn ce kicvkeyvtes, Estit vtotketvn komekon omat, homipats.

11 Ece 'pakvkat, fvccekon fullēt, naken vtotkvkekot, momis nanvkokvlke sasvcuken pohēkv.

12 Momen mv omakan, mahlvpvtket vtotket, tvklike enake mahusan hompvkvren, pu Pucase Cesvs Klist ofvn kicēt, em vcahneyet os.

13 Momis centake, teeakkvte toyatskat, here momeckv ofvn ce hotohsvkats.

14 Momet, estimvt o estomet pum opunvkv heyv cokv ofv ocan apohicekon omat, mvn vketecet, vpahkatskvs, vlsvren.

15 Mome estomis, vnrapv omecekot, tecakkvte omēn em vcahnvkes.

16 Momen herkv e Pucase mahusat estofis, momet estomet o estomis herkvn ce'mvkekvs. Omvlkatskan Pucaset ece 'pakvkekvs.

17 Vne Pal vseketv cv'nke mahusan es hayvyat; mvt cokv omvlkv ofv eskerkvt omes; momēn hoccicis.

$18 \mathrm{Pu}$ Pucase Cesvs Klist en heromkvt omvlkatskan ece 'pakvkekvs. Emen.

I Cokv Pal Resvlonikvlke ohtotvte svhokkolan Arens trlofvn hoccicvtet os. 


\section{COKV ENHVTECESKV VPASTEL PAL.}

\section{TEMORE OHTOTVTE.}

\section{SETENTVCKE I.}

Pal, Hesaketvmese pu Hesayecr, momet Pucase Cesvs Klist pum enhonrkv makvte etehoyvnen Cesvs Klist em vpastel toyvyat;

2 Temore, vkvsvmkv ofv cv'ppuce mahusan ohtotis: Heromkv, merkv herkv esyoman Hesaketvmese Pu'rke, momet Cesvs Klist pu Pucase avtet cem ocekvs.

3 Masetonevn eceyvyof, Efesvn fekhonnvtcvs, momet mvhakv etvn mvhayvkekaren,

4 Momet nakonvkucen, monkat este erhonvpse opunvyeckv meyuksv-sekan, Hesaketrmese ofv yekcvkueckv vkvsvmkv ofv ocan hayekot, nakvpohetv min hahicakan vketecvkekaren ypvlwvn emvcahnvkvtcrs, ce kicvyvte verkvyen momecvs.

5 Momen heyv vhakv em vheckv vnokeckv tes, efeke hvsvtke, eohvkerrickv here, vkvsvmkv mehenwe esyoman aossat;

6 Mvn em fiyecet, opunvkv ehvperkakan ohfulecakat sases.

7 Mvt vhakv mvhayvlke haketvn komakis, nanvke makakan, monkat nanvke opunvyecakan kerrvkekot os.

8 Momis estet vhakv vcrkvyen 'se vfasten omat, vhakv heret omat kerreyet os.

9 Heyvn kerret, vhakvt, este faccv tokon, momis vhakv 
vevkvyen fullekat, mvn apohicrkekat, Hesaketrmese vmoskomvkekat, holwvyecvlke, este hvsvthvkekat, nakhayvkekvlke, erke-prsatvlke, ecke-pvsatvlke, este-prsatvlke,

10 Hoktarvlke, hunvntake hunvntaken etohkvlket nake holwvke vtotkvlke, estenhorkopvlke, laksvlke, fveceko enken kvwvpakat esyomat vrahkvt os, momet mvhakv fvecat vnrvpen nake etvt ocen omat, mv vrahkvt omes.

11 Hesaketvmese vrakkuehocat em opunvkv-herv vrakkat $\mathrm{mv}$ vm wihokvte vevkvyen.

12 Momen Cesvs Klist pu Pucasen vkvsamis, mv mecuye tayen cr yekcicvte, erkenvkv em wiketrn ov huericet, Heromet os, cv komvtet ok';

13 Vne tis, enhomvn vtvklecv, momet assecv momet vhopanv toyvyvtet; momis merkvt vn heckvtes, kerrvkot, momet vkvsvmkv tokat ofvn momecvyvtet ok'.

14 Momen vkvsvmkv momet vnokeckv Klist Cesvs ofv ocakat vpvkaken, pu Pucase en heromkvt tayet omvtes.

15 Heyvt opunvkv mehenwet on, omvlkvt eenakuecakvr tayet os, Klist Cesvs naorkvlken hesahuecvranet yvmv ekvnvn vlakvtes, vnet mv naorkvlke en homahte toyvyat.

16 Mome estomis, heyvt omecicen merkvt vn heckvtes, vc ofv taten Cesvs Klist em momis kometv hofonat omvlkvn kerken hayvren, muev ayat hesaketv yuksv-sekon en heckvre tetaye oren emen oh vkvsvmakat svhopakv en hakaren.

17 Momen Mekko meyuksv-sekat, em clkv-sekat, heckesekat, mv Hesaketvmese hoporrene enhvmkusat, vrakketv vkvsvmkv tepakat estofis meyuksv-sekon cm ocekvs. Emen.

18 Temore, cv'ppuce toyetskat, owvletr hoinvn ec oh vpeyvte vcrkvyen heyv vhakvn cem wikis, $\mathrm{mv}$ eteropotten tepokv heren etepoyetskvren; 
19 Vkvsvmkvn momet eohvkerrickv heren hvlatet, $\mathrm{mv}$ wikakvte saset omen, mvt vkvsvmkv ohfvecrn em perrocuko take vkvhopvnakes.

20 Himenevs Vleksantv tepakat mvn vpvkaket os, mvn Setvnen em wikvyvtes, Hesaketvmese vtvklecvkekaret kerrvkvren.

\section{SETENTVCKE II.}

Moniv nak makyranvyat omvlkv enhvteceskvn, este omvlkv, mekkvlke, este yekcetv ocakat omvlkv esyomat vrahkvn, vpohetv, mekusvpkv, este'm emekusvpkv, momet vkrsvmkv emetv hahoyvren vcahnis,

2 Hesaketvmese ometv, momet fvecetv omvlkv ofvn mahlvpvtket, momet etenherket fulleyvren.

3 Heyvt Hesaketvmese pu Hesayecv ehomvn here momet afveecicat omekv;

4 Mvt este omvlkvt hesahoket, mehenwv kerretvn orievkvren komet os.

5 Hesaketvmese hvmkuset omen, momen Hesaketvmese momet este etenherkepuecv hvmkuset omekv, mvt estehunvnwv Klist Cesvs tes.

6 Mvt omvlkv pvlke-es-neskvn e wikvtes, oketv orof ohkerkuchocvren.

$7 \mathrm{Mv}$ ohfvcevn opunvkv-sarv momet vpastel em wiketv v'mhoyvtes, (Klist ofvn mehenwvn opunayit, laksvkos,) vkvsvmkv mehenwv tepakat Centilvlke em mvhayv toyaren.

8. Monkv, este hunvntaket, enke hvsvthvken kvwvpaket, crprkketv momet en kerkekat sekon, estrmv estomis mekusvpvkvren komis.

9 Matvpomen hoktvkeu, eyasket, emahlvpvtket, accvke senehickv svheremahen accekot, ekvesse hotvnkan, monkat crtokunaplanen, monkat makelitvn, monkat accvke veake mahen 'se nehicet omekot; 
10 Momis, (hoktvke, Mekusapvlke toyēs, maket e ohkerkuecakat momvkrre here tetayat vcvkvyen,) vtotketv herakan 'se nehicvkvren komis.

11 Hoktet cvyayvket, apohice heret, kerrekvs.

12 Momis hokte emvhayet, monkat este-hunvnwvn ohfvnkvie em etektrnecukos, momis cryayvket vrvren komis.

13 Atvme taten hahoyen, mohmof Ef hahoyvtet omekv.

14 Momen Atvme mvkerhoyekon, momis hokte mvkerhoyen mv naorketv ofvn erhuervtes.

15 Momis emahlvpvtketv vpvken, ekvsvmkv, momet vnokeckv, momet efeke hvsvtketv ofvn emomecicaken omat, estuce heckueckv ofvn veayehocvres.

\section{SETENTVCKE III.}

Hervt maketv mehenwvt's, Estimvt mekusvpkv-cuko vheciev em wiketvn eyaeat vtotketv heren eyacet os.

2 Monkv mekusvpkv-cuko vhecicvt, nake em mvttekot, ehiwv hvmkusen ocet, vketecet, emahlvpviket, yvmaskuset, este en cukopericat encaket, mwhayetv emvnvcket:

3 Hacv tokot, tepoyv tokot, cvto-kunawvn vnokecekot; momis momis komet, tepokv em vlostekot, vleskapv tokot;

4 En cuko mahusan heren ohfvnket, en hopuetake emahlvpvtketv omvlkv ofvn em apohicakusen ocet, omvranet os.

5 (Hiyomet omekv, estimvt en cuko mahusan heren em fvyvtetvn kerrekon omat, estomēn Hesaketvmese em mekusvpkv-cukon vhecicvr haks?)

6 Mekusapv hvtuse hakat tokot, ekvsvmkvt hvlwecen, puyvfekev holwakat em ohfvececkv ofvn lvtkekaren.

7 Momen ohvtvlakat mekusvpkv cuko en lvpvtke svpaklat este here omecet opunvyecaket omvranet os, vlesketv 
momet puyvfekev holwakat'sem enkvnhetv ofvn lvtkekaren.

8 Tekvnvlke matvpomen mahlvpvtket, tulaswv hokkolicekot, prrko-opuswrn em vloste mahekot, vlesketv ofv nake 'se ohvtvlvyetvn eyacekot;

9 Vkvsvmkv en nake ehkan eohvkerrickv hvsvtkat ofvn hvlvtaket omvranet os.

10 Momen heyvu eskerrvkhoye en topvrvn nake em mvttvkekon eshechoyen, tekvnvlke em wiketvn vfvstvkekvs.

11 Matvpomen ehitaket fvccet, este ohlaksvlke tokot, mahlvpvtket, nanvke omvlkv ofv heromvket omvranet os.

12 Tekvnvlket, ehiwv hvmkusen ocet, en hopuetaken, momet e hute take heren ohfvnkvket omekvs.

$13 \mathrm{Mv}$ tekvnvlke em wiketvn heren vfrstakvtet hueretv heran, momet vkvsvmkv Klist Cesvs ofv ocat ofvn fekhvmketv rakken e en heckuecakekv.

14 Lvpken cem vlvkares, komvyat enhonrkvn ocit, heyv nanvken cen hoccicis;

15 Momis, vlvkvkon hofunen omat, Hesaketvmese en cuko, mvt Hesaketvmese wenakat em mekusvpkv-cnkot, momet mehenwetv en cukele momet em wakkvt omen, $m v$ ofv estomēn momvre heren vretske tetayat kerretskvren.

16 Momen kerkuset os, mekusvpkv en nake ehkat rakke tes; Hesaketvmeset vpeswv ofvn hechoyen, Puyvfekevt fvccecen, encelvlket hecaken, Centilvlke 'sem erkenvkhoyen, ekvnv ofvn oh vkvswmboyen, hryayvke ofvn a eshoyvtes.

\section{SETENTVCKE IV.}

Momen Puyvfekcvt opunvkv. kerkvken 'sopunayet, hommaket os; Oketv yicvranat ofvn mvkerrvlke em 
puyvfekcvlke momet puyvfekcv holwvhokat em mvhakvin vpohicaket, vkvsvmkvn enkvpakat svsvranet os:

2 Mvkerretv ofvn lakset opunayet, em eohvkerrickv take crto hryyan es nekrihocen ocaket;

3 Etepayetvn vsehet, nakhompetv morahrvkvn estet $\mathrm{e}$ em vsehvkvren kicaket os; Hesaketvmeset, mv mehenwetvn kerrve, vkvsvmakat, vkvsvmkv emetv vprken eenakuecvkvren hahicvtet ont oman.

4 Nake Hesaketvmese hayvte vtekat heret omet, vhepvkhoyvre tokot omekv, vkvsvmkv emetv vprken eenakuehncen omat.

5 Hesaketvmese em opunvkv, momet mekusvpkv eteropotten hrsvtken hahoyet omekv.

6 Heyv nanvken tecakkakvten vkerricvkuecetsken omat, Cesvs Klist em erkenvkv here, vkvsvmkv mvhakv herat tepakat em opunvkv vevkvyetskvte ofvn rakkuehocet ometskvres.

7 Momis nakonvkuce hoktvlvke enanvke momet mekusvpkv vcvkvye tokvkekan vhepvkvs; momis mekusvpkv ohfvecrn e nekeyicrs.

8 Hiyomet omekv, ena nekeyickv estomusen lopicet omekv: momis mekusvpkvt nanvke omrlkv ohfvecrn lopicet omes, hiyome hesahokeyan, momet hesaketv vlvkvranan temfvecetvn ocet omekv.

9 Heyvt maketv mehenwvt's, momen omvlkvt eenakuecakvr tayet os.

10 Hiyomet omekv, heyvt omecicen vtotkēt, vlesketvn kvwapeyet os, Hesaketrmese wenake likat, mv este omvlkv, momet vkvsvmakat mahvkvu Hesahuecvn em enhonrēkv.

11 Heyv nanvken yekcetvn 'sopunayet, 'sestemvhayvs.

12 Cem mvnettetvn estit enhomehcats; momis vkrsvmakan opunvkv, vretv, vnokeckv, puyvfekev, vkvsvm- 
$\mathrm{kv}$, efeke hvsvtketv esyomat ofvn 'sem vhopakvt ometskvres.

13 Cokv ohonvyetv, emvcahnetv, emvhayetv esyoman vfastetske emunken vlvkares.

$14 \mathrm{Mv} c e^{\prime}$ mhoyvte ec ofv ocan, mv owaletv eteropotten momet erkenvkvlke nvkafte e'nke oh wvkecakat etehoyvnen ce'mhoyvten vmoskomekot huyiretskvs.

15 Heyv nanvken oh vkerricvs; $\mathrm{mv}$ of mvketeken huervs; cen lopican omvlkvt hecvkvren.

$16 \mathrm{E}$ vketecet, mvhakvn vketecvs; heyv nanvke ofvn emomecicvs; heyvn momecetskat e hesayecet, cem apohicrlkeu hesahuecetskvres.

\section{SETENTVCKE V.}

Este vculican ehvnekot, ce'rke omēn emvcahnet; este mvnettakan tecakkakvte. omēn;

2 Hoktvlakan $c e$ 'ckvlke omēn; hoktvke mvnettakan ce 'wvntake omēn efeke hvsvtketv omvlkv ofvn emvcahnvkvs. 3 Oyevlken vrakkuecrkvs, mehenwvn oyevlket on omat. 4 Momis oye estomet hopuetaken, monkat em osustaken, echussucvlke tis ocen omat, mvt e hute take ofvn heromkvn heckuecet, e'ckvlke take vfvstakvte vrahkvn vyoposketvn kerrvkekvs, heyvt Hesaketvmese ehomvn here momet afvecicat omekv.

5 Momen hokte oye mehenwe, entvlkuse vhoskat Hesaketvmesen em enhonret, mekusvpkv momet Hesaketvmese em'pohetv ofvn nettvn momet neren emomecicet os. 6 Momis mv afvcketv ofv likat, hesaket omis, elet os. 7 Momen heyv nanvken es emvcahnvkvs, em mvttvkekot svpaklvren.

8 Momis estimvt en nakvlke mahusan, en cuko-vtehkvlke mahvkvu, en hopoyekon omat, vkvsvmkvn helaksehcet, este vkvsvmekat 'sen holwvket os. 
9 Oyet ohrolope pale epakan oricekat sohvhonkvthohyats :

10 Este-hunvnwv hvmkuse e 'hiwvt omvtet, em vtotketv herakat vrahkvn heren opunvyehocen, hopuetaken mahmicrtet on omat, este kerrvkekan evpryvten omat, mekusapvlke e'len em okkosvten omat, este efeknokhokan emahlvpatvten omat, vtotketv herakat vtekan heren vevkayvten omat tvwwon sohvhonkvthoyvres.

11 Momis oyevlke esmvnettakan vhepvkvkvs, Klist vnrvpen afveketr hopoyvlke hakof, ehesetvn eyacvket omekv.

$12 M v t$ ohfvcceckvt en heckaket os, em vkvsvmkv enhvteceskvn vwikakekv.

13 Momen sohvpakat, cukon etohtvlecet, enhorkvn kerret, momet enhorkv tvlkekon, este opunvyecvlke, nanvkokvlke, momet nanvke makēsko tayat opunvyakat haketon kerraket os.

14 Monkv hoktvke esmvnettakat ehesaket, hopuetaken heckuecet, cukon vfastet, $m v$ vnrapvt holwvken opunvyecrre em etektrnecrkekaren komis.

15 Entis Setvnen vevkvpeyetvn fiyecakat sasekv.

16 Este-hunvnwv, monkat hokte vkvsamat oye hoktvken ocen omat, ent emvnicvkekvs, mekusvpkv-cukot sen honnekaren, momat mehenwv oyevlken emvnicuke tayat.

17 Mekusvpkv-cuko em fvyatvlke heren fvyvtakat, vrakkueckv tepvkocket en heckvre heretv orices, komhoyvkekvs, mv opunvkv mvhakv tepakat ofvn vtotkakat mahvkvu.

18 Wakv-vtotkv telekon cesapan yupopihcetskvs, momet, Vtotkvt em feketr en heckvre heretv oret os, Cokat maket omekv.

19 Akerrvlke hokkole monkat tutcene ehomv take tokon 
omat, mekusvpkv-cuko em fvyatv vnrvpen akerkueckvn em apohihcetskvs.

20 Mv naorkakan omvlkv ehomvn em vsehvkvs, vpvltakeu penkvlvkvren.

21 Etemetueckv sekon, temvrahken est' ohvkerricekot heyv nanvken vfvstrs, makit, Hesaketvmese, momet Pucase Cesvs Klist, momet encelvlke enhopoyvkvte. ehomvn yekcen $c e$ 'mvcahnis.

22 Estimvn vyvkhvmkusen $c e$ 'nken oh wvkehcetskvs, momet este etv en naorketv take vpvken naken ohcetskvs : ce hvsvtke emunkv taren e vcayecrs.

$23 \mathrm{Mucv}$ ayat uewv tvlkusen esiketskvs, momis ce 'nvcofv vrahkvn, momet enokketv cem etewolicat omecicen prrko-opuswv estomusen eskvtcrs.

24 Este-hunvntake en naorketv homvn heckvket, homvn ohfvececkvn oh vpeyat svset os, momis vpvlwv en naorketvt yupvn vpeyet os.

25 Momen matvpomen vtotketv herakat heckaket os, momen mvrahkvt o estomis ehkvkeko tayes.

\section{SETENTVCKE VI.}

V'TotKvLke estomomuse nokwv-oh-wakkv elecrn svpaklat eme mahusat e pucasvlken, Vrakkueckv omvlkv en heckvre oricakes, komet oh vkerricvkekvs, Hesaketvmese e hocefkv momet mvhakvn tvklehocekaren.

2 Momen mv epucase vkvsvmvke ocakat, etecakkakvtet omakat vråhkvn enhomecahkats, momis vtotketv min em mecvkekvs, heromet, momet vnokeckvket, momet mv nake herat etencawvlket omakekv. Heyv nanvken vcahnet, emvhayvkes.

3 Estimvt emvrahken mvhayet, opunvkv hoporrenvke, pu Pucase Cesvs Klist em opunvkv, momet mvhakv mekusvpkv vcrkvye ocan apohicekon omat, 
4 Ekvsvmet, naken kerrekot, momis este enokket naken eyacusat omēn, nake vpohetv momet opunvkv setenrvpkv ohfvecrn eyacet os.

5 Vleskvpkvt, etenrvpikvt, tvhiketv, ohvkerrickv holwvhokat, momet mehenwv ncvkekot, vkerrickv holwvken. oce, Eohvtvlvyetv mekusvpkvt's, komakat em opunvkv setenrvpkvt mvn asossaket os; heyv omakan e enkvpvyecicrs.

6 Momis mekusvpliv emahlvpvtketv vpakat nake epoyetv rakke tes.

7 Hiyomet omekv, yvmv ekvnvn naken asceyēkates, momet mehenwvn naken esossēko tayes.

8 Momis hompetv momet accvken oceyat, mvn es pu mahlvpvtket omekvs.

9 Momis mv nake-ocvke haketv komakat nake eskerhoyat ofun pvlatkat, momet nake senkvnhetv, momet eyackv hoporrenkv vpvkeko nokkicakat sulke ofvn pvlatket os, mvt sumketv estemerketv tepakan esten vkpvsatet os.

10 Cvto-kunawv vnokeckv nake holwvhokat omvlkv en yvlonkvt omekv; mvn vleskvpakat svset omof, vkvsvmkvn em fiyecet, feknokketv sulken 'se etekfvnvnicakvtes.

11 Momis cemet, Hesaketvmese em este toyetskat, heyv nanvken en letkvs; momet fvccetvn, mekusvpkvn, vkvsvmkvn, vnokeckvn, momis kometrn, momet eyasketvn vevkvyvs.

12 Vkvsvmkv etepokv heran etepoyvs, hesaketv yuksvseko, mv ec ohhuehhokan hvlvtvs, eohkerkueckv heran, akerrvlke sulke ehomvn es e ohkerkuecetskvtes.

13 Hesaketvmese nak omvlkv wenahuecat ehomvn, momet Klist Cesvs, Pantevs Pilvte ehomvn eohkerkueckv herat eohkerkuecvte ehomvn yekcen ce kicis, 14 Heyv vhakv, svholwvke cotkusis sekon, momet 
mvttes kihoceko tayen pu Pucase Cesvs Klist heckvranat oren vevyecrtcrs:

15 Mvt, Ohfvnke vrakkuehoce enhvmkuse liket, mekkvlke em Mekkot, momet pucasvlke e Pucaset omat, em oketv omof, em vlvketvn hecicvres;

$16 \mathrm{Mv}$ tvlkuset hesaketv emunkvn ocet, hvyayvke estimvt oreko tayat ofvn likat, mvn estimvt hecekatet, hecetv umeko tayes; emen vrakketr momet yekcetv yuksv-sekat em ocekvs. Emen.

- 17 Yvmv ekvnv ohfvecr nake-ocvlken em punayet, Vkerrickvn es hvlhvwecekot, nake-ockv sumke tokon, momis Hesaketvmese wenake, mv esfrcken es afvckeyvren nanvke omvlkv pu'man enhonret,

18 Heren momecet, vtotketv herakat ofvn nake-ocvket, nake enkvpicetv metetaket, nake etekvpicvketv vkvsamuset omvkes,

19 Oketv vlvkvranat vrahkvn enlikv heran hericet, hesaketv yuksv-sekon hvlvtatskvren, maket yekcen kicrkvs.

20 Temore toyctskat, opunvkv ehvpcrkvke, momet hacohakakan, momet mv laksen nakkerretv kihocat em etenrvpkrn munvttepet, mv nake hericetskvren ce'mhoyvten vevyecrs:

$21 \mathrm{Mvn}$ eenakuece omecaket, vkvsvmkv ohfvecrn em muttakvtet svset os. Heromkvt ece 'pakekvs. Emen.

I Pal Peketevnvlke em etvlofv rakke Leotesev hocefkan liket, heyv cokv enhvteceskvn Temore ohtotvte coyvtes. 


\section{COKV VPASTEL PAL}

\section{TEMORE OHTOTVTE SVHOK KOLAT.}

\section{SETENTVCKE I.}

PaL, Hesaketvmese komat eteropotten, hesaketv Klist Cesvs ofv ocat etemfvccetv vcrkvyen Cesvs Klist em vpastel toyvyat:

2 Temore $c v^{\prime}$ ppuce $v m$ vnokeckan ohtotis; Heromkv, merkv momet herkv Erke Hesaketvmese, momet pu Pucase Klist Cesvs avtet cem ocekvs.

3 Hesaketvmesen, mv cv'rkvlke tate eteropotte $v n$ heckvten eohvkerrickv hvsvtkan es em vtotkvyat, vkvsamis,

4 Fekhonnekot, nettr nereu, ce turopuswv prlatkan vkerricit, ce hecetvn cv yacuset, vm mekusvpkv ofvn ece 'kerricvyet os; afvcketvn es cv fvcke taren komit;

5 Vkvsumkv mehenwe ec ofv likan, enhvteceskvn Loes ce 'puse ofvn, momet Yunese ce'cke ofvn likvten vkerricryof; momen mvt cemeu ec ofvn likes, komvyc heres.

6 Monkv Hesaketvmese en nakemkv cv'nke ec oh wvkhokicki eteropotte ec ofv ocan hvtvm vhetecetskvren ece 'kerricepuecis.

7 Hesaketrmeset penkvlkv vkerrickv tokon, momis yekcetv, vnokeckv, momet hoporrenkv em vkerrickvn pu'mvtet omekv.

8 Monkv pu Pucase ohkerkucckvn, monkat vne en 
svlvfke toyryat es ec elset ohmetskvs; momis opunvkvherv em estemerketvn astenkvwapvt omrs, Hesaketvmese en yekcetv vevkvyen.

9 Mvt pu hesahuecet, huehketv hrsvtken es pun huehkvtet os, pum vtotketv vevkvye tokon, momis eme komat, momet en heromkv vevkvyen;

$10 \mathrm{Mv}$ heromkvt ekvnv vliceckv oreko emunken Klist ofvn pu'mhoyvtes, momis hiyomat pu Hesayecv heckat eteropotten hecket os; mvt elkvn sumecihcet, opunvkvherv eteropotten hesaketv, momet hesaketv meyuksvsekan ohhvyayicrtet os:

$11 \mathrm{Mv}$ ohfvecvn vne, erkenvkv, vpastel mumet Centilvlke mvhayvn cr huerihocvtet os.

12 Momen mv vrahkvn heyv nanvken es cv'stemerket os, momis vc vlsekos; hiyomet omekv, estimv ofvn vkvsamvyvten kerrit, mv nake em wikvyvten mv nettv oren vcayece tetayat yekcetv ocet os, komvye heres.

13 Opunvkv etclilvke herake opunvyvye vm pohetskvten, vkvsvmkv vnokeckv tepake Klist. Cesvs ofv ocat ofvn yekcen hvlvtvs.

$14 \mathrm{Mv}$ nak here hericetskvre ce'mhoyvten Puyvfekev Herat ep ofv likat etcropotten veryecvs.

15 Heyvn kerretskes, Esev ofvn vpokat omvlkvt v'nkvprkakvtes; mv vprkakat Ficelvs Hvmocenes tepakat omakes.

16 Hesaketvmeset Onesefolvs en cukon oh merrate, tewolen v'mahlvpatet, vn crto-tetvkkakv es vlsekatet ok'.

17 Momis Lomen arof, vfekcakhet cv hopoyet, es $c v$ hecemvts.

18 Pucase akvpvken mv nettv omof merkv en heckvren Pucaset emate! Momen nanvke en sulke estomomusen Efesvs ofv vm vtotkvten heren kerretskes. 


\section{SETENTVCKE II.}

Monkv cemet, cv'ppuce toyetskat, heromkv Klist Cesvs ofv ocat ofvn yekcet omvs.

2 Momet mv nanvke akerrvlke sulke em etenrvwvn vm pohetskvten, heyvn hunvntake heromvke, etvu emvhayvke tayan, em wikvkvs.

3 Monkv Cesvs Klist en suletawv here omet estemerkvn kvwrpvs.

4 Suletawv are estomet yvmv hesaketv enanvken 'se vpvllvyekot os; mv suletawv hakvre enhopoyvten afvcecicetvn komet omekv.

5 Momen estimvt etemuntvlkv vcevllat, vhakv vcvkvyen vcevllekon omat, kvtvlvkv vcakan es kvpvtuehocekot os.

$6 \mathrm{Mv}$ crpofv oh vtotkat tawvt em etten cvwrranet os.

7 Nake makvyan oh vkerricvs; momen Pucaset nanvke omvlkv ofvn kerretvn ce'mate!

8 Cesvs Klist, Tewe en honvpsen avtet, vm opunvkvherv vcrkvyen, pvsatkvte aenkvpvken akvwvpkvten ce hohsats.

$9 \mathrm{Mv}$ ofvn holwvyecv omēn estemerketvt vn hecket os, svlvfkueckv oren; momis Hesaketvmese em opunvkvn svlvfkuehocekot os.

10 Monkv enhopokvte vrahkvn nanvke omvlkvn kvwapvyèt os, hryayvke emeyuksv sekat vpvken vhesaketr Klist Cesvs ofv ocat en heckvkvren.

11 Heyvt maketv mehenwet os; Vpaket pu prsatkvten omat, vpaket hesahokeyvret ok'.

12 Estemerketvn kvwapēn omat, vpaket mekkeyvres; vhepakēn omat, emeu epu 'hepvkvres :

13 Vkvsamēko estomis, emet mehenwet huere munkvt os; ehelakseceko tayes.

14 Heyv nanvken ohvkerricepuecet, Pucase ehomvn, Opunvkv ocakat ohfvccvn etenrahpatskvs, mvt naken 
lopiceko tayekv, momis pohakan ehosvkueces, kicet, yekcen em vsehvkvs.

15 Hesaketvmese ehomvn vkvsvmvke taye oren e heckuecetskvret vcevllvs, momat vtotkv nak es vlsetv cen sekon, mehenwv em opunvkvn fvccvn tekvpicet hueretskvret.

16. Momis opunvkv ehvperkvke momet hacohakakan em e fulkvs, holwvyeckv em ohvtvlvkvkvret ok'.

17 Momen em opunvkvt hote vkuekvnto omet pvipvkvres; Himenevs Filetvs tepakat mvn vpvkakes.

18 Mvt mehenwetv ohfvecvn em mvttet, Elkv aenkvwvpketv entis hoyvneps, maket, este vnvcome em vkvsvmkv taken lekvfaket os.

19 Mome estomis, Hesaketvmese en likvt yekcen hueret os, heyv eskerkvn ocet, Pucaset enaken kerhoyes, momet, Klist e hocef kvn hocefat vtekat fvccetv tokan enkvpvkekvs.

20 Momis cuko rakke ofvn nake-vcrnkvt ocvket os, cvto-kunaplane, momen cvto-kunaphvtke es hayvkvte tvlkekon, momis eto tis, fakke tis es hayvkvte ocvket os; momet vrakkuehocvranat sasen, vrakkuehocvranekat ocvket os.

21 Monkv heyv nanvken estimvt e en hvsvtecen omat, nake-vcrnkv vrakkuehocvranat, hrsvtke, Pucase es en lopice herat, momet vtotketv herat vtekat emetetake tares.

22 Momis este mvnettvlke em eyackvn en letkvkvs, momet fvecetvn, vkvsvmkvn, vnokeckvn, momet efeke hvsvtke aossen Pucasen oh huehkakat vpvken etenherkvn hopoyvs.

23 Momis vpohetv ehvperkvke hoporrenkv vpvkeko ocakan vhepvkvs, mvt tepokv hayaket oman kerret.

24 Momen Pucase em vtotkvt tepoyekot, este omvlkv em eyaskuset, mvhayetv mvnvcket, momis komet, 
25 Eyasketv ofvn mv. vnrvpakan emvhayet; Hesaketvmeset mehenwetv ohkerkueckv oren vkerrickv-etickvn emake witen omat;

26 Momen mv puyvfekev holwakat komat vevkvyen svlvfkuecvkvtet 'sem enkvnhetvn 'se ossicvkvren.

\section{SETENTVCKE III.}

HeYvu kerrvs, nettv espokakat omof, oketv penkvlket ocvkvres.

2 Hiyomet omekv, estet e vnokecet, crto-kunawvn encaket, opunvkvn 'sekvsamet, ekvsamet, tvhiket, eckvlken em apohicekot, Hesaketvmesen vkvsvmekot, mekusapv tokot,

3 En nakvlken vnokecekot, temfvccetv-kacvlket; esteohlaksvlket, e-em-fryvtekat, sehonecket, este herakan en homecet,

4 Enhonricvlke, ekv-yekcakat, serakken vkerricet, Hesaketvmese senhoyvnen afvcketvn vnokecet;

5 Mekusvpkv en heckvn ocet omis, mv en yekce helaksecakat ocvkvres; mv omakan em e fulkvkvs.

6 Hiyomet omekv, heyv omakat cukon esceyet, hoktvke hacohakvke, naorketv es honhoye eyackv mvrahrvkvn es nekeyihocan,

7 Estofis naken kerrakis, mehenwetv kerretvn estofis umvkeko tayan svlvfkvken evpayaket os.

8 Momen Canese Camplese tepakat Moses vnrvpakvte omēt heyvt mehenwetvn vnrvpaket os; este em vkerrickv holwvhoket omen, vkvsvmkv ohfvecvn etv vhepvkhoyvten.

9 Momis ohvtvlaken vpeyekares, mv en hoporrenkv take sekat hecket omvte etvpomen mvu en hoporrenkv sekat este omvlkv ehomvn hecke taret ok'.

10 Momis vm mvhakvn, vretvn, kometvn, vkvsvm- 
kvn, momis kometv hofunat, vnokeckvn, momis kornetvn,

11 Asseckvn, estemerketvn momet nanvke Anteak, Ikonev Lestlv esyomat ofv vc oh yicvten heren kerretskvtet omes; asseckv estomakan kvwapvyvten kerretskes; momis $m v$ omvlkvn Pucaset a 'svc ossvtes.

12 Momes, momen Klist Cesvs ofv mekusvpkv vcrkvye fulletvn komat omvlkvt asseckvt en heckvkvres.

13 Momis este holwvyecvlket momet ehosicvlket holwryeckv ofvn ohvtvlaket vpeye munkvres, ehosicaket, momet ehosihocen.

14 Momis mv nanvke kerret, heren vkvsametskvte ofvn emomecicvs, estimvt mvn ce mvhayvten kerret;

15 Momet hopuewvt aretskof, cokv vcacakat, vkvsvmkv Klist Cesvs ofv ocat eteropotten vhesaketske taye oren ce hoporrenice tayan kerre vtecicetskvten kerretskes.

16 Cokv vcakat omvlkvt Hesaketvmeset este em vkerrickvn nekeyicen estemhoyvtes, momet mvhakvn, nak est em vsehetvn, vretv-mvherickvn, fvccetv-mvhayetv esyoman lopicet omes :

17 Hesaketvmese em este-hunvnwvt temvpoket, vtotketv herakat omvlkv ohfvecvn metetake heret omvren.

\section{SETENTVCKE IV.}

Monrv Hesaketvmese ehomvn, momet Pucase Cesvs Klist, mv em vlvketv momet em ohmekketv omof, hesahokan momet prsatkvten ohfvccecvranat ehomvn yekcen ce kicis ;

2 Opunvkvn 'serkenvkvs; oketv omof, oketv toko estomis vhuervs; momis kometv mvhakv tepakat omvlkvn 'sest' em vsehet, emvcahnet, em punvyvs.

3 Hiyomet omekv, oketvt vlvkvres, momof muhakv mehenwan kvwvpekot, momis, e hvcko hottopvken ocet, 
eme mahusat em eyackv take vcvkvyen mvhayvlken e em vteloyvkvres;

4 Momet $e$ hrcko taken mehenwetrn 'sem fulotket, nakonvkuce min oh fulotecvkvres.

5 Momis cemet, nanvke omvlkv ofvn vketecvs, estemerketvn kvwapet, opunvkv-herv emvhayv em vtotketvn momecet, cem vtotketvn es fveecvs.

6 Vnet Cesvs vrahkyn cv'lehocvre vm etetaket omen, yvmvn enkvpvkvranvyat oketv vwolet omekv.

7 Tepokv heren tepoyit, temvrkvn poyit, vkvsvmkvn veryecryvtes.

$8 \mathrm{Mucv}$ ayat fvccetv en kvpvtokv:vcakan vn herihocet os, mv Pucase frccecv fvccat mv nettv omof v'mvranat; momet vne tvlkekon, momis em vlvketv vnokecakat omvlkvn emvkvranat.

9 Lvpken vm vlvketskvret vketecvs:

10 Temvs v'nkvprkepekv, yvmv ekvnv mucv ocan vnokecet, yvmvn enkvpaket, Resvlonikvn ayepet os, Klessens Kelesevn ayet, Titvs Talmesevn ayepet os.

11 Luk tvlkuset vc vpakes. Mak evpahyet, ece 'pvken svtes; vtotketv ohfvccvn vn lopicet omekv.

12 Momen Tekekvn Efesvn ohtotvyrnks.

13 Kapv-rakko Kapvs likan Tlov tvlofon wikvyvten, atetskof, 'svtvtcrs, momet cokv ocakan, momet pacments kicetv mahvkvu.

14 Cvto-lane paccv, Vleksantv hocefkat, holwaken tayen cv momecvtet os; em vtotketv vcrkvyen Pucaset em fekate:

15 Momen cemeu mvn vketecrs; pum opunvkvn tayen vnrapvtet omekv.

16 Enhvteceskv em vyoposkvyof este hvmkusis vm vpvlhymken huerekates, momis omvlkvt v'nkvpvkakvtes; Hesaketvmeset mv taken vrvhecekate. 
17 Mome estomis, Pucaset ve vpaket hueret, cv yekcicrtes; momat vm erkenvketvn esfvcken kerhoyen Centilvlke omvlkvt pohvkvren; momen este-papv e cukwvn a ve ossihocvtes.

18 Momen Pucaset vtotketv holwvke vtekan a vc ossihcet, em ohmekketv hvlwe ocan orvye tayen $v c$ vcryecvres; emen vrakketvt estofis emeyuksv-sekon em ocekvs. Emen.

19 Pleskv Akwelv tepakan, momet Onesefolvs en cukon vsekvkvs.

20 Elastvs Kolenrvn fekhonnemvts; momis Tlofimvs enokken Miletvn wikimvts.

21 Rvfo orekon vlvketvn komvs. Yupulvs, Plutens, Linvs, Klatev momet tecakkakvte omvlkvt ece 'sekaket os.

22 Pucase Cesvs Klist cem puyvfekcvn vpvkekvs. Herkvt ece 'pakvkekvs. Emen.

I Cokv esvhokkolat Pal Temore, mv Efesvlke em mekusvpkvcuko vfastv enhvteceskv hahoyvten, ohtotvtet, Lomen likof, Nelo ehomvn svhokkolvn huerihoce omof. 


\section{COKV VPASTEL PAL \\ FILEMVN OHTOTVTE.}

PaL, Cesvs Klist en svlvf ke tecakkvte Temore tepakat pum vnokeckuse, momet vtotkv pum etohkvlketv, Filemvn,

2 Hvtvm Afiv pum vnokeckan, momet Akepvs suletawv pum etohkvlketvn, momet mekusvpkv-cuko cen cuko ofv ocan ohtoteyat:

3 Heromkv, momet herkv Pu'rke Hesaketvmese momet Pucase Cesvs Klist avtet cem ocvkekvs.

4 Cem vnokeckv momet vkvsvmkv mv Pucase Cesvs, momet mekusapvlke omvlkv em ocetskat pohit,

$5 \mathrm{Vn}$ Hesaketvmesen vkvsamis, vm mekusvpkv take ofvn estofis ce hocefet;

6 Nake here Cesvs Klist etehoyvne ec ofv ocat vtekat ohkerkueckv eteropotten cem vkvsvmkv este em etekvpickvt lopickvn yekce taren.

7 Hiyomet omekv, afvcketv mahlvpvtkets tepakat rakken cem vnokeckv ofvn oceyet os, emekusapvlke em envcofv taken tayen mahlvpatetskekv, tecakkvte toyetskat.

$8 \mathrm{Monkv}$ Pal vculat momet ohvtvlakat hiyomat Klist en svlvfket omit, vnokeckv mimv vrahkvn ce'nkusapvyet os,

9 Nake momecrkvr tayat ohfvecv yekcen ce kicare Klist ofv fekhvmketvn ocvyet o estomis.

10 Onesemvs, cv'ppuce, cv svlvfkuehoce omof heckuecryvte ohfvecvn ce'nkusapis :

11 Oketv hoyanvte ofvn cen lopicekat omet, momis hiyomat ceme vne tepakvn pun lopicet omes:

12 Mvn yefulecicvyrnks; monkv evpryvs, cv nvcofv mahusat mvt omes: 
13 Opunvkv-herv eswrnakv ofv ece 'yoposket vm vtotkvren ve vpvkvren fekhonnicetvn komvyvnks.

14. Momis cem vkerrickvt vc vpaket omekat naken momecetvn komvkvnks; vn lopicetskat ec ohyekcihocvtet omekon, ceme kometskat omecice taren.

15 Hiyomet omekv, oketv vcrpke mahekon ce'nkvpahken meyuksv-sekon evpryetskvre ome witvtes :

16 Momen mucvn evpayetskat vtotkv omekon, momis vtotkv senhvlwe, tecakkvte vnokecke, vne mahvkvu vm vnokeckat, momis mv senhoyvnen enavpeswv ofv, momet Pucase ofvn cem vnokeckat omēn evpayvranetsket os.

17 Monkv, Vm etohkvlketv tes, cv kometsken omat, vne omēn evpryvs.

18 Naken cem vhopanen, monkat naken vhuericetsken omat, mvn es ve vrvhecvs:

19 Vne Pal toyvyat cv'nke mahusan es hoccihcis, vnet fekares: mome estomis, ohvtvlakan e mahusan vm e vhuericetskan ce kicvkos.

20 Momis, tecakkvtē, Pucase ofvn ec ofvn vc afvckekvs; Pucase ofvn cv 'nvcofvn mahlvpatrs.

21 Cem apohickvn enhonrit, cen hoccihcis, makvyat senhoyvnen momecvranetskan kerrit.

22 Momen ohvtvlakat, fekhonnvye tayan vm etetakuecrs, cem mekusvpkv eteropotten cem vlvkaren vm etektvnechoyvres, komit enhonrikv.

23 Klist Cesvs ofv svlvf kuehocat vm etohkvlketv, Epeflas hocefkat,

24 Hvtvm Mak, Elestakvs, Temvs, Luk, vtotkvlke vm etohkvlketvt ece 'sekakes.

$25 \mathrm{Pu}$ Pucase Cesvs Klist en heromkvt cem puyvfekcv taken vpvkekvs. Emen.

ๆ Heyv cokv, Pal Lomen likof hoccihcen, vtotkv Onesemvs Filemvn 'sem ayvtes. 


\section{COKV VPASTVL PAL \\ TITVS. OHTOTVTE.}

\section{SETENTVCKE I.}

PaL Hesaketvmese em vtotkv, momet Cesvs Klist em vpastvl toyvyat, Hesaketvmese enhopoyvkvte em vkvsvmkv momet mehenwv ohkerkueckv, mekusvpkv mehenwuse ohfvecv ocat vevkvyen,

2 Hesaketv emeyuksv-seko em enhonrkv, mv Hesaketvmese lakseko taye, ekvnv vliceceko munkof, este temfaccrte ocat,

3 Momet momvre tetaye ocof, erkenvketv eteropotten em opunvkvn kerkuecvte, mv Hesaketvmese pu Hesayecv em vhakv vevkvye vm wihokat ofvn;

4 Titvs, vkvsrmkv tvleme vcrkvyen cv'ppuce mahusat oh 'totis; heromkv, merkv momet herkv Hesaketvmese Erke, momet pu Pucase Cesvs Klist, pu Hesayecv a vtet ece 'pvkekvs.

5 Heyv esvrahkv Kletv ofvn ce wikimvts, nake espokeko ocakan mvhericet, etvlwv omvlkvn erkenvkvlke vculvkuecan em wiketv hayetskvren, ce kicvkvyvte omen;

6 Estimvt nake em mvttekot, ehiwv hvmkusen ocet, hopuetake vfekrakkvke tayen ocet, yvmahkuecv kihocekot, ekv-yekcet omekon omat, mvn hayetskvres.

7 Mekusvpkv-cuko vhecicv, Hesaketvmese en nakvfastvt omet, nake em mvttekot, ekvsvmekot, lvpken cvpvkkekot, tepoyv tokot, hacv tokot, vlesketv ofv nake 'se ohvtvlvyetvn eyacekot omvranet omekv; 
8 Momet este en cukopericat encaket, este herakat vnokecet, emahlvpvtket, fvccvt, hvsvtket, e em fvyatet,

9 Mvhahoyvte vcrkvyen punvkv fvccrn yekcen hvlatet, opunvkv mehenwv ofvn este'm vcahnet, momet a vnrapvlke ohfvnkvre tetayen.

10 Ekv-yekcakat sulket omekv, momet ehvperkv opunvyvlke, este-mvkerrvlke, svkvmsesvn vkvsamvlke mahvkvu.

$11 \mathrm{Mv}$ e cukwv vkhothokvranet os; mv cuko hvmecat rvkpvlricet, momet vlesketv ofv nake 'se ohvtvlvyetvn eyacvket, nake mvhayeko tayat mvhayaket os.

12 Kletvnvlke estofis laksvlke, ponvttv holwvyecvke, - $\quad$ nvrke fekhonnvket omes, Kletvnvlke mahusat hvmket, owalvt, makvtes.

13 Heyv kerkuecrte mehenwvt os, monkv fvsken em vsehvkvs, vkvsvmkv ofvn cvfencvke taren ;

14 Cusvlke en nakonvkuce, momet este mehenwr en kvpvkakat em vhakvn oh hecvkekaren.

15 Hvsvthakat nak' omvlkvt en hvsvthvkēs; momis hvsvthvkekat, momet vkvsvmvkekat naket en hvsvthvkekot, em puyvfekcv, em vkerrickv tis svholwvhoket os.

$16 \mathrm{E}$ oh kerkuecakat, Hesaketvmesen kerres, makaket os; momis vtotketv ofvn Hesaketvmesen ehelaksecet, momet en homechoye tetayat, momet apohicvkekot, vtotketv herakat vtekat ocvkekot omes.

\section{SETENTVCKE II.}

Momis mvhakv temvpoke vcrkvyen nanvken em punvyvketskvres;

2 Vculakat mahlvpvtkvke, fvccrke, hoporrenvke, vkvsvmkv, vnokeckv, momis-kometv esjomat ofv crfencrke taren.

3 Matvpomen hoktvlvke, em fulletv hvsvthvket, este- 
oh-laksvlke tokot, pvrko-opuswv sulken eyacvkekot, nake herakat emvhayvlke taren.

4 Hoktvke mvnettvlken hoporrenkvn mvhayet, ehetake vnokecvke, en hopuetakuceu vnokecvke,

5 Mahlvpvtkvke, efeke hvsvthvke, cukovfekhonnvlke, herake, ehetake em apohicvke taren; momof Hesaketvmese em opunvkvn tvklehocekares.

6 Matvpomen hunvntake mvnettvlke em vcahnvks, emahlvpvtkvke taren.

7 Nake omvlkv ohfvecv vtotketv herakat ofvn vhayetvn e heckuecet, mvhakv ohfvecv fvyvtkeko tayen, okhacekon,

8 Opunvkv ofv temvpoken, ohfvecehoceko tetayen $e$ heckuecvs, estinut vpvlhvimke vpvket on omat, nake holwvke ec opunvyeckv ocekot, vlsvren.

9 Vtolkvlken em vcahnvks, e pucasvlke mahusan apohicet, nak' omvlkv ofvn afvcecicet, pvlken em vyoposkvkekot,

10 Vkonahvkekot, momis omvlkvt heren vkvsvmhoye taye taren; nake omvlkv ofv Hesaketvmese pu Hesayecv em mvhakvn vccece omet herusen hayvkvren.

11 Hesaketvmese en heromkv, vhesaketv sarat, este omvlkv ehomvn hecke hakvtet omekv pu mvhayet,

12 Mekusvpkv-tokon, momet ekvnv em eyackvn e em vsehet, mahlvpvtkvke, fvccrke, mekusapvket yvmv ekvnv oh fulleyvren, pu mvhayet,

13 Enhonrkv afveketv ocan, momet Hesaketvmese rakkat, momet pu Hesayecv Cesvs Klist em vlvketv rakkat en nene hecet,

$14 \mathrm{Mv}$, holwvyeckv omvlkvn en kvpvken pu neset, momet etvlwv veake, vtotketv herakat vfekcakhen e en hayet hvsvtecvret, e pum estemvten,

15 Heyv nanvken opunayet vcahnvtcvs, momet yekcetv 
omvlkvn es opunayet este'm vsehvtevs. Estimvt cen homehcats.

\section{SETENTVCKE III.}

Meknvlken, momet ohfvnkakat en yekcetvn em vkvsvmet, 'mapohicvket, momet vtotketv herakat vtekat emetetakvket omvkvren vkerricepuecvs.

2 Estimvn nanvkokicekot, tepokv en kvpvkvket, yvmaskvket, eyasketv vtekat este omvlkvn em e heckuecvkvren.

3 Pume tis, pu hoporrenvkeko, vkvsvmvkeko, ehosvke, eyackv momet afvcketv mvrahrvkvn vfastet, enhomeckvn, vholvckvn fullet, en homecvke tayet, ete'n homecakeyvte ocet os.

4 Momis Hesaketvmese, pu Hesayecv en heromkv, momet vnokeckv este ohfvecv ocat hecikof,

5 Fvecetv em vtotketv momeceyvte eteropotte tokon, momis em merkv vcvkvyen, svnvcomv-hecketv okkoskv momet Puyvfekcv Vcakat mucrseckv eteropotten pu hesahuecitet os ;

$6 \mathrm{Mv}$ pu Hesayecv Cesvs Klist eteropotte esohfvcken es ep oh kalvte,

7 En heromkv eteropotten pu fvccrke omen, hesaketv meyuksv-seko em enbonrkv vcrkvyen pucasvke taranen pu hahihocvren.

8 Heyv opunvkv mehenwrt's, momen heyv nanvken yekcen est' emvhayetskvren komis, Hesaketvmese ofvn vkvsvinakat vhericet vtotketv herakat vcayecvkvren. Heyv nanvke este en heraket, en lopicvket omes.

9 Momis hoporrenkv-toko tempohetv, momet honvpsetv, momet etenrvpkv, momet vhakv ohfvecr etehvnkvn en kvpvkvtcvs; heyv nanvke esten lopicvkekot, ehvperkvt omekv. 
10 Este vkvsvmkv ofv fvecekat vhvmkvn, momet svhokkolvn em vevhinet, em e fulkvtcrs.

11 Heyvn kerret, este heyv omat fiket, naorket, eme tan e oh fvccecet os.

12 Atemvs monkat Tekekvs ec oh 'tohtvyof, Nikapoles tvlofvn lvpken vm vlvkvtcvs; rvfo mvn fekhonnares komvyet okv.

13 Vhakv-hayv, Senvs, Vpalvs tepakan vcayecet, vtotvkvtcvs, naken en kusvpkekaren.

14 Momen punakeu vtotketv herakat, nake en lopicvke tayat, vcryeckvn eskerrvkekvs, ettvkekot omvkekaren.

$15 \mathrm{Vc}$ vpvkakat omvlkvt ece 'sekakes. Vkvsvmkv ofv epu 'nokecakat vsekvkvtcrs. Omvlkatskan heromkv ece 'pakvkekvs. Momekvs. 



\section{COKV VPASTEL PAL}

\section{HEPLUVLKE OHTOTVTE.}

THE EPISTLE OF PAUL THE APOSTLE TO THE

\section{HEBREWS,}

TRANSLATED

FROM THE ORIGINAL GREEK

INTO THE MUSKOKEE LANGUAGE.

\section{NEW YORK :}

AMERICA $⿱$ N BIBLE SOCIETY,

INSTITUTED IN THE YEAR MDCCCXVI.

1886.

[Muskokee, 16mo.] 


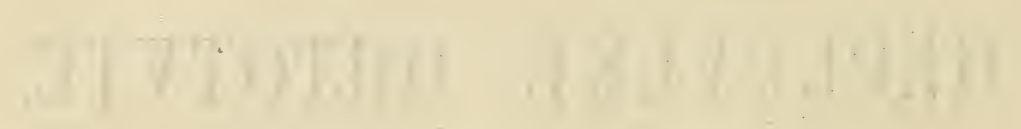

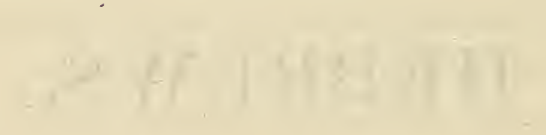




\section{COKV VPASTEL PAL \\ HEPLUVLKE OHTOTVTE.}

\section{SETENTVCKE I.}

Hesaketvmese, mv oketv hoyvnakvte ofvn owalvlke eteropotten oketv sulke momet cahmelike ofvn erkvlken em punayvtet,

2 E'ppuce, mv nanvke omvlkvn epucasvranen ohmellet, momet mv eteropotten ekvnv hahicvte, heyv nettv espokakat ofvn $m v$ eteropotten pum punayvtet os:

3 Mvt Erke en rakketv en hvyayvket, momet en heckv vhakuset omet, momet en yekce em opunvkv eteropotten nanvke omvlkvn kvwapet, e eteropotten pun holwvyeckvn pun hvsvthihcof, Rakketv hvlwe likat em vkvpervn likvtes :

4 Epucase taranat omecicen encelvlke e hocefhokv senrakken hocefkv epoyvte etvpomen $\mathrm{mv}$ encelvlke senrakke hakvtet.

5 Hiyomet omekv, Cemet Cv'ppucet omet, mucv-nettvn ce heckuehcis, momet hvtvm, E'rke toyin, emet Cv'ppucet omvres, encelvlke hvmkis kice em punayvte oce haks? 6 Momet hvtvm, heckvte enhvteceskvn yvmv ekvnvn asceyof, Hesaketvmese em encelvlke omvlkvt vrakkuecvkekvs, maket os.

7 Momet encelvlken opunvyecet, $\mathrm{Mv}$ em encelvlken puyvfekcvlken hayet, em vtotkvlken totkv fenken hayat, maket os.

8 Momis Eppncen em punayet, Mekko-em-ohliketv ce- 
naket estofis emeyuksv-sekot omes, Hesaketvmese toyetskat, fvccetv en septr cem ohmekketv en septvt omes ;

9 Momet, Fvccetvn vnokecet, holwvyeckvn enhomecetskvtes; monkv Hesaketvmese, cen Hesaketvmeset cem etohkvlketv senhoyvnen afvcketv vfekvn es cem vfeyvtet os.

10 Momet, Pucase, cemet hvteceskv omof yvmv ekvnv en likvn hayetskvtes, momen sutvt ce'nke em vtotketvt omakes :

11 Mvt sumecvres, momis cemet liketske munkvt os; momen mv omvlkvt accvke leske hake omet vhassvke hakvres;

12 Momen accvke pvkohlihoce omēn cemet pvkohlicetsken, mvrahke hakvkvres; momis cemet emunkuset liketsken, cem ohrolopet pokvkekares, maket os.

13 Momis, Vm vkvpervn liketske munken cen horrvlke ce'leohhuerickvn cen hayares, encelvlke hvmkis kice em punayvte oce haks?

14 Omvlkvt puyvfekcv vtotkvlke vhesaketv epucasvke taranan em vtotkvkvren atothoyvtet omeko haks?

\section{SETENTVCKE II.}

Monkv nanvke poheyvten ohvtvlaken vketecvraneyet os, estome estomis fihne omet sumecekaren.

2 Hiyomet omekv, opunvkv encelvlke opunahoyvtet yekcet omen, vhakv-kackv momet mapohickv tokat vtekat feketv fvecet en heckakvten omat;

3 Vhesaketv en rakke momvtekan vmoskomēkon omat, estomēt mvnvttepeyvr haks, enhvteceskvn Pucaset onayetvn vlicehcen, pohakvtet pum ohyekcicaken,

4 Momen Hesaketvmeset, eme komat vcrkvyen nakeskerkv, nak estomahakat momet yekcetv cahmelikv, mo- 
men Puyvfekcv Vcakat emkv esyomat eteropotten vpvken a en kerrekv?

5 Encelvlke ekvnv vlvkviane opunvyeceyan ohfvnkvkvren hayekatet ok.

6 Momis este hvmket akerret hoccicvte ocet os, hvmmaket, Estet naket omen vkerricetske haks? monkat este e'ppuce naket omen en cukopericetske haks?

7 Encelvlke estomuse a senkvnevpen hayet, rakketv vrakketv tepakan es kvtvlicetskvtes, momet ce'nke em vtotketv take ohfvnken hayetskvtes.

8 E'le elecvn nanvke omvlkvn hayetskvtes. Hiyomet omekv, nanvke omvlkvn eme elecvn hayvtet on nake elecv hayekat vhoskekates. Momis nanvke omvlkv mv ocakan elecv hvte hecēkos.

9 Momis Cesvs hecēs, mv elkvn kvwapvre vrahkvr encelvlke estomuse a senkvncrpen hahoyvten, rakketv vrakketv tepakan es kvtvlihocvten, momat mvt Hesaketvmese en heromkv etehoyvnen este omvlkv vrahkvn elkvn lasvren.

10 Hiyomet omekv, mv vrahkv nanvke omvlkv ocet, mv etehoyvne nanvke omvlkv ocakat, eppucetake sulken evpayet en hvyayvken 'soh ayat, estemerketv sulke eteropotten em vhesaketv en kvpetvne temvpoke hayetv momvre tetayuset omvtes.

11 Hiyomet omekv, mv hvsvthicat momet mv hvsvthakat omvlkvt hvmkuse asosset os; momen mv vrahkvn tecakkakat kicetvn es vlsekos.

12 Tecakkeyvten ce hocefkvn en kerkuecares; mekusvpkv-cuko em etenrvwrn ece 'kvsamit yvhikares, maket omekv.

13 Momet hvtvm, Vm enhonrkvn eme ofvn ocares. Momet hvtvm, Hecvs, vne momet hopuetake Hesaketvmese a'mvte. 
14 Momekv hopuetaket vpeswv catv tepakat ohfvccrn etohkvlke ocvlket omen, emeu matvpomet vpaket matan ocvtes; elkv eteropotten mv elkv en yekcen ocat en yekcen kvcrret, puyvfekcv-holwakat mvt omen;

15 Momet $\mathrm{mv}$ heckakvte vteken elkv em penkvlkv eteropotten svlvfketv ofvn svpaklan asossicvret.

16 Hiyomet omekv, mehenwvn encelvlke em munkvn accekot, Eplehame en honvpsen accrtes.

17 Monkv nanvke Hesaketvmese ohfvccv ocakat ofvn plest enhomahtv merruset momet herome taren, nanvke omvlkv ofv etecakkakyte omēn hakat, momet este en holwvyeckv ohfvecvn etenherkv hayvren momvre tetayusvtes.

18 Eme mahusat eskerhoyen estemerkvtet omen, mv eskerhoye sasan emvnicetvn ume tayet omekv.

\section{SETENTVCKE III.}

Monkv tecakkvte vcacvke, huehketv hvlwe vte etohkvlke eenakuecvlke toyatskat, pum ohkerkueckv em Vpastel momet Plest Enhomahtv, Klist Cesvn ohvkerricvkes ;

2 Mvt Moses en cuko omvlkv ofvn heromet omvte omēt mv mellvte ohfvccun heromet omvtes.

3 Heyv estet Moses vrakkvte senhoyvnen vrakkuehoce taye heretv ores komhoyvtet ok, cuko vrakkuehocat senhoyvnen $\mathrm{mv}$ cuko hayvte min vrakkuehocet omekv.

4 Cuko vtekat estet hayvte vlket omen, momis mv nanvke omvlkv hayvtet Hesaketvmeset omekv.

5 Momen Moses, vtotkv omēt, mehenwvn mv nanvke mv renyupvn mahokvranat esohkerkueckvn en cuko omvlkv ofvn heromet omvtes;

6 Momis, eppucet omet, Klist eme mahusat en cuko ohfvriken heromet omvtes; mv en cuko toyeyat, enhonrkv 
em vkvsvmkv, momet em afvcketvn meyuksv vteken yekcen hvlatēn omat.

7 Monkv Puyvfekcv Vcakat makat vevkvyen, Mucvnettvn, em punvkv en haken pohatsken omat,

8 Ce'rkvlke take e feke take wonhecakvte omët ce 'feke taken wvnhehcatskvs, crpvkkueckv momet eskerhoye nettv omof, vnrvwr ofvn,

9 Ce'rkvlke taket es cv kerret, vc vketecet, momet vm vtotketv taken ohrolope pale osten hecakvtet omekv.

10 Monkv mv este honvpsen en cvm afvckekot, $E$ 'feke take ofvn estofis fiyecet, vm vyetvtaken kerrvkvte sekos, makvyvtes.

11 Moṇkv vn crpvkke ofvn, Mvt vm fekvpkvn esceyvkekares, makit, fvececvyvtes.

12 Tecakkvte toyatskat, efeke holwvke, vkvsvmekat Hesaketvmese wenake likat enkvpvketv ofvn hvmkusatskis ece 'pikekaren e vketec vkes.

13 Momis, Mucv-nettrn mahokof, nettv omvlkvn ete'mvcahnvkes, holwvyeckv em mvkerretv eteropotten hvmkvtekatskis ce wvnhvket omekaren.

14 Hiyomet omekv, pum enhonrkv em vliceckvn meyuksv vteken yekcen hvlatēn omat, Klist a en cawvlke pu hahoyet os;

15 Mucv-nettvn em opunvkv en haken pohatsken omat, crpvkkueckv nettv tate ofv omēn, ce feke taken wrnhehcatskvs, mahoke emunkof.

16 Pohakof, crpvkkuecakat sasvtet ok; momis Moses evpayet Ecepse asossvke omvlkvt omekates.

17 Momis estimvlken ohrolope pale osten es em afvckekot omvt haks? mv naorkake em enataket vnivwv ofv pvlatkvten mvn omekate te?

18 Momen, Vm fekvpkvn esceyatskekares, estimvn kicet fvceecet omvt haks, mv vkvsvinvkekate tokon omat? 
19 Monkv vkvsvmvkekat omecicen esceyeko tayet omakvten heceyet os.

\section{SETENTVCKE IV.}

Monkv em fekvpkv esceyeyvre temfvccetv pum wikvtet o estomis, hvmkvtekatskis ceye tayat oricekos ce komhoyekaren, penkvlvkeres.

2 Hiyomet omekv, opunvkv-herv es em erkenvkhoyvte etvpomen es pum erkenvkhoyvtet os, momis mv opunvkv 'sem erkenvkhoyvtet en lopicvkekates, mv pohakat vkvsvmkvt vpvken pohvkekatet ok.

3 Pumet vkvsvmeyvtet fekvpkvn esceyet omekv, Hesaketvmeset, em vtotketvt ekvnv en likv ocvte vteken pokakvtet o estomis, Vn crpvkke ofvn makit faccecvyvte omēn makvte etvpomen.

4 Nettv eskolvpakan opunvyeeet hivmmakvte ocet omekv, Momen nettv eskolvpakan Hesaketvmeset em vtotketv omvlkvn pohyet fekapvtes.

5 Momen heyvmvn hvtvm, Vm fekvpkvn esceyvranen omat.

6 Monkv mv fekvpkv esceyvranat tvlkusat sase emunkvt omaten, momis enhomvn mv fekvpkv es em erkenvkhoyvtet vkvsvrnvkekat omecicen esceyvkekatet omen,

7 Hvtvm nettv hvmken mellet, oketv momvteke hoyanen, Tewe eteropotten punayet os, Mucv-nettvn, mahokvte omen, Mucv-nettvn vm punvkv en haken pohatsken omat, ce 'feke taken wrnhehcatskvs.

8 Hiyomet omekv, Caswet fekvpkvn emakvten omat, mv renyupvn nettv etvn opunvyecekvnt os.

9 Monkv Hesaketvmese em esten fekvpkv em ocvke emunkvt omes.

10 Hiyomet omekv, mv em fekvpkvn eceyvtet, emeu 
em vtotketvn wikvtes, Hesaketvmeset em vtotketvn wikvte omēn.

11 Monkv pumet mv fekvpkvn esceyeyvret vfekcakhet omvkeres, estimvt mata vkvsvmkv-sekat vhopake vevkvyen lvtkekaren.

12 Hiyomet omekv, Hesaketvmese em opunvkvt wenaket momet yekcet, eslafkv.crpko em fvske hokkole estomis semfvsket omet, puyvfekcv vkerrickv tepakan, momet etekkekv faluwv tepakan etekvpickv oren sekeyet, efeke koman momet em vkerrickvn kerrvt omes.

13 Momen en renakv ehomvn nake hahoyvte hvmkusis heckekat sekot omen, momis nanvke omvlkvt mv em e ohkerkuecvraneye likat en renakv ehomvn enatvlket, momet enrvmkvket os.

14 Monkv plest enhomahtv rakke, Hesaketvmese E'ppuce Cesvs, hvlhawan eceyvten ocēkv, pum eohkerkueckvn yekcen hvlvtvkeres.

15 Hiyomet omekv, plest enhomahtv pum ehvperketv ocakat vcelakeko tayan ocēkot, momis nanvke omvlkv ohfvccun pume omēn eskerhoyvte estomis, naorkvte sekan oceyet os.

16 Monkv fekhvmket heromkv ohliketvn a oh vwvkeres, merkvt pun hecken, kusvpke oketv omof heromkv pum vnice tetayan esheceyvren.

\section{SETENTVCKE V.}

Hryomes omekv, plest enhomahtv este aenkvpvke enhopohoyvte vtekat, este vrahkv nake Hesaketvmese ohfvecv ocakat ofvn melhoyet omes, naorketv vrahkvn nakemkv momet nake Hesaketvmese ehomvn prsvthoyvranan emvren:

$2 \mathrm{Mv}$ eme mahusat yekcetv tokat em vfulotet on, nak kerrvkekan momet fiyecakan em merre tayat, 
3 Momet $\mathrm{mv}$ vfulotat omecicen, naorketvt omecicen este vrahkvn naken emvranat omen eme mahusat e vrahkvu eme tayet omes.

4 Momen Hesaketvmeset Elvn enhuehkvte omēn enhuehhokekon omat, heyv vrakketvn e oh wrkecat sekot os.

5 Momen matvpomen Klist e vrakkuecekates, plest enhomahtv hahoyvren, momis $\mathrm{mv}$, C'vppuce toyetskes, mucv-nettvn ce heckuehcis, makvtet mecvtes.

6 Momet, Melkesetek plest hakvte vcrkvyen estofis Plest toyetskes, makat etvn ocet omekv.

7 Momen mvt enavpeswv en nettr take omof huehketv yekcen, momet turopuswv pvlatken mv elkv aenkvpvken hesayece tetayan es em mekusapet, momet yekcen enkusapet penkalat of rn em apohihocrten;

8 Eppucet omis, nanvke es estemerkvte eteropotten apohickvn kerrvtes.

9 Momen temvpoke hahkof, mv em apohicakat omvlkvn vhesaketv meyuksv-sekon en heckuecv hakvtet os.

10 Hesaketvmeset plest enhomahtv Melkesetek hakvte vevkvyen kicen.

$11 \mathrm{Mv}$ ohfvecr nanvke sulke momet ce hrckotake pohetv tefnat omecicen ohhvyvyickv yekcakan opunvyetvn oceyet os.

12 Hiyomet omekv, oketv hiyomvteke oren mvhayvlke hakatskvnt omat, nanvke Hesaketvmese kerkuecvte en likv enhvteceskv ocakan ceme min hvtvm ce mvhahoye taye ont os; momet hompetv yekcen hompakat tokon, epese eskakat mi omvke hakatsket os.

13 Hiyomet omekv, epesen eskat vtekat fvccetv em opunvkv ofvn mvnvckekot os, estucet omekv.

14 Momis hompetv yekcat vculkv orice, mv senses kicetv 'sevnicat eteropotten nake here, momet holwakan kerre taye hakvte enake tes. 


\section{SETENTVCKE VI.}

Monkv Klist em mvhakv enhrteceskv ocakan opunvyeckvn wihket, vtotketv prsatkvte enkvpvken, efeke etickv momet vkvsvmkv Hesaketvmese ohfvecv ocat,

2 Momet paptesvm ocakat em muhakv, momet enke oh wrkecetv, momet prsatkvte raenkvwvpketv, momet ohfrececkv emeyuksv-sekat en likvn hvtrm hayëkot, temvpoke haketvn oh vpeyvkeres.

3 Momen Hesaketvmeset etektvnecen omat, heyvn momeceyvres.

4 Hiyomet omekv, mv vhvmkvn em oh hryayihocvtet, momet emkv hvlwe vten lasvkvtet, momet Puyvfekev Veakat en heckvilke hakvtet,

5 Momet Hesaketvmese em opunvkv here, ekvnv vlvkvranat en yekcetv tepakan lasvkvtet,

6 En lvpvtke pvlatken omat, hvtvm vkerrickv etickv oren mucrsvkuecēsko tetayet os, Hesaketvmese E'ppucen emetake e ohfvecvn hvtrm tohweklepkvn svrahet, hecken vlesketvn en hayaket omekv.

7 Hiyomet omekv, ekvnvt, oske tewolen a oh hvtapkan esket, mv vfvstakat en herakvren nake hontakan honticat merkv Hesaketvmese avtet en hecket os;

8 Momis mv nanvke em fvske ocakat, momet eto-fvsken hontican wihoket: os, momet ohtvhihokvranusen, em eyuksvt nekrvranat tes.

9 Momis, vnokeckatskat, hiyomēn opunayeye estomis, nake svmmomakan, momet nanvike vhesaketv vprkakan ec ohfrecrn enhonreyet os.

10 Hiyomet omekv, mekusapvlke em vtotkatskvten, momet hiyome em vtotkatskat, cem vtotketv taken, momet vnokeckv vtotketvn e hocefkv vrahkvn heckuecatskvten ehose tayat Hesaketvmeset fvecekot omekot os. 
11 Momen enhonrkv esfvckat oren meyuksv vteken mata vcvfeknetvn hecken hayatskevlke taren komēs;

12 Momat enhorrvkekot, momis vkvsvmkv momiskometv tepakat eteropotten temfvccetv ocakan en heckakat vevkfullvlket omatskvren.

13 Hiyomet omekv, Hesaketvmeset Eplehamen etemfaccof, eme mahusat e hocefet etemfaccrtes, eme senrakken hocefet etemfacceko tayvtet ok;

14 Mehenwvn ohmerret, ec ohmerrares, momet ohvtvlayet, ec ohvtvlayares, maket.

15 Momen hiyomēn hofunen momis-komvte renyupvn, mv etemfvecetvn eenakuecrtes.

16 Mehenwvn estet emetake senrakkan hocefet etemfvccaken, Hesaketvmese hocefe temfvccetv hahoyat etenrvpkv em eyuksvt omekv.

$17 \mathrm{Mv}$ ofvn Hesaketvmeset, nake komat emvrahke hakeko tayat etemfvccetv en heckvkvranan senyekcen en kerkuecetvn komet, temfvccetv vcakan es yekcicvtes:

18 Momat nake mvrahkv hakeko taye hokkolat, $\mathrm{mv}$ ofv "Hesaketvmeset lakseko tayat eteropotten pume, ehketvn hopoyēt, mv enhonrkv pu homvn huerihocvten hvlvtetvn komēt pefatkeyvtet mahlvpvtketv rakken oceyvren ;

$19 \mathrm{Mv}$ enhonrkv puyvfekcv'sem ehvlvtkv omēn yekcen momet nekeyihoceko tayan, momet setentvckv vcakat ofv eceyan oceyet os;

20 Mvn Cesvs homv-letkv Melkesetek plest hakvte vevkvyen plest enhomahtv like emunkat hakvtet pum eceyvtes.

\section{SETENTVCKE VII.}

Hiyomèt omekv, Heyv Melkesetek Selem tvlofv em mekko, Hesaketvmese hvlwe mahat em plest, mv Eplehamet mekkvlke prsatkv rafulkof etenrahpet, ohmerret; 
2 Momen Eplehamet omvlkv tekvpice tekvpvke espalan emvtet; enhvteceskvn $e$ hocefk $v$ ohyvtekhoyat fvccetv em Mekkot omet, mohmen hvtvm Selem em mekkon, mv herkv em Mekkot omat;

3 Erke seko, eckeu seko, em vhonvpsetvu sekot, momet nettv em vliceckv monkat en hesaketv em eyuksvn ocekot; momis Hesaketvmese E'ppuce vhaken hahoyvtet plest like emunkvt os.

4 Tokvs, heyv hunvnwv en rakketv estomet omvten vkerricvkes, mvn cuko-hvmecvlke em ekv Eplehame tisem, en horre nanvike svpenkvlecvte tekvpvke espalaten emvtes.

5 Momen mehenwrn Lefi e'ppucetake plest em wiketv en heckakvtet vhakv verkvyen tekvprke espalan esten fekepuecetvn vhakvn ocakes, mv estet etecakkvtet omaken, Eplehame etetackvn asossaket o estomis;

6 Momis mv este en honvpse mv Lefivlke en honvpse vpvke vhonkvtkekat tekvpvke espalakan Eplehamen en cawvtes, momet mv etemfvccetvn ocvten merkvn oh opunayvtes.

7 Momen. nake vnrvphoyeko tayaten, rakkatet sencutkan merkvn oh opunayet os.

8 Momen yvmvn este prsatkvlke tekvpvke espalakan en heckaket os, momis min, Hesakes, make opunvyece cohoyat espalakan cvwet os.

9 Momen, makvye tayat vcvkvyen, Lefi, tekvpvke espalakan en heckat, Eplehame eteropotten tekvpvke espalakan fekvtes.

10 E'rke etetackv ofv emunken Melkesetek vnrapvtet ok.

11 Monkv temvpoketv plest em wiketv Lefi vcrkvyet ōn omat, (mv elecvn vhakv est' emhoyvtet omekv,) estomaten plest etvt, Elvne em etecvkvyetv vcrkvye 
kihocekōt, Melkesetek em etecvkvyetv vcvkvye min akvwvpke tayet omvt haks?

12 Plest em wiketv teyoposecihohcen, vhakvu mvrahkuehocvranat tvlkuset omekv.

13. Hiyomet omekv, mv este heyv nanvken es opunvyehocat em vliketv etv, mv altv vfastv aossvte sekan, aosset omes.

$14 \mathrm{Pu}$ Pucaset Cutv em vliketvin aossvtet omat kerkuset omekv, momen Moses plest em wiketv ohfvecvn opunayet mv em vliketv ohfvecrn naken makekates.

15 Momen ohvtrlaken kerket os: Melkesetek vhake vevkvyen plest etvt,

$16 \mathrm{Mv}$ enapeswv vhakv vevkvye tokot, hesaketv eyuksv ocekat en yekcetv vcrkvyen hahoyat akvwrpket omekv. 17 A en kerret, Plest Melkesetek em etecvkvyetv vcvkvyen meyuksv-sekon liketskes, maket omekv.

18 Vhakv homv ocvte en yekce sekat, momet en lopickv sekat omecicen yvmahkuehocet omekv.

19 Hiyomet omekv, vhakvt nake hvmkusis temvpoken hayekon, momis enhonrkv svmmomusan asceyihocat hayvtes ; mv eteropotten Hesaketvmesen vwoliceyet os.

20 (Hiyomet omekv, mv plestvlket etemfvccetv vcakat sekon hahoyvtes, momis heyvt, mv em punayat, Pucaset etemfvecetv vcakan 'sopunayet, momet em vkerrickv etihcekos, Melkesetek em etecvkvyetv vcvkvye estofis plest toyetskes, makvte, etemfvccetv vcakat eteropotten plest hahoyvtes.).

21 Monkv etemfvecetv vcakat sekon Cesvs plest hahoyekate etvpomusen,

22 Etemfvccetv svmmomusat eskerkv hahoyvtet os.

23 Momen mehenwvn entat, elkvt omecicen emomecicetv em etektrnechoyekok, sulket plestvlket omakvtes. 
24 Momis heyv este hunvnwvt plest em wiketv em vkueyihoceko tayen oces, estofis like emunkvt omekv.

25 Monkv mv eme eteropotten Hesaketvmesen a oh awat estofis esfackusen hesahuecetv ume tayes, em emekusapvret estofis like emunkvt omekv.

26 Plest enhomahtv heyv omat; mv vcaket, em mvttekot, svholwvkekot, naorkvlke enkvpvket, hulhawat senhvlwe hakvten oceyvret momvre heret omvtet ok.

27 Plest enhomvhhotat nettv omvlkvn enhvteceskvn eme en naorketv take vrahkvn nanvke Hesaketvmese ehomvn pvsvtakket, momof este en naorketv take vrahkvn momecakvte omēt momecvranat tvlket omekates; heyvn vhrmkvn momecvtet ok, eme mahusan Hesaketvmese ehomvn elehocvren est'em e wikof,

28 Hiyomet omekv, vhakvt este temvpoke mahekan plest enhomvhhotan hahicen; momis heyv etemfvecetv vcakat em opunvkvt, mv vhakv enyupv ocvtet Eppuce meyuksvseko temvpokan plest enhomahtvn hayet os.

\section{SETENTVCKE VIII.}

Mомеs mv nanvke opunvyeceyat nake rakke mahat heyv tes; Pumet plest enhomahtv momẽn oceyet os, mv Rakketv em ohliketv hvlhawat ofv ocat vkvpervn liket omat,

2 Momet liketv vcake mahat, escukohakv vcake mehenwe, este tokot, momis Hesaketvmeset huericvte em vfastvt:

3 Hiyomet omekv, plest enhomahtv vtekat nakemkv momet nake ohnekrickv wiyetvn melhoyet os: monkv heyv plest enhomahtvu nake wiyetvn ocvranat tvlkuset os. 4. Hiyomet omekv, ekvnvn oh are munkvten omat, plest huerekvnt os: plestvlket vhakv vevkvye nakemkv wiýakat fullet omekv : 
5 Mvt nanvke hvlwe ocakat vhaken momet em vpetten vfvstaket os, Moses escukohakv vcakat hayvranof, Hesaketvmese emvcahnvte etvpomen: Vhayetv ekvnhvlwe onvpv ce hecihocvte vcvkvyen nanvke omvlkv hahicetvn vketecvs, maket omekv.

6 Momis hiyomat, etemfrccetv svmmomusat, etemfvecetv svmmomakan oh hahoyvte etenhayv hakvte etvpomen vfvstetv svmmoman en hecket os.

$7 \mathrm{Mv}$ etemfvccetv enhyteceskvt temvpoket omvten omat, svhokkolat em etektvnke hopohoyvre sekvnt omekv.

8 Hiyomet omekv, em muttaken em punayet, Hecvs, nettvt yicet os, Eslevl en cuko momet Cutv en cuko etemfvecetv mucvsen ete'n hayares, Pucaset maket os;

9 Momet $\mathrm{Mr}$ nettr Ecepse ekvnrn sossicetrn enke taken en hvlatvyof etemfvecetv e'rkvlken ete'n hayvyvte vcrkvyen ete'n hayvkares: vm etemfvccetp ofv emomecicvkekat omecicen vnet emetaken vmoskomvkatet ok, Pucaset maket os.

$10 \mathrm{Mv}$ nettr take renyupvn etemfvecetv Eslevl en cuko ete'n hayvranvyat heyvt omekv; Vm vhakvn em vkerrickv taken vtehit, e 'feke take ofvn hoccicares: momet emetake vrahkvn Hesaketvmeset huerares, momen emetaket ve vrahkvn etvlwvt omvres.

11 Momet estimvt em vwolicvn momen etecakkaten mvhayet, Pucasen kerrvs, maket kicake vlket omekares, cotkus mahaten 'svlicecet rakke mahat oren omvlkvt $\mathrm{cv}$ kerrvkvret ok'.

$12 \mathrm{Em}$ fvecetv svkekat ohfvecvn em merret, en naorketv momet en holwvyeckv taken ohvtvlakat vkerricvkaret ok, Pucaset maket os.

13 Etemfvccetv mucvsen makat, enhvteceskan veulen hayvtet os. Momen $\mathrm{mv}$ vhasse momet vcule hakat sumketvn vwolices. 


\section{SETENTVCKE IX.}

Momen mehenwrn etemfvccetv enhvteceskvt Hesakețmese emekusvpkv ohfvecv nanvke melhoyvten, momet nvthof $v$ vcakat yvmv ekvnv enaken ocvtet os.

2 Escukohakv hahoyvtet omekv; enhvteceskv, mv ofv kulke sohhuerickv, ohhompetv, tvklike hecetv esyomet ocvte; mv nvthofv vcake kihocan.

3 Momen setentrcke svhokkolat ofv, escukohakv ofv omvlkv Semvcakat kihocan ocvtes,

4 Nakfvmecuse-es-nekrickv, sensv kicetv, cvtokunaplane es hayvkvte, momet setemfivecetv en tovhahvwv vcakat tokunaplane ohpvcket $\mathrm{mv}$ ofv tokunaplane vrkvswv manv vtehke ocvte, momet Elvne em vhuswv prkpakvtet vpikvtes, momet setemfvecetv en cvto tvpestvke hokkolat vtehkvtes.

5 Momen mv tovhahvwv vcakat onvpusan celupvlke hvyayvhakusat merkv ohliketvn oh vpetticrke tates; hiyomat mvn esfvcken opunvyecēko tayes.

6 Momen heyv nanvken hiyomēn melhohyof, plestvlket, Hesaketvmese em mekusvpkv v́astetvn esfvcecet, estofis escukohakv setentvcke aye homv likan esceyet omvtes.

7 Momis svhokkolan plest enhomahtvt ohrolope ore vtekat vhvmkvn entvlkuset eceyet, catv ocekat ceyekot, momis catvn mv e mahusat vrahkvn, momet este en naorketv take vrahkvn wiyvtes.

8 Puyvfekcv Vcakat heyvn okvtes, escukohakv enhvteceskat huere emunkof escukohakv setentvcke vcake mahat eceyetv hvte heckekates.

9 Mvt oketv mv omof svhopakv em ocvtes, mv omof nakemkv momen nanvke ohnekrickv, mv vfastan eohvkerrickv ohfvccun temvpoken hayvkeko tayan em wiyakvtes. 
10 Mvt hompetv esketv esyomakat ofv, momet uewv es hvsvteckv cahmelikv, momet vtotketv enavpeswv ohfvecv ocakat, mucvseckv oketv oren $\mathrm{mv}$ ohlumhihocvte tvlkuse ofvt ocvtes.

11 Momis Klist nake herake ocvkvranat em plest enhomahtvt, escukohakv senrakke momet senherat, enke es hahoyvte toko, nak hayetr hiyome ocat vcrkvye tokon okis, mv etehoyvnen vlahket;

12 Pvlke neskv meyuksv-sekon pun heckuehcet, cowatv wakucvlke esyomat e catv eteropotte tokon, momis eme mahusat e catv etehoyvnen vhvinkvn cuko vcake mahan esceyvtes.

13 Hiyomet omekv, wakv-hunvntake cowatvlke esyomat e catvt, momet wakv hokte mvnette nekrihocvte em essut enavpeswv hvsvteckv oren hvsvtecen omat;

$14 \mathrm{Mr}$ senhoyvne estrmahen Klist, mv Puyvfekev meyuksv-seko likat eteropotten, holwvke cotkusis vlikekon, Hesaketvmese ehomvn elehocvren e wikvtet e catvt vtotketv pvsatkat enkvpvken cem eohvkerrickv taken hvsvthicekar haks, Hesaketvmese wenake likat em vtotkatskvren?

15 Momen heyvt omecicen etemfvecetv mucrse vevkvyen etenherkepuecvt os, naorketv ctemfvecetv enhvteceskv elecv ocakvte ohfvccrn pvlke neskv hakvre elvte eteropotten enhuehhokat omvlkvt nake meyuksv-seko ocvkvranat etemfvecetv en heckvkvren.

16 Estvmv wel ocen omat, welhayv em elkvt ocvranat tvlkusekv.

17 Hiyomet omekv, este prsatke erenyupvn wel yekcetv ocet os; momis welhayv like emunkof, mv en yekce sckot os.

18 Monkv setemfvccetv enhvteceskv tis catv sekon yekcetvn emhoyekates. 
19 Hiyomet omekv, Moses vhakv vevkvyen vhakv omvlkvn esten 'sem punayahkof, wakucvlke cowatvlke esyomat e catvn, momen uewvn, momen yvpefikv-esse cahtuse hesvpe esyomen cawet, nakcokv momet este omvlkvn es ohfeskvtes,

20 Heyvt mv setemfvccetv Hesaketvmése ce kicvkvte vrahkv ecatv tes, maket.

21 Momet ohvtvlaken escukohakv momet nakvtehkv mekusvpkv svfistetv omvlkv matvpomen catvn es ohfeskvtes.

22 Momen vhakv vcrkayat nanvke omvlkvranuset catvn es hvsvthihocet os; momen cate pvlvtkv sekat holwvyeckv enkvpvyecickv sekos.

23 Monkv nanvke hvlhawat ofv ocakat vhakakat heyv nanvken es hvsvthihocrranat tvlkusvtes; momis hvlwat enanvke mahusat nanvke ohnekrickv heyv nanvke senherakan es hvsvthihocvranvtes.

24 Hiyomet omekv, Klist cuko vcacvke enke es hayvkvte, mehenwakat vhakvket omen, eceyet omekos; momis hiyomat epu 'rahkvn Hesaketvmese ehomvn e heckuecetvn hvlwe tvlofv mahusan eceyvtes.

25 Momet hvtvm, plest enhomahtv ohrolope omvlkvn vhvmkvn setentvcke vcakat ofv, catv enake toko esceyet omen, tewolen e wiyvranat tokates.

26 Momen omat, ekvnv vliceckv vteken etewolen estemerrihocvranvtet omekv; momis hiyomat, ekvnv 'meyuksv omof, vhvmkvn, holwvyeckvn vkueyvranet, Hesaketvmese ehomvn elehocvren e wikvtet os.

27 Momen vhvmkv eletv, momet mv erhoyanat ohfvcceckv este em mellvkhoyvte etvpomen,

28 Klist vhvmkvn elehocvtes sulke en naorketv taken e oh wrkecvren; momet mv en nene hecakan vhesaketvn emvranet, naorketv sekon, svhokkolvn em e heckuecvres. 


\section{SETEN'TVCKE $\dot{\mathrm{X}}$.}

Hiyomet omekv, vhakvt nake herake ocvkvranat vhake mahusat tokon, em vpette tvlkusen ocet, mv nake ohnekrickv ohrolope omvlkvn wihoye emunkv tate eteropotten $\mathrm{mv}$ a oh awat estofvn temvpokvken hayvkeko tayet omekv.

2 Hiyomet omekv, momen omat, mv vcvkvpeyvlket, vhvmkvn hvsvthihohcen, e ohfvecvn naorketvn kerrvkekot omen, mv nake ohnekrickv wihoyat fekhonnvkvranekate te?

3 Momis mv nanvke ohnekrickv ofvn ohrolope omvlkvn naorketv vkerricepueckv hrtvm ocet omekv.

4 Wakv-hunvntake cowatvlke esyomat e catvt naorketvn vkuehueceko tayet omekv.

5 Monkv ekvnvn vlakof, Nake ohnekrickv momet nak wiyetvn kometskekos, momis enan vm etetakuecetskvtes;

6 Nanvke ohnekrickv momet nak wiyetv naorketv vrahkv es ece 'fvckekates;

7 Momof, Hecrs, Hesaketvmesē, vnet naken kometskat momecrranit vlakis, (nak hocce polpokat ofv vc ohfvecrn cohoyet os,) Cesvs makvtes.

8 Enhomvn, Nake ohnekrickv, momen nak wiyetv, momen nanvke ohnekrickv, momen nake holwvyeckv vrah$\mathrm{kv}$, mv vhakv vevkvye $c e$ 'mhoyan kometskekos, momet es ece 'fvckekot os, mahkof,

9 Momof, Hecvs, Hesaketvmesē, vnet nake kometskan momecvranit vlakis, makvtes. Svhokkolan yekcicvret enhvteceskan vkueyet os.

$10 \mathrm{Mv}$ komat eteropotten vcacvken pu hahoyet os, Cesvs Klist ena vhvmkv wihoyvte eteropotten.

11 Momen plest vtekat, nettv omvlkvn mekusvpkvn 
vfastet, tewolen ohnekrickv matan, holwvyeckv estofvn vkueyeko tayan, wiyet hueret omekv:

12 Momis heyv hunvnwrt, nake Hesaketvmese ehomv elehocvre meyuksv-seko hvmken holwvyeckv vrahkvn ehmof, Hesaketvmese em vkvpervn likvtes;

$13 \mathrm{Mucv}$ vtekat en horre ele em ohhuerickv hahoyvre vteken mehaket.

14 Nakwikv hvmke eteropotten,.(elehocvtet mot omen,) mv vcacvke hakat, meyuksv-sekon temvpokvken hayvtet omekv.

15 Momen mv ohfvccvn Puyvfekev Vcakat a pun kerret os:

16 Hiyomet omekv, Etemfvccetv mv nettv take renyupvn ete'n hayvranvyat heyv tes; vm vhakvn efeke take ofvn em vtehit, em vkerrickv take ofvn hoccicares, Pucaset maket os, mahkof, hvmmakvtes ;

17 Momet en naorketv momet en holwvyeckv taken svnvcomvn oh vkerrihcvkos.

18 Momen estvmvn heyv enkvpakickv ocat, ohvtvlaken naorketv vrahkv naken elehocekot os.

19 Monkv, tecakkvte toyatskat, Cesvs e catv eteropotten vyetv mucvse, momet hesakat setentvckv eteropotten, em enavpeswvt mvt omen, emet vcake pun hayvten;

20 Nvthofv veake mahan esceye tetayat fekhvmketvn ocēt;

21 Momet plest enhomahtv Hesaketvmese en cuko ohfvnken ocèt;

$22 \mathrm{Pu}$ 'feke taken eohvkerrickv holwakat enkvpvken ohfeshokvten, momet pu 'na taken uewv kvsvmrusen es okkoskvken, vkvsumkv en kerretv esfvekat ofvn efeke mehenwen es vwolicvkeres.

23 Vkerrickv inelohlekon, vkvsvmkv kerkueckvn yekcen hvlvtvkeres ; mv temfaccutet heromet omekv ; 
24 Momet et'oh vkerricvkeres, vnokeckv momen vtotketv herakat ohfvecv et'oh yekcickvn.

25 Etohkvlketvn wikake sasvnto omēn etohkvlketv wikekot, momis ete'm vcahnet omvkes; momet $\mathrm{mv}$ nettvt vwolicen hecatskat mahvkvu.

26 Mehenwetv keriyen hoyanat, momeyis komēt naorkēn omat, nake holwyvyeckv enkvpvyecickv elehocvre svnvcomvn sekot omekv;

27. Momis ohfvececkv, momen cvpvkketv fenke vnrapvlke sumecicvranat en nene heckv penkvlkat oces.

$28 \mathrm{Mv}$ Moses em vhakvn vhepakat, akerrvlke hokkolet monkat tutcenet akerraken, merkvt en sekon, elvtet os.

29 Mv Hesaketvmese E'ppucen oh hueret, etemfrceetv ecatv, mv eteropotte vcake hahoyvten, vcakekos, komet, heromkv em Puyvfekevn vlesketvn en hayvtet estemerrickv senhoyvne estomomuse en hecke taye ores, komatske haks?

$30 \mathrm{Mv}$, Pvleckvt crnaket's, vnet pvlken fekares, Pucaset maket os, makvten kerrēkv. Momet hvtvm, Pucaset em esten ohfvececvkvres.

31 Hesaketvmese wenake likat enke ofv lvtketv nake penkvlket os.

32 Momis. mv nettv hoyvnakvten, $m v$ ofv ec ohhvyvyakvte renyupvn estemerkv em etepokv rakken kvwvpatskvtet vkerricvkes;

$33 \mathrm{Mv}$ ofv estemerrickv tvhiketv esyomat etēropotten nak huyopkvn ce hahoyvten, momen hvtvm este momẽ momehocvte fullat em etohkvlketv hakatskof.

$34 \mathrm{Cv}$ svlvfkat ofvn vm merratskvtet omekv, momet cen nakockv 'sece 'penkvlehocvkan ece 'facket kvwvpatskvtes, hvlwat ofvn nakockv senhere, momet oce munkan ece 'rahkv ocatskat kerret. 
35 Monkv cem enhonrkv take, mv feketv rakken ocat vwihkatskvs.

36 Momis kometvn ocvranatskat tvlket omekv, momat, Hesaketvmese koman momehcatskof, etemfvecetv cen heckvkvren.

37 Hiyomet omekv, hofunekon mv vlvkvranat vlvkvres, momet vcewicekares.

38 Momen mv fvccat vkvsvmkv eteropotten hesakvres; momis éstimvt vcekellet on omat, $\mathrm{mv}$ este ofvn $\mathrm{vm}$ puyvfekcrt afvcketvn ocekares.

39 Momis mv sumketv oren vcekellecakat tokon, momis mv puyvfekev vevyeckv oren vkvsvmakat vpakvlke toyẽs.

\section{SETENTVCKE XI.}

Momen vkvsvmkvt nanvke en nene hechoyan en likvt ot, nanvke hechoyekan 'sohkerkueckvt os.

$2 \mathrm{Mv}$ eteropotten vkvsamvlke vculvke tate heren ohkerkuehocvtet omekv.

3 Yvmv ekvnv momet etimv sasat Hesaketvmese em opunvkv etehoyvnen tetakuehocvten momat nanvke hechoyan nanvke heckakan es hahoyekatet on vkvsvmkv eteropotten kerreyet os.

4 Vkvsvmkv eteropotten Epelet, nake emetv Kenet Hesaketvmese emvte senheren Hesaketvmesen emvtes, mv etehoyvnen fvecet omat akerkueckvn e en heckuecrtes, Hesaketvmeset en nakemkv ohfvccrn akerren; momen mv eteropotten, elis, punaye munkes.

5 Vkvsvmkv eteropotten Envk, elkvn hecekaren, tohtvlecihocvtes; momen eshechoyekates, Hesaketvmeset tohtvlecicekv; Hesaketvmesen afveecicvtet omat, tohtvlecihoceko emunkof, akerkueckvn ocvtet omekv.

6 Momis vkvsvmkv sekat afvcecicēsko tetayuset os, 
Hesaketvmese a oh atat, Likes, momet mv vhericet hopoyakat em fekvt os, komet vkvsvmvkvranet omekv.

7 Vkvsvrukv eteropotten Nov, Hesaketvmeset nanvke hvte heckvkekat ohfvecvn emvcahnen, penkalet, en cuko hesahueckvn perro-cukon tetakuecvtes; mvn momecat ekvnvn ohfvececet, fvecetv vkvsvmkv. etehoyvne ocat epucase hakvtes.

8 Vkvsvmkv eteropotten Eplehamet osiyet, liketv mv erenyupvn epucasvranat ayvren enhuehhokof, apohicvtes; momet, estvmvn ayat kerrekot ossvtes.

9 Vkvsvmkv eteropotten, tvleporv em ekvnvn are omēt etemfvecetv ekvnvn arvtes, Iseke Cekvpen etohkvlket escukohakv ofvn vpoket, etohkvlket etemfrccetv hvmkuse epucasvlket omakekv;

10 Tvlofv en likv ocvken, $\mathrm{mv}$ tohtarvt momet hayvt Hesaketvmeset omen en nene hecrtet omekv.

11. Vkvsvmkv eteropotten Sele mahusat tis nvrkese taye yekcetrt en heckvtes, momen, hoktvlehpof, hopuewrn heckuecrtes, Mv temfaccrten heromet os, komvtet ok.

12 Monkv hvlwe kococumpv momet oktahv uehvtkv afopke oce vhonkvtēsko tayat en sulke etvpomusen estet este hvmkusen asossvtes, momen $\mathrm{mv}$ estet ele etenhvmket omvtes.

13 Heyv omvlkvt vkvsvmkv ofvn prsatkvtes, etemfvccetvt enheckvkekon, momis hopvyen ocen hecaket, em enhonrvke heret, otakakvtes, momet, Cuneckvlke momet tvleporvlket ekvnvn oh fullēs, maket e oh kerkuecakvtes.

14 Nanvke momē makakat ekvnvn hopoyāket omat heckusen kerkuecaket omekv.

15 Momen mehenwvn, mv ekvnv asossvtet vkerricakvten omat, oh fulecetv em etektrnkvke tayvtes.

16 Momis hiyomat ekvnv svmmome, hvlwe ocan eyacv- 
ket os; monkv en Hesaketvmese take kihocvre Hesaketvmeset em vlsekot os; emet tvlofvn em etetakuecrkvtet omekv.

17 Vkvsvmkv eteropotten Eplehamet, eskerhoyof, Iseken ohnekrickvn emvtes;

18 Momet on $\mathrm{mv}$ temfvecetv take en heckvtet e'ppuce hvmkuse en heckuehocvten, mv, Iseke ofvn cen honvpsen hocefhoyvres, kihocvten emvtes:

19 Hesaketvmeset prsatkvte tis aenkvpvken ahuerice tayes, komet; momet svhopakv ofvn mvn aenkvpvken evpayvtes.

20 Vkvsvmkv eteropotten Iseket, nanvke ocvkvranat ohfvecrn, Cekvpe Esa tepakan merkvn oh opunayvtes.

21 Vkvsvmkv eteropotten Cekvpet, elof, Cose e'ppuce take hokkolen merkvn oh opunayvtes, momet 'sem etekkekvn oh cuneket mekusapvtes.

22 Vkvsvmkv eteropotten Coset, elof, Eslevl en hopuetake sosse ocvranan opunvyecet, e fune ohfvecvn vhakvn emvtes.

23 Vkvsvmkv eteropotten Moset heckof, e'ckvlket hvse tutcenen ehakvtes, estuce en heckv heret on hecakekv; momet mekiko em vhakvn em penkvlvkekates.

24 Vkvsvmkv eteropotten Moset, vculehpof, Feleo e'chuste e'chuswv kihocvren komekates;

25 Naorketv em afvcketvn vkocoknusen es afvcketvn kornekot, Hesaketvmese em esten vpaket estemerketv $\min$ enhopoyet;

26 Klist em vlesketvt nake ockv Ecepse ekvnv. en nakockv senrakket os, komet; feketv em ocvranan vhecet omekv.

27 Vkvsvmkv eteropotten Ecepsen enkrpakvtes, mekko en crpvkketvn em penkvlekot; $m v$ hecēsko tayat hece omēt estemerkvn kvwapet omekv. 
28. Vkvsvmkv eteropotten ohhoyvnkv momet catv ohfesketvn vfastvtes, mv enhvteceskv heckakvten pvsatat emetaken celayekaren.

29 Vkvsvmkv eteropotten Uehvtkv Caten tehoyvnecvtes, ekvnv kvrpe tis tayusen; momen Ecepsvlket mvn mometvn komet fullet, vksumecvtes.

30 Vkvsvmkv eteropotten Cvleko em vtohopke rakkot, nettv kolvpaken vfolotechohyof, lekafkvtes.

31 Lehape hoktarvt vkvsvmkv eteropotten mv vkvsvmvkekat vpvket elekates, ekvnv hecvlke herkv ofvn evpahyof.

32 Momen ohvtvlakat naken makare te? Ketevn, Peleke, Samse, Cefrv, Tewe, Samyvl owalvlke esyomat opunvyecetv vm etektvnkeko tayekv.

$33 \mathrm{Mv}$ vkvsvmkv etcropotten ohmekketv ocakan ohfvnket, fvccetvn vtotket, temfvecetv taket enhecken, estepapvlke e'cukwv taken vkhottet,

34 Totkv en yekcen vslecet, eslafkv-crpko em fvsken mvnvttepet, yekce sekat aossen yekcvke hakepet, horre ofv fekhvmkvke hakepet, momen tvleporvlke en suletawvlke etohkvlkan pefathuecat sasvtes :

35 Hoktvket em pvsatkvten hvtvm hesahoken evpayakvtes; vpvlwvn estemerrihocrtes, e ossicetvn eenakuecekot, elkv aenkvwvpketv svmmomusat en heckvkvren.

36 Nakhayeko momet tephokan kvwrpakat sasvtes; momet ohvpakat eswrnakvn momet cuko-yeker piketrn enheckakvtes :

37 Cvton es nvfhoken, esfokvn 'setewarhoyen, eskerhoyen, eslafkv-cvpkon es pvsvthoyvtes; yvpefikv hvrpe momet cowatr hrrpen vecet tefullvtes; enkusapket, estemerket, estemerrihocen.

38 (Ekvnvt mvt oh vpokvre heretv orekates:) vnrvwvn, 
momen ekvnhvlhawan, ekvnhaukakan momet ekvnv sufsokan etefullvtes.

39 Momen heyv omvlkvt vkvsvmkv eteropotten ohkerkueckv heran epoyaket omis, etemfvccetvt enheckvkekates;

40 Hesaketvmeset nake svmmomusan pum etetakuecrtet omen, vpvkēkon omat temvpoke hakvkekaren.

\section{SETENTVCKE XII.}

Monkv pumeu akerrvlke aholoce rakke momvtekat pum vfulotet omekv, nake honnat vtekat, momet naorketv tewolen pum vfulotat vwikvkeres,

2 Momet, Cesvs, pum vkvsvmkv hayv momet espoyv, mv, afvcketv ehomv ocvte vrahkvn, em vlesketvn moskomekot, etohweklepkv em estemerketvn kvwapvtet, Hesaketvmese em ohliketv vkvperv likan vhecet, etemvrkv pu homv ocan momis komēt pefatkvkeres.

3 Momet omekv opunvkv vnrvpkv mv omēn holwvyecvlket vnrvpen opunvyecakan kvwapvten ohvkerricvkes, ce hotoset, cem vkerrickv take ofvn yekceko ce hakvkekaren.

4 Naorketv vnrvpen vcevllatskat hvte catv pvlvtke oren vnrapatskekos.

5 Momet cem vcahne, hopuctake omece cem pnnayat, Cv'ppucē, Pucase $c e$ 'stemerricat nake cotke omehcetskvs, momet naorketv ohfvccun cem vsehof, ce hotohsats ;

$6 \mathrm{Mv}$ Pucaset vnokecan estemerricet, eppucen evpayat vtekat rukafet omekv, makan ce hosvket os.

7 Ce'stemerrihocan kvwapatsken omat, Hesaketvmeset eppuce taken ec omecvket os; hiyomet omekv, eppuce estomet like te, mv erket estemerricekat?

8 Momis omvlkvt'sestemerrihocar cen heckvkekon omat, eppuce take erke evpayat tokon, evpahoyekat omatskes. 
9 Momen ohvtvlakat pu'navpeswv erkvlke $p u$ 'stemerrvkuecakan oceyvtet os, momet vrakkueceyvtes; pum puyvfekev Erke mahvkvu em apohicet, hesahokēkar haks?

10 Hiyomet omekv, mehenwvn emetaket nettv vnvcomekon, eme komat mahusat vcvkvyen $p u$ 'stemerrvkuecakvtes, momis heyvt pun lopickv vrahkv, en hvsvtketv en heckvlket omeyvren momecet os.

11 Momen estemerrickv estomis, hvte heckof, est' afvcecice taye onkon, momis honne ont os; momis renyupvn fvecetv em ette herken $\mathrm{mv}$ 'sestemerrvkuehocan en heckuecaket os.

12 Monkv enke vtvrtakan, momet torkowv yekcvkekan kvwrpvkes ;

13 Momet vyetv lvpotlvken ce'le taken en hahicvkes, mv ele nokkan fiyecihocekaren; momis em wicehocvre mit omekvs.

14 Este omvlkv etenherketvn hopoyvkes, momet hvsvtketvn, mvn ocekat estimvt Pucasen hehcekot ok;

15 Vhericet hecet omvkes, estimvt Hesaketvmese en heromkvt en yupvklvtkekaren; hometv en yvlonkvt hontet $c e$ naoricen, mv eteropotten sulket svholwvkekaren vketecet;

16 Momet hoktarvt monkat mekusvpkv ehvperkuecv Esa omēt sekaren vketecvks; mvt homv heckvte vrahkvn enaken wiyvtet ok, hompetv hvmkuse vrahkvn.

$17 \mathrm{Mv}$ renyupvn ohmerkv enake taranvten eenakuecetvn komof, vhepvkhoyvten kerratskekv ; vhericet, eturopuswvt pvlatken, hopoyet o estomis, vkerrickv etickv oce tayan eshecekatet ok.

18 Hiyomet omekv, mv ekvnhvlwe celahoye taye, totkv es fenkan a oh awatskekos, monkat lvsten, monkat yomucken, monkat hvnvcofelvn,

19 Monkat pofketv en haken, monkat opunvkv en 
haken; mv haken pohakvtet $m v$ opunvkvn ohvtvlaken 'sem punayvkhoyekaren vpohakvten;

20 (Momen punvttv tis mv ekvnhvlwen vcelaken omat, cvton es nvfhoken, monkat nak fvsken ropotten es sekehoyvres, makan kvwvpvkeko tayvtet ok,

21 Momen en heckvt sehonecke mahet omen, Moset, Tayen $\mathrm{cv}$ penkalit, $\mathrm{cr}$ fekeket os, makvtes.)

22 Momis Sivn ekvnhvlwen, momet Hesaketvmese wenakat em etvlofv Celuslvme hvlwan, momet encelvlke en sulke vhonkvtēsko taye etohkvlkạn,

23 Enhvteceskv heckakvte mv $e$ hocefhokv hvlwe tvlofv ofv hoccakat en nvkvftetv, momet em mekusvpkv-cukon, momet Hesaketvmese este omvlkv en Frccecvn, momet este fvecakat em puyvfekcv temvpokvke hahoyvten;

24 Momet Cesvs etemfvccetv mucrse etenhayvn, momet catv ohfesketv, mv Epel tate e catv nak makvte nake senherake punayan a oh awatsket os.

$25 E$ vketecrkes, mv opunayan vhepvkatskekaren; hiyomet omekv, mv ekvnvn aret punayen vhepvkakat mvnvttvkepekaten omat, pume mahvkvu, mv hvlwe vte punayan em e fulecēn omat, mvnvttehpēkos;

26 Eme em opunvkv en haket momof ekvnvn nekeyicvtes; momis hiyomat, Hvtvm vhvmkv, ekvnv tvlkusekon momis hvlwateu nekeyicaret os, maket etemfaccrtet os.

27 Momen heyv, Hvtvm vhvmkv, makat, mv nanvke hahihocvte omakat mv nekeyihoce tayat vkuehuecvranan oket os, mv nanvke nekeyihoceko tayat oce munkv taren.

28 Monkv ohmekketv nekeyihoceko tayat pun heckekv, vrakkueckv momet em penkvlkv ofvn, heromkv $\mathrm{mv}$ eteropotten Hesaketvmesen afvcecicet, em vtotkeye tayat ocvkeres :

29 Pun Hesaketvmeset totkv nak sumecicvt omekv. 


\section{SETENTVCKE XIII.}

VNoKeCKV tecakkakvte ete'm ocatet oce munkvt omekvs.

2 Este kerkrkekat evpryetvn ce hohsvkates; mvn momecat, kerrekot encelvlke evpayat sasvtet omekv.

$3 \mathrm{Mv}$ svlvfkuehocan, mv vpaket ce svlvfkuehoce omēn vkerricet, mv estemerrvkuehocan centakeu ena ofv fullatsket omat vkerricvkes.

4. Etepvyetv momet topv svholwvkekat este omvlkvt vrakkuecakvr tayet os; momis hoktarvlke momen etepayakvte hoktarvlke Hesaketvmeset ohfvccecrres.

5 Cem fulletv taket crto-kunawv vnokeckv sekot omekvs; momen nanvke ocatskat es ce mahlvpvtkvket omekvs; Estofis ce'nkvpaket, monkat ce wihkvkos, emet makvtet ok.

6 Momèt on, Pucaset vm vnicvt on, estet nake cv momecan en cv penkvlekares, fekhvmket makeye tetayes.

7 Cem fvyatvlke, mv Hesaketvmese em opunvkv cem punayvkvten vkerricvkes; momet em vkvsvmkv taken svhayepvks, em fulletv em eyuksvn vkerricet.

8 Cesvs Klist pvksvnke, mucv-nettv momet meyuksvsekon mvrahke sekot omes.

9 Mvhakv murahrvkv momet estomahakat es ce fuliyvkates, hompetr vcrkvye fullat en lopicvkekate tokon, efeke heromkvn es yekcihocat heret omekv.

10 Altv mv oh ocan mv escukohakv vcake vfvstakat hompvkvre mv em etektvnkekan oceyes.

$11 \mathrm{Mv}$ nak wenahokat e catv plest enhomahtrt naorketv vrahkv escukohakv vcakan esceyvtet, ena taken este-hvpo en lvpvtken nekrihocet omekv.

12 Monkv Cesvs tis, e catv mahusat este vcacvken es 
hayvret, tvlofv em vtohopke 'mvhauke topvrvn estemerkvtes.

13 Monkv este-hrpo en lvpvtken, em vlesketvn kvwapet, emen oh vpeyvkeres.

14 Heyvmvn trlofv like munkan ocēkot, momis ocvranan hopoyēkv.

15 Monkv eme eteropotten, Hesaketvmese e hocefkvn vkvsamet, vkvsvmkv nak emetvn estofis emvkeres, mvt pu cukhvrpe em ettet omekv.

16 Momis est'en lopickv, momen est'en kvpickvn ce hohsvkates; nak emkv mv omakan Hesaketvmeset es afackuset omekv.

17 Cem fvyatvlken em apohicet, elecrn svpvklvkes, este e ohkerkuecvkvrane omēt cem puyvfekcv take ohfvecvn vketecaket ok, efeke es nokkicekot, momis afvcket momecvkvren; cen lopicvkekaret ok, feknokket momecaken omat.

18 Pum emekusvpvkes; nak omvlkv ofv fvccusen fulletvn komēt, eohvkerrickv heren oceyat enhonreyet omekv.

19 Momen heyvn momecatskvren ohvtvlaken cen kusapvkvyet os, senlvpkusen hvtvin cem vlvkvkaren.

20 Momen herkv en Hesaketvmeset, mv pu Pucase Cesvs Klist Yvpefikv Vhecicv rakkan pvsatkvte aenkvpvken a kvwapvtet,

21 Etemfvccetv meyuksv-seko e catv eteropotten eme komat momecatskvren Cesvs Klist eteropotten mv ehomv afvcecicusan ec ofv taken vtotket, vtotketv herakat vtekat of temvpokvken ce hayvkate! vrakketv estofis meyuksv-sekon mvn em ocekvs. Emén.

22 Momen tecakkvte toyatskat, vcahnetv opunvkvn em etektvnecatskvren cen kusapvkis, opunvkv nvcomusen cen hoccicvkikv. 
23 Tecakkeyvte Temoret enrecopkvten kerrvkes; lvpken vlaken omat, mvn vpakit ce hecvkares.

24 Cem fvyatvlke, momet mekusapvlke omvlkvn vsekvkes. Etvle vpokatet ece 'sekakes.

25 Heromkvt omvlkatskan ece 'pakvkekvs. Emén. Heyv cokv Pal Etvlen liket Hepluvlken en hoccicvtes. 


\section{COKV CEMS}

\section{MEKUSAPVLKE OMVLKVN OHTOTVTE.}

\section{SETENTVCKE I.}

Cems, Hesaketvmese em vtotkv, momet Pucase Cesvs Klist em vtotkv, etvlwv pale hokkolohkake vwahakat vseket os.

2 Tecvkkvyvte toyatskat, holwahoke mvrahrvkv ofv pvlatkatskof, afveketv omvlkvt's komvkvtcrs;

3 Cem vkvsvmkv cen celayvkat momis-kometvn hayet omat, kerret.

4 Momis momis-kometvt vtotketv esfrcken ocekvs, ceme temvpoke, herake, naket cen seko ce hakvkvren.

5 Estomatskis hoporrenkvn enkusapken omat, Hesaketvmese, mv encakekot momet Cem mvttes kicekot, este omvlkvn emakan, em 'pohekvs, momen emhoyvres.

6 Momis em vkerrickv melohlekot, vkvsvmkv ofvn vpohekvs, mv em vkerrickv melohlat uehatkv melohlv, hotvle vtohken melohlicat omet omes.

7 Monkv mv estet, Hesaketvmeset nake a'men vn heckvres, kohmats.

8 Este-hunvnwv em vkerrickv hokkolat em vretv omvlkv ofv heren huerekot os.

9 Etecakkat kvncapat hvlwehocat es afvckekvs.

10 Momis este nake-ocat kvncrpe hakat es afvckekvs, pvhe em prkpvke omet sumkvret okv. 
11 Hvse en hiye ocet akvwapkof, pvhen yuknicet omekv, momen em pvkpvket latkes, momen en hecky herusat sumket os. Matrpomen este nake-ocat em vyetv ofvn sumkvres.

12 Este-hunvnwv en heres momis-komat eskerhoyof; eskerhoyat hoyanof, hesaketv en kvpvtukvn en heckvret okv, mv Hesaketvmese vnokecakat ce'mvkares kice etemfaccrte.

13 Este estomet, eskerhoyof, Hesaketvmeset es cv kerret os, mahkats. Hesaketvmese nak' holwvke eskerreko tetayet omekv, momet este estomen naorkepueckv svrahkv eskerrekot os.

14 Momis este estomis, em eyackvt akueyicen, mvkerhoyof, eskerhoyet omes.

15 Momof eyackvt, ofvn vfekhoniyet, holwvyeckvn heckuecet os, momen holwvyeckvt, espokof, elkvn heckuecet os.

16 Cem mvtiyvkats, tecrkkvyvte vm vnokeckvlke toyatskat,

17 Estemkv herakat vtekat, momet estemkv temvpokat vtekat hvlwe vtet os, momet hvyayvke Erke, mv mvrahkv hakvte sekat, momet folotketv em vpette sekaten a hvtapket os.

18 Eme komat pu heckuecrtet os, mehenwv em punvkv eteropotten, mv hayakvte enhvteceskv toyeyvren.

19 Monkvhkvn, tecvkkvyvte vm vnokeckvlke toyatskat, este estomis pvfnen apohicet, yvlohusen opunayet, yulohusen crpakket omekvs.

20 Este-hunvnwv en crpvkketv Hesaketvmese em fvccetvn vtotkekot omekv.

21 Monkv efeke svholwvke omvlkvn, momet holwvyeckv en sulken vpvlvtvks, momet eyasketv ofvn opunvkv vsekke cem puyvfekcr take hesahuece tetayan evpryvks. 
22 Momis, e emvkerrake, opunvkv apohicvlke tvlkekot, opunvkv momecvlket omvkes.

23 Estimvt opunvkv apohicvt omet, opunvkv momecv tokon omat, este-hunvnwv estvkheckvn hecet e turofv emmunkvn hecat omet omekv.

24 E hehcet, em vyetvn ayet os, momet vyvkhvmkvn este estomet omat ehoset omekv.

25 Momis estimvt pucasseko-hueretv em vhakv temvpokan hecet, momet fekhonnen omat, mvt apohicr ehosv tokot, momis vtotketv momecrt omet, mvt em vfrstetv ofv en hervres.

26 Estimvt ece 'pakvkat, emekusapv e omecet omis, e tulaswvn cuksakkuecekot, e feken emvkerren omat, $\mathrm{mv}$ em emekusvpkv ehvperkvt omes.

27 Erke momet Hesaketvmese ehomvn emekusvpkv hrsatkuse, momet svholwrkekat heyv tes, eckemelvlke oyevike esyoman em estemerkv ofvn er hecet, momet ekvnv en holwvken esvholwvkekot e veryecatet.

\section{SETENTVCKE II.}

Tecvkrvyvre toyatskat, pu Pucase Cesvs Klist vrakkat em vkvsvmkv este etemetuece ohcatskvs.

2 Momen este-hunvnwv, estenke-wesakvtehkv cvtokunaplanen ocet, vccvke herateu ocet, cen nvkvftetr take ofvn vlaken, hvtrm estemerkusat, vecvke svholwvken ocet vlaken omat;

3 Momen vccvke here ocan vrakkuecet, Yvmvn heran likvs, kicatsken, momet este estemerkusan, Vsvn huervs, monkat cv'le vm ohhuerickv elecvn likvs, kicatsken omat;

4 Este etemetuecet etefullatskeko haks? momet fvececvlke vkerrickv hervkeko ocet ce hakvkeko haks?

5 Pohvkes, tecrkkvyvte vm vnokeckvlke toyatskat, este 
estemerkakuse ekvnv oh fullat vkvsvmkv ofvn nake-ocvken hayet, momet etvlwv Hesaketvmese vnokecakan ce'mvkares kice temfaccrte epucase hakvkvren Hesaketvmeset enhopoyvtet omeko haks?

6 Momis este estemerkakan enhomecatskvtet os. Este nake-ocvlke ce'stemerrvkuecet, ohfvececkv ohliketv ehomv ce svpvklecvket omeko haks?

7 Eme mv hocefkv vcakat ec oh huehokat tvklecaket omeko haks?

8 Momis Nakhocce vcrkvyen, vhakv mekkvke 'mvhakv omat esfrcecatsken omat, Cem vwolicvn e vnokecetskat etvpomen vnokecrs, makat, heren vfastatsket $\rho$ s.

9 Momis este etemetuecatsken omat, naorketvn vfastatsket os, vhakv eteropotten naorkvlke toyatsken ec ohkerkuecrket os.

10 Este estomis, vhakv omvlkvn vfastis, hvmken kacen omat, omvlkvin kvce omet hueret os.

11 Hoktahretskvs, makat, matat, Elehcetskvs, makvtet omekv. Monkv hoktaretskeko estomis, elecetsken omat, vhakv-kacvt hueretsket os.

12 Pucasseko-hueretv em vhakv eteropotten ohfvccehocvrane omen opunayet, momecrs.

13 Merkvn heckuecekatet merkv en heckekon ohfvecehocvres ; momen ohfvececkv vnrvpen merkv afvcket os.

14 Tecrkkvyvte toyatskat, estimvt vtotketvn ocekot, Vkvsvmkvn ocis, maken omat, naken en lopicet ome haks? Vkvsvmkvt hesayece taye haks?

15 Tecakkakvte hunvnwv hvmket, monkat tecakkakvte hokte hvmket, enatvlkvranuset, momet hompetv nettv omvlkvn eyace enkusapket on omat,

16 Momen hvmkvtekatskat kicaket, Herkv ofvn vpeyvks, ce lekuthvket, momet ce fvckvkekvs; momis nake ena en lopicrke tayat emakatskekot, naken lopice haks? 
17 Matvpomen vkvsvmkv vtotketvn ocekon omat, entvlkuset omet, elet os.

18 Momes, Cemet vkvsvmkvn ocetsken, vnet vtotketvn ocis, cem vkvsvmkvn cr hecicvs, cem vtotketv sekon, vneu vm vkvsvmkvn ce hecicares, vm vtotketv eteropotten, este-hunvnwvt make wites.

19 Hesaketvmese hvmkuset likes, komet, vkvsametsket os, heren vfastetskes, mv vkvsametskat; puyvfekcv holwrhokat tis vkvsamet, fekekaket omes.

20 Momis Aeha, este ehvperkv toyetskat, vkvsvmkv, vtotketv sekat, elet os, kerretvn kometsket o haks?

21 Epleham, purke, eppuce Iseke altvn oh wvkecet, Hesaketvmesen emvranof, vtotketv eteropotten este-faccv en hueretvn hueret omekvt haks?

22 Hecetske haks, vkvsvmkv vtotketv vpvke vtotkvte, momet vtotketv eteropotten vkvsvmkv espokvtes.

23 Momen Nakhocce esfackvtes, miv makat, Epleham Hesaketvmesen vkvsamvtes, momen mv vkvsamat omecicen, fvecetv enaken en hahoyvtes, momen Hesaketvmese en hessen kihocvtes.

24 Monkv hecatskes, este vkvsvmkv eteropotte tvlkekon, vtotketv eteropotten este-faccv en hueretvn hueret os.

25 Matvpomen Lehap, hoktarv, opunvkv-esfullvlken evpayet, etin vtotakof, vtotketv eteropotten este-faccv en hueretvn hueret omekat haks?

26 Monkv ena puyvfekev vpvkekat elat etvpomen, vkvsvmkv vtotketv vpvkekat elet os.

\section{SETENTVCKE III.}

Erecvkкvyvte toyatskat, mvhayvlke sulket ohmatskvs, hoyvnen ec ohfvccehocvkvres kerratskat.

2 Omvlkeyat sulken naorkeyet omekv; estimvt opunvkv 
ofvn naorkekon omat, matat este-hunvnwv hrsvtket omet, ena omvlkvn cuksakkuece tayet os.

3 Hecvks, corakko e cukwv cuksakkvn cuksakkueceyet os, pum vkvsvmvkvren, momet ena omvlkvn foloteciceyet os.

4 Hecvks, perro-cuko matvpomen rvkrvketvt ont omis, momet hotvle yekcakat es vtohkis, estvmin em fvyatv komat semfryatkv cotkusis es folotecihocet os.

5 Matvpomen etulaswr enalvtce cotkuset os, momet nake rvkrvken 'sopunayet os. Hecvks, nake rakke mahis totkv cotkusat vhetecet os.

6 Momen etulaswv totkvt omet, ${ }^{\circ}$ holwvyeckv ekvnv tes; etulaswv pu'nalvtce vpvke hiyomet os, ena omvlkvn vhopanet, pum munkv en nekeyetv vhetecet os, momet tôtkv-rakkot vhetecet os.

7 Nake ele-ostvkusat, fuswr tis, cetto tis, uehatkv vk fullat tis, mvrahrvkv omvlkvn yvmvsvkuehocet, yvmvsvkuehocvtet omekv.

8 Momis etulaswrn estimvt yvmvseceko tayes, nak' holwvke fvyvthoyeko tayet, nake estelece tayat es fvcke tes.

9 Mvn Hesaketvmese, Erke tan, es vkvsamet, momet mvn este Hesaketvmese vhake hahihocvten es tvhikeyet os.

10 Vkvsvmkv tvhiketv tis ecukwv matan esossaket os. Tecvkkvyvte toyatskat, heyv nanvke momvranekot os.

11 Uekiwv mata a fihnat uewv heren uewr home tepaken a fihnece te?

12 Tecrkkvyvte toyatskat, ke-rakko olefv em ette ettuece taye haks? momen prrkofvkv ke-rakkon ettuece taye haks? monkv uekiwv ueokcvncvmpv, uewv herat tepaken a fihneceko tayes.

13 Estimvt ece 'pakvkat hoporrenet, nake kerret ome haks? vretv herat aossen hoporrenkv en yvmasketv ofvn em vtotketvn heckvkuecekvs. 
14 Momis vholvckv home etepoyetv tepakat ce feke ofvn ocatsken omat e ekvsahmatskvs, momet mehenwr vnrvpet lvkisatskvs.

15 Heyv hoporrenkv hvlwe a hvtapkekot omes, momis ekvnv enaket's, ena-vpeswr enaket's, puyvfekev holwrke omet os.

16 Estvmvn vholvckv tepoyetv tepakat ocat, mvn tehvn$\mathrm{kv}$ momet vtotketv holwakat vtekat ocet omekv.

17 Momis hoporrenkv hvlwe a hvtapkat, enhvteceskvn hvsvtket, mohmet tenherkepuecet, yvmaskuset, vkvsamuset, merkv ette herakat tis es fvcket, este etemetuecekot, sahayekot omes.

18 Momen fvccetv em ette herkv ofvn fokahoyen, herkv hayakat fokayet os.

\section{SETENTVCKE IV.}

Horre etepokv tis ece 'pvkvke ocakat estvmin a awe haks? Yvn a aweko haks? Cem eyackv take ce'nalvtce ofvn tepoyakat $a$ aweko haks?

$2 \mathrm{Ce}$ yacvket, ocatskekot os. Elecatsket os, momet ocetv ce yacrket, cen heckvkeko tayet os ; tenrapet etepoyatsket os, momis ocatskekot os, vpohatskekokv.

3 Vpohatskes, momis cen heckvkekot os, cem eyackv take oh sumecicvranet, mvtten vpohatskekv.

4 Hunvntake hoktarvlke, hoktvke hoktarvlke esyomatskat, ekvnv en hesse vretv Hesaketvmese vnrvpkvt omat kerratskeko haks? Monkv estimvt ekvnv en hesse haketvn komen omat, Hesaketvmese vnrapvt os.

5 Puyvfekcv epu 'pike likat vholvckv oren eyacet os, Nakhocce makat ehvperkvn maket os, komatske haks?

6 Momis herkv ohvtvlvken emet os. Monkv Hesaketvmese ekvsamat vnrvpet omet, momet efeke ofv kvncapusat herkvn emet os, maket os. 
7 Monkv Hesaketvmesen em e wikvks. Puyvfekev holwakat vnrvpvks, momen cen letkvres.

8 Hesaketvmesen vnakuecvks, momen ece 'nakuecvkvres; naorkvlke toyatskat, cenke taken hvsvthicvks, . momen vkerrickv hokkolicatskat, ce feke taken hvsathakusen hahicvks.

9 Ce feket cen nokhokusekvs, momet wahoket, hvkihhokvks; cem vpelkv taket waketv hakekvs; afvcketv feknokketv hakekvs.

10 Hesaketvmese ehomvn e kvncrpuecvkes, momen ce hvlwecvkvres.

11 Tecakkvte toyatskat, holwvken et'opunvyehcatskvs. Estimvt tecakkvten holwvken opunvyecet, tecakkvten ohfvececat, vhakvn holwvken opunvyecet vhakvn ohfvececet os; momis vhakvn ohfvececetsken omat, vhakv vfastv tokot, vhakv ohfvececrt ometsks.

12 Vhakv estemv hvmket, hesayece tayet, sumecice tayat liket os; este etv ohfrccecetskat, estimv toyetske haks?

13 Tokvs hiyomat, Mucv-nettv monkat vpvksen mv tvlofvn vpeyet, ohrolope hvmken fekhonnet, wiyet, neset, 'se ohvtvlvyeyvres, makatskat,

14 Vpvksen nake estomvranat kerratskekofvt." Cen hesaketv nake te? Hopere, estomusen heciket, sumkat tes.

15 Momat, Hesaketvmeset komen omat, hesahokeyet, heyvn momecet, monkat mvn momeceyvres, makvranatsket os. 16 Momis hiyomat cem ekvsvmkvtake ofvn ece 'fackvket os; afvcketv mv omat omvlkvt holwvket os.

17 Monkv mv herat mecetvn kerret, mecekat, holwvyeckv em ocet os.

\section{SETENTVCKE V.}

Tokvs hiyomat, nake-ocvlke toyatskat, estemerkv ec oh yicvranvkan es hvkihhoket, wahokvks. 
2 Nake ocatskat lekwvket, cem vccvkeu sukehtvt loket os.

3 Cen cvto-kunaplane take, momet cen cvto-kunaphvtke vhopvnkvket os, momen mv vhopvnkakat ohkerkueckv ece'nrvpvke tares, momet totkv omet cena-vpeswrn pvprres. Nettv espokakat en nak' sulecatskvtet os.

4, Hecrks, vtotkvilke cem ecvpofv taken wucutakvte en feketv em fekhonnicatskvtet a huehken, wucutakvte en huehketvt Sapeot en Hesaketvmese e hrckon 'sen ceyvtet os.

5 Afvcketv ofvn ekvnvn oh fullatsket, yvmahkuecvlke toyatskvtes; vpesprsatkv nettv omen ce feke taken hompicatskvtes.

6 Este-faccrn ohfrccecet, elecatskvtes, ece'nrvpekot oman.

7 Hesaketvmese em vlvketv oren momis-komvks, tecakkvte toyatskat. Hecrks, crpofv vhecicv ekvnv em etten em ehaket, hofunen momis komet emehaken, oske hvten hvtapket momet yupv hvtapkat en hecket os.

8 Cemeu momis-komet, ce feketaken yekcrkuecvks, Hesaketvmese em vlvketv vwolicet omekv.

9 Tecakkvte toyatskat, etem encahkatskvs, ec ohfvecehocekaren; hecrks, Fvccecvt vhauke ehomvn hueret os.

10 Tecrkkvyvte toyatskat, owalvlke, Hesaketvmese e hocefkv ofv opunahoyvte, momis-komet, momet vkvsamusen estemerrihocvte ocat, vhayetvn ocvkes.

11 Hecvks, momis-komet vpeyakat afvckakes, komeyet os. Cop momis-kome ayvten pohatskvtet os, momet Hesaketvmese emmerruset, merkv es fvcket omen, momet naken komat hecatskvtet os.

12 Momis tecrkkvyvte toyatskat, nak' omvlkv en hoyanat, Hesaketvmese e hocefkv ehvperkvn punahyatskvs, hvlwe es hocefekot, ekvnvu es hocefekot; nake etv estomis es hocefekot; momis henka maketskat henka 
tares, momen monkos maketskat monkos tares, ohfvcceckv ce'k pvlvtkvkekaren.

13 Ece 'pakvkat hvmket estemerket on omat, mekusvpekvs. Estimvt afvcket on omat, esvkvsvmkvn yvhikekvs.

14 Ece 'pakvkat hvmket enokket on omat, mekusvpkvcuko vfastvlke en huehkahken, Hesaketvmese en hocefkv ofvn nehen es em vfeyaket oh mekusvpvkekvs.

15 Momen vkvsvmkv mekusvpkvt enokkat hesayecen, Hesaketvmeset a huericvres, momen naorketvn vfastvten omat, en kvpryecihocvres.

16 Etem mvttecatskvten etem e ohkerkuecet, etem emekusvpatskvres, cem wikvkvren. Este-facev em emekusvpkv yekcetv ocat sulken momecet os.

17 Elivs pume pum emunkv etvpomet omvtes; momet osikats komet, heren mekusapvtes; momen ohrolope tutcenen hvse epaken ekvnv oh oskolekates.

18 Momen hvtvm emekusahpen, hvlwet osken ehmen, ekvnvt em etten hayvtes.

19 Tecakkvte toyatskat, hvmkvtekatskis mehenwvn en kvpaket, em matten omat, momen hvmket afulecicen omat,

20 Heyvn kerrekvs, estimvt em vretv em mvttat naorkvn afulecicen omat, elkv en kvpvken puyvfekcvn hesayehcet, naorketv sulken ehvres. 


\section{COKV ENHVTEOESKV}

\section{MEKUSAPVLKE VTEKAT}

- $\quad$ oust

\section{OHTOTVTE.}

\section{SETENTVCKE I.}

MV nake enhvteceskv vteke ocvte, poheyvte, pu turwv es heceyvte, momet oh heceyvte, momen punke celayvte mv hesaketv em opunvkv a en kvprkat.

2 Hesaketv heckvtet os, momen heceyvtet os, momet kerkueceyvtet os, momen mv hesaketv em eyuksv-seko Erke vpake tate, momet pun kerkuecvkvten ce hecicvkeyet os.

$3 \mathrm{Mv}$ nake heceyvte momet poheyvtet, cen kerkuecvkeyet os, cemetaken etohkvlketv pum ocatske taren, momen pum etohkvlketv mehenwusen Erke, momet Eppuce Cesvs Klist vpaket os.

4 Momen cem afveketv taket fveke taren heyv nanvke cen coyvkeyet os.

5 Momen heyvt opunvkv em poheyvtet os, momet kerkuecrkeyat, Hesaketvmeset hryayvkets, momen eme ofv yomucke seko mahet os.

6 Etohkvlketv emen etem oceyēs maket yomucke ofvn yvkvpakēn omat, laksēyet os, momet mehenwrn momecēkot os.

7 Momis eme hryayvke ofv likat omen hvyayvke ofvn Creek. 
yvkvpakēn omat, etohkvlketv etem ocēyēs momen Eppuce Cesvs Klist e catvt naorketv omvlkvn pun hvsvtecet os.

8 Naorketvn ocēks makēn omat, e emvkerreyet os, momen mehenwv epu 'pikekot os.

9 Naorketv pun kvpvyecicvret, momet fvecetv tokat omvlkvn pun hvsvtecvret, mvt vkvsvmvkvre oret fvccet omes, pun naorketvn kerkuecēn omat.

10 Naorkēks makēn omat, emen laksvn hayeyet os, mo. men em opunvkv epu 'pikekot os.

\section{SETENTVCKE II.}

VN hoputetakuce, naorkatskekaren heyv nanvke cen coyvkit omis. Momen este estomet naorken omat, Erke vpvken em viastrn aceyet os, Cesrs Klist fuccan.

2 Momen pun naorketv oh homeckvte emahlvpvtecicvts, momet punake trlkekon, momis ekrnv omvlkv enaken.

3 Momen em vhakvn vcayecēn omat, eme kerreyatet es kerreyet os.

4 Estimvt Kerris makatet em vhakvn vcayecekat, este laksvts, momen fvccetv vpikekot os.

5 Momis estimvt em punvkv vcayecat, mehenwwn eme ofvn Hesaketvmese em vnokeckv esfrcket os, eme ofv toyeyat heyvn es kerreyet os.

6 Estimvt $\mathrm{mv}$ ofvn vfekhonnis makatet mv yukapvte omen emet yvkvpvre tayet os.

7 Etecakkeyvte toyatskat, vhakw mucrse tokon, momis vhakv vcule enhvteceskv vteke ocatskvten cen coyvkvyet os: Mv vhakv veulat enhrteceskv vteke opunvkv pohatskvtet mv tes.

8 Hrtvm vhakv mucrsen cen coyvkryet mv nake eme ofv, momet ceme ofvn mehenwet omes, yomucket hoys- 
nepekv, momen hryayvke mehenwatet hiyomat hryayakekv.

9 Mv este hvyayvke ofvn hueris makat, momet etecakkaten en homecat, hiyomis yomucke ofvn huere munkvt os.

10 Etecakkat vnokecat, hvyayvke ofvn vfekhonnet os, momen naket elentappuecrre sekot os.

11 Momis etecakkvte en homecat yomucke ofvn huērēs, momet yomucke ofvn yvkapēt os, momet estrmin ayat kerrekot os, yomucket e turwvn em vrvnayet onkv.

12 Hopuetakuce, mv en hocefkv vrahkvn cen naorketvt cen kvpvkvkvtet onkv, cen coyvkit omis.

13 Erkvlke toyatskat, mv enhvteceskv vtvte hueran kerratskvtet: onkv, cen coyvkit omis. Este hunvntake mvnettvke toyatskat, mv holwvyecrn ohfvnkatskvtet onkv, cen coyvkit omis. Hopuetake toyatskat, Erke kerratskvtet omekv, cen coyvkit omis.

14 Erkvlke toyatskat, mv enhvteceskv vtvte hueran kerratskvtet onkv, cen coyvkvytet omes. Este-hunvntake mrnettvke toyatskat, ce yekcrket omen, Hesaketvmese em. opunvkvt ec ofvn vfekhonnet omen, mv holwvyecaten ohvnkatskvtet omekv, cen coyvkvyvtet os.

15 Ekvnv vnokehcatskvs, momet nanvke ekvnv vtehkateu. Este estomet ekrnvn vnokecet on omat, Erke vnokeckv vpikekot $o$.

16 Nake ekvnv vtehkat omvlkvt, vpeswr em eyackv, eturwv em eyackv, momet hesaketv em ekvsvirkv, Erke a en kvpvket omeks, momis ekrnv a en kvpvket onkv.

17 Momen ekvnv momet nake em eyackvu hoyvnēpèt os, momis este Hesaketrmese komaten momecatet estofis vfekhonnet os.

18 Hopuetakuce, oketv espokets, momen Klist-vnrapv vlvkvranen pohatskvtet onkv, hiyomis Klist-vnrapvlke sulket omes. Mvn es kerreyet omes, oketv espoke tat. 
19 Pun kvpvket esossvkvtet os, momis epu 'pvke tokates epu 'prkvke taten omat, epu 'pvkake munkvnt omekv, momis esossvkvtet os omvlkvt epu 'pvkekot omat kerkuecvret.

20 Momis centake Vcakat en kvpvken vfeyetvn ocatskes, momet nake omvlkvn kērratsket os.

21 Mehenwv kerratskekat svrahkvn cen coyvkit omvkates, momis kerratskat svrahkvn, momen lvksetv vtekat mehenwrn aossekot omekv, cen coyvkvyvtes.

22 Estimvt este-laksvt o haks, Cesvs Klist tat laksecat mv tokon omat? Mv Erke, momen eppuce helaksecatet Klist-vnrapvt os.

23 Eppuce helaksecat vtekat Erken ocekot os; momis Eppuce oh vkvsamat Erkeu ocet os.

24 Monkv enhvteceskv vteke mv pohatskvtet ec ofvn fekhonnekvs. Mv enhvteceskv vteke pohatskvtet ec ofvn fekhonnen omat, centakeu Eppuce momen Erkeu ofvn fekhonnatskvres.

25 Momen setemfvecetv es pu temfaccrte heyvt mv tes, hesaketv meyuksv sekot.

$26 \mathrm{Mr}$ ce mvkerrvkat ohfvecrn heyv nanvke cen coyvkvyvtet os.

27 Momen emen a en kvprke vfeyetv cen heckvkvte ec ofvn fekhonnet os, momen este estomet ce mvhayvkvre ce yacvkekot os, momis mv vfeyetv mehenwvt omet, lvksetv tokot os, momet nake omvlkvn ce mvhayvket os, momen mv ce muhayvkvte vcrkvyen eme ofvn fekhonnatskvres.

28 Momen hiyomat hopuetakuce toyatskat, eme ofvn fekhonnvkes, hēckof fekhvmketvn oceyvren, momet vlakof ehomv epu'lsekaren.

29 Emet fvccet omen kērratsken omat, fvccetv momecat vtekat, mvn es heokvtet omen kerratsket os. 


\section{SETENTVCKE III.}

VNoKEckv estrmahe Erke pu'mvten hecvkes Hesaketvmese eppucetake pu kihocvren. Monkv ekvnv pu kerrekot os, emen kerrekot omvtet okv.

2 Vnokeckatskat, hiyomat Hesaketvmese eppucetake toyēs, momen nake toyvraneyat hvte heckekot os, momis emet heckofv inv omeye taretut kerrēs, estomet ōmate mv omen heceyrret okv.

3 Momen este heyv enhonrkvn eme ofvn ocat vtekat, eme hvsvtkat omet, e hvsvtecet os.

4 Naorketv vfastat vtekat vhakvu kacet os, naorketvt vhakvn kveetvt omekv.

5 Momen naorketvn pun kvpvyecicvret hecket omvtet on kerratsks, momen eme ofv naorketv sekot os.

6 Eme ofvn vfekhonnat vtekat naorkekot os, naorkat vtekat emen hecekates, momet kerrekates.

7 Hopuetakuce toyatskat, estimvt ce mvkervyvkats; fvccetv vfastat, eme fvecat omet, fvecet os.

8 Naorketr vfastat Este-nekricr enakets. Este-nekricv enhvteceskv vteken naorkvt onkv, momen Este-nekricv em vtotketv vhopvnvre svrahkvt Hesaketvmese Eppuce hecket omvtes.

9 Hesaketvmese es heckat vtekat naorketv vfvstekot omes, e nerkvt eme ofvn fekhonnet onkv, momen naorkeko tayet os 'Hesaketvmesen es hecket omekv.

10 Heyv ofvn Hesaketvmese en hopuetake kerkvket os, momen este-nekricv en hopuetake. Frccetr momecekat vtekat Hesaketvmese vpvkekot os, etecakkate vnokecekateu.

11 Heyv opunvkv, etenokeceyrre makan, enhvteceskv vteken pohatskvtet omekv. 
12 Holwvyecat enake Ken onkot, momet etecakkvte elecrte, momen estomen elecet omvt haks? em vtotketv holwahoket omen momen etecakkvtet fvccrken omekv.

13 Etecakkvyvte toyatskat, ekvnvt cen homecvken omat, estomahes kohmatskvs.

14 Elkvn en kvpahket hesaketvn oriceyvtet omet kerrēs, etecakkvten vnokeceyet okv. Etecakkat vnokecekat elkv ofvn fekhonnet os.

15 Etecakkat en homecat vtekat este-elecv tes, momen este-elecv vtekat hesaketv yuksv sekat vpikekot omen kerratsket omes.

16 Hesaketvmese em vnokeckv heyvn es kerreyet os, en hesaketvn pun wrkecrtet omekv, moinen pumeu pun hesaketvn etecakkeyvte en wrkeceyvre tayet os.

17 Momis estimvt yvmv ekvnv enake heran ocepet omen, momet etecakkvtet en kusvpket on hecis, envcofv em vnokeckun em vkhotten omat, estomet Hesaketvmese vnokeckv vpike haks?

$18 \mathrm{Vn}$ hopuetakuce, opunvkv ofvn monkat tulaswv ofvn vnokecet ohmeyats, momis mehenwv, momet vtotketv ofvn.

19 Momen mehenwr vpvkeye tat heyvn es kerreyet os, momen eme ehomvn pu feke mvhlvprteyvres.

20 Monkv pu feket ep ohfvececen omat, Hesaketvmeset pu feken 'sen rakket omet, nak' omvlkvn kerret omes.

21 Vnokeckatskat, pu feket ep ohfvececekon omat, Hesaketvmese ohfvecr fekhvmketv oceyet os.

22 Monkv naketo estomen vpoheyis eme en kvpvken pun hecket os, em vhakv vcayeceyatet ehomv nake afvccecican momecēkv.

23 Momen heyvt em vhakv tes, vhakv pu'mute vcrkvyen Eppuce Cesvs Klist hocef kvn oh vkvsamet, etenokeceyvret. 
24. Momen em vhakv vcayecat, eme ofvn vfekhonnet os, momen emeu mv este ofvn. Momen Puyvfekev pu'mvte etehoyvnen pume ofv vfekhonnetat es kerreyet os.

\section{SETENTVCKE IV.}

VNoKeCKatskat, puyvfekev omvlkvn oh vkvsahmatskvs, momis puyvfekev Hesaketvmesen a en kvpvkvke tat ohkerrvks, owalv laksvlke sulket ekvnv sohceyvtet onkv.

2 Heyvn Hesaketvmese em Puyvfekcv es kerratsket os. Cesvs Klist vpeswv ofvn vlvkes Puyvfeker makat Hesaketvmese vprket os.

3 Momen Cesvs Klist vpeswv ofv vlvkes puyvfekev makekat vtekat Hesaketrmese vpvkekot os, nomen heyvt Klist-vnrapvts mvt vlvkvrane pohatskvte, momen hiyomis ekvnvn aret os.

4. Hopuetakuce, Hesaketvmese enake toyatsks, momet mv Klist vnrapulken ohfvnkatskvtet os eme ece 'pikatet ekvnv vpikan 'sen rakket omekv.

5 Ekvnv enaket omākes, monkv ekvnvn opunvyecaken, ekvnvt em apohicet os.

6 Hesaketrmese enake toyës; Hesaketvmese kerrat pum apohicet os; Hesaketrmese vprkekat puim apohicekot os. Puyvekev frccan momet Puyvfeker frecekaten heyvn es kerreyet os.

7 Vnokeckatskat, etenokecvkeres; vnokeckr Hesaketrmesen a en kvprket omekv, momen vnokecat vtekat Hesaketrmesen es heckvtet os, momet Hesaketrmesen kerret os.

8 Vnokecekat Hesaketrmese kerrekot os, Hesaketvmeset vnokeckvt okv.

9 Momen Hesaketvmese Eppuce hvmkuse heckuecrte eteropotten hesahokepeyvren ekvnvn ohtotvtet onkv, 
Hesaketrmese ep ohfvecr vnokeckv ocat heyv ofvn hecket os.

10 Momen vnokeckv heyv ofvts; pumet Hesaketrmesen vnokeceyat tokon, momis emet pumen epu'nokecētut Eppucen atotvtet os pun naorketv es feketvn.

11 Vnokeckatskat, Hesaketvmese momën epu'nokecvte乞 on omat, pumeu etenokeceyvre tetayet os.

12 Estimvt estofvn Hesaketvmese hecvte sekos. Etenokeceyet on omat, Hesaketvmeset ep ofvn fekhonnet os, momen em vnokeckvt ep ofvn es fvcket os.

13 Momen eme ofvn fekhonneyet omen, emeu ep ofvn fekhonnet omen kerrës, em Puyvfekev akvpvken pu'mvtet omekv.

14 Momen Erket Eppuce ekvnv Hesayecvn atotvtēt omen heceyvtet omet, momet kerkueceyet os.

15 Estimvt Cesvs Hesaketvmese Eppuce tes make kerkuecat, Hesaketvmeset eme ofvn fekhonnet omen, erneu Hesaketvmese ofvn fekhonnet os.

16 Momen Hesaketvmese vnokeckv pum ocat kerreyvtet omet momet vkvsvmeyvtet os. Hesaketvmeset vnokec$\mathrm{kv}$ tes. Momen vnokeckv ofvn fekhonnat Hesaketvmese ofvn fekhonnet os, momen Hesaketvmeset eme ofvn vfekhonnet os.

17 Momen heyv ofvn pum vnokeckv etemvpoket os; Ohfvcceckv-nettv omof fekhvmketvn oceyvren, eme omet yṿmv ekvnvn pumeu fulleyet omekv.

18 Vnokeckv ofv penkvlkv sekot os, momis vnokeckv etemvpokat penkvlkv ossicet os, penkvlkv tat estemerkvn ocet onkv. Este penkalat vnokeckv ofv etemvpoken hahoyekot os.

19 Emet homvn epu'nokecvtet onkv, vnokeceyet os.

20 Este Hesaketvmesen vnokecryes makat, etecakkan en homecen omat, este-laksv tes. Estomet, etecakkan: 
hecrte vnokecekat, Hesaketvmese hecvte sekatet vnokece taye haks?

21 Momen heyv vhakvn eme a en kvpvken ocēs, este Hesaketvmese vnokecat, etecakkateu vnokecekvs.

\section{SETENTVCKE V.}

Césvs mvt Klist tat vkvsamat vtekat Hesaketvmesen es heckvtet os, momen $\mathrm{mv}$ heckuecvte vnokecat vtekat $\mathrm{mv}$ es heckvten vnokecet os.

2 Hesaketvmesen vnokecēt, em vhakvn vcayeceyofvt, Hesaketvmese en hopuetakuce vnokeceyat, heyvn es kerreyet os.

3 Momen Hesaketvmese vnokeceyat em vhakvn vcayeceyat tes; momen em vhakv honhoyekot os.

4 Momen naket Hesaketrmesen es heckat vtekat ekvnvn ohfvnkēt os; momen pum vkvsvmkvt ohfvnketvts, ekvnv ohfvnkat.*

5 Estimvt ekvnv ohfvnkat to haks, Cesvs, Hesaketvmese Eppuce tat vkvsamat tokon omat?

6 Uewv, momet catvu etehoyvne vlakvtet Cesvs Klist tes, momen newv etehoyvne tvlkekon, momis uewv catv tepakv etehoyvnen, momen Puyvfekcvt akerrv tes, Puyvfekcut mehenwot omekv.

7 Momen Erke, Opunvkv, Puyvfekcv Vcakat estutcenatet hvlwe ofv akerrakat tokv, momen heyv tutcenat hvmkuset os.

8 Momen Puyvfekcr, catv, uewr estutcenat ekvnv akerrakat tes, momen heyv tutcenat etenhrmkuset os.

$9^{*}$ Este kerkuecvten ësēn omat, Hesaketvmese kerknecvtet 'sen rakkes. Hesaketvmese mv Eppuce ohkerkuecrtet Hesaketvmese kerkuecat tokv.

10 Hesaketrmese Eppucen oh vkvsamat eme ofvn ker- 
kueckv ocet os. Hesaketvmesen oh vkvsvmekat laksvn hayvtet os, Hesaketvmese mv Eppuce ohkerkuecan vkvsvmekot omekv.

11 Momen hesaketv meyuksv-sekon Hesaketvmeset pu'mvtet omen heyvt mv kerkueckv tes, momen heyv hesaketv Eppucen vpiket os.

12 Estit mv Eppucen ocat hesaketvn ocet os. Hesaketvmese Eppucen ocekat hesaketvn ocekot os.

13 Hesaketvmese Eppuce hocefkv oh vkvsamatskan heyv nanvke cen coyvkvyvtet os, hesaketr meyuksvsekon ocatskatet kerrepatskvren, momet Hesaketvmese Eppuce hocefkvn oh vkvsrmatskvren.

14 Momen eme komat vevkvyen nake vpohēn omat, pum apohicet os; momen heyvt emen vfekrakketv oceyat tes.

15 Momen pum apohicet on kerrēn omat, nake estomen vpoheyis, mv vpohkv em'poheyvtet oceyet omet kerrēs.

16 Estimvt etecakkvtet naorketv elkv orekon naorken hecen omat, vpohvres, momen mv elkv oreko naorkakan eme vpohan hesaketvn emvres. Elkv oret naorketv oces. IV svrahkvn mekusvprres makvkot os.

17 Fvecetv tokat vtekat naorketvts, momen elkv orekot naorketv ocet os.

18. Este Hesaketvmese es heckvte vtekat naorkekot omen kerrēs, momis mv este Hesaketvmese heckuecvte tat e vcayecet os; momen holwvyecatet celayekot os.

19 Momen Hesaketvmese vprkeyet omēt kerrēs, momen ekvnv omvlkvt holwryeckv ofvt os.

20 Momen Hesaketrmese Eppuce vlvket omen kerrēs, momet mv fvecan kerreyvre tayen vkerrickvn pu'mvtet os, momet fvccan mv Eppuce Cesvs Klist vtehkeyet os. Heyvt Hesaketvmese fvccat tes, momet hesaketv meyuksv-sekat tes.

21 Hopuetakuce, nake vhake em e vcayecrkes. Emen. 


\section{COKV SVHOKKOLAT}

\section{MEKUSAPVLKE VTEKAT}

CANE

\section{OHTOTVTE.}

\section{SETENTVCKE I.}

Veulat toyvyat cuko-pucase hokte enhopokvte toyetskat punvkv ec ohtotis, momen, en hopuetakuceu, mehenwr ofv vnokecakvyat, momen vn tvlkekot, momis mv mehenwv kerrvkvte vtekateu;

2 Mehenwv ep ofv fekhonnat momet estofis epu 'pakvranat svrahkvn.

3 Hesaketvmese Erke, momet Pucase Cesvs Klist Erke Eppuce a en kvpvken heromkv, merkv momet herkvu ece 'pakekvs, mehenwv momet vnokeckv ofvn.

4 Erke a en kvpvke vhakv pun heckvte vevkvyen cen hopuetake mehenwv ofvn yrkvpaken kerrvyat tayen vc afackvtet os.

5 Momen hiyomat cuko-pucase hokte toyetskat, vhakvmucrsen cen coyvyrte omecrkot, momis mv enhrteceskv vteke oceyvte vcvkvyen etenokeceyvren cem vpohvyet os.

6 Momen heyvt vnokeckv tes em vhakv vevkvyen yvkvprkeyvret. Momen mv enhvteceskv vteke ocvte pohetskvte ofvn yvkvpetskvret vhakv tes.

7 Mvkerrvlke sulket ekvnv sohceyvtet omekv Cesvs 
Klist vpeswv ofv vlakat kerkuecvkekat, heyvt mvkerrv tes momet Klist vnrapv tes.

$8 \mathrm{E}$ vketecrkes $\mathrm{mv}$ nanvke vtotkeyvte pun sumecekaren, momis feketv fvcken eseyvren.

9 Este naorkat vtekat, momet Klist em vhakv ofv vfekhonnekat Hesaketvmese ocekot os; Klist em vhakv ofvu vfekhonnat heyvt Erke Eppuce tepakvn ocet os.

10 Estimvt cem vlaket heyv vhakvn svlvkekon omat, cen cuko ofv evpahyetskvs, momet afveecice tayen opunahyetskvs.

11 Estimvt afveecice tayen opunayat en nak vfvstetv holwahokan ohvpvket omekv.

12 Nanvke sulken cen coyetv ocit cokvn lvste tepakat es cen coyetv komvkos; momis ec oh ahtin etehecen ce tempunvyaret $\mathrm{cv}$ 'nhonres, pum afvcketvt fvcke taren.

13 Etecakketskat hokte en hopokvte en hopnetakucet ece 'sekvket os. Emen. 


\section{COKV SVTUCENAT}

\section{MEKUSAPVLKEV'TEKAT'}

CANE

\section{OHTOTVTE.}

\section{SETENTVCKE I.}

KIvs vnokecketske, mehenwv ofv ece 'nokecvyat, vculat toyvyat opunvkv ec ohtotis.

2 Vnokecketskat, nake cem ohvtvlaken, momet ce cvfekne taren cem puyvfekcv em ohvtvlakat etvpomen nake omvlkv'sen hoyanen komvyet os.

3 Etecakkeyvtet yihcet mehenwv ece 'pikan kerkueeakof, tayen ve afackvtet omekv, mehenwv ofv yvkapetskat etvpomen.

$4 \mathrm{Vn}$ hopuetake mehenwv ofvn yvkvpakveuken pohvyat afveketr $\mathrm{mr}$ sen hoyanat ocrkot os.

5 Vnokecketskat, etecakkeyvte momet tvlepurvlke ohfvecv nake estomis em vtotketskat, vfekcakhet momecetsket os.

6 Emekusapvlke ehomv cem vnokeckv kerkuecakvte, Hesaketrmese em en heren erpayetskat heren momecet ometskvres.

7 Eme e hocef kv svrahkvn tvlepurvlke naken en cvwekot esossvkvtet omekv.

8 Monkv mv omakat crweyvre tetayet os, mehenwetv em vnicvlke toyeyvret. 
9 Emekusapvlke cokvn ohtotvyvnks, momis Tiotefes homv huerihocrre em vlostatet pu crwvkekot os.

10 Heyv omecicen, vlakin omat, em vtotketv momecat vkerricares, opuuvkv holwahoken 'sepu'nrapet, momet mvn 'semvhlvpvtkekot, momet eme tecakkeyvte crwekot, momet mv cvwetv komakat tis em vsehet, momet emekusvpkv-cuko sossicet os.

11 Vnokecketskat, holwakat vevkahyetskvs, momis heran vevkvyvs. Este here momecatet, Hesaketvmese vprket os, momis holwake momecatet Hesaketrmese hecvte sekos.

12 Este omvlkvt momet mehenwetwu. Temetevs heren ohkerkuecaket os, momen pumeu ohkerkueceyet os, momen pum ohkerkueckv fvecvt omen kerratsket os.

13 Nanvke sulken coyetv ocvyvnks, momis lvsten escoyetv tepakat es cen coyetv komvkos.

14 Momis hofunekon ce hecares komvyet os, momen etehecet etempunahoyeyvres komit cv'nhonres. Herkvt cem ocekvs. Pun hessvlket ece'seket os. Pun hessvlke hocefet vsekvkes. 


\section{COKV MEKUSAPVLKE VTEKAT}

\section{PETV OHTOTVTE ENHVTECESKV.}

\section{SETENTVCKE I.}

Pantvs, Kelesev, Kapvtosev, Esev, Pirenev esyoman tvleporvlke hvmecicakat,

2 Erke Hesaketrmese en homv-kerretv vcrkvye, Puyvfekcvt vcacrke hayat eteropotte, mapohickv momen Cesvs Klist e catv ohfesketvn enhopokvten Petv, Cesvs Klist em vpastel ohtotakes: Heromkvt cem ocen, momen herkvt cem ohvtvlakvkekvs.

3 Pucase Cesvs Klist E'rke, momet en Hesaketvmesen vrakkuchocekvs; mvt em merkv sulkat vcvkvye Cesvs Klist prsatkvte enkvpvke akvwapvte etehoyvnen, hvtvm enhonrkv hesakat oren pu heckuecrtet,

4 Nake hopvnkeko taye, svholwvkeko, momet sumkvraneko hvlwe-tvlofv ofv epucashoyvranat,

5 Centake vkrsvmkv eteropotte vhesaketv oren Hesaketvmese en yekcet ece 'cayecvken, oketv espoke omof heckuehocvranatet, cen hericvkhoyvkvtet os.

6 Mvn es ece 'fackusvket os, hiyomat vhofune mahekon, nake es ce kerhoyat sulke mahe eteropotten ce feknokhoket o estomis, mvt momvranat tvlket on omat;

7 Momet cem vkvsvmkv take eskerhoyat cvtokunaplane sumke taye eskerhoyat sem vcake estrmahet omet, totkvn eskerhoye estomis, Cesvs Klist e heckuecof, vkvsvmkv, vrakketv, rakketv esyomat oren eshechoyvren:

$8 \mathrm{Mv}$ hecatskvte seko estomis, vnokecet, hiyomat 
hecatskeko estomis, mv ofvn vkvsamet, afvcketv onayēsko tetaye, momet hvyayvke es fvcken es ece 'fackvket os :

9 Nake cem vkvsvmkv take vhecat, cem puyvfekcv take vhesaketvn cen heckvket.

10 Owalvlke heromkv ec oh vlvkvranat ohfvecr owalakvtet, Klist em Puyvfekev emetake ofv likat, Klist em estemerketv, momen vrakketv yupvn ocvranan ohkerkuecof, nake momet oketv estomen okvten hopoyet,

$11 \mathrm{Mv}$ vhesaketv ohfvecvn vpohet, hericet ohelentakvtet os.

12 Heyvn mvn en kerkuehocvtet os, mv nanvken hiyomat Puyvfekcv Vcakat hvlwen atothoyvte etehoyvnen opunvkv-herv es cem erkenvkakat cen kerkuecaken, eme vrahkv tokon, pume epu 'rahkvn vfvstakvtes; mv nanvke encelvlket. kerretvn em vlostaket os.

13 Monkv cem vkerrickv take em etetackvn wvnawicet, ce mahlvpvtket omet, heromkv Cesvs Klist e heckuecof es cem vlvkvianan en nene hecet, meyuksicvkvtcrs;

14 Hopuetake apohicvke omēt, nak kerratskekvnke omof eyackv ocatskvte vcrkvyen e hayekot;

15 Momis, mv ce'nhuehkvkvtet hrsvtkat etvpomen centaket fulletv estomis omvlkv ofvn hvsvthvket omvkes:

16 Vne $c v$ hrsvtket omekv, ce hvsvthvket omekvs, maket hoccet omekv.

17 Momen, Erke, mv este etemetuecekot, este em vtotketv vevkvye vlken ohfvececat oh huehkatsken omat, yomvn fekhonnatskat em oketvn penkvlkv ofvn yvmahkuecrkes :

18 Nake hopvnkake taye, crtokunaphvtke, monkat crtokunaplane omakat tokon,

19 Momis Klist, yvpefikuce nake holwvke, monkat svholwvke oceko, e catv vcakat eteropotten cem fulletv 
take ehvperke, ce'rkvlke tate cen heckvkvte aenkvpvken pvlken ce neshoyvkvtet omat kerratskekv;

20 Mehenwvn ckvnv vliceckv seko emunkof mvt hoinv melhoyvtet os ;

21 Momis heyv oketv espokakat ofvn ceme ece 'rahkv. taken, mv eme eteropotten Hesaketvmese prsatkvte enkvpvken akvwahpet, vrakketvn emvten oh vkvsamatskat; cem vkvsvmkv momet cem enhonrkv taket Hesaketvmese ofv ocvren e heckuecvtet os.

22 Vhericet efeke hvsvtkat aossen yekcen ete 'nokecvles, Puyvfekev eteropotten; mehenwetv em apohickv ofvn tecakkakvte mehenwusen vnokecatskat oren cem vkerrickv taken hvsvthicatskekv :

23 Nerkv hopvnke tayat tokon, nerkv hopvnkeko tayat etehoyvnen svnvcornv heckatsken, Hesakettvmese em opunvkv hesake estofis oce munkat etehoyvnen.

24 Enarpeswr vtekat pvhe omēn, momen este en rakketv omvlkv prhe em pvkpvke omēt omekv. Pvhet yuknen, em prkpvket lvtkepet os ;

25 Momis Hesaketvmese em opunvkvt estofis oce munkvt omes. Momen heyvt mv opunvkv tes, mv opunvkv-herv ofvn cem erkenvkhoyat.

\section{SETENTVCKE II.}

Monkv holwvyeckv omvlkv, mvkerretv omvlkv, here e omeckv, vleskvpkv momet est' opunvyeckv holwvhokat omvlkvn etvn ocet,

2 Hopuetakuce hvte heckakat omēt, opunvkv epese mahhen komvkes, mvn es ce mahvkvren;

3 Pucase heromet omat lasatskvten omat.

4 Emen, crto hesakat, estet mehenwrn vhepvkaken, momis Hesaketvmeset enhopoyvte vcakan, a oh awet, 5 Centakeu Hesaketvmese ehomv nanv̀ke ematskat puyv- 
- fekev enanvke, Cesvs Klist eteropotten Hesaketvmese afvecice taye ematskvren, crto hesahoke omēt, puyvfekev cuko hahoyat, plest em wiketv vcake toyatskes.

6 Hecrs, Sivn ofvn kvnowv crto em ekv enhopoke, veaken licis; momen estit mvn oh vkvsainat vlsehohcekos, maket Cokat ofvn hoccet omekv.

7 Monkv centake vkvsamatskat, cem vrakkvket os, momis apohicvkekat, $\mathrm{mv}$ cvto tohtarvlke wikakvte, matrwvt kvnowv em ekv hakes,

8 Momet crto elentappueckvn, momet cvto esemmvttetvn em ocvket os, $\mathrm{mv}$ apohicvkeko opunvkvn oh elentappakan okis; mrn ohmelhoyvtet omen.

9 Momis centaket este enhopoke, plest em wiketv mekkvlke omvkēt, etvlwv vcake, este Hesaketvmese eenakuecvte toyatskes, mv yomucke asosset en hvyayvke estomahan esceyet ce'nhuehkvte en heretv cahmelikvn kerkuecatskvren :

10 Oketv hoyanvte ofvn, etvlwv tokatet, momis hiyomat Hesıketvmese em etvlwvt; merkvt cen heckvkekatet, momis hiyomat merkvt cen heckvket os.

11 Vnokeckusatskat, cen kusapvkis, tvleporvlke momet cuneckvlke omatskat, enavpeswv em eyackv take puyvfeker vnrvpen tepoyakat enkvpvken e en hvlvtvkes;

12 Centilvlke em etenrvwrn cem fulletv take heret omen; momat holwvyecvlke $e c$ omecvket vnrvpen ec opunvyecaket omis, Hesaketvmese en cukoperickv nettv orof, cem vtotketv herakan hecakat omecicen Hesaketvmesen vrakkuecvkvren.

13 Pucase vrahkvn est' em wiketv vtekat elecvn svpvklvkes; mekko omvlkv en homahtvt ot omēt,

14 Monkat evpayvlke mekkot, holwvyecvlke estemerrvkuecvkvren, monkat heren momecakat vrakkuecvkvren, vtotat omaken omat, em apohicvkes; 
15 Heren momecatskat eteropotten este hoporrenekvlke nakkerrvkekan evyvyicatskvren Hesaketvmeset komat tok.

16 Ce pucassvkekot omis, pucasseko hueretv holwryeckvn esohrvnkvn hayekot, momis Hesaketrmese em vtotkvlke omēt svpvklvkes.

17 Este omvlkvn vrakkuecrkes. Etecakkakvten vnokecvkes. Hesaketvmesen em penkvlvkes. Mekkon vrakkuecrkes.

18 Vtotkvlkē, ce pucasvlke, hervke, yvmvskakat tvlkusekon, momis holwryecakat tis em apohicvkes, penkvlkv 'somvlkvn.

19 Eohvkerrickv Hesaketvmese ohfvecv vrahkvn estehunvnwvt, fveceko estemerrihocen, estemerkvn kvwapen omat, heyvt Hesaketvmesen afveecicet omekv.

20 Hiyomet omekv, vkvsvmkv estomet oce haks, cen naorketv take vrahkvn ce nvfhokvken, momis komet kvwvpatsken omat? momis heren momecatskis, mv vrahkvn ce 'stemerrihoevken, momis komet kvwvpatsken omat, heyvt Hesaketvmesen afveecicet os.

21 Heyvn ec ohhuehhokvtet omekv: Klist tis, es pum vhopakvn pum wiket, elehvpon oh yvkapet vevkfullatskvren, pum estemerkvtet ok:

22 Mvt naorketwn momece sekon, mvkerretv e cukwv ofvn eshechoyekates:

23 Mvt tvklehocof pvlecekates, estemerkof, Ce 'stemerricares, makekot, momis mv faccuse ohfvècecan em $e$ wikvtes ;

24 Eme mahusat eton oh vtarket e'na ofv pun naorketvn kvwapvtes, pumet naorketv ohfvecrn prsatket, fvecetv ohfvecrn hesahokeyvren; momen eme rukaf hoyvte eteropotten cem wicehocvtet os.

25 Hiyomet omekv, yvpefikv ctehoske fulle omatskvtes, 
momis hiyomat cem puyvfekcrtake Vheciev, momet Ohvfastvn ohfulecatskvtet os.

\section{SETENTVCKE III.}

Matvpomen, ehitake toyatskat, ce 'hetaken em apohicvkes, momat opunvkv em apohicvkeko svset on omat, opunvkvt omecicekon, ehitake em vretv eteropotten epohoyvren :

2 Cem vretv hvsatkuse penkvlkvt vpvken hecakof.

$3 \mathrm{Mv}_{\mathrm{v}}$ 'sem enehickv onvpv ocat, ekvesse hotvnkv, monkat crtokunaplane es fulletv, monkat vccvken vccakat ohmates ;

4 Momis efeke ofv este ehke, 'sem enehickv hopvnkeko taye, puyvfekev yvmvske, momet mahlvpatkuset, $\mathrm{mv}$ Hesaketvmese ehomv vcake mahat omekvs.

5 Hofonof hoktvke vcacvke, mv Hesaketvmese enhonrakat, e 'hetaken em apohicet, hiyomēn e nehicakvtet omekv.

6 Matvpomen Selet, Eplehamen, pucasen kicet, em apohicvtes; momen centaket e'chustake toyatskes, heren momecet, penkvlkv estomen es ce penkvlvkekon omat.

7 Matvpomen ehetake toyatskat, hokten, nake vcrnkv yekce mahekat omēn, momen etepvket hesaketv en heromkvn epucasvranatsket omēn vrakkuecet, kerretv vcvkvyen vpvkvkes; cem mekusvpkv taken mvnvttekaren.

8 Espokat, ete'm merret, vkerrickv livmkusen omvlkatskat ete'm ocvkes; est' em merruset, en yvmaskuset, etecakkake ete 'nokecvke omēn vnokecvkes :

9 Holwakan holwvken vyoposkekot, momet tvkleckvn pvlken tvklecekot; momis pvlhvmiken merkv opunvkv min punvyvkes, Hesaketvmeset heyvn ec ohhuehkvkvtet omekv, ohmerretv epucasatskvren.

10 Hiyomet omekv, estit hesaketv vnokecetv, momet 
nettv herakan hecetvn komen omat, holwakat enkvpvken e tulaswvn, momet mvkerretvn punayvkekaren e cukhvrpen en hvlvtvranet omekv :

11 Holwakan enkvpaket, heran momecekvs; herkvn hopoyet, revkvyekvs.

12 Hiyomet omekv, este fvccakan Pucase en renakvt oh ocen, e hvckot em mekusvpkv taken en haukaket os; momis holwvke momecakan Pucase e turofvt vnrvpet os.

13 Momen nak heran vcvkvpeyvlket omatsken omat, estit holwvken ce 'stomecrkvr haks?

14 Momis fvccetv vrahkvn ce 'stemerkvken omat, cen hervkes; momet em penkvlkvn em penkvlekot, momet fekhervkekot ohmatskvs

15 Momis Pucase Hesaketvmesen ce feke take ofvn vcryecet, estimvt o estomis, Naket omecicen mv enhonrkv ec ofv take oce haks? maket cem 'pohvken omat, yvmvsketv penkvlkv tepakat vpvken em vyoposketvn estofis ce metetakvket omekvs :

16 Eohvkerrickv heren ocet; momat holwvyecvlke omecet, holwvken ec opunvyecakis, cem fulletv take here Klist ofv ocan tvklecakat vlsvkvren.

17 Holwvke momeckv vrahkv tokon, here momeckv vrahkv ce 'stemerrvkuehocvre mit heret omekv, Hesaketvmeset momēn komen omat.

18 Klist tis, enavpeswv ofv elehocen, momis Puyvfekevt wenahuecen, fvccat fvccrkeko vyoposket, Hesaketvmesen a 'sep oh vtvret, vhvmkvn naorketv vrahkvn estemerkvtet omekv :

19 Momet mata Puyvfelecv eteropotten puyvfekcvlke cuko-yekcv vtehkan em erkenakvtes;

20 Mvt hofonof apohicvkekot omvtes, vhvmkv Hesaketvmese em momis kometv hofunat mehake Nov en nettv take ofvn perro-cuko-rakkon hahoyof, mvn vtehket 
este nvcomuset, cenvpaken okis, uewv etehoyvnen hesahoket omof.

$21 \mathrm{Mv}$ vhaket, (enavpeswv svholwakat vkueyickv tokon, momis eohvkerrickv here Hesaketvmese ohfvecv em vyoposketvt,) paptiskvn okis, hiyomat pu hesahuecet os, Cesvs Klist aenkvwrpketv eteropotten;

22 Mvt hvlwe tvlofvn ecehyet, encelvlke momet ohfvnketv ocakat, momet yekcetv ocakat eme elecvn svpvklehocen, Hesaketvmese em vkvpervn liket os.

\section{SETENTVCKE IV.}

Monkv Klist epu 'rahkvn enavpeswv ofvn pum estemerkvtet omekv, centakeu mata vkerrickvn setepokvn cvwvkes; estit enavpeswv ofv estemerkvten omat naorketvn wikvtet omekv;

2 Momat oketv enavpeswv ofy em vhoskan este em eyackr take vcrkvye ohvtvlakat vrekot, Hesaketvmese nake komat vevkvyen vrvren.

3 Hiyomet omekv, pun hesaketv yrmahkueceyvte, mv omof, hoktarkv, enavpeswv em eyackv take, hackv, eshvyvtketv, tohkvlke pvrko-opuswv esketv, momet nakvhake emekusvpkv holwvhokat ofvn fullēt, Centilvlke nak komakan vtotkeyvtet pum etetayes.

$4 \mathrm{Mv}$ nak holwvhokat ohfvecvn, vpvkaket mata yvmahkueckv rakkan oh vpeyatskekat estomahes komaket os, holwvken $e c$ opunvyecet:

5 Mvt wenahokat momet. prsatkvten ohfvececetv metetake huerat ehomvn e ohkerkuecvkvres.

6 Hiyomet omekv, heyv vrahkvn opunvkv-hervn pvsatkvten em erkenvkhoyvtes, este vevkvye enavpeswv ofvn ohfvccehocen, momis puyvfekcv ofv Hesaketvmese vevkvye hesahokvren. 
7 Momis nanvke omvlkv em eyuksvt vwolices; monkv emahlvpatket omet, mekusvpkv oren vketecrkes.

8 Momen nanvke omvlkv esemuntalat vnokeckv yekcen ete'm ocvkes; vnokeckvt naorketv sulken ohranet omekv.

9 Etencukoperickvt ecakv sekon cem etenrvwv taken ocekvs.

10 Este vtekat nak emkv en heçkvte vevkvyen matvpomen matan ete'mvkes, Hesaketvmese en heromkv cahmelikv vfastvlke herakat omēn.

11 Estimvt o estomet punayen omat, Hesaketrmese em opunvkvn 'sest' em punaye omēt punayekvs; estimvt nake vfastet on omat, yekcetv Hesaketvmese emvte aossat vcrkvyen momecekvs; momat Hesaketvmesen Cesvs Klist eteropotten nanvke omvlkv ofvn vrakkuehocvren; mvn vrakketv yekcetv tepakat estofis meyuksv-sekon em ocekvs. Emén.

$12 V m$ vnokeckatskat, mv totkv omēt es ce kerrvkat ohfrecr nak estomahet ep oh latket os, komet, estomahet os, kohmatskvs ;

13 Momis afvckvkes, Klist em estemerketv take etenkvwapvlke toyatskekv; momat en hryayvke heckof, centakeu afvcketv rakken es ece 'fvckvkvren.

14 Cesvs Klist e hocefkv vrahkvn holwvken ec opunvyehocrken omat, cen hervkes; Hesaketvmese momet rakketv em Puyvfekcvt ec oh fekhonnvket ornekv; emetaket on omat, Hesaketvmesen holwvken opunvyecakes, momis centaket vrakkuecatsket omes.

15 Momis hvmkusatskis, estelecvt, monkat horkopvt, monkat holwvke momecvt, monkat este eti enanvke 'sem e naorickv omēn es ce,'stemerikats.

16 Momis estimvt emekusapvt omat omecicen estemerrihocen omat, vlisats; momis heyv vrahkvn Hesaketvmesen vrakkuecekvs. 
17 Ohfvececkvt Hesaketvmese en cukon es vlicecvranat oketv vlvket omekv; momen pumen es epu 'licecen omat, mv Hesaketvmese em opunvkv-herv apohicvkekat em eyuksv nake tar haks?

18 Momen en yekcusen este faccrt hesaken omat, este naorkv momet mekusvpekat estvmvn huervr haks?

19 Monkv mv Hesaketvmese komat vcrkvyen estemerkakat here momeckv ofvn em puyvfekcr taken Hesaketvmese, Hayvte heroman em wikvkekvs.

\section{SETENTVCKE V.}

Mekusvprv cuko em fvyatvlke ece'pakvkan, vneu mv toyvyat, momet Klist em estemerketvn a en kerrvt, momet hvyayvke heckvranan etemesv toyvyat, emvcahnvkvyet os;

2 Hesaketvmese en yvpefikv ece 'pake fullan hompvkuecvkes, ec ohyekcihocekon, momis vkvsamuset; vlesketv ofv nake seohvtvlvyetv vrahkv tokon, momis vkerrickv metetakuse aossen ohvfastet;

3 Momet vhecickv Hesaketvmese cem wikvkvte e pucasvlke omēt omekot, momis mv yvpefikv fullat 'sem vhopakvt omvkes.

4 Momen yvpefikv Vhecicv homahtvt e heckuecof, kvpotokv-vcake hvyayvke, sumkeko tayat cen heckvkvres.

5 Matvpomen esmvnettakatskat, es cem vculakat elecvn svpvklvkes. Momes, omvlkatskat ete'm apohicet, efeke kvncvpkvn accrkes; Hesaketvmeset ekvsvmakat vhepaket, efeke ofv kvncvpakusan heromkvn emaket omekv.

6 Monkv Hesaketrmese e'nke yekcat elecvn e kvncvpuecvkes, oketv orof ce hvilwecvkvren.

7 Es cem enaorickv omvlkvn einen oh wvkecet, emet ce moskomet omekv.

8 Emahlvpvtket, vketecet omvkes; ce'nrapv puyvfekcv 


\section{PETV I, 5.}

holwakat, este-papv hehke omēt, estimv loke tayat hopoyet aret omekv:

9 Mvn vnrvpvkes, vkvsvmkv ofv yekcen svpaklet, ce tecakkake ekvnv fullat ofv mata estemerketv esfvcken ocvket omat kerret.

10 Momen heromkv omvlkv en Hesaketvmeset, mv Cesvs Klist etehoyvnen en hryayvke meyuksv-sekan ep oh huehkvtet, vkocoknusen ce'stemerikvkof, yekcen ce svpvklecet, ce yekcvkuecet; vkueyihoceko tayen, momet temvpoken ce hayvkate.

11 Emen vrakketv momet yekcetv estofis meyuksvsekon em ocekvs. Emén.

12 Selfenvs, ce tecakkvte heromat tis omes komvyat, es cem ayvranen, cem vcahnet, momet heyv heromkv, mv ofv svpaklatskat, Hesaketvmese en heromkv mehenwat tes, maket akerrit kocoknusen hoccihcis.

13 Mekusvpkv-cuko Papelvn likat, ece 'pvkvke enhopokvtet ece 'sekaket os, momen Makvs cv'ppuceu ece 'seket os.

14 Vnokeckv afvnketvn 'sete 'sekvkes. Klist Cesvs ofv svpaklatskat omvlkv herkvt cem ocvkekvs. Emén. 


\section{PETVT OHTOTVTE.}

\section{SETENTVCKE I.}

Simvn Petv, Cesvs Klist em vtotkv, momet em vpastel toyvyat, mv Hesaketvmese momet pu Hesayecv Cesvs Klist em frcetv eteropotten vkvsvmkr vcake oceyat epu 'pvkake matan eenakuecakvten ohtotakis :

2 ITeromkvt cem ocvkekvs, momen herkv Hesaketvmese momet pu Pucase Cesvs kerretv ofvn cem ohvtvlakvkekvs, 3 Hvyayvke momet vretv heran mv pu'nhuehkvte kerretv eteropotten nanvke hesaketr momet Hesaketvmese em apohickv ohfvecv ocakat omvlkvn Hesaketvmese en yekcetvt pu'mvte etvpoinen:

$4 \mathrm{Mv}$ nanvke eteropotten setemfvccetv rvkrvke momet vcacrke mahen pu'mhoyet omes; momat enavpeswv eyackv etehoyvne hopvnketv ekvnv ocan minvttehpet, Hesaketvmese em munkv etemesvlke toyatskvren.

5 Momen heyv ohvpakat, vketeckv omvlkvn ocet, cem vkvsvmkv taken heretvn ohvpayet, cen heretv taken nakkerretvn;

6 Nakkerretvn eemfvyatkvn; eemfvyatkvn momis kometvn; momis kometvn Hesaketvmese 'mapohickvn;

7 Momen Hesaketvmese 'mapohickvn etecakkvte vnokeckvn; etecakkvte vnokeckvn vnokeckvn ohvpryvkes.

8 Heyv nanvke ece 'tehket, esfvcken ece 'tehkvken omat, pu Pucase Cesvs Klist kerratskat ofv enhorrvke momet ettvkekot omatskekaren ce hayvket omekv. 
9 Momis estimvt, heyv nanvken ocekot huerat, turheceko, hopvye heceko tayẹt, en naorketv vculvke en hvsvthihocvten ehoset omekv.

10 Monkv, tecakkvte toyatskat, ohvtvlaken vketecvkes, ce'nhuehhokat momet $c e$ 'nhopohoyvte yekcen hayatskvret; heyv nanvken momecatsken omat, estofvn pvlatkatskekaret ok :

11 Momat pu Pucase momet $p u$ Hesayecv Cesvs Klist em etvlwv meyuksv-seko ocan esceyatskvre esfvcken ce'mhoyvret ok.

12 Monkv heyv nanvke ece 'kerricepuecetv vm etetake emunkv tares, mvn kerret, momet mehenwetv hiyomē ocat ofv yekcen svpaklatske estomis.

13 Momen heyv escukohakvn vpikvye vtekait ece 'kerricepuecrkit, ece 'honechuecrkvyat fvccrts, komvyet os ;

14 Hofunekon vm escukohakvn kayvranvyat kerrit, pu Pucase Cesvs Klist vn kerkuecrte etvpomen.

15 Momen vm elkv hoyanof, heyv nanvken estofis vkerricatskvren heren vtotkares.

16 Hiyomet omekv, pu Pucase Cesvs Klist en yekcetv rakketv tepakan cen kerkuecvkeyof, nakonvkuce mvkerretvn es hahoyvten es vcrkvpeyet omēkates, momis en rakketv turwv es hece akerrvlket omeyvtes.

17 Hryayvke here mahat avtet opunvkv en hake hiyomēn em punaye, Heyvt Cv'ppuce vm vnokecke mv ofv vfackusvyat tes, makat vlakof, Erkc Hesaketvmeset vrakketv hvyayvke tepakan emvtet ok.

18 Momen heyv opunvkv en hake hvlwan ahvtapken poheyvtes, emen vpaket ekvnhvlwe vcakan oh vpokeyof.

19 Momen hvtvm mv senyckcen owaletv opunvkvn oceyet os; hvyatken, hvyvtecv ce feke take ofv kvwapkeko emunken hryayvke yomuckofv hvyvyicat omēn mvn vketecatskvre heret omes. 
20 Enhvteceskvn heyvn kerret, owaletv cohoyvte hvmkusis enhvmkusen enrvmkeko tayes.

21 Hiyomet omekv, este komat etehoyvnen owaletv opunvkv estofvn vlvkekates, momis este vcacvke Hesaketvmese enaket Puyvfekcv Vcakat nekehuecat eteropotten opunayakvtes.

\section{SETENTVCKE II.}

Momis owalv laksvlket fullvtes, este em etenrvwvn, mvhayv laksvlke cem etenrvwv taken fullvranat etvpomen; mv mvhayvlket Pucase emetake nesvten helaksecet, sumketv pvfnen e oh vlvkuecet, etekvpvketv esten sumecicvke tayan ehkusen asceyvkvres.

2 Momen em fulletv take holwvhokan sulket vcrkvpeyvkvres; momen mvt omecicen mehenwetv vyetvn tvklehocvres.

3 Momen nak est' em vleskvpkv ofv opunvkv mvkerretv eteropotten nak neskvn es ce hayvkvres; mv em ohfvcceckv hofonvte vteke atvtet fekhonnekon, en sumketvt nocekot os.

4 Hiyomet omekv, Hesaketvmeset encelvlke naorkakan hesahuecekot, momis totkv rakkon tvkpvlatet, yomucke en svlvfke ofvn ohfvececkv oren vpohoyvren wikvten omat;

5 Momet ekvnv vculan vcayecekot, momis Nov este escenvpakat, fvccetv em erkenvkvn hesayecet, fvccvkeko ekvnv oh fullan uelaukon a oh hvtvpecicet;

6 Momet tvlofv Satvme Komalv tepakan essun hahicet, sumecickvn es ohfvececet, mv en yupvn fvccvkeko fullvranat 'sem evhopakvn hayet;

7 Momet Latkv fvece, este holwvyecvlke em fulletv hvsvtkeko es hotosan hesayecrten omat;

$8(\mathrm{Mv}$ este-hunvnwv fvecat, $\mathrm{mv}$ vpvken liket, hece 
momet pohat, nettv omvlkvn em puyvfekcv fvecan em vtotketv holwvhokan es estemerricvtet omekv ;)

9 Este fvccakat nake eskerhoyan estomēn asossicetvn, momet fvccvkekat ohfvcceckv nettvn estemerrvkuehocvren $m v$ oren vcvyecetvn Pucaset kerret omes;

10 Momis hvsvtketv tokat eyackv ofv enavpeswv vevkvye fullat mahvkvts; $\mathrm{mv}$ evpayvlke en yekcetvn enhomecet, tepokvn metetakuset, eme koman ryekcrket, emwiketv hvlhawat tvklecetv penkvlvkekot os.

11 Momis encelvlket, mv yekcetv ohfvnketv tepakat ofv senyekcrket ot omis, Pucase ehomvn tvkleckv ohfvcceckvn vnrvpen esyicvkekot os.

12 Momis heyv estet, nak wenahoke nakkerrvkeko, cawet, sumecihocvranan hahoyvte omaket, nanvke kerrvkekan tvklecaket os; momet eme mahusat em vhopvnketv take ofvn sumecvres:

13 Momet, Afvcketv holwvhokan nettvn 'se afvcecicetv pun heres, komakat etvpomen fvccetv tokat em feketvt en heckvkvres. Nake vholwvhokat momet svholwvhokat omakes, e emvkerretv take ofvn e afvcecicet ece 'pakvket hompetv-rakkon hompakof :

14 Turwv hoktarkv es fvcfake, momet naorketvn wikvkeko tayan ocakes; est' em puyvfekev yekce svpaklekan ehosvkuecet, efeke vleskvpkv fulletv es nekeyihocan ocvket, ohtvhiketv en hopuetaket omakes;

15 Vyetv fvccan enkvpahket, ehosakvtes, Pelvme Posv e'ppuce, mv fvecetv tokat es em feketvn vnokecvte, em vretvn vevkvpeyet;

16 Momis Pelvme eme mahusat vhakv kacat omecicen em vsehohvtes; corakko-wakv opunayekat este em opunvkv haken es opunayet, mv owalv en hacohaketvn em vsehvtes.

17 Heyvt newv en korkv uewv sekat, aholoce hotvle 
yékce svtohkat omakes; mvn yomucke en Ivsten em vcryehocet os, meyuksv-sekon.

18 Nake rakrvke sekvsvmkv ehvperkakan opunayakof, vpeswv em eyackv take, momet seafvcecickv holwakat eteropotten, mv etehoskv ofv vpokan mvnvttepuset fullan a e oh hvlvtaket omekv.

19 Pucassekon svpaklatskvres, kicet etemfvecakis, eme mahusat hopvnketv en svlvfket omakes; estimvt estehunvnwvn ohfvnken omat, mv hunvnwvt mata en svlvfke hakepet omekv.

20 Cesvs Klist Pucase momet Hesayecv kerrakat eteropotten yvmv ekvnv en nak holwvhokan mvnvttvkehpe renyupvn hvtvm mvn seteyokcen eswrnayet, ohfvnhoken omat, mv en svpvkletv espokat hvteceskvn senholwvket omekv.

21 Fvecetv vyetv kerrahke renyupvn mv vhakv vcake emhoyvten em e fulecaken omat, kerrvkekate mit en herakvnt omekv.

22 Momis heyv maketv mehenwat vevkvyen em momaket os, Efvt nake eme mahusat vwotvten ra oh fulken, sukhv en hokte vklopehocvtet vklewvhen vkpvlpakvten oh fulkes, makat.

\section{SETENTVCKE III.}

VNokeckatskat, heyv cokv svhokkolan hiyomat cen hoccicvkvyet os, mv hokkolv ofvn vkerricatskvren cem vkerrickv take mehenwusan nekehuecis.

2 Opunvk owalvlke vcacakat homv etvwrn opunayakvten, momet pume, Pucase momet Hesayecv em vpastelvlke toyeyat nanvke makeyvten vkerricatskvren;

3 Enhvteceskvn heyvn kerret, nettr espokakat ofvn nakhayvkekvlke em ena take mahusat em eyackv vevkvyen fullet, 
4 Momet, Em vlvketv etemfvecetv estvn oce haks? nanvke omvlkvt nak omvl hocackvte enhvteceskv vteken, erkvlke nocicakvte vtekat nekehoye atvte emmunkuset omekv, maket yicvkvres.

5 Hiyomet omekv, hofunof ekvnvt uewv vkfvnvnaken, momet uewv vk ocaten, momen sutvn Hesaketrmese em opunvkvt hayvtes, Kerreyates, komet, heyvn kerrvkekot os :

$6 \mathrm{Mv}$ eteropotten mv ekvnv momof ocvten, uewvt ohbvmecicen, sumkvites:

7 Momis sutv ekvnv tepakat hiyome ocakan mata opunvkv eteropotten totkvn es estemerkvren ohfvececkv nettv, momen este fvccukeko estemerrickv nettv oren vevyehocvket os ;

8 Momis vnokeckatskat, heyv nake hvmkan kerrekot ohmatskvs, Pucase ehomvn nettr hvmkat ohrolope cukpe-rakko hvinke etvpomen, ohrolope cukpe-rakko hvmkat nettr hvmke etvpomet os.

9 Estomēn vcewickv ohvkerrice sushoyat omēn Pucase nake etemfaccute ohfvecv veewice tokot omes; momis hvmkusis sumkvre eyacekot, momis omvlkvt vkerrickv eticvkvren komet, ep ohfvecv taken hofunen momis komet hueret omes.

10 Momis Pucase en nettvt horkopv nere vlakat omēt vlvkvres; mv omof sutvt estoke mahet sumkvres, momen mv nanvke nak omvlkv es hahoyvte hiyye heran es kafkvres; ekvnvu, momet vtotketv ekvnv oh ocakat nekrihocvres.

11 Monkv heyv nanvke omvlkvt sumecvranekv, vretv hvsvtke, fvecetv tepakat ofv este estomvket omvranatske haks,

12 Hesaketvmese en nettvn en nene hecet, momet lvpecicet oh vpeyet, mv omof sulvt vhetket, nekret sumkv- 
res, momen nanvke nak omvl es hahoyvte hiyye heran es kafkvres?

13 Momis puntat em etemfvecetv verkvyen sutv mucvse momet ekvnv mucvse, mv ofv fvecetv liken en nene heceyet os.

14 Monkv, vnokeckatskat, nanvke mv omakan en nene hecatskekv, herkv ofvn vpoket, cem mvttvkeko momen svholwvke hvmkusis ce 'likekon, emet es ce hecvkvren vketecvkes.

15 Momet, pu Pucase em momis kometv hofunat vhesaketv tes, komet vkerricvkes, Pal tecakkeyvte pum vnokeckat hoporrenkv e'mhoyvte vevkvyen cen coyvkvte etvpomen ;

16 Cokv hoccicvte omvlkvu etvpomen, $\mathrm{mv}$ ofv heyv nanvken opunvyecet; momen mv ofv nanvke kerretv yekcakat oces, momen nakkerrvkeko momen yekcen svpaklekat, cokv vpvltakeu opvyet mvrahkuecakat etvpomēn, en sumketv mahusat oren, mvn matvpomecaket os.

17 Monkv vnokeckatskat, heyv nanvken homvn kerratskekv, vketecvkes, holwvyecakat em etehoskvn es ce hvlvthohyen, yekcen svpaklatskat pvlatkatskekaren.

18 Momis heromkv pu Pucase Hesayecv Cesvs Klist kerretv tepakat ofvn mahvkes. Emen vrakketvt $\mathrm{em}$ ocekvs, hiyomat, momet meyuksv-sekon. Emén. 


\section{COKV MEKUSAPVLKE OMVLKVN \\ CUTVSET OHTOTVTE.}

Curvse, Cesvs Klist em vtotkv, momet Cems etecakkvte, mv Erke Hesaketvmeset vcacvke hayvte, momen Cesvs Klist ofv vcryehocvte, momet enhuehhokvten ohtotakvyat;

2 Merkvt cem ocvken, herkv vnokeckv tepakat cem ohvtvlakvkekvs.

3 Vnokeckatskat, vhesaketv tvleme ohfvecv cen hoccicetv fekcakhetv omvlkvn es ohvkerricvyof, Vkvsvmkv vhvmkvn mekusapvlke em wihokvte vrahkvn yekcen $e s t$ ' vnrvpvkes, kicit, cem vcahnit hoccicvranvyat tvlkuset omvnks.

4 Este-hunvntake, homv etvwv hofunof heyv ohfvececkvn em melhoyvtet, este fvccrkeko, pun Hesaketvmese en heromkvn afvcketv holwvken hayet, momet Pucase Hesaketvmese hvmkusat, momet Cesvs Klist pu Pucasen helaksecet, ehkusen asceyakat svset omekv.

5 Monkv vhvmkvn kerratskvtet o estomis, heyvn ece 'kerricepuecetvn komis, momat Pucaset Ecepse ekvnv asossen esten hesahuehce en topvrvn, mv vkvsvmvkekan sumecicvtes.

6 Momet mv encelvlke, en hueretv hvlwan vcryecekot, momis e hute take mahusan wikakat, yomucke elecvn, eswrnakv meyuksv sekon es hvlvthoyen, ohfvececkv nettv rakko omof ocvranat vevyehocvtet os.

7 Satvme Komalv tepakat, momen mv tvlofv em vna- 
kuecakat matvpomen hoktarkvn em e wikat, momet vpeswv etvn hopoyaket, totkv meyuksv-sekon es estemerkaken, esvhopakvn este ehomv svpvklehocvte etvpomen.

8 Matvpomen heyv vpuecvlket enavpeswvn vholwvyecet, ohmekketv en yekcetvn enhomecet, este-emwiketvn tvklecaket os.

9 Momis encelv enhomahtvt, Mikevl hocefket, Moses e'na ohfvccrn puyvfekcv holwakat vnrvpen etempunayet vnra pof, trkleckv ohfvcceckvn es vnrvpe taye oren fekhvmkekates, momis, Pucaset cem vsehekvs, makvtes.

10 Momis heyv este-hunvntaket nanvke kerrvkekan tvklecaket os, momis mv nanvke nak wenahoke hoporrenkv ocvkekat omet enavpeswv vcvkvyen kerraken, inv ofvn e vhopvnaket os.

11 En holwvkakes, Kene em vyetvn vpeyet, foketv vrahkvn afvcketv holwvhokan 'se afvckvkuecet, Pelvme ein etèhoskvten verkpefatket, Kole vnrvpe opunvyetv ofvn estemerkakvtet ok.

12 Vnokeckv cen hompetv-rakko taken ece 'pakvket hompakof, mv ofvn nak vholwvhoket ot, penkvlekot e hompicakat omakes; vholoce newv oceko hotvle vtohkat; eto em ette yuknvke hakat, em ette seko, vhokkolr talvtet, yvlonkv svpakvn cupenhoyvtet os.

13 Uehvtkv em melohlv svheremahecet, em vlesketv take mahusan pvkpvkuecet, kococumpv ehosake, mv yomucke en lvstet meyuksv-sekon em vevyehocat omakes.

14 Momen Envkeu, Atvme vteke eskolvpakat, heyvn opunvyecet owalvtes, hvmmaket,

15 Hecrs, em este vcacakat cukpe rakko palaken sevpayet, omvlkv vnrvpen ohfvececkvn sohfvececvranet, momet mv vpvkakat nak celayvkvte fvccrkeko fvccekon momecakvten, momen opunvkv yekcvke cme vnrvpen holwv- 
yecvlke fvccrkekat fvccekon 'sopunvyecakvte omvlkvn ohfvececvranet, Pucaset vlaket os.

16 Heyvt ecayvlket, estemerkake omēn e opunvyecakat, ein eyackv take vevkvyen fullet os: momet eenlopickv vrahkrn este etemetuecaken, e cukwv taket nake rakrvke sekvsvmkvn opunayaket os.

17 Momis centake, vnokeckatskat; opunvkv pu Pucase Cesvs Klist em vpastelvlke homv opunahoye;

$18 \mathrm{Mv}$, Oketv espokat omof, fvccrkekat, nakhayvkekvlke em eyackv take vcrkvyen yvkvpakat fullvres, makakvten vkerricukes.

19 Heyvt e emrrahkuecet, enavpeswv vcrkvyen fullet, Puyvfekcrn ocvkekat omakes.

20 Momis centake, vnokeckatskat, cem vkvsvmkv take vcake mahat en likvn oh hayet e etohtvrvkes, Puyvfekev Veakat ofvn emekusapet,

21. $\mathrm{Pu}$ Pucase Cesvs Klist em merkv hesaketv meyuksvseko oren en nene hecet, Hesaketvmese em vnokeckv ofvn e revyecvkes.

22 Momet eteinvrahken ohfvececet, vpvlwvn em merret, 23 Momet vprlwrn penkvlkv ofvn hesayecvkes, hulatet, totkvn asossicet, enavpeswr accuke svholwvyecat tis enhomecet.

24 Momen mv ce'lentappvkekaren ece 'cryecet, afvcketv rakken es ece 'fvcken, naorketv cen svkekon en hryayvke ehomvn ce svprklecvke tayan,

$25 \mathrm{Mr}$ pu Hesayeer Hesaketvmese hoporrene hvmkusat rakket, vrakketv tepakat, momen yekcetv obfunketv tepakat em ocvkekvs, hiyomat momet meyuksv-sekon. Emén. 


\section{LEFELES VN,}

(NAKE ENRVMHOYVTE OKETV TES,) MV CANE MEKUSAPV, HESAKETVMESE OPUNVYECV'T HOCCICVTE.

Cesvrs Kuist en Lefelesvn, mv nanvke vhofuneko momvranat, em vtotkvlke hecicvre Hesaketvmese e'mvten; momen vtohten, encelvt em vtotkv Canen hecicvtes:

2 Mvt Hesaketvmese em opunvkv, momet Cesvs Klist em ohkerkueckv, momet nanvke omvlkv hecvten ohkerkuecrtes.

3 Heyv owaletv em opunvkv ohonayat, momet 'mapohicake, $\mathrm{mv}$ ofv nanvke escoke ocan vcayecakat en heraket os ; oketvt awolicekv.

4 CANE toyvyat mekusvpkv-cuko kolvpake. Esev ofv ocakan ohtotakis : Heromkv cem ocvkekvs, momet herkv, mv like, likvte, momet likvranate aenkvpvket; momet Puyvfekev kolvpake em ohliketr vcakat ehomvn svpaklat aenkvpvket ;

5 Momet Cesvs Klist, akerrv mehenwe, pvsatkvte aenkvpvken hvteceskv heckvte, momet ekvnv em, mekkvlke en homahtvn aenkvpvket cem ocvkekvs. $\mathrm{Mr}$ epu 'nokecet, e catv mahusan pun holwvyeckv enkvpvken es pu hrsvtecvte,

6 Momet mekkvlke momet plestvlke E'rke momet Hesaketvmese enaken pu hayvtet, vrakketv olifvnketv tepakat meyuksv-sekon em ocekvs. Emén.

7 Hecrs, vholocen vpaket vlakes; momen eturwv vtekat, momet sckeyakatet hecvkvres; momen mv 
ohfvecvn ekvnv em vliketv omvlkvt wahokvres. Momes, Emén.

8 Vnet Alfv momet Omēkv, momet vliceckv momet meyuksv toyis, Pucase, mv like, likvte momet likvrane, Yekcetv Omvlecat maket os.

9 Vne Cane, ce tecakkat, momet estemerketv ofv, momet Cesvs Klist em ohmekketv, momet em momis kometv ofv cem etohkvlketv take toyvyat, Hesaketvmese em opunvkv, momet Cesvs Klist em ohkerkueckv vrahkvn ote Patmvs hocefkan oh likvyvnks.

10 Pucase en nettv omof, Puyvfekcv ofvn likit, opunvkv en hake rakke, pofketv en hake omēt, cv 'ra topvrvn hakvcuken pohvyvnks,

11 Vnet Alfv momet Omēkv, enhvteceskv momet espokat toyis, maket, momet, Nake hecetskat nakcokv ofvn coyet, mekusvpkv-cuko kolvpake Esev ofv ocakan, Efesvn, Smvnvn, Pvkemvn, Rivtilvn, Satesen, Feletelfevn momet Leotesevn ohtotvkvtcvs :

12 Momen en hake vm punayan hecvranet afulotkvyvnks. Momet fulotikvyof, tokunaplane kulke sohhuerickv kolvpaken hecvyvnks;

13 Momet mv kulke sohhuerickv kolvpakat em ohnvrkvpvn este este E'chushunvnwv omēt, accvke ele oren hvtapken accet, morret ehokpen cvtokunaplane sewvnaketvn e trkkvyen hecryvnks.

14 E'kv momet e'kvesse yvpefikesse hvtkat omvket, hetute en hvtke orvket omvnks; momen e turwv totkv em fenke omvket omvnks.

15 Momen e'le cvtolane hololoccuse omēt, nak rekkickv hute tvk rekkihoce omvket omvnks, momen em opunvkv en hake uewv sulke en hake omēt omvnks.

16 Momen e'nkvperv ofvn kococumpv kolvpaken ocvnks; momen eslafkv-crpko em fvske hokkolen ocet ecukwrn 
afvnket ocen; e turofheckvt hrset en yekce ofvn hvyvyicat omēt omvnks.

17 Momen hehcryof, este ele omit e'le em vnakvn $t v k$ latkvyvnks. Momen e'nkvpervn ve oh wvkècet, hvmmakvnks; Ce penkahlats : vnet enhvteceskv momet espokat toyis :

$18 \mathrm{Mv}$ elvtet hesakat toyis, momen hecvs, meyuksvsekon cv. hesakes, Emén; momet elkv elkv tvlofv tepakat es en hvweckvn ocis.

$19 \mathrm{Mv}$ nanvke hecetskise, momen mv nanvke hiyome ocakat, momen nanvke heyv eryupv ocvkvranat;

$20 \mathrm{Mv}$ kococumpv kolvpake cv'nkvperv vtehken hecetskise momet kulke sohhuerickv kolvpakat enake ehkan hoccicrs. Kococumpv kolvpakat mekusvpkv-cuko em encelvlke take kolvpakat omen; momen kulke sohhuerickv kolvpake hecetskise mekusvpkv-cuko kolvpakat omes.

\section{SETENTVCKE II.}

EFESVLKE em mekusvpkv-cuko em encelvn hvmmaket hoccicvs : Mv kococumpv kolỵpakat e'nkvperv es hvlatat, cvtokunaplane kulke sohhuerickv kolvpakat em etenrvwvn yvkapat heyv nanvken maket os:

2 Cem vtotketv taken kerris, momet cen hotoskvn, momet cem momis kometrn, momet este holwvyecvlke en cem ehereko mahvket omat, momet mv, Vpastelvlke toyēs, makakis mv tokvkekon eskerret, laksvlket omen eshecetskvten kerris;

3 Momet vtotketvn kvwapet, momis kometvn ocet, cv hocefkv vrahkvn vtotketskat, es ce hotosekaten kerris;

4 Momis ece'nrapat nake ocis, cem vnokeckv enhvteceskvn en yupvklatketskvtet omekv.

5 Monkv estvmv a ce $t v k$ latkvten vkerricet, cem vkerrickvn eticet, enhvteceskv cem vtotketrn momecrs; 
monkon omat, lvpken a ec oh vtares, momet cem vkerrickvn eticetskekon omat, cen kulke esohhuerickvn etrn vkueyares.

6 Momis heyvn ocetskes; Nekolitvlke em vtotketv taken enhomecetskat, mvn vneu en $\mathrm{cv}$ homeces.

7 Estit ehvcko acat nake Puyvfekev Veakat mekusvpkv-cuko kolvpakan em punayan pohekvs; $\mathrm{M}_{\mathrm{r}}$ ohfvnkat hesaketv em eto, Hesaketvmese em palvtis ofv huerat em ette hompvren emares.

8 Momen mekusvpkv cuko Smvnv likat em encelvn hvmmaket en hoccicvs; Enhrteceskvt momet espokat, elvtet momet hesakat heyv nanvken maket os;

9 Cem vtotketv take, cem estemerketr cen kusvpketv esyoman kerris, (momis nake ocet ometskes;) momet Cusvlke toyēs makakis, mv tokot, momis Setvne en senekaket onat, em vtvkleckv taken kerris.

10 Nanvke es ce 'stemerkvranat hvmkusis em penkahlatskvs; hecvs, es ce kerhoyvre vrahkvn Setvnet cuko-yekcrn ce 'tehe susecures; momen nettr palen estemerkvn ocatskvres; elkv oren heromet omvtcrs, mo-men hesaketv kvpotokv vcakan ce'mares.

11 Estimvt ehvcko ocat nake Puyvfekevt mekusvpkvcukon em punayakan pohekvs; Estimvt ohfvnkat elkv svhokkolat en nokkicekares.

12 Momen mekusvpkv-cuko Pvkernvse likat em encelvn hrmmaket en hoccicvs; $\mathrm{Mv}$ eslafkv-crpko em fvske hokkole ocen ocat heyv nanvken maket os;

13 Cem vtotketv taken, momet estvmvn liketskat, $\mathrm{mv}$ Setvne em ohliketv ocan kerris; momen cr hocefkvn yekcen hvlatetskes, mv nettv take vm akerrv herome, Anteprse hocefket, ece 'pakvken, Setvne likan, elehocvtet, arof tis vm vkvsvmkv ohfvccrn ehelvksecetskekates.

14 Momis nanvke vnvcomusekon ece'nrvpen ocis, 
Pelvme eme mvhakvn hvlvtakan ocetskekv; mv Pelvmet Pelvke Eslevl en hopuetake nake es elentappetvn ehomvn ocetrn momat nanvke nakvhakvke emhoyan hompvkvren, momet hoktarvkvren emvhayvtes.

15 Momen hvtvm Nekolitvlke eme mvhakv, mvn en ev homecat, hvlvtakan ocetskes.

16 Cem vkerrickvn eticvs; monkon omat, lvpken cem vlakit, cv cukwv em eslafkv-crpkon emetaken 'setepoyares.

17 Estimvt ehvcko ocat Puyvfekevt nake mekusvpkvcukon em punayakan pohekvs; Estit ohfvnkat manv ehken akvpvken hompvren emit, cvto hvtke, nıv cvto hocefkv mucrse 'soh hoccen e'mares, momen mv hocefkv, $\mathrm{mv}$ en heckat tvlket kerren, este etvt kerrekos.

18 Momen mekusvpkv-cuko Rivtilv likat em encelvn hvmmaket en hoccicvs; Hesaketrmese E'ppuce, mv e turwv totkv em fenke omvkēn ocet, momet e'le cvtolane holococcuse omvkēt omen, heyv nanvken maket os ;

19 Cem vtotketvn, momen cem vnokeckvn, momen heromkv vtotketvn, momen vkvsvmkvn, momen cem momis kometvn momen cem vtotketvn kerris; momen vtotketv espokakat enhomv vtotketv sensulket omat kerris.

20 Mome estomis nake ece'nrvpakat vnvcomusekon ocis, 'mv hokte, Cesepel hocefket, owalv hokte e kicat mvhayet, vm vtotkvlke hoktarvkvren, momet nanvke nakvhakvke emhoyvten hompvkvren ehosvkuecvre em etektvnecetsket omekv.

21 Momen en hoktarkv ohfvecv em vkerrickv eticvren em etektvnecryvtes, momis eticekates.

22 Hecrs, emen toprn oh wikares, momet emen vpaket hoktarakat estemerkv rakken vk pvlvtares, nak momecakvte ohfvecr vkerrickv eticvkekon omat. 
23 Momet en hopuetake elkvn es pvsvtares; momen vnet mv este envcofv efeke takeu ofv hopoyvt omvyat mekusvpkv-cuko omvlkvt kerrvkvres; momen omvlkatskan cem vtotketv take vcrkvyen ce'mvkares.

24 Momis centaken, momen vpvlwv Rivtilv vpokat, estomomuset heyv mvkakv ocvkeko, Setvne en sufsokan kerrvkekat, makakat vcvkvyen, heyvn ce kicvkvyet os; Nake honnat etvn ec oh wikvkin omekares.

25 Momis mv ocatskan yekcen hvlvtatske munken vlvkares.

26 Momen estimvt ohfvnket, eyuksv vteken vm vtotketv taken vfastat, mvn Centilvlke ohfvnken yekcetvn emares;

27 Momen emet crto estepketvn es ohmekkvkvres; momen vrkvshayv em vrkvswv omēn lopockusen tvkohlihcrres; Cv'rke a'men vn heckvte etvpomen.

28 Momen hryvtecvn e'mares.

29 Estimvt ehvcko ocat Puyvfekcrt nake mekusvpkvcukon em punayakan pohekvs.

\section{SETENTVCKE III.}

Momen mekusvpkv-cuko Satese likat em encelvn hvmmaket en hoccicvs; Mv Hesaketvmese em Puyvfekcvlke kolvpakan, momet kococumpv kolvpakan ocat heyv nanvken maket os; Cem vtotketvn kerrakit, eletske tat, hesaketske omēn hocefkvn ocetskat kerris.

2 Vketecet omvs, momet nanvke vhoske pvsvtkvranuse ocakan yekcrkuecvs, Hesaketvmese ehomv cem vtotketv esfvcfvken eshecvkot omekv.

3 Monkv nake cen hecken, momet pohetskvten vkerricvs, momet veryecet, cem vkerrickvn eticvs. Monkv vketecetskekon omat, horkopv omēt cem vlvkares, momen hvse-vkerkv estoman cem vlvkvranvyan kerretskekares.

4 Este em accvke svholwvyecvkekat hocef kv nvcomusen 
Satese ofv tis ocetskes, momen hvthvken vchoyet vc vpaket yvkvpvkvres; heretv oricrket omekv.

5 Estimvt ohfvnkat accvke hvtken vecvres, momen e hocefkv hesaketv nakcokv ofv ocan vslecvkares, momet $\mathrm{e}$ hocefkvn Cv'rke, momet em encelvlke ehomvn ohkerkuecares.

6 Estimvt ehvcko ocat Puyvfekcvt nake mekusvpkvcuko em punayakan pohekvs.

7 Momen mekusvpkv-cuko Feletelfev likat em encelvn en hoccicvs, Mv hvsvtkat, mv mehenwat, mv Tewe em eshvweckvn ocat, mv hvwecan momen estit vkhottekon, momet mv vkhottat momet estit hrwecekos, heyv nanvken maket os.

8 Cem vtotketvn kerrakis; hecvs, vhauke hauken ce hoinvn ocis, momen estit vkhotteko tajes; yekcetv estomusen ocet, vm opunvkvn vfastet, momet $\mathrm{cv}$ hocef $\mathrm{kv}$ ohfrecrn ehelvksecetskekatet ok.

9 Hecrs, emis, mv este Setvne en senekake enake svpakle, mv, Cusvlke toyēs, maket, mv toko, lvksakat; hecvs, yicet, ce'le ehomvn vrakkuecet, vnet ece 'nokecvyvten kerraken hayares.

$10 \mathrm{Vm}$ momis kometv em opunvkvn veryecetskvtet omekv, vneu, hvse-vkerkv este eskerretv ekvnv omvlkvn oh vlahket, este ekvnv oh vpokan eskerrvranat enkvpvken ece 'cryecares.

11 Hecvs, lvpken vlakis; mv ocetskat yekcen hvlvtvs, nvpvt cen kvpotokv vcakan ce 'konvhekaren.

12 Estimvt ohfvnkat vn Hesaketvmese en cuko-vcakat ofvn cukelen hayares, momen estofis mvn aossekares; momen vn Hesaketvmese e hocefkvn momet Celuslvme mucvsat vn Hesaketvmese em etvlofv, mv hvlwan aosse, vn Hesaketvmese vtet ahvtapkat e hocefkvn, momet en hocef $\mathrm{kv}$ mucvsen oh hoccicares. 
13 Estimvt ehvcko ocat nake Puyvfekcvt mekusvpkvcukon em punayakan pohekvs.

14 Momen mekusvpkv-cuko Leotesev likat em encelvn en hoccicvs; Emén, mv akerrv herome momet mehenwat, Hesaketrmese en nake hocackvte em ekvt heyv nanvken maket os.

15 Cem vtotketvn kerrakit, kvsvppekot momet biyyekot ometskat kerris; kvsvppet monkat hiyyet ometskate.

16 Monkv kvsvppe monkat hiyye tokot, lekhet ometskat omecicen, ev cukwr aossen ce pockares.

17 Nake ocit, nake ockv sulken ocit naket vn kusvpkekos, maketskat omecicen, momet cen honne, ce 'stemerke, cen kusvpke, ce turheceko, momet enatvlkvranuse toyetsket omat kerretskekat omecicen;

18 Cvtokunaplane totkv ofv eskerhoyvten vn neset nake oce ce hakvren; momet vecet ometskvren, momet ce'natvlkvranusat em vlesketvn heckekaren, accvke hrthvken vn nesvs, ce kicet cem vcahnvyet os; momen este turwv es sikvn ce turwvn 'sen siyvs, heçetskvren.

19 Estomomusen vnokecakvyat em vsehit estemerrvkuecis, monkv vfekcakhet omet, cem vkerrickvn eticrs.

20 Hecvs, vhauken vhuerit, vnafkis, estimvt vm opunvkv en haken pohet, vhauken hrwecen omat, en ceyit, emen vpakit yvfke hompetvn hompares, momen emet ve vpaket hompures.

21 Estimvt ohfvnkat mekko ohliketv crnake ve vpaket oh likvren emares, vnet ohfvnikit, Cv'rke vpakit em ohliketv oh likvyat etvpomen.

22 Estimvt ehvcko ocat Puyvfekcvt nake mekusvpkvcukon em punayakan pohekvs.

\section{SETENTVCKE IV.}

Hexv hoyanof vhecryvnks, momen hecrs, vhauke sutv ofv hvwechoyvten; momen opunvkv en hake enhvieceskv 
pohvyvuke pofketv en hake omēt vm punayet, Yvmvn acemiketsken, heyv nanvke hoyahn of nake ocvkvranan ce hecicares, maken pohvyvnks.

2 Momet moman vpaken Puyvfekev ofvt omvyvnks, momen hecvs, mekko em ohliketv sutv ofvn lihocen, estet mv ohliketvn oh lidkvnks.

3 Momen mv oh likat en heckvt caspv momen satenv cvto omēt omvnks, momen ohliketv vfolotkat oskentacv sinvlvktenv en heckv omet omvnks.

4 Momen ohliketv pale hokkolen ostohkaket mekko em ohliketv vfolotken ocakvnks; momen este vculvke palc hokkolen ostohkaket, accvke hvthvken vchoyet mv ohliketvn oh vpoken hecvyvnks; momen cvtokunaplane kvpotokv vcakan kvpotoyvket omvnks.

5 Momen vtoyehvtte sossen, tenetketv sossen en hake sulket mv mekko em ohliketv sossvnks; momen mv ohliketv ehomvn totkv en kulke kolvpake fenkaket ocakvnks, mvt Hesaketvmese em Puyvfekcv kolvpakat omakes.

6 Momen mekko em ohliketv ehomv estvkheckv uehvtkv, klestelv cvto omēt ocvnks, momen mv ohliketv en nvrkvpvn momet vfolotken nake wenahoke ostet, ehomvn momet eyupvu turwv sulke mahen ocet, svpaklvnks.

- 7 Momen nake wenake enhrteceskvt este-papv omēt omen, nake wenake svhokkolat wakuce omēt on, nake wenake svtutcenat e turofv este eturofv omēn ocvnks, momen esostat lvmhe tvmke omēt omvnks.

8 Momen nake wenahoke ostat etvrpv epaken ocvke vlket omet, ofv eturwv sulke mahen ocvnks; momet netta nereu fekvpekot, Pucase Hesaketvmese Yekcetv Omvlece, hvsvtke, hvsvtke, hvsvtke, mv likvte, like, momet likvranat, makaket os. 
9 Momen mv mekko em ohliketv oh like, estofis meyuksv-seko likaten $\mathrm{mv}$ nake wenahokat vrakkueckv, vcryeckv, vkvsvmkv esyomen emakof,

10 Veulvke pale hokkolen ostohkakat mekko em ohliketvn oh likat ehomvn tvk pvlvtiket, mv estofis meyuksvseko likan vrakkuecet, ohliketv ehomvn en kvpotokv veacakan tvkwikaket,

11 Pucasē, vrakketv, vrakkueckv, yekcetv esyome cen heckvren heretv oretsket omes, nanvke omvlkvn hocacetsken, ceme kometskat eteropotten hocackvtet, momet ocaket omekv, makaket os.

\section{SETENTVCKE V.}

Momen, ofv momet onvpru oh hocce, momet esvlokpickv kolvpaken es vlokpihocen nakcokv mv ohliketv oh likat e'nkvpervn vpiken hecryvnks.

2 Momet encelv yekcet, Estimvt mv nakcokvn enrahmet sohlokpickv kolvpakan enrecopetv heretv ore haks? maket, en hake yekcen es huehken hecryvnks.

3 Momen hvlwat ofv, monkat ekvnv onvpv, monkat ekvnv ofv nvpvt mv nakcokvn enrahmet, hecetv ume tayat sepekvnks.

4 Momen mv nakcokvn enrahmet, ohonayetv monkat hecetv heretv oren nvpvn eshechoyekat omecicen tayen hvkihkvyvnks.

5 Momen mv vculvke hvmket, Hvkihiketskvs, hecvs, Este-papv, Cutv em vliketv aosse, Tewe en Yvlonkvt ohfvniket, mv nakcokvn enramet, 'sem vlokpickv kolvpakan enhvwece tayes, cv kicvnks.

6 Momen hecryvnks, momen hecrs, mekko em ohliketv, nake wenahoke ostat vculvke esyomat en nvrkvpvn, Yrpefikuce elehocvte omēt, eyvpe kolvpaken, momet 
eturwv kolvpaken ocet, mvt Hesaketvmese em Puyvfekev kolvpake ekvnv omvlkv ofv vtothoyat omakes.

7 Momen vlahket, $\mathrm{mv}$ ohliketvn oh like nakcokv e'nkvperv vpikan 'sem esvtes.

8 Momen, nakcokvn ehsof, nake wenahoke ostat, momen vculvke pale hokkolen ostohkakat, kervlv momet crtokunaplane esesketucen fvmecickv here es fvcfvken, mv fvmecickvt mekusapvlke em emekusvkvt omaken, ocvke vlket omet, mv Yvpefikuce ehomvn tvk pvlatkvnks.

9 Momet yvhiketv mucrsen yvhikakvnks, hvmmaket; Cernet, mv nakcokvn ehset, 'sem vlokpickvn hvwecetv heretv oretsket os, ce'lehocvtet omen, este em vliketv, etulaswr, etvlwv, momet etvlwvvlke vtekat asossen ce catvn es pu nesetskvtet omet,

10 Pun Hesaketvmese ehomvn mekkvlke momet plestvlken pu hayetskvtet omekv, momen ekvnvn ohmekkeyvres.

11 Momen hecryvnks, momet nake wenahoke, momen vculvke esyoman, momet mekko em ohliketv em vfolotakat encelvlke sulke em opunvkv en haken pohvyvnks; momen em vhonkvtkvt cukpe-rakko pale cukperakko palet, momet cukpe-rakko cukpe-rakko sulket omvnks;

12 Opunvkv en hake yekcen 'sopunayet, Mv Yvpefikuce elehocvtet ohfvnketv, nake ockv, hopovrenkv, yekcetv, vrakketv, vkvsvmkv esyomat en heckvre heretv oret os, makakvnks.

13 Momen nake hocackvte vteke hvlwe ofv fullat, momet ekvnvn fullat, momet ekvnv ofv, momet uehvtkv vk fullat, momet mv omvlkv ofv fullat omvlkvt hvmmaken pohvyvnks; Vkvsvmkv, vrakketv, vrakkueckv, ohmekketv esyomat $\mathrm{mv}$ Yvpefikuce, momet $\mathrm{mv}$ mekko em ohliketv oh likat em ocvkekvs, estofis meyuksv-sekon. 
14 Momen nake wenahokat, Emén, makakvnks. Momen vculvke pale hokkolen ostohkakat tvk pvlvtiket, mv estofis meyuksv seko likan vrakkuecakvnks.

\section{SETENTVCKE VI.}

Momen Yvpefikucet svlokpe kolvpakat hvmken hvwecof, hecryvnks, momen nake wenahoke ostat hrmke, tenetke en hake oket, Ahtet, hecvs, maken pohvyvnks.

2 Momen hecvyvnks, momen corakko hvtket huervnks; momen $\mathrm{mv}$ oh likat etcrkotaksen ocvnks; momen kvpotokv vcakan e'mhoyvnks; momen ohfvnket momet ohfvnkvranet ayvnks.

3 Momen svlokpe svhokkolan hvwehcof, nake wenake svhokkolat, Ahtet, hecvs, maken pohvyvnks.

4 Momen corakko etv catet ayvnks; momen mv oh likat herkvn ekvnvn vkonahvren, momen estet etepvsvtvre yekcetvn e'mhoyvnks; momen eslafkverpko rakken e'mhoyvnks.

5 Momen svlokpe svtutcenan hvwehcof, nake wenake svtutcenat, Ahtet, hecrs, maken pohvyvnks. Momen hecryvnks, momen, hecrs, corakko lvstet, momen mv oh likat svtarkueckvn enken 'sesvnks.

6 Momen nake wenahoke ostat en nvrkvpvn opunvkv en hake hvmmaken pohvyvnks, Svkerkuce teleko fvcke hvmket tenelyv hvmke enrahkvt omen, momen svkerkuce ketinv fvefvke tutcenet tenelyv hrmke enrahkvt omvres; momen olefv neha pvrko-opuswv tepakvn vhopahnetskvs.

7 Momen svlokpe esostan hrwehcof, nake wenake esostat em opunvkv en haket, Ahtet, hecrs, maken pohvyvnks.

8 Momet hecryvnks, momen hecvs, corakko hvtwet, momen mv oh likat e hocefkv Elkvt omvnks, momen Elkv tvlofvt vcrkayvnks. Momen ekvnv tekvpvke esostan 
eslafkv-cvpko, momet elauko, elkv momet punvttv sehoneckvke ekvnv oh fullat es pvsvtvkvre ohfvnken yekcetvn emvkhoyvnks.

9 Momen svlokpe svcahkepan hvwehcof, altv elecvn mv Hesaketvmese em opunvkv, momet ohkerkueckv ocakvte vrahkvn pvsvthoyvte em puyvfekcv taken hecvyvnks.

10 Momen en hake yekcen es huehkakvnks, Pucase hvsvtke, mehenwetskat, en hofune estomomusen mv ekvnv vpokakat vnrvpen pu catv vrahkvn ohfvececet, em pvlecvranetskeko haks? maket.

11 Momen vccrke hvtke e'mhoye vlket omvnks, momen, Ohvtvlakat estomusen fekapatske emunken vtotkv cem etohkvlketv takeu, momet cem etecakketvlke ce mehocvte omēn prsvthoyrranat oketv es oh fvckekvs, maket em onahoyvnks.

12 Momen hecryvnks, svlokpe sepakan hvwehcof, momen hecvs, ekvnv nekekv rakket ocvnks; momen hvset lvste sukcv hakv esse es hahoyvte omē haken, hvresset catv omē hakvnks;

13 Momen ke-rakko em vpe hotvle rakkot fvmoyicen, em ette lokcvkeko apvlate omēn, hvlwe kococumpvt ekvnvn a tvk pvlatkvnks.

14 Momen nakcokv pvlpihoce omēt sutvt etvn vkuekvnks, momen ekvnhviwe vtekat, momet ote vtekat etvn vkueke vlkvnks.

15 Momen ekvnv em mekkvlke, este en rvkrakv, nake ocvlke, kvpetvne homvhhotat, este yekcvke esyomat, momen svlvflke vtekat, momen pucasseko vtekat omvlkvt ekvnv haukvke, momen ekvnv hvlhvwe crto vlkat ofvn ehkakvnks;

16 Momet, Mv mekko em ohliketv oh likat e turofv, momet Yvpefikuce en crpvkketv enkvpvken pu ehvkes, cvto, ekvnv hvlhawat esyomen kicakvnks. 
17 En cvpvkketv nettv rakkatet vlvket omekv, momen estit hueretv ume taye haks?

\section{SETENTVCKE VII.}

Momen heyv nanvke renyupvn encelv ostet ekvnv en kvnowv ostan oh svpaklen hecvyvnks, momen ekvnv en hotvle ostan hvlvtakvnks, ekvnvn, monkat uehvtkvn, monkat eto estomis oh hotvlekaren.

2 Momen encelv etv, Hesaketvmese wenakat 'sem enkerkv ocet, hvsossv fvecv aossen hecvyvnks, momen encelv oste, ekvnv momet uehvtkv vhopvnetv yekcetv e'mhoyvten em punayet, en hake yekcen es huehkvnks;

3 Ekvnvn, monkat uehvtkvn, monkat eton vhopvnatskeko emunken pun Hesaketvmese em vtotkvlken ekvhomv taken eskerkvn em ohlicvkeres, maket.

4 Momen eskerkv ohlihocat em vhonkvtkvn pohvyvnks; Eslevl en hopuetake em vliketv omvlkv asosse ohlihocat cukpe-rakko cukpe hvmken pale osten ostohkaket omvnks.

5 Cutv em vliketv asosse cukpe-rakko palen hokkolohkaken ohlihocen; Lupe em vliketv asosse cukperakko palen hokkolohkaken ohlihocen; Katv em vliketv asosse cukpe-rakko palen hokkolohkaken ohlihocen;

$6 \mathrm{Asv}$ em vliketv asosse oukpe-rakko palen hokkolohkaken ohlihocen; Nafteli em vliketv asosse cukperakko palen hokkolohkaken ohlihocen; Menasv em vliketv asosse cukpe-rakko palen hokkolohkaken ohlihocen; 7 Semev em vliketv asosse cukpe rakko palen hokkolohkaken ohlihocen; Lefi em vliketv asosse cukperakko palen hokkolohkaken ohlihocen; Esekv em vliketv asosse cukpe-rakko palen hokkolohkaken ohlihocen;

8 Momen Sepulv em vliketv asosse cukpe-rakko palen hokkolohkaken ohlihocen; Cosef em vliketv asosse cukpe- 
rakko palen hokkolohkaken ohlihocen; Penceme em vliketv asosse cukpe-rakko palen hokkolohkaken ohlihocet omvnks.

9 Heyv hoyahnof hecryvnks, momen hecrs, este sulke mahe, nvpvt vhonkvteko tayat, etvlwv, momet em vliketv, momet este, momet etulaswv omvlkv asosse, vecvke hvthvken vcce, talv lvcce enken cawvket, mekko em ohliketv ehomvn, momet Yvpefikuce ehomvn svpaklvnks;

10 Momet huehketv yekcen es huehket, Vhesaketv pun Hesaketvmese mekko em ohliketv oh likat, momet Yvpefikuce enaket os, makakvnks.

11 Momen encelv omvlkvt mekko em ohliketv, nake wenahoke ostat vculvke esyomat vfolotken svpaklet, mekko em ohliketv ehomvn e turofvn oh pvlatket,

12 Emén: Vkvsvinkv, vrakketv, hoporrenkv, vkvsvmkvemetv, vrakkueckv, yekcetv, ohfvnketv esyomat pun Hesaketvmesen em ocvkekvs, estofis meyuksv-sekon; Emén : makaket, Hesaketvmesen vrakkuecakvnks.

13 Momen mv vculvke hvmkat vyoposket, Heyv hvthvke vchoyat estit omet, estrmvn awvt haks? cv kicvnks.

14 Momen, Pucasē, kerretskes, kicvyvnks. Momen, Heyvt estemerketv rakken asosiyet, em vccrken Yvpefikuce e catvn es okkoset, hvthvken hayakvtet omakes, ev kicvnks.

15 Momet, Heyvt omecicen Hesaketvmese em ohliketv ehomvn vpoket, en cuko vcakat ofvn netta nereu em vtotkaket os; momen mv mekko em ohliketr oh likatet vpaket likvres.

16 Svnvcomvn elvwekot, svnvcomvn ewvnhkahkekos, momen hrsohiyyvt momet hiyye estomis en nokhokihcekos.

17 Hiyomet omekv, mv Yvpefikuce mekko ein ohliketv 
en nvrkvpv likat vhecicet, hompvkuecet, uewv hesake nekiwv ocakan evpayet sohayvres; momen Hesaketvmeset eturopuswv omvlkvn e turwv taken en hrsvthicvres, $c v$ kicvnks.

\section{SETENTVCKE VIII.}

Momen svlokpe eskolvpakan hrwehcof, hvse-vkerkv en nvrkvpv ore tayen hvlwat ofvn cryvyakuset omvnks.

2 Momen encelv kolvpakat Hesaketvmese ehomvn svpaklen hecryvnks; momen pofketv kolvpaken e'mhoyonks.

3 Momen encelv etvt, crtokunaplane sensvn ocet, altrn vhuervnks; momen esfvmecickv sulken e'mhoyvnks, mekusapvlke omvlkv em mekusvpkv take vpvken crtokunaplane altv mekko em ohliketv ehomv hueran mvn oh emvren.

4 Momen mekusapvlke em mekusvpkv taket vprken, mv esfvmecickv em ekkucet encelv e'nken osiyet, Hesaketvmese ehomvn kvwapkvnks.

5 Momen encelvt sensvn ehset, totkv altv oh ocan es fvcehcet, ekvnvn es tvk wikvnks; momen en hake sulket tenetketv, vtoyehvtte, ekvnv nekekv esyomat ocakvnks.

6 Momen encelv kolvpake pofketv kolvpake ocakat pofkvranet e etetakuecakvnks.

7 Momen encelv enhvteceskvt pofkvnks, momen hetute nerkv momet totkv catv vpvken ocakvnks, momen ekvnvn oh prlvthoyvnks; momen eto tekvprke svtutcenat nokren, prbe lane omvlkvt nokrvnks.

8 Momen encelv svhokkolat pofkvnks, momen ekvnhvlwe rakke vhetke omën uehvtkvn vk wihokvnks; momen uehvtkv tekvpvke svtutcenat catv hakvnks;

9 Momen nake wenahoke hocackvte uehvtkv vk fullat tekvpvke svtutcenat prsatkvnks, momen perro-cuko tekvpvke svtutcenat vkvhopvnkakvnks. 
10 Momen encelv svtutcenat pofkvnks, momen kococumpv rakke vhetke kulke omēt sutvn a latkvnks, momet hvcce ocakat tekvprke esvtutcenan, momet nekiwv fihnakan oh latkvnks.

11 Momen mv kococumpv e hocefkv Wvmwut kihocet omes; momen uewv etekvpvke svtutcenat wrmwut hakvnks ; momen uewrt omecicen este sulket prsatkvnks, uewv hometv hakvnkekv.

12 Momen encelv esostat pofkvnks, momen hvse tekvpvke svtutcenat, hvresse tekvpvke svtutcenat, kococumpv tekvpvke svtutcenat esyomat nvfhokvnks; mv omvlkv em etekvpvke svtutcenat yomucecihohcen, nettv tekvpvke svtutcenat hryayvkekaren, momen nereu matvpomen.

13 Momen hecryvnks, momet encelv hvmke hvlwen tvmket tehoyanet, en hake yekcen es opunayet, Encelv tutcene pofkvranat em pofketv en hake omecicen ekvnv oh vpokat en holwakes, holwakes, holwakes, maken pohvyvnks.

\section{SETENTVCKE IX.}

Momen encelv svcahkepat pofkvnks, momen kococumpv sutvn avtet ekvnvn oh latken hecvyrnks; momen sufkē-rakko em prtakv sekat es en hvweckvn e'mhoyvnks.

2 Momen mv sufkē-rakkon hvwecvnks; momen ekkuce nakrekkickv hute rakke em ekkuce omēt sufkan aosiyet, kvwapkvnks; momen mv sufke rakkat em ekkuce hrse hotvle tepakan oh yomucecicvnks.

3 Momen tvffonoksvlket ekkuce asosset, ekvnvn oh yicvnks; momen hvcesesterahv ekvnv fulle yekcetv ocakat omēn yekcetvn emhoyvnks.

4 Momet, Ekvnv pvhe ocan, monkat nake lane estomis, 
monkat eto hvmkusis vhopahnatskvs; momis mv este Hesaketvmese em eskerkv ekvhomv take oh ocvkekat tvlken en nokhokicatskēs, kicet en kerkuehocvnks.

5 Momet prsvtekot, momis hvse cahkepen en nokhokicvren em etektvnechoyvnks; momen $\mathrm{mv}$ en nokket hvcesesterahv este rahe en nokke omēt omvnks.

6 Momen $\mathrm{mv}$ nettr take omof estet elkvn hopoyakis, eshecvkekares; momet eletvn komakis, elkvt en letkvkvres.

7 Momen mv tvffonoksvlke en heckvt corakko tepokvn sohayvrane etetakuehocvte en heckv omēt omvnks; momen cvtokunaplane kvpotokv vcacakat omvkēn kvpotoyvket omvnks; momen e turofv taket este e turofv omvkēt omvnks.

8 Momen ekvesse hoktvke em ekvesse omēn ocakvnks, momen e nute taket este-papv e nute omvket omvnks.

9 Momen hokpe sevrvnakv taket hokpe sevrvnakv crto es hahoyvte omvkēn ocakvnks; momen e trrpv take en haket calev sulke, momet calev eme corakko sulke tepokv vpefatke en hake omēt omvnks.

10 Momen hvcesesterahv omvkēt ehvcen ocakvnks; momen ehvcet en ren ocvket omvnks; momen hvse cahkepen est'en nokhokice taye en yekcet omakvnks.

11 Momen sufke-rakko em prtakv seko em encelvn em mekko taken ocakvnks, momen e hocefkvt Hepluvlke em punvkv ofv Epatvnet omen, Klekvlke em punvkv ofv Epalyvnet omes.

12 Este en holwakat hvmket hoyanet os; hecvs, heyv en yupvn holwakat hokkolet vlahokes.

13 Momen encelv sepakat pofkvnks, momen opunvkv en hake cvtokunaplane altv Hesaketvmese ehomv huerat e yvpe ostan aosset, 
14 Encelv sepake pofketv ocan em punayet, $\mathrm{Mv}$ encelv oste hvcce rakke Yufletes hocefken vk wvnahoyan enrecopvkvs, maken pohvyvnks.

15 Momen encelv oste hvse-vkerkv hvmke, momet nettv hvmke, momet hvse hvmke, momet ohrolope hvmke vrahkvn etetakuehocvten, este etekvpvke svtutcenan prsvtvkvren enrecoprehocvnks.

16 Momen corakko oh vpoke sulctawv rakko em vhonkvtkvt cukpe-rakko vcule cukpe hokkolet omvnks, momen em vhonkvtkvn pohvyvnks.

17 Momen nak $c v$ hecihocat ofvn hiyomēn corakkon momet mv oh vpokat, hokpe sevrvnakv totkv, cesenrv, tohottoplane esyomen es hahoyvten ocaken hecryvuks; momen corakko e'kv taket este-papv e'kv take omvkēt omen; totkv, ekkuce, tohottoplane esyomet e cukwv taken asossvnks.

18 Totkv, ekkuce, momet tohottoplane, heyv tutcene e cukwv take asossat este etekvpvke svtutcenan pvsvtakvnks.

19 En yekce e cukwr momet e hvce take ofvt omekv; e hvce take cetto omvkēt, momet ekvri ocvket omvnkekv; momen heyvn es nokkicaket os.

20 Momen este vhoskat, heyv estennokkickvt pvsvtvkekatet, puyvfekev holwvhokat, monkat nake vhakvke cvtokunaplane, monkat cvtokunaphvtke, monkat cvtolane, monkat cvto, monkat eto es hahoyvte, mv heceko taye, monkat poheko taye, yvkvprkeko tayan em mekusapvkekaret, e'nke take em vtotketv ohfvecvn em vkerrickvn eticvkekvnks :

21 Momet este pvsvtakvte, monkat en famvkakvn, monkat en hoktarkvn, monkat en horkopkv take ohfvecvn em vkerrickvn eticvkekvnks. 


\section{SETENTVCKE X.}

Momen encelv etv yekce, aholocen accet, sutv ahvtapken hecvyvnks; momen oskentacvt e'kvn ohcakhen, e turofv hrse omēt omen, e'le totkv escrkcahe omvkēt omvnks :

2 Momen nakcokv cotkuse enrvmken e'nken vpikvnks; momet e'le vkvpervn uehvtkvn vk huericet, e'le vkvskvuvn ekvnvn oh huericvnks,

3 Momet este-papv hehke omēn en hake yekcen es huehkvnks; momen hohikof, tenetke kolvpaket tenetkakvnks.

4 Momen $\mathrm{mv}$ tenetke kolvpakat tenetkahkof, hoccicvranvyvnks: momet punvkv en liake sutv avtet, $\mathrm{Mv}$ nanvke tenetke kolvpakat onayakan hoccicekot, sem oh vlokpicvs, maken pohvyvnks.

5 Momen mv encelv uehvtkv, momet ekvnv oh huere hecryvnke e'nken sutvn vkvwapet,

6 Sutv momen mv ofv fullat, momen ekvnv momen mv oh fullat, momen uehvtkv momen mv vkfullat hayvte, mv estofis meyuksv-seko likan es hocefet, Oketvt vlvkeko emunken,

7 Encelv eskolvpakat hake en nettv take omof, mvt pofketvn vlicecof, Hesaketvmese enake ehkat esfvckvres, em vtotkvlke owalvlke en kerkuecakvte vcvkvyen, makvnks.

8 Momen opunvkv en hake sutv avte pohvyvnke hvtvm vm punayet, Ahyet, nakcokv cotkuse, enrvmke encelv uehvtkv momet ekvnv oh huere e'nke vpikan esvs, makvnks.

9 Momen encelvn oh ahyit, Mv nakcokucen v'mes, kicvyvnks. Momen vm punayet, Ehset, lokvs; momen ce nvrke homen hayvres, momis ce cukwv ofv fo en cvmpe omēt evmpe tares, cv kicvnks. 
1.0 Momen mv nakcokucen encelv e'nken a ein ehset, lokvyvnks; momen cv cukwv ofv fo en crmpe omēt crmpet omvnks; momis lohkvyof, ev nvrke home hakvnks.

11 Momen, Hvtvm este, etvlwr, etulaswv momet mekkvlke sulke ehomvn owalvranetsket os, cv kicvnks.

\section{SETENTVCKE XI.}

Momen kohv setekkekv omēn v'mhoyvnks; momen encelvt hueret, Ahuyiret, Hesaketvmese en cuko vcakan, momet mv ofv emekusvpakan, momet altvn vhopayvs, makvnks.

2 Momis cuko vcakat en tvkfettvn vhopayekot, wiketskvres, Centilvlken emhoyvtet omekv; momen mv tvlofv vcakan hvse pale osten hokkolohkaken oh svpaklvres.

3 Momen a vn kerrvlke hokkolan yekcetvn ernvkares, momen sukcv hakvn vchoyet, nettv cukpe-rakko hvmken cukpe hokkolen pale epaken owalvkvres.

4 Heyvt olefv em vpe hokkolat, momet kulke esohhuerickv ekvnv en Hesaketvmese ehomv sehokat omakes.

5 Momen estimvt eu nokhokicetvn komen omat, totkv. e cukwv taken aosiyet en horrvlken lokaket os; momen estimvt en nokhokicetvn komen omat, hiyomēn elehocvranet os.

6 Heyvt, em owaletv en nettv take ofvn oskekaren sutv em vkhottetv yekcetvn ocakes; momet eticet, catvn haye tayet uewv ocakat ohfvnken yekcetvn ocet, momet etewole estomomusen komakat nake estennokkickv estomis ekvnvn oh vlvkuecetvn yekeetvn ocakes.

7 Momen akerkueckvn es poyahkof, punvttv sufke-rakko em pvtakv sekan aosset eme take vnrvpen horren hayet, ohfvniket, pvsvtvres.

8 Momen e'na take tvlofv rakkat em etehoyvnkvn tvk 
wvkhokvres, mv tvlofv puyvfekcv verkvye Satvme momet Ecepsen kihocet os, momen mvn pu Pucasen elehocvtet omes.

9 Momen este, momet em vliketv, momet etulaswv, momet Centilvlke asosset mv ena taken nettv tutcenen nvrkvpvt vpaken hecvkvres, momet mv ena take sepvlkv vtehkvre em etektvnecvkekares.

10 Momen mv yvmv ekvnv vpokat oh afvcket, afvckvkvres, momet nak emkvn ete'm vtotvkvres; heyv owalv hokkolat yvmv ekvnv vpokan estemerrvkuecakvtet ok.

11 Momen $m v$ nettv tutcene nvrkvpvt vpakat renyupvn hesaketv em Puyvfekcv Hesaketvmese aatat mv enataken eceyaken, asehoket e'le taken oh schokvnks; momen penkvlkv rakket hecakat omvlkvn oh latkvnks.

12 Momen opunvkv en hake yekce sutv avtet, Yvmvn a vcemhokvks, kihocen pohakvnks. Momet aholoce ofv sutvn vkvwrphoken, en horrvlket hecakvnks.

13 Momen mata hvse-vkerkv omof ekvnv nekekv rakket - ocvnks, momen $m v$ tvlofv etekvpvke espalat latkvnks; momen este mv ekvnv nekekv ofv prsatkvte em vhonkvtkv cukpe-rakko kolvpaket omvnks; momen este vhoskat penkvlvket, hvlwe en Hesaketvmesen vrakkuecakvnks.

14 Nake este en holwvke svhokkolat hoyvnet os; hecvs, holwvke svtutcenat lvpken vlaket os.

15 Momen encelv eskolvpakat pofkvnks, momen sutv ofv punvkv en hake yekcvket ocakvnks, Yvmv ekvnv em ohmekketr ocakat pu Pucase momet en Klist enake haket os; momen estofis meyuksv-sekon ohmekkvres, maket.

16 Momen vculvke pale hokkolen ostohkakat Hesaketvmese ehomvn em ohliketv vcacakan oh vpoket, e turofv taken oh prlvtiket, Hesaketvmesen vrakkuecet,

17 Vkvsvmkvn ce'meyet os, Pucase Hesaketvmese 
Yekcetv Omvlece, like, likvte momet likvranat toyetskat; cen yekce rakkan ehset, ohmekketskvtet omekv.

18 Momen Centilvlket crpvkhokvtes, momen crpvkketvt vlvkēs, momen pvsatkvte ohfvececkv oketv, momet owalv cem vtotkvlke, momet emekusapvlke, momet lopockuse momet rvkrvke ce hocefkvn em penkvlakat feketvn emvketskvre; momet ekvnvn sumecicakat sumhuecetskvre oketvt vlvkès, makakvnks.

19 Momen hvlwe ofv Hesaketvmese en cuko vcakat hauket omvnks, momen en cuko vcakat ofv em etemfvccetv tovhahvwr vcakat hecket omvnks; momen vtoyehvtte, momet en hake sulket, tenetke ocakat, ekvnv nekckv momet hetute nerkv tayet ocakvnks.

\section{SETENTVCKE XII.}

Momen eskerkv rakket hvlwe ofvn heckvnks, hoktet mvt omen; momen $m v$ hokte hvsen accet, hvresset e'le elecvn ocen, kvpotokv vcake kococumpv palen hokkolohkake es haken kvpvtoyet omvnks :

2 Momen mv hokte nvrkesēt, estuce heckuecvranuset omen, momet heckuecvrane en nokkan es huehkvnks.

3 Momen eskerkv etvt hvlwe ofvn hecket ocvnks; momen hecrs, tlekonv cate, rakke, ekv kolvpaken, eyvpe palen ocet, momen ekv kolvpakat kvtvlvkv oh ocvke vlket omvnks.

4 Momen e hvcet hvlwe kococumpv ocakat etekvprke svtutcenan hvlatet, ekvnvn tvk wikvnks; momen mv tlekonvt hokte estuce heckuecrranat ehomvn huervnks, estuce heckan vpakusen lokvret.

5 Momen hoktet hunvnwucen heckuecvnks, tvlwvvlke omvlkvn cvto estepketvn es ohmekkvranat; momen em estucen Hesaketvmese likan, momet em ohliketv vcakan vkvwvphoyvnks. 
6 Momen hoktet letket, vnrvwvn eceyvnks, mvn liketv Hesaketvmese em etetakuecvtet ocet omen, invn nettv cukpe-rakko hvmken cukpe hokkolen pale epaken hompicvkvren.

7 Momen hvlwe ofv horret ocvnks; Mikevl momet em encelvlket tlekonv tepoyakvnks; momen tlekonv momet em encelvlke tepoyakvnks,

8 Momis ohfvnkvkekvnks; momen em vpoketv hvlwe ofv svnvcomvn eshechoyekvnks.

9 Momen mv tlekonv rakkan a wihokvnks, mv cetto vcule, Tiapolv momet Setvne kihoce, yvmv ekvnv omvlkvn mvkerrat; mvt ekvnvn tvk wihokvnks, momen em encelvlket vpvkaken apvlathoyvnks.

10 Momen opunvkv en hake yekce hvlwe ofvn hvmmaken pohvyrnks, Vhesaketv, yekcetv, momet pun Hesaketvmese em ohmekketv, momet en Klist em ohfvnketvt hiyomat yices; tecakkeyvte e'nrapv, mv pun Hesaketvmese ehomvn nettvn momet nereu vnrapvten awihokekv.

11 Momen Yvpefikuce e catv eteropotten, momen em ohkerkueckv em opunvkv eteropotten mvn emuntvlakvtes; momen elkv tis oren en hesaketv taken vnokecrkekates.

12 Monkv hvlhawatskat, momet mv ofv vpokatskat, afvckvkepvkes. Ekvnv vpoke, momet uehvtkv $v k$ fulle toyatskat ce'n holwvhokes, Tiapolvt, oketv kocoknuse em ocen kerrat omecicen cvprkke heret, a cen hvtapkvkekr.

13 Momen tlekonvt, ekvnvn a tvk wihoken hehcof, mv hokte hunvnwuce heckuecvten assecvnks.

14 Momen hoktet, en liketv vnrvwv ocan tvmket oh ayvren lvmhe rakke e tvrpv hokkolen emhoyvnks, mvn oketv hvmke, oketv vnvcome, momet oketv en nvrkvpvn mv cetto e turofv enkvpvken vfvshotet os. 
15 Momen uelanken svkvyecicetvn komet, mv cettot e cukwv ossen uewr uelauke omēn hokte eyupvn fihnecrnks;

16 Momen ekvnvt hokten emvnicet, e cukwrn hvwvklehcet, mv uelauke tlekonvt e cukwv aossicvten nokmelvnks.

17 Momen tlekonvt hokten en homecet, en nerkv vhoske, mv Hesaketvmese em vhakvn vfastet, Cesvs Klist ohkerkueckv hvlvtakan horre en hayetvn komet ayvnks

\section{SETENTVCKE XIII.}

Momen uehvtkv em oktahvn oh huervyvnks. Momen ekv kolvpakan, eyvpe palen ocet punvttv uehvtkv aossen hecvyvnks, momen kvtvlvkv vcacvke palet eyvpen oh ocaken, momen Hesaketvmese vtvkleckv e hocefkvt e'kvn oh ocaken hecvyvnks.

2 Momen mv punvttv hecvyvnke uekaccv omēt omen, ele nokuse e'le omvkēt omen, e cukwvt estepapv e cukwv omēt omvnks; momen en yekcetv, momen mekkn em ohliketvn, momen ohfvnketv rakkan tlekonvt e'mvnks.

3 Momen ekv hvmke elkv ore vnvttēhoce omēn hecryvnks; momen elece taye vnvttan em wicehocvnks; momen mv punvttv ohfvecvn ekvnv omvlkvt em estomahet omvnks.

4 Momet $\mathrm{mv}$ tlekonv punvttvn yekcetv emvten vrakkuecakvnks; momet punvttvn vrakkuecakvnks, Estit mv punvttv omēt like haks? estit etepoyetv ume taye haks? makaket.

5 Momen e cukwv nake rakrvken, momet opunvkv Hesaketvmese vtvkleckvn opunayen e'mhoyvnks; momen hvse pale osten hokkolohkaken likvre yekcetvn e'mhoyvnks.

6 Momen Hesaketvmese vtvkleckv ofv e cukwvn hvwvk- 
lecet, e hocefkvn, em escuko-hakv, momet mv hvlwe ofv vpoke vtvklecvranet hvwvklecvnks.

7 Momen mekusapvlke etepoyet, ohfvnketvn e'mhoyvnks; momen este em vliketv, momet etulaswv, momet etvlwvvlke omvlkv ohfvnken yekcetvn e'mhoyvnks.

8 Momen ekvnv vpokat, mv e hocefhokv Yvpefikuce ekvnv vliceckv vteke elehocvte en hesaketv nakcokv ofv cohoyekatet omvlkvt vrakkuecvkvres.

9 Estimvt ehvcko ocen omat, pohekvs.

10 Estit esten svlvfkuecat, emen svlvfkuehocvres; estit eslafkv-cvpkon es est' elecat, eslafkv-cvpkot mvn elecvranet os. Mekusapvlke em vkvsvmkv momet em momis kometvt yvmvn ocakes.

11 Momen punvttr etv, yvpefikuce omēt eyvpe hokkolen ocet, ekvnv aossen hecryvuks, momen tlekonv omēn punayvnks.

12 Momen punvttv enhvteceskv ohfvnketv ocat omvlkvn ocet, mv ehomvn es vtotket omes, momet yvmv ekvnv, momen mv oh vpokat punvttv enhvteceskv elece taye vnattan em wicehocvten vrakkuecaken hayet omes.

13 Momet eskerkv rakrvken momecet os, momat este ehomvn hulwe vten totkvn ekvnvn a oh hrtvpecicet os,

14 Momet mv eskerkv mv punvttv ehomv momecvre e'mhoyat eteropotten este ekvnv vpokan mvkerret os, $M v$ punvttv eslafkv-crpko es vnatte em wikvte vhaken en hayvkes, este ekvnv vpokan kicet.

15 Momet $m v$ punvttv vhakan hesaketv emetv yekcetvn ocvnks, momat $m v$ punvttv vhakat opunayat tvlkekot, momis punvttv vhakan en sulke estomomuset vrakkuecvkekat prsvthoyvre omecice tayes.

16 Momẹn lopocke momet rvkrakat, momet nake ocvlke, momet nake ocvkekvlke, momet pucassvkeko momet 
pucase ocakat omvlkvt e'nkvperv, monkat ekvhomv take ofv eskerkv en heckvren;

17 Momet $m v$ eskerkv, monkat mv punvttv e hocefkv, monkat e hocefkv em vhonkvtkvn ocat tvlket, estimvt o estomet mvn ocekat nesetv monkat wiyetv umeko tayan hayet os.

18 Hoporrenkvt yvmvn ocet os. Estit hoporrenkvn ocat mv punvttv em vhonkvtkvn vhonkvtekvs; este em vhonkvtkvt omekv: momen Cukpe epaken pale epaken epohkaket em vhonkvtkvt omes.

\section{SETENTVCKE XIV.}

Momen hecvyvnks, momen hecrs, Yvpefikucet ekvnhvlwe Sivn oh hueren, cukpe-rakko cukpe hrmken pale osten ostohkake, E'rke e hocefkv e'kvhomv taken oh hoccat vprkaken hecvyvnks.

2 Momen uewv sulke en hake, momet en hake hvlwe vten tenetke rakke en hake omēn pohvyvnks: momet kervlv hayecvlke en kervlv taken hayecaken pohvyvnks.

3 Momen mekko em ohliketv, nake wenahoke ostat, momet mv vculvke ehomvn yvhiketv mucvse omēn yvhikakvnks; momen mv yvhiketvn estit kerreko tayen, mv cukpe-rakko. cukpe hvmken pale osten ostohkake, ekvnv asosse prlken neshoyvte tvlket kerrake tayes.

4 Heyvt hoktvken vpaket e vholwvyecvkekatet omes; fvcenvlket omakekv. Heyvt Yvpefikuce estrmv estomēn ayis, vcrkvpeyat omakes. Heyvt ette enhvteceskv Hesaketvmese momet en Yvpefikuce enake este asosse neshoyvtet omakes.

5 Momen e cukwv take ofv mvkerretv eshechoyekvnks: Hesaketvmese em ohliketv vcakat ehomvn em mvttekot omakekv.

6 Momen encelv etvt, opunvkv herv meyuks̊v-sekon, 
ekvnv vpokan, etvlwr, em vliketv, etulaswv, momet este vtekat 'sem erkenvketvn ocet, hvlwan ivmken hecvyvnks;

7 Momen opunvkv yekcen 'sopunayet, Hesaketvmesen em penkalet, vrakketvn emvkes; em ohfvececkv en hvsevkerkvt vlakekv; momet mv hvlwe, ekvnv, uehvtkv momet nekiwv ocakat hayvten: vrakkuecvkes, makvnks.

8 Momen encelv etvt, eyupvn ayet, Papelv, mv tvlofv rakkat, lvtkēs, lvtkēs, tvlwvvlke omvlkvn en hoktarkv en cvpvkketv em pvrko-opuswrn eskvkuecrtet omekv.

9 Momen encelv svtutcenat heyv hokkolan eyupvn ayet, hvmmakvnks; Estimvt mv punvttv momen vhakan vrakkuecet, momet e'kvhomvn, monkat e'nke ofvn hoccen eskerkvn en hecket on omat,

10 Matat Hesaketvmese en cvpvkketv em pvrko-opuswv nake seteyvmkeko en crpvkke sem esketvn avcrnhoyan eskvres ; momet totkv, tohottoplane tepakat ofvn, encelvlke hvsvthakat ehomv, momet Yvpefikuce ehomvn estemerrihocvres :

11 Momen em estemerketv take em ekkucet estofis meyuksv-sekon kvwapket os; momen mv punvttvn momet vhakan vrakkuecakat, momen estimvt o estomet $\mathrm{e}$ hocef $\mathrm{kv}$ es en kerkvn en heckat nettvn monkat neren fekvpvkekos.

12 Emekusapvlke em momis kometvt yvinvn oces: Hesaketvmese em vhakv ocakan, momet Cesvs ofv vkvsvmkvn vfrstakat yvmvn svpakles.

13 Momen opunvkv en hake hvlwe vtet, Hoccicrs, cv kicen pohvyvnks; $\mathrm{Mv}_{v}$ prsatke Pucase ofv prsvtkat en herakes, mucv ayat; Momes, em vtotketr take enkvpvken fekvpvkvren; momen em vtotketv taket vcrkvpeyes, Puyvfekevt maket os, maket hoccicvs.

14 Momen hecryvnks, momen hecrs, aholoce hvtken, momen este E'chushunvnwv omēt aholocen oh liket 
kvpotokv vcakan kvpotoyet, momet estackv fvsken e'nken es hvlaten hecvyvnks.

15 Momen encelv etvt, cuko-vcakan aosiyet, mv aholoce oh likan opunvkv en hake yekcen 'sem punayet, Cem estackvn ceyihcet, tvcvs; hiyomèt omekv, tvcvranetskat hvse-vkerkv vlvkēs, momen ekvnv en naklokcet hiyohakes, maket huehkvnks.

16 Momen mv aholoce oh likatet em estackvn ekvnvn a oh hvtrpecihcet, ekvnv en lokcen vteloyvnks.

17 Momen encelv etvt, estackv fvsken ocet, cuko-vcakat hvlwe ofv likan aossvnks.

18 Momen encelv, totkv ohfvnke yekcetv ocat, altv likan aosiyet, mv estackv fvsken ocan opunvkv en hake yekcen 'sem punayet huehkvnks, Cem estackv fvskan ohceyicet, ekvnv em pvrko em vpe em etten vteloyvs; em prrko lokcvke mvketeket omekv, maket.

19 Momen encelvt em estackvn ekvnvn ohceyicet, ekvnv em pvrko em vpen vteloyvnks, momet Hesaketvmese en crpvkketv em prrko es opecetv rakkan vtehvnks. 20 Momen pvrko es opecetv tvlofv en lvpvtken ohsvpaklet cetakhoyvnks; momet catvt pvrko es opecetvn corakko en cuksakkv tis oren afihnat vkerkv cukpe hokkolen orvnks.

\section{SETENTVCKE XV.}

Momen hvlwe ofvn nake eskerkv etv rakke momet estomahen hecit, encelv kolvpake nake este 'sestemerrickv espokake kolvpakan ocaken hecryvnks; $\mathrm{mv}$ ofv Hesaketvmese en crpvkketvt esfvcket omekv.

2 Momen uehvtkv estrkheckv totkv setekyvmke omēn hecit, mv punvttv, vhakat, es en kerkv, momet e hocefkv em vhonkvtkvn ohfvnkakvtet, Hesaketvmese en kervlvn ocaket, mv estvkheckv nehvtkvn oh svpaklen hecvyvnks.

3 Momen Hesaketvmese em vtotkv Moses en yvhiketv, 
momen Yvpefikuce en yvhiketrn yvhikaket os, Pucase Hesaketvmese Yekcetv Omvlecetskat, cem vtotketv rakrvke, estomahvket omes; mekusapvlke em Mekko toyetskat, cem vretv fvccuke, mehenwvket omakes, maket.

4 Pucase toyetskat, estimvt cem penkalet, ce hocefkvn vrakkuecekar haks? ceme tvlkuset hvsvtket ometskekv; momen tvlwvvlke omvlkvt yicet ece 'rakkuecvkvret ok: cem ohfvececkvt heckaken hahoyekv.

5 Momen mv hoyanat hecvyvnks, momen hecvs, ohkerkueckv em escukohakv en cuko vcakat hvlwe tvlofv ofv ocat haukvnks :

6 Momen encelvlke kolvpakat este 'sestemerrickv kolvpakan ocet, hvnvwelv tvrke, hvtke, hvsatkusen accvket, momet e hokpen cvtokunaplane sewvnvketvn 'sewvnvkaket cuko vcakan asossvnks.

7 Momen nake wenahoke ostat hvmket cvtokunaplane sesketv kolvpake, Hesaketvmese estofis meyuksv-seko likat en cvpvkketv es fvefvken encelvlke kolvpakan emakvnks.

8 Momen cuko vcakat Hesaketvmese en rakketv momet en yekcetv em ekkucen es fackvnks; momen encelvlke kolvpakat em este sestemerrickv kolvpakat esfvckvkeko emunkat estimvt cuko vcakat eceyeko tayvnks.

\section{SETENTVCKE XVI.}

Momen cuko vcakat avtet opunvkv en hake yekce encelvlke kolvpakan em punayet, Vpehyet, sesketv Hesaketvmese en crpvkketv es fucfakan ekvnvn a oh pvlvtvkes, maken pohvyvnks.

2 Momen encelv enhrteceskvt ahyet, sesketv sesan ekvnvn a oh prlatvnks; momen lekhowe holwvke, nok- 
kice mahe mv este punvttv es en kerkv ocet, vhakan vrakkuecakan vlumhvnks.

3 Momen encelv esvhokkolat sesketv sesan uehvtkvn a oh pvlahten, mv uehvtkv este elvte e catv omē hahken', nake wenake omvlkvt nehvtkvn vk prsatkvnks.

4 Momen encelv svtutcenat sesketv sesan hrcee momet uekiwv ocakan a oh pvlahten, catv hakvnks.

5 Momen uewv em encelvt, Pucasē, like, likvte, momet likvranetskat, fvecet ometskes, hiyomēn ohfvececetskekv ;

6 Mekusapvlke momet owalvlke e catvn pvlvtakvtet omekv, momen catvn eskvkuehcetskes; mehocvre orvket omekv, maken pohvyvnks.

7 Momen etv, altv avtet, Momes, Pucase Hesaketvmese Yekcetv Omvlecetskat, cem ohfvcceckvt mehenwe momet fvccuket omakes, maken pohvyvnks.

8 Momen encelv esostat sesketv sesan hvsen oh pvlahten, este totkvn es nekricvre yekcetvn e'mhoyvnks.

9 Momen estet hiyye heran es nekrihohcen, Hesaketvmese heyv este sestemerrickv ohfvnken yekcetv ocat e hocefkvn vtvklecakvnks; momet em vkerrickvn eticet vrakketv emvkekvnks.

10 Momen encelv svcahkepat sesketv sesan punvttv em ohliketrn a oh pvlatvnks, momen em ohmekketv yomucket fvciken, en nokkat omecicen e tulaswr taken vkkakvnks,

11 Momet en nokhoke, momet en lekhowat omecicen hvlwat en Hesaketvmesen vtvklecaket, em vtotketv take ohfvccun em vkerrickvn eticvkekvnks.

12 Momen encelv sepakat sesketv sesan, Yufletes, mv hvcce rakkan vk pvlahten, mv em uewvt vkvrpepvnks, hvsossv-fvecv em mekkvlke em vyetv metetake taren. 
13 Momen puyvfekev hvsvthvkeko kute omvke tutcene tlekonv e cukwv, momet punvttv e cukwv, momet owalv laksv e cukwv asossen hecryvnks.

14. Mvt puyvfekcv holwvhokat em puyvfekcvlke, nakeskerkvn vtotket, ekvnv mekkvlke, momet ekvnv omvlkv em mekkvlken oh vpeyat omet, Hesaketvmese Yekcetv Omvlecat en nettv rakkat en horren oh vteloyvranct omekv.

15 Hẹcvs, horkopv omēt vlakis. Enatvlkvranuset yvkapen, estet em vlesketvn hecvkekaren, mv e vketecet, em accvken vcayecat en heres.

16 Momen ekvnv Hepluvlke em punvkv ofv Amvkētv kihoce likan tohvteloyvnks.

17 Momen oncelv eskolvpakat sesketv sesan hotvle ofvn pvlahten, opunvkv en hake yekce mekko em ohliketv, cuko vcake hvlwe ofv ocan aatvnks, Pokes, maket.

18 Momen en hake sulket, vtoyehvtte momet tenetke ocakvnks; momen ekvnv nckekv rakke, este yvmv ekvnv vpokvte vtekat mv ekvnv nekekv omēt sekon, en yekce momen en rakke mv omēt sekates.

19 Momen tvlofv rakkat tekvpvke tutcenen tekvpihocvnks, momen Centilvlke eme tvlofvt prlatkvnks; momen Papelv rakkat Hesaketvmese em vkerrickv ehomvn vlakvnks, sesketv en homeckv en cvpvkketv em pvrko-opuswvn esemvren.

20 Momen ote vtekat pefatken, ekvnv hvlhawan eshechoyekvnks.

21 Momen hvlwen asosset, hetute nerkv rakrvket esten oh pvlatkvnks, hetute nerkv homke en honnet talentv en honne oren; momen heyv hetute nerkv este estemerrvkuecat omecicen estet Hesaketvmesen vtvklecakvnks; mv este sestemerrickv rakke mahet omvnkekv. 


\section{SETENTVCKE XVII.}

Momen encelv kolvpake sesketv kolvpake ocakat hvmket vlahket, vm punayet, Yvmvn ahtetsken, mv hoktarv rakke uewv sulke oh likat em ohfrececkvn ce hecicares.

2 Mvn vpvkaket ekvnv em mekkvlke hoktaraken, ekvnv vpokat en hoktarkv em pvrko-opuswvn esiket es hacakvnks, makvnks.

3 Momen Puyvfekcr ofvn vurvwrn 'svc ayvnks; momen hoktet punvttv cahtuse, vtvkleckr em vhocefhokvt e'na hvmecicen, ekv kolvpaken, eyvpe palen ocen oh liken hecryvnks.

4 Momen hoktet nake cahtuse momet okholatten accê, crtokunaplane, crto vcacrke, momet makelitvn 'senehicet, cvtokunaplane sesketv en hoktarkv en holwvhokat, momet hvsvtketv tokat es fvcken e'nken es hvlatvnks.

5 Momen hocefkv ekvhomvn oh hoccet ocvnks, heyvt omen, NAKE-EHKE, PAPELV RAKKAT, EKVNV EN HOKTARVLKE MOMET VRETV HOLWVHOKE MAHAT E'CKET.

6 Momen mv hokte mekusapvlke e catv, momet Cesvs a en kerrvlke e catvn es hacen hecryvnks; momet hecvyof, $v m$ estomahetv rakken vm estomahvnks.

7 Momen encelvt hvmmaket cv kicvnks, Estomēn cem estomahet omisa? Mv hokte, momen punvttv ekv kolvpaken, momet eyvpe pale oce hokte oh likan enake ehkan cem onayares.

$8 \mathrm{Mv}$ punvttv hecetskise, likvte tis, likeks; momet sufke rakko em pvtakv sekan aosset, estemerkvn eceyvres. Momen ekvnv vpoke, mv e hocefkv hesaketv nakcokv ofv ekvnv vliceckv vteke hoccvkekaten, em 
estomahvke tares, mv punvttv, likvte tis, likekot omat, mome estomis likat hecakof.

9 Momen vkerrickv hoporrenkv ocat heyv tes. Ekv kolvpakat ekvnhvlwe kolvpakat tes, hokte oh likat.

10 Momen mekkvlke kolvpaket os, cahkepet pvlatken, hvmket liken, hvmkat hvte vlvkekot, momis vlakof, vkocoknusen likvranet os.

11 Momen mv punvttv likvte tis, likekat, mvt escenvpakat omes, momet mv kolvpakan vevkayat tes, momet sumketvn oh ayes.

12 Momen eyvpe pale hecetskise mekkvilke pale em ohmekketv hvte enheckvkekat omakes; momis punvttv vpvken hvse-vkerkv hvmke oren mekkvlke omēt yekcetv en heckaket omes.

13 Heyvt vkerrickv hvmkusen ocet, en yekce em ohfvnketv tepakan punvttvn emvkvres.

14 Heyvt Yvpefikuce vnrvpet horren hayaken, Yvpefikucet ohfvnkvkvres; pucasvlke e Pucaset, momet mekkvlke em Mekkot omekv; momen eme vpvkakatet enhuehket, enhopohoyvtet, momet heromvket omakes.

15 Momen hvmmaket, Hoktarvt oh liken mv uewv sulken hecetskise este, momet este sulsokat, momet etulaswv, momet etvlwvvlke taket omakes.

16 Momen eyvpe pale punvttr oh crkcahe hecetskise, heyvt hoktarvn en homecet, nakoceko, enatvlkvranusen hayet, em vpeswrn papvkvres, momet totkvn és nekricvkvres.

17 Hesaketvmeset naken komat momecetv, momet vkerrickv hvmkusen ocet, Hesaketvmese em opunvkv esfvcfvkeko vtekat em ohmekketv take punvttvn emvkvren efeke taken em vpikakvtet omekv.

18 Momen mv hokte hecetskise mv tvlofv rakke ekvnv em mekkvlke ohmekkat omes; cv kicvnks. 


\section{SETENTVCKE XVIII.}

Momen heyv nanvke renyupvn encelv etv, yekcetv rakken ocet, hvlwan ahvtapken hecryvnks; momen en hvyayvket ekvnvn hvyayicvnks.

2 Momen yekcicet, opunvkv en hake yekcen es huehket, Papelv rakkat lvtkēs, lvtkēs, momet puyvfekcy holwvhokat e hute, momet puyvfekev hvsvtkeko omvlkv em vpoketv, momet fuswv hvsvtkeko, momet holwvke vtekat em vpoketv haket omes.

3 Trlwvvlke omvlkvt en hoktarkv en evprkketv em pvrko-opuswrn eskaken, ekvnv em mekkvlke vpaket hoktaraken, en nake seafvcecickv sulkat eteropotten esnesvlket ekvnv vpokat nake ocvke hakvtet omekv.

4 Momen opunvkv en hake etv hvlwe vtet hrmmaken pohvyvnks, Vm este toyatskat, Papelvn asossvkes, vpaket naorkatskekaren, momet vpaket em este sestemerrickvt cen heckvkekaren;

5 En naorketvt hvlwan oricaken, Hesaketvmeset en holwvyeckv sulkan vkerricekv.

6 Ce'mvkvte etvpomen e'mvkes, momet em vtotketv vcrkvyen tepvkocken e'mvkes; sesketv fvcecvten hokkolvn en fvcecvkes.

7 Estomomusen e vrakkuecet, nake 'se afvcecicvte, mv etvpomusen estemerkv efeknokketv tepakan e'mvkes; Oye toyvkot, mekko hoktet likit, feknokketvn hecvkares, e feke ofvn maket omekv.

8 Heyvt omecicen estemerrickv nettv hvmkuse ofvn oh cakkakvres, elkv, feknokketv, elauko esyomat; momen totkv ofvn nekret sumkvres; Pucase Hesaketvmese mv ohfvececat yekcet omekv.

9 Momen ekvnv em mekkvlke vpaket hoktaret, seafvcecickv ofv vpaket fullvtet, nekrat em ekkucen hecakof, 
10 Em estemerkvn em penkvlakat omecicen hopvyen svpaklet, Holwakes, holwakes, Papelv mv tvlofv rakkat, mv tvlofv yekcat! hvse-vkerkv hvmkuse ofvn cem ohfvececkvt vlaket omekv, maket, oh wahoket, en hvkihhokvres.

11 Momen ekvnv em esnesvlket, eme ohfvecvn feknokhoket, oh hvkihhokvres; nvpvt en nakwiyetvn svnvcomvn en nehsekot ok:

12 Cvtokunaplane, cvtokunaphvtke, cvto vcacakat, makelitv, hvnvwelv tvrke telekmuse, naktvrke okholatte, afusherakv tvrke, naktvrke cahtuse, ruinv eto omvlkv, nakvtehetv yupo-lowake enute es hahoyvte omvlkv, nakvtehetv eto vcake mahat es hahoyvte, momen nake cvtolane, monkat crto, monkat esechetv-cvto es hahoyvte omvlkv,

13 Momet senemv, nak fvmecakusat, vfekv vcacakat, lipenv helukwv, pvrko-opuswv, olefv neha, hockvte hvtke herat, teleko, wakv, yvpefikv, corakko, calev, vtotkvlke, est' em puyvfekcv esyomat omvlkv nakneskv hahoyvten nvpvt svnvcomvn nehsekos.

14 Momen naklokce cem puyvfekcr em vlostat ce'nkvprkaket os, momet nake hompetv herakat, momet nak vcacakat ce'nkvpvkahken svnvcomvn eshehcetskekos.

15 Heyv nanvke esnesvlke, mv eteropotte nake ocvlke hakakvtet, em estemerketv em penkvlakat omecicen hopvyen svpaklet, hvkihket, wahokvres,

16 Holwakes, holwakes, mv tvlofv rakke, hvnvwelv tvlekmuse, okholatte, momet cahtusen accrtet, momet crtokunaplane, cvto vcacvke momet makelitvn 'se nehicvtet! Hvse-vkerkv hvmkuse ofvn nake-ockv en rakke momvteke sumkepekv, maket.

17 Momen perro-cuko en kvpetvne vtekat, perro-cuko vtehkvlke omvlkvt, perro-cuko vfastvlke, momet uehvtkv 
svkfulle nake esyoposkvlke estomomusat omvlkvt hopvyen svpaklet,

18 Nekrat em ekkucen hecakof, huchkakvnks, Tvlofv estomet heyv tvlofv rakkat omēt ome haks? maket.

19 Momet essu mokkvn e'kv taken oh tusayet, hvkihket, wahoket huehkakvnks, Holwakes, holwakes, mv tvlofv rakke mv ofv perro-cuko uehvtkv vk fulle ocakat omvlkvt em vcaketv eteropotte nake ocvke hakakvtet! hvse-vkerkv hvmkuse ofvn sumkepekv.

20 Hvlwat toyetskat, momet vpastelvlke fvccuke, momet owalvlke toyatskat, oh afvckvkes, Hesaketrmeset cem empvlecekv.

21 Momen encelv yekcet, enfulotkv en crto rakkat omēn cvton ehset, uehvtkvn vk wikvnks, Hiyomēn Papelv, mv tvlofv rakkat, lekvfhohyen, en lvtketv rakke tares, momen svnvcomvn eshechohyekos, maket.

22 Momet, Kervlv hayecvlke, hayecvlke estomis, fehpv hayecrlke, pofketv pofkvlke esyomat en hake taket ec ofvn svnvcomvn pohkekares; momen vtotketv estomis vtotkakat hvmkusis ec ofvn svnvcomvn eshechoyekares; momen enfulotkv en hake ec ofv svnvcomvn pohkekares;

23 Momen kulke en hryayvke ec ofvn svnvcomvn hvyayvkekares; momen hokte epayvranat ehiwv taranat tepakat en hake ec ofvn svnvcomvn pohkekares; cem esnesvlke ekvnv en rvkrakvt omen, cem famvkakv eteropotten tvlwvvlke omvlkvn mvkerhoyvtet omekv.

24 Momen owalvlke, mekusapvlke, momet este ekvnv oh pvsvthoyvte omvlkv e catvn eme ofvn eshechoyvnks.

\section{SETENTVCKE XIX.}

Momen heyv nanvke renyupvn hvlwe tvlofv ofv este sulke mahe em opunvkv en hake yekcet, Aleluyv: 
Vhesaketv, rakketv, vrakketv momet yekcetvt Pucase pun Hesaketvmese em ocekvs ;

2 Em ohfvcceckvt mehenwet, fvecvket omekv; mv hoktarv rakke, en hoktarkv eteropotte ekvnv hopanvten ohfvececet, em vtotkvlke e catvn e'nke pvlatvten em pvlecekv, maken pohvyvnks.

3 Momen hvtvm, Aleluyv, makakvnks. Momen em ekkucet estofis meyuksv-sekon kvwapket omes.

4 Momen vculvke pale hokkole ostohkakat, momet nake wenahoke ostat tvkpvlvtiket, Hesaketvmese mekko em ohliketv oh likaten vrakkuecet, Emén; Aleluyv, maket, vrakkuecakvnks.

5 Momen opunvkv en hake mekko em ohliketvn aosset, Pun Hesaketvmesen, em vtotkvlke omvlkatskat, momet lopockuse momet rakrvke em penkvlakatskat omvlkvt vkvsvmvkes, makvnks.

6 Momen este sulke mahe em opunvkv en hake omēt, momen uewr sulke en hake omēt, momen tenetke yekcakat en hake omēt, Aleluyv; Hesaketvmese Yekcetv Omvlecat mekket omekv.

7 Epu 'fvcket omekvs, momet afvckvkepvkeres, momet vrakketvn e'mvkeres, Yvpefikuce em etepvyetv em oketvt vlahken, e 'hiwvt e em etetakuecet omekv; makaken pohvyvnks.

8 Momen emet hvnvwelv telekme hvsvtke, momen hvtken accrren e'mhoyvnks; mv hvnvwelv telekmat mekusapvlke em fvecetvt omekv.

9 Momen, Hoccicvs, Mv Yvpefikuce em etepryetv en yaf ke-hompetvn enhuehhokvtet en herakes, cv kicvnks. Momet, Heyvt Hesaketvmese em maketv mehenwakat omes, cr kicvnks.

10 Momen vrakkuecvranit e'le ehomvn tvk latkvyvnks. Momen, Momecetskekaren vketecrs; vnet ceme momen 
etecakkaketskat Cesvs em ohkerkueckv ocakat vtotkv cem etohkvlketv toyis; Hesaketrmesen vrakkuecvs; Cesvs em ohkerkueckvt owaletv em puyvfekcvt omekv, cv kicvnks.

11 Momen hvlwat hauken hecvyvnks, momen hecrs, corakko hvtke; momen mv oh likat, Herome momet Fvecen kihocvnks: momen fvecetv ofv ohfvececet, horren hayet omes.

$12 \mathrm{E}$ turwvt totkv en fenke omēt on, kvpotokv vcacvke sulket e'kvn oh ocakvnks; momen hocefkv hoccen ocet, nvpvt kerrekon, eme tvlkuset kerret omes.

13 Momen kapv catv vik 'tehkvten accrnks; momen e hocef kvt Hesaketvmese em Opunvkvn kihocet os.

14 Momen suletawvlke hvlwe ofvn fullat hvnvwelv telekme hvtke momen hvsvtken accet, corakko hvthvken oh vpoket vevkvpeyvnks.

15 Momen eslafkv-crpkot e cukwrn afvnket os, mvn tvlwvvlken es caskvren; momen cvto estepketvn es ohmekkvkvres; momet Hesaketvmese Yekcetv Omvlecat en crpvkketv momet en homeckv em prrko es opecetv nakvtehkan e'len es cetaket omes.

16 Momen en kapvn momen e hvfen oh hocce hocefkvn ocet omes, MEKKVLKE EM MEKKOT, MOMET PUCASVLKE E PUCASE, makat.

17 Momen encelv hvmket hvse ofv hueren hecryvnks; momen opunvkv en hake yekcen es huehket, fuswv hvlwe tvmece fullat omvlkvn em punayet, Ahwet, Hesaketvmese rakkat en yafke-hompetv ocan tohvtelokvkes;

18 Mekkvlke e'navpeswvn, momet kvpetvnvlke e'navpeswrn, momet este yekcakat e'uavpeswrn, momet corakko vpeswrn, momet mv ohvpokat e'navpeswv, momet este omvlkv, pucassvkeko momet pucase ocakat, lopoc- 
kuse momet rvkrakat em enavpeswv taken hompatskvren, makvnks.

19 Momen mv punvttv, ekvnv em mekkvlke en suletawvlke taken esyomet, mv corakko oh likat, momet en suletawvlke vnrvpen horren hayvranet, vteloken hecvyvnks.

20 Momen punvttv momet owalv laksv mv punvttv ehomvn nake eskerkv mv eteropotte este punvttv es en kerkv en heckvkvten, momet vhakat vrakkuecakan mvkerret vtotkvtet vpaken eshoyvnks: heyv hokkolv hesahoken vkhvse totkv tohottoplane 'setenekran vk kahoyvnks.

21 Momen vhoskat corakko oh likat em eslafkverpko e cukwv afvnkan es pvsvthoyvnks; momen fuswv omvlkvt e'navpeswv taken es fvcfakvnks.

\section{SETENTVCKE $X X$.}

Momen sufke-rakko em prtakv sekat es en hvweckvn ocet, momet cvto-tetvkkakv rakken cvwet, encelvt livlwan a hvtapken hecryvnks.

2 Momen tlekonv, cetto vcule, mvt Puyvfekcv holwakat, momet Setvnet omen, mon hvlahtet, ohrolope cukperakko hvmken likvren wvnayvnks,

3 Momet sufke-rakko em pvtakv sekan vk wihket, em vkhotiyet, 'sem oh vlokpicrnks, ohrolope cukpe-rakko hvmkat esfvckeko vtekat tvlwvvlken ohvtvlakat mvkerrvkekaren: momen $\mathrm{mv}$ enyupvn oketv vkocoknusen enrecopkvranat tvlkes.

4 Momen mekko em ohliketvt ocaken hecvyvnks, momen mvn oh vpohokvnks, momen ohfvcceckvn emvkhoyvnks: momen Cesvs em ohkerkueckv vrahkv, momet Hesaketvmese em opunvkv vrahkv ekv take en warhoyvte, momet punvttv monkat vhakan vrakkuecvkekate, momet es en kerkv e'kvhomv take, monkat e'nke take 
ofvn vlicvkekat em puyvfekcvlken hecryvnks; momen Klist vpvkaket ohrolope cukpe-rakko hvmken ohmekkakvnks.

5 Momis pvsatkvte vpvlwvt awenahokeko emunken ohrolope cukpe-rakko hvmkat esfvckvnks. Heyvt elkv aenkvwvpketv enhvteceskv tes.

$6 \mathrm{Mv}$ elkv aenkvwvpketv enhvteceskv vpvke enake ocat hvsvtket momet en heres; heyv ohfvnken elkv svhokkolat yekcetvn ocekon, momis plestvlke Hesaketvmese enake, momet Klist enaket omvkvres, momet vpvkaket ohrolope cukpe-rakko hvmke oren ohmekkvkvres.

7 Mornen, mv ohrolope cukpe-rakko hvmket hoyanof, Setvne enrecopket en cuko-yekcvn aossvres,

8 Momet Kak Mekak tepake tvlwvvlke ekvnv em vkvnowv oste oh vpokat, mv em vhonkvtkv uehvtkv em oktahv omēt on, mvn emvkerret, horren hayvranet tohvteloyvret ayvres.

9 Momen yvmv ekvnvn hvmecicet, mekusapvlke e hvpo, momet tvlofv vnokeckan em vfolotakvnks; momen totkv Hesaketvmese avtet hvlwan ahvtapket lokakvnks.

10 Momen puyvfekcv holwakat mv emvkerrakvten vkhvse totkv tohottoplane setenekran vk wihokvnks; punvttv owalv laksv tepakat kakan, momet netta, neren estemerrvkuehocvres, estofis meyuksv-sekon.

11 Momen mekko em ohliketv rakke, hvtken, momen mv oh liken hecit, ekvnv sutv tepakat estvmv kake tayat eshechoyekon, $m v$ oh likat e turofv enkvpvken sumhoken hecvyonks.

12 Momet pvsatkvte, lopocke momet rvkrakat Hesaketvmese ehomv svpaklen hecryvnks: momen nakcokvn enrvmrihocvnks: momen nakcokv etv, hesaketv nakcokvn enrvmhoyvnks: momen prsatkvtet nanvke mv 
nakcokv ofv hoccakat aossen em vtotketv take vevkvyen ohfvececvkhoyvnks.

13 Momen uehvtkvt prsatkan sossicvnks : momen elkv momet prsatkvte e hutet prsatkvten afulhuecrnks; momen em vtotketv vcvkvyen ohfvccehoce vlket omvnks.

14 Momen elkv momet prsatkvte e hute totkv vkhvsen vk pvlvthoyvnks. Heyvt elkv svhokkolat tes.

15 Momen estimvt $e$ hocefkv hesaketv nakcokv ofv hoccen eshechoyekan totkv vkhvsen vk wihokvnks.

\section{SETENTVCKE XXI.}

Momen ekvnv mucvse momen sutv mucvsen hecryvnks; ekvnv enhvteceskv momet sutv enhvteceskv ocvtet sumhokepekv; momen uehvtkv sekot omvnks.

2 Momen vne, Cane toyvyat Celuslvme mucvse, tvlofv vcakat, este'hiwv tarane e nehice e'he em etetakat omēt, Hesaketvmesen aatet, sutv aosset hvtapken hecryvnks.

3 Momet sutv aosse opunvkv en hake yekcet, Hecvs, Hesaketvmese em escukohakv vcakat este em etenrvwvn liken, momen emet vpaket liken, em estet omakvres; momet Hesaketvmese mahusat vpaket liket, en Hesaketvmese take tares.

4 Momet Hesaketvmeset turopuswv omvlkvn e turwv taken en hvsvthicvres; momen elkvt svnvcomv sekares, momen feknokketv monkat hvkihketvt sekon, nak nokke svnvcomv sekares; nanvke enhvteceskv ocakvtet hoyvnecepekv, maken pohvyvnks.

5 Momen mekko em ohliketv oh likat, Hecvs, nanvke omvlkvn mucvsvken hayis, makvnks. Momet, Hoccicvs ; heyv opunvkv fvccet, mehenwrket omekv, cv kicvnks.

6 Momet, Pokes, Alfv momet Omēkv, enhvteceskv momet yuksv toyis. Mv ewvnhkan hesaketv newv em uekiwv akvpvken enrahkv sekon emares. 
7 Estimvt ohfvnkat nanvke omvlkvn epucasvres; momet vnet en Hesaketvmese toyin, emet cv'ppucet omvres.

8 Momis penkvlakat, vkvsvmvkekat, este enhomechoye tetayat, estelecvlke, hoktarvlke, famvkayvlke, nakvhake mekusapvlke, momet laksvlke omvlkvt vkhvse totkv tohottoplane setenekrat ofvn enaken ocvkvres: mvt elkv svhokkolat omen, maket ev kievnks.

9 Momen encelv kolvpake, mv sesketv este sestemerrickv espoke kolvpake es fvcfvke ocakat hvmket vm vlaket, $c v$ kicet, Yvmvn vtes, ehiwv taranat, Yvpefikuce e hiwvn ce hecicvranis, maket vm punayvnks.

10 Momet Puyvfekcv ofvn ekvnhvlwe rakke hvlwen es vc ohcemket, Celuslvme vcake, mv tvlofv rakke Hesaketvmesen avtet, sutv aosse ahvtapket,

11 Hesaketvmese en hvyayvken ocet hvyvyaken cv hecicvnks: momen en hryayvke cvto vcake mahat omēt, cvto caspv kihoce, klestelv hvyvyakat omēt hvyvyaket omvnks.

$12 M v$ tvlofv vtohopke rakke, hvlwen ocet, tohopkvhauke palen hokkolohkaken ocen, encelv palen hokkolohkaket v svpaklvnks, momen hocefhokv Eslevl en hopuetake em vliketv pale hokkolohkakat e hocefhokv vhoccvken ocvnks.

13 Hvsossv fvecvn tohopkvhauke tutcenen; honerv fvecrn tohopkvhauke tutcenen; wahvlv fvecvn tohopkvhauke tutcenen; hvsvklatkv fvecvn tohopkvhauke tutcenet omvnks.

14 Momen mv tvlofv em vtohopke en likv palen hokkolohkaken ocen, Yvpefikuce em vpastelvlke palen hokkolohkakat $\Theta$ hocef hokvt vlumhen ocvnks.

15 Momen $\mathrm{mv} \mathrm{cv}$ tempunayat, mv tvlofv, momen en tohopkvhauke em vtohopke esyoman es vhopayetvn kohvn ocvnks. 
16 Momen mv tvlofv vkvnowv oste temmomvlken ocat likes, momen en crpke momet en tvphet temmomvlket omes; momen tvlofv vfolotken kohvn es vhopayen, vkerkv cukpe-rakko hvmken cukpe cahkepet omvnks; momen en crpke, en tvphe en hvlwe esyomat temmomvlket omvnks.

17 Momen mv em vtohopken vhopayen, kupetv cukpe hvmken pale osten ostohkaket omvnks, este hunvnwv em vhopakv vcvkayat, mvt encelv em vhopakvt omen.

18 Momen em vtohopke es hahoyat caspvt omen; tvlofv cvtokunaplane mahhet, estvkheckv hvyvyakat omēt omvnks.

19. Momen tvlofv em vtohopke en likv crto vcacvke omvlkv es nehihocet omvnks. En likv enhvteceskv caspvt omen; svhokkolat sefilv; svtutcenat kalsetonv; esostat smvlvktenv;

20 Svcahkepat satvnekse; sepakat satev; eskolvpakat klesolitv; escenvpakat pelelv; esostvpakat topasv; espalat klesoplesv; espalen hvmkontvlakat cesenrv; espalen hokkolohkakat amerestvt omvnks.

21 Momen tohopkvhauke palen hokkolohkakat makelitv palen hokkolohkaket omakvnks: tohopkvhauke hvmkat makelitv hvmkusen es hake vlket omvnks; momen tvlofv etehoyvnkv cvtokunaplane mahhet omen, estvkheckv hvyvyakat omēt omvnks.

22 Momen ofv cuko-vcakat hvmkusis hecvkvnks; Pucase Hesaketvmese Yekcetv Omvlecat momet Yvpefikuce en cuko.vcakat omakekv.

23 Momen tvlofvt hvse, monkat hvresse ofv hvyayvhokvre eyacvkekos; Hesaketvmese en hvyayvket hryayicen, Yvpefikucet $\mathrm{mv}$ en hvyayvket omekv.

24 Momen hesahokvte em etvlwvvlket en hryayvke ofvn yvkvpvkvres; momen yvmv ekvnv em mekkvlket

Muskokee. 
en hvyayvke momet em vrakketv taken mvn esceyaket omes.

25 Momen mv em vtohopkvhauke nettvn vkhotkvkekares; neret mvn sekaret ok.

26 Momen tvlwvvlke en hvyayvke momet em vrakketv taken mvn esceyvkvres.

27 Momen nake hvsvtkeko, monkat nake enhomechoye tayan, momet lvksetvn hayat vtekat mvn eceyekon; momis mv hesaketv nakcokv Yvpefikuce enake ofvn cohoyat tvlkuset esceyvkvres.

\section{SETENTVCKE XXII.}

Momen emet hesaketv uewv en hvcce hvyryakat, klestelv omēt hvyryaket, Hesaketvmese Yvpefikuce tepakat em ohliketv a fihnen cv hecicvnks.

2 Em etehoyvnkv en nvrkvpvn, momet hvcce afopke tempvlusen hesaketv em etot, em ette mvrahrvkv vpalen hokkolohkaken hayet, hvse omvlkvn ettet svpaklvnks; momen $\mathrm{mv}$ eto em esset tvlwvvlke es em wiceckvt omakvnks.

3 Momen mvn ohtvhiketvt ohvtvlakat sekares; momis Hesaketvmese Yvpefikuce tepakat em ohliketv vcakat mv ofvt omvres; momen em vtotkvlket em vtotkvkvres.

4 Momen e turofvn hecaken, e hocef kvt e'kvhomv taken vhoccvket omvres.

5 Momen mvn neret sekares; momen kulke, monkat hvse en hvyayvken eyacvkekos; Pucase Hesaketvmeset hvyayvken e'makekv: momen estofis meyuksv-sekon ohmekkvkvres.

6 Momen, Heyv opunvkv fvccet, mehenwvket omes; momen Pucaset owalvlke vcacakat en Hesaketvmese taket; nanvke lvpken hoyvnvranat em vtotkvlke hecvkuecvren ern encelvn vtotvtet omes. 
7 Hecvs, lvpken vlakis; mv heyv nakcokv em owaletv em opunvkvn veryecat en heres.

8 Momen vne, Cane toyvyat, heyv nanvken hecet, pohvyvnks. Momet pohit momet hehcryof, mv encelv heyv nanvken cr hecicat vrakkuecvranit e'le ehomvn tvk latkvyvnks.

9 Momof, Vketecet momehcetskvs; vtotketv cem etohkvlketv toyit, owalvlke etecakkaketskat, momet heyv nakcokv em opunvkvn vfvstakat homkat toyikv: Hesaketvmesen vrakkuecrs, cv kicvnks.

10 Momet, Heyv nakcokv em owaletv em opunvkvn semvlokpihcetskvs; oketvt vwolusekv.

11 Estimvt fvccekat fvcceko emunket omekvs; momen vholwakat vholwvke emunket omekvs; momen mv fvccat fvcce emunket omekvs; momen hvsvtkat hvsvtke emunkvt omekvs.

12 Momen hecvs, lvpken vlakis; momet feketvn ehopakis, omvlkv em vtotketv vcrkvyen e'maren.

13 Alfv momet Omēkv, vliceckv momet espoke, enhvteceskv momet meyuksv toyis.

$14 \mathrm{Mv}$ em vhakv vfvstakat en herakes, hesaketv em eto oh vwetv em etektvniken, tohopkvhauken esceyet, tvlofvn esceyvkvren.

15 Momen efvt, famvkayvlke, hoktarvlke, estelecvlke, momet nakvhake mekusapvlke, momet estimvt lvksetvn vnokecet momet hayat $m v$ tvlofv en lvpvtken fulles.

16 Vne, Cesvs toyvyat, mekusvpkv-cuko ocakat ofv heyv nanvken cen kerkuecvren vm encelvn vtotvyvtes. Tewe en yvlonkv, momet en honvpse toyit, hvyvtecv kococumpv hvyvyakat toyis.

17 Momen ehiwv taranat momet Puyvfekcvt, Vtes, makaket os. Momen pohat, Vtes, makekvs. Momen 
estit ewvnhkat vtekvs. Momen estit komat hesaketv uewvn enrahkv sekon eenakuecekvs.

18 Heyv nakcokv em owaletv em opunvkvn pohat vtekat heyvn en kerkuecikv; Estit heyv nanvken ohvpayen omat, Hesaketvmeset nake este sestemerrickv heyv nakcokv ofv cohoyan mv esten 'sem ohvpryvres:

19 Momen heyv nakcokv em owaletv em opunvkv vkueye sasen omat, Hesaketvmeset, hesaketv nakcokv akvpvken, momet tvlofv vcakat ossen, momet nanvke heyv nakcokvn cohoyat akvpvken enaken em vkueyvres; maket cv kicvnks.

20 Heyv nanvke kerkiecat, Mehenwvn lvpken vlakis, maket os. Emén. Momes, Cesvs Pucasē, vtes.

$21 \mathrm{Pu}$ Pucase Cesvs Klist en heromkvt omvlkatskat ece 'pakvkekvs. Emén. 


\section{C or 1 ig e n d a.}

\section{V H E R I C K V.}

MARO $2: 11$. Momen cuko seceyof, hopuewuce e'cke Mele vpaken hecakvtes, momet tvkpvlahtet vrakkuecakvtes; momet nakvtehkv en hauhicof, nake emakvtes, cvtokunaplane, lipenv, smvnv esyomen.

CANE 1:3. Nanvke omvlkvn mvt hayvtes, momen mvt sekate nake hakvte hvmkusis hakekates.

CANE 1: 7. Matrwvt ohkerkuecvt vlaket omvtes, Hvyayvke ohkerknecvranatet, $m v$ eteropotten omvlkvt, vkvsvmvkvren.

CANE 1: 34. Momen hecryvnks, momet, Heyvt Hesaketvmese E'ppuce tes, makit ohkerkuecryvnks.

CANE 2: 15. Momet afushunvke vnvcomen estepketvn es hahyof, omvlkvn cuko-vcakan sossicvtes, moinen yvpefikv wakv svpvken, momet esyoposkvlke en cvtokunawv a em pvlatet, em ohhompetv cektulcecvtes.

CANE 3:4. Este vculehpof, estomet hecke taye haks? svhokkolv ecke e uvrken ecehyet, hecke taye haks? Nekotemvset kicvtee.

CANE 5: 43. Cv'rke hocefkv ofvn vlakis, momen cv evpayatskekos ; etvt eme e hocefkv ofvn vlaken omat, mvn evpayatskvres.

CANE 6:7. Felvpe em vyoposket, Tvklike tenelyv cukpe hokkole enrahkv tis em etetahyvkekos, cotkuse vlken ehset o estomis, kicvtes.

CANE 6: 9. Cepanet likes, ketinv tvklike-vnore cahkepen, momet rvro lopuckuse hokkolen ocet; momis heyv naket o haks, este sulke momat em etenrvwv?

CANE 6: 62. Monkv enhomv likvtet este Echuswv $\mathrm{v}$ kvwapken hecatsken omat, estomvre te?

CANE 7:4. Este hecke kerhoyvren eyacet, ehke 
naken celayate sabsekot omekv. Heyv nanvke vfastetsken omat, ekvnvn em e heckuecrs, kicakvtes.

CANE 7: 9. Heyv opunvkv kicakof, Kalvle ofvn fekhonnvtes.

CANE 7:31. Momen este sulket ohvkvsvmakat, Klist vlakofvn nake eskerkv heyv este momecat senrakrvken momecvr haks? makakvtes.

CANE 11: 12. Momof en kerrepvlke em punayat, Pucasē, nocet on omat, en hervres, kicakvtes.

CANE 14: 2. Cvrke hute ofv liketv sulket os; momekaten omat, cem onvyvkvyvnt os. Liketvn ce metetakuecvranvkit ayis.

CANE 14: 26. Momis Emvhlvpatv, Puyvfekcv Vcakat, Erket cv hocefkv ofv vtotvranat, mvt nanvke omvlkvn ce mvhayet, nanvke omvlkv ce kicvkvyvten ece 'kerricepuecvlkvres.

CANE 20:11. Momis Melet hvkihket, cvto-kofken fettvn vhuervtes, momet hvkihkof, ohcunehket, crtokofke ofvn vfvnnakvtes.

CANE 20: 23. Momekv heyv opunvkv, Mv enkerrepv elekares, makat etecakkvte em etenrvwvn pohkvtes. Momis Cesvs, Elekares, makekot, momis, Fekhonne munken vlvkaret komin omat, mvt cem'estoma? makvtes.

CANE I: 4. 10. Momen vnokeckv heyv ofvts, pumet Hesaketvmese vnokeceyvtet tokon, momis emet pumen epu'nokecet ot, Eppucen vtotvte tat pun naorketv esvrahkv nakwikv taren.

CANE I: 5. 6. Uewv momet catvu etehoyvne vlakvtet heyv tes, Cesvs Klist mvt ot os, momen uewv etehoyvne tvlkekon, momis uewv catv tepakv etehoyvnen. Momen Puyvfekcvt akerrv tes, Puyvfekcvt mehenwvt omekv.

EFESVLKE I: 3. Cesvs Klist pu Pucase Erke, momet en Hesaketvmese vrakkuehocekvs, mvt hvlhawat ofv Klist ofvn puyvfekev merretv omvlkvn es ep ohmerrvtes. 




SMITHSONIAN INSTITUTION LIBRARIES

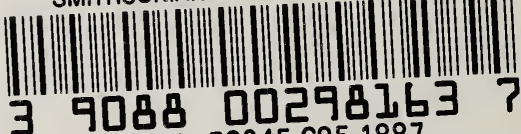

3 9088 Q0298146

nhanth BS345.
Pu pucase momet pu hesayecv Cesvs Klist 